\title{
A Replanning Algorithm for Decision Theoretic Hierarchical Planning: Principles and Empirical Evaluation
}

\author{
Guido Boella guido@di.unito.it \\ Rossana Damiano rossana@di.unito.it \\ Dipartimento di Informatica, Universita' di Torino \\ C.so Svizzera 185, 10149 Torino Italy \\ August 4, 2005
}

\begin{abstract}
In this paper, we present a replanning algorithm for a decision-theoretic hierarchical planner, and illustrate the experimental methodology we designed to investigate its performance. The methodology relies on an agentbased framework, in which plan failures emerge from the interplay of the agent and the environment. Given this framework, the replanning algorithm is compared with planning from scratch by executing experiments in different domains. The empirical evaluation shows the superiority of replanning with respect from planning from scratch. However, the observation of significant differences in the data collected across planning domains confirm the importance of empirical evaluation in practical systems.
\end{abstract}




\section{Introduction}

Replanning is a central research issue for a variety of AI applications involving the execution of plans in realistic contexts, ranging from real-time architectures for automation to agent-based and multi-agent systems. Although Nebel and Koehler [Nebel and Koehler, 1993] have shown that some forms of replanning are harder than planning itself, the interest for replanning is motivated by both theoretical and practical considerations. In real time systems, the interleaving of deliberation and execution is an effective strategy to tackle non-determinism and incomplete knowledge, but does not eliminate completely the need to replan from time to time.[Myers, 1999, Lemai and Ingrand, 2004]. More importantly, the stability of commitment remains a key property of agents in social contexts. For example, in multi-agent systems, a certain amount of a priori coordination on individual plans cannot be set aside, together with the necessity for the agents to keep to their plans as much as possible during the joint activity. So, efficient and effective replanning techniques are required to guarantee the stability and predictability of the behaviour of agents.

In this paper, we present a replanning algorithm and propose an agent-based methodology and framework for its empirical evaluation. The replanning algorithm extends the planning algorithm of the DRIPS system [Haddawy and Hanks, 1998], that conjugates hierarchical planning with Decision Theory [Luce and Raiffa, 1957]. The evaluation methodology rests on an experimental framework that simulates the occurrence of plan failures as a result of the interaction between an autonomous agent and a non-deterministic environment. The replanning algorithm is compared with the alternative of planning from scratch based on time performance and quality of output plans.

The paper is structured as follows: Section 2 describes the replanning algorithm; the evaluation framework and methodology are described in Section 
3. Section 4 reports the experiments performed according to the methodology, discussed in Section 5. Conclusions end the paper.

\section{Extending Decision-Theoretic Hierarchical Plan- ning to Replanning}

Hierarchical planning [Sacerdoti, 1977] lends itself to the design of resourcebounded agents as the levels of abstraction encoded in hierarchical plans can be directly mapped to the elaboration of partial plans. Partial plans are a key notion in the theory of practical reasoning [Bratman, 1990], since they allow avoiding premature commitment to overdetailed plans. In the context of replanning, they provide a useful instrument to manipulate commitment at the lower levels of detail, leaving it unmodified at the higher levels.

Decision-theoretic planning allows an agent to make a distinction between the achievement of a goal and the utility of the states in which the goal is achieved. States are represented as sets of attribute-value pairs and the individual preferences of an agent are expressed as functions which map the value of single attributes to real numbers, representing the utility values. By releasing the "all or nothing" goal satisfaction paradigm of classical planning [Blythe, 1999], in decision-theoretic planning the achievement of a goal can be weighted against the costs of achieving it, and goals can be partially satisfied.

\subsection{The Planning Algorithm}

The representation of the world in DRIPS consists of attribute-value pairs. Attributes can have boolean values as well as continuous or discrete ones (e.g., the consumption of fuel is represented by a continuous quantity). In order to account for the role of uncertain knowledge about the world in planning, each 
attribute is associated with a probability distribution on its values. Since the attributes are assumed to be independent, this representation is equivalent to a set of alternative worlds in which each attribute has a certain value with a certain probability. Formally, the representation of the current state of the world $W_{s}$ is a set of pairs $\left\{A_{i}, P D_{i}\right\}$, where $A_{i}$ is an attribute and $P_{i}$ is a probability distribution on its values:

$$
W_{s}=\left\{\left\{A_{1}, P D_{1}\right\}, \ldots,\left\{A_{n}, P D_{n}\right\}\right\}
$$

For each attribute $A_{i}$, the probability distribution on its values is a set of pairs $\left\{V_{i}^{j}, P_{i}^{j}\right\}$, where $V_{i}^{j}$ is one of the possible values, and $P_{i}^{j}$ is the probability of that value:

$$
P D_{i}=\left\{\left\{V_{i}^{1}, P_{i}^{1}\right\}, . .,\left\{V_{i}^{n}, P_{i}^{n}\right\}\right\}
$$

where $\sum\left(P_{i}^{1}, \ldots, P_{i}^{n}\right)=1$.

The effects of an action are non-deterministic and depend on a set mutually exclusive and exhaustive conditions. For example, the definition of the action go-X-room-4-to-2 in Figure 1 states that, when the action is executed while the agent is in room 4 and the door is open, there is one chance out of ten that the agent ends up being in the same room. ${ }^{1}$ Notice that there is no proper goal in the action representation: the DRIPS system provides an inter-subjective representation of actions, and relies on the agent's utility function to represent individual goals. In order to account for the notion of action failure, we augmented the original representation with a field that specifies the action goal (see the :goal field).

\footnotetext{
${ }^{1}$ The representation of effects in DRIPS allows representing the uncertainty associated with both complex effects, defined on multiple attributes (see the :effs field in Figure 1), and elementary effects (defined on individual attributes, see the :eff field), by posing separate probability distributions on the two levels.
} 


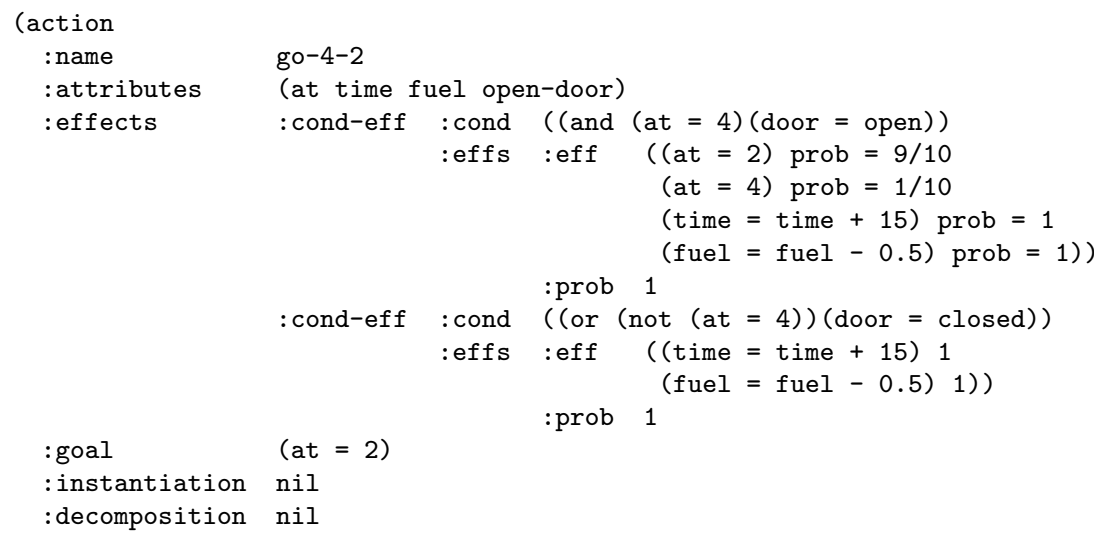

Figure 1: The definition of a primitive action, go-4-2, going from room 4 to room 2 , in the office domain (see Section 4.3 for description of the domains).

A plan library is an action hierarchy that includes two kinds of abstraction relations, as described by [Haddawy and Suwandi, 1994, Haddawy and Hanks, 1998]. The sequential abstraction relation is a task decomposition relation: an action type of this kind (complex action type) is a macro-operator that the planner can replace by a sequence of action types. The specification relation describes how an abstract action type specializes into more specific action types. A primitive action is a directly executable action.

The input to the planner is a plan that contains only the root of the action hierarchy; this plan is recursively refined by substituting complex actions with their decomposition and abstract actions with the more specific actions they subsume, until a set of primitive plans is obtained, i.e., a set of plans composed of primitive actions. At each cycle, the input to planning algorithm is the result of the refinement performed in the previous cycle, i.e. a set of more detailed plans, which subsume a smaller set of alternatives. Before execution, the utility of a plan is not known, but its expected utility can be computed on the set of states obtained by projecting the plan onto the current state of the world. 
The expected utility of a plan that has not been fully refined (a partial plan) is an interval that encompasses the expected utilities of all the alternatives plans it subsumes. Suboptimal plans are plans whose expected utility upper bound is lower than the lower bound of some other plan. In order to restrict the search space and to identify optimal plans, suboptimal plans are pruned at each refinement step.

\subsection{The Replanning Algorithm}

In classical planning, the validity of a plan is evaluated in terms of success or failure, intended as the truth or falsity of a logical expression. In decisiontheoretic planning, the outcome (or expected outcome) of a course of action is mapped to an utility value, whose acceptability cannot be established a priori. The replanning algorithm presented here delegates to the agent model the task of defining the acceptable utility values, and assumes that a threshold is sufficient to identify the acceptable values. In this work, we consider as failure the situation in which the expected utility of plan drops below the acceptability threshold of the agent while the plan is being executed.

When a plan fails, it is possible that the current plan is 'close' to a similar feasible solution, where "closeness" is represented by the fact that the current plan and a new valid one are subsumed by the same partial plan at some level of abstraction in the plan space. The key idea of the replanning algorithm is to retract a refinement choice operated during planning (partialization), and then to restart the refinement process on the partial plan thus obtained, in the attempt to verify if an alternative refinement is acceptable in the current context ([Boella and Damiano, 2002a, Boella and Damiano, 2002b, Boella and Damiano, 2003]). After a plan has been partialized, the expected utility of the resulting plan is computed. Since this value encompasses the util- 


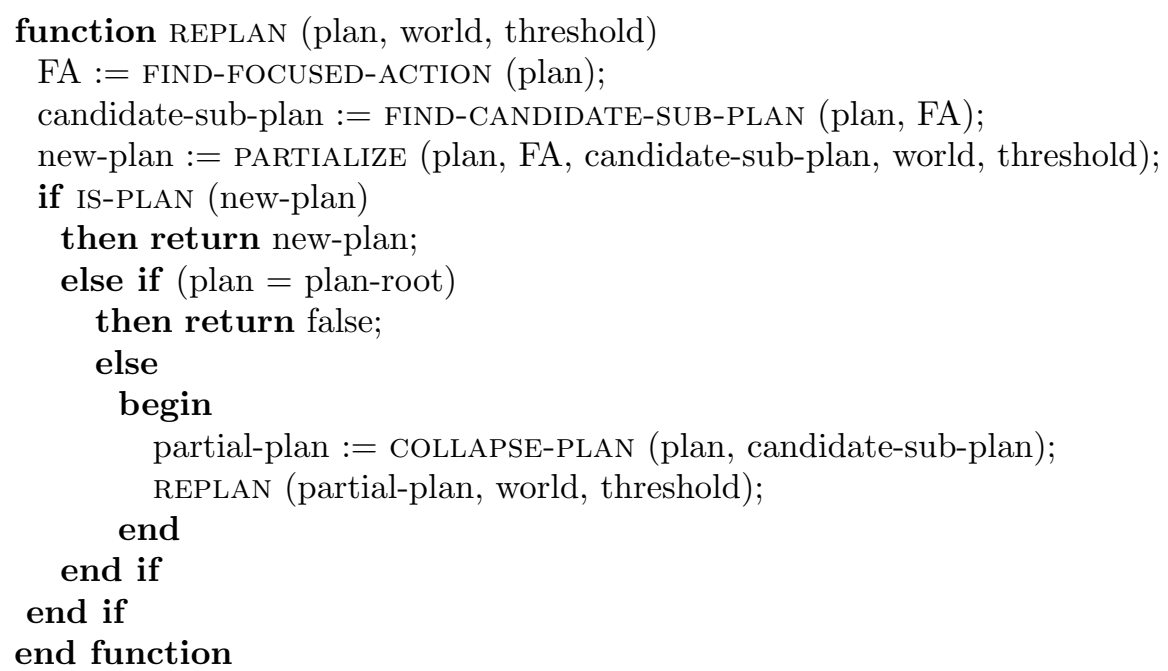

Figure 2: The main function of the replanning algorithm, REPLAN

ity of all its refinements, it is possible to verify if it is a promising candidate, i.e., if it encompasses a refinement whose utility is above the acceptability threshold.

The abstraction and the decomposition hierarchies play complementary roles in the replanning algorithm: the abstraction hierarchy allows identifying alternatives to the steps of the failed plan, while the decomposition hierarchy focusses the partialization process on a sub-plan of the current plan, as described below. The partialization process is incremental: if a new valid refinement of the failed plan is not found after the first partialization step, the process is iterated, until either a new valid plan is found or the root of the action hierarchy is reached. The functioning of the algorithm is the following:

1. The starting point of the replanning process (see the replan procedure in Figure 2) is the first non-executed step whose preconditions do not hold. This step becomes the initially focused action (FA). ${ }^{2}$

2. FIND-CANDIDATE-SUB-PLAN identifies the sub-plan of the input plan on

\footnotetext{
${ }^{2}$ Although the representation of actions does not explicitly report the preconditions of an action, they can be univocally identified based on the action goal.
} 


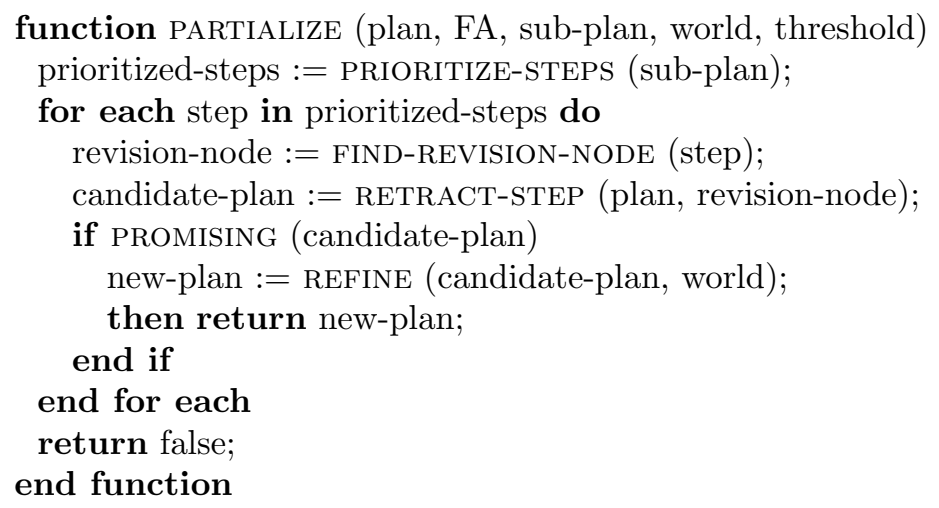

Figure 3: The PARTialize function

which it will focus the search for alternatives. In order to do so, it consults the derivation tree of the plan to identify the lowest-level decomposition the FA appears into; then, it marks the steps which appear this decomposition as candidate sub-plan. The rationale is to ensure that only the steps of the plan which are most directly related to the focused step (i.e. that are part of the same, lowest-level decomposition) are potentially affected by the revision process.

The subtree of the action hierarchy dominated by the root of the candidate sub-plan constitutes the local context of the FA; the local context is the portion of the action hierarchy that the algorithm will search for an alternative refinement of the input plan (see next step).

3. The partialization process (see the PARTIALIZE function in Figure 3) tries to replace the steps of the candidate sub-plan with alternatives, in an incremental way, until no candidates are left or it succeeds. Each candidate step is replace with a more abstract action type, so as to retract a refinement choice operated by the planning algorithm when the plan was created; then, after each retraction, the resulting plan is refined to verify if the retraction has made it possible to bring back the failed plan to a 
new valid plan. In detail:

(a) The PRIORITIZE-STEPS function establishes the order in which the steps of the candidate sub-plan will be examined for partialization: the FA is examined first; then, the steps on its right (the ones that temporally follow it) are examined; finally, the steps on its left (the ones that precede it) are examined. This order reflects the assumption that it is preferable to modify actions that have not been executed yet.

(b) The revision cycle is the core of the algorithm: for each step in the candidate sub-plan, the algorithm searches the current local context of the action hierarchy for an abstract action that subsumes that step (FIND-REVISION-NODE), and marks it as the revision node; if more than one abstract actions are found, the lowest level one is selected, in the attempt to limit the extent of the modification performed on the input plan. The step to be revised is then replaced by the revision node in the input plan (RETRACT-STEP), obtaining the candidate plan. If the plan candidate plan is promising, i.e., it subsumes a valid refinement, it is refined. The PROMISING predicate is evaluated by removing from the candidate plan the steps that correspond to executed actions: in this way, the utility evaluation becomes sensitive to the execution context.

The revision process is incremental: the input to the subsequent revision cycle is the result of the previous revision.

4. If the partialization process has failed to generate a new valid plan, all the actions in the candidate sub-plan are removed from the plan and replaced by the complex action which subsumes them in the action hierarchy (COLLAPSE-PLAN). The plan thus obtained becomes the new input to the 
replanning algorithm, and the former root of the candidate sub-plan becomes the new FA (see Figure 2). In this way, as the input plan becomes more partial, the context within which alternatives are sought for becomes wider, until the plan eventually collapses onto the root of the action hierarchy and the refinement process is restarted from scratch.

In order to illustrate how the replanning algorithm works, we will resort to a portion of the office domain (see Section 4.3). This domain consists of four interconnected rooms, where a robot accomplishes tasks like moving objects and delivering mail. Consider the situation in which a robot is in room 4 has the goal of getting the mail from room 2 to room 1, but wrongly believes that the door between 4 and 2 is open. In order to satisfy the goal to get the mail from room 2 to room 1, the robot has devised a plan composed of the steps: GO-4-2-door TAKE-MAIL GO-2-1 PUT-MAIL (see Figure 4). After executing the step GO-4-2-door, the robot realizes that it is in room 4 and its plan is not valid anymore: executing the remaining plan steps from there will not bring about the desired state of affairs, i.e. that the mail is in room 1 . So, the robot starts replanning:

- The replanning algorithm identifies the focused action (FA), the first nonexecuted action whose preconditions are not satisfied. In this example, TAKE-MAIL is marked as the FA, as it requires the agent to be in the same room as the mail (room 2).

- The replanning algorithm identifies the complex action which directly subsumes FA in the action hierarchy, GET-MAIL, and identifies the candidate sub-plan, composed of the steps GO-4-2-door, GO-2-1 and PUT-MAIL. The local context of the revision is the portion of the action hierarchy subsumed by GET-MAIL. 
- The partialization function examines the right siblings of GO-4-2-door, GO-2-1 and PUT-MAIL, in search of an abstract ancestor. None of the two is subsumed by any abstract actions in the local context. So, the only left-side sibling of the $F A$ in the derivation tree hierarchy, GO-4-2-door, is examined: this action is subsumed in the action hierarchy by the abstract action GO-4-2, that is marked as the revision node.

- The step GO-4-2-door is replaced by the revision node (GO-4-2) in the plan, obtaining the candidate partial plan GO-4-2 TAKE-MAIL GO-2-1 PUT-MAIL. This plan is promising, as it subsumes an alternative refinement of the initial plan which is more appropriate than the failed plan (GO-4-2-long does not require the door to be open, as it consists of a longer path through rooms 3 and 1).

- When the planner refines the new partial plan, it generates the following refinement: (see the second box of Figure 4): GO-4-3 GO-3-1 GO-1-2 TAKE-MAIL GO-2-1 PUT-MAIL.

\subsubsection{Considerations}

The replanning algorithm rests on the assumption that a plan failure can normally be ascribed to a disrupted dependence relation between actions situated in the same, lowest-level decomposition. If this assumption holds in empirical contexts, we expect that, in most cases, the revision of the failed plan will affect only a sub-part of it; more precisely, it will affect only the steps which are closer to the initially focused step, i.e. the first non executed step whose preconditions do not hold. Confirming this hypothesis is one of the goals of the empirical evaluation illustrated in this work (see Section 3.2).

As it has been remarked by [Nebel and Koehler, 1993], reusing existing plans raises complexity issues. They show that modifying existing plans is advanta- 

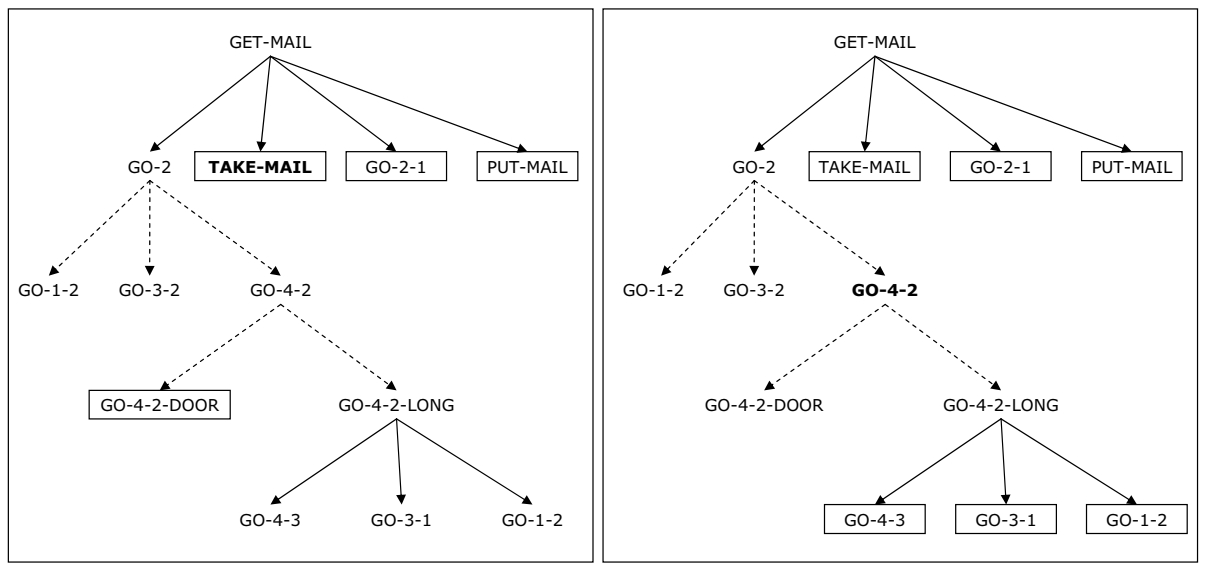

Figure 4: A representation of the steps performed by the replanning algorithm on the action hierarchy given the robot's plan. The original plan (1); the new plan (2).

geous only under some conditions, like replanning, in which it is crucial to retain as many steps as possible of the plan the agent is committed to. According to [Liberatore, 1998], conservative plan modification leads replanning to be harder than planning itself, while non conservative plan modification - under some assumptions - is always less expensive than planning in computational terms. The replanning algorithm we propose is not conservative: it tends to limit the extent of the modifications to the failed plan, thus limiting the amount of computation required to repair the failed plan and promoting the reuse of plan steps, but it does not guarantee that the modifications to the failed plan are minimal, and does not generate optimal plans, in line with the notion of bounded optimality expressed by [Russell and Subramanian, 1995].

The replanning algorithm addresses complexity issues in two main ways: the refinement process is restarted on a candidate partial plan only if the latter is promising, and the refinement process benefits from the same pruning heuristic applied by DRIPS during planning. In the worst case, the complexity of the 
replanning algorithm is the same as the planning algorithm, as the complexity of the latter will simply be increased by a factor of $n$, where $n$ is the number of new refinements attempted. Most importantly, the replanning algorithm is complete: in the worst case, it reaches the root of the action hierarchy (and so will have explored all the plan space) before finding a solution.

Several approaches have been proposed to replanning and to the partially similar task of plan adaptation. Although all approaches can be ultimately reduced to a form of back-tracking of the search operated by planning algorithm in the plan space, the comparison is not always straightforward, due to the differences among the planning frameworks on which individual proposals are based. In particular, the bulk of work in replanning concerns partial order planning: to cite some, see [Wilkins et al., 1994], [Haigh and Veloso, 1996], [van der Krogt et al., 2000], [Lemai and Ingrand, 2003]). An exception is represented by [Gerevini and Serina, 2000] and [Koenig et al., 2002], who extend heuristic search planning to heuristic search replanning. Our proposal is complementary to these approaches, as it relies on the paradigm of decision-theoretic hierarchical planning, in which dependence relations between actions are compiled out in abstract operators trough the use of sequential abstraction.

The research on replanning is not necessarily concerned with plan failures: for example, in the seminal work by [Kambhampati and Hendler, 1992, Hanks and Weld, 1995], plan modification is exploited as a planning strategy to adapt existing plans to new problems; in [van der Krogt et al., 2000], replanning is carried out to make the plan of different agents compatible in a multi-agent, distributed planning environment; in the work by [Miksch and Seyfang, 2000], or [Haigh and Veloso, 1996] replanning is an instrument to account for new user goals, by incorporating them into the system's plans. In these situations, the tradeoff between costs and benefits of replanning is different from the standard 
plan failure situation, making a proper comparison impossible.

\section{An Agent-Based Evaluation Framework for Re- planning}

The framework for the empirical evaluation of the performance of the replanning algorithm is based on a utility-based agent architecture, presented in [Boella and Damiano, 2002a, Boella and Damiano, 2002b]. The framework nondeterministically generates a planning problem, then simulates the execution of the plan devised by the agent. When a plan failure occurs, the alternatives of replanning and planning from scratch are attempted and evaluated based on their time performance, and the quality of output plans. Since no other replanning algorithms are available for decision-theoretic, hierarchical planning, we resorted to planning from scratch as a benchmark to evaluate the performance of the replanning algorithm.

The agent is situated in a dynamic environment, i.e. the world can change independently of the agent's actions, and actions can have non-deterministic effects. The framework does not assume full observability of the world, thus a perfect correspondence between the actual state of the environment and the agent's representation of it is not guaranteed. Plan failures arise either from the agent's incorrect knowledge of the world, or from execution failures; the nondeterminism of the framework is also an independent source of failures. Preliminary work is reported in [Boella and Damiano, 2003, Boella and Damiano, 2004].

\subsection{The Agent Architecture}

The agent is based on a BDI model [Rao and Georgeff, 1991, Georgeff et al., 2000]; its internal state is defined by its beliefs about the current world, its desires, 
and the intentions (or plans) it has formed in order to achieve a subset of its desires. Since the deliberation component of the agent is provided by the DRIPS planning system, it inherits the ontological commitments made by DRIPS in the representation of the world and the actions (the agent's beliefs), of the plans (the agent's intentions), and the utility function (the agent's desires, concretized into goals and preferences). We refer to [Boella and Damiano, 2002a, Boella and Damiano, 2002b] for a detailed description of the architecture.

The intentions of the agent are dynamic, and can be modified as a result of redeliberation. Following [Rao and Georgeff, 1995, Wooldridge and Parsons, 1999], the architecture is structured in two levels: the object level has the role of devising plans and executing them, performing sensing actions after each step to update the agent's representation of the world; the meta-deliberation level controls the object level by selecting input goals to the deliberation process and monitoring the success of the plan being executed. Since the agent's representation of the world is uncertain, and actions are non-deterministic, the expected utility of the initial plan may significantly vary as the execution of the plan proceeds. The meta-deliberation module relies on the agent's initial expectations about the utility of the plan to detect plan failures: after the execution of each step, the agent computes the expected utility of the remaining steps; if the difference between the initial expected utility and the new one is above a certain threshold (acceptability threshold), this counts as a failure of the current plan, and replanning is performed. The acceptability threshold is obtained by applying a ratio to the higher bound of the initially expected utility interval. This ratio (acceptability ratio), together with the agent's utility function, forms the preference structure of the agent. 


\subsection{Empirical Evaluation: Methodology}

In order to generate replanning problems in an automated way, we arranged two different scenarios in which a plan may fail. Both scenarios simulate, though in a different way, the occurrence of a misalignment between the agent's representation of the world and the representation maintained in the simulator. In particular, we distinguish misalignments that occur when the execution of one or more plan actions fail (failure-determined misalignment) from misalignments that occur in the absence of an execution failure (execution-determined misalignment). In the first case, the mismatch between the agent's expectations about the plan effects and the actual effects is caused by an execution failure, while, in the second case, the mismatch is determined by the fact that the world changes unexpectedly before or during the execution of a plan (similarly to the distinction between precondition failures and action failures in [Myers, 1999]). ${ }^{3}$

The relation between plan failure, replanning and planning from scratch is independent of the type of failure. Every time a plan fails, the replanning algorithm is invoked on the failed plan. If the replanning algorithm outputs a new plan, the planner is launched on the failed plan by simulating the world state holding at the time of failure. In this way, the performance of the two strategies - replanning and planning from scratch - are compared as if they were carried on in parallel by the same agent in the same situation. Since the replanning algorithm is complete, there is no point in attempting to plan from scratch if no plan has been found by replanning, so planning from scratch is attempted only if the replanning algorithm has succeeded. On the contrary, planning from scratch when replanning has succeeded is not pointless: since the replanning algorithm does not generate optimal plans, planning from scratch

\footnotetext{
${ }^{3}$ From the agent's point of view, these two situations are indistinguishable: the agent simply realizes, at a certain point of the plan execution, that executing the remaining plan steps will yield a significantly lower utility than initially expected.
} 
may generate a better plan, or employ less time to generate a plan of the same quality.

\subsubsection{Experiment Setting Definition}

The execution of a set of replanning experiments according to the methodology described above is performed by the Experiment Manager. Given the specification of an experiment setting, the Experiment Manager automatically generates a planning problem, then invokes the Scenario Manager to generate plan failures. When the agent's meta-deliberation component detects a failure, the Experiment Manager invokes the replanning algorithm to repair the plan. If the replanning algorithm succeeds, the planner is assigned the task to find a new plan given the execution context at the failure time. During the entire process, the Experiment Manager records all the relevant data about the experiment: the initial representation of the world in the agent's beliefs and in the simulator, the execution trace, and the data about replanning and planning from scratch - if the latter has occurred.

The specification of an experiment setting includes the following elements:

- The agent definition (see Section 3.1)

- The environment definition (see Section 3.1).

- A planning problem specification:

- A domain specification: a plan library (see Section 2.1), and a specification of the world (a set of attributes, each with a range of possible values);

- An agent preference structure (see Section 3.1);

- The execution scenario specification according to which the execution 
of the agent's plan will be carried out by the Experiment Manager (see Section 3.2.2, below).

\subsubsection{Defining Experiment Scenarios}

Given an experiment setting definition, the Experiment Manager generates the initial representations of the world for the agent and the simulator in a nondeterministic way. At this stage, the two representations are consistent, i.e., the world represented in the simulator is simply one of the possible words listed in the agent's probabilistic representation of the world (see Section 2.1). Then, the Experiment Manager invokes the agent loop on the agent's initial representation of the world; the agent devises a plan to achieve the input goal and starts to execute it in the simulated world.

The Scenario Manager forces the occurrence of misalignments between the agent's representation of the world and the world represented in the simulator by altering the execution mechanism and the agent's representation of the world. Notice that the occurrence of a misalignment does not necessarily result in a plan failure: a plan failure occurs only if the misalignment affects the plan in a significant way, making its expected utility drop significantly with respect to the agent's acceptability threshold.

The first scenario, Execution Failure, depicts the situation in which the execution of a plan step fails, i.e. it does not result in the intended effects. According to the terminology introduced in Section 3.2, this situation can be classified as a failure-determined misalignment. The second scenario, Incorrect Representation, reproduces the situation in which the actual world is incorrectly represented in the agent's beliefs, i.e., it simulates a representationdetermined misalignment.

In the first scenario, Execution Failure, the initial subjective and objective representations of the world coincide, but the effects of the plan steps are 
non-deterministically altered when they are executed in the simulator. Implementing this scenario involves modifying the action execution mechanism in the simulator so that the effects of actions can be non deterministically altered, i.e., the value of the attributes affected by the execution of the action differs from the value encoded in the action definition (see the "effects" field in the action definition reported in Figure 1). The new value is constrained to the range of the values included in the interval between the expected value of the attribute (encoded in the action definition) and its value in the world state that precedes the action execution. In this way, the failure simulation process mimics the way the execution of an actions fails in the real world: an action may completely fail (the value of the affected attributes remain unchanged, or, in our implementation, it is brought back to its previous value), or it may yield a partial achievement (the affected attributes take an intermediate value between the initial value and the expected one).

1. The algorithm computes the effects of the action in the current world state, obtaining a probability distribution on a set of outcomes; then, it selects an outcome in a way that accounts for this probability distribution.

2. A new value is non-deterministically drawn in the range given by attribute value prior to the execution and its value after the execution. Formally, if $V_{i}^{m}$ and $V_{i}^{n}$ are the values of attribute $A_{i}$ before and after the execution of a step, the failure generation algorithm $F_{f}$ non-deterministically generates a new value $V_{x}$, where $n \leq x \leq m$.

3. In the absence of a statistical model of action failure, the number of steps to be altered is determined by the scenario specification, while the choice of the steps to be altered, and the number of attributes affected, are non deterministic. 
The second scenario, Incorrect Beliefs, depicts the situation in which the agent has incorrect initial beliefs about the world. Executing experiments according to this scenario requires generating a subjective representation of the initial world that differs from the representation of the initial world in the simulator.

1. First, a random number of attributes are non-deterministically selected for alteration.

2. For each attribute, its value is replaced by a new value, drawn from the range of values specified for that attribute in the agent's specification of the world (see Section 3.2.1).

\section{Empirical Evaluation}

Given the experimental framework described in the previous section, we consider successful replanning instances the experiment runs in which the agent's plan fails during execution and the agent's attempt to repair it by replanning succeeds. For each experiment setting, we applied the experimental procedure for a number of runs sufficient to obtain a statistically appropriate set of successful runs. Consequently, the overall number of runs required to gather the same number of successful runs may not be same for all experiment settings.

\subsection{Comparing Replanning and Planning from Scratch}

Before introducing the data on which the comparison is performed, further clarification is needed about the differences between the two strategies. Although, in the worst case, the replanning algorithm ends up searching the entire plan space from which the original plan has been derived, this is not true in general. At each partialization level, the replanning algorithm tries to modify only a 
subpart of the failed plan, the candidate sub-plan, so the search operated by the replanning algorithm is performed is simpler than the standard search operated by the planning algorithm. Moreover, this search is performed in a way that accounts for the execution context, so it tends to re-use executed steps. Reusing executed steps is more advantageous than starting a brand new plan from scratch, and the advantage is reflected by the expected utility.

In order to compare the efficiency and the effectiveness of the strategies of replanning and planning from scratch, the following data are recorded:

- First of all, we record the replanning time (RT) and the planning from scratch time (ST), i.e. the time employed by the replanning algorithm and by the planning algorithm to generate the result.

In order to assess the extent to which one strategy is more efficient than the other, for each set of experiments, we compute the mean of the computation times employed by the two strategies and we compare these values by obtaining the replanning time ratio. This value indicates how much time is saved (or wasted) by replanning - instead of planning from scratch. Finally, we run tests to assess the statistical significance of data; we assumed that the distribution is normal for all the populations of data, based on the motivation that the replanning problems were generated non-deterministically by the experimental methodology given the initial specifications.

As the replanning algorithm that we propose tends to limit the extent of the plan modification, we expected to find that the replanning algorithm is more efficient than planning from scratch. This expectation is based on the assumption that, in most cases, there is a new valid plan that is similar to the failed one (see Section 2.2), and finding it does not require exploring the entire plan space. 
- In order to evaluate which strategy is more effective, we record and compare the expected utility of the plans generated by replanning and by planning from scratch as a measure of plan quality. ${ }^{4}$

In order to assess to what extent one strategy is better than the other in terms of the quality of output plans, we compute the mean values of the expected utility of the two sets of plans and we compare them, by obtaining the expected utility ratio. This value indicates to what extent the quality of the plans generated by replanning is better (or worse) than the plans generated by planning from scratch. Again, we run statistical tests on the data to assess if there is a statistically significant difference between the two.

Concerning plan quality, our expectation was that replanning would generate better plans than planning from the start, since it incorporates a preference for the modifying the steps that have not been executed yet.

\subsection{Evaluating the Replanning Algorithm}

Beside collecting data for comparing replanning and planning from scratch, we also record a set of specific data about the behavior of the replanning algorithm, in order to verify if the conservative assumption on which the algorithm is based holds in empirical contexts. The replanning algorithm consults the decomposition relations encoded in the action hierarchy to focus the revision process on a subpart of the failed plan, and gradually increases the scope of the revision, until it reaches the top of the action hierarchy (see Section 2.2). Clearly, the time performance of the replanning algorithm is negatively affected by the number of times it repeats the partialization process, and by the overall number of

\footnotetext{
${ }^{4}$ As the notion of plan failure incorporated in the experiment framework refers to the higher expected utility of the current plan, we record the higher expected utility of the generated plans.
} 
retractions - and tentative refinements - it performs.

- For each successful replanning instance, we recorded the overall number of refinements (NR) it attempted during the partialization process, and the number of times it collapsed the focussed sub-plan, i.e., it enlarged the scope of the partialization process by collapsing the focussed sub-plan onto the complex action which constitutes its root in the decomposition hierarchy (NC). We expected that a local revision of the failed plan would be sufficient to restore it in most cases, i.e., that the focus of the revision would be enlarged only a few times and that few refinements would be necessary to find a new valid plan.

For each set of experiments, we collected some data which characterize the role of the setting in the occurrence of plan failure. Although a statistical assessment of the relation between the experiment setting and the occurrence of plan failures is out of the scope of this work, we think that these data raise some general issues for the management of replanning in real domains.

- For each experiment setting, we measured the failure rate, the number of plan failures encountered by the agent in that setting, given the overall set of runs. This value allows making an approximate evaluation of the frequency with which a certain agent would be likely to resort to replanning in that setting. Since plan libraries and initial world specifications differ by the degree of non-determinism, the rate of plan failures was expected to be affected by the domain specification.

- In order to verify the effectiveness of the replanning algorithm in each setting we measured the relative successful replanning rate, i.e., the number of times the replanning algorithm outputs a valid plan given the number of plan failures, and the absolute successful replanning rate, 
i.e. the number of successful replanning instances given the number of experiments in the set. In practice, given a planning problem specification and a scenario, the former value tells us how frequently replanning was able to overcome the failures it encountered, while the latter provides a means for evaluating the relevance of successful replanning in an experiment setting.

We expected to find no correlation between these two values: an experimental setting may generate more plan failures than another, but they may be harder to repair than those generated by setting that generated less failures.

\subsection{Domains and Settings}

In order to investigate the impact of plan failures and the trade-off between planning and replanning in a domain-independent way, the scenarios described above have been applied to three planning problem specifications (see Section 3.2.1), consisting of a domain specification (a plan library and a world specification) and of an agent preference structure. Domains differ along two main dimensions: the complexity of the plan library (measured in terms of maximal and minimal plan length and action hierarchy height), and the complexity of the representation of the world (the number of attributes involved in world definition). For sake of brevity, in the following, we will refer to an experiment setting as a domain-scenario pair.

In order to limit the factors involved in the experimentation, we made some simplifications. Concerning the agent preference structure specification, the acceptability ratio has been set to 0.1 for all settings. Moreover, the maximum number of steps affected by an execution failure in the Execution Failure scenario has been set to the arbitrary number of 2 . 
The first two domains (domain $A$ and domain $B$ ) concern industrial brewing planning [Haddawy and Suwandi, 1994] and are characterized by non-deterministic actions. Although these two domains include similar action definitions, or reuse the same action definitions, they differ as they concern the brewing of different beer types: so, the plans they generate differ for the type of actions they include. In practice, given the same action hierarchy, different portions of it are relevant depending on the utility function employed to trade off the resource consumption against the quality parameters. For these reasons, we decided to consider them as two different domains. They both contain 34 action types organized along in a hierarchy which includes three decomposition levels and four specification levels, for a total of seven abstraction levels. The initial world is defined by nine attributes for both domains, and the output plans contain from 4 to 6 steps. The overall number of primitive plans is 1728 .

The third domain (domain $C$ ) has been designed for this study. It represents an office toy world (a type of domain adopted also by [Dastani et al., 2003]) and concerns the planning of a complex mail delivery task. The plan library of domain $C$ includes 32 operators and is characterized by a lower height than the other two domains (three decomposition levels and three specification levels, yielding six abstraction levels); differently from the other two domains, it contains non deterministic actions. The agent utility function accounts for time and resource consumption in the achievement of the goal. In domain $C$, the initial world representation is characterized by a lower number of defining attributes with respect to domains $A$ and $B$ (6 attributes); the length of output plans varies between 9 and 12 steps. The overall number of primitive plans is 288.

In order to compare the performance of our replanning algorithm with the strategy of planning from scratch, we applied to each domain the two scenarios 


\begin{tabular}{|c|c|c|c|}
\hline $\begin{array}{c}\text { Experiment } \\
\text { setting }\end{array}$ & $\begin{array}{c}\text { Replanning } \\
\text { time } \\
\text { ratio }\end{array}$ & $\begin{array}{c}\text { Executions } \\
\text { where } \\
R T<S T\end{array}$ & $\begin{array}{c}\text { Z-test on } R T \text { and } S T \\
\text { hypothesized difference }=0 \\
\text { (two-tailed, } p<, 001, \\
\text { critical } z=1,959961)\end{array}$ \\
\hline A Execution Failure & 2,86 & 30 & $\mathrm{z}=353,44 P(Z \leq z)=0$ \\
\hline A Incorrect Beliefs & 0,57 & 30 & $\mathrm{z}=244,75 P(Z \leq z)=0$ \\
\hline B Execution Failure & 1,55 & 30 & $\mathrm{z}=236,14 P(Z \leq z)=0$ \\
\hline B Incorrect Beliefs & 0,48 & 30 & $\mathrm{z}=163,85 P(Z \leq z)=0$ \\
\hline C Execution Failure & 26,43 & 30 & $\mathrm{z}=29,33 P(Z \leq z)=0$ \\
\hline C Incorrect Beliefs & 18,64 & 30 & $\mathrm{z}=34,00 P(Z \leq z)=0$ \\
\hline
\end{tabular}

Figure 5: Computation time. Second column: the ratio between the time required for replanning (RT) and planning from scratch (ST); third column: the number of executions in which planning from scratch takes longer than replanning; fourth column: statistical significance of the difference between the two series of data.

presented above (see Section 3.2.2), the Execution Failure scenario and the Incorrect Belief Scenario. For each setting consisting of a domain-scenario pair, we performed as many experiments as necessary to collect a set of 30 instances of successful replanning. ${ }^{5}$

\section{Discussion of the Results}

In this section, we describe the results of the experiment we performed by applying the methodology presented above, and we discuss the results obtained.

\subsection{Comparing Replanning and Planning from Scratch:}

\section{the Results}

Concerning the time performance of the two repair strategies, the replanning algorithm is by and large more efficient than the planning from scratch strategy in all settings (see Figure 5, second column). Moreover, replanning always employed less time than planning from scratch. The ratio between the mean

\footnotetext{
${ }^{5}$ The number of 30 was chosen for it is conventionally considered to be the boundary between "small" and "large" samples [Cohen, 1995].
} 
time employed by the replanning algorithm, and the mean time required to plan from scratch, ranges from 26,43\% (domain $C$ with Execution Failure scenario) to $0,57 \%$ (domain $A$ with the Incorrect Beliefs scenario).

In particular, the difference between the two strategies is bigger in more complex plan libraries, like domains $A$ and $B$. This can be explained by considering the functioning of the replanning algorithm: since it embodies a preference for local repair, the most complex is the library (i.e., the deepest is the specialization library), the better is the performance of the algorithm, in comparison with the top-down search operated by the planning algorithm on the entire plan library. In fact, while the partial revision performed by the former tends to remain confined to one or two levels of the specialization hierarchy (as discussed below), the latter necessarily deals with all the levels of the action hierarchy.

In order to test the significance of these data from a statistical point of view, for each set of data, we applied the Z-test to the computation time of the two strategies. Since the Z-value is, for all sets, higher than the critical value of $1,644853^{6}$ with a $\mathrm{P}<0,01$, it is possible to affirm, with an acceptable probability (99\%) that the difference between the computation times of the two strategies is not due to chance.

Regarding the quality of the plans generated by the two strategies, the data show the superiority of the replanning algorithm, although in a less clear-cut way. The expected utility ratio shows the superiority of the replanning algorithm in all settings (see Figure 6, second column), but only in two settings (domains $A$ and $B$ with the Incorrect Beliefs scenario) the quality of plans generated by replanning is always higher than the quality of the plans generated by planning from scratch (for all the 30 cases constituting the set). In the two settings obtained by associating the Execution Failure scenario with domains $A$ and $B$,

\footnotetext{
${ }^{6}$ The test is one tailed since the difference between the replanning time and scratch planning time is always positive.
} 


\begin{tabular}{|c|c|c|c|}
\hline $\begin{array}{c}\text { Experiment } \\
\text { setting }\end{array}$ & $\begin{array}{c}\text { Expected utility } \\
\text { ratio }\end{array}$ & $\begin{array}{c}\text { Executions } \\
\text { where } \\
R E U>S E U\end{array}$ & $\begin{array}{c}\text { Z-test on } R E U \text { and } S E U \\
\text { hypothesized difference }=0 \\
\text { (two-tailed, } p<, 001, \\
\text { critical } z=1,959961)\end{array}$ \\
\hline A Execution Failure & 152,06 & 28 & $\mathrm{z}=-4,78 P(Z \leq z)=1,78$ \\
\hline A Incorrect Beliefs & 276,45 & 30 & $\mathrm{z}=14,38 P(Z \leq z)=0$ \\
\hline B Execution Failure & 149,32 & 25 & $\mathrm{z}=3 P(Z \leq z)=6$ \\
\hline B Incorrect Beliefs & 292 & 30 & $\mathrm{z}=14,84 P(Z \leq z)=0$ \\
\hline C Execution Failure & 119,59 & 16 & $\mathrm{z}=1,48 P(Z \leq z)=0,14$ \\
\hline C Incorrect Beliefs & 100,2 & 12 & $\mathrm{z}=-0,04 P(Z \leq z)=0,96$ \\
\hline
\end{tabular}

Figure 6: Expected utility of plans. Second column: the ratio between the expected utility of plans obtained by replanning (REU) and planning from scratch (SEU); third column: the number of executions in which the expected utility (EU) of the plans obtained by replanning is higher than the expected utility of the plans obtained by planning from scratch; fourth column: statistical significance of the difference between the two series of data.

the quality of the plans generated by replanning is higher in most cases, but non in all cases. By contrast, in both settings with domain $C$, planning from scratch outperforms the replanning algorithm by the quality of plans.

Again, the scenario does not seem to influence the relative quality of the output plans, while the domain seems to play a central role. Action hierarchies that have been optimized to generated plans for batch execution in a well-defined initial states (like domains $A$ and $B$ ) yield a poor performance on initial states in which one or more plan steps have already been executed. On the contrary, the action hierarchy contained in domain $C$ has been purposely designed with an eye on replanning: the possibility for actions to have conditional effects has been exploited to account for a wider range of initial states. For this reasons, the expected utility values obtained by planning from scratch in domain $C$ (with both scenarios) are comparable if not equal to the expected utility values obtained by the replanning algorithm.

As for the computation time, in order to test the significance of these data from a statistical point of view, we applied the Z-test to the expected utility of the plans generated by the two strategies. Not surprisingly, the Z-test indicates 
a significant difference only when the difference in expected utility between replanning and planning from scratch is sharp in 30 cases out of 30, (like in domains $A$ and $B$ with the Incorrect Belief scenario). In the remaining settings, there is no statistically significant difference between the two populations of expected utility values obtained by replanning and by planning from scratch.

In conclusion, the data collected empirically substantially confirm the expectation that the replanning algorithm generates better plans (i.e. plans with a higher expected utility) than the strategy of replanning from start, although they do not show a complete dominance of replanning over planning from scratch for what concern the quality of output plans, and the difference is not statistically significant in all settings. It is worth noting that preliminary experiments conducted on a simplified version of domain $C$ [Boella and Damiano, 2003] yielded opposite results: the strategy of performing from scratch outperformed the replanning algorithm by time and plan quality, confirming the hypothesis that the complexity of the plan library plays a crucial role in determining the advantage of replanning with respect to planning from scratch.

\subsection{Evaluating the Replanning Algorithm: the Results}

The data concerning the behavior of the replanning algorithm confirm the expectation that a solution is generally found at a local level. The number of times the replanning algorithm enlarged the scope of the revision by collapsing the focussed sub plan is very low in all settings (see Figure 7, third column, NC), ranging from 0,1 (domain $A$ with Execution Failure Scenario) to 1,2 (domain $C$ with Incorrect Beliefs scenario). In the large majority of cases, the scope of the plan revision was enlarged only 0 or 1 times, meaning that the replanning algorithm repaired the failed plan by operating at a very local level. While in the settings built by using domain $A$ and $B$ the value of $\mathrm{NC}$ is at most 1 , a value 


\begin{tabular}{|c|c|c|c|c|}
\hline $\begin{array}{l}\text { Experiment } \\
\text { setting }\end{array}$ & $\begin{array}{l}\text { Avg. } \\
\text { NR }\end{array}$ & $\begin{array}{l}\text { Avg. } \\
\text { NC }\end{array}$ & $\begin{array}{c}\text { Correlation } \\
\text { NR / } \\
\text { time } \\
\text { (Pearson) }\end{array}$ & $\begin{array}{c}\text { Correlation } \\
\text { NC / } \\
\text { time } \\
\text { (Pearson) }\end{array}$ \\
\hline $\begin{array}{c}\mathrm{A} \\
\text { Execution Failure }\end{array}$ & $\begin{array}{c}2,1 \\
(\max 4-\min 1)\end{array}$ & $\begin{array}{c}0,1 \\
(\max 1-\min 0)\end{array}$ & $0,749(0,000)$ & $-0,145(0,444)$ \\
\hline $\begin{array}{c}\mathrm{A} \\
\text { Incorrect Beliefs }\end{array}$ & $\begin{array}{c}2,43 \\
(\max 3-\min 1)\end{array}$ & $\begin{array}{c}0,67 \\
(\max 1-\min 0)\end{array}$ & $0,168(0,374)$ & $-0,072(0,704)$ \\
\hline $\begin{array}{c}\text { B } \\
\text { Execution Failure }\end{array}$ & $\begin{array}{c}1,93 \\
(\max 3-\min 1)\end{array}$ & $\begin{array}{c}0,03 \\
(\max 1-\min 0)\end{array}$ & $0,537(0,002)$ & $-0,065(0,732)$ \\
\hline $\begin{array}{c}\mathrm{B} \\
\text { Incorrect Beliefs }\end{array}$ & $\begin{array}{c}2,4 \\
(\max 3-\min 1)\end{array}$ & $\begin{array}{c}0,67 \\
(\max 1-\min 0)\end{array}$ & $0,379(0,039)$ & $0,180(0,342)$ \\
\hline $\begin{array}{c}\mathrm{C} \\
\text { Execution Failure }\end{array}$ & $\begin{array}{c}1,33 \\
(\max 4-\min 1)\end{array}$ & $\begin{array}{c}1,2 \\
(\max 4-\min 0)\end{array}$ & $0,883(0,000)$ & $0,698(0,000)$ \\
\hline $\begin{array}{c}\mathrm{C} \\
\text { Incorrect Beliefs }\end{array}$ & $(\max 4-\min 1)$ & $\begin{array}{c}0,6 \\
(\max 3-\min 0)\end{array}$ & $0,909(0,000)$ & $0,702(0,000)$ \\
\hline
\end{tabular}

Figure 7: Correlation between the behavior of the replanning algorithm and its time performance. Second column: the average number of alternative refinements attempted (NR); third column, the average number of times the scope of the revision was enlarged by collapsing the focussed sub-plan onto a complex action (NC); fourth and fifth columns: Pearson's correlation between NR and $\mathrm{NC}$ and the computation time.

of 4 has been been episodically observed in domain $C$ (in only one case), suggesting that the structure of the plan library is a crucial factor in determining the possibility of finding a local solution to plan failures.

The number of refinements (see Figure 7, second column, NR) is very low as well, ranging from 1,33 (domain $C$, Execution Failure scenario) to 2,44 (domain $B$, Incorrect Beliefs scenario). The number of alternative refinements executed by the replanning algorithm after the retraction of one or more steps is small and does not seem to vary significantly across settings.

In order to test the of correlation between the number of refinements executed by the replanning algorithm and the computation time, we resorted to Pearson's correlation coefficient (see Figure 7, fourth column, $p \leq 0,005$ ): a significant correlation was obtained only in three data sets out of six (domain $A$ with Execution Failure Scenario and domain $C$ with both scenarios). Concerning the correlation between the value of $\mathrm{NC}$ and the computation time (see 


\begin{tabular}{|c|c|c|c|}
\hline Experiment & Failure & \multicolumn{2}{|c|}{ Successful replanning } \\
\hline setting & rate & $\%$ (relative) & $\%$ (absolute) \\
\hline A Execution Failure & 57,64 & 36,14 & 20,83 \\
\hline A Incorrect Beliefs & 38,68 & 16,57 & 6,41 \\
\hline B Execution Failure & 43,49 & 20,41 & 8,88 \\
\hline B Incorrect Beliefs & 34,82 & 22,57 & 7,85 \\
\hline C Execution Failure & 39,58 & 52,63 & 20,83 \\
\hline C Incorrect Beliefs & 42,86 & 100 & 42,86 \\
\hline
\end{tabular}

Figure 8: The replanning rate in each domain-scenario pair and the successful replanning rate, relative and absolute.

Figure 7, fifth column), the experiments did not generate a sufficiently large set of data to perform a statistical evaluation. However, the fact itself that,in most runs, the algorithm was able to find a new valid plan by modifying only the initially focussed sub-plan, makes the assessment of this relation somewhat less relevant than we expected prior to the empirical evaluation.

There are considerable differences among experiment settings for what concern the rate of plan failures (see Figure 8), confirming the theoretical expectation that the probability of encountering a failure is not the same in all settings. The failure rate ranges from 34,82\% (domain $B$ with Incorrect Beliefs scenario) to $57,64 \%$ (domain $A$ with Execution Failure scenario). Neither the scenario specification nor the planning problem specification determine, taken separately from each other, a higher or lower failure rate.

The successful replanning rate shows a larger variation across experiment settings, meaning that, in some settings, plan failures are more easily repaired than in other settings: the successful replanning rate ranges from 16,57\% (domain $A$, Incorrect Beliefs scenario) to $100 \%$ (domain $C$, Incorrect Beliefs scenario). This value does not depend on the failure rate: for instance, the setting where the failure rate is the highest, domain $B$ with the Incorrect Beliefs scenario, is not the one in which the success rate of replanning is the highest. The variation 
of the successful replanning rate in turn affects the absolute successful replanning rate, which ranges from $6,41 \%$ (domain $A$, Incorrect Beliefs scenario) to $42,86 \%$ (domain $C$, Incorrect Beliefs scenario). The lower complexity of domain $C$ seems to affect also the successful replanning rate, that is significantly higher in both settings that include this domain.

\section{Conclusions and Future Work}

In this paper, we presented an algorithm that extends decision-theoretic hierarchical planning to replanning and we proposed an empirical methodology for its evaluation. The replanning algorithm consults the decomposition relations between actions in the action hierarchy to focus the revision process on a subpart of the failed plan; then, it retracts refinement choices within the focused sub-plan, in search for new, valid refinements. If a new valid plan cannot be found, the scope of the revision is enlarged, and the process is restarted on it [Boella and Damiano, 2002b] [Boella and Damiano, 2002a]. The algorithm is based on the assumption that a solution to the replanning problem can be found in most cases by searching the portion of the space of possible plans which is "local" to the failed one in the plan space. Beside limiting the computational effort required to the agent, this approach is in line with the fact that intentions, according to theories of rational agents cite above, are characterized by stability. The algorithm is complete: eventually, the plan collapses onto the root of the action hierarchy, and planning is attempted from scratch. However, it is not conservative, according to definition provided by [Nebel and Koehler, 1993], i.e. it does not perform minimal modifications to the failed plan.

The second research issue we pursued in this work is the design and implementation of an empirical methodology for evaluating the performance of the replanning algorithm within an agent-based framework. Starting from an agent 
specification and a planning problem specification, the methodology consists in defining execution scenarios in which the plan of an agent fails and replanning is performed. Planning from scratch is attempted in parallel with replanning, and the performance of the two is compared in terms of computation time and quality of output plans. It is important to notice that this methodology is independent of the replanning strategies compared. While the methodology to compare the performance of planners described in [Long and Fox, 2003] involves elaborating a number of planning problems in several domains, the goal of the methodology we propose is somehow different: the focus is not on the formulation of the planning problems; rather, our proposal focusses on the definition of the experimental framework for the automated generation of the plan failures on which different replanning strategies are tested. Since it is agent-based, this framework provides a principled way to address the difficulties observed by [Howe and Dahlman, 2002] in relation with problem sampling in planner comparison.

In order to limit the complexity of the experimental framework, in the experiments discussed here, we set some parameters to default values, leaving to future work the task of investigating how the experimental framework affects the performance of the replanning algorithm.

The replanning algorithm was largely faster than replanning from scratch in all settings and in all experiment runs. The quality of the plans obtained by replanning was on average equal or higher than the quality of the plans obtained by planning from scratch, but the superiority of the replanning algorithm was not as clear-cut as for the time performance. This fact, together with the observation that the rate of plan failures and the performance of the replanning algorithm vary across settings, leads to hypothesize a methodology according to which it is essential to assess the impact on plan failures in a certain domain by 
performing extended simulations (and the effectiveness of different replanning strategies) before committing to a redeliberation strategy.

\section{References}

[Blythe, 1999] Blythe, J. (1999). Decision-theoretic planning. AI Magazine, $20(2)$.

[Boella and Damiano, 2002a] Boella, G. and Damiano, R. (2002a). An architecture for normative reactive agents. In Kawabara, K. and Lee, J., editors, Intelligent Agents and Multi-Agent Systems (Proc. of Prima 02), LNAI 2413, pages $1-17$, Tokyo.

[Boella and Damiano, 2002b] Boella, G. and Damiano, R. (2002b). A replanning algorithm for a reactive agent architecture. In Scott, D., editor, Artificial Intelligence: Methodology, Systems, and Applications (Proc. of Aimsa 02), LNCS 2443, pages 183-192, Varna.

[Boella and Damiano, 2003] Boella, G. and Damiano, R. (2003). Empirical evaluation of a replanning algorithm. In ICAPS Workshop on plan execution, Trento.

[Boella and Damiano, 2004] Boella, G. and Damiano, R. (2004). A decisiontheoretic replanning algorithm. In Milani, A., editor, Proceedings of the $3 d$ Italian Workshop on Planning and Scheduling, Perugia.

[Bratman, 1990] Bratman, M. E. (1990). What is intention? In Cohen, P. R., Morgan, J., and Pollack, M. E., editors, Intentions in communication, pages 15-32. MIT Press.

[Cohen, 1995] Cohen, P. R. (1995). Empirical Methods for Artificial Intelligence. The Mit Press, Cambridge, Massachusetts. 
[Dastani et al., 2003] Dastani, M., Dignum, V., and Dignum, F. (2003). Roleassignment in open agent societies. In Procs. of $A A M A S^{\prime} 03$, pages 489-496, Melbourne. ACM Press.

[Georgeff et al., 2000] Georgeff, M., Pell, B., Pollack, M., Tambe, M., and Wooldridge, M. (2000). The belief-desire-intention model of agency. In Mller, J., Singh, M., and Rao, A., editors, Intelligent Agents V. Proc. of Agent Theories, Architectures, and Languages: 5th International Workshop (ATAL98), LNAI, Paris. Springer-Verlag.

[Gerevini and Serina, 2000] Gerevini, A. and Serina, I. (2000). Fast plan adaptation through planning graphs: Local and systematic search techniques. In Proceedings of the 5th International Conference on Artificial Intelligence Planning Systems (AIPS-00). AAAI Press.

[Haddawy and Hanks, 1998] Haddawy, P. and Hanks, S. (1998). Utility models for goal-directed, decision-theoretic planners. Computational Intelligence, $14: 392-429$.

[Haddawy and Suwandi, 1994] Haddawy, P. and Suwandi, M. (1994). Decisiontheoretic refinement planning using inheritance abstraction. In Proc. of 2nd AIPS Int. Conf., pages 266-271, Menlo Park, CA.

[Haigh and Veloso, 1996] Haigh, K. Z. and Veloso, M. (1996). Interleaving planning and robot execution for asynchronous user requests. In Planning with Incomplete Information for Robot Problems: Papers from the 1996 AAAI Spring Symposium, pages 35-44. AAAI Press, Menlo Park, California.

[Hanks and Weld, 1995] Hanks, S. and Weld, D. S. (1995). A domainindependent algorithm for plan adaptation. Journal of Artificial Intelligence Research, 2:319-360. 
[Howe and Dahlman, 2002] Howe, A. E. and Dahlman, E. (2002). A critical assessment of benchmark comparison in planning. Journal of Artificial Intelligence Research, 17:1-33.

[Kambhampati and Hendler, 1992] Kambhampati, S. and Hendler, J. (1992). A validation-structure-based theory of plan modification and reuse. Artificial Intelligence, 55:193-238.

[Koenig et al., 2002] Koenig, S., Furcy, D., and Bauer, C. (2002). Heuristic search-based replanning. In Proc. of AIPS 02.

[Lemai and Ingrand, 2003] Lemai, S. and Ingrand, F. (2003). Interleaving temporal planning and execution: Ixtex-exec. In Proc. of ICAPS 03 Workshop on Execution, Trento.

[Lemai and Ingrand, 2004] Lemai, S. and Ingrand, F. (2004). Interleaving temporal planning and execution in robotics domains. In Proc. of AAAI 04.

[Liberatore, 1998] Liberatore, P. (1998). On non-conservative plan modification, young research paper. In Procs. of ECAI 98. John Wiley and Son ltd.

[Long and Fox, 2003] Long, D. and Fox, M. (2003). The third international planning competition: Results and analysis. Journal of Artificial Intelligence Research, 20:1-59.

[Luce and Raiffa, 1957] Luce, D. and Raiffa, H. (1957). Games and Decisions. John Wiley and Sons, Inc.

[Miksch and Seyfang, 2000] Miksch, S. and Seyfang, A. (2000). Continual planning with time-oriented, skeletal plans. In Proc. of ECAI 00.

[Myers, 1999] Myers, K. L. (1999). Cpef: Continuous planning and execution framework. AI Magazine, 20(4):63-69. 
[Nebel and Koehler, 1993] Nebel, B. and Koehler, J. (1993). Plan modification versus plan generation: A complexity-theoretic perspective. In Proceedings of of the 13th International Joint Conference on Artificial Intelligence, pages 1436-1441, Chambery, France.

[Rao and Georgeff, 1995] Rao, A. and Georgeff, M. (1995). BDI agents: From theory to practice. In Proceedings of the First International Conference on Multi-Agent Systems (ICMAS'95).

[Rao and Georgeff, 1991] Rao, A. and Georgeff, M. P. (1991). Modeling rational agents within a BDI-architecture. In Proc. 2th Int. Conf. Principles of Knowledge Representation and Reasoning (KR:91), pages 473-484, Cambridge, MA.

[Russell and Subramanian, 1995] Russell, S. J. and Subramanian, D. (1995). Provably bounded-optimal agents. Journal of Artificial Intelligence Research, 2:575-609.

[Sacerdoti, 1977] Sacerdoti, E. D. (1977). A Structure for Plans and Behavior. American Elsevier, New York.

[van der Krogt et al., 2000] van der Krogt, R., Bos, A., de Weerdt, M., and Witteveen, C. (2000). An algorithm for replanning. In van den Bosch, A. and Weigand, H., editors, Proceedings of the Twelfth Belgium-Netherlands Artificial Intelligence Conference BNAIC '00, pages 21-28, Tilburg.

[Wilkins et al., 1994] Wilkins, D., Myers, K., Lowrance, J., and Wesley, L. (1994). Planning and reacting in uncertain and dynamic environment. Journal of Experimental and Theoretical AI, 6:197-227. 
[Wooldridge and Parsons, 1999] Wooldridge, M. and Parsons, S. (1999). Intention reconsideration reconsidered. In Müller, J., Singh, M. P., and Rao, A. S., editors, Proc. of ATAL-98), volume 1555, pages 63-80. Springer-Verlag. 


\title{
Introduction to the special issue on normative multiagent systems
}

\author{
Guido Boella • Leendert van der Torre • \\ Harko Verhagen
}

Published online: 30 April 2008

Springer Science+Business Media, LLC 2008

\begin{abstract}
This special issue contains four selected and revised papers from the second international workshop on normative multiagent systems, for short NorMAS07 (Boella et al. (eds) Normative multiagent systems. Dagstuhl seminar proceedings 07122, 2007), held at Schloss Dagstuhl, Germany, in March 2007. At the workshop a shift was identified in the research community from a legal to an interactionist view on normative multiagent systems. In this editorial we discuss the shift, examples, and 10 new challenges in this more dynamic setting, which we use to introduce the papers of this special issue.
\end{abstract}

Keywords Norms - Multiagent Systems - Normative multiagent systems - Social mechanism design $\cdot$ Artifical social systems

\section{Towards a more dynamic interactionist view}

Traditionally normative systems have been studied in philosophy, sociology, law, and ethics, and during the past two decades they have been studied in deontic logic in computer science $(\triangle \mathrm{EON})$. Normative multiagent systems is a research area where the traditional normative systems and $\triangle \mathrm{EON}$ research fields meet agent research. The proposed solutions to the $\triangle \mathrm{EON}$ research problems are changing, and solutions based on multiagent systems are increasing. Gradually the $\triangle \mathrm{EON}$ research focus changes from logical relations among norms, to, for

\footnotetext{
G. Boella

Dipartimento di Informatica, Università di Torino, Torino, Italy

e-mail: guido@di.unito.it

L. van der Torre $(\varangle)$

Computer Science and Communication Research Unit, University of Luxembourg, Luxembourg, Luxembourg

e-mail: leendert@vandertorre.com

H. Verhagen

Department of Computer and Systems Sciences, Stockholm University/KTH, Kista, Sweden

e-mail: verhagen@dsv.su.se
} 
example, agent decision making, and to systems in which norms are created and in which agents can play the role of legislators. The eighth conference on Deontic Logic in Computer Science in 2006 in Utrecht, the Netherlands had as special focus "artificial normative systems" [10,11], and the seventh conference [14,15] in 2004 in Madeira, Portugal had as special theme "deontic logic and multiagent systems." Continuing this trend, the third workshop on normative multiagent systems is co-located in Luxembourg in July 2008 with the ninth conference on Deontic Logic in Computer Science [11,23], which has as special topic "security and trust," and the fourth workshop will again be a Dagstuhl seminar to be held in March 2009.

The Agentlink Roadmap [16, Fig. 7.1] observes that norms must be introduced in agent technology in the medium term for infrastructure for open communities, reasoning in open environments and trust and reputation. After four days of discussion, the participants of the second workshop on normative multiagent systems agreed to the following consensus definition:

A normative multiagent system is a multiagent system organized by means of mechanisms to represent, communicate, distribute, detect, create, modify, and enforce norms, and mechanisms to deliberate about norms and detect norm violation and fulfilment.

The shift towards a more dynamic interactionist view on normative multiagent systems is reflected in the way this definition builds on its predecessor which emerged at the first workshop on normative multiagent systems held in 2005 as a symposium of the Artificial Intelligence and Simulation of Behaviour convention (AISB) in Hatfield, United Kingdom: "A normative multiagent system is a multiagent system together with normative systems in which agents on the one hand can decide whether to follow the explicitly represented norms, and on the other the normative systems specify how and in which extent the agents can modify the norms" [3].

The emphasis has shifted from representation issues to the mechanisms used by agents to coordinate themselves, and in general to organize the multiagent system. Norms are communicated, for example, since agents in open systems can join a multiagent system whose norms are not known. Norms are distributed among agents, for example, since when new norms emerge the agent could find a new coalition to achieve its goals. Norm violations and norm compliance are detected, for example, since spontaneous emergence norms of among agents implies that norm enforcement cannot be delegated to the multiagent infrastructure.

This shift of interest marks the passage of focus from the more static legalistic view of norms (where power structures are fixed) to the more dynamic interactionist view of norms (where agent interaction is the base for norm related regulation. This ties in to what Strauss [20] called "negotiated order," Goffmans [12] view on institutions, and Giddens' [9] structuration theory). The workshop vote on next generation scenarios for normative multiagent systems clearly preferred social scenarios like virtual communities and Second Life (over $50 \%$ ) to more classical e-commerce settings where centralized solutions like e-institutions are used (less than 20\%).

The legalistic view of normative multiagent systems is a top-down view which considers the normative system as a regulatory instrument to regulate emerging behavior of open systems without enforcing the desired behavior. Agents are often motivated by sanctions to stick to norms, rather than by their sharing of the norms. Even if agents are allowed some freedom to create norms, this freedom is mostly restricted to the possibility for agents to create contracts to regulate the interaction among them. 
The interactionist view on normative multiagent systems represents a bottom-up view. In this autonomous individually oriented view norms can be seen, e.g., as regularities of behavior which emerge without any enforcement system because agents conform to them either because their goals happen to coincide, or because they feel themselves as part of the group or because they share the same values of other agents. Sanctions are not always necessary, where by sanctions we mean formal measures towards norm violating agents carried out by agents whose task it is to sanction norm violations, because social blame and spontaneous exclusion of non-conforming agents are often enough.

This interactionist view, which has been promoted in the multiagent systems community by Castelfranchi [7], becomes essential in applications related to virtual communities. In Second Life, for example, communities emerge in which the behavior of its members show increasing homogeneity.

To put this shift from legal to interactionist view into perspective, we can identify five levels in the development of normative multiagent systems. At level 1 of off-line norm design [19], norms are imposed by the designer and automatically enforced, and agents cannot organize themselves by means of norms. At level 2 of norm representation, norms are explicitly represented, they can be used in agent communication and negotiation, and a simple kind of organizations and institutions can be created. At level 3 of norm manipulation, a legal reality is created in which agents can add and remove norms following the rules of the normative system. Whereas existing normative multiagent systems are still at one of these first three levels of norm autonomy (for an introduction to norm autonomy in multiagent systems, see [24]), multiagent system research is now moving to level 4 of social reality, and is concerned with the 10 challenges discussed in Sect. 3 below. We believe that there is at least one more level to be dealt with in the future. At level 5, the norms create a new moral reality. This goes beyond present studies in machine ethics [1]. Machine ethics is more concerned with agent decision making in the context of norms, which is an issue dealt with at each level of normative multiagent systems, than with creating a new ethics.

Clearly, for each level the development of the normative multiagent system will take a much larger effort than the development of similar systems at lower levels. For example, if norm are explicitly represented (level 2) rather than built into the system (level 1), then the system has to be much more flexible to deal with the variety of normative systems that may emerge. However, it may be expected that normative multiagent systems realized at higher levels will have a huge effect on social interaction, in particular on the web. Before introducing the papers of this special issue, we discuss some examples and several research needs that arise in this more dynamic interactionist view on normative multiagent systems.

\section{Examples of an interactionist view}

We illustrate the more dynamic interactionist viewpoint on normative multiagent systems using virtual communities in virtual reality settings like Second Life. In these virtual communities, human agents interact with artificial agents in a virtual world. The new communication instruments offered by the internet have resulted in the creation of virtual communities of users sharing information, emotions, or hobbies. When the interaction possibilities are multiplied in applications like Second Life or multi-player online games, new scenarios emerge.

In particular, given the higher degree of freedom of behavior with respect to the real world, and the unaccountability offered by anonymity, on the one hand, as said above, spontaneous 
communities emerge showing regularities of behavior. However, to preserve the autonomy of the members of these communities, interactionist mechanisms for regulating behavior are needed. Thus, members of communities should be endowed with tools to make the community norms explicit and communicable to preserve their members' autonomy.

The participants will eventually end up creating their own norms and rules, even if in virtual communities like Second Life and in multi-player games normative infrastructure is imposed by the designers. Sometimes, the rules created by the participants counter the designers' objectives and rules and players start to play in ways unforeseen by the game designers.

An example is discussed by Ludlow [17] from Sony's EverQuest. EverQuest is a multiplayer online game where gamers are supposed to fight each other in a world of snakes, dragons, gods, and the Sleeper. Sony intended the Sleeper to be unkillable and gave it extreme high hit points. However, a combined combat of close to 200 players nearly succeeded to kill the 'animal.' Unfortunately, Sony decided to intervene and rescue the Sleeper. Most of the discussion on this example has highlighted the decrease in trust of the game players in Sony, despite the fact that the next day Sony let the game players beat the Sleeper. However, in this paper we would like to highlight what this story tells us about the goals of game players, and its consequences for necessary technology in games. The following quote illustrates the excitement in killing the Sleeper.

A supposedly [player-vs.-player] server banded together 200 people. The chat channels across the server were ablaze, as no less than 5,000 of us listened in, with OMG theyre attempting the Sleeper! Good luck d00dz! Everyone clustered near their screens, sharing the thrill of the fight, the nobility of the attempt and the courage of those brave 200. Play slowed to a crawl on every server as whispers turned to shouts, as naysayers predicted, It can't be done or It will drop a rusty level 1 sword and most of us just held our breath, silently urging them forward. Rumors abounded: If they win, the whole EQ world stops and you get the text from the end of Wizardry 1, or If they win, the president of Sony will log on and congratulate them. With thousands watching and waiting, the Sleeperć6s health inched ever downward.

...

[EverQuest player] Ghenwivar writes, On Monday, November 17th, in the most amazing and exciting battle ever, [EverQuest guilds] Ascending Dawn, Wudan and Magus Imperialis Magicus defeated Kerafyrm, also known as The Sleeper, for the first time ever on an EverQuest server. The fight lasted approximately three hours and about 170-180 players from [EverQuest server] Rallos Zeks top three guilds were involved. Hats off to everyone who made this possible and put aside their differences in order to accomplish the impossible. Congratulations RZ!!! [17]

Normative multiagent systems study multiagent technology to support the emergent cooperation in online multi-player games like EverQuest [2]. The example illustrates that the game had been so well wrought that a real coalition of communities of players had formed, one that was able to set aside the differences between the communities, at least for a night, in pursuit of a common goal. This was not intended nor foreseen by Sony, and getting two hundred people to focus on accomplishing the same task is a challenge.

Why, you might ask, would anyone waste four hours of their life doing this? Because a game said it couldn't be done.

This is like the Quake freaks that fire their rocket launchers at their own feet to propel themselves up so they can jump straight to the exit and skip $90 \%$ of the level and finish in 2 seconds. Someone probably told them they couldn't finish in less than a minute. 
Games are about challenges, about hurdles or puzzles or fights overcome. To some players, the biggest hurdle or challenge is how to do what you (the designer) said couldn't happen. If you are making a game, accept this. [17]

A typical problem in virtual communities is caused by the ease in which new participants can enter the community, known as "newbies." The virtual communities should be able to defend itself from dangerous new players, and normative systems are a way to pose virtual gates to such communities. "Griefers would also maintain numerous alts that were sent out into greater Alphaville in attempts to scam and disrupt other houses. Because alts were usually abandoned soon after they had been created, they appeared to others as new characters, and this had the effect of making many players highly suspicious of newbies, and of generating virtual gated communities in response." [17] However, a virtual space should be able to deal with honest new participants. It has been noted that existing communities establish practices which tend to exclude newly entered participant in the virtual space: "Processes of norm building were visible, resulting in patterns of established users versus outsiders; new bonds were created, and users experience an appropriation of this newly created virtual public space: parts of the Digital City were 'taken over' by active established users who behaved as a closed community and were perceived accordingly by the outsiders." [22]

As illustrated by the "newbies" example, there are some aspects in which normative systems for virtual communities are more challenging than traditional regulations. For example, the construction of autonomous virtual communities cannot ground itself on an external legal system - apart from most serious cases like frauds going beyond the virtual environmentas in e-commerce applications that ground the validity of online contracts on the relevant human regulations. Consequently, these normative systems should be developed separately, in the same way as different national systems are created independently. Another issue is related to the possibility to augment actions in virtual scenarios: in these scenarios characters can be created with their own behavior that have more abilities then humans in the real world (e.g., flying, walking through wall), objects nor existing in reality, and even places. Moreover, the abilities of characters are not only related to the ones of their players: e.g., an avatar in Second Life entering a dancing room can acquire new dancing abilities which it did not have before and will lose afterwards. Thus the autonomy of characters assumes new dimensions.

\section{Ten research challenges for the interactionist view}

For the 10 challenges posed by the interactionist viewpoint, we take the perspective from an agent programmer, and consider which kinds of tools like programming primitives, infrastructures, protocols, and mechanisms she needs to deal with norms in the example scenario. Similar needs exist at the requirements analysis level, or the design level, but we have chosen for the programming level since it makes the discussion more concrete, and this level is often ignored when norms are discussed. The list is not exhaustive, and there is some overlap between the challenges. Our aim is to illustrate the range of topics which have to be studied, and we therefore do not attempt to be complete.

Challenge 1 Tools for agents supporting communities in their task of recognizing, creating, and communicating norms to agents.

Even if social norms emerge informally, e.g., when a community becomes more complex and more open, an explicit representation of norms becomes necessary. There are still numerous philosophical problems for the representation of norms, see, for example, [13]. However, 
the new problem is the role of the agents and humans involved in the interaction with the multiagent system.

Challenge 2 Tools for agents to simplify normative systems, recognize when norms have become redundant, and to remove norms.

Challenge 2 is the counterpart of Challenge 1, because the natural tendency of overregulation creates the need for a counterbalance. Since all norms come with a cost, for example to process them, to communicate them, to maintain them, or to enforce them, norms should only be introduced when they are really needed, and they should be removed as soon as they are no longer needed. For example, when the number of violations is increasing, this is typically a case where norms must be changed or removed, rather than where norm enforcement has to be increased.

\section{Challenge 3 Tools for agents to enforce norms.}

If we allow communities of agents to create their own normative multiagent systems, then the issue of how to enforce the norms arises. In case a centralized approach is needed, the infrastructure should support the enforcement of norms created by the communities. In a distributed approach, roles should be defined for agents in charge of monitoring and sanctioning. The virtual environment can offer new opportunities for norm enforcement not found in the usual environments. For example, evidence about agent behaviors can be collected via the logfiles of the system.

\section{Challenge 4 Tools for agents to preserve their autonomy.}

Challenge 4 is the counterpart of Challenge 3, because there is a natural tendency to enforce norms by regimenting them into the system. The danger highlighted by Castelfranchi [8] is related to the "formalization" of the informal. Norms have the nature of general directives which cannot cover all cases nor avoid all conflicts with other norms. Thus, normative multiagent systems need to preserve the autonomy of agents regarding the making of decisions about norm compliance and norm violation. Agents in charge of monitoring and enforcing norms should be flexible enough to preserve the autonomy of the agents subject to the norms with respect to norm violations, for instance in circumstances that differ from the circumstances which the norms have been defined to preserve and where norm compliance is not advantageous for the normative multiagent system.

\section{Challenge 5 Tools for agents to construct organizations.}

As the example about EverQuest example shows, cooperation among the participants of virtual reality can result in coalitions which can achieve results which go beyond the ones reachable by their members. This is of great interest for participants in virtual reality, also because Second Life is becoming a place where business takes place. Thus, participants should be given some facilities and tools which allow the construction and management of organizations to achieve their goals. Note that in the real world such mechanisms exist, first of all the laws which allow the creation of organizations and attribute the responsibilities to different entities. E-institutions as proposed in multiagent systems can be a starting point, but they are often too flat-i.e., not hierarchically organized-and they usually do not support the dynamics of the underlying normative systems by allowing the creation of new norms.

Challenge 6 Tools for agents to create intermediate concepts and normative ontology, for example to decide about normative gaps. 
In real institutions norms have a fuzzy character in the sense that they are not able to cover all possible situations. In particular because new situations can arise, e.g., due to technological advancement (for instance: is a digital signature the same as an handwritten signature?) This problem increases exponentially in virtual worlds where all kind of new behaviors and objects can be defined. The solution in real normative systems is to endow some agents with powers to decide whether a new concept is subsumed by another one. The role of agents in the logical reasoning of a normative system is something which is still missing in the state of the art of the field.

\section{Challenge 7 Tools for agents to decide about norm conflicts.}

This challenge is related to Challenge 6 since norms do not cover all possible cases and conflicts between norms are possible. Thus agents need a mechanism to take decisions in situations of conflicting norms. The mechanism cannot always be automated, for example because the degree of freedom in virtual world to create new behaviors and objects norms may become underspecified. Thus, the problem is to define normative systems, where, like in human normative systems, roles are defined and role keepers are empowered to take decision when automated reasoning alone is not enough. At some point, the view of the normative system as a self contained logical system is not viable anymore.

Challenge 8 Tools for agents to voluntarily give up some norm autonomy by allowing automated norm processing in agent acting and decision making.

In many examples, the autonomy of the agent must be adjusted to the context. In general avatars are graphical representations of users of a system and can be seen as interface agents. Avatars living in Second life are interface agents for human players but also increasingly for autonomous agents. Consider the example above, where new abilities like dancing are automatically added to the avatar. Moreover, even if now prohibited, autonomous agents should be allowed to on the player's behalf cope with events that occur when the player is not online. It is possible to envisage a scenario where avatars are partially programmed to take autonomous decisions when the player is off-line. Among these decisions is whether to comply with norms of the community the avatar is acting into.

Note that these mechanisms are useful not only when the avatar is acting autonomously on behalf of its off-line owner, but also during the activity of the player. In real life norms are often violated just by distraction, ignorance or by lack of resources and the violator does not gain anything by its deviant behavior. The same will eventually happen in virtual worlds, especially when norms to be respected will not be necessarily intuitive or similar to the ones of real world. In these cases, the decision to conform to norms can be left to the avatar and the player can be relieved from this task. E.g., consider the case of communities where nudity of avatars is prohibited. The player could simply leave to its avatar the burden to conform to the norms by automatically disabling actions which are deviant with respect to the norms.

\section{Challenge 9 Tools for conviviality.}

Since scenarios like Second life are aiming at people having pleasant social interactions, and norms may interfere with the goals of the players, the impact of norms on this dimension must be considered. Norms should not constrain the freedom of participants too much and allow to avoid unpleasant behavior from other agents, but there is also a more subtle effect to be considered. Social interaction is regulated by social conventions, which can be modeled as a sort of institution. Part of the fun of "living" in Second life, like when participating in a carnival or when embodying a character of a drama depends-according to Taylor [6,21] 
who calls this effect "conviviality" - is the temporary displacement with respect to the usual norms of social life. In particular, in the sense that in social relations the player acquires new social powers which he does not have in his first life.

The tools for conviviality should study social dependencies among players and indicate how these dependencies can be made less unbalanced by attributing more social powers to some players. Note that, as in the example about automatic learning of dancing abilities in Sect. 2, adding social powers in a virtual reality can take a more extended sense, since in the real world physical abilities cannot be added. Tools for conviviality should also facilitate the introduction of new participants in a virtual community by addressing the "newbies" problems.

\section{Challenge 10 Tools for legal responsibility of the agents and their principals.}

Nowadays, agents become subjects of human legislation. For example, it is debated if agents have responsibilities beyond the ones attributed to their owner, or if agents can be really attributed mental states which are to be taken into account in the attribution of responsibilities. However, in scenarios like Second life, new questions arise. Participants accept the rules of the game and they should be made aware whether following the rules of some communities leads to infringement of real legislations.

\section{Papers in this issue}

This special issue on Normative Multiagent Systems includes four papers, selected from over thirty papers presented at the second workshop on normative multiagent systems in Dagstuhl. The papers start to address the 10 challenges of the interactionist view, covering different dimensions of the normative multiagent systems field: the first one is a recent evolution of the traditional deontic logic approach, the second paper concerns the interaction between mental attitudes and norms, the third article addresses the ontological point of view on collectives of agents who are regulated by norms, and the fourth one provides an automatic translation from norm specification to a rule based implementation.

"Prioritized Conditional Imperatives: Problems and a New Proposal" by Jörg Hansen observes a problem with reasoning about prioritized imperatives. It surveys various ways in which conflicts among conflicting imperatives are resolved, and concludes that none of them satisfies the relevance postulate that "any imperative should be considered relevant (included) as long as it is not violated or, in the given situation, conflicts with other imperatives that are also considered relevant (included) and do not rank lower." A logical framework for conditional imperatives is introduced in the tradition of input/output logic, extended with priorities. In this formal framework, a new conflict resolution mechanism satisfying the postulate is defined. The relevance of this result extends to reasoning with conditionals in other domains such as reasoning about prioritized default rules.

"BIO Logical Agents: Norms, Beliefs, Intentions in Defeasible Logic" by Guido Governatori and Antonino Rotolo studies an exemplary problem of interaction among norms and mental attitudes. The authors follow the BOID (Belief, Obligation, Intention, Desire) architecture [5] approach to describe agents and agent types. In this view the behavior of agents is the outcome of a rational balance among their (possibly conflicting) mental states and also normative (external) factors such as obligations. The paper addresses several issues: first of all the definition of policy-based motivations, as rules, which are triggered by potentially recurring circumstances in agent's life. Secondly, in contrast with the idea that side effects are never intended, the authors argue that some side effects could be intended, which 
is particularly important for the notion of responsibility in legal contexts. Thirdly, in the formal mechanism the concept of rule conversion is introduced, which allows to derive some motivations by using rules devised for inferring different motivations (e.g., intentions from beliefs). Fourthly, the notion of agent type has been analyzed from the complexity point view. To deal with the side-effect problem, conflict resolution turns out to be very expensive in case of social agents. Finally, the paper is important also for its methodological choice: the formalism used to analyze the problem is Defeasible Logic, a non-monotonic logic-since it has to deal with conflicts—which is computationally feasible—-linear complexity — so that it provides a realistic tool also to implement agents and not only to study their properties.

In "Norms and Plans as Unification Criteria for Social Collectives" Aldo Gangemi builds an ontology for "normative intentional collectives." If collective acceptance as discussed by Searle [18] is the basis of social reality, and thus also of normative multiagent systems, then the notion of collective has not yet received enough attention. This paper fills this gap providing a first order formalization as well as an OWL (i.e., Description Logic) representation of the relation between norms and collectives. The ontology is based on the distinction between descriptions and situations to separate relations between 'conceptual elements,' like norms, from relations between 'observable elements,' like cases, states of affairs, etc. described by norms. Formally, a reification mechanism allows to have descriptions and situations in the same domain of quantification (i.e. at the same logical level), and to relate them by means of a reified relation of satisfiability. Gangemi criticizes the definition of normative multiagent system given in [3] (see Sect. 1) in that it detaches the normative system from the multiagent system. Norms are a specification of a conceptualization whose objective is regulatory, and social agents use norms as constraints within their own plans. This view leads to the definition of intentional normative collectives as knowledge communities unified by a plan that, in turn, is entrenched with norms according to the possible interaction between norms and plans. Here knowledge communities are collections of agents unified by descriptions that are shared by the member agents.

The paper "From the Specification to the Implementation of Norms: An Automatic Approach to Generate Rules from Norms to Govern the Behavior of Agents" by Viviane Torres da Silva deals with the specification of norms and their implementation by defining an automatic transformation to generate the implementation based on the specification. In this way specifications of norms can be given without having to also learn the implementation language. The specification language provided extends previous proposals under several respects: not only dialogical actions are the object of norms, conditions and temporal situations related to norms are introduced, sanctions pointing out the authorized agents that can apply the punishments are defined, and norms activate other norms conditioned to their activation, deactivation, fulfillment or violation. All these features of the specification of a norm find support in the implementation provided in Jess: the translation from the norm specification to the Jess rules is made via an automated translator. The Jess system can be used by the agents who must be aware of the active norms as well as by the governance mechanism to become aware of the fulfilled norms and norms violations in order to apply the corresponding sanctions.

\section{Future research}

The four papers selected from NorMAS07 touch upon the issues we described in the section on 10 challenges for the interactionist view of normative multiagent systems. We expect the papers of NorMAS08 to further delve into these issues and that NorMAS09 will clarify the 
challenges even more, hopefully even with implementations of interactionalist mechanisms in hybrid social games.

\section{References}

1. Anderson, M., \& Leigh Anderson, S. (2007). Machine ethics: Creating an ethical intelligent agent. AI Magazine, 28(4):15-26.

2. Boella, G., Caire, P., \& van der Torre, L. (to appear). Norm negotiation in online multi-player games. Knowledge and Information Systems.

3. Boella, G., van der Torre, L., \& Verhagen, H. (2006). Introduction to normative multiagent systems. Computation and Mathematical Organizational Theory, Special Issue on Normative Multiagent Systems, 12(2-3), 71-79.

4. Boella, G., van der Torre, L., \& Verhagen, H. (Eds.) (2007) Normative multiagent systems. Dagstuhl Seminar Proceedings (Vol. 07122). Schloss Dagstuhl, Germany: Internationals Begegnungs- und Forschungszentrum für Informatik (IBFI).

5. Broersen, J., Dastani, M., Hulstijn, J., \& van der Torre, L. (2002). Goal generation in the BOID architecture. Cognitive Science Quarterly, 2(3-4), 428-447.

6. Caire, P., Villata, S., Boella, G., \& van der Torre, L. (2008). Conviviality masks in multiagent systems. In Proceedings of the Seventh International Conference on Autonomous Agents and Multiagent Systems (AAMAS'08).

7. Castelfranchi, C. (1998). Modeling social action for AI agents. Artificial Intelligence, 103(1-2), 157-182.

8. Castelfranchi, C. (2003). Formalising the informal? Dynamic social order, bottom-up social control, and spontaneous normative relations. Journal of Applied Logic, 1(1-2), 47-92.

9. Giddens, A. (1984). The constitution of society. University of California Press.

10. Goble, L., \& Meyer, J. J. Ch. (Eds.) (2006). Deontic logic and artificial normative systems. In Proceedings of the 8th International Workshop on Deontic Logic in Computer Science, DEON 2006, Utrecht, The Netherlands, July 12-14, 2006, Proceedings. Lecture Notes in Computer Science (Vol. 4048). Springer.

11. Goble, L., \& Meyer, J. J. Ch. (Eds.). (in press). Revised versions of papers presented in at the proceeding of the eighth international workshop on deontic logic in computer science (DEON06). Journal of Applied Logic.

12. Goffman, E. (1959). The presentation of self in everyday life. Doubleday.

13. Hansen, J., Pigozzi, G., \& van der Torre, L. (2007). Ten philosophical problems in deontic logic. In G. Boella, L. van der Torre, \& H. Verhagen (Eds.), Normative multi-agent systems. Dagstuhl Seminar Proceedings (Vol. 07122). Schloss Dagstuhl, Germany: Internationales Begegnungs- und Forschungszentrum für Informatik (IBFI).

14. Lomuscio, A., \& Nute, D. (Eds.) (2004). Deontic logic in computer science. In 7th International Workshop on Deontic Logic in Computer Science, DEON 2004, Madeira, Portugal, May 26-28, 2004. Proceedings. Lecture Notes in Computer Science (Vol. 3065). Springer.

15. Lomuscio, A., \& Nute, D. (2005). Revised versions of papers presented in the proceeding of the seventh international workshop on deontic logic in computer science (DEON04). Journal of Applied Logic, 3(3-4).

16. Luck, M., McBurney, P., \& Preist, C. (2003). Agent technology: Enabling next generation computing (A roadmap for agent based computing). AgentLink.

17. Ludlow, P., \& Wallace, M. (2007). The second life herald. Cambridge, MA: MIT Press.

18. Searle, J. R. (1995). The construction of social reality. New York: The Free Press.

19. Shoham, Y., \& Tennenholtz, M. (1995). On social laws for artificial agent societies: Off-line design. Artificial Intelligence, 73(1-2), 231-252.

20. Strauss, A. (1978). Negotiations: Varieties, contexts, processes and social order. Jossey-Bass: San Francisco.

21. Taylor, M. (2004) Oh no it isn't: Audience participation and community identity. Trans, Internet Journal for cultural sciences, $1(15)$.

22. van den Besselaar, P. (2001). E-community versus e-commerce: The rise and decline of the Amsterdam digital city. AI and Society, 15(3), 280-288.

23. van der Meyden, R., \& van der Torre, L. (Eds.) (in press). Deontic logic in computer science. In 9th International Conference on Deontic Logic in Computer Science, DEON 2008, Luxembourg, July 16-18, 2008, Proceedings. Lecture Notes in Computer Science (Vol. 5076). Berlin: Springer.

24. Verhagen, H. (2000). Norm autonomous agents. PhD thesis, Department of System and Computer Sciences, The Royal Institute of Technology and Stockholm University, Sweden. 


\title{
Norm Negotiation in Online Multi-Player Games
}

\author{
Guido Boella ${ }^{1}$ \\ Patrice Caire ${ }^{2}$ \\ Leendert van der Torre ${ }^{2}$ \\ 1 Dipartimento di Informatica, Università di Torino, Italy. \\ 2 University of Luxembourg - Luxembourg.
}

\begin{abstract}
In this paper we introduce an agent communication protocol and speech acts for norm negotiation. The protocol creates individual or contractual obligations to fulfil goals of the agents based on the so-called social delegation cycle. First, agents communicate their individual goals and powers. Second, they propose social goals which can be accepted or rejected by other agents. Third, they propose obligations and sanctions to achieve the social goal, which can again be accepted or rejected. Finally, the agents accept the new norm by indicating which of their communicated individual goals the norm achieves. The semantics of the speech acts is based on a commitment to public mental attitudes. The norm negotiation model is illustrated by an example of norm negotiation in multi-player online gaming.
\end{abstract}

Keywords: Multi-player online games, normative multi-agent systems, dependence networks, social delegation cycle, agent communication protocols.

\section{Introduction}

Online multi-player games are online spaces in which human and artificial agents interact with each other every day [4]. For example, EverQuest has more than four hundred thousand subscribers and has developed into a complex system of social dynamics, like The Sims Online or Second Life, something more than just a game. Massively multiplayer online games have now become among the most complex and sophisticated online social spaces in existence [34]. One aspect in which online games move towards 
social spaces is that players become more independent and autonomous with respect to the game designers, in the sense that they start to play in ways unforeseen by the game designers.

"Why, you might ask, would anyone waste four hours of their life doing this? Because a game said it couldn't be done.

This is like the Quake freaks that fire their rocket launchers at their own feet to propel themselves up so they can jump straight to the exit and skip $90 \%$ of the level and finish in 2 seconds. Someone probably told them they couldn't finish in less than a minute.

Games are about challenges, about hurdles or puzzles or fights overcome. To some players, the biggest hurdle or challenge is how to do what you (the designer) said couldn't happen. If you are making a game, accept this." [34]

An important aspect of autonomy, both for individuals and groups, is the creation of one's own norms, as reflected by the literal meaning of autonomy: auto-nomos. In online games, players can create their own norms to coordinate their activities, as we illustrate by a running example in this paper. Moreover, autonomy is a central concept in agent theory, and therefore multi-agent systems technology can be used to develop online games. However, existing social mechanisms for norm creation and change in multiagent systems are restricted in the sense that either norms are not explicitly represented, or there are no communication primitives, protocols and social mechanisms to create or change norms. Normative multi-agent systems [21, 13, 8, 9] provide agents with abilities to automatically devise organizations and societies coordinating their behavior via obligations, norms and social laws. A distinguishing feature from group planning is that also sanctions and control systems for the individual or contractual obligations can be created. Since agents may have conflicting goals with respect to the norms that emerge, they can negotiate amongst each other which norm will be created. In [10] we assume that the social goals emerge, and in [11] we propose a two step negotiation process:

- Propose the social goal and argue about that (instead of merging), and

- Negotiate the norm and sanction.

The research question of this paper is how to define a communication protocol for online multiplayer games and social spaces based on the social delegation cycle $[11,10]$ explaining the negotiation of new social norms from cognitive agent goals in three steps. First individual agents or their representatives negotiate social goals, then a social goal is negotiated in a social norm, and finally the social norm is accepted by an agent [20] when it recognizes it as a norm, the norm contributes to the goals of the agent, and it is obeyed by the other agents. A model of norm negotiation explains also what it means, for example, to recognize or to obey a norm, and how new norms interact with existing ones. This breaks down in the following three sub-questions:

1. How to use agent technology for the social delegation cycle referring to the individual goals of the agents, which are not accessible for other agents and which are not represented in the agent environment? We propose an additional step before the social delegation cycle, in which the agents communicate their individual goals and powers. In our communication model, the social delegation cycle does not refer to the individual private goals of the agents, but to the communicated and therefore public goals.

2. How do agents publicly accept a norm in the social delegation cycle based on public mental attitudes? We assume that each agent has to commit itself to the social norm by indicating which of its publicly communicated goals the norm achieves. 
3. How to use the communication protocol in multi-player online games like EverQuest and social spaces like The Sims Online or Second Life, where the kind of norms that must be used cannot be anticipated beforehand, norms are created by human players and users, and we cannot expect that users are able to specify logical formulas as used in most agent communication languages. We use an abstract power and dependence based representation of the social delegation cycle.

The semantics of the speech acts is based on public mental attitudes. To keep the formal details of this paper within limits, we do not detail the operational semantics of the speech acts, but sketch informally their effects and illustrate them by a running example. Formalizations of speech acts with public mental attitudes can be found in $[26,39,25]$.

An important subtask of the social delegation cycle is to break down goals in subgoals, using a goal hierarchy. However, to focus on the main social mechanism, in this paper we do not detail the sub-protocol for subgoaling. Therefore, we assume that the individual goals communicated by the agents are such that they can be seen to by other agents. The extension with a protocol for subgoaling is left for further research.

The layout of this paper is as follows. In Section 2 we discuss norm creation in online games and social spaces, and we introduce our running example. In Section 3 we define our social-cognitive conceptual model of multiagent systems and in Section 4 we discuss the kind of agent communication language in which we study and formalize the social delegation cycle. In Section 5 we formalize individual goal and power communication. In Section 6 we define the negotiation protocol, which we use in Section 7 to formalize goal negotiation, and in Section 8 we formalize norm negotiation. Finally, in Section 9 we formalize the acceptance relation. In Section 10 we discuss related research of an abstract model of the social delegation cycle in Tennenholtz' game-theoretic artificial social systems, our own more detailed social-cognitive model, and we discuss why these models are less suitable for communication models in online games and social spaces than our power and dependence based model.

\section{Multi player online games}

As a running example we use in this paper an example discussed by Peter Ludlow [34] from Sony's EverQuest.

\subsection{EverQuest}

EverQuest is a multiplayer online game where gamers are supposed to fight each other in a world of snakes, dragons, gods and the Sleeper. Sony intended the Sleeper to be unkillable and gave it an extreme high hit points. However, a combined combat of close to 200 players nearly succeeded to kill the animal. Unfortunately, Sony decided to intervene and rescue the monster. Most of the discussion on this example has highlighted the decrease in trust of the game players in Sony, despite the fact that the next day Sony let the game players beat the Sleeper. However, in this paper we would like to highlight what this story tells us about the desires of game players, and its consequences for necessary technology in games. The following quote illustrates the excitement in killing the Sleeper.

A supposedly [player-vs.-player] server banded together 200 people. The chat channels across the server were ablaze, as no less than 5,000 of us listened in, with OMG theyre 
attempting the Sleeper! Good luck d00dz! Everyone clustered near their screens, sharing the thrill of the fight, the nobility of the attempt and the courage of those brave 200. Play slowed to a crawl on every server as whispers turned to shouts, as naysayers predicted, It cant be done or It will drop a rusty level 1 sword and most of us just held our breath, silently urging them forward. Rumors abounded: If they win, the whole EQ world stops and you get the text from the end of Wizardry 1, or If they win, the president of Sony will $\log$ on and congratulate them. With thousands watching and waiting, the Sleepers health inched ever downward.

[EverQuest player] Ghenwivar writes, On Monday, November 17th, in the most amazing and exciting battle ever, [EverQuest guilds] Ascending Dawn, Wudan and Magus Imperialis Magicus defeated Kerafyrm, also known as The Sleeper, for the first time ever on an EverQuest server. The fight lasted approximately three hours and about 170180 players from [EverQuest server] Rallos Zeks top three guilds were involved. Hats off to everyone who made this possible and put aside their differences in order to accomplish the impossible. Congratulations RZ!!!"” [34]

The example illustrates that the game had been so well wrought that a real community of players had formed, one that was able to set aside its differences, at least for a night, in pursuit of a common goal. This was not intended or foreseen by Sony, and getting two hundred people to focus on accomplishing the same task is a challenge. In this paper we study multiagent technology to support these emerging cooperation in online games.

\subsection{Multiagent systems}

Computer games have traditionally implemented empirical solutions to many AI problems and are now turning to more traditional AI algorithms. Cavazza [18] introduces the role of AI in gameplay, reviews the main techniques used in current computer games such as Finite-State Transition Networks, rule-based systems and search algorithms, describes the implementation of AI in several commercial computer games, as well as academic research in AI targeting computer games applications, and discusses future trends and proposing research directions.

"Another important challenge for AI in computer games is the development of on-line and multi-player gaming. In theory, the availability of human opponents around the clock would seal the fate of AI in on-line games. However, this is unlikely to be the case, as for instance autonomous actors would still be needed for the most mundane tasks in large-scale simulations and on-line games, as human players are unwilling to play such parts.

The recent advances in graphic rendering will leave more room for the future development or AI in games. With the level of realism currently achieved, further improvements in realism will come from physics and AI, The development of AI should also foster the development of new game genres based on more sophisticated user interaction with artificial actors." [18]

Laird and van Lent [30] propose that artificial intelligence for interactive computer games is an emerging application area in which this goal of human-level AI can successfully be pursued. Interactive computer games have increasingly complex and realistic worlds and increasingly complex and intelligent computer-controlled characters. They motivate their proposal of using interactive computer games, review previous research 
on $\mathrm{AI}$ and games, present the different game genres and the roles that human level AI could play within these genres, describe the research issues and AI techniques that are relevant to each of these roles. Their conclusion is that interactive computer games provide a rich environment for incremental research on human-level AI.

"From a researcher's perspective, even if you are not interested in human-level AI, computer games offer interesting and challenging environments for many, more isolated, research problems in AI. We are most interested in human-level AI, and wish to leverage computer games to rally support for research in human-level AI. One attractive aspect of working in computer games is that there is no need to attempt a Manhattan Project approach with a monolithic project that attempts to create human-level intelligence all at once. Computer games provide an environment for continual, steady advancement and a series of increasingly difficult challenges. Just as computers have inexorably gotten faster, computer game environments are becoming more and more realistic worlds, requiring more and more complex behavior from their characters. Now is the time for AI researchers to jump in and ride the wave of computer games." [30]

\subsection{Agent communication protocol}

We use the EverQuest example in this paper to illustrate our communication model. It consists of the following four steps.

1. Agents communicate their individual goals and powers. They say which monster they would like to kill, how they want it to be killed, and which tasks they are able to see to given their powers.

2. They propose social goals which can be accepted or rejected by other agents, based on the communicated goals of the agents. The negotiation can be done by a subset of all players involved.

3. They propose obligations and sanctions to achieve the social goal, which can again be accepted or rejected. Again, the negotiation can be done by a subset of the agents involved in the coordinated attack.

4. Finally, the agents who like to participate in the coordinated attack accept the new norm by indicating which of their communicated individual goals the norm achieves.

\section{Power viewpoint on normative multiagent systems}

In this paper we follow the definition of power as the ability of agents to achieve goals. Thus, an agent is more powerful than another agent if it can achieve more goals.

For example, in the so-called power view on multi-agent systems [5], a multi-agent system consists of a set of agents $(A)$, a set of goals $(G)$, a function that associates with each agent the goals the agent desires to achieve (goals), and a function that associates with each agent the sets of goals it can achieve (power). To be precise, since goals can be conflicting in the sense that achieving some goals may make it impossible to achieve other goals, the function goals returns a set of set of goals for each set of agents. Such abstract structures have been studied as qualitative games by Wooldridge and Dunne [53], though they do not call the ability of agents to achieve goals their power. To model trade-offs among goals of agents, we introduce a priority relation among goals.

Definition 1. Let a multiagent system be a tuple $\langle A, G$, goals, power, $\geq\rangle$ where: 
-the set of agents $A$ and the set of goals $G$ are two finite disjoint sets;

-goals : $A \rightarrow 2^{G}$ is a function that associates with each agent the goals the agent desires to achieve;

-power : $2^{A} \rightarrow 2^{2^{G}}$ is a function that associates with each set of agents the sets of goals the set of agents can achieve;

$-\geq: A \rightarrow \subseteq 2^{G} \times 2^{G}$ is a function that associates with each agent a partial pre-ordering on the sets of his goals;

The following example illustrates the notion of a multiagent system for the running example.

Example 1. The set of agents are the players that participate in the coordinated attack. We assume that the individual agents have various ways to attack the Sleeper, and they disagree about which is the best way to kill the beast. The goals of the agents are different individual ways to attack the animal. The powers of the agents are the goals each agent can achieve. The priorities of the agents are their preferences for the ways to attack the animal.

To model the role of power in norm negotiation, we extend the basic power view in a couple of ways. To model obligations we introduce a set of norms, we associate with each norm the set of agents that has to fulfill it, and for each norm we represent how to fulfill it, and what happens when it is not fulfilled. In particular, we relate norms to goals in the following two ways.

- First, we associate with each norm $n$ a set of goals $O(n) \subseteq G$. Achieving these normative goals $O(n)$ means that the norm $n$ has been fulfilled; not achieving these goals means that the norm is violated. We assume that every normative goal can be achieved by the group, i.e., that the group has the power to achieve it.

- Second, we associate with each norm a set of goals $V(n)$ which will not be achieved if the norm is violated (i.e., when its goals are not achieved), this is the sanction associated with the norm. We assume that the group of agents does not have the power to achieve these goals.

Since we accept norms without sanctions, we do not assume that the sanction affects at least one goal of each agent of the group the obligation belongs to.

Definition 2. Let a normative multi-agent system be a tuple $\langle M A S, N, O, V\rangle$ extending a multiagent system $M A S=\langle A, G$, goals, power, $\geq\rangle$ where:

-the set of norms $N$ is a finite set disjoint from $A$ and $G$;

$-O: N \times A \rightarrow 2^{G}$ is a function that associates with each norm and agent the goals the agent must achieve to fulfill the norm; We assume for all $n \in N$ and $a \in A$ that $O(n, a) \in \operatorname{power}(\{a\})$;

$-V: N \times A \rightarrow 2^{G}$ is a function that associates with each norm and agent the goals that will not be achieved if the norm is violated by agent $a$; We assume for each $B \subseteq A$ and $H \in \operatorname{power}(B)$ that $\left(\cup_{a \in A} V(n, a)\right) \cap H=\emptyset$.

The normative multiagent system is illustrated by our running example.

Example 2. The obligations of each agent are the attacks each individual player must make. Sanctions can be that someone is removed from the coalition, has to pay a penalty (if this is possible in the game), gets a lower reputation, and so on.

An alternative way to represent normative multiagent systems replaces the function 
power by a function representing dependencies between agents. For example, a function of minimal dependence can be defined as follows. Agent $a$ depends on agent set $B \subseteq A$ regarding the goal $g$ if $g \in \operatorname{goals}(a), g \notin \operatorname{power}(\{a\}), g \in \operatorname{power}(B)$, and there is no $C \subset B$ such that $g \in \operatorname{power}(C)$. Note that dependence defined in this way is more abstract than power, in the sense that we have defined dependence in terms of power, but we cannot define power in terms of dependence.

\section{Agent communication languages}

Agent communication languages share the idea that agents communicate by performing speech acts $[3,40]$, and that these actions can be modeled as some types of planning operators with preconditions and effects $[19,2]$. Longer stretches of dialogue can be explained by plans which structure attempts of participants to reach their joint goals, e.g., [32]. However, many variations of agent communication languages have been designed on these common speech act foundations, thus making the standardization effort difficult.

There is a distinction in terms of communication primitives between two main traditions in agent communication. The agent's mental attitudes like beliefs and intentions are included in preconditions and effects of speech acts used in information seeking dialogues using speech acts such as inform and request. This side has been popularized by the standardization efforts of the Foundation for Intelligent Physical Agents [23], though the concepts of desire, goal and intention are not used uniformly, and desire and goal are often used interchangeably. Below the agent social semantics tradition, developed as a reaction to FIPA, is depicted [45]. The agent can either be a creditor or a debtor of his social commitments. The semantics of speech acts used in negotiation or persuasion refer to these commitments. Though both kinds of dialogue use the terminology of commitment, requesting an action in negotiation means something else than defending a proposition in persuasion after a challenge. We therefore distinguish action commitment from propositional commitment, where the former is used to give a semantics of speech acts like request or propose in negotiation [45, 24], and the latter is used for speech acts like assert or challenge in persuasion [28, 52].

In this paper we use an approach bridging the two traditions based on a reinterpretation of the beliefs and intentions. The idea that meaning cannot be private, but is inter-subjective or based on a common ground, has been accepted for a long time in the philosophy of language. This idea of public meaning has been discussed, for example, by [31] or [46], who stress the importance of the common ground. The crucial point is that a private semantics does not make it possible for a language user to be objectively wrong about an interpretation from a third person point of view. In contrast to FIPA, beliefs and intentions are not interpreted as private mental attitudes, but as some kind of public mental attitudes, for example as grounded beliefs [26] or as ostensible beliefs or public opinions [39].

\section{Individual goal and power communication}

The first phase consists of communicating the individual goals and powers of the agents. Since existing agent communication languages do not cover inform speech acts whose content is a public goal, we introduce the speech act desire. The semantics of the speech act

$$
\text { desire }(a, \phi)
$$


is that agent $a$ has the public goal $\phi$. It means that he is committed to this public goal, and, for example, he cannot say later that he desires the opposite.

If hundreds of agents have to communicate their goals, then the communication will become long and cumbersome. So, when an agent informs the others that he desires $\phi$, the other agents can respond by a simple 'Me too!'. Moreover, we do not have only speech acts implying that the speaking agent has a goal, but also speech acts implying that all agents have the same goal. Agent $a$ says that everyone has the desire for $\phi$ :

desireall $(a, \phi)$

The agents can respond to this speech act by saying 'Not me!'. If none of them answers in this way, then the desireall act is equivalent to agent $a$ stating his goal for $\phi$, and all the other agents saying 'Me too!'. The principle that by default all agents agree, is sometimes called 'silence means consent'.

An optional parameter of these two speech acts is the priority of the individual goals. In this running example we assume that there are five priorities: essential, important, intermediate, useful, possible.

Example 3. Suppose that three agents $\{a, b, c\}$ are going to negotiate the attack strategy to kill the Sleeper. Moreover, we assume there are various ways in which the individual players can attack the animal: hitting the feet, throwing stones at the animal, shooting between the eyes, and so on. The three agents are negotiating for three kinds of game players with specific powers. For example, agent $a$ is negotiating for all agents that have the power to hit the feet, throw stones, and have guns. In this example, each negotiator communicates goals they cannot see to by themselves. For example, agent $a$ will communicate the desire to scare the Sleeper to death.

The negotiators begin by communicating their individual goals. Each player can type individual or shared goals by entering a text string into his computer. In the text box is also a list of goal priorities, which is default at intermediate. When someone types an individual goal, other agents can press the 'Me too!' button, When an agent enters a shared goal, other agents can press the 'Not me!' button, or change the suggested priority of the goal. For example, when agent $a$ enters his goal and agent $b$ accepts it too, and agent $c$ communicates a shared goal but agent $d$ refuses it, then the system translates it into the following speech acts.

desire ( $b$, 'hitting the feet')

desire ( $b$, 'hit him with stones', intermediate)

desire me too( $c$, 'hit him with stones', important)

desire (c, 'shoot him in the eyes', possible)

desire ( $a$, 'show him a picture of M.')

desireall ( $a$, 'scare him to death')

desire not me ( $c$, 'scare him to death')

Each agent can indicate which goals it can see to. We use the new speech act power $(a, \phi)$, indicating that agent $a$ communicates that he has the power to see to goal $\phi$. As before, the other agents can respond by 'Me too!' and there is a speech powerall which agents can respond to by saying 'Not me!'.

Example 4. Consider the following three speech acts.

power ( $a$, 'hitting the feet')

powerall ( $a$, 'hit him with stones')

power not me ( $c$, 'hit him with stones')

powerall ( $c$, 'shoot him in the eyes')

... 


\begin{tabular}{ccccc} 
\# goal & speaker & agents and priorities & goal description & powers \\
\hline 1 & $b$ & $b, c$ & hitting the feet & $a$ \\
2 & $b$ & $b:$ intermediate, $c:$ important & hit him with stones & $a, b$ \\
3 & $c$ & $c:$ possible & shoot him in the eyes & $a, b, c$ \\
4 & $a$ & $a$ & show him a picture of M. & $b, c$ \\
5 & $a$ & $a, b$ & scare him to death & $c$ \\
$\ldots$ & $\ldots$ & $\ldots$ & $\ldots$ & $\ldots$
\end{tabular}

Table 1. Communicated goals and powers

The communicated goals and powers are represented in a single table for each player, as shown in Table 1 . In case the agents are negotiating a norm for a large set of players, then not all individual names of the agents are given, but only aggregate information such as the number of players having the power to see to a goal.

\section{Generic negotiation protocol}

A negotiation protocol is described by a set of sequences of negotiation actions which either lead to success or failure. In this paper we only consider protocols in which the agents propose a so-called deal, and when an agent has made such a proposal, then the other agents can either accept or reject it (following an order $-\succ-$ of the agents). Moreover, they can also end the negotiation process without any result.

Definition 3 (Negotiation Protocol). A negotiation protocol is a tuple $\langle A g$, deals, actions, valid, finished, broken, $\succ\rangle$, where:

-the agents $A g$, deals and actions are three disjoint sets, such that actions $=\{$ propose $(a, d), \operatorname{accept}(a, d), \operatorname{reject}(a, d) \mid a \in A g, d \in$ deals $\} \cup$ $\{\operatorname{breakit}(a) \mid a \in A g\}$.

-valid, finished, broken are sets of finite sequences of actions.

We now instantiate this generic protocol for negotiations in normative multiagent systems. We assume that a sequence of actions (a history) is valid when each agent does an action respecting the order defined on agents. Then, after each proposal, the other agents have to accept or reject this proposal, again respecting the order, until they all accept it or one of them rejects it. When it is an agent's turn to make a proposal, it can also end the negotiation by breaking it. The history is finished when all agents have accepted the last deal, and broken when the last agent has ended the negotiations.

Definition 4 (NMAS protocol). Given a normative multiagent system $\langle M A S, N, O, V\rangle$ extending a multiagent system $M A S=\langle A, G$, goals, power, $\geq\rangle$, a negotiation protocol for $N M A S$ is a tuple $N P=\langle A$, deals, actions, valid, finished, broken, $\succ\rangle$, where:

$-\succ \subseteq A \times A$ is a total order on $A$,

-a history $h$ is a sequence of actions, and $\operatorname{valid}(h)$ holds if:

- the propose () and breakit () actions in the sequence respect $\succ$,

- each propose () is followed by a sequence of accept () or reject () actions respecting $\succ$ until either all agents have accepted the deal or one agent has rejected it,

- there is no double occurrence of a proposal propose $(a, d)$ in the same deal by any agent $a \in A g$, and 
the sequence $h$ ends iff either all agents have accepted the last proposal (finished $(h)$ ) or the last agent has broken the negotiation $(\operatorname{broken}(h))$ instead of making a new proposal.

In theory we can add additional penalties when agents break the negotiation. However, since it is in the interest of all agents to reach an agreement, we do not introduce such sanctions. In this respect norm negotiation differs from negotiation about obligation distribution [7], where it may be the interest of some agents to see to it that no agreement is reached. In such cases, sanctions must be added to the negotiation protocol to motivate the agents to reach an agreement.

Example 5. Assume three agents and the following history.

action $_{1}: \operatorname{propose}\left(a, d_{1}\right)$

action $_{2}:$ accept $\left(b, d_{1}\right)$

action $_{3}: \operatorname{reject}\left(c, d_{1}\right)$

action $_{4}: \operatorname{propose}\left(b, d_{2}\right)$

$\operatorname{action}_{5}: \operatorname{accept}\left(c, d_{2}\right)$

action $_{6}: \operatorname{accept}\left(a, d_{2}\right)$

We have $\operatorname{valid}(h)$, because the order of action respects $\succeq$, and we have accepted $(h)$, because the history ends with acceptance by all agents (action ${ }_{5}$ and action $_{6}$ ) after a proposal $\left(\right.$ action $\left._{4}\right)$.

The open issue of the generic negotiation protocol is the set of deals which can be proposed. They depend on the kind of negotiation. In social goal negotiation the deals represent a social goal, and in norm negotiation the deals contain the obligations of the agents and the associated control system based on sanctions. This is illustrated in our running example in the following sections.

\section{Social goal negotiation}

We characterize the allowed deals during goal negotiation as a set of goals which contains for each agent a goal it desires. Moreover, we add two restrictions. First, we only allow goals the agents have the power to achieve. Moreover, we have to consider the existing normative system, which may already contain the norms that look after the goals of the agents. We therefore restrict ourselves to new goals. Additional constraints may be added, for example excluding goals an agent can see to itself. However, since such additional restrictions may be unrealistic in some applications (e.g., one may delegate some tasks to a secretary even when one has the power to see to these tasks oneself), we do not consider such additional constraints.

Definition 5 (Deals in goal negotiation). In the goal negotiation protocol, a deal $d \in$ deals is a set of goals satisfying the following restrictions:

1. $d \in \operatorname{power}(A)$

2. for all $a \in A$ there exists some $g \in d$ such that

(a) $g \in \operatorname{goals}(a)$

(b) there does not exist a norm $n$ in $N$ such that $g \in \cup_{a \in A} O(n, a)$

The following example illustrates social goal negotiation in the running example.

Example 6. The agents proposing a deal select a couple of individual communicated goals from Table 1 by clicking on them. The system then send them to the other agents, 


\begin{tabular}{cccccc} 
\# goal proposal & speaker & goals & accepted & rejected & reason \\
\hline 1 & $a$ & 2,5 & $a$ & $b$ & $\ldots$ \\
2 & $b$ & $2,4,5$ & $a, b, c$ & & \\
$\ldots$ & $\ldots$ & $\ldots$ & $\ldots$ & $\ldots$ &
\end{tabular}

Table 2. Social Goal Negotiation

which can either accept them, or reject them. When they reject a communicated goal, they can add a reason to it, which is communicated to the other agents.

Let $M A S=\left\langle\{a, b, c\},\left\{g_{1}, g_{2}, g_{3}, g_{4}, g_{5}\right\}\right.$, goals, power, $\left.\geq\right\rangle$ be a multiagent system representation of Table 1, where:

power: $\operatorname{power}(a)=\left\{\left\{g_{1}\right\},\left\{g_{2}\right\},\left\{g_{3}\right\}\right\}, \operatorname{power}(b)=\left\{\left\{g_{2}\right\},\left\{g_{3}\right\},\left\{g_{4}\right\}\right\}$, $\operatorname{power}(c)=\left\{\left\{g_{3}\right\},\left\{g_{4}\right\},\left\{g_{5}\right\}\right\}$, if $G_{1} \in \operatorname{power}(A)$ and $G_{2} \in \operatorname{power}(B)$ then $G_{1} \cup G_{2} \in \operatorname{power}(A \cup B)$. Agent $a$ has the power to achieve goals $g_{1}, g_{2}, g_{3}$, agent $b$ has the power to achieve goals $g_{2}, g_{3}, g_{4}$, and agent $c$ can achieve goals $g_{3}, g_{4}, g_{5}$. There are no conflicts among goals.

goals: goals $(a)=\left\{g_{4}, g_{5}\right\}$, goals $(b)=\left\{g_{1}, g_{5}\right\}$, goals $(c)=\left\{g_{1}, g_{2}\right\}$. Each agent desires the tasks it cannot perform itself.

Moreover, let $N M A S=\langle M A S, N, O, V\rangle$ be a normative multiagent system with $N=\{n\}, O\left(n, a_{1}\right)=\left\{g_{1}\right\}$. Since there has to be some benefit for agent $b$ and $c$, the goals $g_{5}$ and $g_{2}$ have to be part of the social goal. Therefore, social goals (i.e., possible deals) are $\left\{g_{2}, g_{5}\right\}$ and $\left\{g_{2}, g_{4}, g_{5}\right\}$.

Finally, consider the negotiation. Assuming agent $a$ is first in the order $\succ$, he may propose $\left\{g_{2}, g_{5}\right\}$. The other agents may accept this, or reject it and agent $b$ will them propose $\left\{g_{2}, g_{4}, g_{5}\right\}$. The latter would be accepted by all agents, as they know that according to the protocol no other proposals can be made.

The example illustrates that the negotiation does not determine the outcome, in the sense that there are multiple outcomes possible. Additional constraints may be added to the negotiation strategy to further delimit the set of possible outcomes.

\section{Social norm negotiation}

We formalize the allowed deals during norm negotiation as obligations for each agent to see to some goals, such that all goals of the social goal are included. Again, to determine whether the obligations imply the social goal, we have to take the existing normative system into account. We assume that the normative system only creates obligations that can be fulfilled together with the already existing obligations.

Definition 6 (Fulfillable NMAS). A normative multiagent system $\langle M A S, N, O, V\rangle$ extending a multiagent system $M A S=\langle A, G$, goals, power, $\geq\rangle$ can be fulfilled if there exists a $G^{\prime} \in \operatorname{power}(A)$ such that all obligations are fulfilled $\cup_{n \in N, a \in A} O(n, a) \subseteq G^{\prime}$.

Creating a norm entails adding obligations and violations for the norm.

Definition 7 (Add norm). Let NMAS be a normative multiagent system $\langle M A S, N, O, V\rangle$ extending a multiagent system $M A S=\langle A, G$, goals, power, $\geq\rangle$. Adding a norm $n \notin N$ with a pair of functions $\left\langle o_{1}, o_{2}\right\rangle$ for obligation $o_{1}: A \rightarrow 2^{G}$ and for sanction $o_{2}: A \rightarrow 2^{G}$ leads to the new normative multiagent system $\left\langle M A S, N \cup\{n\}, O \cup o_{1}(n), V \cup o_{2}(n)\right\rangle$.

Moreover, if every agent fulfills it obligation, then the social goal is achieved. 


\begin{tabular}{cccccc} 
\# goal & speaker & agents and priorities & goal description & powers & obligation \\
\hline 2 & $b$ & $b:$ intermediate, $c$ :important & hit him with stones & $a, b$ & $a$ \\
4 & $a$ & $a$ & show him a picture of M. & $b, c$ & $b$ \\
5 & $a$ & $a, b$ & scare him to death & $c$ & $c$
\end{tabular}

Table 3. Negotiated norm

Definition 8 (Deals in norm negotiation). In the norm negotiation protocol, a deal $d \in$ deals for social goal $S$ is a pair of functions $\left\langle o_{1}, o_{2}\right\rangle$ for obligation $o_{1}: A \rightarrow 2^{G}$ and for sanction $o_{2}: A \rightarrow 2^{G}$ satisfying the following conditions:

1. Adding $\left\langle o_{1}, o_{2}\right\rangle$ to $N M A S$ for a fresh variable $n$ (i.e., not occurring in $N$ ) leads again to a normative multiagent system $N M A S^{\prime}$;

2. $N M A S^{\prime}$ achieves the social goal, $\cup_{a \in A} O_{1}(a)=S$.

3. If $N M A S$ is fulfillable, then $N M A S^{\prime}$ is too.

The running example illustrates the norm negotiation protocol.

Example 7. For the social goal $\left\{g_{2}, g_{5}\right\}$, there will always be one of the agents who does not have to see to any goal. Consider norm for the social goal $\left\{g_{2}, g_{4}, g_{5}\right\}$ in Table 3. A possible solution here is that each agent sees to one of the goals.

Sanctions can be added in the obvious way. In the norm negotiation as defined thus far, the need for sanctions has not been formalized yet. For this need, we have to consider the acceptance of norms.

\section{Norm acceptance}

An agent accepts a norm when it believes that the other agents will fulfill their obligations, and the obligation implies the goals the cycle started with. For the former we use the quasi-stability of the norm (e.g., if the norm is a convention, then we require that the norm is a Nash equilibrium). Each agent $b$ fulfills the norm given that all other agents fulfill the norm. Again we have to take the existing normative system into account, so we add the condition that all other norms are fulfilled. In general, it may mean that an agent does something which it does not like to do, but it fears the sanction more than this dislike. We use the trade-off among goals $\geq$.

Definition 9 (Stability). A choice $c$ of agent $b \in A$ in NMAS with new norm $n$ is $c \in$ power $(b)$ such that $\cup_{m \in N \backslash\{n\}} O(m, b) \subseteq d$. The choices of the other agents are $o c=\cup_{a \in A \backslash\{b\}, m \in N} O(n, a) \cup V(n, a)$. The effect of choice $c$ is $c \cup o c \cup V(n, a)$ if $O(n) \subseteq c, c \cup o c$ otherwise. NMAS is stable if $\forall b \in A$, there is a choice $c$ such that $O(n, b) \subseteq c$, and there is no choice $c^{\prime} \geq(b) c$ with $O(n, b) \nsubseteq c^{\prime}$.

Finally, we have to test whether the new situation is better than the old one for all agents. For example, we may request that the outcome in both the original multiagent system as in the new multiagent system is a Nash equilibrium, and we demand that each Nash outcome in the new system is better than each Nash outcome in the original normative multiagent system. The formalization of these concepts is along the same lines as the definition of acceptance in Definition 9.

We ask that each agent communicates to the other agents why it accepts the norm. By making a public statement, it thus commits to the norm and gives an explanation of its acceptance. Agent $a$ accepts the norm $n$ for goal $g$ : 


\begin{tabular}{|c|c|c|c|c|c|c|}
\hline \# goal & speaker & agents and priorities & goal description & powers & obligation & norm acceptance \\
\hline 2 & $b$ & $b$ :intermediate, $c:$ important & hit him with stones & $a, b$ & $a$ & $c$ \\
\hline 4 & $a$ & $a$ & show him a picture of M. & $b, c$ & $b$ & $a$ \\
\hline 5 & $a$ & $a, b$ & scare him to death & $c$ & $c$ & $b$ \\
\hline
\end{tabular}

Table 4. Norm Acceptance

accept-norm $(a, n, g)$

Norm acceptance is illustrated in our running example.

Example 8. Let $n$ stand for the norm accepted by the agents. Assume that every agent can select the goal for which he accepts the norm from the list of all goals of the social goal. This can be communicated to the other agents using the following three speech acts.

accept-norm $(a, n, 4)$

accept-norm $(b, n, 5)$

accept-norm $(c, n, 2)$

The resulting table is given in Table 4 .

\section{Related work}

In this section we discuss the existing three formalizations of the social delegation cycle.

\subsection{Norms are a class of constraints deserving special analysis}

Meyer and Wieringa define normative systems as "systems in the behavior of which norms play a role and which need normative concepts in order to be described or specified" [38, preface]. Alchourròn and Bulygin [1] define a normative system inspired by Tarskian deductive systems:

"When a deductive correlation is such that the first sentence of the ordered pair is a case and the second is a solution, it will be called normative. If among the deductive correlations of the set $\alpha$ there is at least one normative correlation, we shall say that the set $\alpha$ has normative consequences. A system of sentences which has some normative consequences will be called a normative system." [1, p.55].

Jones and Carmo [29] introduce agents in the definition of a normative system by defining it as "sets of agents whose interactions are norm-governed; the norms prescribe how the agents ideally should and should not behave. [...] Importantly, the norms allow for the possibility that actual behavior may at times deviate from the ideal, i.e., that violations of obligations, or of agents' rights, may occur." Since the agents' control over the norms is not explicit here, we use the following definition.

A normative multi-agent system is a multi-agent system together with normative systems in which agents can decide whether to follow the explicitly represented norms or not, and the normative systems specify how and in which extent the agents can modify the norms. [13]

Note that this definition makes no presumptions about the internal architecture of an agent or of the way norms find their expression in agent's behavior. 


\subsubsection{Representation of norms}

Since norms are explicitly represented, according to our definition of a normative multiagent system, the question should be raised how norms are represented. Norms can be interpreted as a special kind of constraint, and represented depending on the domain in which they occur. However, the representation of norms by domain dependent constraints runs into the question what happens when norms are violated. Not all agents behave according to the norm, and the system has to deal with it. In other words, norms are not hard constraints, but soft constraints. For example, the system may sanction violations or reward good behavior. Thus, the normative system has to monitor the behavior of agents and enforce the sanctions. Also, when norms are represented as domain dependent constraints, the question will be raised how to represent permissive norms, and how they relate to obligations. Whereas obligations and prohibitions can be represented as constraints, this does not seem to hold for permissions. For example, how to represent the permission to access a resource under an access control system? Finally, when norms are represented as domain dependent constraints, the question can be raised how norms evolve.

We therefore believe that norms should be represented as a domain independent theory. For example, deontic logic [51, 49, 48, 35, 36, 37] studies logical relations among obligations and permissions, and more in particular violations and contrary-to-duty obligations, permissions and their relation to obligations, and the dynamics of obligations over time. Therefore, insights from deontic logic can be used to represent and reason with norms in multi-agent systems. Deontic logic also offers representations of norms as rules or conditionals. However, there are several aspects of norms which are not covered by constraints nor by deontic logic, such as the relation among the cognitive abilities of agents and the global properties of norms. Meyer and Wieringa explain why normative systems are intimately related with deontic logic.

"Until recently in specifications of systems in computational environments the distinction between normative behavior (as it should be) and actual behavior (as it is) has been disregarded: mostly it is not possible to specify that some system behavior is nonnormative (illegal) but nevertheless possible. Often illegal behavior is just ruled out by specification, although it is very important to be able to specify what should happen if such illegal but possible behaviors occurs! Deontic logic provides a means to do just this by using special modal operators that indicate the status of behavior: that is whether it is legal (normative) or not" [38, preface].

\subsubsection{Norms and agents}

Conte et al. [20] distinguish two distinct sets of problems in normative multi-agent systems research. On the one hand, they claim that legal theory and deontic logic supply a theory of norm-governed interaction of autonomous agents while at the same time lacking a model that integrates the different social and normative concepts of this theory. On the other hand, they claim that three other problems are of interest in multi-agent systems research on norms: how agents can acquire norms, how agents can violate norms, and how an agent can be autonomous. Agent decision making in normative systems and the relation between desires and obligations has been studied in agent architectures [15], which thus explain how norms and obligations influence agent behavior.

An important question in normative multi-agent systems is where norms come from. Norms are not necessarily created by legislators, but they can also be negotiated among agents, or they can emerge spontaneously, making the agents norm autonomous [50]. 
In electronic commerce research, for example, cognitive foundations of social norms and contracts are studied [8]. Protocols and social mechanisms are now being developed to support such creations of norms in multi-agent systems. Moreover, agents like legislators playing a role in the normative system have to be regulated themselves by procedural norms [12], raising the question how these new kind of norms are related to the other kinds of norms.

When norms are created, the question can be raised how they are enforced. For example, when a contract is violated, the violator may have to pay a penalty. But then there has to be a monitoring and sanctioning system, for example police agents in an electronic institution. Such protocols or roles in a multi-agent system are part of the construction of social reality, and Searle [41] has argued that such social realities are constructed by constitutive norms. This raises the question how to represent such constitutive or counts-as norms, and how they are related to regulative norms like obligations and permissions [9].

\subsubsection{Norms and other concepts}

Not only the relation between norms and agents must be studied, but also the relation between norms and other social and legal concepts. How do norms structure organizations? How do norms coordinate groups and societies? How about the contract frames in which contracts live? How about the relation between legal courts? Though in some normative multi-agent systems there is only a single normative system, there can also be several of them, raising the question how normative systems interact. For example, in a virtual community of resource providers each provider may have its own normative system, which raises the question how one system can authorize access in another system, or how global policies can be defined to regulate these local policies [9].

\subsection{Social delegation cycle using artificial social systems}

The problem studied in artificial social systems is the design, emergence or more generally the creation of social laws. Shoham and Tennenholtz [42] introduce social laws in a setting without utilities, and they define rational social laws as social laws that improve a social game variable [43]. We follow Tennenholtz' presentation for stable social laws [47]. In Tennenholtz' game-theoretic artificial social systems, the goals or desires of agents are represented by their utilities. A game or multi-agent encounter is a set of agents with for each agent a set of strategies and a utility function defined on each possible combination of strategies. The social goal is represented by a minimal value for the social game variable. Tennenholtz [47] uses as game variable the maximin value. This represents safety level decisions, in the sense that the agent optimizes its worst outcome assuming the other agents may follow any of their possible behaviors.

The social norm is represented by a social law, characterized as a restriction to the strategies available to the agents. It is $u s e f u l$ with respect to an efficiency parameter $e$ if each agent can choose a strategy that guarantees it a payoff of at least $e$. A social law is quasi-stable if an agent does not profit from violating the law, as long as the other agent conforms to the social law (i.e., selects strategies allowed by the law). Quasi-stable conventions correspond to Nash equilibria. The efficiency parameter can be seen as a social kind of utility aspiration level, as studied by Simon [44]. Such aspiration levels have been studied to deal with limited or resource-bounded reasoning, and have led to the development of goals and planning in artificial intelligence; we therefore use a goal based ontology in this paper. The three steps of the social delegation cycle in this classi- 
cal game-theoretic setting can be represented as follows. Goal negotiation implies that the efficiency parameter is higher than the utility the agents expect without the norm, for example represented by the Nash equilibria of the game. Norm negotiation implies that the social law is useful (with respect to the efficiency parameter). The acceptance relation implies that the social law is quasi-stable.

The game-theoretical model has several drawbacks as a basis of our communication model. Due to the uniform description of agents in the game-theoretic model, it is less clear how to distinguish among kinds of agents. For example, the unique utility aspiration level does not distinguish the powers of agents to negotiate a better deal for themselves than for the other agents. Moreover, the formalization of the social delegation cycle does neither give a clue on how the efficiency parameter is negotiated, nor on how the social law is negotiated. For example, the goals or desires of the agents as well as other mental attitudes may play a role in this negotiation. There is no sanction or control system in the model (adding a normative system to encode enforceable social laws to the artificial social system complicates the model [6]). Finally, an additional drawback is that the three ingredients of the model (agent goals, social goals, and social laws) are formalized in three completely different ways.

\subsection{Power and dependencies}

The second formalization of the social delegation cycle is the highly detailed model we have proposed within normative multiagent systems [10]. The challenge to define social mechanisms, as we see it, is to go beyond the classical game theoretic model by introducing social and cognitive concepts and a negotiation model, but doing so in a minimal way. In the model proposed in this paper we therefore keep goals and obligations abstract and we do not describe them by first-order (or propositional) logic or their rule structure, we do not introduce decisions, actions, tasks, and so on. Similar concerns are also mentioned by Wooldridge and Dunne in their qualitative game theory [53].

In [11] we introduce a norm negotiation model based on power and dependence structures. Power may affect norm negotiation in various ways, and we therefore propose to analyze the norm negotiation problem in terms of social concepts like power and dependence. Power has been identified as a central concept for modeling social phenomena in multi-agent systems by various authors [14, 16, 22, 33], as Castelfranchi observes both to enrich agent theory and to develop experimental, conceptual and theoretical new instruments for the social sciences [17].

The model in [11] is based on a distinction between social goal negotiation and the negotiation of the obligations with their control system. Roughly, the social goals are the benefits of the new norm for the agents, and the obligations are the costs of the new norm for the agents in the sense that agents risk being sanctioned. Moreover, in particular, when representatives of the agents negotiate the social goals and norms, the agents still have to accept the negotiated norms. The norm is accepted when the norm is quasi-stable in the sense that agents will act according to the norm, and effective in the sense that fulfilment of the norm leads to achievement of the agents' desires - i.e., when the benefits outweigh the costs.

Our new model is based on a minimal extension of Tennenholtz' game theoretic model of the social delegation cycle. We add a negotiation protocol, sanction and control, and besides acceptance also effectiveness. It is minimal in the sense that, compared to our earlier model [10] in normative multiagent systems, we do not represent the rule structure of norms, we do not use decision variables, and so on. Also, as discussed in 
this paper, we do not add goal hierarchy, definitions, etc. The model therefore focusses on various uses of power: the power as ability to achieve goals and in negotiation.

\section{Summary}

Massively multiplayer online games have now become among the most complex and sophisticated online social spaces in existence $[4,34]$. As the lives people lead in virtual worlds become more and more connected with their offline lives such online spaces are shedding significant light on questions of liberty and responsibility, and on how an online existence reverberates in what we think of as a gamers real life $[34,27]$. A restricted version of norm creation is built in some of the games, such as for example A Tale in the Desert, but we envision in this paper a gaming environment in which the players can create arbitrary norms to coordinate their behaviors.

In this paper we introduce an agent communication protocol and speech acts for norm negotiation. It creates individual or contractual obligations fulfilling goals of the agents based on the social delegation cycle. The first problem is that the social delegation cycle refers to the individual goals of the agents, which are not accessible to other agents and which are not represented in the agent environment. This problem is solved by the first step of our communication protocol.

1. First, agents communicate their individual goals and powers.

2. Second, they propose social goals which can be accepted or rejected by other agents.

3. Third, they propose obligations and sanctions to achieve the social goal, which can again be accepted or rejected.

4. Finally, the agents accept the new norm by indicating which of their communicated individual goals the norm achieves.

Consequently, the social delegation cycle does not refer to the individual private goals of the agents, but to the communicated public goals.

The second problem, to define norm acceptance, is solved by the final step of our norm negotiation protocol, in which each agent has to commit itself to the social norm by indicating which of its publicly communicated goals the norm achieves.

Finally, the solution of the third problem, that is, how to use the norm negotiation protocol in online multi-player games and social spaces like The Sims Online or Second Life, is to use an abstract power and dependence based representation of the social delegation cycle. Clearly the game players cannot detail their individual probabilities and utilities as required by Tennenholtz' game-theoretic model, and they cannot detail the decision variables of the model in [10]. However, they can detail their goals and powers, as they can use natural language to describe their goals, and detail their powers by selecting the relevant goals. This solution is illustrated by our running example of norm negotiation in multi-player online gaming, where the goals are lines of text entered in a table, powers are cross-tables, social goals are sets of individual goals, and norms are distributions of the goals over the agents.

An important subtask of the social delegation cycle is to break down goals in subgoals, using a goal hierarchy. However, to focus on the main social mechanism, in this paper we do not detail the sub-protocol for subgoaling. Therefore, we assume that the individual goals communicated by the agents are such that they can be seen to by other agents. The extension with a protocol for subgoaling is left for further research. 


\section{References}

[1] C.E. Alchourrón and E. Bulygin. Normative Systems. Springer, Wien, 1971.

[2] J. F. Allen and C. R. Perrault. Analyzing intention in utterances. Artificial Intelligence, 15:143-178, 1980.

[3] J.L. Austin. How to do things with words. Harvard University Press, Cambridge Mass., 1962.

[4] F. Beau. Culture d'Univers. FYP editions, 2007.

[5] G. Boella, L. Sauro, and L. van der Torre. Social viewpoints on multiagent systems. In Procs. of the 3rd International Joint Conference on Autonomous Agents and Multi Agent Systems (AAMAS'04), pages 1358-1359, 2004

[6] G. Boella and L. van der Torre. Enforceable social laws. In Procs. of the 4th International Joint Conference on Autonomous Agents and Multiagent Systems (AAMAS'05), pages 682-689, 2005.

[7] G. Boella and L. van der Torre. Fair distribution of collective obligations. In Procs. of the 17th European Conference on Artificial Intelligence (ECAI'06), pages 721-722, 2006.

[8] G. Boella and L. van der Torre. A game theoretic approach to contracts in multiagent systems. IEEE Transactions on Systems, Man and Cybernetics - Part C, 36(1):68-79, 2006.

[9] G. Boella and L. van der Torre. Security policies for sharing knowledge in virtual communities. IEEE Transactions on Systems, Man and Cybernetics - Part A, 36(3):439-450, 2006.

$[10] \mathrm{G}$. Boella and L. van der Torre. Norm negotiation in multiagent systems. International Journal of cooperative Information Systems (IJCIS), 16(1), 2007.

[11]G. Boella and L. van der Torre. Power in norm negotiation. In Agent and Multi-Agent Systems: Technologies and Applications, First KES International Symposium, KES-AMSTA 2007, volume 4496 of LNCS, pages 436-446, Berlin, 2007. Springer.

[12]G. Boella and L. van der Torre. Substantive and procedural norms in normative multiagent systems. Journal of Applied Logic, in press.

[13]G. Boella, L. van der Torre, and H. Verhagen. Introduction to normative multiagent systems. Computational and Mathematical Organizational Theory, Special issue on Normative Multiagent Systems, 12(2-3):71-79, 2006

[14]S. Brainov and T. Sandholm. Power, dependence and stability in multi-agent plans. In Procs. of the 21st National Conference on Artificial Intelligence (AAAI'99), pages 11-16, 1999.

[15]J. Broersen, M. Dastani, J. Hulstijn, and L. van der Torre. Goal generation in the BOID architecture. Cognitive Science Quarterly, 2(3-4):428-447, 2002.

[16]C. Castelfranchi. Modeling social action for AI agents. Artificial Intelligence, 103(1-2):157-182, 1998

[17]C. Castelfranchi. Micro-macro constitution of power. ProtoSociology, 18-19, 2003.

[18]M. Cavazza. AI in computer games: Survey and perspectives. Virtual Reality, 5:223-235, 2000.

[19]P. R. Cohen and C. R. Perrault. Elements of a plan-based theory of speech acts. Cognitive Science, 3:177-212, 1979 .

[20]R. Conte, C. Castelfranchi, and F. Dignum. Autonomous norm-acceptance. In Intelligent Agents V (ATAL'98), LNAI 1555, pages 99-112. Springer, 1999.

[21]R. Conte, R. Falcone, and G. Sartor. Agents and norms: How to fill the gap? Artificial Intelligence and Law, 7(1):1-15, 1999.

[22]R. Conte and J. Sichman. Multi-agent dependence by dependence graphs. In Procs. of the 1st International Joint Conference on Autonomous Agents and Multiagent Systems (AAMAS'O2), pages 483-490, 2002.

[23]FIPA. FIPA ACL communicative act library specification. Technical Report SC00037J, Foundation for Intelligent Physical Agents, 2002.

[24]N. Fornara and M. Colombetti. A commitment-based approach to agent communication. Applied Artifcial Intelligence, 18(9-10):853-866, 2004.

[25]J. Hulstijn G. Boella, R. Damiano and L. van der Torre. A common ontology of agent communication languages: Modeling mental attitudes and social commitments using roles. Applied Ontology, 2(3-4):217 $-265,2007$.

[26]B. Gaudou, A. Herzig, and D. Longin. A logical framework for grounding-based dialogue analysis. Electronic Notes in Theoretical Computer Science, 157(4):117-137, 2006.

[27]James Grimmelmann. Virtual borders: The interdependence of real and virtual worlds. First Monday, 11(2), 2006

[28]C. L. Hamblin. Fallacies. Methuen \& Co, London, 1970.

[29]A. Jones and J. Carmo. Deontic logic and contrary-to-duties. In D. Gabbay and F. Guenthner, editors, Handbook of Philosophical Logic, volume 3, pages 203-279. Kluwer, Dordrecht (NL), 2001.

[30]J. Laird and M. van Lent. Human-level AI's killer application: Interactive computer games. AI Magazine, 22(2):15-25, 2001

[31]David Lewis. Convention: A Philosophical Study. Harvard University Press, Cambridge, 1969. 
[32]Karen E. Lochbaum. A collaborative planning model of intentional structure. Computational Linguistics, 24(4):525-572, 1998.

[33]F. Lopez y Lopez. Social Power and Norms: Impact on agent behaviour. PhD thesis, University of Southampton, 2003

[34]P. Ludlow and M. Wallace. The Second Life Herald. MIT Press, Cambridge (MA), 2007.

[35]D. Makinson and L. van der Torre. Input-output logics. Journal of Philosophical Logic, 29:383-408, 2000.

[36]D. Makinson and L. van der Torre. Constraints for input-output logics. Journal of Philosophical Logic, 30:155-185, 2001.

[37]D. Makinson and L. van der Torre. Permissions from an input-output perspective. Journal of Philosophical Logic, 32(4):391-416, 2003.

[38]J. Meyer and R. Wieringa. Deontic Logic in Computer Science: Normative System Specification. John Wiley and Sons, Chichester, England, 1993.

[39]M. Nickles, F. Fischer, and G. Weiss. Communication attitudes: A formal approach to ostensible intentions, and individual and group opinions. Electronic Notes in Theoretical Computer Science, 157(4):95$115,2006$.

[40]J.R. Searle. Speech Acts: an Essay in the Philosophy of Language. Cambridge University Press, Cambridge, UK, 1969

[41]J.R. Searle. The Construction of Social Reality. The Free Press, New York, 1995

[42]Y. Shoham and M. Tennenholtz. On social laws for artificial agent societies: off-line design. Artificial Intelligence, 73 (1-2):231 - 252, 1995.

[43]Y. Shoham and M. Tennenholtz. On the emergence of social conventions: Modeling, analysis and simulations. Artificial Intelligence, 94(1-2):139-166, 1997.

[44]H.A. Simon. A behavioral model of rational choice. The Quarterly Journal of Economics, 69:99-118, 1955.

[45]M.P. Singh. A social semantics for agent communication languages. In Issues in Agent Communication (AC'O0), LNCS 1916, pages 31 - 45. Springer, Berlin, 2000.

[46]R.C. Stalnaker. Common ground. Linguistics and Philosophy, 25:701-719, 2002.

[47]M. Tennenholtz. On stable social laws and qualitative equilibria. Artificial Intelligence, 102(1):1-20, 1998.

[48]L. van der Torre. Contextual deontic logic: Normative agents, violations and independence. Annals of Mathematics and Artificial Intelligence, 37(1-2):33-63, 2003.

[49]L. van der Torre and Y. Tan. Contrary-to-duty reasoning with preference-based dyadic obligations. Annals of Mathematics and Artificial Intelligence, 27(1-4):49-78, 1999.

[50]H. Verhagen. On the learning of norms. In Procs. of Modelling Autonomous Agents in a Multi-Agent World (MAAMAW'99), 1999.

[51]G. H. von Wright. Deontic logic. Mind, 60:1-15, 1951.

[52]Douglas N. Walton and Erik C. Krabbe. Commitment in Dialogue: Basic Concepts of Interpersonal Reasoning. State University of New York Press, New York, 1995.

[53]M. Wooldridge and P.E. Dunne. On the computational complexity of qualitative coalitional games. Artificial Intelligence, 158(1):27-73, 2004.

\section{Author Biographies}

boella.jpg $\mathrm{He}$ is currently professor at the Department of Computer Science of the University of Torino. His research interests include multi-agent systems, in particular, normative systems, institutions and roles using qualitative decision theory. He organized the first workshops on normative multi-agent systems NorMAS, on coordination and organization CoOrg, and the AAAI Fall Symposium and ECOOP Roles.

Parice Caire is currently PhD student at the University of Luxembourg. She is interested in digital cities, virtual communities and tools for conviviality. 
Leendert van der Torre is currently full professor in intelligent systems at the University of Luxembourg. He received the $\mathrm{PhD}$ degree from Erasmus Univer-

sity Rotterdam in 1997. He has developed the so-called input/output logics and the BOID agent architecture. His current research interests include deontic logic, qualitative game theory and coordination in normative multiagent systems. $\mathrm{He}$ is responsible for priority P1 on security and reliability of University of Luxembourg.

Correspondence and offprint requests to: Leendert van der Torre, Computer Science and Communication, University of Luxembourg, Luxembourg. Email: leendert@ vandertorre.com 


\title{
Substantive and Procedural Norms in Normative Multiagent Systems
}

\author{
Guido Boella ${ }^{a}$ \\ Leendert van der Torre ${ }^{b}$ \\ ${ }^{a}$ Dipartimento di Informatica - Università di Torino - Italy. email: guido@di.unito.it \\ ${ }^{\mathrm{b}}$ Computer Science and Communications - University of Luxembourg - Luxembourg. \\ e-mail: leendert@vandertorre.com
}

Procedural norms are instrumental norms addressed to agents playing a role in the normative system, for example to motivate these role playing agents to recognize violations or to apply sanctions. Procedural norms have first been discussed in law, where they address legal practitioners such as legislators, lawyers and policemen, but they are discussed now too in normative multiagent systems to motivate software agents. Procedural norms aim to achieve the social order specified using regulative norms like obligations and permissions, and constitutive norms like counts-as obligations. In this paper we formalize procedural, regulative and constitutive norms using input/output logic enriched with an agent ontology and an abstraction hierarchy. We show how our formalization explains Castelfranchi's notion of mutual empowerment, stating that not only the agents playing a role in a normative system are empowered by the normative system, but the normative system itself is also empowered by the agents playing a role in it. In our terminology, the agents are not only institutionally empowered, but they are also delegated normative goals from the system. Together, institutional empowerment and normative goal delegation constitute a mechanism which we call delegation of power, where agents acting on behalf of the normative system become in charge of recognizing which institutional facts follow from brute facts.

\section{Introduction}

The distinction between substantive and procedural norms is well known in legal theory [35], but it seems that thus far procedural norms have not been formalized in deontic logic or introduced in formal models of normative multiagent systems. Substantive norms define the legal relationships of people with other people and the state in terms of regulative and constitutive norms, where regulative norms are obligations, prohibitions and permissions, and constitutive norms define what counts as institutional facts in the normative system. Procedural norms are instrumental norms, addressed to agents playing roles in the normative system, aiming to achieve the social order specified in terms of substantive norms [35]. In the Italian law, for example, it is obligatory for an attorney to start a prosecution process when he comes to know about a crime (art. 326 of Codice di procedura penale). Procedural law encompasses legal rules governing the process for settlement of disputes (criminal and civil). Procedural and substantive law are comple- 
mentary. Procedural law brings substantive law to life and enables rights and duties to be enforced and defended. For example, procedural norms explain how a trial should be carried out and which are the duties, rights and powers of judges, lawyers and defendants.

Substantive norms are discussed not only in law, but also in normative multiagent systems like electronic institutions. They are multiagent systems together with normative systems in which agents on the one hand can decide whether to follow the explicitly represented norms, and on the other the normative systems specify how and in which extent the agents can modify the norms [17].

In this paper we formalize procedural, regulative and constitutive norms using the following formal methods:

1. Input/output logic to define the various kinds of norms as logical relations between brute facts, decisions or actions and institutional facts in a logical framework without committing to one particular deontic logic. Various logical properties of the norms we define in this paper follow from general results in input/output logic (e.g., soundness and completeness with respect to the semantics of input/output logic) and are not discussed in this paper.

2. A multiagent ontology to define the concepts used in the system, not only to define the role playing agents in the normative system, but also to define a normative system as an organization or a legal actor.

3. An abstraction hierarchy to compare our detailed model with more abstract models and formalisms in the literature. For example, most deontic logics abstract from procedural norms or even from norms at all, and some normative multiagent systems assume that norm enforcement is done by cooperating or controlled agents only.

A success criterion for formal models of procedural norms, is which aspects of Castelfranchi's notion of mutual empowerment they can explain. Mutual empowerment says that not only the agents playing a role in a normative system are empowered by the normative system, but the normative system itself is also empowered by the agents playing a role in it. In our model, the agents are not only institutionally empowered, but they are also delegated normative goals from the system. In other words, the role playing agents empower the normative system by accepting its normative goals. Together, institutional empowerment and normative goal delegation constitute a mechanism which we call delegation of power, where agents acting on behalf of the normative system become in charge of recognizing which institutional facts follow from brute facts.

The paper is organized as follows. In Section 2 we informally discuss the concepts and mechanisms formalized in our logical framework. We motivate the introduction of procedural norms and the notion of delegation of power by means of an example, we discuss the role of procedural norms in regulative and constitutive norms, we define delegation of power and we compare it to goal delegation and institutionalized power. In Section 3 we introduce and explain our methodology of the abstraction hierarchy in which we distinguish five levels of abstraction at which normative multiagent systems can be represented. In Section 4 we introduce the formal model explained by examples of regulative, constitutive and procedural norms at the different levels of abstraction. 


\section{Procedural norms and delegation of power}

In this section we informally discuss the concepts and mechanisms formalized in our logical framework. Section 2.1 motivates the introduction of the notions of procedural norms and delegation of power in normative multiagent systems by means of an example. Section 2.2 discusses the role of procedural norms in regulative and constitutive norms. Section 2.3 discusses in which sense normative systems are empowered by agents playing a role in it. Section 2.4 defines delegation of this power and compares it to goal delegation and delegation of power.

\subsection{Cars with a catalytic converter count as ecological vehicles}

To illustrate the notion of procedural norms and delegation of power playing a central role in the logical framework, we start with an example about traffic norms involving one of the authors. It is used as a running example thoughout this paper.

Due to increased levels of pollution, on some precisely defined days, only ecological vehicles are allowed in major towns of Italy. One author of this paper bought many years ago one of the first catalytic cars, and since cars with a catalytic converter count as ecological vehicles, he felt permitted to always go around by car. On some day, he was stopped by the police and fined for driving around a non-ecological vehicle, because the car was bought before the local law recognized catalytic cars as ecological vehicles. The police agreed that the car had a catalytic converter: they could see it, the car worked only with unleaded fuel, both the manual and the licence of the car said it has a catalytic converter. However, there was a missing rubber stamp by some office declaring that the car counts as an ecological vehicle.

The problem is not simply that only catalytic cars bought after a certain date are considered as ecological, but that a catalytic car is not ecological unless an agent officially recognizes it as such. The police has no power to consider the car as ecological, the evidence notwithstanding. The policeman may even argue that it is not the case that cars with a catalytic converter count as ecological vehicles, but that cars with the right stamps count as ecological vehicles - though this ignores the reason why the constitutive rule was introduced. The moral of the story is that even if a brute fact is present and could allow the recognition of an institutional fact, the institutional fact is the result of the action of some agent who is empowered to make institutional facts official.

For the layman, as the unlucky driver of the example, it is natural to describe the normative system as specifying the fact that catalytic cars are ecological. However, this is true only at a coarse level of detail. If the details of the functioning of the normative systems are entered, then a more complex description is necessary and agents with the specific role of making true some institutional facts under some circumstances must be introduced.

There may be many reasons why an institutional fact is the result of action of an empowered agent, for example for separation of duties, because it is not easy to identify whether a car has a catalytic converter, or simply for representational efficiency. We do not consider these reasons further in this paper. 


\subsection{Regulative, constitutive and procedural norms}

Regulative norms specify the ideal and varying degrees of sub-ideal behavior of a system by means of obligations, prohibitions and permissions. Deontic logic [3,44] considers logical relations among obligations and permissions and focuses on the description of the ideal or optimal situation to achieve, driven by representation problems expressed by the so-called deontic paradoxes, most notoriously the contrary-to-duty paradoxes, see, for example, $[30,43]$. If we formalize the running example using regulative norms, we might say that only with a rubber stamp, cars are permitted to drive on these special days. However, norms are defined referring to more abstract states of affairs than catalytic converters and rubber stamps, like being an ecological vehicle.

Constitutive norms are based on the notion of counts-as and are used to support regulative norms by introducing institutional facts in the representation of legal reality. The notion of counts-as introduced by Searle [41] has been interpreted in deontic logic in different ways and it seems to refer to different albeit related phenomena [28]. For example, Jones and Sergot [31] consider counts-as from the constitutive point of view. According to Jones and Sergot, the fact that A counts-as B in context C is read as a statement to the effect that A represents conditions for guaranteeing the applicability of particular classificatory categories. The counts-as guarantees the soundness of that inference, and enables "new" classifications which would otherwise not hold. An alternative view of the counts-as relation is proposed by Grossi et al. [27]: according to the classificatory perspective A counts-as B in context $\mathrm{C}$ is interpreted as: A is classified as B in context $\mathrm{C}$. In other words, the occurrence of $\mathrm{A}$ is a sufficient condition, in context $\mathrm{C}$, for the occurrence of B. Via counts-as statements, normative systems can establish the ontology they use in order to distribute obligations, rights, prohibitions, permissions, etc. In $[10,11]$ we propose a different view of counts-as which focuses on the fact that counts-as often provides an abstraction mechanism in terms of institutional facts, allowing the regulative rules to refer to legal notions which abstract from details. In all of these approaches, we can formalize the running example with a constitutive norm that a rubber stamp counts as an ecological vehicle, and a regulative norm stating that ecological vehicles are permitted to drive on the special days.

However, at the level of abstraction of these approaches we cannot express that cars with a catalytical converter count as ecological vehicles, since the evidence presents a counterexample. As long as we consider only the perspective of the unfortunate author, it may seem that there is also no need to do so. Clearly the evidence shows that cars with a catalytical converter not necessarily count as ecological vehicles, and there does not seem to be a reason why we should want to represent this norm. However, at the level of the normative multiagent system, for example from the perspective of the designer, it makes a lot of sense to state that catalytical cars count as ecological vehicles, since this may hold in the social order the normative system aims to achieve. Moreover, it is easier to communicate a norms like this to citizens.

The role that agents have in enforcing the social order the normative system aims to by creating norms has been recognized in normative multiagent systems [11,13], and agents are considered which are in charge of sanctioning violations on behalf of the normative system [6,7]. Moreover, obligations are associated with procedural norms which are instrumental - to use Hart [29]'s terminology - to distribute the tasks to agents like judges 
and policemen, who have to decide whether and how to fulfill them. In the example, there are procedural norms that cars with a catalytical converter should count as ecological vehicles, and that drivers of non-catalytic cars that drive on the given days should be fined. Thus, at least informally, procedural norms can be seen as nestings of regulative and constitutive norms.

A formal framework for procedural norms has to explain how regulative and constitutive norms are related. Usually, constitutive norms are used in normative multiagent systems to define intermediate concepts like marriage or money, because they enable a more efficient representation to relate brute facts with deontic ones [38]. Another relation between the two kinds of norms is that an obligation to see to some state of affairs can be defined as "the absence of the state of affairs counts as a violation," generalizing Anderson's well known reduction of deontic logic to alethic modal logic. Therefore, constitutive rules enter in the definition of sanction based obligations, since the recognition of a violation results in an institutional fact. Finally, with procedural norms, some agents may be obliged to recognize violations, to sanction violations, or to recognize some facts as institutional facts. These actions are carried out by agents playing roles in the normative system like judges and policemen.

\subsection{Mutual empowerment}

The normative system is an immaterial entity which exists due to the collective acceptance by the agents of a community and has the purpose to coordinate their activity. Thus, it cannot act in the environment, but it can act only by means of representatives. For example, there must be an agent which counts behavior as a violation, and one that applies the sanction in case of a violation. However, once there are such agents playing a role in it, we may say that the system is empowered by these agents, in the sense that it can act via these agents to achieve its goals. Since the normative system also gives some power to act in the normative system to the role playing agents, Castelfranchi has called the relation between a normative system and its agents mutual empowerment [22]. Procedural norms play an essential role in mutual empowerment, since they are the mechanism to see to it that the empowered agents empower the normative system, and do not abuse their power.

To represent that obligations are used to achieve social order, obligations can be defined as goals of the normative system that a certain state of affairs is achieved and that, if it is not achieved, that situation is recognized as a violation and sanctioned. Note the asymmetry between considering something as a violation and sanctioning. Sanctions can create new obligations like paying a fine, but they can also be physical actions like putting into jail, while a violation has always an institutional character. So, while a sanction of the latter type like putting into jail can be directly performed by a policeman, the recognition of a violation, and sanctions of the former type, can only be performed indirectly by means of some action which counts as the recognition of a violation, e.g., a trial by a judge which establishes that a violation happens and creates new obligations the violator is subject to.

Moreover, the same idea that norms can be defined as goals of the normative system can be used for constitutive norms. However, despite the fact that an action of an agent is necessary to create the institutional fact in case of ecological cars and in case of violations, 
there is an apparent asymmetry between constitutive rules and regulative ones. These cases can be modelled by a counts-as relation between the action of an agent (putting a stamp on the car licence or recognizing a violation) and the institutional fact (being an ecological vehicle or having violated an obligation), rather than by a direct counts-as relation between the brute facts and the institutional facts. But at first sight the two cases also have a difference: the recognition of a violation is wanted by the normative system to achieve its social order. In this case besides the counts-as rule between the action and the recognition as a violation there is also the goal of the normative system that this recognition contributes to the social order. As illustrated in the running example, in many circumstances facts which in principle should be considered as institutional facts are not recognized as such. In such circumstances, considering a fact as an institutional fact may depend on the action of some agent who is the representative of the normative system: we say that this agent has been delegated the power do recognize the fact as an institutional fact. We can see this as an instance of resource bounded reasoning: the assumption made above on constitutive rules, even if useful in some circumstances, is not realistic in all circumstances.

In other words, as a first step to explain why and how procedural norms are created, we should consider the motivational aspects behind constitutive norms required by agent theory. Constitutive norms are modelled as counts-as conditionals which allow to infer which institutional facts follow from brute facts and from existing institutional facts. E.g., a car counts as a vehicle for the traffic law. None of the models discussed in the previous section considers this issue. The inference from facts to institutional facts is considered as automatic, i.e., it is assumed not to need any agent or resource to perform it. Agent theory, instead, considers also the resources needed to perform inferences, since it is aware that agents are usually resources bounded and that inferences have a cost too. Calculating the consequences following from some premises has a cost which must be traded off against the benefit of making the inferences. Thus in Georgeff and Ingrand [24] inferences are considered as actions which are planned and subject to decision processes as any other action: there must be an agent taking the decision about which inference to perform and executing the inference.

This mechanism allows also to give flexibility to the law, since the law cannot specify all possible cases in advance both as concerns regulative and constitutive rules. In this way, the decision to apply a constitutive rule is performed by an agent who can evaluate carefully the circumstances.

\subsection{Delegation of power}

Delegation of power involves two different phenomena, which are at first sight unrelated, institutional empowerment and goal delegation:

Institutional empowerment: how an action of some agent can establish an institutional fact.

Goal delegation: how this agent can carry out a goal of the delegator that the institutional fact holds in certain situations, and how it can be motivated to perform the action which establish the institutional fact. 
Empowerment means that an agent is made in the condition to satisfy the goal of some agent. In general, it has no institutional character. However, institutional empowerment is by nature an institutional phenomenon which is based on the counts-as relation: an agent is empowered to perform an institutional action - a kind of institutional fact - if some of its actions counts as the institutional action. The institutional action has some effects on the institution which empowers the agent. For example, a director can commit by means of his signature his institution to purchase some goods. Thus it is essentially related to counts-as rules, albeit restricted to actions of agents. Consider as a paradigmatic case the work by Jones and Sergot [31]. However, it is not sufficient to explain mutual empowerment either, since it does not explain how it empowers the normative system. In particular, the empowerment of the normative system is not institutional, but it consists in the actions which agents carry out on behalf of the normative system, which, by itself, cannot act.

According to Castelfranchi [21], goal delegation is relying on another agent for the achievement of one own's goal: "in delegation an agent A needs or likes an action of another agent $\mathrm{B}$ and includes it in its own plan. In other words, $\mathrm{A}$ is trying to achieve some of its goals through B's behaviours or actions; thus A has the goal that B performs a given action/behaviour." Goal delegation by itself is not sufficient to explain mutual empowerment, since it is not an institutional phenomenon but a basic capability of agents which enables them to interact with each other.

Bottazzi and Ferrario [18] argue that the two phenomena are related, because an agent which is institutionally empowered, is also delegated the goal of the institution of making true an institutional fact by exercising his power in the specified situations. The connection between goal delegation and institutional empowerment is not a necessary one. There are situations where an agent is delegated but not institutionally empowered. For example, the agent in charge for sanctioning an obligation is delegated the goal of sanctioning, but there is no need of institutional powers in case of physical sanctions: the agent's physical powers are sufficient, for instance, to put the violator into jail. Viceversa, the law institutionally empowers agents to stipulate private contracts which have the force of law, without being delegated by the law to do so, since contracting agents act for their own sake [11].

This connection, which we call delegation of power, can be used to explain the running example. For the normative system, catalytic cars have to be considered as ecological vehicles. There are three possibilities: first, recognizing all catalytic cars as ecological vehicles by means of a constitutive norm. This solution, however, does not consider the actual performance of the inference and the possible costs related to it. Second, the normative system can rely on some agent to recognize catalytic cars as ecological vehicles. As said above, this can be done by means of a counts-as relation between an action of an agent and its effects. This solution, however, fails to account for the motivations that the agent should have to perform the action of recognizing ecological vehicles as such. Third, also a goal of the normative system is added to motivate his action: there is an agent who has the institutional power to recognize cars as ecological vehicles and the normative system has delegated it the goal that it does so in order to motivate it. If this motivation is not sufficient since the agent is not cooperative, an obligation to achieve this goal of the normative system must be added. 


\section{Level of abstractions in the definition of norms}

Normative Multiagent Systems can be described at different levels of abstraction, using definitions of obligations and counts-as relations which differ at their level of detail. We identify five different levels of abstraction, which are suited for different applications, depending on the role attributed to the system infrastructure or on specialized agents. The abstraction dimension is the detail at which we consider agents acting for the normative system: at the higher abstraction level agents have no role, at the next abstraction level only actions of the normative system are considered but agents are not considered; then at more concrete level, where agents are in charge of the actual functioning of the normative system concerning regulative and constitutive rules, delegation of power enters the picture; finally, at the more concrete level of abstraction, procedural norms are introduced to motivate the agents playing roles in the system. In the next section we will provide the formal definition of obligations and counts-as relations at the different levels.

1. The higher level abstracts from the fact that violations, sanctions and institutional facts are the result of the action that some agent desires and decides to perform. The obligations are defined as in Anderson's reduction [2], in the sense that the recognition of the violation and the sanction follow necessarily from the violation. This abstraction level for regulative rules is adopted also by [4,36] and we use it in [15]. For constitutive rules, up to our knowledge, this is the only level considered. The responsibility of monitoring, sanctioning and making counts-as inferences is completely delegated to the infrastructure.

2. At the second level the normative system is in some way personified and is attributed mental attitudes, thus, the recognition of violations and sanctions are wanted by the normative system itself. If obligations are goals of the normative system, the institutional facts follow from the beliefs of the normative system: they are still logical consequences of facts, but in the beliefs of the normative system. This is the solution adopted in [10].

3. The third level not only abstracts from the role of agents in the normative system, but the recognition of violations and sanctions are considered as the actions of the normative system itself. We adopt this level of representation for regulative norms in $[11,13]$. Analogously, institutional facts follow from actions of the normative system: they are not anymore logical consequences of beliefs, but consequences of decisions of the normative systems which are traded-off against other decisions. They, thus, do not follow automatically, since the normative system can take a different decision due to conflicts with other goals or to lack of resources. The normative system can decide to enforce norms or not in specific situations, thus allowing interpretation of them.

4. The fourth level takes into account the actions of the agents acting on behalf of the normative system. Concerning regulative norms, some agents are delegated the goal to sanction violations and the goal and power of recognizing violations. I.e., they are delegated the power to do so. Concerning constitutive norms, the agents are delegated the goal to recognize some facts as institutional facts and the power 
to do so by means of their actions. I.e., they are delegated the power to do so. The problem of agents recognizing violations has been partially addressed in [6], but the recognition action was considered as a physical action like the sanction. In this paper we add the counts-as relation to the recognition of violations.

5. The fifth level introduces procedural norms to motivate the agents playing roles in the system. These norms oblige agents playing roles to achieve the goals of the normative system. Concerning regulative norms, some agents are obliged to achieve the delegated goal to sanction violations and the goal and power of recognizing violations. Concerning constitutive norms, the agents are obliged to achieve the goal to recognize some facts as institutional facts by exercising the delegated powers. In the Italian law, for example, it is obligatory for an attorney to start a prosecution process when he comes to know about a crime (art. 326 of Codice di procedura penale).

Our logical framework of the game-theoretic approach to normative multiagent systems is based on the so-called agent metaphor: social entities like normative systems can be conceptualized as agents by attributing them mental attitudes like beliefs and goals. The agent metaphor underlying our framework is based on cognitive motivations [9]. Beliefs model the constitutive rules of the normative system, while goals model regulative rules. Thus, in the normative system the interaction between constitutive and regulative rules is the same as the interaction of beliefs and goals in an agent. However, differently from a real agent, the normative system is a socially constructed agent. It exists only because of the collective acceptance by all the agents and, thus, it cannot act in the world. Its actions are carried out by agents playing roles in the normative system, like legislators, judges and policemen. It is a social construction used to coordinate the behavior of agents.

Obligations are not only modelled as goals of the normative system, but they are also associated with the instrumental goals that the behavior of the addressee of the norms is considered as a violation and that the violation is sanctioned. Considering something as a violation and sanctioning are actions which can be executed by the normative system itself, or, at a more concrete level of detail, by agents playing roles in it.

The counts-as relation in our model is modelled as a conditional belief of the normative system to provide an abstraction of reality in terms of institutional facts. Regulative norms can refer to this level, thus decoupling them from the details of reality. For example, the institutional fact that traffic lights are red must be distinguished from the brute fact that red light bulbs in the traffic lights are on: in the extreme case the institutional fact can be true even if all the red bulbs are broken. In the following section, we consider how countsas can be used to define delegation of power. Counts-as relations are not used in this case to directly connect brute facts to institutional facts, but only to express the institutional effects of actions of agents empowered by the normative system. This is analogous to the act of signing of the director counts as the commitment of the institution he belongs to. Moreover, constitutive rules specify also how the normative system can change [11]. Since it is a socially constructed agent, it cannot directly change itself, but it relies on the actions of agents playing roles in it, like legislators, which count as changes of the system. 


\section{The formal model}

The definition of the agents is inspired by the rule based BOID architecture [20], though in our theory, and in contrast to BOID, obligations are not taken as primitive concepts. Beliefs, desires and goals are represented by conditional rules rather than in a modal framework. Intentions have been introduced as a form of bounded rationality: since an agent has not enough resources to make the optimal decision at each moment, he maintains its previous choices. In this paper we consider only one decision, so we do not need to introduce intentions to model decisions which persist over time.

\subsection{Input/output logic}

To represent conditional mental attitudes we take a simplified version of the input/output logics introduced in [33,34]. Though the development of input/output logic has been motivated by the logic of norms, the same logic can be used for other conditionals like conditional beliefs and conditional goals - which explains the more general name of the formal system. Moreover, Bochman [5] also illustrates how the same logic is used for causal reasoning and various non-monotonic reasoning formalisms.

A rule set is a set of ordered pairs $P \rightarrow q$. For each such pair, the body $P$ is thought of as an input, representing some condition or situation, and the head $q$ is thought of as an output, representing what the rule tells us to be believed, desirable, obligatory or whatever in that situation. In this paper, to keep the formal exposition simple, input and output are respectively a set of literals and a literal.

Definition 1 (Input/output logic) Let $X$ be a set of propositional variables, the set of literals built from $X$, written as Lit $(X)$, is $X \cup\{\neg x \mid x \in X\}$, and the set of rules built from $X$, written as $\operatorname{Rul}(X)=2^{\text {Lit(X) }} \times$ Lit $(X)$, is the set of pairs of a set of literals built from $X$ and a literal built from $X$, written as $\left\{l_{1}, \ldots, l_{n}\right\} \rightarrow l$. We also write $l_{1} \wedge \ldots \wedge l_{n} \rightarrow l$ and when $n=0$ we write $\top \rightarrow l$. For $x \in X$ we write $\sim x$ for $\neg x$ and $\sim(\neg x)$ for $x$. Moreover, let $Q$ be a set of pointers to rules and $R D: Q \rightarrow \operatorname{Rul}(X)$ is a total function from the pointers to the set of rules built from $X$.

Let $S=R D(Q)$ be a set of rules $\left\{P_{1} \rightarrow q_{1}, \ldots, P_{n} \rightarrow q_{n}\right\}$, and consider the following proof rules strengthening of the input (SI), disjunction of the input (OR), cumulative transitivity (CT) and Identity (Id) defined as follows:

$$
\frac{p \rightarrow r}{p \wedge q \rightarrow r} S I \quad \frac{p \wedge q \rightarrow r, p \wedge \neg q \rightarrow r}{p \rightarrow r} O R \quad \frac{p \rightarrow q, p \wedge q \rightarrow r}{p \rightarrow r} C T \quad \frac{}{p \rightarrow p} I d
$$

The following output operators are defined as closure operators on the set $S$ using the rules above.

out $_{1}:$ SI (simple-minded output) out ${ }_{3}: S I+C T$ (reusable output) out $_{2}: S I+O R$ (basic output) out $_{4}: S I+O R+C T$ (basic reusable output)

Moreover, the following four throughput operators are defined as closure operators on the set $S$. out $t_{i}^{+}$: out ${ }_{i}+I d$ (throughput) We write out $(Q)$ for any of these output operations and out $^{+}(Q)$ for any of these throughput operations. We also write $l \in \operatorname{out}(Q, L)$ iff $L \rightarrow l \in$ out $(Q)$, and $l \in$ out $^{+}(Q, L)$ iff $L \rightarrow l \in$ out $^{+}(Q)$. 
Example 1 Given $R D(Q)=\{a \rightarrow x, x \rightarrow z\}$ the output of $Q$ contains $x \wedge a \rightarrow z$ using the rule $S I$. Using also the CT rule, the output contains $a \rightarrow z$. $a \rightarrow$ a follows only if there is the Id rule.

A technical reason to distinguish pointers from rules is to facilitate the description of the priority ordering we introduce in the following definition.

The notorious contrary-to-duty paradoxes such as Chisholm's and Forrester's paradox have led to the use of constraints in input/output logics [34]. The strategy is to adapt a technique that is well known in the logic of belief change - cut back the set of norms to just below the threshold of making the current situation inconsistent.

Definition 2 (Constraints) Let $\geq: 2^{Q} \times 2^{Q}$ be a transitive and reflexive partial relation on the powerset of the pointers to rules containing at least the subset relation and $R D$ : $Q \rightarrow \operatorname{Rul}(X)$ a function from the pointers to the set of rules. Moreover, let out be an input/output logic:

- maxfamily $(Q, P)$ is the set of $\subseteq$-maximal subsets $Q^{\prime}$ of $Q$ such that out $\left(Q^{\prime}, P\right) \cup P$ is consistent.

- $\operatorname{preffamily}(Q, P, \geq)$ is the set of $\geq$-maximal elements of maxfamily $(Q, P)$.

- outfamily $(Q, P, \geq)$ is the output under the elements of preffamily, i.e., $\left\{\operatorname{out}\left(Q^{\prime}, P\right) \mid Q^{\prime} \in \operatorname{preffamily}(Q, P, \geq)\right\}$.

- $P \rightarrow x \in$ out $_{\cup}(Q, \geq)$ iff $x \in \cup$ outfamily $(Q, P, \geq)$

$P \rightarrow x \in$ out $_{\cap}(Q, \geq)$ iff $x \in \cap \operatorname{noutfamily}(Q, P, \geq)$

Example 2 Let $R D(\{a, b, c\})=\{a=(\top \rightarrow m), b=(p \rightarrow n), c=(o \rightarrow \neg m)\},\{b, c\}>$ $\{a, b\}>\{a, c\}$, where by $A>B$ we mean as usual $A \geq B$ and $B \nsupseteq A$.

$\operatorname{maxfamily}(Q,\{o\})=\{\{a, b\},\{b, c\}\}$,

$\operatorname{preffamily}(Q,\{o\}, \geq)=\{\{b, c\}\}$,

$\operatorname{outfamily}(Q,\{o\}, \geq)=\{\{\neg m\}\}$

The maxfamily includes the sets of applicable compatible pointers to rules together with all non applicable ones: e.g., the output of $\{a, c\}$ in the context $\{o\}$ is not consistent. Finally $\{a\}$ is not in maxfamily since it is not maximal, we can add the non applicable rule $b$. Then preffamily is the preferred set $\{b, c\}$ according to the ordering on set of rules above. The set outfamily is composed by the consequences of applying the rules $\{b, c\}$ which are applicable in $o(c): \neg m$.

The semantics of input/output logic given by Makinson and van der Torre [33] is an operational semantics, which characterizes the output as a function of the input and the set of norms. Makinson and van der Torre illustrate how to recapture input/output logic in modal logic, and thus give it a classical possible worlds semantics. Bochman [5] illustrates how the operational semantics of input/output logic can be rephrased as a bimodel semantics, in which a model of a set of conditionals is a pair of partial models from the base logic (here, propositional logic). Due to space limitations we have to be brief on details with respect to input/output logics, see $[33,34]$ for the semantics of input/output logics, further details on its proof theory, its possible translation to modal logic, alternative constraints, and examples. 


\subsection{Multiagent systems}

We assume that the base language contains Boolean variables and logical connectives. The variables are either decision variables of an agent, which represent the agent's actions and whose truth value is directly determined by it, or parameters, which describe both the state of the world and institutional facts, and whose truth value can only be determined indirectly. Our terminology is borrowed from Lang et al. [32] and is used in discrete event systems, and many formalisms in operations research.

Given the same set of mental attitudes, agents reason and act differently: when facing a conflict among their motivations and beliefs, agents may prefer to fulfill distinct goals and desires. We express these agent characteristics by a priority relation on the mental attitudes which encode how the agent resolves its conflicts [20]. The priority relation is defined on the powerset of the mental attitudes such that a wide range of characteristics can be described, including social agents that take the desires or goals of other agents into account. The priority relation contains at least the subset-relation which expresses a kind of independence among the motivations.

Background knowledge is formalized by a set of effects $E$ represented by rules.

Definition 3 (Agent set) An agent set is a tuple $\left\langle A, X, B, D, G, A D, E, \geq_{,} \geq_{E}\right\rangle$, where:

- the agents $A$, propositional variables $X$, agent beliefs $B$, desires $D$, goals $G$, and effects $E$ are six finite disjoint sets. $B, D, G$ are sets of mental attitudes. We write $M=D \cup G$ for the motivations defined as the union of the desires and goals. The set of effects $E$ represents the background knowledge of all agents.

- an agent description $A D: A \rightarrow 2^{X \cup B \cup M}$ is a total function that maps each agent to sets of variables (its decision variables), beliefs, desires and goals, but that does not necessarily assign each variable to at least one agent. For each agent $b \in A$, we write $X_{b}$ for $X \cap A D(b)$, and $B_{b}$ for $B \cap A D(b), D_{b}$ for $D \cap A D(b)$, etc. We write parameters $P=X \backslash \cup_{b \in A} X_{b}$.

- a priority relation $\geq: A \rightarrow 2^{B} \times 2^{B} \cup 2^{M} \times 2^{M}$ is a function from agents to a transitive and reflexive partial relation on the powerset of the motivations containing at least the subset relation. We write $\geq_{b}$ for $\geq(b)$.

- a priority relation $\geq_{E}: 2^{E} \times 2^{E}$ is a transitive and reflexive partial relation on the powerset of effects containing at least the subset relation.

Example $3 A=\{a\}, X_{a}=\{$ drive $\}, P=\{$ s, catalytic $\}, D_{a}=\left\{d_{1}, d_{2}\right\}$, $\geq_{a}=\left\{d_{2}\right\} \geq\left\{d_{1}\right\}$. There is a single agent, agent a, who can drive a car. Moreover, it can be sanctioned and the car can be catalytic. It has two desires, one to drive $\left(d_{1}\right)$, another one not to be sanctioned $\left(d_{2}\right)$. The second desire is more important.

In a multiagent system, beliefs, desires, goals and effects are abstract concepts which are described by rules built from literals.

Definition 4 (Multiagent system) A multiagent system, written as NMAS, is a tuple $\left\langle A, X, B, D, G, A D, E, R D, \geq, \geq_{E}\right\rangle$, where $\quad\left\langle A, X, B, D, G, A D, E, \geq_{,} \geq_{E}\right\rangle \quad$ is 
an agent set, and the rule description $R D:(B \cup M \cup E) \rightarrow \operatorname{Rul}(X)$ is a total function from the sets of beliefs, desires and goals, and effects to the set of rules built from $X$. For a set of pointers $S \subseteq B \cup M \cup E$, we write $R D(S)=\{R D(q) \mid q \in S\}$.

Example 4 (Continued) $R D\left(d_{1}\right)=\top \rightarrow$ drive, $R D\left(d_{2}\right)=\top \rightarrow \neg s$.

In the description of the normative system, we do not introduce norms explicitly, but we represent several concepts which are illustrated in the following sections. Institutional facts $(I)$ represent legal abstract categories which depend on the beliefs of the normative system and have no direct counterpart in the world. $F=X \backslash I$ are what Searle calls "brute facts": physical facts like the actions of the agents and their effects. $V(x, a)$ represents the decision of agent $\mathbf{n}$ that recognizes $x$ as a violation by agent $a$. The goal distribution $G D(a) \subseteq G_{\mathbf{n}}$ represents the goals of agent $\mathbf{n}$ the agent $a$ is responsible for.

Definition 5 (Normative system) A normative multiagent system, written as NMAS, is a tuple $\left\langle A, X, B, D, G, A D, E, R D, \geq, \geq_{E}, \mathbf{n}, I, V, G D\right\rangle$ where the tuple $\left\langle A, X, B, D, G, A D, E, R D, \geq, \geq_{E}\right\rangle$ is a multiagent system, and

- the normative system $\mathbf{n} \in A$ is an agent.

- the institutional facts $I \subseteq P$ are a subset of the parameters.

- the norm description $V: \operatorname{Lit}(X) \times A \rightarrow X_{\mathbf{n}} \cup P$ is a function from the literals and the agents to the decision variables of the normative system and the parameters.

- the goal distribution $G D: A \rightarrow 2^{G_{\mathbf{n}}}$ is a function from the agents to the powerset of the goals of the normative system, such that if $L \rightarrow l \in R D(G D(a))$, then $l \in \operatorname{Lit}\left(X_{a} \cup P\right)$.

Agent $\mathbf{n}$ is a normative system with the goal that non catalytic cars are not driven, i.e., with the aim to achieve the social order in which such cars are not driven.

Example $\mathbf{5}$ (Continued) There is agent $\mathbf{n}$, representing the normative system.

$P=\{s, V($ drive, $a)$, catalytic $\}, D_{\mathbf{n}}=G_{\mathbf{n}}=\left\{g_{1}\right\}$,

$R D\left(g_{1}\right)=\{\neg$ catalytic $\rightarrow \neg$ drive $\}, G D(a)=\left\{g_{1}\right\}$.

The parameter $V($ drive, a) represents the fact that the normative system considers a violation agent a's action of driving. It has the goal that non-ecological vehicles should not be driven by $a$ and it has distributed this goal to agent a.

In the following, we use an input/output logic out to define whether a desire or goal implies another one and to define the application of a set of belief rules to a set of literals; in both cases we use the out $t_{3}$ operation since it has the desired logical property of not satisfying identity.

We now define obligations and the counts-as relation at the five levels of abstraction. 


\subsection{First level of abstraction (highest)}

Regulative norms are conditional obligations with an associated sanction. At the higher level of abstraction, the definition contains three clauses: the first two clauses state that recognitions of violations and sanctions are a consequence of the behavior of agent $a$, as it is represented by the background knowledge rules $E$. For an obligation to be effective, the third clause states that the sanction must be disliked by its addressee.

Definition 6 (Obligation (level 1)) Let NMAS be a normative multiagent system $\left\langle A, X, B, D, G, A D, E, R D, \geq, \geq_{E}, \mathbf{n}, I, V, G D\right\rangle$.

Agent $a \in A$ is obliged to see to it that $x \in \operatorname{Lit}\left(X_{a} \cup P\right)$ with $V(\sim x, a) \in \operatorname{Lit}(P)$ and sanction $s \in \operatorname{Lit}(P)$ in context $Y \subseteq \operatorname{Lit}(X)$ in $N M A S$, written as $N M A S \models O_{a \mathbf{n}}^{1}(x, s \mid Y)$, if and only if:

1. $Y \cup\{\sim x\} \rightarrow V(\sim x, a) \in \operatorname{out}\left(E, \geq_{E}\right)$ : if $Y$ and $x$ is false, then it follows that $\sim x$ is a violation by agent $a$.

2. $Y \cup\{V(\sim x, a)\} \rightarrow s \in \operatorname{out}\left(E, \geq_{E}\right)$ : if $Y$ and there is a violation by agent a, then it is sanctioned.

3. $Y \rightarrow \sim s \in \operatorname{out}\left(D_{a}, \geq_{a}\right)$ : if $Y$, then agent a desires $\sim s$, which expresses that it does not like to be sanctioned.

Example 6 Let: $E=\left\{e_{1}, e_{2}\right\}, D_{a}=\left\{d_{2}\right\}$

$R D\left(e_{1}\right)=\{\neg$ catalytic, drive $\} \rightarrow V($ drive,$a)$

$R D\left(e_{2}\right)=\{\neg$ catalytic,$V($ drive,$a)\} \rightarrow s$

$R D\left(d_{2}\right)=\neg$ catalytic $\rightarrow \sim s$

$N M A S \models O_{a \mathbf{n}}^{1}(\neg$ drive, $s \mid \neg$ catalytic $)$, since:

1. $\{\neg$ catalytic, drive $\} \rightarrow V($ drive,$a) \in \operatorname{out}\left(E, \geq_{E}\right)$

2. $\{\neg$ catalytic,$V($ drive,$a)\} \rightarrow s \in$ out $\left(E, \geq_{E}\right)$

3. $\neg$ catalytic $\rightarrow \sim s \in \operatorname{out}\left(D_{a}, \geq_{a}\right)$

To determine what the agent a will do, we can define a qualitative decision problem that considers the consequences of its decisions, and chooses the decision which achieves the goals with the highest priority. Here it will choose not to drive a non-catalytic car, because it does not want to be sanctioned.

Constitutive norms introduce new abstract categories of existing facts and entities, called institutional facts. In [10] we formalize the counts-as conditional as a belief rule of the normative system $\mathbf{n}$. However, since at the first level of abstraction we do not consider yet the normative system, counts-as conditionals are part of the background knowledge rules $E$.

The condition $x$ of the rule is a variable which can be an action of an agent, a brute fact or an institutional fact. So, the counts-as relation can be iteratively applied. 
Definition 7 (Counts-as relation (level 1)) Let NMAS be a normative multiagent system $\left\langle A, X, B, D, G, A D, E, R D, \geq, \geq_{E}, \mathbf{n}, I, V, G D\right\rangle . A$ literal $x \in \operatorname{Lit}(X)$ counts-as $y \in \operatorname{Lit}(I)$ in context $C \subseteq \operatorname{Lit}(X), \quad N M A S \models$ counts-as $_{\mathbf{n}}^{1}(x, y \mid C)$, iff $C \cup\{x\} \rightarrow y \in \operatorname{out}\left(E, \geq_{E}\right): y$ is a consequence of $C$ and $x$.

Example $7 \quad P \backslash I=\{$ catalytic $\}, I=\{e c o\}, X_{a}=\{$ drive $\}, E=\left\{b_{1}\right\}, R D\left(b_{1}\right)=$ catalytic $\rightarrow$ eco

Consequently, NMAS $=$ counts-as $\mathbf{n}_{\mathbf{n}}$ (catalytic, eco|T). This formalizes that for the normative system a catalytic car counts as an ecological vehicle. The presence of the catalytic converter is a physical "brute" fact, while being an ecological vehicle is an institutional fact. In situation $S=\{$ catalytic $\}$, given $E$ we have that the consequences of the constitutive norms are out $\left(E, S, \geq_{E}\right)=\{$ eco $\}$ (since out $_{3}$ does not include Id).

Note that the institutional facts can appear in the conditions of regulative norms.

Example 8 A regulative norm which forbids driving non catalytic cars can refer to the abstract concept of ecological vehicle rather than to catalytic converters:

$N M A S \models O_{a \mathbf{n}}^{1}(\neg$ drive, $s \mid \neg e c o)$

To define the optimal decision of an agent in a normative system incorporating both the regulative and the constitutive norm, the consequences of its decisions first take into account which institutional effects follow from the brute facts, and thereafter the decisions of the normative system.

As the system evolves, new cases can be added to the notion of ecological vehicle by means of new constitutive norms, without changing the regulative norms about it. E.g., if a car has fuel cells, then it is an ecological vehicle: fuelcell $\rightarrow e c o \in R D(E)$.

\subsection{Second level of abstraction}

At the second level of abstraction we introduce the normative system as an agent described by mental attitudes like beliefs and goals. We do not consider, however, how its goals are achieved in the multiagent system. Thus, it represents only an abstract specification of the ideal behavior of the system.

The first and central clause of our definition of obligation defines obligations of agents as goals of the normative system, following the "your wish is my command" metaphor. It says that the obligation is implied by the desires of the normative system $\mathbf{n}$, implied by the goals of agent $\mathbf{n}$, and it has been distributed by agent $\mathbf{n}$ to the agent. The latter two steps are represented by out $\left(G D(a), \geq_{\mathbf{n}}\right)$.

The second and third clause can be read as the normative system has the goal that the absence of $p$ is considered as a violation. The third clause says that the agent desires that there are no violations, which is stronger than that it does not desire violations, as would be expressed by $\top \rightarrow V(\sim x, a) \notin$ out $\left(D_{\mathbf{n}}, \geq_{\mathbf{n}}\right)$.

The fourth and fifth clause relate violations to sanctions. The fifth clause says that the normative system is motivated not to sanction as long as their is no violation, because otherwise the norm would have no effect. Finally, for the same reason the last clause says that the agent does not like the sanction. 
Definition 8 (Obligation (level 2)) Let NMAS be a normative multiagent system $\left\langle A, X, B, D, G, A D, E, R D, \geq, \geq_{E}, \mathbf{n}, I, V, G D\right\rangle$. Agent $a \in A$ is obliged to see to it that $x \in \operatorname{Lit}\left(X_{a} \cup P\right)$ with $V(\sim x, a) \in \operatorname{Lit}(P)$ and sanction $s \in \operatorname{Lit}(P)$ in context $Y \subseteq \operatorname{Lit}(X)$ in $N M A S$, written as $N M A S \models O_{a \mathbf{n}}^{2}(x, s \mid Y)$, if and only if:

1. $Y \rightarrow x \in \operatorname{out}\left(D_{\mathbf{n}}, \geq_{\mathbf{n}}\right) \cap \operatorname{out}\left(G D(a), \geq_{\mathbf{n}}\right)$ : if $Y$ holds then agent $\mathbf{n}$ desires and has as a goal that $x$, and this goal has been distributed to agent $a$.

2. $Y \cup\{\sim x\} \rightarrow V(\sim x, a) \in \operatorname{out}\left(D_{\mathbf{n}}, \geq_{\mathbf{n}}\right) \cap$ out $\left(G_{\mathbf{n}}, \geq_{\mathbf{n}}\right)$ : if $Y$ holds and $\sim x$, then agent $\mathbf{n}$ has the goal and the desire $V(\sim x, a)$ : that it is recognized as a violation by agent a.

3. $\top \rightarrow \neg V(\sim x, a) \in \operatorname{out}\left(D_{\mathbf{n}}, \geq_{\mathbf{n}}\right)$ : agent $\mathbf{n}$ desires that there are no violations.

4. $Y \cup\{V(\sim x, a)\} \rightarrow s \in \operatorname{out}\left(D_{\mathbf{n}}, \geq_{\mathbf{n}}\right) \cap \operatorname{out}\left(G_{\mathbf{n}}, \geq_{\mathbf{n}}\right)$ : if $Y$ holds and agent $\mathbf{n}$ decides $V(\sim x, a)$, then agent $\mathbf{n}$ desires and has as a goal that agent $a$ is sanctioned.

5. $Y \rightarrow \sim s \in \operatorname{out}\left(D_{\mathbf{n}}, \geq_{\mathbf{n}}\right)$ : if $Y$ holds, then agent $\mathbf{n}$ desires not to sanction. This desire of the normative system expresses that it only sanctions in case of violation.

6. $Y \rightarrow \sim s \in$ out $\left(D_{a}, \geq_{a}\right)$ : if $Y$ holds, then agent a desires $\sim s$, which expresses that it does not like to be sanctioned.

The beliefs, desires and goals of the normative agent - defining the obligations - are not private mental states of an agent. Rather they are collectively attributed by the agents of the normative system to the normative agent: they have a public character, and, thus, which are the obligations of the normative system is a public information.

In [10] we formalize the counts-as conditional as a belief rule of the normative system $\mathbf{n}$. At the second level we do the same, to separate brute facts and their relations $E$ from institutional facts. Note that in our model the counts-as relation does not satisfy the identity rule. See [10] for a discussion of the motivations.

Definition 9 (Counts-as relation (level 2)) Let NMAS be a normative multiagent system $\left\langle A, X, B, D, G, A D, E, R D, \geq \geq_{E}, \mathbf{n}, I, V, G D\right\rangle$. A literal $x \in$ Lit $(X)$ counts-as $y \in \operatorname{Lit}(I)$ in context $C \subseteq \operatorname{Lit}(X), \quad N M A S \models$ counts-as $_{\mathbf{n}}^{1}(x, y \mid C)$, iff $C \cup\{x\} \rightarrow y \in \operatorname{out}\left(B_{\mathbf{n}}, \geq_{\mathbf{n}}\right)$ : if agent $\mathbf{n}$ believes $C$ and $x$ then it believes $y$.

Example $9 \quad P \backslash I=\{$ catalytic $\}, I=\{e c o\}, X_{a}=\{$ drive $\}, B_{\mathbf{n}}=\left\{b_{1}\right\}$, $R D\left(b_{1}\right)=$ catalytic $\rightarrow$ eco

Consequently, NMAS $\models$ counts-as $\mathbf{n}_{\mathbf{n}}^{1}$ catalytic, eco|T). This formalizes that for the normative system a catalytic car counts as an ecological vehicle. The presence of the catalytic converter is a physical "brute" fact, while being an ecological vehicle is an institutional fact. In situation $S=\{$ catalytic\}, given $E$ we have that the consequences of the constitutive norms are out $\left(B_{\mathbf{n}}, S, \geq_{\mathbf{n}}\right)=\{$ eco $\}$ (and not catalytic, since out $t_{3}$ does not include Id). 


\subsection{Third level of abstraction}

At the next level of abstraction, also for constitutive rules actions of the normative systems are added in the definition of the obligations: the recognition of a violation and sanctions. Since the actions undergo a decision process, desires and goals of the normative system are added like at the previous level.

Definition 10 (Obligation (level 3)) $N M A S \models O_{a \mathbf{n}}^{3}(x, s \mid Y)$, iff $N M A S \models O_{a \mathbf{n}}^{2}(x, s \mid Y)$ and $s \in \operatorname{Lit}\left(X_{\mathbf{n}}\right)$ and $V(\sim x, a) \in \operatorname{Lit}\left(X_{\mathbf{n}}\right)$.

The rules in the definition of obligation are only motivations, and not beliefs, because a normative system may not recognize that a violation counts as such, or that it does not sanction it: it is up to its decision. Both the recognition of the violation and the application of the sanction are the result of autonomous decisions of the normative system that is modelled as an agent. In this way, regulative norms can be interpreted by the normative system, who takes a decision depending on the circumstances.

Example 10 Let: $G_{\mathbf{n}}=\left\{g_{1}, g_{2}, g_{4}\right\}, G_{\mathbf{n}} \cup\left\{g_{3}, d_{2}\right\}=D_{\mathbf{n}},\left\{g_{1}\right\}=G D(a),\left\{d_{2}\right\}=D_{a}$

$$
\begin{aligned}
& R D\left(g_{2}\right)=\{\neg \text { catalytic, drive }\} \rightarrow V(\text { drive }, a) \quad R D\left(g_{3}\right)=\top \rightarrow \neg V(\text { drive }, a) \\
& R D\left(g_{4}\right)=\{\neg \text { catalytic, } V(\text { drive }, a)\} \rightarrow s \\
& N M A S \models O_{\text {an }}^{3}(\neg \text { drive, } s \mid \neg \text { catalytic }) \text {, since: } \\
& \text { 1. } \neg \text { catalytic } \rightarrow \neg \text { drive } \in \operatorname{out}\left(D_{\mathbf{n}}, \geq_{\mathbf{n}}\right) \cap \operatorname{out}\left(G D(a), \geq_{\mathbf{n}}\right) \\
& \text { 2. }\{\neg \text { catalytic, drive }\} \rightarrow V(\text { drive }, a) \in \operatorname{out}\left(D_{\mathbf{n}}, \geq_{\mathbf{n}}\right) \cap \operatorname{out}\left(G_{\mathbf{n}}, \geq_{\mathbf{n}}\right) \\
& \text { 3. } \top \rightarrow \neg V(\text { drive }, a) \in \operatorname{out}\left(D_{\mathbf{n}}, \geq_{\mathbf{n}}\right) \\
& \text { 4. }\{\neg \text { catalytic, } V(\text { drive }, a)\} \rightarrow s \in \operatorname{out}\left(D_{\mathbf{n}}, \geq_{\mathbf{n}}\right) \cap \operatorname{out}\left(G_{\mathbf{n}}, \geq_{\mathbf{n}}\right) \\
& \text { 5. } \neg \text { catalytic } \rightarrow \sim s \in \operatorname{out}\left(D_{\mathbf{n}}, \geq_{\mathbf{n}}\right) \\
& \text { 6. } \neg \text { catalytic } \rightarrow \sim s \in \operatorname{out}\left(D_{a}, \geq_{a}\right)
\end{aligned}
$$

It is only at this level of abstraction that we formalize the game-theoretic approach to normative systems, in the sense that to determine the optimal decision of an agent, we have to take the response of the normative system into account by recursively modelling it [25]. Moreover, we can also play more complex games, for example one in which the normative system decides which norm to introduce by recursively modelling agents recursively modelling the normative system again, and so on.

At the third level of abstraction, we introduce in the model the actions of the normative system. The beliefs of the normative system are restricted to the connections between actions and the consequences of these actions for the normative system. The normative system has the desire and goal that the institutional fact $y$ holds if the fact $x$ holds in context $C$. The normative system believes that to make $y$ true it has to perform an action $z$. Thus the fact $x$ holding in context $C$ is not sufficient for the institutional fact $y$ to be true: a decision is necessary also to do $z$ by the normative system. 
Definition 11 (Counts-as relation (level 3)) Let NMAS be a normative multiagent system $\left\langle A, X, B, D, G, A D, E, R D, \geq, \geq_{E}, \mathbf{n}, I, V, G D\right\rangle$. A literal $x \in$ Lit $(X)$ counts-as $y \in \operatorname{Lit}(I)$ in context $C \subseteq \operatorname{Lit}(X), N M A S \models$ counts-as $s_{\mathbf{n}}^{3}(x, y \mid C)$, iff:

1. $C \cup\{x\} \rightarrow y \in \operatorname{out}\left(D_{\mathbf{n}}, \geq_{\mathbf{n}}\right) \cap \operatorname{out}\left(G_{\mathbf{n}}, \geq_{\mathbf{n}}\right)$ : it is a desire and goal of the normative system that in context $C$ the fact $x$ is considered as the institutional fact $y$.

2. $\exists z \in X_{\mathbf{n}}$ such that $C \cup\{z\} \rightarrow y \in$ out $\left(B_{\mathbf{n}}, \geq_{\mathbf{n}}\right)$ : there exists an action $z$ of the normative system $\mathbf{n}$ such that if it decides $z$ in context $C$ then it believes that the institutional fact $y$ follows (i.e., counts-as $\mathbf{n}_{\mathbf{n}}^{2}(z, y \mid C)$ at the second level of abstraction).

Example $11 P \backslash I=\{$ catalytic $\}, I=\{$ eco $\}, X_{a}=\{$ drive $\}, X_{\mathbf{n}}=\{$ stamp $\}$

$D_{\mathbf{n}}=G_{\mathbf{n}}=\left\{d_{3}\right\}, R D\left(d_{3}\right)=$ catalytic $\rightarrow$ eco

$B_{\mathbf{n}}=\left\{b_{1}\right\}, R D\left(b_{1}\right)=$ stamp $\rightarrow$ eco

Consequently, NMAS $=$ counts-as $\mathbf{n}_{\mathbf{n}}^{2}$ catalytic, eco|T). This formalizes that the normative system wants that if a car is catalytic, then it is considered as an ecological vehicle and the normative believes that from system putting a stamp on a catalytic car licence follows the fact that the car is catalytic. In situation $S=\{$ catalytic $\}$, given $B_{\mathbf{n}}$ we have that the consequences of the constitutive norms are out $\left(B_{\mathbf{n}}, S, \geq_{\mathbf{n}}\right)=\emptyset$ and thus the goal $d_{3}$ remains unsatisfied, while in situation $S^{\prime}=\{$ catalytic, stamp $\}$ they are out $\left(B_{\mathbf{n}}, S^{\prime}, \geq_{\mathbf{n}}\right)=\{$ eco $\}$ and the goal $d_{3}$ is satisfied.

This level of abstraction supposes that the normative system is an agent acting in the world. For example, a specialized agent introduced by the designer of the infrastructure. This abstraction can be detailed by introducing agents acting on behalf of the normative system: the normative system wants that an agent $a$ makes the institutional fact $y$ true if $x$ holds in context $C$ and believes that the effect of action $z$ of agent $a$ is the institutional fact $y$.

\subsection{Fourth level of abstraction}

Before introducing the next concrete level of abstraction in obligations we discuss the fourth level of constitutive norms which is based on the notion of delegation of power, since it is used in obligations.

Definition 12 (Counts-as relation (level 4) and delegation of power) Let NMAS be a normative multiagent system $\left\langle A, X, B, D, G, A D, E, R D, \geq, \geq_{E}, \mathbf{n}, I, V, G D\right\rangle$.

$a \in A$ is an agent, $z \in X_{a}$ an action of agent $a, x \in \operatorname{Lit}(X)$ is a literal built out of a variable, $y \in \operatorname{Lit}(I)$ a literal built out of an institutional fact, $C \subseteq \operatorname{Lit}(X)$ the context. Agent a has been delegated the power to consider $x$ in context $C$ as the institutional fact $y$, $N M A S \models$ delegated $_{\mathbf{n}}^{4}(a, z, x, y \mid C)$, iff:

1. $C \cup\{x\} \rightarrow y \in \operatorname{out}\left(D_{\mathbf{n}}, \geq_{\mathbf{n}}\right) \cap \operatorname{out}\left(G D(a), \geq_{\mathbf{n}}\right)$ : it is a desire of the normative system and a goal distributed to agent a that in context $C$ the fact $x$ is considered as the institutional fact $y$.

2. $\exists z \in X_{a}$ such that $C \cup\{z\} \rightarrow y \in \operatorname{out}\left(B_{\mathbf{n}}, \geq_{\mathbf{n}}\right)$ : there exists an action $z$ of agent a such that if it decides $z$ then the normative system believes that the institutional fact $y$ follows (i.e., counts-as $s_{\mathbf{n}}^{2}(z, y \mid C)$ at the second level of abstraction). 
If $N M A S \models$ delegated $_{\mathbf{n}}^{4}(a, z, x, y \mid C)$, then $N M A S \models \operatorname{counts}_{-} a s_{\mathbf{n}}^{4}(x, y \mid C)$,

Example $12 b \in A, P \backslash I=\{$ catalytic $\}, I=\{$ eco $\}, X_{a}=\{$ drive $\}, X_{b}=\{$ stamp $\}$

$D_{\mathbf{n}}=G D(b)=\left\{d_{3}\right\}, R D\left(d_{3}\right)=$ catalytic $\rightarrow$ eco

$B_{\mathbf{n}}=\left\{b_{1}\right\}, R D\left(b_{1}\right)=$ stamp $\rightarrow$ eco

Thus, $N M A S \models$ delegated $_{\mathbf{n}}^{4}(b$, stamp, catalytic, eco|T). Note that with respect to Example 11 , the goal $d_{3}$ is distributed to agent $b$ and stamp is an action of agent $b$.

We can now define obligations where agents have been delegated the power of recognizing violations by means of actions which count as such. Differently from the obligation of level 3, clause 2 distributes a goal to agent $b$ who is in charge of recognizing violations and whose action $z$ is believed by the normative system $\mathbf{n}$ to be the recognition of a violation (clause 7).

Definition 13 (Obligation (level 4)) Let NMAS be a normative multiagent system $\left\langle A, X, B, D, G, A D, E, R D, \geq, \geq_{E}, \mathbf{n}, I, V, G D\right\rangle$. Agent $a \in A$ is obliged to see to it that $x \in \operatorname{Lit}\left(X_{a} \cup P\right)$ with $V(\sim x, a) \in \operatorname{Lit}(P)$ and sanction $s \in \operatorname{Lit}\left(X_{c} \cup P\right)$ in context $Y \subseteq \operatorname{Lit}(X)$ in $N M A S$, written as $N M A S=O_{a \mathbf{n}}^{4}(x, s \mid Y)$, if and only if $\exists b, c \in A$ and $a$ decision variable $z \in X_{b}$ such that Definition 10 holds except that:

2. $Y \cup\{\sim x\} \rightarrow V(\sim x, a) \in \operatorname{out}\left(D_{\mathbf{n}}, \geq_{\mathbf{n}}\right) \cap$ out $\left(G D(b), \geq_{\mathbf{n}}\right)$ : if $Y$ holds and $\sim x$ is true, then agent $\mathbf{n}$ has distributed to agent $b$ the goal $V(\sim x, a)$ : that it is recognized as a violation in context $Y$.

4. $Y \cup\{V(\sim x, a)\} \rightarrow s \in \operatorname{out}\left(D_{\mathbf{n}}, \geq_{\mathbf{n}}\right) \cap \operatorname{out}\left(G D(c), \geq_{\mathbf{n}}\right)$ : if $Y$ holds and agent $\mathbf{n}$ decides $V(\sim x, a)$, then agent $\mathbf{n}$ has distributed the goal to sanction agent a to agent $c$

7. $Y \cup\{z\} \rightarrow V(\sim x, a) \in \operatorname{out}\left(B_{\mathbf{n}}, \geq_{\mathbf{n}}\right)$ : from action $z$ of agent $b \mathbf{n}$ believes it follows the recognition of the violation.

From clause 2 and 7 it follows that agent $b$ has been delegated the power to recognize violations by means of his action $z$ :

if $N M A S \models O_{a \mathbf{n}}^{4}(x, s \mid Y)$ then

$N M A S \models \exists b \in A, z \in X_{b}$ delegated $4(b, z, \sim x, V(\sim x, a) \mid Y)$

\subsection{Fifth level of abstraction (most detailed)}

Clauses 2 and 4 of the definition above are like the first clauses of obligations $O_{b \mathbf{n}}\left(V(\sim x, a), s^{\prime} \mid Y \cup\{\sim x\}\right)$ and $O_{c \mathbf{n}}\left(s, s^{\prime \prime} \mid \sim x \wedge V(\sim x, a) \wedge Y\right)$. The model can thus be extended with procedural norms directed towards agents which have to take care of the procedural aspects of law, like prosecuting violations and sanctioning violators. These additional obligations are procedural norms and provide a motivation for the agents in charge of prosecuting and sanctioning. In this way, we introduce the distinction between a defender agent $b$ who has the duty to monitor a norm and a defender agent $c$ who has to enforce the norm by applying sanctions: they are subject to procedural norms of the normative system. 
Definition 14 (Obligation with procedural norms (level 5)) Let NMAS be a normative multiagent system $\left\langle A, X, B, D, G, A D, E, R D, \geq, \geq_{E}, \mathbf{n}, I, V, G D\right\rangle$. Agent $a \in A$ is obliged to see to it that $x \in \operatorname{Lit}\left(X_{a} \cup P\right)$ with $V(\sim x, a) \in \operatorname{Lit}(P)$ and sanction $s \in \operatorname{Lit}\left(X_{c} \cup P\right), s^{\prime}, s^{\prime \prime} \in \operatorname{Lit}\left(X_{\mathbf{n}} \cup P\right)$ in context $Y \subseteq \operatorname{Lit}(X)$ in NMAS, written as $N M A S \models O_{a \mathbf{n}}^{5}(x, s \mid Y)$, if and only if $\exists b, c \in A$ and a decision variable $z \in X_{b}, 1 \geq i \geq 4$, such that Definition 13 holds except that:

2. $N M A S \models O_{b \mathbf{n}}^{i}\left(V(\sim x, a), s^{\prime} \mid \sim x \wedge Y\right)$ : if $Y$ holds and $\sim x$ is true, then agent $b$ is obliged by $\mathbf{n}$ that $V(\sim x, a)$ is true: that $\sim x$ is recognized as a violation in context $Y$.

4. $N M A S \models O_{c \mathbf{n}}^{i}\left(s, s^{\prime \prime} \mid V(\sim x, a) \wedge Y\right)$ : if $Y$ and $\sim x$ hold and $b$ has recognized $\sim x$ as a violation done by agent $a$, then agent $c$ is obliged by agent $\mathbf{n}$ that $V(\sim x, a)$ to sanction agent a with $s$.

Note that the procedural norms cannot be obligations defined at level 5 to avoid an infinite recursion. The idea underlying this definition is that less complex methods of control are needed to deal with the behavior of agents playing roles in the normative system. As Firozabadi and van der Torre [40] claim, at higher levels the control routines become less risky and require less effort, there is no need of a infinite regression of authorities controlling each other.

Obligations at Items 2 and 4 imply by their definitions the following goals:

1. $Y \cup\{\sim x\} \rightarrow V(\sim x, a) \in \operatorname{out}\left(D_{\mathbf{n}}, \geq_{\mathbf{n}}\right) \cap \operatorname{out}\left(G_{\mathbf{n}}, \geq_{\mathbf{n}}\right)$

2. $Y \cup\{V(\sim x, a)\} \rightarrow s \in \operatorname{out}\left(D_{\mathbf{n}}, \geq_{\mathbf{n}}\right) \cap \operatorname{out}\left(G_{\mathbf{n}}, \geq_{\mathbf{n}}\right)$

Given that these two goals are of the normative system $\mathbf{n}$ we have that:

$N M A S \models O_{a \mathbf{n}}^{5}(x, s \mid Y)$ implies $N M A S \models O_{a \mathbf{n}}^{4}(x, s \mid Y)$

Moreover, it follows that agent $b$ has been delegated the power to recognize violations by means of his action $z$ :

$N M A S \models \exists b \in A, z \in X_{b}$ delegated $_{\mathbf{n}}^{4}(b, z, \sim x, V(\sim x, a) \mid Y)$

Similar definitions can be introduced for obligations where sanctions are institutional facts which have been delegated to defender agent $c$.

Procedural norms enters the definition of delegation of power by obliging some agent to recognize a fact as an institutional fact by exercising the delegated power:

Definition 15 (Delegation of power with procedural norms and counts-as (level 5)) Let $N M A S$ be a normative multiagent system $\left\langle A, X, B, D, G, A D, E, R D, \geq, \geq_{E}, \mathbf{n}, I, V, G D\right\rangle$.

$a \in A$ is an agent, $z \in X_{a}$ an action of agent $a, x \in \operatorname{Lit}(X)$ is a literal built out of $a$ variable, $y \in \operatorname{Lit}(I)$ a literal built out of an institutional fact, $C \subseteq \operatorname{Lit}(X)$ the context, sanction $s \in \operatorname{Lit}\left(X_{\mathbf{n}} \cup P\right)$ and $1 \geq i \geq 4$. Agent a has been delegated the power to consider $x$ in context $C$ as the institutional fact $y, N M A S \models \operatorname{delegated~}_{\mathbf{n}}^{5}(a, z, x, y \mid C)$, iff Definition 12 holds, except that:

1. $N M A S \models O_{b \mathbf{n}}^{i}(y, s \mid C \wedge x)$ : agent $b$ is obliged by the normative system $\mathbf{n}$ that in context $C$ the fact $x$ is considered as the institutional fact $y$. 
Agent a can fulfil his obligations by exercising his powers specified in clause 2. If $N M A S \models$ delegated $_{\mathbf{n}}^{5}(a, z, x, y \mid C)$, then $N M A S \models \operatorname{counts}^{-a s_{\mathbf{n}}^{5}}(x, y \mid C)$.

Moreover, if $N M A S \models$ delegated $_{\mathbf{n}}^{5}(a, z, x, y \mid C)$, then $N M A S \models \operatorname{delegated}_{\mathbf{n}}^{4}(a, z, x, y \mid C)$.

When procedural norms and agent playing roles in the system like defender agents are introduced, games become more complex. In particular an agent subject to an obligation has to predict the behavior of the defender agent of monitoring and sanctioning him. However, he knows that the defender agent will take his decision by foreseeing the reaction of the normative system too, since he is subject to procedural norms. In [13] we discuss such more complex games.

Procedural norms allow to realize one of the key concepts of the organization of modern societies: the separation of powers as proposed in the Montesquieu's trias politica: the representative, executive and judicial authorities should be kept distinct [7]. Moreover decentralizing the control of the policies regulating a society supports the view that tasks can be better performed if they are dealt with by the local level in an autonomous way. As we discussed before, this delegation allows agents to interpret norms, making the system more flexible.

\section{Related work}

Most work in normative multiagent systems gives an abstract description of the normative system in terms of substantive norms only, specifying which is the desired social order in terms of obligations, permissions and, sometimes, counts-as norms. At this level of abstraction, the monitoring and enforcement of norms is made automatically and implicitly at the infrastructure level. Alternatively, special agents are introduced for monitoring and enforcing norms, but they are assumed to comply with their role without further motivations [19].

Moreover, no declarative representation of the monitoring and enforcement mechanisms is done. However, when the system is examined at more detailed levels of abstraction new problems arise, like which actions are necessary to establish whether an obligation has been violated, which sanctions are to be applied and, at even more detailed levels where agents acting for the normative system are introduced, who is in charge of executing these actions and which are his motivations which lead him to perform these actions. It is an open problem, moreover, how constitutive norms behave at these more detailed levels of abstraction where agents playing roles in normative systems are considered.

The framework in this paper further refine the logical framework of our game-theoretic approach to normative multiagent systems $[11,13]$ which explicitly takes into account the activity of agents in the definition of sanction based obligations. The basic assumptions of our model are that beliefs, goals and desires of an agent are represented by conditional rules, and that, when an agent takes a decision, it recursively models the other agents interfering with it in order to predict their reaction to his decision as in a game. Most importantly, the normative system itself can be conceptualized as a socially constructed agent with whom it is possible to play games to understand what will be its reaction to the agent's decision. The actions of the normative system can be seen as abstractions of the actions of the agents to whom some of the powers of the system have been delegated.

The abstraction hierarchy explains also how the normative systems as autonomous agent 
metaphor introduced in our earlier work can be explained. In the model presented in [11], regulative norms are represented by the goals of the normative system and constitutive norms as its beliefs. However, when actions of the normative system and agents playing roles in it are considered new facts must be added to norms. For example, the goals of recognizing violations and applying sanctions must be added to obligations [11]. The abstraction hierarchy explains this metaphor, since at level 5 we model all details of a normative multiagent system. The abstraction at which a normative system acts as an agent is given at level 3 .

Aldewereld [1] introduces an abstraction hierarchy for norms for norm design, that is, to refine abstract norms such that they can be implemented. His approach is based on so-called "root enforcers," which we believe are not necessary [8]. It is not clear how we can map our five layers to his hierarchy. Moreover, Aldewereld does not formally define procedural norms, he only suggests that they are a kind of nested norms. Given the problems with nested norms in the context of von Wright's so-called "transmission of will", it is not clear whether nested deontic conditionals defined in the usual way are sufficient, see [13].

The levels of abstraction allow a comparison with existing models of norms in deontic logic and normative systems. Most works in normative multiagent systems give an abstract description of the normative system in terms of substantive norms only, specifying which is the desired social order in terms of obligations, permissions and, sometimes, counts-as norms, and can therefore be compared with level 1. We adopt level 2 and 3 for regulative norms in $[11,13]$.

The notion of empowerment in normative multiagent systems is widely discussed, but it has not been related yet with the notion of goal delegation.

Pacheco and Santos [37], for example, discuss the delegation of obligations among roles. In particular, they argue that when an obligation is delegated, a corresponding permissions must be delegated too. This rationality constraint inside an institution parallels our notion of delegation of power: when the goal to make true an institutional fact is delegated, the agent must be empowered to do so too. Moreover, in our model we can add to the notion of delegation of power also the permission for the delegated agent to perform the action which counts as the delegated institutional fact. This can be done using the definition of permission given in [13].

Pacheco and Santos consider the delegation process among roles rather than among agents. This feature can be added to our model too, using the framework for roles we discuss in [16]. Note that our model of roles describes roles by means of beliefs and goals; it is, thus, compatible with the distribution of goals to agents described by clause 2 of Definition 13.

Gelati et al. [23] combine obligations and power to define the notion of mandate in contracts: "a mandate is a proclamation intended to create the obligation of exercising a declarative power". However, they do not apply their analysis to the definition of constitutive rules but to the normative positions among agents.

Comparison with other models of counts-as is discussed in [10] and [12]. 


\section{Further research}

Some points for further research follow from our discussion on related work. For example, the use of our model for norm design could be studied, taking the work of Aldewereld as a starting point [1].

Another issue is the relation between regulative rules and delegation of power. The challenge here is to define how it is possible to create global policies [13] obliging or permitting other agents to delegate their power. Since we propose to model delegation of control by means of obligations concerning what is obligatory and what must be sanctioned, our framework can be extended with meta-policies. We can extend this framework for representing obligations by the central authority that local authorities permit of forbid access as well as permissions to forbid or permit access.

Other subjects of research can be explored when we combine the refined logical framework in this paper with other aspects of our normative multiagent systems, such as our model of roles. This allows to structure organizations in sub-organizations and roles to make a multiagent system modular and thus manage its complexity, is described in [16].

We can consider abstraction also in the underlying input/output logic. Abstraction in the input/output logic framework has been left for lions or input/output networks. In such networks each black box corresponding to an input/output logic is associated with a component in an architecture. A discussion can be found in [12].

Finally, further work is to study the use of the new refinements in applications. For example, this approach allows facing the problem of controlling distributed systems, such as virtual communities, by delegating to defender agents the task of monitoring and sanctioning violations. However, these agents are not assumed to be fully cooperative so that they are kept under the control of a judicial authority. Since, as Firozabadi and van der Torre [40] claim, at higher levels the control routines become less risky and require less effort, there is no need of a infinite regression of authorities controlling each other. As Firozabadi and Sergot [39] discuss, centralized control is not feasible in virtual communities where each participant is both a resource consumer and a resource provider. In fact, there is no authority which is in control of all the resources. Rather the central authority can only issue meta-policies [42] concerning the policies regulating the access to the single resources: for example, the central authority can oblige local authorities to grant access to their resources to authorized users, who are thus entitled to use the resources.

The model introduced in this paper also can be used for an analysis whether constitutive norms such as "cars with a catalytic converter count as ecological vehicles" can be 'violated'. The fact that obligations can be violated has been driving much research in deontic logic due to the notorious contrary-to-duty paradoxes, as well as work in normative multiagent systems where norms are considered as soft constraints rather than hard constraints. Usually it is assumed that constitutive norms cannot be violated, since they operate as a kind of definitions of the normative system. However, at a more detailed analysis proposed in this paper, it may be argued as well that the constitutive norm is 'violated' when the agent who is delegated the power to count catalytic converter as ecological vehicles does not do so. We believe that the notion of 'violation' of constitutive norms can drive further understanding and development of constitutive norms in partic- 
ular and normative multiagent systems in general, and one of the results of this paper is that it is made precise in what sense and at which level of abstraction we can say that constitutive norms are 'violated'. Despite the fact that in some sense and at some level of abstraction both regulative and constitutive norms can be violated, there are still various distinctions between them, made precise by our formal analysis.

At the lower levels it becomes possible to answer the question not only of whether constitutive norms can be violated like it happens for regulative ones, but also whether constitutive norms can be interpreted. Interpreting an obligation means that the agent in charge for monitoring violations or applying sanction decides that in a given situation it is not worthwhile to apply the norms. This is not necessarily an abuse of his position, since norms cannot foresee all possible cases where they can applied, nor all the possible exceptions. In the same way, it is possible to interpret also constitutive rules, since the institutional facts follow from actions delegated to some agent. This agent can decide to execute the action in the required context or not. For example, a constitutive norm applying to signatures can be extended to new form of signatures, like digital ones. Conversely, a signature by a fifteen years person may not be considered as a case where the same rule can be applied, even if the law does no say explicitly anything about this case. The possibility to interpret constitutive norms, however, gives rise to new possibilities that constitutive norms are violated or abused. A constitutive norm can be violated in the sense that the normative system or the agent who is delegated the goal to recognize the institutional fact and empowered to do so fails to achieve the delegated goal. In our running example the office could fail or refuse to recognize the car as an ecological vehicle. The reason can be the inability to perform the necessary actions, laziness, bribing, etc., like it happens for regulative norms. Moreover, constitutive rules can be abused, in the sense that the delegated agent can exercise its power without being delegated to do so in the given circumstances. This possibility assumes that the institutional power can be exercised beyond the conditions under which it has been delegated the goal to exercise it.

A further issue of future research is how to define norms at different level of abstraction, rather then the normative system itself. Abstract norms can be then refined in specific institutions as discussed by [26].

\section{Summary}

In this paper we refine the logical framework of our game-theoretic approach to normative multiagent systems. Starting from our previous model of regulative norms $[11,14]$, which already considers some procedural aspects, we study how also counts-as constitutive norms are associated with procedural norms. In this context we introduce the notion of delegation of power. Counts-as relations do not always provide directly an abstract recognition of brute facts in terms of institutional facts. We argue that in many cases the inference that concludes institutional facts from brute facts is the result of actions of agents acting on behalf of the normative systems and who are in charge of recognizing which institutional facts follow from brute facts. These agents make the institutional facts "official", i.e., "legally binding". The delegation of this responsibility opens space for interpretation of constitutive norms, thus making the system more flexible. Delegation of power is composed of a direct counts-as relation specifying that the effect of an action of an agent is an institutional fact and by a goal of the normative system that 
the fact is considered as an institutional fact. Constitutive norms are used not only to define intermediate concepts at each level, but they also enter in the definition of sanction based obligations, to define what counts as the recognition of a violation at level 4 , and regulative norms are used to see to motivate agents to apply constitutive norms at level 5 . The notion of delegation of power is necessary to achieve mutual empowerment, in the sense that the agents playing a role in a normative system empower this system, and the system delegates again this power to the agents. We show how counts-as relations in some cases depend on the action of agents which are in charge of recognizing facts as institutional facts. Moreover, we show that these agents are motivated to do so by a goal delegated to them by the normative system. We add procedural norms to both delegation of power and obligations. In the resulting logical framework, we can consider the optimal decision of an agent at the following five levels of abstraction (for various applications of normative multiagent systems):

1. The optimal decision of an agent follows from a simple decision problem, because it abstract from the fact that violations, sanctions and institutional facts are the result of the action that some agent decides to perform. The recognition of the violation and the sanction logically follow from the violation. The responsibility of monitoring, sanctioning and making counts-as inferences is completely delegated to the infrastructure.

2. The optimal decision takes into account the beliefs of the normative system, because the normative system is in some way personified and is attributed mental attitudes. The recognition of violations and sanctions are wanted by normative system itself. If obligations are goals of the normative system, the institutional facts follow from the beliefs of the normative system: they are still logical consequences of facts, but in the beliefs of the normative system.

3. The optimal decision can only be calculated by taking the reaction of the normative system into account, because institutional facts follow from actions of the normative system, and the recognition of violations and sanctions are considered as the actions of the normative system itself. The normative system can decide to enforce norms or not in specific situations, thus allowing interpretation of them.

4. The optimal decision takes into account the actions of the agents acting on behalf of the normative system. Some agents are delegated the goal and power to sanction violations, to recognize violations, and to recognize some facts as institutional facts.

5. The optimal decision takes into account the actions of other agents acting on behalf of the normative system, and thereafter again the normative system, because procedural norms are used to motivate the agents playing roles in the system. These norms oblige agents playing roles to achieve the goals of the normative system. Some agents are obliged to achieve the delegated goal to sanction violations, the goal and power of recognizing violations, and to achieve the goal to recognize some facts as institutional facts by exercising the delegated powers. In the Italian law, for example, it is obligatory for an attorney to start a prosecution process when he comes to know about a crime (art. 326 of Codice di procedura penale). 


\section{REFERENCES}

1. H. Aldewereld, Autonomy vs. conformity: an institutional perspective on norms and protocols, Ph.D. thesis, Utrecht University (2007).

2. A. Anderson, A reduction of deontic logic to alethic modal logic, Mind 67 (1958) 100-103.

3. L. Aqvist, Deontic logic, in: D. Gabbay, F. Guenthner (eds.), Handbook of Philosophical Logic: Volume II: Extensions of Classical Logic, Reidel, Dordrecht, 1984, pp. 605-714.

4. A. Artikis, M. Sergot, J. Pitt, An executable specification of an argumentation protocol., in: Procs. of 9th International Conference on Artificial Intelligence and Law, ICAIL 2003, ACM Press, New York (NJ), 2003.

5. A. Bochman, Explanatory Nonmonotonic Reasoning, World Scientific Publishing, London (UK), 2005.

6. G. Boella, L. van der Torre, Norm governed multiagent systems: The delegation of control to autonomous agents, in: Proceedings of the 2003 IEEE/WIC International Conference on Intelligent Agent Technology (IAT'03), IEEE, 2003.

7. G. Boella, L. van der Torre, Game specification in normative multiagent system: the trias politica, in: Procs. of IEEE/WIC/ACM International Conference on Intelligent Agent Technology (IAT'04), IEEE, 2004.

8. G. Boella, L. van der Torre, Enforceable social laws, in: Procs. of 4th International Joint Conference on Autonomous Agents and Multiagent Systems (AAMAS'05), ACM, New York (NJ), 2005.

9. G. Boella, L. van der Torre, From the theory of mind to the construction of social reality, in: Procs. of the 27th Annual Conference of the Cognitive Science Society (CogSci'05), Lawrence Erlbaum, Mahwah (NJ), 2005.

10. G. Boella, L. van der Torre, Constitutive norms in the design of normative multiagent systems, in: Computational Logic in Multi-Agent Systems, 6th International Workshop (CLIMA VI), vol. 3900 of LNCS, Springer, Berlin, 2006.

11. G. Boella, L. van der Torre, A game theoretic approach to contracts in multiagent systems, IEEE Transactions on Systems, Man and Cybernetics - Part C: Applications and Reviews 36 (1) (2006) 68-79.

12. G. Boella, L. van der Torre, A logical architecture of a normative system, in: Deontic Logic and Artificial Normative Systems, 8th International Workshop on Deontic Logic in Computer Science ( $\left.\triangle \mathrm{EON}^{\prime} 06\right)$, vol. 4048 of LNCS, Springer, Berlin, 2006.

13. G. Boella, L. van der Torre, Security policies for sharing knowledge in virtual communities, IEEE Transactions on Systems, Man and Cybernetics - Part A: Systems and Humans 36 (3) (2006) 439-450.

14. G. Boella, L. van der Torre, Institutions with a hierarchy of authorities in distributed dynamic environments, Artificial Intelligence and Law Journal (AILaw).

15. G. Boella, L. van der Torre, Norm negotiation in multiagent systems, International Journal of cooperative Information Systems (IJCIS) Special Issue: Emergent Agent Societies 16 (2) (2007) 97-122.

16. G. Boella, L. van der Torre, The ontological properties of social roles in multi-agent systems: Definitional dependence, powers and roles playing roles, Artificial Intelli- 
gence and Law Journal (AILaw).

17. G. Boella, L. van der Torre, H. Verhagen, Introduction to normative multiagent systems, Computation and Mathematical Organizational Theory, Special issue on Normative Multiagent Systems 12 (2-3) (2006) 71-79.

18. E. Bottazzi, R. Ferrario, A path to an ontology of organizations, in: Procs. of EDOC Int. Workshop on Vocabularies, Ontologies and Rules for The Enterprise (VORTE 2005), 2005.

19. R. Brafman, M. Tennenholtz, On partially controlled multi-agent systems., Journal of Artificial Intelligence Research (JAIR) 4 (1996) 477-507.

20. J. Broersen, M. Dastani, J. Hulstijn, L. van der Torre, Goal generation in the BOID architecture, Cognitive Science Quarterly 2(3-4) (2002) 428-447.

21. C. Castelfranchi, Modeling social action for AI agents, Artificial Intelligence 103(1-2) (1998) 157-182.

22. C. Castelfranchi, The micro-macro constitution of power, Protosociology 18 (2003) 208-269.

23. J. Gelati, A. Rotolo, G. Sartor, G. Governatori, Normative autonomy and normative co-ordination: Declarative power, representation, and mandate, Artificial Intellingence and Law 12(1-2) (2004) 53-81.

24. M. Georgeff, F. F. Ingrand, Decision-making in an embedded reasoning system, in: Procs. of 11th International Joint Conference on Artificial Intelligence (IJCAI'89), Morgan Kaufmann, San Mateo (CA), 1989.

25. P. J. Gmytrasiewicz, E. H. Durfee, Formalization of recursive modeling, in: Procs. of the 1st International Conference on Multiagent Systems (ICMAS'95), AAAI/MIT Press, Cambridge (MA), 1995.

26. D. Grossi, H. Aldewereld, J. Vazquez-Salceda, F. Dignum, Ontological aspects of the implementation of norms in agent-based electronic institutions, Computational \& Mathematical Organization Theory 12 (2-3) (2006) 251-275.

27. D. Grossi, F. Dignum, J. Meyer, Contextual terminologies, in: Computational Logic in Multi-Agent Systems, 6th International Workshop (CLIMA VI), vol. 3900 of LNCS, Springer, Berlin, 2006.

28. D. Grossi, J.-J. Meyer, F. Dignum, Counts-as: Classification or constitution? an answer using modal logic, in: Deontic Logic and Artificial Normative Systems, 8th International Workshop on Deontic Logic in Computer Science ( $\Delta$ EON'06), vol. 4048 of LNCS, Springer, Berlin, 2006.

29. H. Hart, The Concept of Law, Clarendon Press, Oxford, 1961.

30. A. Jones, J. Carmo, Deontic logic and contrary-to-duties, in: D. Gabbay, F. Guenthner (eds.), Handbook of Philosophical Logic, vol. 3, Kluwer, Dordrecht (NL), 2001, pp. 203-279.

31. A. Jones, M. Sergot, A formal characterisation of institutionalised power, Journal of IGPL 3 (1996) 427-443.

32. J. Lang, L. van der Torre, E. Weydert, Utilitarian desires, Autonomous Agents and Multiagent Systems 5(3) (2002) 329-363.

33. D. Makinson, L. van der Torre, Input-output logics, Journal of Philosophical Logic 29 (4) (2000) 383-408.

34. D. Makinson, L. van der Torre, Constraints for input-output logics, Journal of Philo- 
sophical Logic 30(2) (2001) 155-185.

35. Merriam-Webster, Dictionary of Law, Merriam-Webster, 1996.

36. J. J. C. Meyer, A different approach to deontic logic: Deontic logic viewed as a variant of dynamic logic, Notre Dame Journal of Formal Logic 29(1) (1988) 109-136.

37. O. Pacheco, F. Santos, Delegation in a role-based organization, in: Procs. of $\triangle \mathrm{EON} 04$, Springer, Berlin, 2004.

38. A. Ross, Tû-tû, Harvard Law Review 70 (5) (1957) 812-825.

39. B. Sadighi Firozabadi, M. Sergot, Contractual access control, in: Security Protocols, 10th International Workshop, vol. 2845 of LNCS, Springer, Berlin, 2004.

40. B. Sadighi Firozabadi, L. van der Torre, Formal models of control systems, in: Procs. of 13th European Conference on Artificial Intelligence (ECAI'98), John Wiley and Sons, Chichester, 1998.

41. J. Searle, The Construction of Social Reality, The Free Press, New York, 1995.

42. M. S. Sloman, Policy driven management of distributed systems, Journal of Network and Systems Management 2(4) (1994) 333-360.

43. L. van der Torre, Y. Tan, Contrary-to-duty reasoning with preference-based dyadic obligations, Annals of Mathematics and Artificial Intelligence 27 (1-4) (1999) 49-78.

44. G. H. von Wright, Deontic logic, Mind 60 (1950) 1-15. 


\title{
Institutions with a Hierarchy of Authorities in Distributed Dynamic Environments
}

\author{
Guido Boella (guido@di.unito.it) \\ Dipartimento di Informatica - Università di Torino - Italy \\ Leendert van der Torre (leendert@vandertorre.com) \\ University of Luxembourg
}

\begin{abstract}
A single global authority is not sufficient to regulate heterogenous agents in multiagent systems based on distributed architectures, due to idiosyncratic local situations and to the need to regulate new issues as soon as they arise. On the one hand institutions should be structured as normative systems with a hierarchy of authorities able to cope with the dynamics of local situations, but on the other hand higher authorities should be able to delimit the autonomy of lower authorities to issue valid norms. In this paper we study the interplay of obligations and strong permissions in the context of hierarchies of authorities using input/output logic, because its explicit norm base facilitates reasoning about norm base maintenance, and it covers a variety of conditional obligations and permissions. We combine the logic with constraints, priorities and hierarchies of authorities. In this setting we observe that Makinson and van der Torre's notion of prohibition immunity for permissions is no longer sufficient, and we introduce a new notion of permission as exception and a new distinction between static and dynamic norms. We show how strong permissions can dynamically change an institution by adding exceptions to obligations, provide an explicit representation of what is permitted to the subjects of the normative system and allow higher level authorities to limit the power of lower level authorities to change the normative system.
\end{abstract}

\section{Introduction}

Technological developments in multiagent systems like peer to peer systems, grid architectures, virtual organizations, and electronic institutions, pose new challenges for normative systems regulating the heterogeneous agents interacting with each other. These normative systems should be structured in a hierarchical way, because a global authority neither has complete knowledge about local situations, nor the resources to regulate new issues dynamically arising. Moreover, norms should be represented explicitly for transparency of the normative system and norm base maintenance, facilitating the introduction and removal of norms. Therefore, two branches of legal theory in general and deontic logic in particular are of crucial importance for the development of normative multiagent systems.

(C) 2007 Kluwer Academic Publishers. Printed in the Netherlands. 
First, the theory of normative multiagent systems builds on the study of the use of hierarchies in normative systems. The sources of law give rise not only to norms, but also to hierarchical criteria that determine the relative importance of various norms that form part of a legal order. The identification of the material of which a legal system is composed must include a series of relations determining the relative weight of norms. The clearest example is the criterion based on the level of authority that enacted the norm. The authority hierarchy is an example of a method for filtering out the applicable set of norms from a given set of norms. Other strategies to determine the valid norms are valid in a situation are being studied as meta-norms by, e.g., (Prakken and Sartor, 1996).

Second, the theory of normative multiagent systems builds on the study of the promulgation or introduction of norms and the derogation or removal of norms and their consequences, such as the indeterminate derogation or the 'Unbestimmtheit des Normensystems' (Bulygin, 1996), the problem with inconsistent systems, and the role of global authorities to provide general policies which cannot be overruled by lower authorities. Higher authorities, thus, delimit the autonomy of lower authorities of issuing valid norms. Lewis (1979) and Bulygin (1986) argue convincingly that in such cases the notion of obligation is not sufficient, as we discuss in Section 3, but we need a strong kind of permission too.

However, these theories developed mainly in the seventies and the eighties of the previous century cannot directly be used for normative multiagent systems, mainly because they do not make the roles the agents play within the normative system explicit, and consequently they lack detailed computational social mechanisms to maintain and reason with the norm base. Normative multiagent systems (Boella et al., to appear) seek to provide a more general framework for normative reasoning, incorporating amongst others the above multiagent issues. For example, we propose constitutive norms to define a mechanism for agents to exercise their power to update the normative system at various levels (Boella and van der Torre, 2006a), and Von Wright's transmission of will to formalize policies about policies in the scope of knowledge management (Boella and van der Torre, 2006b) (it is not sufficient that there is an obligation for the lower authority to oblige or permit the user, but the norm of the lower authority has to be enforced too). In this paper we address the following questions:

1. How to define conditional permission as exception to conditional obligations in order to deal with the mechanism by which higher authorities can limit the norm-making of lower authorities? 
2. How does the notion of strong conditional permission relate to other proposals of permission discussed in the literature?

3. How to distinguish static and dynamic norms using hierarchies of normative systems, where the former concern the derogation from (existing) obligations, while the latter regard how to block the possibility that lower authorities introduce conflicting valid norms, and how to define the competence and redundancy of norms?

We use Makinson's and van der Torre's framework of input/output logic (Makinson and van der Torre, 2000), because its explicit norm base facilitates reasoning about norm base maintenance, and it covers a variety of conditional obligations and permissions. Thus far the dynamics of normative systems have been studied mainly in the framework of Alchourròn, Makinson and Gardenfors' (AGM) theory of belief revision, since Alchourron's study of retraction in legal systems was one of its constituents. However, the AGM theory seems better suited for belief revision than for norm revision, since it does not cover particularities of normative reasoning such as the distinction between norms and normative propositions, the conditional nature of norms, and the distinction between obligations and strong permissions.

Makinson and van der Torre propose input/output logic to formalize conditional norms, obligations, goals, etc. (Makinson and van der Torre, 2000), negative, static and dynamic permissions (Makinson and van der Torre, 2003), and contrary-to-duty reasoning (Makinson and van der Torre, 2001). In this paper we observe that the notion of dynamic permission no longer corresponds to so-called prohibition immunity, and that the notion of permission as exception has not been covered. We introduce priorities and to model institutions we introduce normative systems as hierarchies of authorities in input/output logic. If a normative system is structured in a hierarchical way, then norms from different authorities can conflict with each other. Moreover, authorities may change the normative status only of behaviors they are competent of.

The layout of this paper is as follows. In Section 2 we discuss normative multiagent systems and in Section 3 we summarize legal theory on permission. In Section 4 we present input/output logic and in Section 5 we introduce permissions as exceptions using conflict resolution. In Section 6 we make the distinction between static and dynamic norms using hierarchies of normative systems, discussing the notion of competence and of redundancy of norms. 


\section{Normative multiagent systems}

There are numerous approaches of social norms and laws in multiagent systems (Dignum, 1999; Dignum et al., 2004; Esteva et al., 2001; Esteva et al., 2004; Lopez y Lopez et al., 2002; Shoham and Tennenholtz, 1995). Jones and Carmo (2001) define a normative system as "Sets of agents whose interactions are norm-governed; the norms prescribe how the agents ideally should and should not behave. [...] Importantly, the norms allow for the possibility that actual behavior may at times deviate from the ideal, i.e., that violations of obligations, or of agents' rights, may occur." Since the agents' control over the norms is not explicit here, we use the following definition: "A normative multiagent system is a multiagent system together with normative systems in which agents can decide whether to follow the explicitly represented norms, and the normative systems specify how and in which extent the agents can modify the norms" (Boella et al., to appear). Note that this definition makes no presumptions about the internal architecture of an agent or of the way norms affect agent's behavior.

The question should be raised how the explicit norms are represented. Norms can be interpreted as a special kind of constraint, and represented depending on the domain in which they occur. However, this representation runs into the question what happens when norms are violated. Not all agents behave according to the norm, and the system has to sanction violations or reward good behavior. Thus, the normative system has to monitor the behavior of agents and enforce the sanctions. Also, when norms are represented as domain dependent constraints, the question will be raised how to represent permissive norms, and how they relate to obligations. Whereas obligations and prohibitions can be represented as constraints, this does not seem to hold for permissions. For example, how to represent the permission to access a resource under an access control system? Finally, when norms are represented as domain dependent constraints, how do norms evolve?

We therefore believe that norms should be represented as a domain independent theory. For example, deontic logic (von Wright, 1951; van der Torre and Tan, 1999; van der Torre, 2003; Makinson and van der Torre, 2000; Makinson and van der Torre, 2001; Makinson and van der Torre, 2003) studies logical relations among obligations and permissions, and more in particular violations and contrary-toduty obligations, permissions and their relation to obligations, and the dynamics of obligations over time. Therefore, insights from deontic logic can be used to represent and reason with norms. Deontic logic also offers representations of norms as rules or conditionals. However, there are several aspects of norms which are not covered by constraints nor 
by deontic logic, such as the relation between the cognitive abilities of agents and the global properties of norms.

Conte et al. (1998) say that normative multiagent systems research focuses on two different sets of problems. On the one hand, they claim that legal theory and deontic logic supply a theory for of norm-governed interaction of autonomous agents while at the same time lacking a model that integrates the different social and normative concepts of this theory. On the other hand, they claim that three other problems are of interest in multiagent systems research on norms: how agents can acquire norms, how agents can violate norms, and how an agent can be autonomous. For artificial agents, norms can be designed as in legal human systems, forced upon, for example when joining an institution, or they can emerge from the agents making them norm autonomous (Verhagen, 1999). Agent decision making in normative systems and the relation between desires and obligations has been studied in agent architectures (Broersen et al., 2002), which thus explain how norms and obligations influence agent behavior.

An important question is where norms come from. Norms are not necessarily created by a single legislator, they can also emerge spontaneously, or be negotiated among the agents. In electronic commerce research, for example, cognitive foundations of social norms and contracts are studied (Boella and van der Torre, 2006a). Protocols and social mechanisms are now being developed to support such creations of norms in multiagent systems. When norms are created, the question can be raised how they are enforced. For example, when a contract is violated, the violator may have to pay a penalty. But then there has to be a monitoring and sanctioning system, for example police agents in an electronic institution. Such protocols or roles in a multiagent system are part of the construction of social reality, and Searle (1995) has argued that such social realities are constructed by constitutive norms. This again raises the question how to represent such constitutive or countsas norms, and how they are related to regulative norms like obligations and permissions (Boella and van der Torre, 2006b).

Not only the relation between norms and agents must be studied, but also the relation between norms and other social and legal concepts. How do norms structure organizations? How about the relation between legal courts? Though in some normative multiagent systems there is only a single normative system, there can also be several of them, raising the question how normative systems interact. For example, in a virtual community of resource providers each provider may have its own normative system, which raises the question how one system can authorize access in another system, or how global policies can be defined to regulate these local policies (Boella and van der Torre, 2006b). 


\section{Permissions and hierarchical normative systems}

\subsection{Permissions are Weak or Strong?}

The first question is whether permission is an autonomous normative category. The question arises because of the interdefinability of the deontic operators of permission and obligation: $P(q)$ iff $\neg O(\neg q)$; is permission only the mere absence of obligation or something which can be positively expressed by a norm?

According to von Wright there are two types of permissions, weak permissions and strong permissions: "An act will be said to be permitted in the weak sense if it is not forbidden; and it will be said to be permitted in the strong sense if it is not forbidden but subject to norm. Strong permission only is a norm-character." (von Wright, 1963, p.86). (Bobbio, 1980) explains that "the difference between weak and strong permission becomes clear when we think about the function of permissive norms. Permissive norms are subsidiary norms: subsidiary in that their existence presupposes the existence of imperative norms [...] a permissive norms is necessary when we have to repeal a preceding imperative norm or to derogate to it. That is to abolish a part of it (that in this case it is not necessary preexisting because a law itself may prescribe a limit to its own extension)", p. 891-892. ${ }^{1}$

Moreover, (Bobbio, 1958) discusses the temporal relations existing between permissions and obligations: "the function of permissive norms is to abolish an imperative in certain circumstances or with reference to certain persons [...] permissive norms are distinguished according to the fact that they abolish an imperative which precedes in time and in this case are called abolishing norms, or a contemporary imperative, and in this case they generally have the function of derogating norms."

For Bulygin there is more to permissive norms than this: "the role played by permissive norms is not exhausted by derogation of former prohibition: an act of permitting an action which has not been hitherto prohibited is not at all pointless as has been suggested by those who deny the importance of permissive norms" (Bulygin, 1986, p.213).

\subsection{INTERACTION GAMES IN HIERARCHICAL NORMATIVE SYSTEMS}

Bulygin explains why permissive norms are not superfluous even if there is no corresponding prohibition. What is lacking in the previous discussion is the idea that a normative system is not composed of only a single authority which enacts norms. Rather, a normative system is

\footnotetext{
1 Authors' translation from Italian text.
} 
composed of many authorities which are linked by hierarchical relations, as the "Rex, Minister and Subject" game shows. Moreover, a normative system has a dynamic character: norms are added to the system one after the other and this operation is performed by different authorities at different levels of the hierarchy.

The notion of permission alone is not enough to build a normative system, as (Lewis, 1979)'s "master and slave" game has shown: for only obligations divide the possible actions into two categories or spheres: the sphere of prohibited actions and the sphere of permitted (i.e., not forbidden) actions or "the sphere of permissibility".

(Bulygin, 1986) challenges the view that permission is only the lack of obligation: in a dynamic view of the normative system, illustrated by the games between "Rex" and "Subject", it is sensible to use permissions to derogate to former prohibitions. In this game, however, it is pointless to issue permissions which do not refer to a preexisting obligation.

Thus, (Bulygin, 1986) devices a new game, showing the role of permissions which do not refer to a preexisting prohibition: "suppose that Rex, tired of governing alone, decides one day to appoint a Minister and to endow him with legislative power. [...] an action commanded by Minister becomes as obligatory as if it would have been commanded by Rex. But Minister has no competence to alter the commands and permissions given by Rex." If Rex permits hunting on Saturday and then Minister prohibits it for the whole week, its prohibition on Saturday remains with no effect.

\subsection{Permissions in hierarchical Legal Systems}

Also (Alchourron and Makinson, 1981) support this view: "when we consider the regulations in legal or administrative code, we can often discern some kind of hierarchy among them. Some are regarded as more basic or fundamental than others", p.125.

Once we have a plurality of authorities we need to cope with conflicts: the solution is to use meta-norms. The meta-norms of the normative system ascribe to each level of authority an area of competence (a set of propositions they can permit or forbid) and prescribe that the system must respect normative principles like "lex superior derogat inferiori" ("norms have the function of preventing - inhibit, preclude - the creation of imperative norms by subordinated sources of law", (Guastini, 1998, p.29)), "lex posterior derogat priori" (the function of abrogating preexisting imperative norms or to derogate to them), etc. 


\section{Input/output logic}

Makinson and van der Torre see a normative code as a set $G$ of conditional norms, which is a set of ordered pairs $(a, x)$. For each such pair, the body $a$ is thought of as an input, representing some condition or situation, and the head $x$ is thought of as an output, representing what the norm tells us to be desirable, obligatory or whatever in that situation. Moreover, given any universe $L$ such that $G \subseteq L^{2}$ and an input $A \subseteq L$, they suggest that the output of $A$ under $G$ may be understood simply as

$$
G(A)=\{x \mid(a, x) \in G \text { for some } a \in A\}
$$

Input/output logic investigates what happens to this basic picture when we pass to the logical level, i.e. when $L$ is a propositional language, closed under at least the usual truth-functional connectives, and $G$ a set of ordered pairs $(a, x)$ of formulae in $L$. Since this investigation is relevant not only for deontic logic, Makinson and van der Torre refer to $G$ not as a normative code, but as a generating set. To avoid all confusion, the generators $G$ are not treated as formulae, but simply as ordered pairs $(a, x)$ of purely boolean (or eventually first-order) formulae. They read a pair $(a, x)$ forwards, i.e. with $a$ as body and $x$ as head; and they call the corresponding truth-functional formula $a \rightarrow x$ its materialization, echoing the old name material implication for the connective involved.

Suppose that we are also given a set $A$ of formulae. The problem studied in input/output logic is: how may we reasonably define the set of propositions $x$ making up the output of $A$ under $G$, or one might also say, of $G$ given $A$, which we write $\operatorname{out}(G, A)$ ? Makinson and van der Torre emphasize that the task of logic is seen as a modest one. It is not to create or determine a distinguished set of norms, but rather to prepare information before it goes in as input to such a set $G$, to unpack output as it emerges and, if needed, coordinate the two in certain ways. A set $G$ of conditional norms is thus seen as a transformation device, and the task of logic is to act as its 'secretarial assistant'.

\subsection{Obligations}

The basic intuition is that input and output are both under the sway of the operation $C n$ of classical consequence. Makinson and van der Torre's simplest response to their problem is to put

$$
\operatorname{out}(G, A)=C n(G(C n(A)))
$$

where the function $G($.$) is defined as on the pre-logical level above, and$ $C n$ alias $\vdash$ is classical consequence. In other words, given a set $A$ of 
formulae as input, they first collect all of its consequences, then apply $G$ to them, and finally consider all of the consequences of what is thus obtained. They also define various variants to deal with disjunctive inputs intelligently, and making outputs available for recycling as inputs.

DEFINITION 1 (Obligations). (Makinson and van der Torre, 2000) Let $L$ be a propositional logic with $\top$ a tautology, and let $G$ be a set of ordered pairs of $L$ (called the generators). A generator $(a, x)$ is read as 'if input a then output $x$ '. The following logical systems have been defined, where $v$ ranges over boolean valuations or the function that puts $v(b)=1$ for all formulae $b$, and $V=\{b \mid v(b)=1\}$.

out $_{1}(G, A)=C n(G(C n(A)))$,

out $_{2}(G, A)=\cap\{C n(G(V)) \mid v(A)=1\}$,

out $_{3}(G, A)=\cap\{C n(G(B)) \mid A \subseteq B=C n(B) \supseteq G(B)\}$,

$\operatorname{out}_{4}(G, A)=\cap\{C n(G(V)): v(A)=1$ and $G(V) \subseteq V\}$.

The following example illustrates and compares the four input/output logics. The most characteristic property is that inputs are not in general outputs; that is, we do not have $A \subseteq$ out $_{1}(G, A)$.

EXAMPLE 1. (Makinson and van der Torre, 2000) Put generators $G=\{(a, x),(b, x),(x, y)\}$, where $a, b, x$ and $y$ are distinct elementary letters, and put $A=\{a\}$. Inputs are not in general outputs, since $G(C n(a))=\{x\}$ so a $\notin$ out $_{1}(G, a)=C n(G(C n(a)))=C n(x)$. Contraposition also fails, for although $x \in$ out $_{1}(G, a)$ we have $\neg a \notin$ out $_{1}(G, \neg x)$ : since $a \in C n(\neg x)$ we have $G(C n(\neg x))=\emptyset$ so that $\neg a \notin$ out $_{1}(G, \neg x)=C n(G(C n(\neg x)))=C n(\emptyset)$.

$W e$ do not have $y \in$ out $_{1}(G, A)$. However, in certain situations, it may be appropriate for outputs to be available for recycling as inputs. For example, the elements $(a, x)$ of $G$ may be conditional norms of a kind that say that any configuration in which a is true is one in which $x$ is desirable. In some contexts, we may wish to entertain hypothetically the items already seen as desirable, in order to determine what is in turn so. We do have $y \in$ out $_{3}(G, A)$ and $y \in$ out $_{4}(G, A)$.

Finally, put $A=\{a \vee b\}$. Then $C n(A) \cap b(G)=\emptyset$ where we write $b(G)$ for the set of all bodies of elements of $G$, i.e. in this example the set $\{a, b\}$. Hence also $G(C n(A))=\emptyset$ so that out $(G, A)=$ $C n(G(C n(A)))=C n(\emptyset)$. However, in many contexts we would want to put $x$ in the output, as it can be obtained from each of the two disjuncts of the input. We do have $x \in$ out $_{2}(G, A)$ and $x \in$ out $_{4}(G, A)$. 
Input/output logic is axiomatized as a kind of conditional logic, where one is used to ask the following question. Suppose we are given only the generating set $\mathrm{G}$ : how may we define the set of input/output pairs $(A, x)$ arising from $\mathrm{G}$, written as out $(G)$ ? Makinson and van der Torre suggest that this is the same question as asking what is $\operatorname{out}(G, A)$, because they define $(A, x) \in \operatorname{out}(G)$ iff $x \in \operatorname{out}(G, A)$ or conversely. They also suggest that the two formulations give a rather different gestalt, and one is sometimes more convenient rather than the other. Whereas the latter tends to be clearer in semantic contexts, the former is easier to work with when considering derivations in a syntactic context. They move freely from one to the other, just as one moves between $C n$ and $\vdash$ for classical consequence.

THEOREM 1. (Makinson and van der Torre, 2000) Let $L$ be a base logic with $\top$ a tautology, and let $G$ be a set of ordered pairs of $L$ (called the generators). Input/output logic out (out $_{2} /$ out $_{3} /$ out $_{4}$ ) is a closure operation on $G \cup\{(\top, \top)\}$ under replacement of logical equivalents and the rules $S I, W O$ and $A N D$ (and $O R / C T / O R$ and $C T$ ).

$$
\begin{array}{cccccc}
S I & \frac{(a, x)}{(a \wedge b, x)} & W O & \frac{(a, x)}{(a, x \vee y)} & A N D & \frac{(a, x),(a, y)}{(a, x \wedge y)} \\
\text { OR } & \frac{(a, x),(b, x)}{(a \vee b, x)} & C T & \frac{(a, x),(a \wedge x, y)}{(a, y)} & \text { ID } & \frac{\overline{(a, a)}}{(a, a)}
\end{array}
$$

EXAMPLE 2. Given $G=\{(a, x),(a, y),(x, z)\}$ the output of $G$ contains $(a \wedge b, x),(a \wedge x, z),(a, x \vee y),(a, a \vee x)$, and $(a, x \wedge y)$ using rules $S I, W O$ and $A N D$. Using also the CT rule, the output contains $(a, z)$.

\subsection{PERMissions}

Permissions are more ambiguous than obligations, and various notions have been defined. Makinson and van der Torre (2003) distinguish three notions of permission. First, negperm is the negation of an prohibition, it corresponds to what is called weak permission. Second, statperm guides the citizen in the deontic assessment of specific actions, and behaves like a weakened obligation: given what is obligatory and what is strongly permitted the actual permissions of an agent are computed. If $P$ is the set of permissive norms, then we have $\operatorname{statperm}(P, G) \subseteq$ out $(P \cup G)$, see (Makinson and van der Torre, 2003) for details. Third, dynperm guides the legislator by describing the limits on what may be prohibited without violating static permissions, which is called prohibition immunity: "on the other hand, dynamic permission corresponds to the needs of the legislator, who needs to anticipate the effect of adding a prohibition to an existing corpus of norms. If prohibiting $x$ in condition $a$ would commit us to forbid something that has been 
positively permitted in a certain realizable situation, then adding the prohibition is inadmissible under pain of a certain kind of incoherence, and the pair $(a, x)$ is to that extent immune from prohibition. For this reason, dynamic permission could also be called prohibition immunity" (Makinson and van der Torre, 2003).

DEFINITION 2 (Permissions). Let $G$ and $P$ be two sets of generators, where $P$ stands for permissive norms, and let out be an input/output logic.

$$
\begin{aligned}
& \text { - }(a, x) \in \operatorname{negperm}(G) \text { iff }(a, \neg x) \notin \operatorname{out}(G) ; \\
& \text { - }(a, x) \in \operatorname{statperm}(P, G) \text { iff }(a, x) \in \operatorname{out}(G \cup Q) \text { for some singleton } \\
& \text { or empty } Q \subseteq P ; \\
& \text { - }(a, x) \in \operatorname{dynperm}(P, G) \text { iff }(c, \neg z) \in \operatorname{out}(G \cup\{(a, \neg x)\}) \text { for some } \\
& \text { pair }(c, z) \in \operatorname{statperm}(P, G) \text { with c consistent. }
\end{aligned}
$$

EXAMPLE 3. It is obligatory to make homework, but if one does homework he is permitted to watch the television, $G=\{(\top, h)\}$ and $P=\{(h, w)\}$. Then $(\top, h) \in$ negperm $(G)$, since what is obligatory is permitted and $(a, b) \in$ negperm $(G)$ since given a there is no restriction about b. Moreover, $(h, w) \in \operatorname{statperm}(P, G)$ since this is explicitly permitted and $(a, w) \in \operatorname{dynperm}(P, G):(a \wedge h, \neg w) \in \operatorname{out}(G \cup\{(a, \neg w)\})$ for some pair $(a \wedge h, w) \in \operatorname{statperm}(P, G)$.

\subsection{Constraints}

The main problem of reasoning with obligations and permissions is the question how to deal with violations and obligations resulting from violations, known as contrary-to-duty reasoning. It has been discussed in the context of the notorious contrary-to-duty paradoxes such as Chisholm's and Forrester's paradox. It has led to the use of constraints in input/output logics (Makinson and van der Torre, 2001).

The strategy is to adapt a technique that is well known in the logic of belief change - cut back the set of norms to just below the threshold of making the current situation contrary-to-duty. In effect, input/output logic carries out a contraction on the set $G$ of generators. In case of contrary-to-duty obligations, the input represents something which is inalterably true, and an agent has to ask himself which obligations (output) this input gives rise to: even if the input should have not come true, an agent has to "make the best out of the sad circumstances" (Hansson, 1969). 
In input/output logics under constraints, a set of generators and an input does not have a set of propositions as output, but a set of sets of propositions. We can infer a set of propositions by for example taking the join (credulous) or meet (sceptical), or something more complicated. Besides, we can adopt an output constraint (the output has to be consistent) or an input/output constraint (the output has to be consistent with the input). In this paper we consider only the input/output constraints.

DEFINITION 3 (Constraints). Let $G$ be a set of generators and out be an input/output logic. Moreover, we write $x \in$ out $(G, a)$ iff $(a, x) \in$ out $(G)$. We define:

- maxfamily $(G, a)$ is the set of $\subseteq$-maximal subsets $G^{\prime}$ of $G$ such that $\operatorname{out}\left(G^{\prime}, a\right) \cup\{a\}$ is consistent.

- outfamily $(G, a)$ is the output under the elements of maxfamily, i.e., $\left\{\operatorname{out}\left(G^{\prime}, a\right) \mid G^{\prime} \in \operatorname{maxfamily}(G, a)\right\}$.

- $(a, x) \in$ out $_{\cup}(G)$ iff $x \in \cup$ outfamily $(G, a)$ $(a, x) \in$ out $_{\cap}(G)$ iff $x \in \operatorname{noutfamily}(G, a)$

Makinson and van der Torre (2001) consider the following example.

EXAMPLE 4. Multiple level of violation may be analyzed. For example, put $G=\{(\top, \neg a),(a, x),(a \wedge \neg x, y)\}$ where $a$ is read as 'you break your promise', $x$ as 'you apologize' and $y$ as 'you are ashamed'. Consider the input $a \wedge \neg x$. On the one hand, out $(G, a \wedge \neg x)=C n(\neg a, x, y)$, which is consistent. On the other hand, out $(G, a \wedge \neg x)$ is inconsistent with input $a \wedge \neg x$, so that maxfamily $(G, a \wedge \neg x)=\{(a \wedge \neg x, y)\}$ and outfamily $(G, a \wedge \neg x)=\{C n(y)\}$.

Permissions under constraints can be formalized by replacing in Definition 2 each occurrence of out by out or out $t_{\cap}$. In this paper we make two observations.

First, negperm and statperm still behave the same, and in particular statperm behaves like a weakened obligation and guides the citizen in the deontic assessment of specific actions. However, dynperm no longer formalizes prohibition immunity, because adding a conflicting obligation does not necessarily create an incoherence. For example, Sartre argues that it is coherent that it is forbidden to kill $((T, \neg k) \in G)$, but a soldier is obliged to kill in wartime $((w, k) \in G)$. We have $(w, k),(w, \neg k) \in$ out $_{\cup}(G)$ and $(w, k),(w, \neg k) \notin o u t_{\cap}(G)$. The process of adding norms to a normative system must be seen in its diachronic character: norms are added to derogate the previous ones. Rather than 
checking the consistency of a new norm the question is which norms are still materially valid after a new one has been added.

Second, the three notions of permission do not cover permissions as exceptions, while, as we have seen in Section 3 this is the principal role of permissive norms in legal systems. Most exceptions in the criminal code can be understood as such permissions, e.g., consider "it is forbidden to kill $((T, \neg k) \in G)$, but it is permitted to kill in selfdefense $((s, k) \in P)$, unless a policeman is killed $((s \wedge p, \neg k) \in G)$ ". In input/output logic with constraints, these norms still imply the prohibition to kill in case of self-defense $\left((s, \neg k) \in\right.$ out $\left._{\cup / \cap}(G)\right)$, because maxfamily and outfamily do not take permissions into account.

\section{Priorities and hierarchy}

Without permissions, a conflict can be defined in input/output logic as a case in which outfamily contains more than one element. Conflict resolution has been studied in defeasible deontic logic. It can be formalized by an ordering on the powerset of generators, such that a preffamily selects from maxfamily only the preferred elements. An extension needed here is the distinction between generator pointers and the generators themselves, because the same generator may occur several times in the ordering. In fact, the same generator can be the object of norms enacted by different authorities: however, all these instances of the generator may have different priorities. So, we consider each norm, i.e., each instance of a generator, as a different generator pointer. As usual we assume that the ordering is at least a partial pre-order, i.e., antisymmetric and transitive, and that it contains the subset-ordering.

DEFINITION 4 (Permissions as exceptions). Let $G$ and $P$ be disjoint sets of generators pointers, $V$ a function that associates with every generator pointer a generator, and $\leq$ a partial pre-order on the powerset of $G \cup P$ that contains the subset-ordering. We read $A \leq B$ as $B$ is at least as preferred as $A$.

- maxfamily $(G, P, V, a)$ is the set $\subseteq$-maximal $G^{\prime} \cup P^{\prime}$ such that $G^{\prime} \subseteq G, P^{\prime} \subseteq P$ and $\operatorname{out}\left(V\left(G^{\prime}\right) \cup V(Q), a\right) \cup\{a\}$ is consistent for every singleton or empty $Q \subseteq P^{\prime}$.

- preffamily $(G, P, V, \leq, a)$ is the set of $\leq$ maximal elements of maxfamily $(G, P, V, a)$. 
- outfamily $(G, P, V, \leq, a)$ is the set of outputs of preffamily, i.e., the sets out $\left(V\left(G^{\prime}\right), a\right)$ such that $G^{\prime} \cup P^{\prime} \in \operatorname{preffamily}(G, P, V, \leq, a)$, $G^{\prime} \subseteq G$, and $P^{\prime} \subseteq P$.

- statpermfamily $(G, P, V, \leq, a)$ is defined analogously, i.e. the set of out $\left(V\left(G^{\prime} \cup Q\right), a\right)$ such that $G^{\prime} \cup P^{\prime} \in \operatorname{preffamily}(G, P, V, \leq, a)$, $G^{\prime} \subseteq G, Q \subseteq P^{\prime} \subseteq P$, and $Q$ is a singleton or empty.

- out $/ \cap(G, P, V, \leq)$ are analogous as in Definition 3.

The following example illustrates permissions as exceptions, but also obligations as exceptions to permissions.

EXAMPLE 5. Let $G=\{a=(\top, \neg k), b=(s \wedge p, \neg k)\}, P=\{c=$ $(s, k)\},\{a, c\}<\{a, b\}<\{b, c\}$, where by $A<B$ we mean as usual $A \leq B$ and $B \not \leq A$.

It is forbidden to kill, but it is permitted to kill in case of self-defence, unless a policeman is killed.

$\operatorname{maxfamily}(G, P, V, \leq, s)=\{\{a, b\},\{b, c\}\}$, $\operatorname{preffamily}(G, P, V, \leq, s)=\{\{b, c\}\}$,

$\operatorname{outfamily}(G, P, V, \leq, s)=\{C n(\{\top\})\}$,

statpermfamily $(G, P, V, \leq, s)=\{C n(\{k\})\}$

The maxfamily includes the sets of applicable compatible generators together with all non applicable ones: e.g., the output of $\{a, c\}$ in the context $s$ is not consistent. Even if $b$ could conflict with $c, c$ is not applicable in situation $s$ whereas $b$ is, hence $\{b, c\}$ is consistent. Finally $\{a\}$ is not in maxfamily since it is not maximal, we can add the non applicable rule $b$. Then preffamily is the preferred set $\{b, c\}$ according to the ordering on set of rules above. The set outfamily is composed of the consequences of applying the generators whose pointer is in $G$ which are included in $\{b, c\}$ and applicable in $s: b$ is the only obligation, but it is not applicable. The set statpermfamily is the result of the application of obligations in $G$ together with a permission at a time from $P$, in this case $c$.

Conflicts between obligations and permissions are traditionally studied using hierarchies (Alchourron and Makinson, 1981), which we formalize as an ordering on the generator pointers, in which each cluster represents an authority.

\subsection{HIERARCHY}

Alchourròn and Makinson (1981) define a hierarchy of regulations in this way: "a hierarchy of regulations to be a pair $(A, \leq)$ where $A$ is a 
non-empty set of propositions, called a code, and $\leq$ is a partial ordering of $A$ ", p.126. Moreover, "[the judge] need[s] to compare, whenever possible, one set of regulations with another. In other words, given a relation $\leq$ that partially orders $A$, we need to envisage ways which $\leq$ induces some kind of ordering of $2^{A} "$, p.127. Our definition above considers a relation $\leq$ on set of rules and not a relation on rules. Thus, given a relation on rules, how can it be lifted to a relation on sets of rules? As Alchourròn and Makinson (1981) notice, "there is no a priory way of determining what the most suitable definitions will be".

So we adopt the following definition of lifting, inspired by (Brass, 1993): " $E_{1} \leq E_{2} \Longleftrightarrow$ for every $\delta_{2} \in E_{2} \backslash E_{1}$ there is a $\delta_{1} \in E_{1} \backslash E_{2}$ with $\delta_{1} \preceq \delta_{2}$." ${ }^{2}$ Hence, we give the following way in which priorities and a hierarchy can be related.

DEFINITION 5 (Hierarchy of norms). A hierarchy is a partial preorder $\preceq$ on generator pointers. A priority ordering on set of rules $\leq$ respects $\preceq$ when $B \leq C$ if for every $b \in B \backslash C$ there is $a c \in C \backslash B$ with $b \preceq c$. We write $a \prec b$ for $a \preceq b$ and $b \npreceq a$.

EXAMPLE 6 . The hierarchy $a \prec c \prec b$ respects the relation on set of rules used in Example $5\{a, c\}<\{a, b\}<\{b, c\}$. The norm a is a general obligation which is derogated by $c$ in a situation of self-defence. Obligation $b$ is an exception to permission $c$, so it must have precedence over $c$. The restriction above is respected: e.g., $\{a, b\} \backslash\{a, c\}=\{b\}$, $\{a, c\} \backslash\{a, b\}=\{c\}$ and $a \preceq c$.

\section{Static and Dynamic norms}

We now consider the distinction between static and dynamic norms. We say that a norm is weakly redundant, when the output of a given set of norms does not change when we remove the norm. We say that a norm is strongly redundant when we the norm is weakly redundant for any extension of the set of norms.

\subsection{STATIC NORMS}

The static interpretation makes sense if the system contains a single authority: then norms should not be weakly redundant. ${ }^{3}$

\footnotetext{
${ }^{2}$ Where with respect to (Brass, 1993) we reverse the meaning of the $\preceq$ relation: for Brass $B \leq C$ means that $B$ is preferred over $C$.

3 Alternative notions can be based on out $/ \cap$. Due to space limitations in this and the following definition we do not detail the changes in $\leq$ and $V$, which have to shrink or grow in the obvious way with the removal or addition of norms. See (Brewka and Eiter, 2000) for a definition of agreement between two priority relations.
} 
DEFINITION 6 (Static norms). $g \in G \cup P$ is weakly redundant iff $\forall a \in L: \operatorname{outfamily}(G, P, V, \leq, a)=\operatorname{outfamily}(G \backslash\{g\}, P \backslash\{g\}, V, \leq, a)$.

In a static normative system permissions are meaningful only if they derogate some other obligations, in part or in toto: in the definition to decide whether $g$ is weakly redundant we compute the set of what is obligatory in the normative system, or outfamily; adding a permission which does not affect the material validity of some obligation does not affect outfamily. In the same way as permissions, obligations can have a role of derogating some permission (as in Example 5) or even another obligation, as the following example shows:

EXAMPLE 7. $G=\{a=(\top, \neg f), b=(p, f)\}, P=\emptyset,\{a\}<\{b\}$,

No one should have firearms, policemen should have one. $\operatorname{maxfamily}(G, P, V, \leq, p)=\{\{a\},\{b\}\}$, $\operatorname{preffamily}(G, P, V, \leq, p)=\{\{b\}\}$, outfamily $(G, P, V, \leq, p)=\{C n(\{f\})\}$, statpermfamily $(G, P, V, \leq, p)=\{C n(\{f\})\}$

\subsection{DYNAMIC NORMS}

We say that a norm is strongly redundant when it is weakly redundant for any extension of the set of norms. The new norms may have any priority, and the priority relation among the old norms remains unchanged.

DEFINITION 7 (Dynamic norms). If $G \cup P$ is a set of norms extended with $G^{\prime} \cup P^{\prime}$, then we say that $\leq^{\prime}$ extends $\leq$ if $\leq \subseteq \leq$ and for all $g_{1} \leq^{\prime} g_{2}$ without $g_{1} \leq g_{2}$, we have $g_{1} \in G^{\prime} \cup P^{\prime}$ or $g_{2} \in G^{\prime} \cup P^{\prime}$.

The norm $g \in G \cup P$ is strongly redundant if and only if $\forall a \in$ $L, \forall G^{\prime}, P^{\prime}$ and $\leq^{\prime}$ extending $\leq$, we have outfamily $\left(G \cup G^{\prime}, P \cup P^{\prime}, V, \leq^{\prime}\right.$ ,a) $=$ outfamily $\left(G \backslash\{g\} \cup G^{\prime}, P \backslash\{g\} \cup P^{\prime}, V, \leq^{\prime}, a\right)$.

Intuitively, an authority may introduce a weakly but not strongly redundant norm to block the possibility that lower level authorities introduce conflicting and materially valid norms. Roughly, norms are strongly redundant when they are logically implied, i.e., derived by the input/output logic. To model the scenarios of Bulygin and Lewis, we further detail the model of hierarchical normative systems by making the authorities and their competence explicit. In such a setting, we may say that the lower and higher levels of authorities play a game against each other. 
In the Bulygin/Lewis games, the hierarchy on rules is due to the "lex superior" principle. Other principles are discussed in (Prakken and Sartor, 1996). These principles play the roles of meta-norms which, according to (Mazzarese, 1991), "establish which norms do constitute a given legal order", i.e., in our terminology, meta-norms establish which norms are materially valid. ${ }^{4}$

DEFINITION 8 (Competence and formal validity). Let $A$ be a set of authorities, and $\succeq_{A}$ an ordering on $A$, aut $: G \cup P \rightarrow A$ a function that associates an authority with each rule, and $C: A \rightarrow 2^{L}$ the competence of authority expressed by a set of propositional formulas of $L$. We say that:

- The hierarchy $\preceq$ reflects $\preceq_{A}$ if and only if aut $\left(g_{1}\right) \prec_{A} \operatorname{aut}\left(g_{2}\right)$ implies $g_{1} \prec g_{2}$.

- The normative system respects the competence of the authorities if and only if for each norm $g=(x, y) \in G \cup P$ we have that $y \in C(\operatorname{aut}(g))$.

- $g \in G \cup P$ is strongly redundant with respect to $\left\langle A\right.$, aut, $\left.\preceq_{A}, C\right\rangle$ if and only if it is strongly redundant for all normative systems respecting the competence of the authorities.

The following example illustrates the static/dynamic distinction.

EXAMPLE 8 (Continued from Example 5). If there is a single authority, and we only consider the rules $\{a, b\}$, then $b$ is strongly redundant. If there are multiple authorities $a_{1}$ and $a_{2}, a_{1} \prec_{A} a_{2}$ such that $a \prec b$, then $b$ is again redundant.

However, if we consider all three rules $\{a, b, c\}$ with $a \prec c \prec b$, then none of the rules is redundant. Hence, with multiple authorities and the rules $\{a, b\}, b$ is not strongly redundant if there is a lower level authority who has the competence to enact c.

The latter definition of strong redundancy supports Bulygin's argument in favor of permissions which do not presuppose explicitly existing obligations, because the new permission prevents lower level authorities to forbid the permitted behavior in toto or under some circumstances; such a permission creates a (would be) prohibition immunity (with respect to lower level authorities).Analogously, an obligation creates a

\footnotetext{
${ }^{4}$ In contrast with the notion of systemic validity of (Mazzarese, 2000), we keep separate the problems of formal and material validity since in this paper we do not address the first problem, apart from the limited treatment of competence.
} 
"permission immunity" with respect to lower level authorities.

EXAMPLE 9. Consider the sets of obligations and permissions $G=$ $\{a=(\top, \neg p), b=(q, r), c=(s, p), d=(s, \neg t)\}$ and $P=\{e=(\top, t)\}$ with $a \prec b, a \prec c, e \prec d$

The norm $b$ derogates to the negative permission $(\top, \neg r)$, due to the fact that $r$ is indifferent without $b$. The norm $c$ derogates to permission $(\top, \neg p)$, a negative permission following the corresponding obligation a. Finally, $d$ derogates the strong permission e.

We have:

$\operatorname{maxfamily}(G, P, V, \leq, q \wedge s)=\{\{a, b, e\},\{a, b, d\},\{b, c, d\},\{b, c, e\}\}$, $\operatorname{preffamily}(G, P, V, \leq, q \wedge s)=\{\{b, c, d\}\}$,

outfamily $(G, P, V, \leq, q \wedge s)=\{C n(\{r, p, \neg t\})\}$,

Note that in our model, a non-redundant permission which does not derogate a preexisting obligation does not correspond to a limitation of the competence of a lower level authority. Competence is defined as a set of propositions, while a higher level permission is expressed as a conditional rule. So a permission can derogate an eventual norm only in part and not in toto, thus non hindering the competence of the lower level authority in those cases where the condition of the permission is false.

We analyze this possibility in the following example:

EXAMPLE 10. Consider a hierarchical normative system where an authority $a_{2}$ has the competence about $d\left(d \in C\left(a_{2}\right)\right)$, e.g. it can permit or forbid to download programs from the web. However, the higher rank authority $a_{1}, a_{2} \prec_{A} a_{1}$ which also has the competence $d$, decides to permit downloading of antivirus programs, $(a, d) \in P$. This permission does not hinder $a_{2}$ 's competence $d$ in that $a_{2}$ can still forbid the download of different types of programs, e.g. crackers $(c, \neg d) \in G$, but also more general ones, e.g. every program $(\top, \neg d) \in G$. These norms are both formally valid, because $d \in C\left(a_{2}\right)$ is still true, and also materially valid. For example, we have $\neg d \in$ outfamily $(\{(c, \neg d)\},\{(a, d)\}, V, \leq, c)$.

However, the prohibition $(\top, \neg d)$ does not cancel the higher level permission in case the condition a, the program is an antivirus, is true (even if a implies $\mathrm{T}$ ):

$\neg d \notin$ outfamily $(\{(\top, \neg d)\},\{(a, d)\}, V, \leq, a)$

$d \in \operatorname{statpermfamily}(\{(\top, \neg d)\},\{(a, d)\}, V, \leq, a)$ 


\section{Related work}

Alchourròn and Makinson (1981) first addressed in a formal way the notion of derogation: "the [legislative] body may decide to reject $y$, with the intention of thereby rejecting implicitly whatever in $A$ implies $y$, retaining the remainder. This we shall call derogation", p.127. Moreover "in the special case where $Y$ is a subset $D$ of $A$, to derogate it, in the sense we have specified is to do much more than merely rescind or abrogate it. For when we abrogate a subset $D$ of $A$, we merely $d r o p$ it from the code, leaving $A-D$ intact even if it implies some of the regulations in $D$ ", p.130. This model subsequently lead to a solution of the belief revision problem by (Alchourron et al., 1985).

In this paper, we are interested in a complementary problem. We are not interested in computing the remainder of a set of propositions given a norm to be derogated. Rather, we focus on the point of view of the legislator who can change the normative system by adding permissions and obligations. Given a new permission or obligation we deal with the problem of computing what is now permitted or obligatory.

Royakkers and Dignum (1997) present a formalization of the distinction between weak and strong permission. The focus of the work is the fact that a permission which is enacted, i.e., a strong permission, implies that it is strongly permitted also the negation of what is permitted. The reason to this claim is that "a regulation concerning a permission is always an exception of an obligation or a prohibition [...]. Otherwise the permission would be superfluous, because of the sealing principle "whatever is not forbidden is permitted'." Hence a strong permission "implies a choice for the norm subjects to perform that act or not, without a liability to sanction."

Our treatment of permissions raises the question whether permissions can be related to undercutters in argumentation theory, when obligations are related to arguments and rebutters.

In (Pollock, 1987) rebutters are a kind of defeat of an argument which attacks a reason by supporting an opposite conclusion; undercutters are arguments that attack the fact that another reason supports its conclusion: given a argument $P \rightarrow Q$, an undercutter is a reason for $\neg(P \rightarrow Q)$. However, when we consider conditional concerning norms, as input/output logic, we should not speak of their truth. So (Pollock, 1987)'s definition is not adequate for obligations and permissions.

More recently, Prakken (1997) proposes a treatment of undercutters in terms of premises which contains weak negation $(\sim)$ in conditionals. An argument like $P \wedge \sim R \Rightarrow Q$ can be undercutted by an argument supporting $R$. 
Verheij (2001) criticizes this approach since he argues that it limits the possibility to add further undercutting arguments once the conditional has been created. Hence Verheij (2001) proposes a different treatment of undercutters, by introducing the unary $\times \phi$ operator with the meaning that $\phi$ is defeated. Undercutters are represented as conditionals which defeat other conditionals; $\phi \rightarrow \times(\psi \rightarrow \chi)$ means that $\phi$ is an undercutter of $\psi$ supporting $\chi$. This allows to model the sequential character of law making, where new permissions can be added after the obligation they are exceptions to. Our model offers the same flexibility, with the restriction that we model only undercutters of formulas instead of defeating conditionals.

\section{Summary}

Normative multiagent systems study general and domain independent properties of norms. It builds on results obtained in deontic logic, the logic of obligations and permissions, for the representation of norms as rules, the application of such rules, contrary-to-duty reasoning and the relation to permissions. However, it goes beyond logical relations among obligations and permissions by explaining the relation among social norms and obligations, relating regulative norms to constitutive norms, explaining the evolution of normative systems, and much more.

The dynamic arrival of new issues and the need to introduce new norms to regulate them is a crucial problem for multiagent systems becoming open and more and more autonomous. Moreover, in such systems social laws are not only designed off-line, but they are also developed at run-time. In such systems, it is of crucial importance that the user still can control the development of social laws by restricting them in advance.

In this paper we study institutions with hierarchies of authorities. Higher authorities in the hierarchy should delimit the legislative autonomy of lower ones. In particular, we study the interplay between obligations and permissions in the (Bulygin, 1986)'s "Rex, Minister and Subject" games.

To study permissions and obligations we use input/output logic. Recent developments in the input/output logic framework distinguished various forms of permissions, but we show that the notion of prohibition immunity cannot be extended to the constrained setting, and that thus far the permissions as exceptions are not formalized in this framework. We introduce such a notion, and discussed some rationality constraints which can be imposed on such definitions. Thus the notion of "prohi- 
bition immunity" (and a symmetric notion of "permission immunity") has been relativized to the different levels of authorities.

Our work supports (Bulygin, 1986)'s suggestion that "permissive norms are normative relevant in an indirect way", they "are not superfluous", i.e., "they play a characteristic role in legal discourse which could not be possibly performed by mandatory norms alone", p.211. Without permissions as exceptions "there would be no possibility of normative change emerging from acts of authority" p.213. Changing a normative system would not amount to adding new obligations or permissions but only to replacing the existing norms by means of alternative ones. Permissions are used to block other (lower) authorities to issue norms. This is a motivation behind the dynamic interpretation of norms.

Elsewhere we have formalized obligations associated with sanctions (Boella and van der Torre, 2006a; Boella and van der Torre, 2006b), extending (Boella and Lesmo, 2002)'s proposal in a qualitative decision theory. Future work concerns the definition of institution with hierarchies of authorities using the sanction-based definition of norms.

\section{References}

Alchourron, C., P. Gärdenfors, and D. Makinson: 1985, 'On the logic of theory change'. Journal of Symbolic Logic 50(2), 510-530.

Alchourron, C. E. and D. Makinson: 1981, 'Hierarchies of regulations and their logic'. In: R. Hilpinen (ed.): New studies in deontic logic. Dordrecht: D. Reidel, pp. $125-148$.

Bobbio, N.: 1958, Teoria della norma giuridica. Torino: Giappichelli.

Bobbio, N.: 1980, 'Norma'. In: Enciclopedia Einaudi, Vol. 9. Torino, pp. 876-907, Einaudi.

Boella, G. and L. Lesmo: 2002, 'A game theoretic approach to norms'. Cognitive Science Quarterly 2(3-4), 492-512.

Boella, G. and L. van der Torre: 2006a, 'A Game Theoretic Approach to Contracts in Multiagent Systems'. IEEE Transactions on Systems, Man and Cybernetics Part C: Applications and Reviews 36(1), 68-79.

Boella, G. and L. van der Torre: 2006b, 'Security Policies for Sharing Knowledge in Virtual Communities'. IEEE Transactions on Systems, Man and Cybernetics Part A: Systems and Humans 36(3), 439-450.

Boella, G., L. van der Torre, and H. Verhagen: to appear, 'Introduction to normative multiagent systems'. Computation and Mathematical Organizational Theory, special issue on normative multiagent systems.

Brass, S.: 1993, 'Deduction with supernormal defaults'. In: G. Brewka, K. P. Jantke, and P. H. Schmitt (eds.): Nonmonotonic and inductive logics, Vol. 659 of LNAI. Berlin: Springer, pp. 153-174.

Brewka, G. and T. Eiter: 2000, 'Prioritizing Default logic'. In: Intellectics and Computational Logic. Kluwer, pp. 27-45. 
Broersen, J., M. Dastani, J. Hulstijn, and L. van der Torre: 2002, 'Goal generation in the BOID architecture'. Cognitive Science Quarterly 2(3-4), 428-447.

Bulygin, E.: 1986, 'Permissive Norms and Normative Systems'. In: A. Martino and F. S. Natali (eds.): Automated Analysis of Legal Texts. Amsterdam: Publishing Company, pp. 211-218.

Bulygin, E.: 1996, 'Logische Fragen der Gesetzgebungstechnik'. In: J. Rodig (ed.): Studien zu einer Theorie der Gesetzgebung. Berlin: Springer, pp. 612-627.

Conte, R., C. Castelfranchi, and F. Dignum: 1998, 'Autonomous norm-acceptance'. In: Intelligent Agents $V$ (ATAL'98), Vol. 1555 of LNCS. Berlin: Springer, pp. 99-112.

Dignum, F.: 1999, 'Autonomous agents with norms'. Artificial Intelligence and Law 7, 69-79.

Dignum, V., J. Vázquez-Salceda, and F. Dignum: 2004, 'A Model of Almost Everything: Norms, Structure and Ontologies in Agent Organizations'. In: Procs. of 3rd International Joint Conference on Autonomous Agents and Multiagent Systems (AAMAS'04). New York (NJ), pp. 1498-1499, ACM.

Esteva, M., J. Padget, and C. Sierra: 2001, 'Formalizing a language for institutions and norms'. In: Intelligent Agents VIII (ATAL'01), Vol. 2333 of LNCS. Berlin, pp. 348-366, Springer.

Esteva, M., J. Rodriguez-Aguilar, C. Sierra, and W. Vasconcelos: 2004, 'Verifying Norm Consistency in Electronic Institutions'. In: Procs. of Workshop on Agent Organizations at AAAI'04. San Jose (CA).

Guastini, R.: 1998, Teoria e dogmatica delle fonti. Milano: Giuffrè.

Hansson, B.: 1969, 'An analysis of some deontic logics'. Nồs 3, 373-398.

Jones, A. and J. Carmo: 2001, 'Deontic logic and Contrary-to-Duties'. In: D. Gabbay and F. Guenthner (eds.): Handbook of Philosophical Logic, Vol. 3. Dordrecht (NL): Kluwer, pp. 203-279.

Lewis, D.: 1979, 'A problem about permission'. In: E. Saarinen (ed.): Essays in Honour of Jaakko Hintikka. Dordrecht: D. Reidel, pp. 163-175.

Lopez y Lopez, F., M. Luck, and M. d'Inverno: 2002, 'Contraining autonomy through norms'. In: Procs. of 2nd International Joint Conference on Autonomous Agents and Multiagent Systems (AAMAS'02). New York (NJ), pp. 674-681, ACM.

Makinson, D. and L. van der Torre: 2000, 'Input-output logics'. Journal of Philosophical Logic 29(4), 383-408.

Makinson, D. and L. van der Torre: 2001, 'Constraints for input-output logics'. Journal of Philosophical Logic 30(2), 155-185.

Makinson, D. and L. van der Torre: 2003, 'Permissions from an input-output perspective'. Journal of Philosophical Logic 32(4), 391-416.

Mazzarese, T.: 1991, 'Deontic Logic as Logic of Legal Norms: Two Main Sources of Problems'. Ratio Juris 4, 374-392.

Mazzarese, T.: 2000, 'Permesso forte e permesso debole: note a margine'. Analisi e diritto.

Pollock, J. L.: 1987, 'Defeasible reasoning'. Cognitive Science 11, 481-518.

Prakken, H.: 1997, Logical tools for modelling legal argument. Dordrecht: Kluwer Academic Publisher.

Prakken, H. and G. Sartor: 1996, 'A Dialectical Model of Assessing Conflicting Arguments in Legal Reasoning.'. Artificial Intelligence and Law 4(3-4), 331-368.

Royakkers, L. and F. Dignum: 1997, 'The logic of enactment'. In: Procs. of Fourth International Conference on Artificial Intelligence and Law (ICAIL'97). ACM, p. 257.

Searle, J.: 1995, The Construction of Social Reality. New York: The Free Press. 
Shoham, Y. and M. Tennenholtz: 1995, 'On Social Laws for Artificial Agent Societies: Off-Line Design'. Artificial Intelligence 73(1-2), 231-252.

van der Torre, L.: 2003, 'Contextual Deontic Logic: Normative Agents, Violations and Independence'. Annals of Mathematics and Artificial Intelligence 37(1-2), 33-63.

van der Torre, L. and Y. Tan: 1999, 'Contrary-To-Duty Reasoning with Preferencebased Dyadic Obligations'. Annals of Mathematics and Artificial Intelligence 27(1-4), 49-78.

Verhagen, H.: 1999, 'On the learning of norms'. In: Procs. of Modelling Autonomous Agents in a Multi-Agent World (MAAMAW'99).

Verheij, B.: 2001, 'Legal decision making as dialectical theory construction with argumentations schemes'. In: Procs. of Eighth International Conference on Artificial Intelligence and Law (ICAIL'01). pp. 225-226.

von Wright, G. H.: 1951, 'Deontic logic'. Mind 60, 1-15.

von Wright, G. H.: 1963, Norm and Action. A logical Inquiry. London: Routledge and Kegan Paul. 
perm.tex; 20/06/2007; $13: 57 ;$ p.24 


\title{
Interaction between Objects in power Java
}

\author{
Matteo Baldoni, Università degli Studi di Torino - Italy \\ Guido Boella, Università degli Studi di Torino - Italy \\ Leendert van der Torre, University of Luxembourg - Luxembourg
}

\begin{abstract}
In this paper we start from the consideration that high level interaction between entities like web services has very different properties with respect to the interaction between objects at the lower level of programming languages in the object oriented paradigm. In particular, web services, for security, usability and user adaptability reasons, offer different operations to different users by means of access control and keep track of the state of the interaction with each user by means of sessions. The current vision in object orientation, instead, considers attributes and operations of objects as being objective and independent from the interaction with another object, which is sessionless. To introduce these features in the interaction between objects directly in object oriented programming languages, we take inspiration from how access control is regulated by means of roles. Roles allow objects to offer different operations depending on the type of the role, of the type and identity of the player of the role, and to define session-aware interaction.

We start from a definition of roles given in ontologies and knowledge representation and we discuss how this definition of roles can be introduced in Java, building our language powerJava.
\end{abstract}

\section{INTRODUCTION}

Object orientation is a leading paradigm in programming languages, knowledge representation, modelling and, more recently, also in databases. The basic idea of object orientation is that the attributes and operations of an object should be associated with it. The interaction with the object is made possible by the public attributes specified by the class which the object is an instance of and by its public operations. The implementation of an operation is encapsulated in the class of the object and can access its private state. This structure allows programs to fulfill the data abstraction principle: the public attributes and operations are the only possibility to manipulate an object and their implementation is not visible to the other objects manipulating it; thus, the implementation of the class can be changed without changing the interaction capabilities of its instances.

This view can be likened with the way we interact with objects in the world: the same operation of switching a device on by pressing a button is implemented in different manners inside different kinds of devices, depending on their functioning.

However, in computer science, other kinds of interaction between entities have been devised at levels higher than programming languages. In particular, the inter-

Cite this document as follows: http://www.jot.fm/general/JOT_template_LaTeX.tgz 
action at the level of web services has different properties, in order to satisfy security, usability and user adaptation requirements: different operations are offered to different users, the execution of an operation depends on the identity of the caller of the operation, and the state of the interaction with a user is maintained in a session.

Albeit these features can be introduced by programming, e.g., by means of patterns, the lack of these abstractions at the lower level limits sometimes the potentialities of object oriented languages:

1. Despite the method invocation mechanism is based on the metaphor of sending messages to objects, there is no notion of the sender of a message. Thus, the caller object (e.g., the this in Java) invoking a method of another object (the callee) is not taken into account for the method execution. Hence, when an operation is invoked its meaning can not depend on the caller's identity and class.

2. All caller objects of whatever classes can access all the public attributes and invoke all the public operations of every other callee object. Hence, it is not possible to distinguish which attributes and operations are visible for which classes of caller objects.

3. The callee object can exhibit a single interface to all the callers, and methods can have only one implementation in the callee.

4. The values of the private and public attributes of a callee object are the same for all other caller objects. Hence, the callee object exhibits only one state.

5. The interaction with a callee object is sessionless since the invocation of an operation does not depend on the identity of the caller (1) and there is only one state (4). Hence, the value of attributes and, consequently, the meaning of operations cannot depend on the previous interactions between the callee and each caller object.

6. Finally, the operational interface of abstract data types induces an asymmetrical semantic dependency of the callers of operations on the operation provider: the caller takes the decision on what operation to perform, passes the values of the parameters, and then it relies on the provider to carry out the operation, without further interaction.

The limitations 2-4 hinder modularity, since it would be useful to keep distinct the core behavior of an object from the different interaction possibilities which it offers to different kinds of objects. Some programming languages offer ways to give multiple implementations of interfaces, but the dependance from the caller cannot be taken into account, unless the caller is explicitly passed as a parameter in all methods. The limitation 5 complicates the modelling of distributed scenarios where communication is based on protocols and sessions are required. The first and last ones complicate coordination of components: method invocation does not allow 
objects to reach a minimum level of "control from the outside" of the participating objects [3].

The reason of these problems rests in the philosophy behind object orientation, which is based on the ontological assumption that attributes and operations of objects are objective: they are the same whatever is the caller object, unless it is passed as an explicit parameter. To solve this problem at the level of programming constructs we take inspiration from the solution used to control access to the services of a system. For security reasons, it is necessary to make explicit the notion of caller of an operation. In particular, in the role based access control model (RBAC) [38], access rights are associated with roles and users - the callers of operations - are made members of appropriate roles, thereby acquiring the roles' permissions. Moreover, sessions are mappings between a user and an activated subset of roles that are assigned to the user.

Like in the RBAC model, in our paper roles are based on an organizational metaphor: roles describe the way persons can interact with and within an organization, assigning them institutional powers. The particular way of interaction with an organization depends on the properties of the person it is interacting with and to what the organization allows him to do in the role he plays. So, an organization does not offer only a direct single way of interacting with it, but it is possible to interact with the organization only via roles, where roles are defined by the organization itself.

Instead, most other works on roles in programming languages adopt a different perspective: roles are seen as a way to extend the behavior of an object (e.g., [16, 21, 23, 26, 37]) and not as a way to model how an object offers different possibilities of interaction to different kind of players and maintains the session of interaction. Thus, we pass from a player-centered vision of roles to an organizationalcentered one.

Our definition of roles emerges from the analyses of organizational roles made in ontologies and knowledge representation [13, 15, 28, 29]. Thus, we not only introduce roles in object oriented programming languages motivated by practical considerations, as discussed above, but we also introduce a notion of role which is well founded and on which there is wide agreement among authors in ontologies and knowledge representation.

The methodology we use is to introduce roles in a real programming language, Java, one of the most used object oriented languages. To prove the feasibility of this approach, and give a semantics, we translate the new language, called powerJava, to pure Java by means of a pre-compilation phase.

The structure of the paper is as follows. First we introduce our ontological definition of roles taking inspiration from a running example. Then we present the powerJava language and its translation. Related work and summary close the paper. 


\section{THE INTERACTION WITH OBJECTS VIA ROLES}

Despite several proposals have been made about how to introduce roles in programming languages, there is little consensus on which are their properties and their purpose. A possible reason for this divergence is that the notion of role is a commonsense one, and, thus, it is fuzzy: everyone has an intuitive understanding of this notion, but partially different from the others.

To avoid resorting to intuition only, in this paper we introduce roles in object orientation starting from the analyses performed in the field of ontology and knowledge representation, also by the authors of this paper $[13,15]$, so to have a precise definition of roles on which there is widespread agreement and which is justified independently from the practical problems we want to solve with it. This definition of roles uses a metaphor taken directly from organizational management. Organizations, and more generally institutions, are not like standard objects which can be manipulated from the outside (e.g., a radio can be switched on). Rather, institutions are objects belonging to the social reality, and the interaction with them can be performed only via the roles which they offer [14].

Roles are useful not only to model domains that include institutions and organizations. Rather, every object can be considered as an institution or an organization structured in roles, if it is necessary to model in different ways the interaction of this callee object with different types of caller objects, depending on their class and on their aims, or to keep track of the interaction with each caller object.

To make an example, let us suppose to model a class Printer. The interaction possibilities offered by the class are different and depend on which objects invoke its methods. For example, some objects have more privileges than other ones, and thus they can invoke methods which are not available to other objects interacting with the same printer. Moreover, some methods keep track of the interaction with each specific object invoking them. For example, print counts the number of pages printed by each object invoking it to check whether the quota assigned to the object is respected. However, objects with more privileges do not have a quota of printed pages.

The Printer can be seen as an institution which supplies two different roles for interacting with it (the set of methods a caller can invoke): one role of normal User, and the other role of SuperUser. The two roles offer some common methods (roles are classes) with different implementations, but they also offer other different methods to their players (and there is no direct way to interact with the Printer). For example, Users can print their jobs and the number of printable pages is limited to a given maximum; thus, the number of pages is counted (the role associates new attributes with the player): each User should be associated with a different state of the interaction (the role has an instance with a state which is associated with its player). The User can print since the implementation of its methods has access to the private methods of the Printer (the methods of the User access the private attributes and operations of another object, the institution). SuperUsers have the 
method print with the same signature, but with a different implementation: they can print any number of pages; moreover, they can reset the page counter of Users (a role can access the state of another role, and, thus, roles coordinate the interaction).

A role like SuperUser can access the state of the other User roles and of the callee object (the institution Printer) in a safe way only if it encapsulated in the institution Printer. Thus the definition of the role must be given by the same programmer who defines the institution (the class of the role belongs to the same namespace of class of the institution, or, in Java terminology, it is included in it).

In order to interact as User or SuperUser it is necessary to exhibit some requested behavior. For example, in order to be a User a caller object must have an account (it must be a Accounted), which is printed on the pages (returned by a method offered by the player of the role). A SuperUser can have more demanding requirements.

Finally, a role User can be played only when there is an instance of Printer and an instance of a class implementing Accounted which can play the role.

This example highlights the following properties that organizational roles have in our ontological model we discuss in $[13,15]$ :

- Foundation: a (instance of) role must always be associated with an instance of the institution it belongs to, besides being associated with an instance of its player (extending Guarino and Welty [22]).

- Definitional dependence: the definition of the role must be given inside the definition of the institution it belongs to.

- Institutional empowerment: the operations defined for the role in the definition of the institution have access to the attributes and operations of the institution and of the other roles: thus, we call them powers. Instead, the operations that a class must offer for playing a role are called requirements.

These features are considered also by other authors in ontologies and knowledge representation, as discussed in Section 5.

Contrary to natural classes like person, roles lack rigidity: a player can enter and leave a role without losing its identity; a person can stop being a student but not being a person. Finally, a role can be played by different kinds of players. For example, the role of customer can be played by instances of person and of organization, two classes which do not have a common superclass. The role must specify how to deal with the different properties of the possible players. This requirement is in line with UML, which relates roles and interfaces as partial descriptions of behavior.

Hence, we propose quite a general definition of roles, independently from programming languages. But the example above illustrates how these features can be mapped on an object oriented scenario to solve the problems described in Section 1, as we discuss in the next section. 


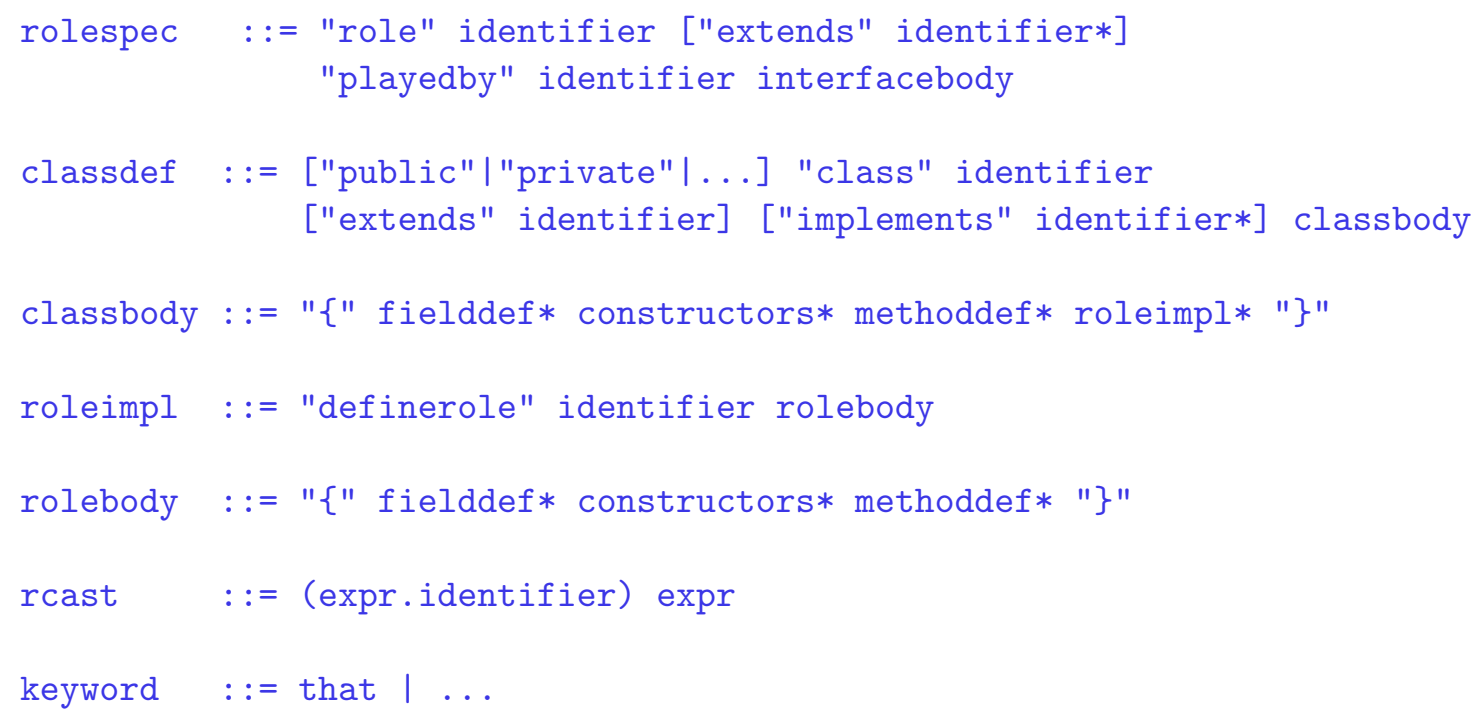

Figure 1: The extension of the Java (1.4) syntax in powerJava.

\section{INTRODUCING ROLES IN JAVA: POWERJAVA}

In this section we introduce our extension of Java by following three steps. First of all, roles are classes with private and public attributes and methods, but we will also describe how to specify them in an abstract way in terms of the signatures of the "requirements" and "powers", independently from their implementation in an institution. We call this description a role specification. A role specification is like a double-faced interface, reflecting the two aspects of the role. Second, we will introduce the way in which a role is implemented by a class in an institution with a particular attention to the definitional dependence of a role with respect to the institution within which it exists. We will do this inspired by Java inner classes. Last, we will show how an object can enter a role and, by playing the role, it can exercise its powers. The syntax of powerJava is illustrated in Figure 1.

\section{Specification of powers and requirements}

A role should be specified, for the sake of modularity, independently from its possible implementations as classes in specific institutions. To promote the view "program to an interface, not to an implementation" [19], we introduce role abstract specifications which must be respected by their implementations. Moreover, for Steimann and Mayer [41], roles define a certain behavior (or protocol) demanded in a context independently of how or by whom it is to be delivered. Thus, in order to make role systems reusable, it is necessary that a role can be played by more than one class only.

Specifying a role implies specifying both what is required to a caller in order to 
play it, and which powers the player acquires in the institution in which the role is implemented. Thus, a role specification has a list of abstract signatures of the methods offered to objects playing the role (powers) and a list of abstract signatures of the methods required to be implemented by the objects in order to be able to play the role (requirements). The latter ones are modelled as an interface associated with the role construct by the keyword playedby.

In this way, objects offering a role and objects which can play it can be developed independently of each other. In particular, any class implementing the requirement interface can play the role.

Let us consider again the Printer of the previous section: a normal User acquires the powers to print and to know the number of printed pages getPrintedPages, and it is required to provide its login (getLogin). The role specification for the user is the following, where the keyword playedby associates with an interface body specifying the powers the requirements specified by the separate interface Accounted:

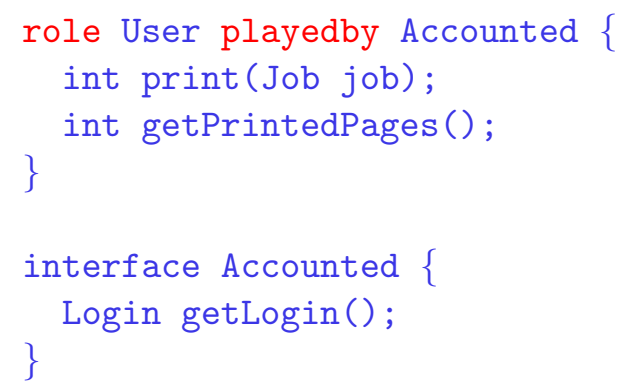

The SuperUser, instead, must have both an account and a certificate to guarantee its profile, and it has additional powers:

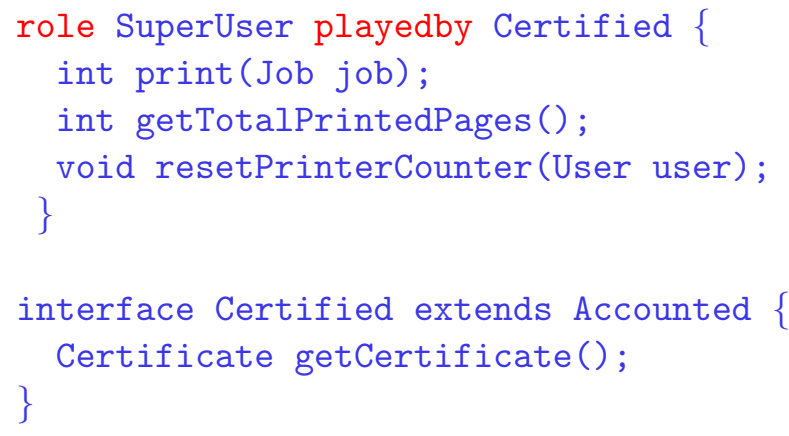

\section{Institutions and role implementation}

As discussed in Section 2, roles are always associated with an instance of an institution, and are definitionally dependent on it. We call the methods offered by roles "powers" because they offer the possibility to modify the private state and access 
private methods of the institution which defines them and the state of the other roles defined in the same institution. In this way roles allow a player to interact with(in) an institution. In our running example, the method print, both of a User and a SuperUser, will access a private counter of the printer for updating the total number of printed pages. The method resetPrinterCounter of SuperUser, instead, allows a player of the role SuperUser to change the state of a different role. Powers, thus, are methods which seem to violate the standard encapsulation principle, where the private variables are visible only to the class they belong to. However, here, the encapsulation principle is preserved by the definitional dependence property: the definition of all the roles of an institution depends on the definition of the institution; so it is the institution itself which gives to the roles access to its private fields and methods. Since it is the programmer of institution itself which implements its roles, there is no risk of abuse by part of the role of its access possibilities. Enabling a class to belong to the namespace of another class without requiring it to be defined as "friend", and thus endangering modularity, is achieved in Java by means of the inner class construct. The construct definerole allows the programmer to define a sort of inner class in order to implement a role specification inside an institution (the outer class). For example, the code in Figure 2 defines the class Printer, which contains the implementations of the above mentioned User and SuperUser role. The name of the class of these role implementation is respectively Printer. User and Printer.SuperUser. Note that, role specifications cannot be implemented in different ways in the same institution and we do not consider the possibility of extending role implementations (possible with inner classes). Even if in a preliminary version [8] this possibility has been considered, we omit it here because it would introduce some problems in requirement handling. If a role extends another role, the most specific inherits also the requirements of the other but, as we will better see in the next section, our translation cannot handle multiple requirements.

In order for an object to play a role it is necessary that it conforms to the role requirements. Since the role requirements are a Java interface, it is sufficient that the class of the object implements the methods of such an interface, e.g.:

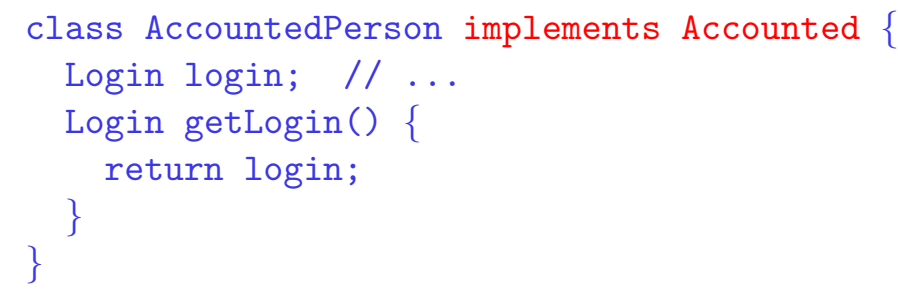

A CertifiedPerson is defined in a similar way by implementing the Certified interface. Note that also other classes can implement the requirement interface and thus play the roles.

Since roles are classes which can be instantiated and the behavior of a role instance depends on its player, in the role method implementation, the player instance can be retrieved via a new reserved keyword: that. So this keyword refers to that 


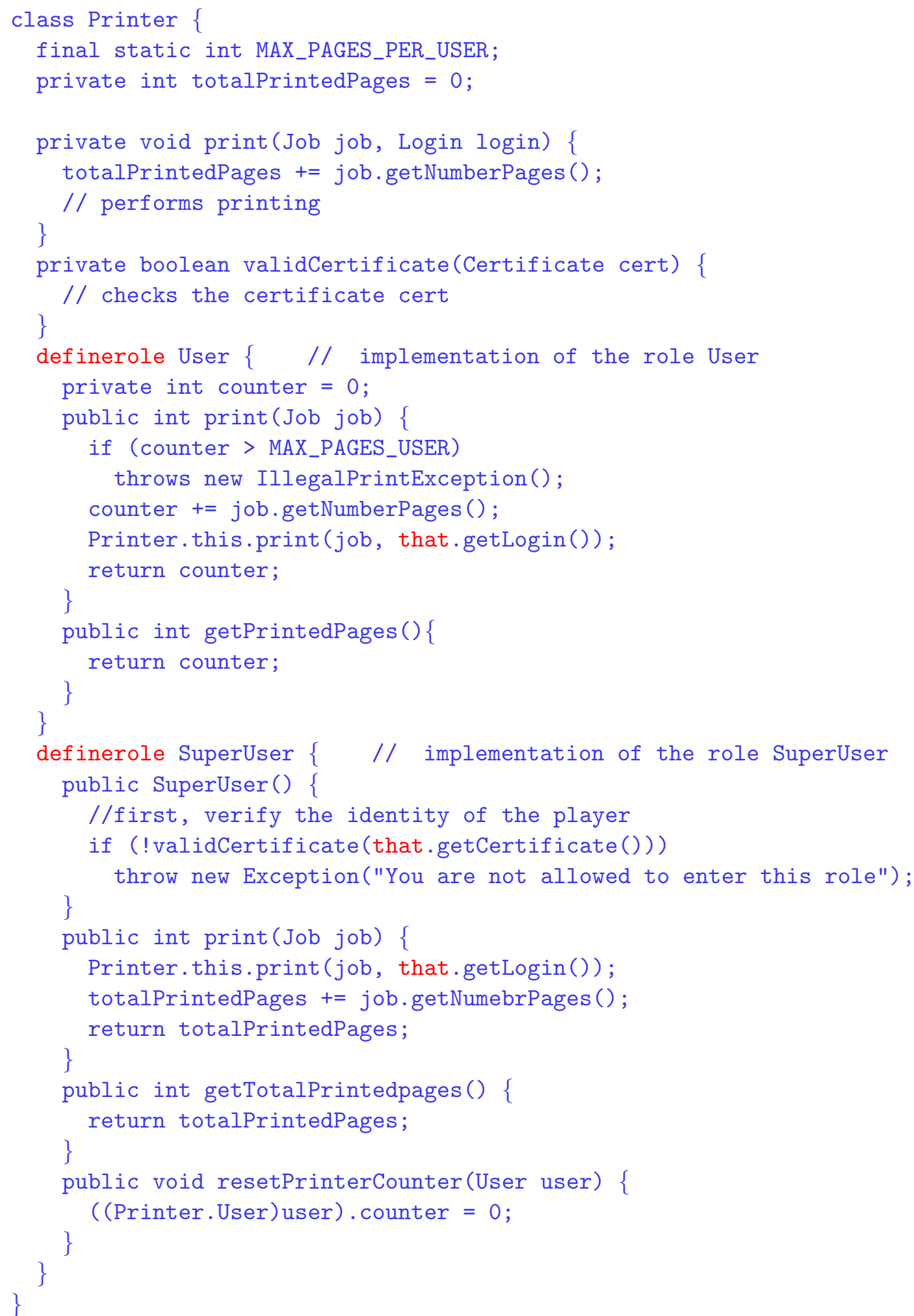

Figure 2: The class Printer with its User and SuperUser roles. 
object which is playing the role at issue, and it is used only in the role implementation. An example is the invocation of that.getLogin() as a parameter of the method print in Figure 2. The value of that is initialized when the constructor of the role implementation is invoked. The referred object has the type defined by the role requirements given by the playedby keyword in the role specification. The fact of having two links, one to the player (that) and one to the institution (Printer.this), is actually an invariant of every role in our extension of Java.

For creating instances of the inner classes implementing roles, we use the Java inner class syntax: starting from an institution instance the keyword new allows the creation of an instance of the role like it were an instance of the inner class, for example:

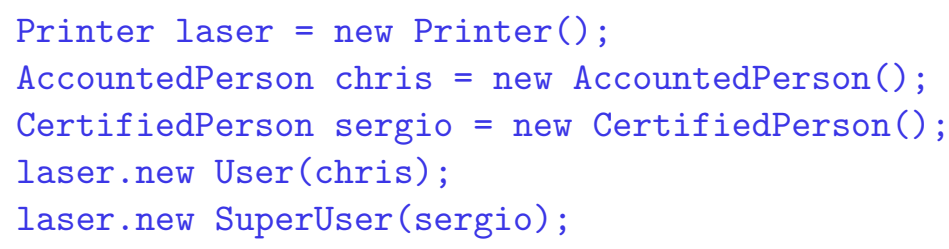

The first instructions create a Printer object laser and two objects, chris (an AccountedPerson) and sergio (a CertifiedPerson). chris becomes a normal User while sergio becomes a SuperUser. Indeed, the last two instructions define the roles of these two objects with respect to the created Printer. Note that all the constructors of role implementations have a first (implicit) parameter which must be bound to the player of the role and whose value becomes the value of that.

\section{Playing a role}

When an object is seen under the perspective of a role, it has a specific state for it, which is different from the player's one. This state is associated with the pair of objects "institution" and "player". It is the state of the interaction between the caller and the callee object and it evolves as a consequence of the invocation of methods on the role (or on other roles of the same institution as we have seen in the running example). In the printer example the variable counter of User keeps track of the number of printed pages for each different user. We will come back to this a little ahead.

When an object uses the methods offered by a role, it should be able to invoke them without any explicit reference to the instance of the role. In this way the association between the object instance and the role instance is transparent to the programmer. The object should only specify in which role it is invoking the method. For example, if an AccountedPerson is a User and it has to print something, it must be able to invoke the method print on the AccountedPerson as a User without referring to the role instance. Note that this does not exclude the possibility of assigning the reference to a role instance to a variable then using the variable for 
invoking the role methods (see the variable user in the code below). Roles belong always to an institution. Hence, an object can play at the same moment the same role more than once, albeit in different institutions. Instead, we do not consider the case of an object playing the same role more than once in the same institution. An object can play several roles in the same institution. In order to specify the role under which an object is referred, we evocatively use the same terminology used for casting by Java: we say that there is a casting from the object to the role. However, to refer to an object in a certain role, both the object and the institution where it plays the role must be specified, thus reflecting the foundation property. We call this methodology role casting. Role casting is a means for stating that an object will act according to the powers that allow it to interact in a given institution. In the following the two Users invoke method print on laser. Notice that the page counter is maintained in the role state and persists through different calls to methods performed by a same player towards the same institution as long as it plays the role.

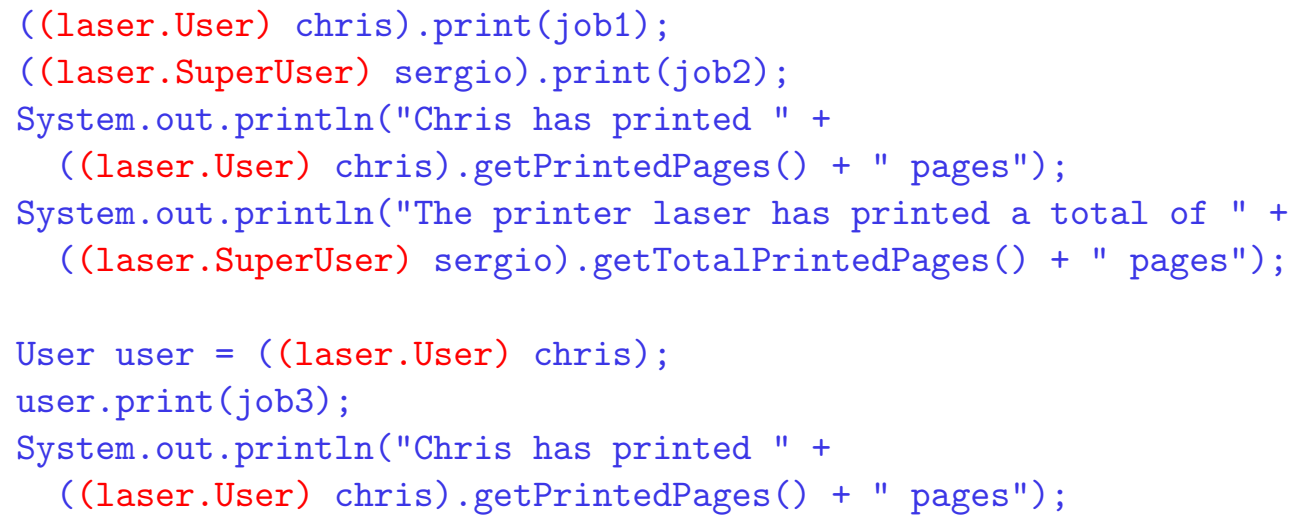

Supposing that job1 consists of ten pages, job2 of twenty pages and job3 of fifteen, the first output operation will print ten, the second one thirty (the sum of the lengths of job1 and job2), the third one twentyfive (the sum of job1 and job3).

By maintaining a state, a role can be seen as realizing a session-aware interaction, in a way that is analogous to what done by cookies or Java sessions for JSP and Servlet. So in our example, it is possible to visualize the number of currently printed pages by the user chris.

Since an object can play multiple roles, the same method will have a different behavior, depending on the role which the object is playing when the method is invoked. However, there will be no conflict among roles, since only the powers of one role at a time can be exercised. To play a role it is sufficient to specify which is the role of a given object we are referring to. In the next example sergio becomes also a normal User of laser, besides being a SuperUser, since a CertifiedPerson is also an implementation of the interface Accounted:

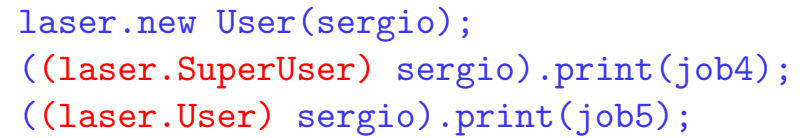




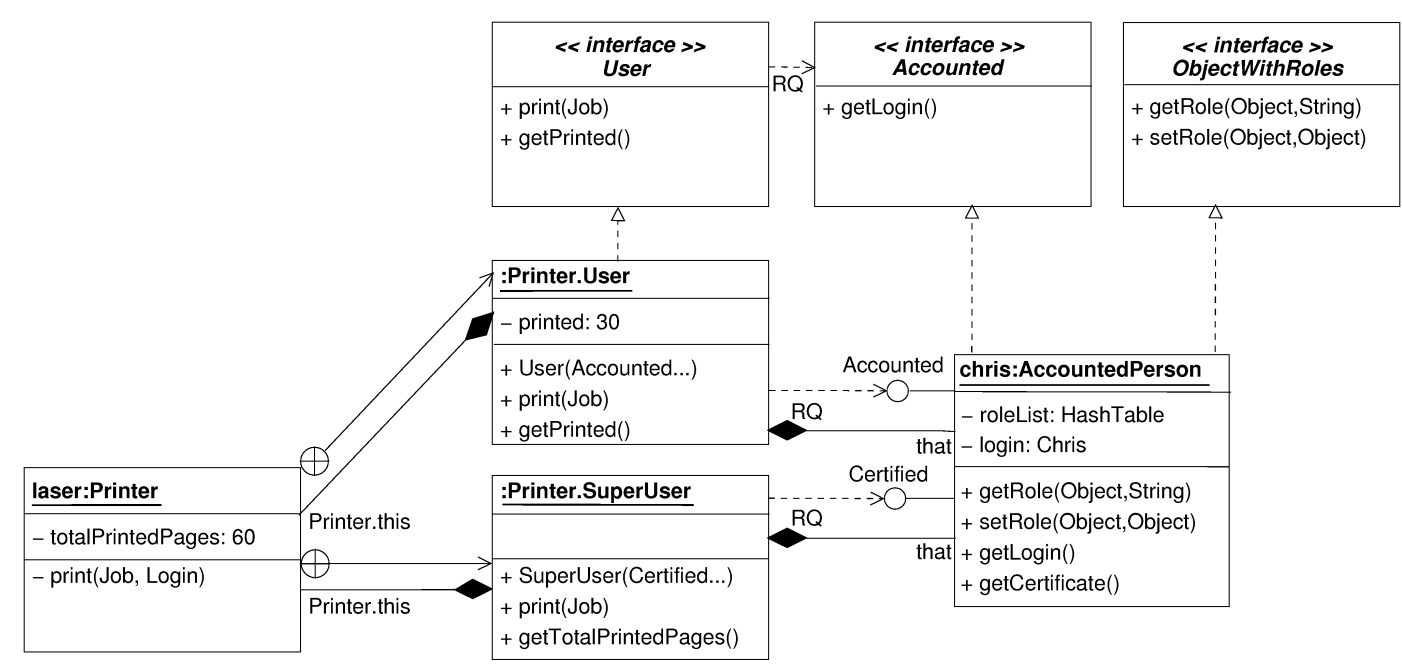

Figure 3: The UML diagram representing our running example.

Notice that in this case two different sessions will be kept: one for sergio as normal User and the other for sergio as SuperUser. Only when it prints its jobs as a normal User the page counter in the role instance is incremented.

A role instance can be left by a player or transferred to another player satisfying the requirements. In the first case, the invariant imposing the foundation of a role on its player is violated. The invocation of a method on such a role instance (which is possible since the role instance could have been assigned to a variable before the player gives up its role or it is destroyed) gives raise to an exception. However, we do not deal with these issues in this work, see [34] for a discussion.

\section{ROLE REPRESENTATION AND TRANSLATION INTO PURE JAVA}

In this section we will present the translation of powerJava in pure Java illustrating it with a UML diagram, showing a portion of our example, see Figure 3.

For what concerns the translation phase into pure Java, this is done by means of a pre-compilation phase. The pre-compiler has been implemented by means of the tool javaCC, provided by Sun Microsystems.

First of all, role requirements are an interface, specifying which methods must be defined in a class whose instances play the role. Powers, instead, are a new concept related to the role construct, representing the methods acquired by role players: the abstract signatures of power specifications are translated into an interface too, related to the interface requirements by the relation $R Q$ in Figure 3: 


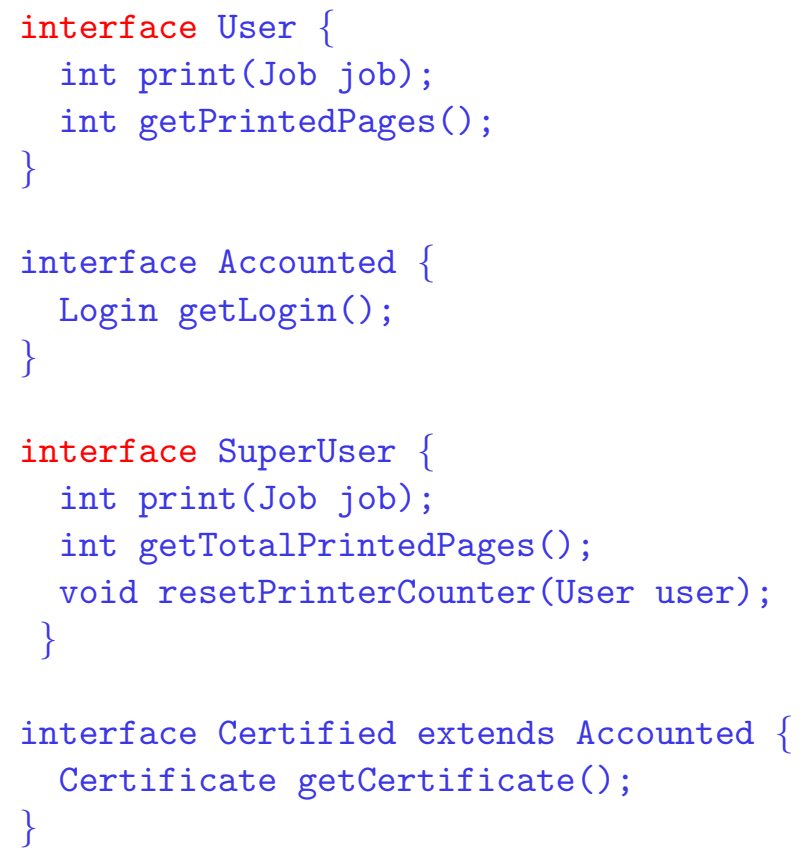

Second, the implementation of roles inside the institution is translated as inner classes, which implement the interface which results from the translation of the role power specification. Inner classes express the fact that the namespace of the institution is visible from the role implementation. For instance, in Figure 3, in the institution Printer the roles User and SuperUser are inner classes. The fact that inner classes belong to the namespace of the outer class is represented in UML by the arrow with a plus sign within a circle at the end attached to the namespace.

The difference with inner classes is that while an inner class can be instantiated given an instance of its outer class, an inner class defining the implementation of a role does not create an object which exists independently also from the object which plays the role. In other words, the instance of the role must be connected both with its player and its institution. In Figure 3 the references to such unnamed objects corresponding to the role instances are respectively represented by the composition arrows with the labels Printer.this and that. The following is an excerpt of the translation of the class Printer:

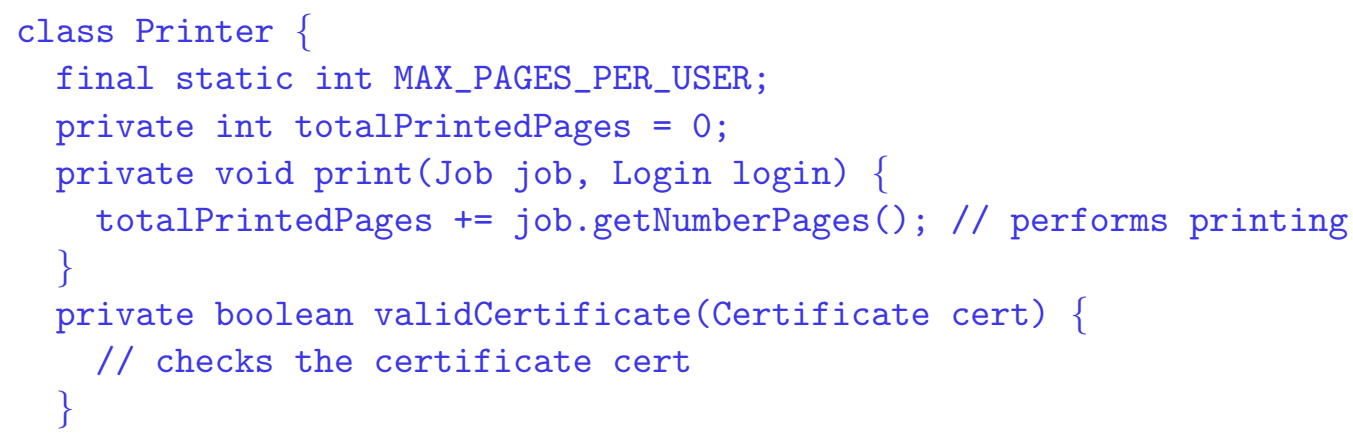




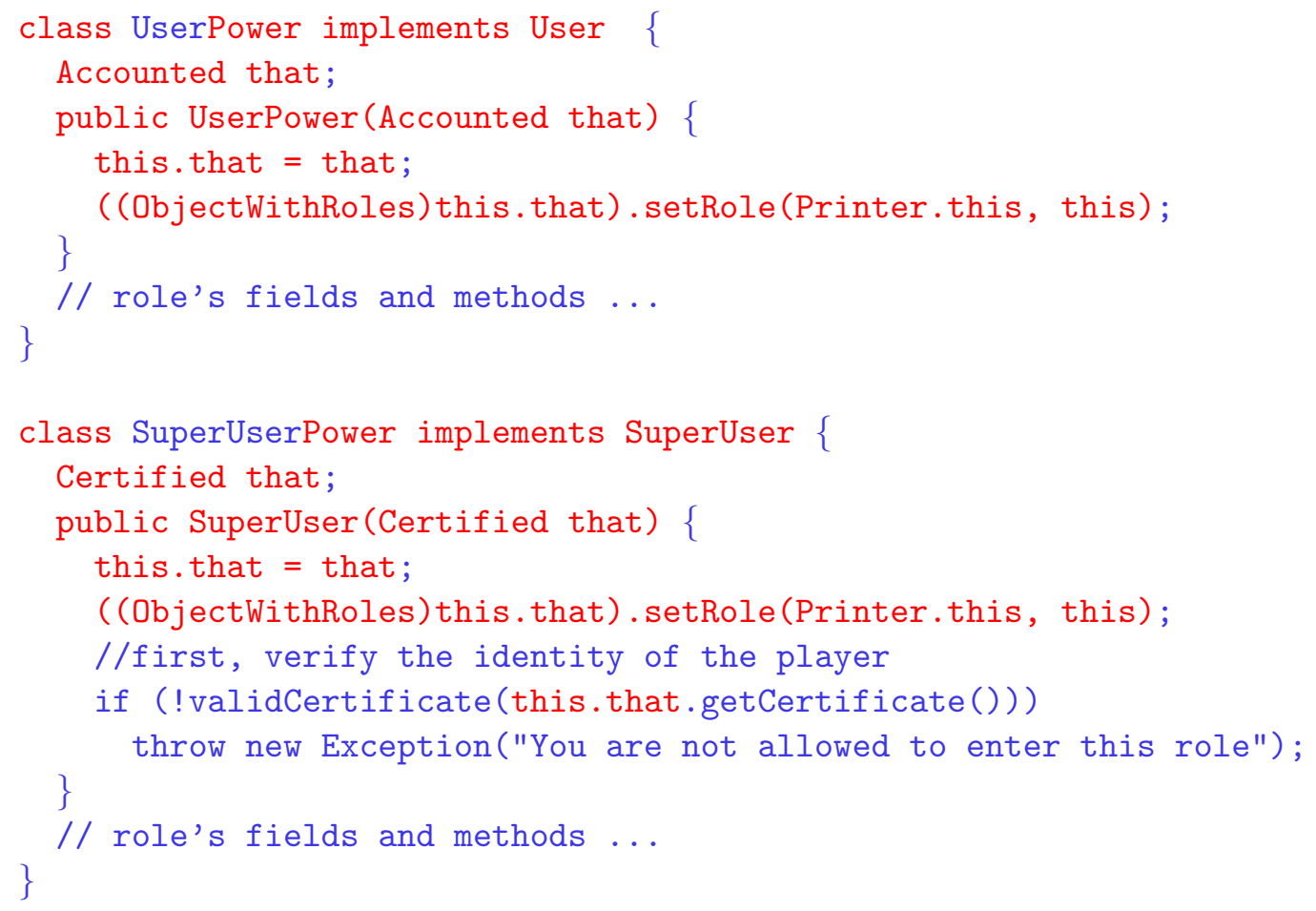

When an inner class implements a role, the role name specified by the definerole keyword is simply added to the interfaces implemented by the inner class. The correspondence between the player and the role instance, represented by the construct that, is pre-compiled in a field called that of the inner class. This field is automatically initialized by means of the constructors of role classes which are extended by the pre-compiler by adding a first parameter to pass the suitable value. The constructor also adds to the role player referred by that a reference to the role instance. The remaining link between the instance of the inner class and the outer class defining it is provided automatically by the language Java (e.g., Printer.this). Note that this translation also explains why the keyword playedby can be followed by just one identifier. The reason is that it would not be possible to assign a correct static type to that. One possibility for overcoming this limitation would be to rely on union types, as proposed, for example, by Igarashi and Nagira [24].

To play a role an object must be enriched by some methods and fields to maintain the correspondence with the different role instances it plays in the different institutions. In this way, in role casts the role instance can be retrieved from its player given the role name and a reference to the institution. This is obtained by adding, at pre-compilation time, to every class a structure for book-keeping its role instances. This structure can be accessed by the methods whose signature is specified by the ObjectWithRole interface (see Figure 3). Since every object can play a role, it is worth noticing that the ideal solution would be that the Object class itself implements ObjectWithRole: 


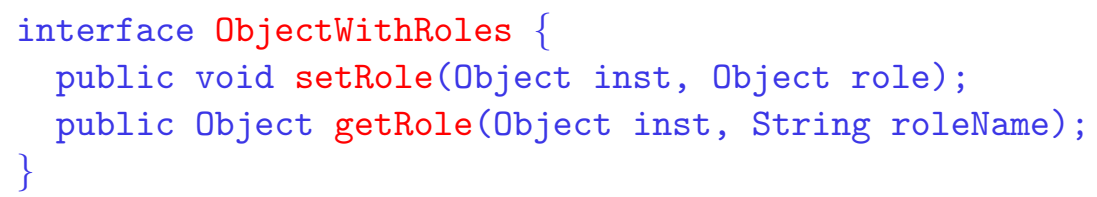

The two methods that are introduced by the pre-compiler are setRole and getRole which, respectively, adds a role instance to an object, specifying where the role is played, and returns the role instance played in the institution passed as parameter together with the role name. Further methods can be added for leaving a role, transferring it, etc.:

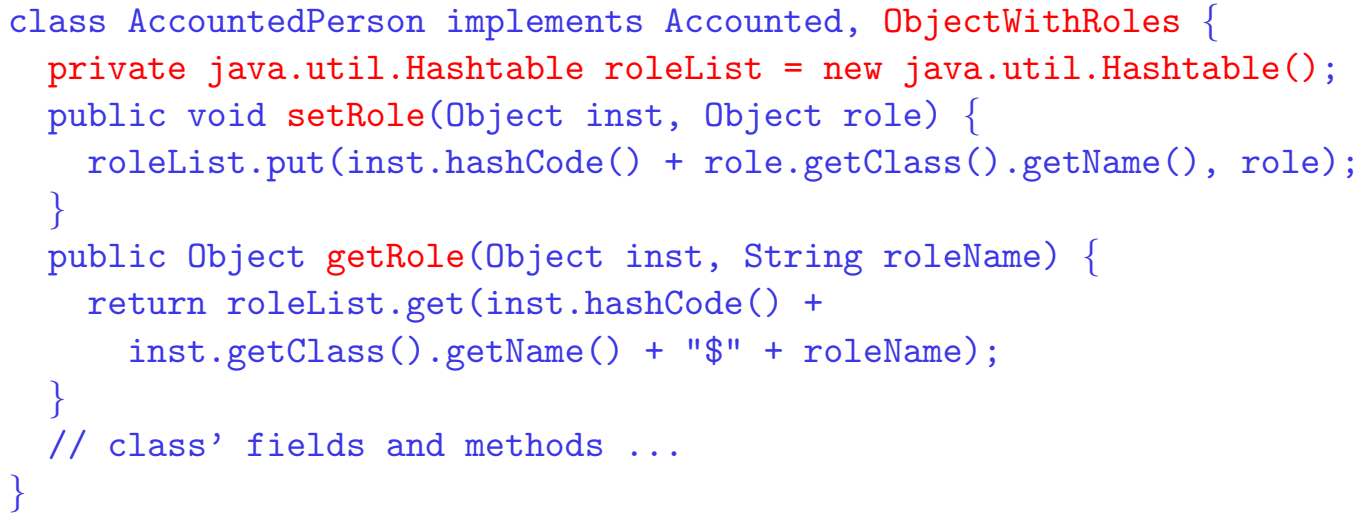

The setRole and getRole methods make use of a private hash-table roleList. As key in the hash-table we use the institution instance address and the name of the inner class. As an example, the class AccountedPerson plays the role User via the interface Accounted. So its instances will have a hash-table that keeps the many roles played by them. Role casting is pre-compiled using these methods. The expression referring to an object in its role (an AccountedPerson as a User, e.g., (laser.User) chris) is translated into the selector returning the reference to the inner class instance, representing the desired role with respect to the specified institution. The translation will be chris.getRole(laser, "UserPower"). The string "UserPower" is provided because in our solution the name of the role class is used as a part of the key of the hash-table:

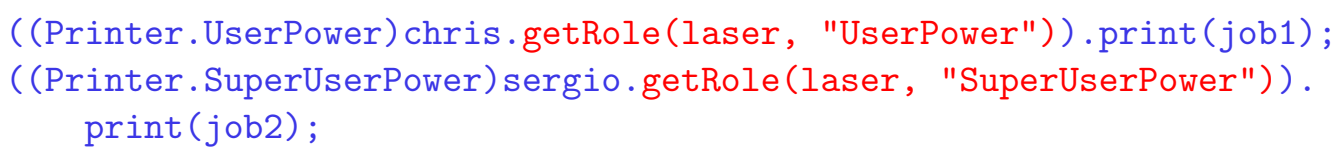

With respect to Java, additional checking is introduced to verify the consistency of the newly introduced constructs. For example, allowing classes prefixed by variables (e.g., laser.User), in the style of the Scala language [35], in the role cast constructs, introduces ambiguities which pure Java is not aware of. 


\section{RELATED WORK}

The concept of role is used quite ubiquitously in Computer Science: from databases to multiagent systems, from conceptual modelling to programming languages. According to Steimann [40], the reason is that even if the duality of objects and relationships is deeply embedded in human thinking, yet there is evidence that the two are naturally complemented by a third, equality fundamental notion: that of roles. Although definitions of the concept of role abound in the literature, Steimann maintains that only few are truly original, and that even fewer acknowledge the intrinsic role of roles as intermediaries between relationships and the objects that engage in them. There are three main views of role: (a) names for association ends, like in UML or in Entity-Relationship diagrams; (b) dynamic specialization, like in the Fibonacci [2] programming language; (c) adjunct instances, like in the DOOR programming language [44] or ObjectTeams [23]. The two last views are more relevant for modelling roles in programming languages.

We stick to the adjunct instance perspective, with an important difference with most previous work, with the partial exception of $[23,30,42]$ : the role instance is always associated with both the player of the role and the callee object which the role belongs to.

Most other works on roles in programming languages adopt a different perspective: roles are seen as a way to extend the behavior of an object and not as a way to model how an object offers different possibilities of interaction to different kind of players. Thus, there is a deep difference with our approach: we pass from a playercentered vision of roles to an organizational-centered one. The different perspective is also signalled by the terminology used. When other works use the phrase "the role of an object" they mean "the role played by a (caller) object" (since there is no explicit context offering that role). Instead, by "the role of an object" we mean the role a callee offers to play to a caller object in order to enable the caller to interact with the callee. In the player-centered approach, the printer example we propose could be modelled only by adding roles like user and superuser to instances of the class person without any systematic relation with the class printer, thus preventing the possibility that the role and the institution share their namespace. The reason of this difference is that these proposals, even when they share some similarities with our work, aim at solving different kind of practical problems than allowing callee objects to exhibit specific behaviors to specific callers.

In this respect our perspective is more similar to the use of role in security, e.g., in the role based access control (RBAC) model [38]. In RBAC roles are used to distinguish different set of authorizations to interact with the resources of a system, and sessions are mappings between a user and an activated subset of roles that are assigned to the user. However, in our model, methods of roles do not exist without the role offering them. For this reason attributes and operations are described by means of classes. In the RBAC model, instead, roles are only groupings of rights concerning operations defined directly in the system, and the operations have the 
same meaning for all roles. Since role classes provide a different instance for each caller which plays the role, they can represent the state of the interaction between the caller object playing a role and the callee which offers the role. Hence, there can be more than one session for each caller object playing different roles. This is again different from the RBAC model where sessions are associated with users and not with role instances.

Many works on the introduction of roles in programming languages [2, 16, 21, 37] consider roles as a specialization of classes, e.g., a customer is seen as a (dynamic) specialization of the class person. In our example, user and superuser would have been subclasses of person. This methodology, as Steimann [40] notices, does not capture the intuition that a role like customer can be played both by a person and by an organization. If customer were a subclass of person, it could not be at the same time a subclass of organization, since person and organization are disjoint classes. Symmetrically, person and organization cannot be subclass of customer, since a person can be a person without ever becoming a customer.

Multiple inheritance does not help, since it becomes impossible to have context dependent access, as Dahchour et al. [16] notice. Fibonacci [2], e.g., is a language which introduces a hierarchy of role types to specialize an object class. This language also supports a radical view of separation of concerns by imposing that the interaction with an object always passes through a role.

Roles as specializations prevent realizing that roles are always associated not only with a player, but to an institution, which defines them, too. This intuition sometimes implicitly emerges also in these frameworks: in [37] the authors say "a role is visible only within the scope of the specific application that created it", but such contexts are not first class citizens like institutions are in our model.

Kristensen and Osterbye [26] recognize the fact that a role depends on its player but they fail to recognize the dependency of a role from the institution. Moreover, they consider roles as a form of specialization, albeit one distinguishing the role as an instance related to, but separated from, its player. As a consequence, the properties of the role include the properties inherited from its player. This idea conflicts with our position we adopt from Steimann [40]: roles are partial descriptions of behavior, thus they shadow the other properties of their players.

Wong et al. [44] recognize that roles are adjunct instances. They introduce a parallel role class hierarchy connected by a player relationship to the object class hierarchy. Moreover, in their model a role player qualification specifies which classes can play the role. This corresponds to our idea of associating requirements to a role, which we model as interfaces. However, like many of the previous approaches, Wong et al. [44] fail to capture the intuition that a role depends on the context defining it. Another major difference with their approach is that we reject the method lookup as delegation. This methodology has a troublesome implication: when a method is invoked on some object in one of its roles, the meaning of the method can change depending on all the other roles played by the object. We do not consider this as a 
desirable feature in the context of a language like Java.

Our approach shares the idea of gathering roles inside wider entities with Object Teams [23] and Caesar [30]. These languages emerge as refinements of aspect oriented languages aiming at resolving practical limitations of aspect programming. In contrast, our language starts from different practical problems and introduce an ontologically founded definition of roles. Differently from $[23,30]$ in our work the method calls make explicit in which role the caller is invoking the method. Moreover, it is not only the meaning of methods which changes, but also the possible methods that can be invoked. If one would like to integrate the aspect paradigm within our view of object oriented programming, the natural place would be use aspects to model the environment where the interaction between objects happens. Consider the within construct in Object Teams/Java [23] which specifies aspects as the context in which a block of statements has to be executed. Since, when defining the interaction possibilities of an object it is not possible to foresee all possible contexts in which the interaction happens, the effect on the environment can be better modelled as a crosscutting concern. Thus, aspect programming is a complementary approach with respect to ours.

Other works which recognize the dependence of roles from a context are [42] and [27]. In Tamai [42], the concepts of context (roughly corresponding to our institution) and role are related in the language EpsilonJ. However, the approach that we propose better adheres to the Java programming style because it relies onto features that are already in this language (roles are implemented using inner classes and institutions are classes), basically adding just the concept of role interface and role casting. In contrast, in EpsilonJ roles and contexts are introduced as new constructs, and their relation with classes and objects is not explicit. Moreover, their proposal is not implemented in Java but in Ruby, limiting the applicability of their approach and not taking into account typing issues.

Lee and Bae [27] introduce the notion of role system and model it by means of a special class with the function of maintaining the coherence among roles and among roles and their players. However, they propose a pattern and not a language.

Baumer et al. [11] propose the role object pattern to solve the problem of providing context-specific views of the key abstractions of a system, since different context-specific views cannot be integrated in the same class. They propose to model context-specific views as role objects which are dynamically attached to a core object, thus forming what they call a subject. This adjunct instance should share the same interface as the core object, so the role cannot have methods different from those of their players.

Mossè [32] presents several patterns related to roles, but none of them considers the problem of roles belonging to institutions for offering access to them.

Molina et al. [31] revise the OOram methodology for modelling roles, translating it to UML. Even if also in this case roles do not belong to institutions, there are some similarities in that role diagrams specify the interaction among a set of roles. 
Even if the notion of role is not mentioned explicitly, in Aksit et al. [1] the notion of abstract communication types has some similarities with our approach, in that communication can be specifies separately from the core behavior of an object. However, they use a reflection based mechanism which makes the approach very flexible but difficult to control. Methods are associated with a set of conditionals acting as guards which prevent inappropriate methods to be called in certain states of the object. Our approach instead sticks to the philosophy of Java trying to overcome the underlying vision of object orientation.

Our notion of role, as a double-face interface, bears some similarities with Traits [39] and Mixins. However, Mixins are distinguished because they are used to extend classes and not instances, with a few exceptions like, e.g., [12]. In contrast, roles extend instances.

Similarities and differences can be found also in our ontological definition of roles with respect to other approaches. Our main aim is to stick to a widely acceptable definition in knowledge representation, so that we left out controversial issues like roles playing roles, which we discuss in [15]. For example, Masolo et al. [29] accept the notion of foundation, of definitional dependence, albeit a weaker one, where the definition of a role must use the concept of the institution. Loebe [28] also considers roles as dependent on contexts, but he does not stress the fact that roles can be seen as different ways of interacting with an object; Viganò and Colombetti [43] also consider powers as an essential feature of roles. Moreover, we are inspired from Guarino and Welty [22] in considering roles as antirigid and dynamic. Also Steimann [40] is an important source of inspiration, when he highlights that a role can be played by different kinds of actors. However, differently from Steimann in our model roles cannot be reduced only to interfaces and have an identity, in the sense that they can become the value of a variable, even if role instances are not independent as they are founded.

The six problems we discussed in the introduction have been already identified, even if separately, but they have been addressed only in partial or indirect ways.

Programming languages like Fickle [18] address the second and third problem by means of dynamic reclassification: an object can change class dynamically, and its operations change accordingly. However, Fickle does not represent the dependence of attributes and operations from the interaction, and all the subclasses share the same interface. StateJ [17] offers different implementations of methods according to a feature called the state of the object. However, an object has only one state and the state does not depend on the caller of a method and different callers or caller types cannot correspond to different states. Aspect programming focuses too on enhancing the modularity by means of crosscutting concerns, but it is less clear how it addresses all the six concerns.

Some patterns partially address the above mentioned issues; for example, the strategy design pattern [19] allows objects to dynamically change the implementation of a method. However, it is complex to implement and it does not address the 
problem of having different methods offered to different types of callers and of maintaining the state of the interaction between caller and callee. So more patterns must be combined with additional problems such as the lack of modularity and increased complexity.

Sessions are dealt with by distributed programming constructs, like the JSP, Servlets or Enterprise Java Beans, but these solutions do not consider the other problems of having different methods and states offered to different types of callers, beside the fact that sessions are not integrated in the method invocation syntax. Rather, interaction happens often only via a web service.

Sessions are also considered in the agent oriented paradigm, which bases communication on protocols [25]. In agent orientation a protocol is the specification of the possible sequences of messages exchanged between two agents, and not simply an interface. Since not all sequences of messages are legal, the state of the interaction between two agents must be maintained in a session. Moreover, not every agents can interact with other ones using whatever protocol. Rather, the interaction is allowed only between agents playing certain roles. Thus, an agent displays different possibilities of interaction to different types of interactants by playing different roles in the interaction with them, and it can even play different roles at the same time in the interaction with the same agent. However, the notion of role in multi-agent systems is rarely related with the notion of session of interaction ([36]).

\section{SUMMARY}

In this paper we identify some problems in the current view of object orientation, namely, the facts that callers of methods are not made explicit, attributes and operations associated with callee objects do not depend on the caller's identity or class, and that there is no session keeping track of the interaction between a callee and a caller object. To overcome these limitations, we introduce the view on roles adopted at higher level in access control in web services and we transfer it at lower level in object oriented programming languages. We base on an ontological analysis of the notion of role to understand which are its properties.

We introduce this model of roles in an extension of Java, called powerJava. First, roles are implemented by classes which can be instantiated only in presence of an instance of the player of the role (caller) and of an instance (callee) of the class representing the institution (foundation). The role instance represents the session of the interaction between caller and callee. Second, the implementation of a role is included in the class definition of the institution the role belongs to using inner classes (definitional dependence). Thirdly, the players of roles have powers since methods of roles can access private fields and methods of the institution they belong to and of the other roles of the same institution (institutional empowerment). Finally, to express the fact that an object can be seen in one of the roles it plays we introduce the notion of role casting. 
As discussed in Section 4, powerJava is more than just the transfer of a pattern in a language. Additional benefits come from the introduction of roles directly in the language, like the management of role instance invariants, the introduction of suitable exceptions, or the fact that roles can be type checked, partly by delegating this task to the Java translation.

Details about the implementation can be found in [4] and the pre-compiler is available at http://www. power java.org.

Roles allow the programmer to adopt different interaction possibilities between callers and a callee, which do not exclude the traditional direct interaction with the object when roles are not necessary. Other possibilities like sessions shared by different objects are not considered for space reasons.

First of all, an object can interact with another one by means of the role offered by it. This is, for instance, the case of chris being a User of laser in Section 3.

Second, a caller object (e.g., sergio in our example) can interact in two different roles with a callee object. This situation is used when a callee object implements two different interfaces for interacting with it, which have methods (like print) with the same signature but with different meaning. In our model the methods of the interfaces are implemented in the roles offered by the objects to interact with them. The role represents also the different sessions of the interaction with the same object.

Third, two caller objects can interact with each other by means of the (possibly different) roles of an institution. This is the original case powerJava has been developed for [9]: achieving separation of concerns, taking apart the core behavior of a class from the dynamically acquired behavior in an unforeseen context; in that paper, we used as a running example the well-known five philosophers scenario. The institution is the table, at which philosophers are sitting and coordinate to take the chopsticks and eat since they can access the state of each other. $\mathrm{s}$

Fourth, two objects can interact with each other, each playing a role offered by the other. This is often the case of interaction protocols: e.g., an object can play the role of initiator in the Contract Net Protocol if and only if the other object plays the role of participant.

Our view of roles inspires a new vision of the object oriented paradigm, whose object metaphor has been accepted too acritically and it has not been subject to a deep analysis. In particular, it adopts a naive view of the notion of object and it does not consider the analysis of the way humans conceptualize objects performed in philosophy and above all in cognitive science [20]. In particular, cognitive science has highlighted that properties of objects are not objective properties of the world, but they depend on the properties of the agent conceptualizing the object: objects are conceptualized on the basis of what they "afford" to the actions of the entities interacting with them. Thus, different entities conceptualize the same object in different ways. We translate this intuition in the fact that an object offers different methods according to which type of object it is calling it: the methods offered (the powers of a role) depend on the requirements offered by the caller. This perspective 
is analysed in $[6,7]$.

powerJava has also been used as a coordination language dealing with concurrency [9]. We discuss how to use powerJava to implement protocols in multi-agent systems in [5].

Future work concerns, first of all, the relational nature of roles which often come into pairs, like client/customer in a business exchange, manager/bidder in a negotiation protocol, etc. In [10] we use roles to model how objects can participate to relationships and, thus, acquire new properties and behaviors. We add roles to the existing relationship as attribute and relationship object patterns proposed by Noble and Grundy [33]. Second, some issues concerning the translation of powerJava in Java must be deepened. In particular, type checking should be clarified, and introduced also during the pre-compilation phase instead of relying only on the Java compiler, so that more explicit errors can be signalled to the user. Finally, the lifecycle of roles should be studied (see, e.g., Odell et al. [34]), and a corresponding systems of exceptions developed to signal which are the possible errors related to roles at runtime.

\section{ACKNOWLEDGEMENTS}

Matteo Baldoni has partially been funded by the 6th Framework Programme project REWERSE number 506779, and by MIUR PRIN 2005 "Specification and verification of agent interaction protocols" national project. Guido Boella has partially been founded by the MIUR PRIN 2006 project "Documentalità. Ontologie e tecnologie per la cittadinanza e la democrazia".

The authors thank the anonymous reviewers for their useful suggestions.

\section{REFERENCES}

[1] M. Aksit, K. Wakita, J. Bosch, L. Bergmans, and A. Yonezawa. Abstracting object interactions using composition filters. In Procs. of ECOOP' 93 Workshop on Object-Based Distributed Programming, volume 791 of LNCS, pages 152-184, Berlin, 1994. Springer Verlag.

[2] A. Albano, R. Bergamini, G. Ghelli, and R. Orsini. An object data model with roles. In Procs. of Very Large DataBases (VLDB'93), pages 39-51, 1993.

[3] F. Arbab. Abstract behavior types: A foundation model for components and their composition. In Formal Methods for Components and Objects, volume 2852 of LNCS, pages 33-70. Springer, Berlin, 2003.

[4] M. Baldoni, G. Boella, and L. van der Torre. Social roles, from agents back to objects. In Procs. of From Objects to Agents Workshop (WOA'05), Bologna, 2005. Pitagora. 
[5] M. Baldoni, G. Boella, and L. van der Torre. Bridging agent theory and object orientation: Interaction among objects. In Procs. of PROMAS'06 workshop at AAMAS'06, 2006.

[6] M. Baldoni, G. Boella, and L. van der Torre. Interaction among objects via roles: sessions and affordances in powerJava. In Procs. of Principles and Practice of Programming in Java (PPPJ'06), pages 188-193, New York (NY), 2006. ACM.

[7] M. Baldoni, G. Boella, and L. van der Torre. Modelling the interaction between objects: Roles as affordances. In Procs. of Knowledge Science, Engineering and Management, KSEM'06, volume 4092 of LNCS, pages 42-54. Springer, 2006.

[8] M. Baldoni, G. Boella, and L. van der Torre. powerJava: ontologically founded roles in object oriented programming language. In Procs. of ACM Symposium on Applied Computing (SAC'06), Track Object Oriented Programming Languages and Systems (OOPS'06), pages 1414-1418. ACM, 2006.

[9] M. Baldoni, G. Boella, and L. van der Torre. Roles as a coordination construct: Introducing powerJava. Electronic Notes in Theoretical Computer Science, 150(1):9-29, 2006.

[10] M. Baldoni, G. Boella, and L. van der Torre. Relationships meet their roles in object oriented programming. In Procs. of FSEN'0'7, 2007.

[11] D. Baumer, D. Riehle, W. Siberski, and M. Wulf. The role object pattern. In Procs. of PLOP'02, 2002.

[12] L. Bettini, V. Bono, and S. Likavec. A core calculus of mixin-based incomplete objects. In Procs. of Foundations and Developments of Object Oriented Languages Workshop (FOOL'04), pages 29-41, 2004.

[13] G. Boella and L. van der Torre. An agent oriented ontology of social reality. In Procs. of Formal Ontologies in Information Systems (FOIS'04), pages 199-209, Amsterdam, 2004. IOS Press.

[14] G. Boella and L. van der Torre. A foundational ontology of organizations and roles. In Procs. of DALT'06 workshop at AAMAS'06, 2006.

[15] G. Boella and L. van der Torre. The ontological properties of social roles in multi-agent systems: Definitional dependence, powers and roles playing roles. Artificial Intelligence and Law, 2007.

[16] M. Dahchour, A. Pirotte, and E. Zimanyi. A generic role model for dynamic objects. In Procs. of Conference on Advanced Information Systems Engineering (CAiSE'02), volume 2348 of LNCS, pages 643-658. Springer, 2002. 
[17] F. Damiani, E. Giachino, P. Giannini, and E. Cazzola. On state classes and their dynamic semantics. In Procs. of International Conference on Software and Data Technologies (ICSOFT'06), pages 5-12. INSTICC, 2006.

[18] S. Drossopoulou, F. Damiani, M. Dezani-Ciancaglini, and P. Giannini. More dynamic object re-classification: Fickle $I I$. ACM Transactions On Programming Languages and Systems, 24(2):153-191, 2002.

[19] E. Gamma, R. Helm, R. Johnson, and J. Vlissides. Design Patterns: Elements of Reusable Software. Addison-Wesley, 1995.

[20] J. Gibson. The Ecological Approach to Visual Perception. Lawrence Erlabum Associates, New Jersey, 1979.

[21] G. Gottlob, M. Schrefl, and B. Rock. Extending object-oriented systems with roles. ACM Transactions on Information Systems, 14(3):268 - 296, 1996.

[22] N. Guarino and C. Welty. Evaluating ontological decisions with Ontoclean. Communications of ACM, 45(2):61-65, 2002.

[23] S. Herrmann. Object teams: Improving modularity for crosscutting collaborations. In Procs. of Net.ObjectDays, 2002.

[24] A. Igarashi and H. Nagira. Union types for object-oriented programming. In Procs. of ACM symposium on Applied Computing (SAC '06), pages 1435-1441, New York, NY, USA, 2006. ACM.

[25] T. Juan, A.R. Pearce, and L. Sterling. ROADMAP: extending the GAIA methodology for complex open system. In Procs. of Autonomous Agents and Multiagent Systems Conference (AAMAS'04), pages 3-10, 2002.

[26] B.B Kristensen and K. Osterbye. Roles: conceptual abstraction theory and practical language issues. Theory and Practice of Object Systems, 2(3):143160, 1996.

[27] Joon-Sang Lee and Doo-Hwan Bae. An enhanced role model for alleviating the role-binding anomaly. Software: Practice and Experience, 32(14):1317 - 1344, 2002.

[28] F. Loebe. Abstract vs. social roles - a refined top-level ontological analysis. In Procs. of AAAI Fall Symposium Roles'05, pages 93-100. AAAI Press, 2005.

[29] C. Masolo, L. Vieu, E. Bottazzi, C. Catenacci, R. Ferrario, A. Gangemi, and N. Guarino. Social roles and their descriptions. In Procs. of Conference on the Principles of Knowledge Representation and Reasoning (KR'04), pages 267277. AAAI Press, 2004.

[30] M. Mezini and K. Ostermann. Conquering aspects with Caesar. In Procs. of International Conference on Aspect-Oriented Software Development (AOSD), pages 90-100. ACM Press, 2004. 
[31] J.G. Molina, M.J. Ortin, B. Moros, and J. Nicolas. Transforming the OOram three-model architecture into a UML-based process. Journal of Object Technology, 1(4):119-136, 2002.

[32] F.G. Mosse. Modeling roles: A practical series of analysis patterns. Journal of Object Technology, 1(4):27-37, 2002.

[33] J. Noble and J. Grundy. Explicit relationships in object-oriented development. In Procs. of Technology of Object-Oriented Languages and Systems (TOOLS), 1995.

[34] J. Odell, H. Van Dyke Parunak, S. Brueckner, and J. Sauter. Changing roles: Dynamic role assignment. Journal of Object Technology, 2(5):77-86, 2003.

[35] M. Odersky. The Scala Experiment - can we provide better language support for component systems? In Procs. of ACM Symposium on Principles of Programming Languages, pages 166-167, 2006.

[36] A. Omicini, A. Ricci, and M. Viroli. An algebraic approach for modelling organisation, roles and contexts in MAS. Applicable Algebra in Engineering, Communication and Computing, 16(2-3):151-178, 2005.

[37] M.P. Papazoglou and B.J. Kramer. A database model for object dynamics. The Very Large DataBases Journal, 6(2):73-96, 1997.

[38] R. Sandhu, E. Coyne, H. Feinstein, and C. Youman. Role-based access control models. IEEE Computer, 2:38-47, 1996.

[39] N. Scharli, S. Ducasse, O. Nierstrasz, and A. Black. Traits: Composable units of behavior. In Procs. of European Conference on Object Oriented Programming (ECOOP'03), volume 2743 of LNCS, pages 248-274, Berlin, 2003. Springer.

[40] F. Steimann. On the representation of roles in object-oriented and conceptual modelling. Data and Knowledge Engineering, 35:83-848, 2000.

[41] F. Steimann and P. Mayer. Patterns of interface-based programming. Journal of Object Technology, 4(5):75-94, 2005.

[42] T. Tamai. Evolvable programming based on collaboration-field and role model. In Procs. of International Workshop on Principles of Software Evolution (IWPSE'02), pages 1-5. ACM, 2002.

[43] F. Viganò and M. Colombetti. Specification and verification of institutions through status functions. In COIN@AAMAS'06 Workshop, 2006.

[44] R.K. Wong, H.L. Chau, and F.H. Lochovsky. A data model and semantics of objects with dynamic roles. In Procs. of IEEE Data Engineering Conference, pages 402-411, 1997. 


\section{ABOUT THE AUTHORS}
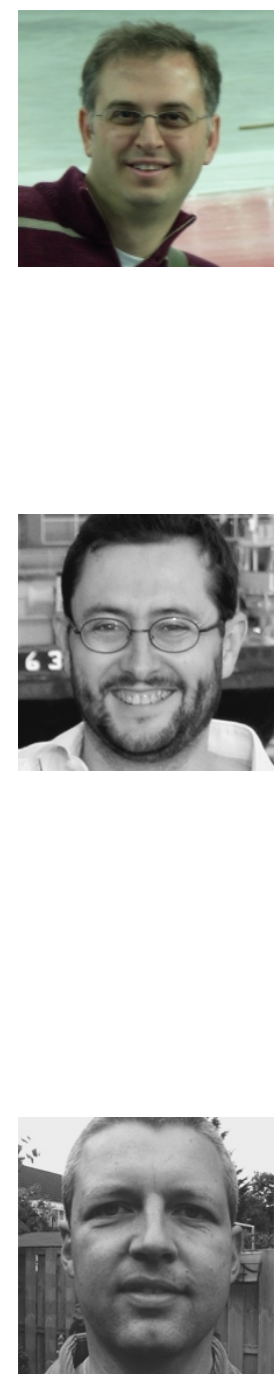

Matteo Baldoni received his Ph.D. in Computer Science in May 1998 from the University of Torino. He is currently associate professor at the Department of Computer Science of the University of Torino, Italy. He has a background in computational logic, modal and nonmonotonic extensions of logic programming, multimodal logics, reasoning by actions and change. His current research interests include issues in communication protocol design and implementation, conformance and interoperabilty for agents and web services, agent programming languages, personalization by reasoning in the semantic web. He organized the last three editions of the Declarative Agent Languages and Technologies International Workshop. He can be reached at baldoni [ at ] di.unito.it. See also http://www.di.unito.it/ ${ }^{\text {baldoni. }}$

Guido Boella received the PhD degree at the University of Torino in 2000. He is currently professor at the Department of Computer Science of the University of Torino. His research interests include multi-agent systems, in particular, normative systems, institutions and roles using qualitative decision theory. He organized the first two workshops on normative multi-agent systems, on coordination and organization, and the first AAAI Fall Symposium on roles. guido [ at ] di.unito.it. See also http://www.di.unito.it/ guido.

Leendert W. N. van der Torre received the $\mathrm{PhD}$ degree from Erasmus University Rotterdam in 1997. He is currently full professor at the University of Luxembourg. He has developed the so-called input/output logics and the BOID agent architecture. He has written over one hundred scientific papers, and he has organized the first two workshops on normative multi-agent systems and the firsts two workshop on coordination and organization. His current research interests include deontic logic, qualitative game theory and coordination in normative multi-agent systems. leon.vandertorre [ at ] uni.lu. See also http://agamemnon.uni.lu/ILIAS/vandertorre. 
International Journal of Cooperative Information Systems

(C) World Scientific Publishing Company

\title{
NORM NEGOTIATION IN MULTIAGENT SYSTEMS
}

\author{
GUIDO BOELLA \\ Dipartimento di Informatica - Università di Torino - Italy.E-mail: guido@di.unito.it \\ LEENDERT VAN DER TORRE \\ University of Luxembourg. E-mail: leendert@vandertorre.com
}

Received (Day Month Year)

Revised (Day Month Year)

\begin{abstract}
Normative multiagent systems provide agents with abilities to autonomously devise societies and organizations coordinating their behavior via social norms and laws. In this paper we study how agents negotiate new social norms and when they accept them. We introduce a negotiation model based on what we call the social delegation cycle, which explains the negotiation of new social norms from agent desires in three steps. First individual agents or their representatives negotiate social goals, then a social goal is negotiated in a social norm, and finally the social norm is accepted by the agents when it leads to fulfilment of the desires the cycle started with. We characterize the allowed proposals during social goal negotiation as mergers of the individual agent desires, and we characterize the allowed proposals during norm negotiation as both joint plans to achieve the social goal (obligations associated with the norm) and the associated sanctions or rewards (a control system associated with the norm). The norm is accepted when the norm is stable in the sense that agents will act according to the norm, and effective in the sense that fulfilment of the norm leads to achievement of the agents' desires. We also compare norm negotiation with contract negotiation and negotiation of the distribution of obligations.
\end{abstract}

\section{Introduction}

Social norms and laws can be used to guide the emergent behavior of multiagent systems. Moreover, these norms guiding the emergent behavior of the system can also emerge themselves, leading to a challenge for models of normative multiagent systems. To deal with the apparent recursion in the definition of the emergence of norms, we need a model making it precise when and how norms can emerge. Since agents may have conflicting goals with respect to the norms that emerge, they try to negotiate amongst each other which norm will emerge. Moreover, we may say that a norm has emerged when it has been accepted by the agents, as has been studied, for example, by Conte et al. ${ }^{14}$

The negotiation and acceptance of norms has to obey various principles. For example, to accept a norm, an agent has to recognize it as a norm, the norm must contribute to the goals or desires of the agent, and it must be obeyed by the other agents. Consequently, the challenge for a model of norm negotiation is how to explain what it means, for example, to recognize or to obey a norm. Moreover, there are additional challenges, for example how 
new norms interact with existing ones.

In this paper we study norm emergence and acceptance using a model of norm negotiation. It is based on a distinction between social goal negotiation and the negotiation of the obligations with their control system. Roughly, the social goals are the potential benefits of the new norm for the agents, and the obligations are the potential costs of the new norm for the agents in the sense that agents risk being sanctioned if they do not respect the norm. Moreover, in particular when representatives of the agents negotiate the social goals and norms, the agents still have to accept the negotiated norms. ${ }^{14}$ The norm is accepted when the norm is stable in the sense that agents will act according to the norm, and effective in the sense that fulfilment of the norm leads to achievement of the agents' desires - i.e., when the benefits outweigh the costs. Summarizing, norm negotiation can be described by the so-called social delegation cycle visualized in Figure 1.

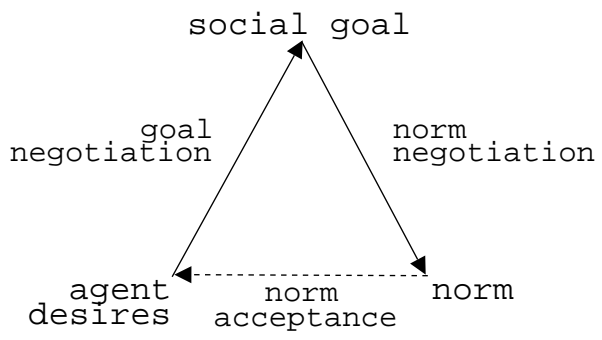

Fig. 1. $\Delta$ : the social delegation cycle.

The conceptual model we use to study and formalize the social delegation cycle is based on a formal characterization of normative multiagent systems we have developed in several other papers. ${ }^{7,8}$ This characterization combines a game-theoretic approach with cognitive agents, rule based systems and input/output logics. The advantage given by this formal system is that it combines a qualitative theory of agent decision-making with a detailed ontology of normative systems. The decision making is used to explain how agents negotiate goals and norms, and when they accept norms.

Within the model of normative multiagent systems, we have to choose a protocol for negotiation. Since we are not interested in the intricacies of negotiation itself, but in the relation among goal negotiation, norm negotiation and acceptance, we choose a relatively simple abstract turn-taking model which we can use for both negotiation steps. The central issue in such an abstract negotiation model is the definition of an allowed proposal for a social goal or for a norm, for which we define additional conditions.

The layout of this paper is as follows. In Section 2 we give an introduction to normative multiagent systems, and in Section 3 we discuss an abstract model of the social delegation cycle. In Section 4 we define the conceptual model in which we study and formalize the social delegation cycle, and in Section 5 we define our version of the negotiation protocol and the logic of rules. In Section 6 we formalize goal negotiation, in Section 7 we formalize norm negotiation, and in Section 8 we formalize the acceptance relation. 


\section{Normative multiagent systems}

Jones and $\mathrm{Carmo}^{21}$ define a normative system as "Sets of agents whose interactions are norm-governed; the norms prescribe how the agents ideally should and should not behave. [...] Importantly, the norms allow for the possibility that actual behavior may at times deviate from the id eal, i.e., that violations of obligations, or of agents' rights, may occur." Since the agents' control over the norms is not explicit here, we use the following definition.

A normative multiagent system is a multiagent system together with normative systems in which agents can decide whether to follow the explicitly represented norms, and the normative systems specify how and in which extent the agents can modify the norms. ${ }^{9}$

Note that this definition makes no presumptions about the internal architecture of an agent or of the way norms find their expression in agent's behavior.

Since norms are explicitly represented, according to our definition of a normative multiagent system, the question should be raised how norms are represented. Norms can be interpreted as a special kind of constraint, and represented depending on the domain in which they occur. However, the representation of norms by domain dependent constraints runs into the question what happens when norms are violated. Not all agents behave according to the norm, and the system has to deal with it. In other words, norms are not hard constraints, but soft constraints. For example, the system may sanction violations or reward good behavior. Thus, the normative system has to monitor the behavior of agents and enforce the sanctions. Also, when norms are represented as domain dependent constraints, the question will be raised how to represent permissive norms, and how they relate to obligations. Whereas obligations and prohibitions can be represented as constraints, this does not seem to hold for permissions. For example, how to represent the permission to access a resource under an access control system? Finally, when norms are represented as domain dependent constraints, the question can be raised how norms evolve.

We therefore believe that norms should be represented as a domain independent theory. For example, deontic logic ${ }^{40,38,37,25,26,27}$ studies logical relations among obligations and permissions, and more in particular violations and contrary-to-duty obligations, permissions and their relation to obligations, and the dynamics of obligations over time. Therefore, insights from deontic logic can be used to represent and reason with norms. Deontic logic also offers representations of norms as rules or conditionals. However, there are several aspects of norms which are not covered by constraints nor by deontic logic, such as the relation between the cognitive abilities of agents and the global properties of norms.

Conte et al. ${ }^{14}$ say that normative multiagent systems research focuses on two different sets of problems. On the one hand, they claim that legal theory and deontic logic supply a theory for of norm-governed interaction of autonomous agents while at the same time lacking a model that integrates the different social and normative concepts of this theory. On the other hand, they claim that three other problems are of interest in multiagent systems research on norms: how agents can acquire norms, how agents can violate norms, and how an agent can be autonomous. For artificial agents, norms can be designed as in legal human 
systems, forced upon, for example when joining an institution, or they can emerge from the agents making them norm autonomous. ${ }^{39}$ Agent decision making in normative systems and the relation between desires and obligations has been studied in agent architectures, ${ }^{10}$ which thus explain how norms and obligations influence agent behavior.

An important question is where norms come from. Norms are not necessarily created by a single legislator, they can also emerge spontaneously, or be negotiated among the agents. In electronic commerce research, for example, cognitive foundations of social norms and contracts are studied. ${ }^{7}$ Protocols and social mechanisms are now being developed to support such creations of norms in multiagent systems. When norms are created, the question can be raised how they are enforced. For example, when a contract is violated, the violator may have to pay a penalty. But then there has to be a monitoring and sanctioning system, for example police agents in an electronic institution. Such protocols or roles in a multiagent system are part of the construction of social reality, and Searle ${ }^{32}$ has argued that such social realities are constructed by constitutive norms. This again raises the question how to represent such constitutive or counts-as norms, and how they are related to regulative norms like obligations and permissions. ${ }^{8}$

Not only the relation between norms and agents must be studied, but also the relation between norms and other social and legal concepts. How do norms structure organizations? How do norms coordinate groups and societies? How about the contract frames in which contracts live? How about the relation between legal courts? Though in some normative multiagent systems there is only a single normative system, there can also be several of them, raising the question how normative systems interact. For example, in a virtual community of resource providers each provider may have its own normative system, which raises the question how one system can authorize access in another system, or how global policies can be defined to regulate these local policies. ${ }^{8}$

Summarizing, normative multiagent systems study general and domain independent properties of norms. It builds on results obtained in deontic logic, the logic of obligations and permissions, for the representation of norms as rules, the application of such rules, contrary-to-duty reasoning and the relation to permissions. However, it goes beyond logical relations among obligations and permissions by explaining the relation among social norms and obligations, relating regulative norms to constitutive norms, explaining the evolution of normative systems, and much more.

Within multiagent systems, norms and social laws are used to design social mechanisms, and in normative systems, agent theory is used to analyze and simulate social phenomena. Various examples can be found in a forthcoming double special issue of Computational and Mathematical Organizational Theory on normative multiagent systems. ${ }^{9}$ The models most closely related to the one presented in this paper can be found in work on artificial social systems by Tennenholtz and colleagues, and the work on social theory by Castelfranchi, Conte and colleagues, which we discuss in the following section. The detailed model of normative systems including a sanction-based control system together with the game-theoretic aspects like acceptance and backward induction distinguishes our approach from numerous other approaches of social norms and laws in multiagent systems. ${ }^{16,17,18,19,24,33}$ 


\section{Social delegation cycle}

To motivate our model, we start with an abstract description of the social delegation cycle using artificial social systems. The problem studied in artificial social systems is the design, emergence or more generally the creation of social laws. Shoham and Tennenholtz ${ }^{33}$ introduce social laws in a setting without utilities, and they define rational social laws as social laws that improve a social game variable ${ }^{34}$ We follow Tennenholtz' presentation of artificial social systems for stable social laws. ${ }^{36}$

The desires of the agents are represented by their utilities. A game or multi-agent encounter is a set of agents with for each agent a set of strategies and a utility function defined on each possible combination of strategies. Tennenholtz only defines games for two agents to keep the presentation of artificial social systems as simple as possible, but he observes also that the extension to the multi-agent case is straightforward. ${ }^{36}$

Definition 3.1. A game (or a multi-agent encounter) is a tuple $\left\langle N, S, T, U_{1}, U_{2}\right\rangle$, where $N=\{1,2\}$ is a set of agents, $S$ and $T$ are the sets of strategies available to agents 1 and 2 respectively, and $U_{1}: S \times T \rightarrow \mathbb{R}$ and $U_{2}: S \times T \rightarrow \mathbb{R}$ are real-valued utility functions for agents 1 and 2 , respectively.

The social goal is represented by a minimal value for the social game variable. Tennenholtz ${ }^{36}$ uses as game variable the maximin value. This represents safety level decisions, in the sense that the agent optimizes its worst outcome assuming the other agents may follow any of their possible behaviors. See Tennenholtz' paper for a discussion why this is natural in many multi-agent systems, where a payoff corresponds to the achievement of a particular user's specification.

Definition 3.2. Let $S$ and $T$ be the sets of strategies available agent 1 and 2, respectively, and let $U_{i}$ be the utility function of agent $i$. Define $U_{1}(s, T)=\min _{t \in T} U_{1}(s, t)$ for $s \in S$, and $U_{2}(S, t)=\min _{s \in S} U_{2}(s, t)$ for $t \in T$. The maximin value for agent 1 (respectively 2 ) is defined by $\max _{s \in S} U_{1}(s, T)$ (respectively $\max _{t \in T} U_{2}(S, t)$ ). A strategy of agent $i$ leading to the corresponding maximin value is called a maximin strategy for agent $i$.

The social norm is represented by a social law, which has been characterized as a restriction of the strategies available to the agents. It is useful with respect to an efficiency parameter $e$ if each agent can choose a strategy that guarantees it a payoff of at least $e$.

Definition 3.3. Given a game $g=\left\langle N, S, T, U_{1}, U_{2}\right\rangle$ and an efficiency parameter $e$, we define a social law to be a restriction of $S$ to $\bar{S} \subseteq S$, and of $\bar{T} \subseteq T$. The social law is useful if the following holds: there exists $s \in \bar{S}$ such that $U_{1}(s, \bar{T}) \geq e$, and there exists $t \in \bar{T}$ such that $U_{2}(\bar{S}, t) \geq e$. A (useful) convention is a (useful) social law where $|\bar{S}|=|\bar{T}|=1$.

A social law is quasi-stable if an agent does not profit from violating the law, as long as the other agent conforms to the social law (i.e., selects strategies allowed by the law).

Definition 3.4. Given a game $g=\left\langle N, S, T, U_{1}, U_{2}\right\rangle$, and an efficiency parameter $e$, a quasi-stable social law is a useful social law (with respect to $e$ ) which restricts $S$ 
to $\bar{S}$ and $T$ to $\bar{T}$, and satisfies the following: there is no $s^{\prime} \in S-\bar{S}$ which satisfies $U_{1}\left(s^{\prime}, \bar{T}\right)>\max _{s \in \bar{S}} U_{1}(s, \bar{T})$, and there is no $t^{\prime} \in T-\bar{T}$ which satisfies $U_{2}\left(\bar{S}, t^{\prime}\right)>\max _{t \in \bar{T}} U_{2}(\bar{S}, t)$.

The three steps of the social delegation cycle in this classical game-theoretic setting can be identified as follows.

Goal negotiation implies that the efficiency parameter is higher than the utility the agents expect without the norm, for example represented by the Nash equilibria of the game.

Norm negotiation implies that the social law is useful (with respect to the efficiency parameter).

Acceptance relation implies that the social law is quasi-stable.

An important class of examples from game theory are coordination problems, like the choice between driving on either the right hand or the left hand side of the road. Such problems are characterized by the fact that it does not matter which option we choose, as long as everyone chooses the same option. Another classic example from game theory is the prisoner's dilemma, which is characterized by the fact that optimal situations can be reached only when we introduce sanctions on behaviors (such as defecting). Both of these examples can be modeled in artificial social systems. Without norms the agents expect accidents and defects, and therefore in goal negotiation the efficiency parameter is set such that there are no accidents, and no defections. The norm negotiation implies that the agent should all drive on the right hand side of the street and they should not defect (otherwise they are sanctioned), and finally the agents accept the norm, because they know that another agent will obey it (if all other agents do so too).

However, there are also drawbacks of this game-theoretic model of the social delegation cycle. Due to the uniform description of agents in the game-theoretic model, it is less clear how to distinguish among kinds of agents. For example, the unique utility aspiration level does not distinguish the powers of agents to negotiate a better deal for themselves than for the other agents. Moreover, the formalization of the social delegation cycle does neither give a clue how the efficiency parameter is negotiated, nor how the social law is negotiated. For example, the desires of the agents as well as other mental attitudes may play a role in this negotiation. Finally, an additional drawback is that the three ingredients of the model (agent desires, social goals, and social laws) are formalized in three different ways. These drawbacks also seem to hold when a normative system to encode enforceable social laws is added to the artificial social system model. ${ }^{6}$

To make a more realistic representation of the above notions, some models use a more detailed cognitive agent theory, in which agents are motivated by desires and goals, and norms are explicitly represented inside agents. For example, Conte et al. ${ }^{14}$ discuss under which conditions new norms can be acquired by cognitive agents by recognizing them and accepting that they become goals. Conte et al.'s first condition for norm recognition concerns the evaluation of the source. They say that "if the norm is not based upon a recognized norm, the entity $y$ that has issued the norm is evaluated. If $y$ is perceived to 
be entitled to issue norms (it is a normative authority), then [the obligation issued by $y$ and addressed to the set of agents $X$ to obtain $q$, written as] $O_{y X}(q)$, can be accepted as a norm." Their second relevant issue in the recognition of a norm is the evaluation of the motives for which the norm has been issued. " $O_{y X}(q)$ is issued for $y$ 's personal/private interest, rather than for the interest $y$ is held to protect: if $x$ believes that $y$ 's prescription is only due to some private desire, etc., $x$ will not take it as a norm. $x$ might ignore what the norm is for, what its utility is for the group or its institutions, but may expect that the norm is aimed at having a positive influence for the group; at least, it is necessary that $x$ does not have the opposite belief, that is, that the norm is not aimed to be 'good for' the group at large, but only for $y . \ldots y$ is entitled only to deliver prescriptions and permissions that are aimed at the general rather than at its own private interest."

Conte et al. claim that for the acceptance of norms as goals to be considered in the decision process requires, instead, the following stronger condition.

"A norm-autonomous agent accepts a norm $q$, only if it sees accepting $q$ as a way of achieving (one of) its own further goal(s)."

This condition is formalized as follows, where $O B T_{X}(q)$ means that the agents $X$ obtain $q$. It "states that $x$ forms a normative goal $O B T_{X}(q)$ (i.e. accepts the norm q) if $x$ believes that the norm exists (for agents in set $X$ ) and that fulfilling the norm (i.e. $\left.O B T_{X}(q)\right)$ is instrumental to one of its own goals."

$$
\begin{array}{r}
B E L_{x}\left(O_{y X}(q) \wedge \operatorname{INSTR}\left(O B T_{X}(q), p\right) \wedge G O A L_{x}(p \mid r)\right) \supset \\
N-\operatorname{GOAL}_{x}\left(O B T_{X}(q) \mid G O A L_{x}(p \mid r) \wedge r\right)
\end{array}
$$

Conte et al. emphasize that they "do not require $q$ to be instrumental for the goal $p$, but rather $O B T_{X}(q)$. With $O B T_{X}(q)$ in this context we mean the fulfilment of the norm $q$ by all members of $X$. The difference is that in this case we only try to fulfil the norm, because it is a norm. We could also have the much stronger case in which we believe that the norm itself is to the benefit of our goal $p$. This is somehow 'internalising' the norm and making it our own goal. This would formally be described by:

$$
B E L_{x}\left(O_{y X}(q) \wedge \operatorname{INSTR}(q, p) \wedge G O A L_{x}(p \mid r)\right) \supset C-G O A L_{x}\left(q \mid G O A L_{x}(p \mid r) \wedge r\right) \quad "
$$

Conte et al. observe that the achievement of the norm can be instrumental to some goal of the agent in different ways:

(1) Arbitrated instrumentality: " $x$ sees accepting $q$ as a means to obtain a non-natural consequent reward. In particular, avoidance of punishment, social praise, etc.," where they distinguish among avoidance of external or internal punishment, and achievement of external or internal reward.

(2) Natural instrumentality, where they distinguish self-interested from value-oriented.

Finally, Conte et al. observe that a norm must have general recognition. "Without a general recognition, a social norm is not a norm. (At the legal level it is sufficient that the authority is recognised as authority and that the norm is recognised as correctly issued by 
it). Autonomous agents subject to norms are in fact autonomous norm creators. They create norms through their evaluation and recognition, through their compliance, and through their interpersonal issuing, monitoring and judging."

From this cognitive setting we learn the following for the social delegation cycle:

Goal negotiation: To be a norm, a norm must not be issued in the interest of the authority only. Thus for an agent to recognize a norm as such, the norm must be aimed to be good for the group at large.

Norm negotiation: The creation of a norm must lead to a norm which achieves some agent own's goals: for example, the goal not to be considered as a violator and sanctioned, or to be rewarded.

Acceptance relation: To be a norm the norm must be recognized, accepted and complied with in general. Thus, the norm is created also by means of the agents' behavior.

Given the cognitive perspective, Conte et al.'s model allows a deeper understanding of the acquisition of norms. It leaves, however, several issues informal. In particular, what does it mean that a norm is "good for" the group at large"? What does it means that it is recognized in general? In general, how can a game-theoretic analysis be added to a cognitive one to understand how norms are created?

In this paper we answer these questions in a cognitive agent framework. In our model agent desires, social goals and social laws are all represented by qualitative rules, formalized using input/output logics. ${ }^{25}$ To formalize and characterize the negotiation of social goals and of obligations together with their sanctions we have to formalize the negotiation protocol including the allowed proposals: proposals which do not meet general interests cannot be made. Our model builds on several existing formal theories to define the acceptable proposals:

Goal negotiation is based on proposals where the social goal is obtained using merging operators, which have been proposed as generalizations of belief revision operators inspired by social choice theory.

Norm negotiation is based on proposals that create for each social goal a set of obligations (or revisions of existing obligations) together with sanctions or rewards.

Acceptance relation accepts or rejects a norm based on the stability criterion of artificial social systems and the general acceptance of cognitive theories, together with a cost-benefit analysis of the agents that the costs of having to comply to the norm are outweighed by the benefit of the social goal of the norm.

As a running example, we consider three sets of agents, who can work together in various ways. They can make a coalition to each perform a task, or they can distribute five tasks among them and obtain an even more desirable social goal. There can be free riders, so they need sanctions to ensure that the norm is accepted.

We define in the following sections the conceptual framework based on rule based systems, the generic negotiation protocol used both for social goal negotiation and norm negotiation, and the logic of rules based on input/output logics. 


\section{Conceptual model of normative multiagent systems}

The conceptual model used in this paper is visualized in Figure 2, in which we distinguish the multiagent system (straight lines) and additions for the normative system (dotted lines). Following the usual conventions of for example class diagrams in the unified modelling language (UML), $\square$ is a concept or set, - and $\rightarrow$ are relations or associations among concepts, and $\longrightarrow$ is the "is-a" or subset relation. The logical structure of the associations is detailed in the definitions below.

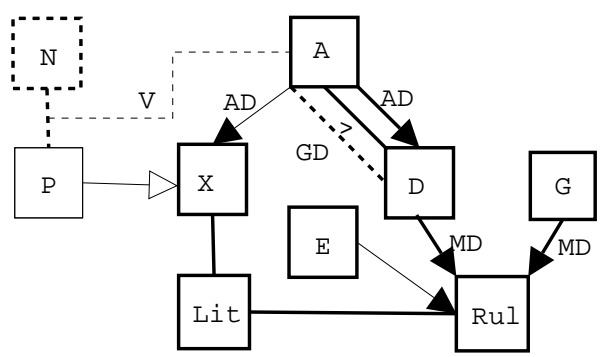

Fig. 2. Conceptual model of normative multiagent system.

The model consists of a set of agents $(A)$ described $(A D)$ by a set of boolean variables $(X)$, including decision variables it can perform, and desires $(D)$ guiding its decision making. Desire rules can be conflicting, and the way the agent resolves its conflicts is described by a priority relation $(\geq)$ that expresses its agent characteristics ${ }^{10}$. The priority relation is defined on the powerset of the motivations such that a wide range of characteristics can be described, including social agents that take the desires of other agents or social goals into account. The priority relation contains at least the subset-relation which expresses a kind of independence between the motivations. Variables which are not decision variables are called parameters $(P)$.

Definition 4.1. (AS) An agent set is a tuple $\langle A, X, D, A D, \geq\rangle$, where:

- the agents $A$, variables $X$ and agent desires $D$ are three finite disjoint sets.

- an agent description $A D: A \rightarrow 2^{X \cup D}$ is a complete function that maps each agent to sets of variables (its decision variables) and desires, but that does not necessarily assign each variable to at least one agent. For each agent $a \in A$, we write $X_{a}$ for $X \cap A D(a)$, and $D_{a}$ for $D \cap A D(a)$. We write parameters $P=X \backslash \cup_{a \in A} X_{a}$.

- a priority relation $\geq: A \rightarrow 2^{D} \times 2^{D}$ is a function from agents to a transitive and reflexive relation on the powerset of the desires containing at least the subset relation. We write $\geq_{a}$ for $\geq(a)$.

The motivational state of the society is composed of its social goals $(G)$, and background knowledge is formalized by a set of effect rules $(E)$. Desires and social goals are abstract concepts which are described by - though conceptually not identified with - rules 
( $R u l$ ) built from literals ( $L i t)$. They are therefore not represented by propositional formulas, as in some other approaches to agency. ${ }^{13,29}$ Agents may share decision variables, or desires, though this expressive power is not used in this paper.

Definition 4.2. (MAS) A multiagent system is a tuple $\langle A S, G, E, M D\rangle$, where $A S=$ $\langle A, X, D, A D, \geq\rangle$ is an agent set, and:

- the set of goals $G$ is a set disjoint from $A, X$, and $D$. The motivations $M=D \cup G$ are defined as the union of the desires and social goals.

- the set of literals built from $X$, written as $\operatorname{Lit}(X)$, is $X \cup\{\neg x \mid x \in X\}$, and the set of rules built from $X$, written as $\operatorname{Rul}(X)=2^{\operatorname{Lit}(X)} \times \operatorname{Lit}(X)$, is the set of pairs of a set of literals built from $X$ and a literal built from $X$, written as $\left\{l_{1}, \ldots, l_{n}\right\} \rightarrow l$. We also write $l_{1} \wedge \ldots \wedge l_{n} \rightarrow l$ and when $n=0$ we write $\top \rightarrow l$. Moreover, for $x \in X$ we write $\sim x$ for $\neg x$ and $\sim(\neg x)$ for $x$.

- the set of effects $E \subseteq \operatorname{Rul}(X)$ is a set of rules built from $X$.

- the motivational description $M D: M \rightarrow \operatorname{Rul}(X)$ is a complete function from the sets of desires and goals to the set of rules built from $X$. For a set of motivations $S \subseteq M$, we write $M D(S)=\{M D(s) \mid s \in S\}$.

To describe the normative system, we introduce a set of norms $(N)$ and a norm description that associates violations with variables $(\mathrm{V})$.

Definition 4.3. ( $N M A S)$ A normative multiagent system is a tuple $\langle M A S, N, V\rangle$, where $M A S=\langle A S, G, E, M D\rangle$ is a multiagent system, $A S=\langle A, X, D, A D, \geq\rangle$ is an agent set, and moreover:

- the set of norms $N$ is a set disjoint from $A, X, D$, and $G$.

- the norm description $V: N \times A \rightarrow P$ is a complete function that maps each pair of a norm and an agent to the parameters, where $V(n, a) \in P$ represents that a violation by agent $a$ of the norm $n$ has been recognized.

We define sanction- and reward-based obligations in the normative multiagent system using an extension of Anderson's well-known reduction ${ }^{2,28}$ : violations and sanctions are the consequences of not fulfilling a norm. It covers a kind of ought-to-do and a kind of ought-to-be obligations.

Definition 4.4. (Obligation) Let $N M A S=\langle M A S, N, V\rangle, M A S=\langle A S, G, E, M D\rangle$ and $A S=\langle A, X, D, A D, \geq\rangle$. We say that:

- $x$ is obligatory for agent $a$ in $N M A S$ iff $\exists n \in N$ with $\sim x \rightarrow V(n, a) \in E$,

- $s$ is a sanction for agent $a$ in $N M A S$ iff $\exists n \in N$ with $V(n, a) \rightarrow s \in E$, and

- $r$ is a reward for agent $a$ in $N M A S$ iff $\exists n \in N$ with $\neg V(n, a) \rightarrow r \in E$.

More sophisticated notions within this kind of framework are developed elsewhere. ${ }^{7}$ In this paper we now turn to the negotiation protocol. 


\section{Generic negotiation protocol and logic of rules}

A negotiation protocol is described by a sequence of negotiation actions which either lead to success or failure. In this paper we only consider protocols in which the agents propose a so-called deal, and when an agent has made such a proposal, then the other agents can either accept or reject it. Moreover, they can also end the negotiation process without any result. Such negotiation protocols can be represented by a finite state machine, but we do not consider such representations in this paper. For turn-taking, we assume that the agents involved are ordered $(\succeq)$.

Definition 5.1. (Protocol) A negotiation protocol is a tuple $N P=\langle$ Ag, deals, actions, valid, finished, broken, $\succeq\rangle$ where:

- the agents $A g$, deals and actions are three disjoint sets, such that actions $=\{$ propose $(a, d)$, accept $(a, d)$, reject $(a, d) \mid a \in A g, d \in$ deals $\}$ $\cup\{\operatorname{breakit}(a) \mid a \in A g\}$.

- valid, finished, broken are three sets of finite sequences of actions.

- $\succeq \subseteq A g \times A g$ is a total order on $A g$,

We instantiate this generic protocol for negotiations in normative multiagent systems. We assume that a sequence of actions (a history) is valid when each agent does an action respecting its turn in the ordering of agents. Then, after each proposal, the other agents have to accept or reject this proposal, again respecting the ordering of agents, until they all accept it or one of them rejects it. When it is an agent's turn to make a proposal, it can also end the negotiation by breaking it. The history is finished when all agents have accepted the last deal, and broken when the last agent has ended the negotiations.

Definition 5.2. (NMAS protocol) Let $N M A S$ be a normative multiagent system $\langle M A S, N, V\rangle$ together with $M A S=\langle A S, G, E, M D\rangle$ and $A S=\langle A, X, D, A D, \geq\rangle$. A negotiation protocol $N P=\langle$ Ag, deals, actions, valid, finished, broken $\rangle, \succeq\rangle$ for $N M A S$ satisfies the following constraints:

- the set of agents $A g \subseteq A$ consists of the negotiators representing the agents $A$;

- A sequence $h$ ends iff either all agents have accepted the last proposal $($ finished $(h)$ ) or the last agent has broken the negotiation (broken $(h)$ ) instead of making a new proposal.

- a history $h$ is a sequence of actions, and $\operatorname{valid}(h)$ holds if:

- the propose and breakit actions in the sequence respect $\succeq$,

- each propose is followed by a sequence of accept or reject actions respecting $\succeq$ until either all agents have accepted the deal or one has rejected it,

- there is no double occurrence of a proposal propose $(a, d)$ of a deal $d \in$ deals by any agent $a \in A g$, and

- the sequence $h$ ends.

The open issue of the generic negotiation protocol is the set of deals which can be proposed. They depend on the kind of negotiation. In social goal negotiation the deals represent a social goal, and in norm negotiation the deals contain the obligations of the 
agents and the associated control system based on sanctions. To define the deals, we have to be more precise about the logic of rules we use.

We use a simplified input/output logic. ${ }^{25,26}$ A rule base is a set of rules, i.e., a set of ordered pairs $p \rightarrow q$. For each such pair, the body $p$ is thought of as an input, representing some condition or situation, and the head $q$ is thought of as an output, representing what the norm tells us to be desirable, obligatory or whatever in that situation. We use input/output logics since they do not necessarily satisfy the identity rule. Makinson and van der Torre write $(p, q)$ to distinguish input/output rules from conditionals defined in other logics, to emphasize the property that input/output logic does not necessarily obey the identity rule. In this paper we do not follow this convention.

In this paper, input and output are respectively a set of literals and a literal. We use a simplified version of input/output logics, since it keeps the formal exposition simple and it is sufficient for our purposes here. In Makinson and van der Torre's input/output logics, the input and output can be arbitrary propositional formulas, not just sets of literals and literal as we do here. Consequently, in input/output logic there are additional rules for conjunction of outputs and for weakening outputs.

Definition 5.3. (Input/output $\operatorname{logic}^{25}$ ) Let a rule base $B$ be a set of rules $\left\{p_{1} \rightarrow q_{1}, \ldots, p_{n} \rightarrow q_{n}\right\}$, read as 'if input $p_{1}$ then output $q_{1}$ ', etc., and consider the following proof rules strengthening of the input (SI), disjunction of the input (OR), and cumulative transitivity (CT) defined as follows:

$$
\frac{p \rightarrow r}{p \wedge q \rightarrow r} S I \quad \frac{p \wedge q \rightarrow r, p \wedge \neg q \rightarrow r}{p \rightarrow r} O R \quad \frac{p \rightarrow q, p \wedge q \rightarrow r}{p \rightarrow r} C T
$$

The following four output operators are defined as closure operators on the set $B$ using the rules above.

$$
\begin{array}{llll}
\text { out }_{1}: \mathrm{SI} & \text { (simple-minded output) } & \text { out }_{3}: \mathrm{SI}+\mathrm{CT} & \text { (reusable output) } \\
\text { out }_{2}: \mathrm{SI}+\mathrm{OR} & \text { (basic output) } & \text { out }_{4}: \mathrm{SI}+\mathrm{OR}+\mathrm{CT} & \text { (basic reusable output) }
\end{array}
$$

We write $\operatorname{out}(B)$ for any of these output operations, we write $B \vdash_{i o l} p \rightarrow q$ for $p \rightarrow q \in \operatorname{out}(B)$, and we write $B \vdash_{i o l} B^{\prime}$ iff $B \vdash_{i o l} p \rightarrow q$ for all $p \rightarrow q \in B^{\prime}$.

The following definition of the so-called input-output constraint checks whether the derived conditional goals are consistent with the input.

Definition 5.4. (Constraints ${ }^{26}$ ) Let $B$ be a set of rules, and $C$ a set of literals. $B$ is consistent with $C$, written as cons $(B \mid C)$, iff there do not exist two contradictory literals $p$ and $\neg p$ in the set $C \cup\left\{l \mid B \vdash_{i o l} C \rightarrow l\right\}$. We write $\operatorname{cons}(B)$ for $\operatorname{cons}(B \mid \emptyset)$.

The semantics of input/output logics, further details on its proof theory, the extension with the identity rule, alternative constraints, permissions, and examples are described elsewhere. ${ }^{25,26,27}$ We do not consider the negotiation of permissions or constitutive norms in this paper. 


\section{Social goal negotiation}

We characterize the allowed deals during goal negotiation as a merger or fusion of the desires of the agents, which may be seen as a particular kind of social choice process. ${ }^{23}$ Technically, we use the merging operators for merging desires into goals in the context of beliefs, ${ }^{15,23}$, which are generalizations of belief revision operators. ${ }^{1,20,22}$. We simplify these operators, because we do not use beliefs, and we make them more complex, because we extend the operators defined on propositional formulas to merge rules.

Definition 6.1. A rule base $B$ is a set of desire rules of an agent, a rule set $S$ of a multiagent system is a multi-set of rule bases. Two rule sets $S_{1}$ and $S_{2}$ are equivalent, written as $S_{1} \leftrightarrow S_{2}$, iff there exists a bijection $f$ from $S_{1}=\left\{B_{1}^{1}, \ldots, B_{1}^{n}\right\}$ to $S_{2}=\left\{B_{2}^{1}, \ldots, B_{2}^{n}\right\}$ such that $\operatorname{out}\left(f\left(B_{1}^{i}\right)\right)=\operatorname{out}\left(B_{1}^{i}\right)$ for $i=1 \ldots n$. We write $\bigwedge S$ for the union of all rules in $S$, and $\sqcup$ for union with multi-sets.

A merging operator is defined by a set of postulates the result of the merger has to obey. For example, the social goal should not violate the integrity constraints (R0 in Definition 6.2.), when the integrity constraints are consistent we should always manage to extract a coherent social goal from the rule set (R1), and if possible, the social goal is simply the conjunction of the rule bases of the rule set with the integrity constraints (R2). Moreover, the principle of irrelevance of syntax says that if we change the syntax but not the meaning of the desire rules of the agents, the social goal does not change (R3).

Koniecnzy ${ }^{23}$ introduces the fairness postulate, which ensures that when merging two rule bases, the operator cannot give full preference to one of them (R4). Pareto's conditions in Arrow's social choice theory ${ }^{3}$ detail how mergers of sets of rules are related (R5 and R6). Finally there are conditions on the conjunction of integrity constraints (R7 and R8). See the above mentioned papers for further details and motivations. In the following definition, as well as in all following definitions, we assume that a logic of rules has been fixed.

Definition 6.2. Let $\vdash_{i o l}$ be an output operation, $S$ be a rule set, $E$ a rule base, and $\nabla$ an operator that assigns to each rule set $S$ and rule base $E$ a rule base $\nabla_{E}(S) . \nabla$ is a rule merging operator if and only if it satisfies the following properties:

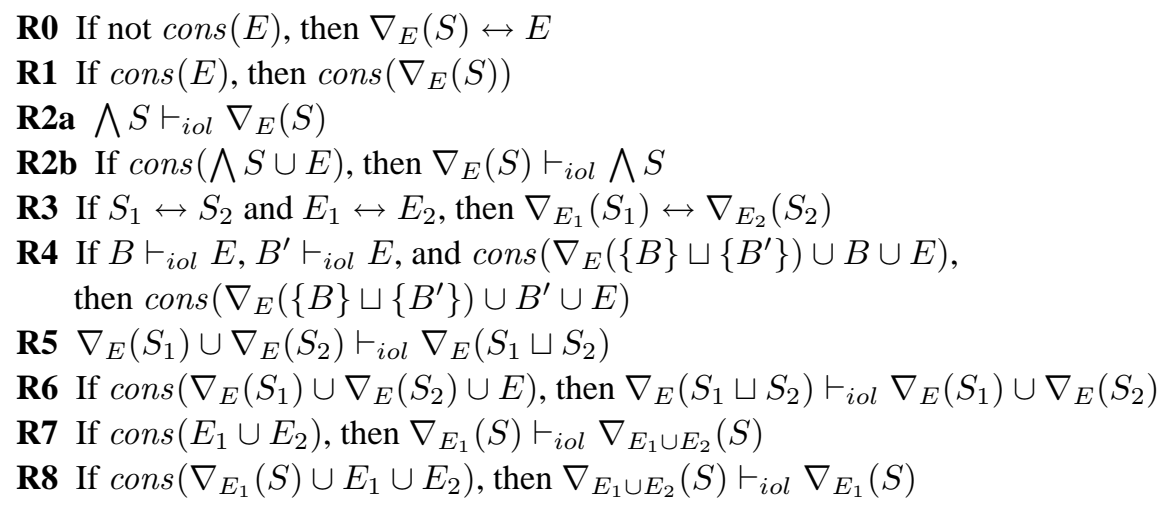

Additional properties and the semantics of merging operators have been defined in the 
literature. ${ }^{23}$ In this paper, we only consider social goals consisting of one rule. At first sight this may seem like a strong restriction, but more complex examples can be modeled using effect rules in $E$.

Definition 6.3. (Deals in goal negotiation) In the goal negotiation protocol, the type of the allowed deals is that deals are a rule base consisting of a single rule.

Let $N M A S$ be a normative multiagent system $\langle M A S, N, V\rangle$ together with $M A S=\langle A S, G, E, M D\rangle$ and $A S=\langle A, X, D, A D, \geq\rangle$. We have $d \in$ deals iff there is a rule merging operator $\nabla$ such that $M D(d) \subseteq \nabla_{E}(M D(D(x)) \mid x \in A)$.

The following example illustrates that the allowed deals depend on the chosen input/output logic.

Example 6.1. Let $\vdash_{i o l}$ be $o u t_{3}$, and consider four agents with desires represented by rule bases each consisting of a single rule $S=\{\{\top \rightarrow p\},\{\top \rightarrow q\},\{p \rightarrow r\},\{q \rightarrow \neg r\}\}$. We cannot take all rules in $S$ as the social goal, since they are not consistent. Formally, due to (R1) we cannot propose the deal $\nabla_{\emptyset}(S)=\bigwedge S$, because we do not have cons $(\bigwedge S)$. We can take for example either the deal $\nabla_{\emptyset}(S)=\{\top \rightarrow p, \top \rightarrow q, p \rightarrow r\}$, or the deal $\nabla_{\emptyset}(S)=\{\top \rightarrow q, p \rightarrow r, q \rightarrow \neg r\}$, since they are consistent and they satisfy the other postulates. For example, they satisfy (R4) since there are no two rule bases directly conflicting with each other. In general, checking whether a social goal base satisfies the merging postulates is non-trivial, and can be based on the semantics of merging operators. ${ }^{23}$ If we assume that $\vdash_{i o l}$ is out $_{1}$, then $\operatorname{cons}(\bigwedge S)$, and due to (R2b) we have $\nabla_{\emptyset}(S)=\bigwedge S$.

Social goal negotiation is illustrated by our running example. We consider three sets of agents, who can work together in various ways. They can make a coalition to each perform a task, or they can distribute five tasks among them and obtain an even more desirable social goal. Variables in the example are syntactic sugar. Quantification over rules means that rules are schemata: there is a set of rules, one for each agent involved. Note that the set of agents $A$ is finite.

Example 6.2. (Running example) Let $N M A S=\langle M A S, N, V\rangle$ together with $M A S=\langle A S, G, E, M D\rangle$ and $A S=\left\langle A_{1} \cup A_{2} \cup A_{3}, X, D, A D, \geq\right\rangle$ be a normative multiagent system with, amongst others, the following ingredients:

variables: $X=\left\{\operatorname{task}_{1}(a), \operatorname{task}_{2}(a), \operatorname{task}_{3}(a) \mid a \in A_{1}\right\} \cup \ldots$

Each agent in $A_{1}$ can perform task 1,2,3, each agent in $A_{2}$ can perform task 2,3,4, and each agent in $A_{3}$ can perform task 3,4 , and 5 .

effect rules: $E=\left\{\ldots \cup \wedge_{i=1 \ldots 5}\right.$ task $_{i} \rightarrow$ best_state $\}$

Performing task 1, 3 and 5 will lead to some good result, and all five tasks will lead to the best result.

desires: for $a \in A_{1}, M D\left(D_{a}\right)=\left\{t_{a s} k_{4}, t_{a s} k_{5}\right\}, \ldots$.

Each agent desires the tasks it cannot perform itself.

Social goal $M D(G)=\{\top \rightarrow$ best_state $\}$.

The agents negotiate the social goal to perform all five tasks for NMAS. 


\section{Norm negotiation}

We formalize the allowed deals during norm negotiation as solutions of a planning problem, distinguishing between the obligations and the associated sanctions and rewards. The planning problem for the obligations is that the obligations of the agents must imply the social goal. We represent a norm $n$ by an obligation for all agents in the multiagent system, that is, for every agent $a$ we introduce an obligation $\sim x \rightarrow V(n, a)$. Moreover, since goals can only be in force in a context, e.g., $Y \rightarrow g$, we introduce in context $Y$ an obligation $Y \wedge \sim x \rightarrow V(n, a)$. Roughly, the condition is that the conjunction of all obligations $x$ imply the social goal $g$.

However, to determine whether the obligations imply the social goal, we have to take the existing normative system into account. We assume that the normative system only creates obligations that can be fulfilled together with the already existing obligations. Moreover, for the test that the social goal $g$ will be achieved, we propose the following condition: if every agent fulfills its obligation, and it fulfills all its other obligations, then $g$ is achieved. We define a global violation constant $\mathbf{V}$ as the disjunction of all indexed violation constants like $V(n, a)$, i.e., $\mathbf{V}=\bigvee_{n \in N, a \in A} V(n, a)$.

Note that the type of the proposed deals in norm negotiation is more complex than the type in goal negotiation. In goal negotiation each agent can propose a social goal, which is a rule base. In norm negotiation each agent can propose a norm, which contains obligation rules together with sanction rules (for each agent).

Definition 7.1. (Deals in norm negotiation with sanctions and rewards) In norm negotiation, the type of deals is a pair of rule bases.

Let $N M A S$ be a normative multiagent system $\langle M A S, N, V\rangle$ together with $M A S=\langle A S, G, E, M D\rangle$ and $A S=\langle A, X, D, A D, \geq\rangle$, and let $Y \rightarrow g \in M D(G)$ be a social goal. We assume that the parameters contain the global violation constant $\mathbf{V} \in P$ and $E$ contains the following set of rules:

$$
\{V(n, a) \rightarrow \mathbf{V} \mid n \in N, a \in A\} \cup\{\neg \mathbf{V} \rightarrow \neg V(n, a) \mid n \in N, a \in A\}
$$

We have $\left\langle E^{\prime}, E^{\prime \prime}\right\rangle \in$ deals when $E^{\prime}$ is a set of obligations and $E^{\prime \prime}$ is a set of sanctions and rewards, defined as follows,

$$
\begin{gathered}
E^{\prime} \subseteq\left\{Y \wedge x \rightarrow V\left(n^{\prime}, a\right) \mid a \in A, x \in \operatorname{Lit}(X)\right\} \\
E^{\prime \prime} \subseteq\left\{Y \wedge V\left(n^{\prime}, a\right) \rightarrow s \mid a \in A, s \in \operatorname{Lit}(X)\right\} \cup\left\{Y \wedge \neg V\left(n^{\prime}, a\right) \rightarrow r \mid a \in A, r \in \operatorname{Lit}(X)\right\}
\end{gathered}
$$

and, moreover:

(1) The norm $n^{\prime}$ is not already part of $N$;

(2) $E^{\prime}$ is a set of obligations for each $a \in A$ such that $E \cup E^{\prime} \vdash_{i o l} \neg \mathbf{V} \wedge Y \rightarrow g$, if all norms are fulfilled, then the social goal is fulfilled;

(3) $\operatorname{cons}(E \mid Y \wedge \neg \mathbf{V})$, it is possible that no norm is violated.

(4) $E^{\prime \prime}$ is a set of sanctions and rewards for each $a \in A$ such that for all such $s$ and $r$ we have $D_{a} \vdash_{i o l} Y \rightarrow \neg s$ or $D \vdash_{i o l} Y \rightarrow r$, sanctions are undesired and rewards are desired. 
If the norm $n^{\prime}$ with obligations $E^{\prime}$ and sanctions and rewards $E^{\prime \prime}$ to achieve social goal $Y \rightarrow g$ in deals is accepted, then it leads to the updated system $\left\langle M A S, N \cup\left\{n^{\prime}\right\}, V\right\rangle$ with $M A S=\left\langle A S, E \cup E^{\prime} \cup E^{\prime \prime}, M D\right\rangle$.

The following example illustrates norm negotiation in our running example. There can be free riders, so they need sanctions to ensure that the norm is accepted.

Example 7.1. (Continued) The normative multiagent system is further detailed as follows (we assume that the same social goal is negotiated):

Desires: $M D(D)=\ldots \cup\left\{\top \rightarrow \neg \operatorname{task}_{1}(a), \top \rightarrow \neg \operatorname{task}_{2}(a), \ldots \mid a \in A_{1}\right\} \cup \ldots$

Each agent desires not to perform any tasks. These desires are weaker (have lower priority in $\succeq$ ) than the desire that the other tasks are performed. Moreover, each agent desires not to be sanctioned, written as $s(a)$.

\section{Effect rules}

We now assume that not all agents have to contribute to a task, but only most of them.

Thus there can be free riders.

E.g., a possible deal contains the obligations $E^{\prime}=\left\{\neg \operatorname{task}_{1}(a) \rightarrow V\left(n^{\prime}, a\right) \mid a \in A_{1}\right\}$ and sanctions $E^{\prime \prime}=\left\{V\left(n^{\prime}, a\right) \rightarrow s(a) \mid a \in A\right\} . \neg t_{a s k}(a)$ counts as a violation of norm $n^{\prime}$ by agent $a$, and is sanctioned with $s(a)$.

The following example illustrates the negotiation protocol.

Example 7.2. (Continued) Consider three agents that have to negotiate a task consisting of five subtasks, with social goal $g=\top \rightarrow$ best_state, the NMAS protocol is:

$A g=\left\{a_{1}, a_{2}, a_{3}\right\}$ with $a_{1} \in A_{1}, a_{2} \in A_{2}, a_{3} \in A_{3}$,

deals $=$ the sets of pairs $\left\langle\tau_{\delta}, \tau_{\sigma}\right\rangle$ where the set of obligations $\tau_{\delta}$ belongs to the set $\Delta=\left\{\delta=\left\{\operatorname{task}_{1}\left(a_{1}\right), \operatorname{task}_{2}\left(a_{2}\right), \operatorname{task}_{3}\left(a_{3}\right), \operatorname{task}_{4}\left(a_{4}\right), \operatorname{task}_{5}\left(a_{5}\right)\right\} \mid a_{1}, \ldots, a_{5} \in A g\right\}$. Moreover, $\tau_{\sigma}$ is a tuple of $|A|$ elements specifying a sanction $s_{i} \in X$ for every agent in $A$. Here is a history $h$, where $a_{1}$ proposes something which is not accepted, but $a_{2}$ thereafter proposes a distribution which is accepted:

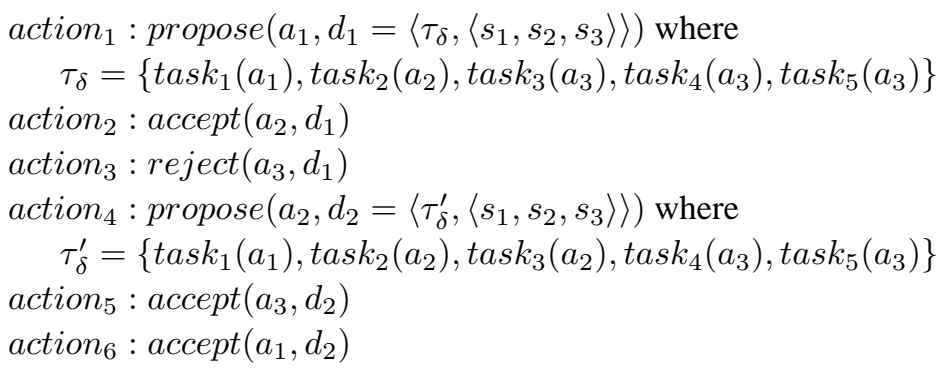

We have $\operatorname{valid}(h)$, because the order of action respects $\leq$, and we have accepted $(h)$, because the history ends with acceptance by all agents (action ${ }_{5}$ and action $_{6}$ ) after a proposal $\left(\right.$ action $\left._{4}\right)$. 


\section{Norm acceptance and agent decision making}

An agent accepts a norm when the obligation implies some desire the cycle started with, and moreover, it believes that the other agents will fulfill their obligations. We propose the following games: agent $a$ plays a game with arbitrary agent $b$ and accepts the norm if agent $b$ fulfills the norm given that all other agents fulfill the norm, and this fulfilment leads to fulfillment of agent $a$ 's desire the cycle started with. This implies that the fulfillment of the goal $g$ is kind of normative equilibrium. The details and examples of the decision model and the notion of unfulfilled desires for conditional rules can be found in our work on normative multiagent systems. ${ }^{7,8}$

Definition 8.1. (Decision) Let $N M A S$ be a normative multiagent system $\langle M A S, N, V\rangle$ with $M A S=\langle A S, G, E, M D\rangle$ and $A S=\langle A, X, D, A D, \geq\rangle$. The optimal decision of agent $b \in A$ given a set of literals $C$ is defined as follows.

- The set of decisions $\Delta$ is the set of subsets of $\operatorname{Lit}\left(X_{b}\right)$ that do not contain a variable and its negation. A decision is complete if it contains, for each variable in $X_{b}$, either this variable or its negation.

- The unfulfilled desires of decision $\delta$ for agent $b \in A$, written as $U(\delta, b)$, are the desires whose body is part of the decision, but whose head is not.

$$
\left\{d \in D_{b} \mid M D(d)=L \rightarrow l, E \vdash_{i o l} C \cup \delta \rightarrow l^{\prime} \text { for } l^{\prime} \in L \text { and } E \nvdash_{i o l} C \cup \delta \rightarrow l\right\}
$$

- A decision $\delta$ is optimal for agent $b$ if and only if there is no decision $\delta^{\prime}$ such that $U(\delta, b)>_{b} U\left(\delta^{\prime}, b\right)$.

We use the definition of optimal decision to define the acceptance relation. We define a variant of the global violation constant $\mathbf{V}_{\sim b}$ as the disjunction of the violation constants of all agents except agent $b$. We assume here that the agents only consider typical cases. In reality there are always exceptions to the norm, but we do not take this into account.

Definition 8.2. (Acceptance) Let $N M A S$ be a normative multiagent system $\langle M A S, N, V\rangle$ with $M A S=\langle A S, G, E, M D\rangle$ and $A S=\langle A, X, D, A D, \geq\rangle$, and let $N M A S^{\prime}=\left\langle M A S^{\prime}, N \cup\left\{n^{\prime}\right\}, V\right\rangle$ with $M A S^{\prime}=\left\langle A S, E \cup E^{\prime} \cup E^{\prime \prime}, M D\right\rangle$ be the system after the creation of a norm. The parameters contain the global violation constants $\mathbf{V}_{\sim b} \in P$ and $E$ contains the following rules:

$$
\left\{V(n, x) \rightarrow \mathbf{V}_{\sim b} \mid n \in N, x \in A \backslash\{b\}\right\} \cup\left\{\neg \mathbf{V}_{\sim b} \rightarrow \neg V(n, x) \mid n \in N, x \in A \backslash\{b\}\right\}
$$

An agent $a \in A$ accepts the norm if:

(1) There is a desire in $D$ which is not fulfilled in $N M A S$, but it is fulfilled in $N M A S^{\prime}$.

(2) For all other agents $b \in A$, we have that the optimal decision of agent $b$ assuming $\neg \mathbf{V}_{\sim b}$ implies $\neg \mathbf{V}$. 
Norms do not always need to be accepted in order to be fulfilled, since the sanction provides a motivation to the agents. However, for a norm to be really effective must be respected due to its acceptance, and not only due to fear of sanctions.

Decision making is not restricted to the normative multiagent system, but it can be extended to the negotiation protocol. We do not consider an equilibrium analysis as in the acceptance relation, but we use the game-theoretic notion called backward induction. In the literature on multi-agent systems it is sometimes referred to as recursive modelling.

The games we define are as follows. First the agents negotiate the joint goal and the norm, then they make a decision in the normative multiagent system to either fulfill the obligations or accept the associated sanctions.

Definition 8.3. A history $h_{1}$ dominates a history $h_{2}$ at step $i$ if they have the same set of actions at step $1 \ldots i-1$, are optimal for step $i+1 \ldots$, and outcomes $\left(h_{1}\right) \succ_{a}$ outcomes $\left(h_{2}\right)$, agent $a$ performing action $i$ prefers the set of possible outcomes of $h_{1}$ to the set of possible outcomes of $h_{2}$.

A history is optimal at step $i$ if it is not dominated by another history, and it is optimal at all steps $j>i$.

A history is optimal if it is optimal at step 1.

The behavior of agents in the negotiation protocol is illustrated in the following example.

Example 8.1. (Continued) Reconsider Example 6.3 with history $h$, together with history $h^{\prime}$ which is like $h$ until action $_{3}$ while it continues in this way:

action $_{4}$ : propose $\left(a_{3}, d_{3}=\left\langle\tau_{\delta}^{\prime \prime},\left\langle s_{1}, s_{2}, s_{3}\right\rangle\right\rangle\right)$ where

$\tau_{\delta}^{\prime \prime}=\left\{\operatorname{task}_{1}\left(a_{1}\right), \operatorname{task}_{2}\left(a_{2}\right), \operatorname{task}_{3}\left(a_{2}\right), \operatorname{task}_{4}\left(a_{2}\right), \operatorname{task}_{5}\left(a_{3}\right)\right\}$

action $_{5}: \operatorname{accept}\left(a_{1}, d_{3}\right)$

action $_{6}: \operatorname{reject}\left(a_{3}, d_{3}\right)$

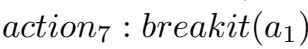

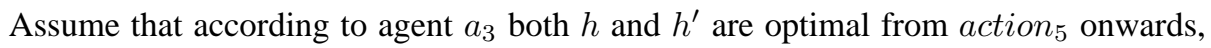
then agent $a_{5}$ compares the two histories to decide how to choose between the two histories. Likewise, the agents of the first three actions decide their optimal actions based on the optimal choice of agent $a_{3}$ for action $_{4}$.

There are a couple of issues with backward induction. First, the length of the histories has to be bounded, because otherwise there is no starting point for the backward induction. Second, if the agents' desires are not common knowledge, then we have to add the desires of agent $a_{i}$ according to $a_{j}$, the desires of agent $a_{i}$ according to agent $a_{j}$ according to $a_{k}$, etc. An efficient representation of such nested modalities is still an open problem. For this reason, in game theory it is usually assumed that the game is common knowledge. 


\section{Negotiation and related work in normative multiagent systems}

In this section we compare norm negotiation in the social delegation cycle with other work on normative multiagent systems.

\subsection{The origin of norms}

Social norms and laws can be used to guide the emergent behavior of multiagent systems. ${ }^{12}$ But where do these social norms and laws come from? The following four possibilities have been discussed or suggested in the literature on multiagent systems.

(1) Norms are off-line designed by the agent programmer. ${ }^{33}$ However, off-line design has been criticized for open systems.

(2) Norms are created by a single agent in a "legislator" role. ${ }^{11}$ The legislator faces the decision problem to decide which norm, sanction and control system has to be created. However, the agent playing the legislator role is vulnerable for attacks or fraud.

(3) Norms emerge spontaneously like conventions. ${ }^{4,34}$ However, conventions can be criticized as a coordination mechanism for dynamic systems, because they develop only after a long time (if at all) and they are difficult to change.

(4) Norms are negotiated by (a subset of) the agents in a "democracy", which raises the problem to devise fair and efficient norm negotiation protocols.

Other approaches of norm emergence are based on simulation techniques. Verhagen, ${ }^{39}$ for example, considers the problem of the learning of norms. He does not focus however on the acceptance of norms but rather on the influence of normative comments on previous choices. Thus this approach is outside the scope of this paper.

\subsection{Negotiation of obligation distribution}

The social delegation cycle distinguishes norm negotiation from other kinds of negotiation in normative multiagent systems. In contract negotiation agents create new regulative and constitutive norms in a legal context ${ }^{7}$ and in negotiation of obligation distribution a group of agents negotiates how to distribute a joint task. ${ }^{5}$ These other kinds of negotiation are not based on a social delegation cycle, and therefore give other powers to the agents.

Norm negotiation is related to the problem how a group obligation together with a group sanction can be distributed among the members of the group. In particular, negotiation of obligation distribution ${ }^{5}$ is analogous to the second step of the social delegation cycle, extended with a penalty $(\pi)$ for the agent who breaks the negotiations (which may depend on the agent, e.g., older boys may be punished harder for breaking negotiations than the younger ones). The model distinguishes among three types of sanctions:

- the sanction associated with the group obligation, which is imposed when the obligation is violated, regardless which agent is responsible for it;

- the sanctions associated with the negotiated deal, which are imposed if one of the agents does not fulfill its part of the deal;

- the sanctions associated with the break penalty $\pi$. 
Moreover, the negotiation is driven by fear of a group sanction, including the sanction for breaking the negotiation. In the model of norm negotiation developed in this paper, the negotiation is driven by the agents' desires. Agents are cooperative in the sense that they have a social goal to achieve.

In some cases it is a drawback to be the only agent able to see to the fulfilment of part of an obligation, but in other cases it may be an advantage, because of the power it gives to the agent over the other agents during the negotiation. This is illustrated by a backward induction argument.

\subsection{Contract negotiation}

As the philosopher of law Ruiter ${ }^{30}$ shows, from the legal point of view, legal effects of actions of the members of a legal system are complex and contracts do not concern only the regulative aspects of a legislation (i.e., the rules of behavior specified by obligations), or the constitutive part of it (i.e., the rules introducing institutional facts such bidding in an auction). Rather, contracts are legal institutions: "systems of [regulative and constitutive] rules that provide frameworks for social action within larger rule-governed settings". ${ }^{30}$ In our model of contract negotiation games, ${ }^{7}$ the larger setting is represented by a normative system which establishes the set of possible contracts.

Contract negotiation concerns only two or a few other agents, and therefore there is no need for the three phases of the social delegation cycle, and in particular there is no social goal. Moreover, there is the additional problem not present in social norm negotiation about the choice of contract partners. This choice of contract partners gives a lot of power to agents who have unique or rare abilities. The social delegation cycle however suggests that the powers of agents are less important. In social goal generation, the situation is symmetric for all agents. Only in norm negotiation, like in negotiation of obligation distribution, the powers of agents may be a strategic advantage (or disadvantage!).

\subsection{Normative system as an agent}

In our other work we discuss also applications of normative multiagent systems. ${ }^{7,8}$ In those papers the agents consider the normative system as an agent, and they attribute mental attitudes to it, because the agents are playing games with the normative system to determine whether to fulfill or violate norms. We refer to this use of the agent metaphor as "your wish is my command": the goals of the normative agent are the obligations of the normal agents. As an agent with goals, the normative system has to decide whether to consider behavior as a violation and to sanction violations.

In the present paper, however, the agents play games with other agents, and the attribution of mental attitudes to normative system is not a necessary assumption. We thus abstract from the role of the normative system in recognizing violations and sanctioning them: here we assume that violations and sanctions are a direct consequence of the behavior of the agents. 


\section{Further research}

\subsection{Mechanisms}

Within the proposed general model of the social delegation cycle, actual procedures can be defined and studied. In this paper, we propose a fairly general formal model of the social delegation cycle, which delimits the kind of norms that can be created, but that does not give an actual procedure to create norms. The reason is that we aim to capture the fundamental properties of the social delegation cycle, which later can be used to design actual procedures. However, compared to informal characterizations of the construction of social reality, such as in the work of Searle ${ }^{32}$, our model is fairly limited as we do not introduce for example beliefs or institutions. Desirable properties may be soundness (compliance with our framework), completeness (for each possible goal there is a goal generated), conciseness of goals and norms generated, generality of goals and norms generated, strictness of goal generation and norm creation, etc.

\subsection{Balancing goal negotiation, norm negotiation and acceptance}

We define three steps in the social delegation cycle, i.e., goal negotiation, norm negotiation and acceptance, which do not have to be done sequentially but also can be done iteratively. Moreover, norm negotiation could have been split into obligation negotiation and sanction negotiation. We have not formalized how the elements of the social delegation cycle are balanced. For example, strictly defined norm creation procedures only create norms that will always be accepted, and analogously strictly defined goal generation procedures generate only goals for which a norm can be created that is accepted.

\subsection{Trust}

For more realistic but also more complex social trust, we have to enrich the model with beliefs. We have to extend the merging operators to merging in the context of beliefs. ${ }^{15}$ Consequently, we have to introduce beliefs in norm creation, and we have to make the acceptance relation relative to beliefs.

\subsection{The negotiation of permissive norms}

It is not directly clear how the social delegation cycle can explain the creation of permissive norms. One way to proceed is to define permissions as exceptions within hierarchical normative systems. ${ }^{11}$ However, also other kinds of permissions have been proposed,${ }^{27}$ and it is unclear which kind of permission should be used in the social delegation cycle.

\subsection{Social institutions and the negotiation of constitutive norms}

How to take social institutions into account in the social delegation cycle? Based on Searle's construction of social reality, we may introduce besides the obligations or regulative norms also constitutive norms, which are definitions of the normative system based on a counts-as conditional. $^{7}$ 


\section{Summary}

The social delegation cycle gives cognitive foundations to a social phenomenon, as it relates agent desires to the norms of the society. Individual agents have desires, which turn into social (or joint) goals. A social goal is individualized by a social norm. The individual agents accept the norm, together with its associated sanctions and rewards, because they recognize that it serves to achieve their desires the cycle started with. The cycle thus explains the creation of norms from desires from a rational perspective, because norms are only accepted if they are respected by the other agents, which explains also why sometimes sanctions are needed. In this sense we explain norms in the tradition of Kant, who emphasized the rational aspects of norms.

The social delegation cycle may be seen as a generalization of single agent decision making, in which also the two steps of deriving goals and deriving plans for goals can be distinguished. For example, in the BOID architecture ${ }^{10}$ there are components for goal generation and for goal-based planning. Additional issues in the social delegation cycle are the role of sanctions and rewards, the acceptance relation, and the implicit assumption of fairness in goal and norm negotiation. Moreover, though we have not considered this extension in this paper, in the social delegation cycle institutions may play a role. Sanctions may be interpreted either as cues that the other agents see to their task, or as decommitment possibilities for the agents themselves. ${ }^{31,35}$ In some cases sanctions must be associated with the norms to ensure that agents fulfill the norm, and therefore to ensure that the agents accept the norm, but in some other cases this is not necessary.

The two following prototypical examples of coordination games and prisoner's dilemma can be represented in our model. ${ }^{4}$ First, agents do not want to crash into each other, and the norm to drive on the right side of the road (or the left side, for that matter) is accepted by all members. In this coordination game, no sanction is necessary and the norm may be called a convention. Second, agents want to cooperate in a prisoner's dilemma, so the norm to cooperate is accepted by all members. In this case, a sanction must be associated with the norm, because otherwise the agent will defect (as game theory shows). In this paper we discuss a more complicated example in which there are various kinds of agents, each capable to perform various kinds of task, and they have to negotiate which tasks the group will perform (social goal), and which agents perform which task (the norm). Sanctions are added to avoid free riding.

We formalize the social delegation cycle combining theories developed in a generalization of belief revision called merging operators, planning and game theory. First, we formalize allowed proposals in social goal negotiation as a merging process of the individual agent desires, for which we extend existing merging operators to deal with rules. Second, we formalize allowed proposals in norm negotiation as a planning process for both the obligation and the associated sanctions or rewards. Third, we formalize the acceptance relation as both a belief of agents that the norm leads to achievement of their desires, and the belief that other agents will act according to the norm, introducing a notion of normative equilibrium which states that agents fulfill norms when other agents do so. 


\section{References}

1. C. Alchourron, P. Gärdenfors, and D. Makinson. On the logic of theory change. Journal of Symbolic Logic, 50(2):510-530, 1985.

2. A. Anderson. A reduction of deontic logic to alethic modal logic. Mind, 67:100-103, 1958.

3. K.J. Arrow. Social choice and individual values. Wiley, New York, second edition, 1963.

4. G. Boella and L. van der Torre. $\Delta$ : The social delegation cycle. In Deontic Logic: 7th International Workshop on Deontic Logic in Computer Science (DEON'04), volume 3065 of LNAI, pages 29-42, Berlin, 2004. Springer.

5. G. Boella and L. van der Torre. The distribution of obligations by negotiation among autonomous agents. In Procs. of 16th European Conference on Artificial Intelligence (ECAI'04), pages 1317, Amsterdam, 2004. IOS Press.

6. G. Boella and L. van der Torre. Enforceable social laws. In Procs. of 4th International Joint Conference on Autonomous Agents and Multiagent Systems (AAMAS'05), pages 682-689, New York (NJ), 2005. ACM Press.

7. G. Boella and L. van der Torre. A game theoretic approach to contracts in multiagent systems. IEEE Transactions on Systems, Man and Cybernetics - Part C: Applications and Reviews, 36(1):68-79, 2006.

8. G. Boella and L. van der Torre. Security policies for sharing knowledge in virtual communities. IEEE Transactions on Systems, Man and Cybernetics - Part A: Systems and Humans, 36(3):439450, 2006.

9. G. Boella, L. van der Torre, and H. Verhagen. Introduction to normative multiagent systems. Computation and Mathematical Organizational Theory, special issue on normative multiagent systems, to appear.

10. J. Broersen, M. Dastani, J. Hulstijn, and L. van der Torre. Goal generation in the BOID architecture. Cognitive Science Quarterly, 2(3-4):428-447, 2002.

11. E. Bulygin. Permissive norms and normative systems. In A. Martino and F. Socci Natali, editors, Automated Analysis of Legal Texts, pages 211-218. Publishing Company, Amsterdam, 1986.

12. C. Castelfranchi. Modeling social action for AI agents. Artificial Intelligence, 103(1-2):157-182, 1998.

13. P. R. Cohen and H. J. Levesque. Intention is choice with commitment. Artificial Intelligence, 42(2-3):213-261, 1990.

14. R. Conte, C. Castelfranchi, and F. Dignum. Autonomous norm-acceptance. In Intelligent Agents $V$ (ATAL'98), volume 1555 of $L N C S$, pages 99-112. Springer, Berlin, 1998.

15. M. Dastani and L. van der Torre. Specifying the merging of desires into goals in the context of beliefs. In Procs. of The First Eurasian Conference on Advances in Information and Communication Technology (EurAsia ICT'02), volume 2510 of LNCS, pages 824-831, Berlin, 2002. Springer.

16. F. Dignum. Autonomous agents with norms. Artificial Intelligence and Law, 7:69-79, 1999.

17. Virginia Dignum, Javier Vázquez-Salceda, and Frank Dignum. A model of almost everything: Norms, structure and ontologies in agent organizations. In Procs. of 3rd International Joint Conference on Autonomous Agents and Multiagent Systems (AAMAS'04), pages 1498-1499, New York (NJ), 2004. ACM.

18. M. Esteva, J. Padget, and C. Sierra. Formalizing a language for institutions and norms. In Intelligent Agents VIII (ATAL'01), volume 2333 of LNCS, pages 348-366, Berlin, 2001. Springer.

19. M. Esteva, J.A. Rodriguez-Aguilar, C. Sierra, and W.W. Vasconcelos. Verifying norm consistency in electronic institutions. In Procs. of Workshop on Agent Organizations at AAAI'04, San Jose (CA), 2004.

20. P. Gärdenfors. Knowledge in flux. MIT Press, Cambridge (Massachusetts), 1988.

21. A. Jones and J. Carmo. Deontic logic and contrary-to-duties. In D. Gabbay and F. Guenthner, editors, Handbook of Philosophical Logic, volume 3, pages 203-279. Kluwer, Dordrecht (NL), 
2001.

22. H. Katsuno and A.O. Mendelzon. Propositional knowledge base revision and minimal change. Artificial Intelligence, 52(3):263-294, 1992.

23. S. Konieczny and R.P. Pérez. On the frontier between arbitration and majority. In Procs. of 8th International Conference on the Principles of Knowledge Representation and Reasoning (KR'02), pages 109-120, San Mateo (CA), 2002. Morgan Kaufmann.

24. F. Lopez y Lopez, M. Luck, and M. d'Inverno. Contraining autonomy through norms. In Procs. of 2nd International Joint Conference on Autonomous Agents and Multiagent Systems (AA$M A S^{\prime} 02$ ), pages 674-681, New York (NJ), 2002. ACM.

25. D. Makinson and L. van der Torre. Input-output logics. Journal of Philosophical Logic, 29(4):383-408, 2000.

26. D. Makinson and L. van der Torre. Constraints for input-output logics. Journal of Philosophical Logic, 30(2):155-185, 2001.

27. D. Makinson and L. van der Torre. Permissions from an input-output perspective. Journal of Philosophical Logic, 32(4):391-416, 2003.

28. J. J. Ch. Meyer. A different approach to deontic logic: Deontic logic viewed as a variant of dynamic logic. Notre Dame Journal of Formal Logic, 29(1):109-136, 1988.

29. A. S. Rao and M. Georgeff. The semantics of intention maintenance for rational agents. In Procs. of 14th International Joint Conference on Artificial Intelligence (IJCAI'95), pages 704-710, San Mateo, CA, 1995. Morgan Kaufmann.

30. D.W.P. Ruiter. A basic classification of legal institutions. Ratio Juris, 10(4):357-371, 1997.

31. T. Sandholm, S. Sikka, and S. Norden. Algorithms for optimizing leveled commitment contracts. In Procs. of 16th International Joint Conference on Artificial Intelligence (IJCAI'99), pages 535-541, San Mateo, CA, 1999. Morgan Kaufmann.

32. J.R. Searle. The Construction of Social Reality. The Free Press, New York, 1995.

33. Y. Shoham and M. Tennenholtz. On social laws for artificial agent societies: Off-line design. Artificial Intelligence, 73(1-2):231-252, 1995.

34. Y. Shoham and M. Tennenholtz. On the emergence of social conventions: Modeling, analysis and simulations. Artificial Intelligence, 94(1-2):139-166, 1997.

35. V. Teague and L. Sonenberg. Investigating commitment flexibility in multiagent contracts. In S. Parsons, P. Gymtrasiewicz, and M. Wooldridge, editors, Game Theory and Decision Theory in Agent-Based Systems, pages 267-292. Kluwer, Dordrecht (NL), 2002.

36. M. Tennenholtz. On stable social laws and qualitative equilibria. Artificial Intelligence, 102(1):1-20, 1998.

37. L. van der Torre. Contextual deontic logic: Normative agents, violations and independence. Annals of Mathematics and Artificial Intelligence, 37(1-2):33-63, 2003.

38. L. van der Torre and Y. Tan. Contrary-to-duty reasoning with preference-based dyadic obligations. Annals of Mathematics and Artificial Intelligence, 27(1-4):49-78, 1999.

39. H. Verhagen. On the learning of norms. In Procs. of Modelling Autonomous Agents in a MultiAgent World (MAAMAW'99), 1999.

40. G. H. von Wright. Deontic logic. Mind, 60:1-15, 1951. 


\title{
A Common Ontology of Agent Communication Languages: Modeling Mental Attitudes and Social Commitments using Roles
}

\author{
Guido Boella ${ }^{\mathrm{a}, *}$, Rossana Damiano ${ }^{\mathrm{a}}$, Joris Hulstijn ${ }^{\mathrm{b}}$ and Leendert van der Torre ${ }^{\mathrm{c}}$ \\ a Universitàdi Torino, Italy - email: \{guido,rossana\}@di.unito.it \\ b Vrije Universiteit, Amsterdam, The Netherlands - email: jhulstijn@feweb.vu.nl \\ ${ }^{\mathrm{c}}$ University of Luxembourg, Luxembourg - email: leendert@vandertorre.com
}

\begin{abstract}
There are two main traditions in defining a semantics for agent communication languages, based either on mental attitudes or on social commitments. These traditions share speech acts as operators with preconditions and effects, and agents playing roles like speaker and hearer, but otherwise they rely on distinct ontologies. They refer not only to either belief and intention or various notions of social commitment, but also to distinct speech acts and distinct kinds of dialogue. In this paper, we propose a common ontology for both approaches based on public mental attitudes attributed to role instances. Public mental attitudes avoid the unverifiability problem of private mental states, while reusing the logics and implementations developed for FIPA compliant approaches. Moreover, a common ontology of communication primitives allows for the construction of agents which do not need separate reasoning modules to participate in dialogues with both mental attitudes and social commitments compliant agents. Moreover, a common ontology of communication primitives allows for the construction of agents participating in and combining the full range of dialogues covered by the individual approaches without having to redefine the existing protocols to cope with new dialog types. We illustrate how to extend the ontology to a semantics for agent communication and how to define mappings from existing semantics to the new one.
\end{abstract}

Keywords: Agent Communication Languages, dialogue, speech acts, communication primitives, roles

\section{Introduction}

Agents are components in modern software that can be added to a dynamic and complex environment. Agents can sense the state of the environment and alter it by acting. Agents are autonomous, in the sense that they direct there own behavior, according to a set of goals or tasks. Agents may be designed to achieve goals and tasks of different stakeholders. Moreover, the agents in an environment can be heterogeneous in the sense that they are built in different ways. But although agents may have different goals and may be built in different ways, they can interact with other agents in the surrounding environment. The interaction mechanism is based on an agent communication language. Because an agent communication language should make heterogeneous agents able to communicate, there is a need for standardization of both the agent communication languages and the languages for expressing the content of the messages that are communicated.

In this paper, the ontology of agent communication languages is studied for standardization. Agent communication languages share the idea that agents communicate by performing speech acts (Austin, 1962; Searle, 1969), and that these actions can be modeled as a kind of planning operators with preconditions and effects (Cohen and Perrault, 1979; Allen and Perrault, 1980). Longer stretches of dialogue can be explained by plans, that structure attempts of participants to reach their joint goals, e.g. (Lochbaum, 1998). However, many variations of agent communication languages have been designed on these common speech act foundations, thus making the standardization effort difficult.

*Corresponding author: Guido Boella, Università di Torino, Italy - email: guido@ di.unito.it 
The partial ontology visualized in Figure 1 highlights the distinctions in terms of communication primitives between two main traditions in agent communication. To the left of the agent are his mental attitudes like beliefs and intentions, which are included in preconditions and effects of speech acts used in information seeking dialogues based on speech acts such as inform and request. This side has been popularized by the standardization efforts of the Foundation for Intelligent Physical Agents (FIPA, 2002a). To the right of the agent, we find the social semantics tradition, developed as a reaction to FIPA (Singh, 2000). The agent is related to his social commitments both as creditor and as debtor, which are adopted in the semantics of speech acts used in negotiation or persuasion. Though both kinds of dialogue rely on the terminology of commitment, requesting an action in negotiation means something else than defending a proposition in persuasion after a challenge. In Section 5 we therefore distinguish action commitment to give a semantics of speech acts like request or propose in negotiation (Singh, 2000; Fornara and Colombetti, 2004), from propositional commitment used for speech acts like assert or challenge in persuasion (Hamblin, 1970; Walton and Krabbe, 1995).

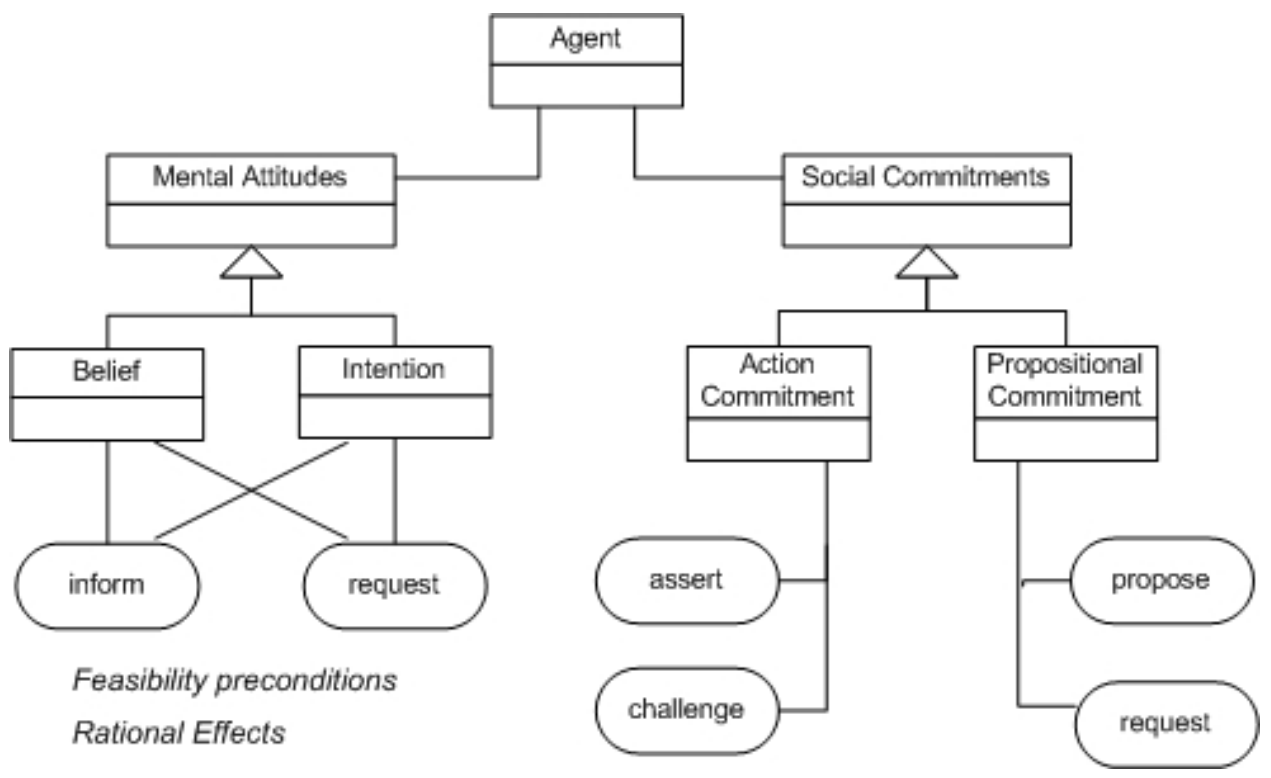

Fig. 1. Partial ontology of Mental Attitudes (FIPA) and Social Commitments based approaches

The comparison given in Table 1, further discussed in Section 7 on related research, illustrates that both traditions are distinct in most aspects. Not only the speech acts and dialogue types of the mental attitudes and social commitment based approaches are distinct, but they are based also on distinct kinds of semantics, agent attitudes, and underlying assumptions. Consequently, building a bridge between them to create a common ontology is a challenging problem. Moreover, it illustrates that for combined dialogues this problem has to be addressed. For example, consider a negotiation or persuasion dialogue that is intertwined with information seeking dialogues, like requesting the details of the object under negotiation. The separation of the two traditions makes it difficult to interpret the interaction among negotiation and information seeking in such a mixed dialogue.

The approaches that bridge the two traditions are based on a reinterpretation of the beliefs and intentions in Figure 1. The idea that meaning cannot be private, but is inter-subjective or based on a common ground, has been accepted for a long time in the philosophy of language. This has been discussed, for example, by Lewis (1969) or Stalnaker (2002) from the analytic tradition, who stress the importance of the common ground. The crucial point is that a private semantics does not make it possible for a language user to be objectively wrong about an interpretation from a third person point of view. In contrast to FIPA, beliefs and intentions are not interpreted as private mental attitudes, but as a kind of public mental attitudes, for example as grounded beliefs (Gaudou et al., 2006a) or as ostensible beliefs, or public opinions (Nickles et al., 2006). 


\begin{tabular}{|c|c|c|}
\hline & Mental Attitudes (FIPA) & Social Commitments \\
\hline Attitudes & beliefs, intentions & propositional commitments, action commitments \\
\hline Speech acts & inform, request & propose, assert, challenge \\
\hline Semantics & model theoretic semantics & operational semantics \\
\hline Dialogue types & information seeking & negotiation, persuasion \\
\hline Attitude & cooperative & competitive \\
\hline Assumptions & sincerity, cooperativity & fear of social disapproval or sanction \\
\hline
\end{tabular}

In this paper we build an ontology for public mental attitudes in agent communication centered on the concept of role. Communication in multi-agent systems is often associated with the roles agents play in the social structure of the systems (Zambonelli et al., 2003; Ferber et al., 2003), but existing approaches to the semantics of agent communication languages do not take roles into account, and thus far roles have not been associated with public mental attitudes in agent communication languages. Roughly, the basis of the idea to build the ontology on roles is that we associate beliefs and intentions with a role instance played by an agent, and that these role-based mental attitudes have a public character. The various alternatives for public mental attitudes can be compared only when it is shown how existing approaches can be mapped onto them. In this paper we show how this can be done for our own role-based semantics. In particular, we answer the following four questions:

1. What is a role-based ontology for agent communication and how can it be extended to a semantic language?

2. How can the agent communication language of FIPA be mapped onto the role-based language?

3. How can languages based on action commitments be mapped onto the role-based language?

4. How can languages based on propositional commitments be mapped onto the role-based language?

In this paper, a mapping from one communication language to another, means that for all expressions in the first language, the semantic representation for the first language can be replaced by a semantic representation in the representation format that is normally used for the second language. Ideally, this mapping should respect the intended meaning of the speech acts, but in some cases, we will see that information may get lost in the process. Therefore we do not aim for a complete mapping, in the sense that the original semantics is reflected completely in our role-based semantics, but we aim to maintain the spirit of the other language. The success criteria are that all dialogues in the original agent communication languages can be given a corresponding meaning in our role-based language.

We explain the main challenges in these four questions below. The ontology of agent communication answering the first question is a list of concept definitions and relations among them to describe the semantics of agent communication. As an illustrative example, in Figure 2 we visualize the speech act inform.

This figure visualizes two agents playing respectively roles of type $r_{1}$ and $r_{2}$ in a dialogue game, and should be read as follows. Since agents can play the same role several times, either simultaneously or sequentially, a role instance is characterized by an agent $x, y$, a role type $r_{1}, r_{2}$ and a session $s$ of a dialogue game type. The lefthand side visualizes agent $x$ (bottom) with role instance $x r_{1} s$ (top), and the righthand side visualizes agent $y$ (bottom) with role instance $y r_{2} s$ (top). Thus, compared to most existing models, we distinguish the private state from the public face, for each agent playing a role. We associate beliefs $(B)$ and intentions $(I)$ with the role instances too; these are the public mental attitudes. The beliefs and intentions of the role instance are constructed by the introduction or removal of beliefs and intentions by the speech acts, according to the rules describing the dialogue game type, called constitutive rules. Since the performance of a speech acts is public for those participating in a dialogue, also the changes are public.

Agent $x$ in role $r_{1}$ informs agent $y$ in role $r_{2}$ that $\varphi$, and therefore $\varphi$ becomes part of his public beliefs, as specified by the feasibility precondition of inform in FIPA, under the assumption that this belief persists. Moreover, the reason that he informs the other agent is that he wants him to believe $\varphi$, and therefore 

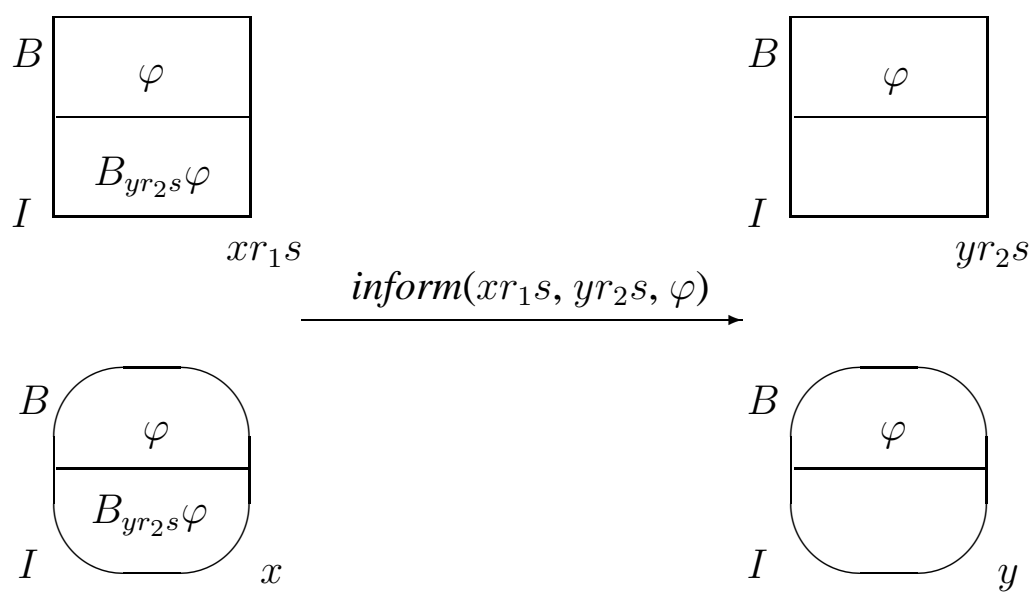

Fig. 2. Private and public mental attitudes.

$B_{y r_{2} s} \varphi$ is part of public intentions of agent $x$ in role $r_{1}$, according to the constitutive rules of the dialogue game type. If agent $y$ (publicly) believes that agent $x$ is reliable, then his public beliefs will contain $\varphi$ too, as visualized by $\varphi$ in the top right square of the Figure 2 .

The public beliefs and intentions of a dialogue participant in a particular role may differ in interesting ways from his private beliefs and intentions, but if the agents are sincere, then they will privately believe $\varphi$ too, as visualized by $\varphi$ in both the left and bottom right squares of Figure 2. The figure is explained in more detail in Section 3.1. The ontology has to clearly define the notion of a role, given the many interpretations of roles in computer science and in other sciences, and it has to distinguish role from role instance, and from the agent itself. The challenge in defining roles is to explain how mental attitudes can be attributed to role instances, and how these mental attitudes can be made public. As part of this problem, it has to be clarified which mental attributes should be assigned to the role instance and which to the agent. Finally, the set of concepts has to be extended to a semantic language, with all the constructs and variables needed to describe a semantics of speech acts.

A challenge in defining the mapping from FIPA to our role-based semantic language to answer the second question is to deal with the notion of precondition. For example, if an agent $x$ informs another agent that $\varphi$, for example with inform $\left(x r_{1} s, y r_{2} s, \varphi\right)$ in Figure 2, then in the original FIPA semantics, agent $x$ has a so called feasibility precondition, that he should already believe the proposition $\varphi$. If not, $x$ would be insincere. With public mental attitudes, however, it is not the case that an agent must already publicly believe $\varphi$ in his role $r_{1}$. To see why, assume that this precondition would hold. When two agents meet there are no public attitudes besides the common knowledge associated with the roles of the agents being played. So in that case, an agent would not be able to make an inform. Moreover, if the information were believed by the roles and thus were already public, then there would be no need for an inform in the first place. Clearly, the notion of precondition has to be reinterpreted in the context of public, role-based semantics.

Another challenge of this mapping is that in FIPA notions like reliability, cooperativity and sincerity are built into the conceptual system. We have to relax these properties if we want to integrate the social commitment approach, and deal for example with non-cooperative types of dialogue like argumentation or negotiation. Therefore, we have to make properties like reliability, cooperativity and sincerity explicit in the formal language, to distinguish between different contexts of use, and we have to explain how these properties can be associated with the role instances. The following formula explains the use of a reliability concept. Detailed explanation of this rule is given in Section 3.1. Since all modal operators are indexed by the same session $s$, we leave the session out of the rules.

$$
B_{y r_{2}}\left(I_{x r_{1}} B_{y r_{2}} \varphi \wedge \text { reliable }\left(x r_{1}, \varphi\right)\right) \rightarrow B_{y r_{2}} \varphi
$$


An initial problem of the mapping from the social commitment approach to answer the last questions is that there is not a single social commitment approach. There are approaches based on action commitment, and approaches based on propositional commitment. Moreover, the social commitment approaches focusing on action commitments (Singh, 2000; Fornara and Colombetti, 2004), are usually given an operational semantics. An additional challenge in how to use the proposed bridges between the two traditions to compare them is that we need either to translate one approach into the other, for all possible pairs of alternatives, or to have a common framework of reference. Moreover, it is necessary to be able to compare the individual speech acts which are not always defined for precisely the same purpose, to understand which are more general or specific in one approach or the other, and to make the hidden assumptions of the models explicit. Finally, we need to define not only commitments but also their evolution and the rules describing their evolution.

For extending our ontology into a semantic language we take a logical language that is directly derived from the FIPA semantic language SL (FIPA, 2002a), which we extend with roles. We call this language Role-SL. We extend it also with two rules to express that mental attitudes are public. We isolate a minimal notion of role, needed for agent communication. This minimal notion of role possesses what we call the characteristic property of roles: the fact that we can attribute mental attitudes to it. We take mental attitudes of a role as our ontological primitive, and derive role from it. The ontological question is then no longer "what is a role?", but rather "what are role-based mental attitudes, and how do they evolve?" To answer this question we consider why multiple sets of mental attitudes are needed, for each role the agent plays, instead of a single set for the agent itself. We define role-based mental attitudes, and explain why they are public. We discuss how it builds upon the notion of role we developed in our earlier work on normative multiagent systems (Boella and van der Torre, 2004). For the mapping from FIPA to the role-based ontology we define translation schemes for FIPA speech acts to Role-SL. For mapping of social commitment based approaches to a role-based ontology we take a similar approach, translating the states of the operational semantics, to sets of public mental attitudes in Role-SL.

The paper is structured as follows. In Section 2 we introduce our role-based ontology for agent communication, and we extend it to a semantic language. In Section 3 and 4 we map respectively the FIPA agent communication language and a social semantics (Fornara and Colombetti, 2004) into role-based semantics. In Section 5 we deal in particular with the difference between action commitments and propositional commitments, and we show how we can account for persuasion dialogues in our approach. In Section 6 we translate an instance of the propose interaction protocol from FIPA and from social semantics into our role model, to compare the two approaches. The paper ends with a discussion of related research and conclusions.

\section{Role-based ontology for agent communication}

Whereas usually in agent communication only a semantic language is given to describe the meaning of speech acts, we distinguish between an ontology and such a language. In Section 2.1 we define the ontology we use in this paper, that is, the set of concepts and the relations among them. In Section 2.2 the set of concepts is related to the semantic language SL used by FIPA, extended with the distinction between private mental attitudes of agents and public mental attitudes associated with role instances, and extended with rules reflecting that mental attitudes associated with roles are public.

\subsection{The ontology}

An agent can be part of multiple communication sessions at the same time. For example, consider an agent who informs another agent that his web services can be used by all other agents, informs a third agent that his services can only be used by agents with the appropriate certificates, requests from a fourth agent a document, and informs a fifth agent that he does not have an intention to obtain this document. Intuitively, we can make another 'copy' of the agent for each session, and we may refer to each 'copy' as 
a role playing agent or a role enacting agent (Dastani et al., 2003). In practice an agent may be required to maintain a minimal consistency among these 'copies', but in this paper we do not consider this consistency problem, because it depends on the application domain of the language.

For each role playing agent, we distinguish between the private beliefs and intentions of the agent, and the public beliefs and intentions attributed to the role instance. For example, when a role playing agent believes that the secret key of the safe used when playing a role in the organization enrolling him is "123456", then this belief is not known to everybody and therefore is not attributed to the role instance, but is part of private mental attitudes of the agent.

The public beliefs and intentions attributed to the role instance are known to everybody overhearing the conversation and aware of the rules of the dialogue game. They represent what the agent is publicly held responsible for. If an agent does not adhere to his role by for example refusing what he has said before, then he can be sanctioned or blamed. Consequently, once an agent starts lying, he has to continue the dialogue consistently with these lies, independently of his real beliefs. We consider beliefs and intentions only, because knowledge may be considered as a strong kind of belief, and goals have a very minor role in the agent communication languages we consider in this paper such as the FIPA language.

Besides the uniqueness of the private mental attitudes and the variance of the public mental attitudes according to the roles an agent is playing, there are various other good reasons why one might want to distinguish the mental attitudes of a role instance from the mental attitudes of the agents themselves:

- If we wish to model that an agent is bluffing (telling something he is not sure of) or lying (telling something he believes is false), then we have to account for divergences between the private mental attitudes and the public ones;

- In a negotiation situation the agents only have the intention of reaching an agreement in common, but they do not have to communicate truthful information or their real preferences. For example, in a negotiation a bid does not need to correspond to the actual reservation price of the agent.

- In persuasion, if the aim is to win a dispute, an agent can adopt a point of view "for the sake of the argument", in order to show its inconsistency or counterintuitive consequences, without actually endorsing the view himself (Caminada (2004)).

- It is necessary to keep apart the motivations for playing a role from the rules of the game which affect the state of the roles. For example, an agent may be sincere, in the sense that he really acts as expected from his role, for pure cooperativity or for the fear of a sanction.

Since the mental attitudes of role instances are the foundation of our ontology, they have to be precisely defined. In particular, we have to clearly distinguish the mental attitudes attributed to a role instance from mental attitudes attributed to the agent playing the role. The mental attitudes attributed to a role instance consist of two parts. First, they contain those public beliefs and intentions following from uttering speech acts. Second, the mental attitudes of a role consist of commonly held beliefs about the attitudes of roles. For example, a buyer in negotiation is expected to prefer lower prices. Moreover, agents in an organization may be expected to be sincere, cooperative and reliable when communicating with other agents in the same organization. No other mental attitudes are attributed to role instances.

Moreover, we distinguish two kinds of mental attitudes attributed to role instances as the result of speech acts. For example, consider an agent informing another one that "it is raining". We can derive that the agent publicly believes that it is raining, and that he has the public intention that the other agent believes it is raining, because these mental attitudes are presupposed when making such an action. More precisely, the agent's belief that it is raining is presupposed, and the belief that the other agent will believe it is the desired effect.

Definition 1 Mental attitudes of role instances $(R)$ consist of beliefs $(B)$ and intentions $(I)$, and are precisely:

1. Consequences of speech acts on the mental attitudes of agents overhearing the uttering of the speech act, representing what the agent is publicly held responsible for, distinguishing between: 
(a) Presuppositions of the speech act; these are public mental attitudes from which we can infer that they held already before the speech act was uttered;

(b) Other public mental attitudes following from the speech acts; from these we cannot infer that they held already before the speech act was uttered.

\section{Common knowledge associated with the roles being played by the agents.}

We build our ontology on the concept of "mental attitudes of a role instance" in Definition 1, rather than on the concept of role instance or role. However, the ontology has to clarify also what we mean by role instance, given the many interpretations of roles in computer science and in other sciences. This is a non-trivial problem, since there are numerous incompatible notions of role available in the literature (Baldoni et al., 2007; Loebe, 2007; Masolo et al., 2005; Herrmann, 2007). In this paper we are interested in role instances mostly, where a role is a generic class and a role instance is associated with an agent playing the role in a dialogue session.

Definition 2 The characteristic property of a role instance is that it is channel end of communication, and contains mental attitudes representing the state of the session of interaction. It may or may not refer to other properties of roles such as expertise needed to play the role, powers associated with the role, expectations associated with the role, and so on.

The starting point of the common ontology for agent communication consists of the ontology of mental attitudes approach to agent communication: agents, beliefs, intentions, utterances, speech act types, also called performatives, and dialogue types. Then we make two decisions. The first decision is to include sessions rather than leaving them out of the ontology. We could leave them out of the ontology, since the rules of the game always refer to a single session only, and we do not consider cross-session rules. Though leaving the sessions out of the ontology would simplify our ontology a little, it obscures the fact that agents can play multiple sessions of the same dialogue type at the same time. In particular, we like to compare the entities to which we attribute the private and public mental attitudes. Since, at some moment in time, a private mental attitude is attributed to an agent, and a public mental attitude is attributed to an agent playing a role in a session, we include the sessions in the ontology too.

The second decision concerns the representation of distinct abstractions, in particular role types and role instances, dialogue types and dialogue session instances, and speech act types and utterances. As usual there are various ways to represent them in a conceptual model. We choose to represent them all as first class citizens in our model to clarify the precise relations among them, and again to express that mental attitudes are attributed to agents and role instances. Therefore, we extend the basic ontology with dialogue sessions and roles to represent role instances with their public mental attitudes. We add several relations between these concepts. A role instance is played by an agent in a session, a dialogue session has a dialogue type, and agents and role instances are described by their beliefs and intentions. Moreover, utterances are made by role instances, directed towards role instances, and have a speech act type.

Moreover, we introduce rules defining the effects of speech acts on the mental attitudes of role instances. We call them the constitutive rules or norms of the dialogue game played by the agents. The constitutive rules describe a speech act in terms of the beliefs and intentions of agents, in the context of a dialogue type. Therefore, a speech act may mean something else in sessions of another dialogue type. Finally, in the ontology we do not consider the content of speech acts.

We have chosen to exclude various other concepts and relations from our ontology. For example, the belief of an agent that it is raining, and the belief of another agent that the first agent has this belief, are related. These complex relations among beliefs and intentions are not represented in the ontology. To detail such relations, a modal language is used in the more detailed semantic languages describing the mental attitudes. Moreover, also the content of the beliefs and intentions, and the constitutive norms are described in a semantic language referring to beliefs and intentions. 
Definition 3 A role-based ontology for agent communication is a tuple

$$
\langle A, R T, S, R, P L, D T, S D T, R D T, B, I, A D, S A, U, U S A, U R S, U R A, C R, C R D, C R M\rangle
$$

where:

- A is a set of agents, like $x$ and $y$.

- RT is a set of role types, like $r, r_{1}, r_{2}, \ldots$

- $S$ is a set of sessions, like $s, s_{1}, s_{2}, \ldots$

- $R$ is a set of role instances, like $i$ or $j$.

- PL : $A \times R N \times S \mapsto R$ is a role playing function, such that for each role instance $i$ there is precisely one agent $x$, role type $r$ and session $s$ with $i=P L(x, r, s)$. We also say that agent $(i)=x$, roletype $(i)=r$, and $\operatorname{session}(i)=s$.

- DT is a set of dialogue types, such as information seeking, negotiation and persuasion.

- SDT :S DT if a function giving the dialogue type of a dialogue session.

- $R D T \subseteq R T \times D T$ if a relation describing the role types that can participate in a dialogue session of a dialogue type.

- $B$ and I are sets of beliefs and intentions respectively, either of individual agents or of role instances. We write $M=B \cup I$ for the set of all mental attitudes.

- $A D: A \cup R \mapsto B \cup I$ is a function associating mental attitudes with agents and role instances.

- SA is a set of speech act types: inform, request, propose, etc.

- $U$ is a set of utterances or messages, i.e., instances of uttering a speech act.

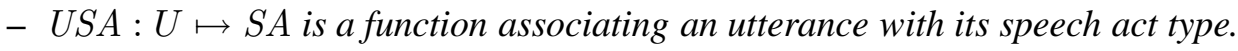

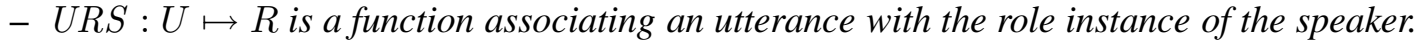

- URA : U $\mapsto 2^{R}$ is a function associating an utterance with the (set of) role instances of the addressees.

- CR is a set of constitutive rules of dialogue games.

- CRD :CR SA $\times D T$ is a function that associates with each constitutive rule a speech act and dialogue type.

- CRM : CR $\mapsto 2^{M}$ is a function detailing which mental attitudes are affected by a constitutive rule.

The ontology is visualized in Figure 3. For readability we have also added the full names of the concepts, and ways to read some of the relations. It is instructive to compare our ontology in Figure 3 with the one in Figure 1. Besides the extension with roles and sessions, we have now only beliefs and intentions, and no longer commitments. Moreover, the class of speech acts in Figure 3 and the individual speech acts in Figure 1 represents that we have a single uniform way to provide a semantics for them, and therefore a uniform way to formalize dialogue sessions of varying dialogue types.

Finally, we explain in which sense the mental attitudes of role instances of the kind defined in Definition 3 are public. This is based on our notion of public communication, defined as follows. Communication is public for every agent who overhears the speech acts, and has the same prior knowledge of roles and dialogue types. Consequently, for public communication, the mental attitudes of role are public. The public beliefs and intentions attributed to the role instances are known to everybody overhearing the conversation according to the constitutive rules or norms. Constitutive rules explain how roles can be autonomous, in the literal sense of the term: auto-nomos, the behavior of roles is described by their own norms. The constitutive rules exist only since roles as descriptions are collectively accepted by the agents. Moreover, since the constitutive rules which change the beliefs and intentions publicly attributed to roles are accepted by all agents, the beliefs and intentions resulting from the application of a constitutive rule after a speech act are public too. This issue of the construction of social reality is discussed in (Boella and van der Torre, 2006a). 


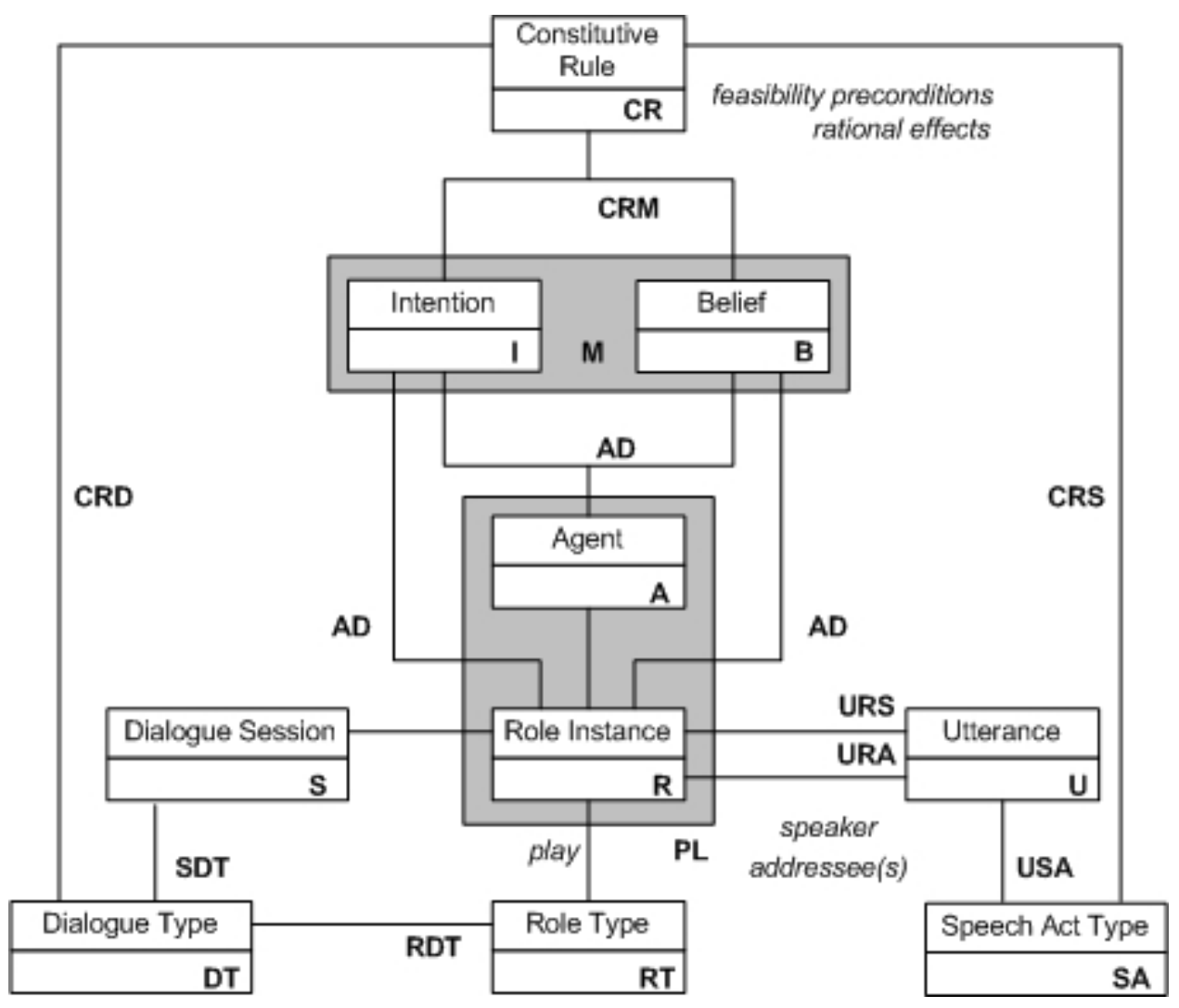

Fig. 3. The role-based ontology of communication.

Definition 4 Communication is public in a group $S \subseteq A$, if and only if:

1. every agent in $S$ hears the speech acts that are uttered, and has the same knowledge of roles and dialogue types.

2. every agent in $S$ knows which role is played by the utterers of the speech acts.

3. every agent in $S$ knows which beliefs are typically attributed to roles types.

The constitutive rules may describe also the rules of the protocol the role is defined for, or the roles agents play in the social structure of the systems. See Section 7.2 for a discussion.

\subsection{The semantic language Role-SL}

We now sketch how a BDI logic can be designed based on our ontology, which can be used as a semantic language. In principle, we can use any existing modal and temporal predicate logic, but we have to introduce the following two important extensions.

1. We have to introduce role instances, and therefore roles and sessions. More precisely, we have to replace the agent identifiers in the BDI logic by a superclass consisting of agents and role instances. Another way to look at this, is that the set of agents in the BDI logic is partitioned into agents and role instances.

2. We have to make mental attitudes of role instances public. This is done by adding rules that state that if a role instance has a belief or intention, then the other role instances will believe this.

The distinction between agents and role instances raises the question how the agent and its role instances are related. Since this issue is relevant for our mapping of FIPA speech acts to our Role-SL, where we have to formalize FIPA property of sincerity. We discuss this issue in Section 3.1. 
There are various BDI logics which can be used, or we can design a new language from scratch. Since our aim in this paper is to show the generality of our ontology, we show how to extend FIPA's specification language (FIPA, 2002a) and its predecessor (Sadek, 1991; Sadek et al., 1997; Sadek, 1999). However, this language has some limitations, which have been discussed in the literature (Pitt and Mamdani, 1999; Wooldridge, 2000). The three main problems are that the logic is based on a rather complex relation between desires, goals and intentions, that there is no explicit representation of a conditional or rule (simulated by material implication according to (FIPA, 2002a)), and that there is no explicit representation of time. In particular, the absence of explicit time has the consequence that there is no formal way to reason about the persistence of mental attitudes over time, for example which preconditions persist after the speech act has been uttered. Though solutions to some of these problems have been proposed (Herzig and Longin, 2000), there is no commonly accepted standard formalism, and we therefore continue to use FIPA's SL. In this paper we are not concerned with these limitations, our aim is to show only how to adapt this language for our ontology.

\subsubsection{Basis of the Semantic Language Formalism}

In FIPA-SL, propositions about mental attitudes and actions are formalized in a first order modal logic with identity. We follow the exposition in Annex $A$ of (FIPA, 2002a) and refer to (Sadek, 1991) for details of this logic. The components of the formalism used in our Role-SL are as follows, where we extend the set of agents with role instances. Since we use the variables $i$ and $j$ to refer to role instances, we introduce new variables $e$ and $f$ to refer to the superclass of agents and role instances.

$-p, q, \ldots$ are taken to be closed formulas denoting propositions,

- $\varphi$ and $\psi$ are formula schemas, which stand for any closed proposition,

- $e, f \in A \cup R$ are schematic variables denoting agents and role instances, and,

$-\models \varphi$ means that $\varphi$ is valid.

The mental model of an agent or role instance in SL or Role-SL is based on the representation of three primitive attitudes: belief, uncertainty and choice (or, to some extent, goal). They are respectively formalized by the modal operators $B, U$, and $D$. FIPA-SL uses the letter $C$ for the latter, but to avoid confusion with commitments later in this paper, we use the letter $D$. Formulas using these operators can be read as follows:

- $B_{e} p: e$ (implicitly) believes (that) $p$,

- $U_{e} p: e$ is uncertain about $p$ but thinks that $p$ is more likely than $\neg p$, and,

- $D_{e} p: e$ desires that $p$ currently holds.

The logical model for the belief operator $B$ in both SL and Role-SL is a KD45 Kripke structure (see, for example, (Halpern and Moses, 1985)) with the fixed domain principle (see, for example, (Garson, 1984)).

To enable reasoning about action, the universe of discourse of FIPA-SL involves, in addition to individual objects and agents, sequences of events. To talk about complex plans, events (or actions) can be combined to form action expressions, noted as $a$ :

- $a_{1} ; a_{2}$ is a sequence in which $a_{2}$ follows $a_{1}$

- $a_{1} \mid a_{2}$ is a nondeterministic choice, in which either $a_{1}$ happens or $a_{2}$, but not both.

FIPA-SL introduces the operators Feasible, Done and Agent to enable reasoning about actions:

- Feasible $(a, p)$ means that $a$ can take place and if it does $p$ will be true just after that,

- Done $(a, p)$ means that $a$ has just taken place and $p$ was true just before that,

- Agent $(e, a)$ means that $e$ denotes the only subject (agent or role) that ever performs (in the past, present or future) the actions which appear in action expression $a$,

- Single $(a)$ means that $a$ denotes an action that is not a sequence. Any individual action is Single. The composite act $a ; b$ is not Single. The composite act $a \mid b$ is Single iff both $a$ and $b$ are Single.

From belief, choice and events, the concept of persistent goal is defined. An agent or role instance $e$ has $p$ as a persistent goal, if $e$ has $p$ as a goal and is self-committed toward this goal until $e$ comes to believe that 
the goal is achieved or to believe that it is unachievable. Intention is defined as a persistent goal imposing the agent to act. Formulas as $P G_{e} p$ and $I_{e} p$ are intended to mean that " $e$ has $p$ as a persistent goal" and " $e$ has the intention to bring about $p$ ", respectively. The definition of $I$ entails that intention generates a planning process. See Sadek (1992); Cohen and Levesque (1990) for the details of a formal definition of intention.

The FIPA documentation notes that there is no restriction on the possibility of embedding mental attitude or action operators. For example, formula $U_{e} B_{f} I_{f}$ Done $\left(a, B_{e} p\right)$ informally means that agent or role instance $e$ believes that, probably, agent or role instance $f$ thinks that $f$ has the intention that action $a$ be done before which $e$ has to believe $p$.

FIPA-SL also uses various abbreviations, including the following ones:

1. Feasible $(a)=$ Feasible $(a$, True $)$

2. Done $(a)=$ Done $(a$, True $)$

3. Done $(j, a)=\operatorname{Done}(a) \wedge \operatorname{Agent}(j, a)$.

4. Possible $(\varphi)=(\exists a)$ Feasible $(a, \varphi)$

5. Bif $_{e} \varphi=B_{e} \varphi \vee B_{e} \neg \varphi$

The mapping of our ontology on Role-SL is as follows:

- The set of agents $A$ and role instances $R$ are mapped to indexes of modal operators, and can be used in the description of actions. The sets of roles types $R T$ and sessions $S$ can be used in the description of role instances using the function $P L$. We write $x r s$ for $P L(x, r, s)$.

- The set of dialogue types $D T$ and the function $S D T$ giving the dialogue type of a dialogue session are represented by a special proposition dialogue $(s, d)$, and similarly for the other relations. The relation between roles and dialogue types can be used as a constraint: if an agent plays a role in a session, then the role must be allowed given the dialogue type of the session. Similarly other kinds of constraints can be expressed in Role-SL.

- The beliefs $B$ and intentions $I$ as well as the function $A D: A \cup R \mapsto B \cup I$ associating mental attitudes with agents and role instances are represented by modal formulas.

- Speech act utterances $U$ are included in the set of actions.

- The constitutive rules $C R$ are represented by formulas of Role-SL.

- The function $C R D$ associating a speech act type and dialogue type with each constitutive rule, is represented by prefixing all rules with the relevant speech act type and dialogue type.

- The function $C R M: C R \mapsto 2^{M}$ detailing which mental attitudes are affected by a constitutive rule is made explicit by the description of the mental attitude in the constitutive rule.

Moreover, the semantic model of FIPA-SL leads to the properties of FIPA-SL in Table 2. In Role-SL, we have to decide whether we accept the properties for the role instances only, or also for the agents. Property 0,1 and 2 are related to the logic of action and we therefore accept it both for role instances as for the agents, $e$ or $f$. Property 3, 4 and 5 are related to the pre and postconditions of speech acts, and we therefore accept them only for role instances, $i$ and $j$. The following three properties are of particular interest for the role model.

- Each role has correct knowledge about its own mental states, in particular, its beliefs about its intentions are correct. These rules correspond to FIPA's (2002a) schema $\varphi \leftrightarrow B_{e} \varphi$, (called Property 0 in Table 2, since it has no explicit name in FIPA) where $\varphi$ is starting by an operator formalizing a mental attitude of agent or role instance $e$.

- Property 1 and 5 hold only under some conditions, and we therefore add a condition $\Phi$ to the properties of FIPA-SL. For example, Property 5 holds only for feasibility preconditions that persist. We do not discuss these conditions in this paper, see the FIPA documentation for details. Properties 3 , 4 and 5 are discussed in Section 3, when we present the speech acts together with their feasibility preconditions $F P$ and rational effects $R E$. 


\begin{tabular}{|l|l|l|}
\hline Property 0 & $\left(\left(B_{e} I_{e} p\right) \leftrightarrow I_{e} p\right) \wedge\left(\left(B_{e} \neg I_{e} p\right) \leftrightarrow \neg I_{e} p\right)$ & (RL1) \\
& $\left(\left(B_{e} B_{e} p\right) \leftrightarrow B_{e} p\right) \wedge\left(\left(B_{e} \neg B_{e} p\right) \leftrightarrow \neg B_{e} p\right)$ & (RL2) \\
Property 1 & $\Phi \wedge I_{e} p \rightarrow I_{e}$ Done $($ act $1|\ldots|$ act \\
PL $)$ & (RL3) \\
Property 2 & $I_{e}$ Done $($ act $) \rightarrow B_{e}$ Feasible $($ act $) \vee I_{e} B_{e}$ Feasible(act) $)$ & (RL4) \\
Property 3 & $I_{i}$ Done $($ act $) \rightarrow I_{i} R E($ act $)$ & (RL5) \\
Property 4 & $B_{i}\left((\right.$ Done $($ act $) \wedge$ Agent $(j$, act $)) \rightarrow I_{j} R E($ act $\left.)\right)$ & (RL6) \\
Property 5 & $B_{i}(\Phi \wedge$ Done $($ act $) \rightarrow F P($ act $))$ & (RL7) \\
\hline
\end{tabular}

Table 2

The properties of FIPA-SL

\subsubsection{Extensions for public mental attitudes}

In our model, we have to add axioms which are necessary due to the separation of role instances from agents. Adding axioms to a logical system raises the question whether the system is still consistent, and even when the system remains consistent, whether the new axioms do not interfere with the existing properties. This is the main logical issue in this paper; the other issues are of a more conceptual nature. We do not give a formal proof of the consistency of our system, because the full logical details of FIPA-SL and therefore also Role-SL are beyond the scope of this paper, but we rely on the following intuitive argument. The axioms which make mental attitudes public are the most challenging ones, since they imply properties not found in FIPA: if a role instance believes some mental attitude of a role instance, then also the other role instances engaged in the same session will believe it. First note that SL and therefore Role-SL only models what holds after a speech act; there is no reference to the past (or, in general, reference to time). Then, if we consider what can be derived from speech acts only, these properties can be interpreted in FIPA too. It means that an agent has communicated all its beliefs, and that agents believe of other agents only what has been communicated to them. Consequently, since our model can be interpreted as a special case in which agents immediately tell all they know to the other agents, and FIPA is generally applicable, so also to this special case, our axioms do not lead to inconsistency. The remaining axioms are harmless, since they only derive beliefs for particular kinds of formulas.

The first two properties concern the public character of mental attitudes when they are attributed to roles. Since the constitutive rules $C R$ have as effect public mental attitudes, all inferences on the basis of speech acts are public. This motivates rules (RL8) and (RL9), expressing that each role instance has complete knowledge about the other role instances' beliefs and intentions. For example, from left to right, if an agent $y$ in role $r_{2}$ in session $s$ has the belief that $p$, expressed by $B_{y r_{2} s} p$, then this implies that any other agent $x$ playing any role $r_{1}$ in the same session $s$ believes this, $B_{x r_{1} s} B_{y r_{2} s} p$. Since properties always refer to the same session, we leave out the session to increase readability, and we write $B_{y r_{2}} p$ implies $B_{x r_{1}} B_{y r_{2}} p$, or that $B_{i} \neg p$ implies $B_{j} B_{i} \neg p$, where $i=x r_{1}$ and $j=y r_{2}$. The reading from right to left is analogous. If role instance $y r_{2}$ believes something about $x r_{1}$ 's beliefs, then role instance $x r_{1}$ actually believes that. Note that if $x r_{1}=y r_{2}$, the rule coincides with Property 0 of FIPA-SL in Table 2 reflecting positive and negative introspection:

$$
\begin{aligned}
& \left(B_{y r_{2}} p \leftrightarrow B_{x r_{1}} B_{y r_{2}} p\right) \wedge\left(\neg B_{y r_{2}} p \leftrightarrow B_{x r_{1}} \neg B_{y r_{2}} p\right) \\
& \left(I_{y r_{2}} p \leftrightarrow B_{x r_{1}} I_{y r_{2}} p\right) \wedge\left(\neg I_{y r_{2}} p \leftrightarrow B_{x r_{1}} \neg I_{y r_{2}} p\right)
\end{aligned}
$$

Another aspect of the fact that communication is public, is that the agents are aware of the communication. So if a belief is associated with a role instance, then it is believed by all agents participating in the same dialogue session. Under the same conditions as in the previous two rules, if an agent $y$ in role $r_{2}$ in session $s$ has the public belief that $p$, expressed by $B_{y r_{2} s} p$, then this implies that any other agent $x$ playing any role $r_{1}$ in the same session $s$ believes this privately, $B_{x} B_{y r_{2} s} p$.

$$
\begin{aligned}
& \left(B_{y r_{2}} p \wedge \operatorname{play}\left(x, r_{1}\right) \leftrightarrow B_{x} B_{y r_{2}} p\right) \wedge\left(\neg B_{y r_{2}} p \wedge \operatorname{play}\left(x, r_{1}\right) \leftrightarrow B_{x} \neg B_{y r_{2}} p\right) \\
& \left(I_{y r_{2}} p \wedge \operatorname{play}\left(x, r_{1}\right) \leftrightarrow B_{x} I_{y r_{2}} p\right) \wedge\left(\neg I_{y r_{2}} p \wedge \operatorname{play}\left(x, r_{1}\right) \leftrightarrow B_{x} \neg I_{y r_{2}} p\right)
\end{aligned}
$$


In FIPA the architecture used for the exchange of messages is not part of the logical formalism, but it is described in a separate document, FIPA (2002b). A speech act is put inside a message which is exchanged between agents on an agent platform. We need the following rule to link the logical model with such a message architecture and information about the roles of agents. The action send() corresponds to (part of) the FIPA message structure specification, with parameters speech act type ( $s a \in S A$ ), sender $(x \in A)$, receiver $(y \in A)$, content $(p)$, and conversation-id $(s \in S)$. Information about the roles that agents are playing in the current session $s$ is stored in relation $P L$.

$$
\operatorname{Done}(\operatorname{send}(s a, x, y, p, s, \ldots)) \wedge P L\left(x, r_{1}, s\right) \wedge P L\left(y, r_{2}, s\right) \rightarrow \operatorname{Done}\left(s a\left(x r_{1} s, y r_{2} s, p\right)\right)
$$

\section{From FIPA to roles}

For the role-based semantics for FIPA communicative acts we have to define the constitutive rules $C R$ of our dialogue game. Using Role-SL, the mapping from FIPA to roles may seem straightforward, since we have defined Role-SL as an extension of FIPA-SL. The constitutive rules are therefore simply the instantiations of property (RL6) and (RL7) of FIPA. However, we have to define the sincerity and cooperativity assumption of FIPA, and we have to show that the resulting formalization using public mental attitudes instead of private mental attitudes has the expected behavior in the FIPA protocols.

In Section 3.1 we formalize the sincerity, cooperativity and reliability assumption in Role-SL, in Section 3.2 we compare the formalization the FIPA speech acts in Role-SL with their formalization in FIPASL, and finally in Section 3.3 we illustrate the formalization by an example.

\subsection{The formalization of sincere cooperative agents, and other agent types}

In this section we formalize sincerity, cooperativity and reliability in Role-SL. In the following Section 3.2, we consider in more detail how these kinds of properties are modeled in FIPA-SL. Roughly, sincerity is explicit in FIPA-SL, but cooperativity and reliability are represented more implicitly.

FIPA assumes sincerity, in the sense that an agent can make an inform speech act only when it believes the content is true, and it requests or proposes something only when it has the intention that it be achieved. This roughly corresponds to the version of sincerity given by Sadek, inspirator of the FIPA approach.

"Sincerity: An agent $i$ cannot have the intention that an agent $j$ comes to believe a proposition $p$ without believing $p$ herself or without having the intention to come to believe it. (Sadek, 1999, p. 182)"

In our role-based model, we model a weaker kind of sincerity. In our model, sincerity means that the public beliefs or intentions of the role instances are attributed also to the agents themselves. This is a common assumption in everyday life (Goffman, 1959). In our visualization of role-based communication in Figure 2, this kind of sincerity represents an implication from the top level to the bottom level. We model it in three steps. First, we have preconditions and effects, attached to speech acts. These generate public beliefs about role instances, similar to the original sincerity properties for agents in FIPA. Second, we add an explicit property of sincerity, modeled by rule (RL13) and (RL14). Third, we assume that whenever an agent is a FIPA agent, then it must be sincere. Using these steps, an agent can be sincere in one role, or in one session, and insincere in another. As before, we do not explicitly represent the session in the properties, so it is implicitly assumed that all references to role instances refer to the same session.

$$
\begin{aligned}
& B_{x r} \varphi \wedge \operatorname{sincere}(x, r, \varphi) \rightarrow B_{x} \varphi \\
& I_{x r} \varphi \wedge \operatorname{sincere}(x, r, \varphi) \rightarrow I_{x} \varphi
\end{aligned}
$$

However, this does not imply that our model of Role-SL, together with the sincerity assumption, coincides with the FIPA model, since we do not have an implication the other way around. In other words, like FIPA we model the implication of public mental attitudes towards the private ones, but in the constitutive rules of the game we refer only to public mental attitudes. We do not assume that private mental attitudes 
are made public, and therefore we do not assume that the private mental attitudes can be reused to check the preconditions of speech acts. In other words, the preconditions of speech acts are exclusively used to infer the public mental attitudes attributed to role instances and not to plan speech acts. In FIPA, preconditions are used to plan a speech act. Insincere speech acts are not possible since the speaker must believe the preconditions before executing a speech act. In our approach, instead, the private mental state of the speaker is kept separate from the public mental attitudes, unless sincerity is explicitly assumed. In this way it is possible to perform a speech act where neither preconditions are believed nor effects are intended by the speaker.

Cooperativity is implicit in FIPA. Although there is no formal property in FIPA-SL or in the speech acts discussed in the next section, if agents are not cooperative, there is no communication. It is assumed that agents recognize the intentions of other agents, and try to help them in reaching their intentions. Historically, the implicit assumption of cooperativity makes sense, because part of the FIPA framework was developed originally for designing cooperative natural language dialogue systems (Sadek, 1999), on the basis of the plan-based theory of speech acts, and a theory of rational agents (Cohen and Perrault, 1979; Allen and Perrault, 1980; Lochbaum, 1998). Such approaches propose general principles for planor intention recognition: based on the actions of the speaker, the hearer can try to recognize the underlying intention or plan, and subsequently adjust her (cooperative) response. The most obvious cooperative principle is intention adoption:

"The minimal principle of cooperation (or the intention adoption principle) states that agents must not only react when they are addressed but, more than that, they must adopt the interlocutor's intention whenever they recognize it, and if there have no objection to adopt it." (Sadek, 1999, p. 182)

However, when agent communication languages are used in applications on the internet, or for noncooperative interaction types like negotiation and debate, cooperativity can no longer be assumed as a general principle. Only in specific circumstances it makes sense to assume cooperativity, for example among agents that are known or trusted.

In our role-based model, we can make implicit cooperativity assumptions explicit. In particular, a successful request may add an intention to the intentions of the hearer, but only when we have a rule such as (RL15), that specifies that the hearer is cooperative. Cooperativity is a role-role relationship, which depends on the social context in which an interaction takes place.

$$
B_{y r_{2}}\left(I_{x r_{1}} \text { Done }(\text { act }) \wedge \text { cooperative }\left(y r_{2}, x r_{1} \text {, Done }(\text { act })\right)\right) \rightarrow I_{y r_{2}} \text { Done }(\text { act })
$$

Besides sincerity and cooperativity, the FIPA documentation also mentions reliability, though it neither assumes nor formalizes reliability:

"Whether or not the receiver does, indeed, adopt belief in the proposition will be a function of the receiver's trust in the sincerity and reliability of the sender." (FIPA, 2002a)

We explain the role of reliability in FIPA, using our model of role-based communication in Figure 2. The constitutive rules of the communication game operate on the mental states of the role instances. Because of the feasibility precondition, an inform creates a proposition $\varphi$ in the beliefs of the speaker $x r_{1}$, and a intention that $y r_{2}$ will come to believe $\varphi$. Only if the hearer $y r_{2}$ believes that the speaker is reliable, rule (RL16) will transfer the belief to the hearer's beliefs:

$$
B_{y r_{2}}\left(I_{x r_{1}} B_{y r_{2}} \varphi \wedge \text { reliable }\left(x r_{1}, \varphi\right)\right) \rightarrow B_{y r_{2}} \varphi
$$

In particular, we assume that all roles are assumed to be reliable about their own mental states; however, this assumption does not extend to the private beliefs of agents, but remains confined to the beliefs of the roles they play. In our framework, when a speaker says something about its own beliefs or intentions, the belief that it believes what has said will be immediately added to the role of the hearer as a belief of the speaker role, without implying that the agent playing the hearer role believes it as well.

For more about issues of trust and reliability, we refer to Demolombe (2001); Liau (2003); Dastani et al. (2004). 


\begin{tabular}{|c|c|c|}
\hline$\langle x$, inform $(y, \varphi)\rangle$ & $\begin{array}{l}\text { FP: } B_{x} \varphi \wedge \neg B_{x}\left(\text { Bif }_{y} \varphi \vee \text { Uif }_{y} \varphi\right) \\
\mathrm{RE}: B_{y} \varphi\end{array}$ & $(1)$ \\
\hline$\langle x$, request $(y$, act $)\rangle$ & $\begin{array}{l}\text { FP: } F P(a c t)[x \backslash y] \wedge B_{x} \text { Agent }(y, a c t) \wedge \neg B_{x} I_{y} \text { Done }(a c t) \\
\text { RE: Done }(\text { act })\end{array}$ & (2) \\
\hline $\begin{array}{l}\langle x, \operatorname{agree}(y,\langle x, \text { act }\rangle, \varphi)\rangle \equiv \\
\left\langle x, \operatorname{inform}\left(y, I_{x} \operatorname{Done}(\langle x, \text { act }\rangle, \varphi)\right)\right\rangle\end{array}$ & $\begin{array}{l}\text { FP: } B_{x} \alpha \wedge \neg B_{x}\left(\text { Bif }_{y} \alpha \vee \text { Uif }_{y} \alpha\right) \\
\mathrm{RE}: B_{y} \alpha \\
\alpha=I_{x} \text { Done }(\langle x, a c t\rangle, \varphi)\end{array}$ & (3) \\
\hline $\begin{array}{l}\langle x, \text { refuse }(y,\langle x, \text { act }\rangle, \varphi)\rangle \equiv \\
\langle x, \text { disconfirm }(y, \text { feasible }(\langle x, \text { act }\rangle))\rangle ; \\
\quad\langle x, \text { inform }(y, \varphi \wedge \neg \text { Done }(\langle x, \text { act }\rangle) \wedge \\
\left.\left.\quad \neg I_{x} \text { Done }(\langle x, \text { act }\rangle)\right)\right\rangle\end{array}$ & $\begin{aligned} & \text { FP: } B_{x} \neg \text { feasible }(\langle x, \text { act }\rangle) \wedge B_{x}\left(B_{j} \text { feasible }(\langle x, \text { act }\rangle) \vee\right. \\
& U_{x} \text { feasible }(\langle x, \text { act }\rangle) \wedge B_{y} \alpha \wedge \neg B_{x}\left(\text { Bif }_{y} \alpha \vee \text { Uif }_{y} \alpha\right) \\
& \mathrm{RE}: B_{y}\left(\neg \text { feasible }(\langle x, \text { act }\rangle) \wedge B_{y} \alpha\right) \\
& \alpha=\varphi \wedge \neg \text { Done }(\langle x, \text { act }\rangle) \wedge \neg I_{x} \text { Done }(\langle x, \text { act }\rangle)\end{aligned}$ & (4) \\
\hline $\begin{array}{l}\langle x, \operatorname{propose}(y,\langle x, \text { act }\rangle, \varphi)\rangle \equiv \\
\quad\left\langle x, \text { inform }\left(y, I_{y} \text { Done }(\langle x, \text { act }\rangle, \varphi)\right.\right. \\
\left.\left.\quad \rightarrow I_{x} \text { Done }(\langle x, a c t\rangle, \varphi)\right)\right\rangle\end{array}$ & $\begin{array}{l}\text { FP: } B_{x} \alpha \wedge \neg B_{x}\left(\text { Bif }_{y} \alpha \vee \text { Uif }_{y} \alpha\right) \\
\text { RE: } B_{y} \alpha \\
\alpha=I_{y} \text { Done }(\langle x, a c t\rangle, \varphi) \rightarrow I_{x} \text { Done }(\langle x, a c t\rangle, \varphi)\end{array}$ & (5) \\
\hline $\begin{array}{l}\langle x, \text { accept proposal }(\langle y, \text { act }\rangle, \varphi)\rangle \equiv \\
\left\langle x, \text { inform }\left(y, I_{x} \text { Done }(\langle y, \text { act }\rangle, \varphi)\right)\right\rangle\end{array}$ & $\begin{array}{l}\text { FP: } B_{x} \alpha \wedge \neg B_{x}\left(\text { Bif }_{y} \alpha \vee \text { Uif }_{y} \alpha\right) \\
\text { RE: } B_{y} \alpha \\
\alpha=I_{x} \text { Done }(\langle y, \text { act }\rangle, \varphi)\end{array}$ & (6) \\
\hline $\begin{array}{l}\langle x, \text { reject proposal }(y,\langle y, \text { act }\rangle, \varphi, \psi)\rangle \equiv \\
\left\langle x, \operatorname{inform}\left(y, \neg I_{x} \text { Done }(\langle y, \text { act }\rangle, \varphi) \wedge \psi\right)\right\rangle\end{array}$ & $\begin{array}{l}\text { FP: } B_{x} \alpha \wedge \neg B_{x}\left(\text { Bif }_{y} \alpha \vee U i f_{y} \alpha\right) \\
\text { RE: } B_{y} \alpha \\
\alpha=\neg I_{x} \text { Done }(\langle y, \text { act }\rangle, \varphi) \wedge \psi\end{array}$ & (7) \\
\hline
\end{tabular}

Table 3

The definition of speech acts in FIPA-SL

\subsection{The translation from FIPA to roles}

FIPA-SL contains twenty-two speech acts. Most of them are defined in terms of inform and request. The purpose of an inform act for the speaker is to get the hearer to believe some information. The purpose of a request is to get the hearer to do some act. This requires that the speaker does not already believe that the hearer has such an intention.

In this paper we consider seven speech acts. The FIPA definitions of speech acts in terms of feasibility preconditions (FP) and rational effects (RE) are summarized in Table 3. The ones we have selected are the most basic and often used ones, and we choose these to illustrate both the translation and two protocols, the request interaction protocol (FIPA, 2002c), and the propose interaction protocol. The request protocol uses request, agree and refuse, and the propose protocol uses propose, accept proposal and reject proposal. The difference between a request and a propose is that a request concerns an action of the hearer, whereas a propose concerns an action of the speaker.

Note that while in Table 3 the definitions are given on agents $x$ and $y$, in Table 4 they are given on role instances $i$ and $j$.

The definitions we use in Role-SL are given in Table 4, which are based on the following simplifications:

1. We have left out preconditions concerning uncertain beliefs, as represented by the modal operator Uif in FIPA-SL, since they are not relevant for the general principle, and in most uses of FIPA-SL they are removed. For example, the feasibility precondition of inform in the FIPA documentation is $B_{i} \varphi \wedge \neg B_{i}\left(\right.$ Bif $\left._{j} \varphi \vee \operatorname{Uif}_{j} \varphi\right)$, but we left out the $\operatorname{Uif} \varphi$ (and we wrote $\operatorname{Bif}_{i} \varphi$ in full). However, if needed for some application, the extension with uncertain beliefs is straightforward.

2. In request, we drop the felicity precondition from FIPA that $i$ thinks it feasible for $j$ to perform act.

3. We use Done $(j, a c t)$ rather than Done (act) as RE, which means that Agent $(j, a c t)$ is a RE rather than a FP.

4. Note that unlike the speech act definitions based on inform, request does not have a sincerity precondition: $B_{i} I_{i}$ Done $(j, a c t)$. This does not matter, because agents are considered to be reliable about 


\begin{tabular}{|c|c|c|}
\hline$\langle i, \operatorname{inform}(j, \varphi)\rangle$ & $\begin{array}{l}\text { FP: } B_{i} \varphi \wedge \neg B_{i}\left(B_{j} \varphi \vee B_{j} \neg \varphi\right) \\
\text { RE: } B_{j} \varphi\end{array}$ & (8) \\
\hline$\langle i$, request $(j$, act $)\rangle$ & $\begin{array}{l}\text { FP: } \neg B_{i} I_{j} \text { Done }(j, a c t) \\
\text { RE: Done }(j, a c t)\end{array}$ & (9) \\
\hline $\begin{array}{l}\langle i, \operatorname{agree}(j, \text { act })\rangle \equiv \\
\left\langle i, \operatorname{inform}\left(j, I_{i} \text { Done }(i, \text { act })\right)\right\rangle\end{array}$ & $\begin{array}{l}\text { FP: } B_{i} I_{i} \text { Done }(i, a c t) \wedge \\
\quad \neg B_{i}\left(B_{j} I_{i} \text { Done }(i, a c t) \vee B_{j} \neg I_{i} \text { Done }(i, a c t)\right) \\
\text { RE: } B_{j} I_{i} \text { Done }(i, a c t)\end{array}$ & (10) \\
\hline $\begin{array}{l}\langle i, \operatorname{refuse}(j, \text { act })\rangle \equiv \\
\left\langle i, \operatorname{inform}\left(j, \neg I_{i} \text { Done }(i, a c t)\right)\right\rangle\end{array}$ & $\begin{aligned} & \text { FP: } B_{i} \neg I_{i} \text { Done }(i, \text { act }) \wedge \\
& \neg B_{i}\left(B_{j} \neg I_{i} \text { Done }(i, \text { act }) \vee B_{j} I_{i} \text { Done }(i, a c t)\right) \\
& \text { RE: } B_{j} \neg I_{i} \text { Done }(i, a c t) \\
&\end{aligned}$ & (11) \\
\hline $\begin{array}{l}\langle i, \operatorname{propose}(j, \text { act })\rangle \equiv \\
\left\langle i, \operatorname{inform}\left(j, I_{j} \text { Done }(i, a c t) \rightarrow I_{i} \operatorname{Done}(i, \text { act })\right)\right\rangle\end{array}$ & $\begin{aligned} \text { FP: } & B_{i}\left(I_{j} \text { Done }(i, \text { act }) \rightarrow I_{i} \text { Done }(i, \text { act })\right) \wedge \\
\neg & B_{i}\left(B_{j}\left(I_{j} \text { Done }(i, \text { act }) \rightarrow I_{i} \text { Done }(i, \text { act })\right) \vee\right. \\
& \left.B_{j} \neg\left(I_{j} \text { Done }(i, \text { act }) \rightarrow I_{i} \text { Done }(i, \text { act })\right)\right) \\
\text { RE: } & B_{j}\left(I_{j} \text { Done }(i, \text { act }) \rightarrow I_{i} \text { Done }(i, \text { act })\right)\end{aligned}$ & (12) \\
\hline $\begin{array}{l}\langle i, \text { accept proposal }(j, \text { act })\rangle \equiv \\
\left\langle i, \text { inform }\left(j, I_{i} \text { Done }(j, \text { act })\right)\right\rangle\end{array}$ & $\begin{aligned} \text { FP: } & B_{i} I_{i} \text { Done }(j, a c t) \wedge \\
& \neg B_{i}\left(B_{j} I_{i} \text { Done }(j, \text { act }) \vee B_{j} \neg I_{i} \text { Done }(j, a c t)\right) \\
\text { RE: } & B_{j} I_{i} \text { Done }(j, \text { act })\end{aligned}$ & (13) \\
\hline $\begin{array}{l}\langle i, \text { reject proposal }(j, \text { Done }(j, \text { act }))\rangle \equiv \\
\left\langle i, \text { inform }\left(j, \neg I_{i} \operatorname{Done}(j, \text { act })\right)\right\rangle\end{array}$ & $\begin{aligned} \text { FP: } & B_{i} \neg I_{i} \text { Done }(j, a c t) \wedge \\
& \neg B_{i}\left(B_{j} \neg I_{i} \text { Done }(j, \text { act }) \vee B_{j} I_{i} \text { Done }(j, \text { act })\right) \\
\text { RE: } & B_{j} \neg I_{i} \text { Done }(j, a c t)\end{aligned}$ & (14) \\
\hline
\end{tabular}

Table 4

The definition of speech acts in Role-SL

their own intentions (see RL16), so we can derive this condition whenever it is needed from the rational effect.

5. We also drop the conditions under which requests or proposals are agreed to be carried out. These are better dealt with in Section 4 below, where we explain conditional proposals, which are easier to understand starting from the Social Commitments approach.

6. In the definition of refuse we also leave out some additional idiosyncrasies of FIPA, like the fact that a refusal is expressed as a disconfirm, which is similar to inform. ${ }^{1}$ In FIPA, the reason for encoding refusal as an inform lies in the fact that FIPA assumes that a refusal should be motivated by the infeasibility of the requested act, and that the agent who cannot perform the requested act should inform the requester about the reasons for the infeasibility. In our perspective, this move is in line with the fact that we dropped this feasibility requirement from requests. In fact we have reinterpreted refuse, as the opposite of an agree.

Despite the compact representation in Table 3 and 4, often it is easier to consider the instantiations of FIPA properties as they appear in this Table 3 . We therefore list the instantiations of the speech acts in Table 5.

In the instantiation it must be noted that:

- The consequent of the instantiated rules starts always with a public mental attitude of a role: this means that every role believes the effect of the rule. For this reason we leave out the belief modality in the speech acts in instances of Property (RL6) and (RL7).

- $\Phi$ in precondition: these properties hold only if they persist in time, as in FIPA.

- We assume reliability about the mental states of roles. For example, for our interpretation of the $R E$ of inform above in (CR19), we translated the $R E$ to an intention of the speaker. In that case, we would end up with $B_{j}$ Done $(\operatorname{agree}(i, j$,Done $(i$, act $))) \rightarrow I_{i} B_{j} I_{i}($ Done $(i$, act $))$. So it is the communicative intention of $i$, that $j$ will believe that $i$ wants to do the action. Following Sadek et al. (1997)), we can simplify this kind of $I_{i} B_{j} I_{i}(\ldots)$-formula into $I_{i} \ldots$, if we assume that the hearer takes

${ }^{1}$ The difference depends on the precondition of the hearer being uncertain about the proposition or its negation. 


\begin{tabular}{|c|c|c|c|}
\hline inform & $\begin{array}{l}\text { FP } \\
\text { FP } \\
\text { RE }\end{array}$ & $\begin{array}{l}\text { Done }(\operatorname{inform}(i, j, \varphi)) \rightarrow B_{i} \varphi \\
\text { Done }(\operatorname{inform}(i, j, \varphi)) \rightarrow \neg B_{i}\left(B_{j} \varphi \vee B_{i} \neg \varphi\right) \\
\text { Done }(\operatorname{inform}(i, j, \varphi)) \rightarrow I_{i} B_{j} \varphi\end{array}$ & $\begin{array}{l}(\mathrm{CR} 17) \\
(\mathrm{CR} 18) \\
(\mathrm{CR} 19) \\
\end{array}$ \\
\hline request & $\begin{array}{l}\text { FP } \\
\text { RE }\end{array}$ & $\begin{array}{l}\text { Done }(\text { request }(i, j, \text { Done }(j, \text { act }))) \rightarrow \neg B_{i} I_{j} \text { Done }(j, \text { act }) \\
\text { Done }(\text { request }(i, j, \text { Done }(j, \text { act }))) \rightarrow I_{i} \text { Done }(j, \text { act })\end{array}$ & $\begin{array}{l}(\mathrm{CR} 20) \\
(\mathrm{CR} 21)\end{array}$ \\
\hline agree & $\begin{array}{l}\text { FP } \\
\text { FP } \\
\text { RE }\end{array}$ & $\begin{aligned} \text { Done }(\operatorname{agree}(i, j, \text { Done }(i, \text { act }))) & \rightarrow B_{i} I_{i} \text { Done }(i, \text { act }) \\
\text { Done }(\operatorname{agree}(i, j, \text { Done }(i, \text { act }))) \rightarrow & \neg B_{i}\left(B_{j} I_{i} \text { Done }(i, \text { act }) \vee\right. \\
& \left.B_{j} \neg I_{i} \text { Done }(i, \text { act })\right) \\
\text { Done }(\operatorname{agree}(i, j, \text { Done }(i, \text { act }))) \rightarrow & I_{i} \text { Done }(i, \text { act })\end{aligned}$ & $\begin{array}{l}(\mathrm{CR} 22) \\
(\mathrm{CR} 23) \\
(\mathrm{CR} 24)\end{array}$ \\
\hline refuse & $\begin{array}{l}\text { FP } \\
\text { FP } \\
\text { RE }\end{array}$ & $\begin{aligned} & \text { Done }(\operatorname{refuse}(i, j, \text { Done }(i, \text { act }))) \rightarrow B_{i} \neg I_{i} \text { Done }(i, \text { act }) \\
& \text { Done }(\operatorname{refuse}(i, j, \text { Done }(i, \text { act }))) \rightarrow \neg B_{i}\left(B_{j} \neg I_{i} \text { Done }(i, \text { act }) \vee\right. \\
&\left.B_{j} I_{i} \text { Done }(i, \text { act })\right) \\
& \text { Done }(\operatorname{refuse}(i, j, \text { Done }(i, \text { act }))) \rightarrow \neg I_{i} \text { Done }(i, \text { act })\end{aligned}$ & $\begin{array}{l}(\mathrm{CR} 25) \\
(\mathrm{CR} 26) \\
(\mathrm{CR} 27)\end{array}$ \\
\hline propose & $\begin{array}{l}\text { FP } \\
\text { FP }\end{array}$ & $\begin{array}{l}\text { Done }(\text { propose }(i, j, \text { Done }(i, \text { act }))) \rightarrow B_{i}\left(I_{j} \text { Done }(i, \text { act }) \rightarrow I_{i} \text { Done }(i, \text { act })\right) \\
\text { Done }(\text { propose }(i, j, \text { Done }(i, \text { act }))) \rightarrow \\
\neg B_{i}\left(\left(B_{j} I_{j} \text { Done }(i, \text { act }) \rightarrow I_{i} \text { Done }(i, \text { act }) \vee\right.\right. \\
\left.\left.\quad B_{j} \neg\left(I_{j} \text { Done }(i, \text { act }) \rightarrow I_{i} \text { Done }(i, \text { act })\right)\right)\right) \\
\text { Done }(\operatorname{propose}(i, j, \text { Done }(i, \text { act }))) \rightarrow B_{j}\left(I_{j} \text { Done }(i, \text { act }) \rightarrow I_{i} \text { Done }(i, \text { act })\right)\end{array}$ & $\begin{array}{l}\text { (CR28) } \\
(\mathrm{CR} 29) \\
(\mathrm{CR} 30)\end{array}$ \\
\hline $\begin{array}{l}\text { accept } \\
\text { proposal }\end{array}$ & $\begin{array}{l}\text { FP } \\
\text { FP } \\
\text { RE }\end{array}$ & $\begin{aligned} & \text { Done }(\text { accept proposal }(i, j, \text { Done }(j, \text { act }))) \rightarrow B_{i} I_{i} \text { Done }(j, \text { act }) \\
& \text { Done }(\text { accept proposal }(i, j, \text { Done }(j, \text { act }))) \rightarrow \neg B_{i}\left(B_{j} I_{i} \text { Done }(j, \text { act }) \vee\right. \\
&\left.B_{j} \neg I_{i} \text { Done }(j, \text { act })\right) \\
& \text { Done }(\text { accept proposal }(i, j, \text { Done }(j, \text { act }))) \rightarrow I_{i} \text { Done }(j, \text { act })\end{aligned}$ & $\begin{array}{l}(\mathrm{CR} 31) \\
(\mathrm{CR} 32) \\
(\mathrm{CR} 33)\end{array}$ \\
\hline $\begin{array}{l}\text { reject } \\
\text { proposal }\end{array}$ & $\begin{array}{l}\text { FP } \\
\text { FP } \\
\text { RE }\end{array}$ & $\begin{aligned} & \text { Done }(\text { reject proposal }(i, j, \text { Done }(j, \text { act }))) \rightarrow B_{i} \neg I_{i} \text { Done }(j, \text { act }) \\
& \text { Done }(\text { reject proposal }(i, j, \text { Done }(j, \text { act }))) \rightarrow \neg B_{i}\left(B_{j} \neg I_{i} \text { Done }(j, \text { act }) \vee\right. \\
&\left.B_{j} I_{i} \text { Done }(j, \text { act })\right) \\
& \text { Done }(\text { reject proposal }(i, j, \text { Done }(j, \text { act }))) \rightarrow \neg I_{i} \text { Done }(j, \text { act })\end{aligned}$ & $\begin{array}{l}(\mathrm{CR} 34) \\
(\mathrm{CR} 35) \\
(\mathrm{CR} 36)\end{array}$ \\
\hline
\end{tabular}

Table 5

Rules expressing preconditions and effects of speech acts in the FIPA translation

the speaker to be reliable concerning reports about its own mental states. By rule (RL16) we derive (CR24) above.

Analogously in rule (CR30), the effect of a propose is directly believed by the addressee $j$, since it concerns a mental attitude of role $i$, who is thus considered reliable about it. Note the contrast with for example the effect of agree in (CR24). This effect is directly expressed by a public intention of the speaker $i$, which is, thus also believed by the hearer $j$. In contrast, the effect of a propose is expressed as a material implication between two intentions, since it represent the way FIPA-SL allows to model conditional intentions. For this reason, the implication must be nested inside a belief operator.

- Moreover, the second precondition is usually canceled by the intended effect if it is successful.

\subsection{Example}

We have made substantial changes to the FIPA semantics, and we therefore have to illustrate that our mapping works. We illustrate it using an example.

To illustrate the rules, we give a example of a short negotiation about household tasks, that we will use to illustrates the distinction between the FIPA approach and the social commitment approach.

$\mathrm{x}-1$ : Could you do the dishes?

$\mathrm{y}-1$ : Only if you put the garbage out.

$\mathrm{x}-2$ : OK.

The dialogue can be interpreted as follows. Initially, agent $x$ makes a request in role $i=x r_{1} s$. Then $y$ makes a counter proposal in role $j=y r_{2} s$, but because there are no speech acts to represent counter 
proposals, we model this as an implicit refusal, followed by a conditional proposal from $y$. Note that the original FIPA definition of propose does not allow conditional proposals, however, as shown in the next section, this is the interpretation of proposals given by social commitments approach. So in order to make the two languages comparable we drop the condition that the agent of the proposed action is the speaker and we allow to propose joint plans which are sequences of actions by different agents. We discuss this issue in more depth in Section 6.2.

In order to deal with composite actions, and actions executed by various agents, we have to make explicit some rationality assumptions which are assumed in the FIPA model (p. 38). Typically, such assumptions depend on the agent planning architecture:

- A rationality constraint concerning the intention that other agents perform an action: if $i$ wants that action act is done by $j$, then $i$ has the intention that $j$ has the intention to do act:

$$
\left.\left.I_{i} \text { Done }(j, a c t)\right) \rightarrow I_{i} I_{j} \text { Done }(j, a c t)\right)
$$

- Some FIPA axioms concern the execution of complex actions. For example Property 1 in Table 2. In a similar vein, we add two rules concerning the distribution of tasks. Taking inspiration from (Cohen and Levesque, 1990), we add a rule to express that if an agent intends a joint action, then it intends that each part is done at the right moment. If $a c t=a c t_{1} ; \ldots ; a c t_{n}$, where ";" is the sequence operator, act is a joint action:

$$
I_{i} \text { Done }(a c t) \rightarrow\left(\text { Done }\left(\operatorname{act}_{1}\left(i_{1}\right) ; \ldots ; \operatorname{act}_{k}\left(i_{k}\right)\right) \rightarrow I_{i} \text { Done }\left(\operatorname{act}_{k+1}(i)\right)\right)
$$

If $\operatorname{act}_{k+1}(j)$ and $i \neq j$, then by axiom RL37:

$$
\left.\left.I_{i} \text { Done }(\text { act }) \rightarrow\left(\text { Done } \operatorname{act}_{1}\left(i_{1}\right) ; \ldots ; \operatorname{act}_{k}\left(i_{k}\right)\right) \rightarrow I_{i} I_{j} \text { Done } \operatorname{act}_{k+1}(j)\right)\right)
$$

Each agent in the group wants that the others do their part.

In our example, we introduce a composite plan which is the concatenation of $j$ 's action of doing the dishes and the action by $i$ of moving the garbage out: kitchen $(i, j) \equiv \operatorname{dishes}(j) ; \operatorname{garbage}(i)$.

\section{Example 1}

$\mathrm{x}-1$ : Could you do the dishes? $\quad \operatorname{request}(i, j$, Done $(j$, dishes $))$

$\mathrm{y}-1$ : Only if you put the garbage out? refuse $(j, i$, Done $(j, \operatorname{dishes}))$

$\mathrm{x}-2$ : OK. $\quad$ accept $\operatorname{proposal}(i, j, \operatorname{Done}(\operatorname{kitchen}(i, j)))$

We start with an empty set of facts about the mental attitudes of $x$ and $y$. In Table 6 we depict the evolution of the public mental states of agents $x$ and $y$ in their roles $i$ and $j$.

After turn x-1 we apply rules (CR20) and (CR21). So that it adds to the public mental state of $i$ the precondition of a request and its rational effect. Since the mental states are public, from $I_{i}$ Done $(j$, dishes $)$ (by RL37), we could also write $I_{i} I_{j}$ Done $(j$, dishes))) we can derive that also $j$ is aware of this intention $B_{j} I_{i}$ Done ( $j$,dishes) (RL9) and from Proposition 0 (RL1) that also $i$ is aware of his own intentions $B_{i} I_{i}$ Done $(j$, dishes $)$.

If we assume persistence for the sincerity precondition, and for the mental attitudes which are not immediately contradicted by later updates, rules (CR25), (CR26) and (CR27) produce the next state of affairs after the implicit refusal of $y-1$. The second precondition from (CR26) is canceled by the success of the effect. A proper representation of this kind of reasoning requires an explicit notion of time and persistence, and rules about changes as a result of actions and events. See Boella et al. (2007) for an initial attempt based on defeasible logic with temporal operators.

The implicit proposal of $\mathrm{y}-1$ is interpreted using rules (CR28) - (CR30). It adds a public belief of $j$ about a relation between the intentions of $i$ and $j$ of doing the kitchen work together.

$$
B_{j}\left(I_{i} \operatorname{Done}(\operatorname{kitchen}(i, j)) \rightarrow I_{j} \text { Done }(\operatorname{kitchen}(i, j))\right)
$$




\begin{tabular}{|c|c|c|}
\hline & Role instance $i$ & Role instance $j$ \\
\hline \multirow[t]{2}{*}{$\begin{array}{l}\mathrm{x}-1: \\
\text { request }\end{array}$} & $\begin{array}{l}\text { BELIEFS } \\
\neg B_{i} I_{j} \text { Done }(j, \text { dishes })\end{array}$ & BELIEFS \\
\hline & $\begin{array}{l}\text { INTENTIONS } \\
\text { Done }(j, \text { dishes })\end{array}$ & INTENTIONS \\
\hline \multirow{2}{*}{$\begin{array}{l}\mathrm{y}-1: \\
\text { refuse }\end{array}$} & BELIEFS & BELIEFS \\
\hline & $\begin{array}{l}\text { INTENTIONS } \\
\text { Done }(j, \text { dishes })\end{array}$ & $\begin{array}{l}\text { INTENTIONS } \\
\neg I_{j} \text { Done }(j, \text { dishes })\end{array}$ \\
\hline \multirow[t]{2}{*}{$\begin{array}{l}\mathrm{y}-1: \\
\text { propose }\end{array}$} & $\begin{array}{l}\text { BELIEFS } \\
I_{i} \text { Done }(\text { kitchen }(i, j)) \rightarrow I_{j} \text { Done }(\text { kitchen }(i, j))\end{array}$ & $\begin{array}{l}\text { BELIEFS } \\
I_{i} \text { Done }(\text { kitchen }(i, j)) \rightarrow I_{j} \text { Done }(\text { kitchen }(i, j))\end{array}$ \\
\hline & $\begin{array}{l}\text { INTENTIONS } \\
\text { Done }(j, \text { dishes })\end{array}$ & INTENTIONS \\
\hline \multirow[t]{2}{*}{$\begin{array}{l}\mathrm{x}-2: \\
\text { accept } \\
\text { proposal }\end{array}$} & $\begin{array}{l}\text { BELIEFS } \\
I_{i} \text { Done }(\text { kitchen }(i, j)) \rightarrow I_{j} \text { Done }(\text { kitchen }(i, j)) \\
I_{i} \text { Done }(\text { kitchen }(i, j)) \\
I_{j} \text { Done }(\text { kitchen }(i, j)) \\
\left.I_{j} \text { Done }(j, \text { dishes })\right) \\
\text { Done }(j, \text { dishes }) \rightarrow I_{i} \text { Done }(i, \text { garbage })\end{array}$ & $\begin{array}{l}\text { BELIEFS } \\
I_{i} \text { Done }(\text { kitchen }(i, j)) \rightarrow I_{j} \text { Done }(\text { kitchen }(i, j)) \\
I_{i} \text { Done }(\text { kitchen }(i, j)) \\
I_{j} \text { Done }(\text { kitchen }(i, j)) \\
I_{j} \text { Done }(j, \text { dishes }) \\
\text { Done }(j, \text { dishes }) \rightarrow I_{i} \text { Done }(i, \text { garbage })\end{array}$ \\
\hline & $\begin{array}{l}\text { INTENTIONS } \\
\text { Done }(\text { kitchen }(i, j))\end{array}$ & $\begin{array}{l}\text { INTENTIONS } \\
\text { Done }(\text { kitchen }(i, j)) \\
\text { Done }(j, \text { dishes })\end{array}$ \\
\hline
\end{tabular}

Table 6

An example of dialogue interpretation in Role-SL. Note that kitchen $(i, j) \equiv \operatorname{dishes}(j) ; \operatorname{garbage}(i)$

Since this belief concerns the mental states of $j$, and every role is considered reliable about his own mental states (CR30), then also $i$ publicly adopts this belief:

$$
B_{i}\left(I_{i} \text { Done }(k i t c h e n(i, j)) \rightarrow I_{j} \text { Done }(\text { kitchen }(i, j))\right)
$$

Note the implication here. The implication derives from the fact that the proposal still needs to be accepted by $i$, before $j$ will really adopt the conditional intention.

Finally, after acceptance $\mathrm{x}-2$ by rules (CR31) - (CR33) the state of the public mental attitudes is updated. Note that rule (CR33) implicitly implements rule (RL16) so that the intention of conditionally moving the garbage is immediately public:

$$
I_{i} \operatorname{Done}(\operatorname{kitchen}(i, j))
$$

At this point more information can be inferred than the one provided by those rules.

First of all, by modus ponens, given the intention of $i$, it is possible to derive the intention of $j$ to do the kitchen work:

$$
I_{j} \operatorname{Done}(\operatorname{kitchen}(i, j))
$$

Moreover, it is possible to infer from the intention of $j$ of doing the kitchen work that $j$ has the intention to do his part of the plan, since it is the right moment (from rule RL38):

$$
I_{j} \text { Done }(j, \operatorname{dishes}(j))
$$

Moreover, from rule (RL37), we can infer that after $j$ does the dishes (Done( $j$, dishes)), $j$ will adopt the intention of moving the garbage out:

$$
\text { Done }(j \text {, dishes }) \rightarrow I_{i} \text { Done }(i, \text { garbage })
$$

Note that $\neg I_{j}$ Done $(j$, dishes $)$, which persists from the refusal, is now overridden by the new inference $I_{j}$ Done $(j$, dishes $)$. 


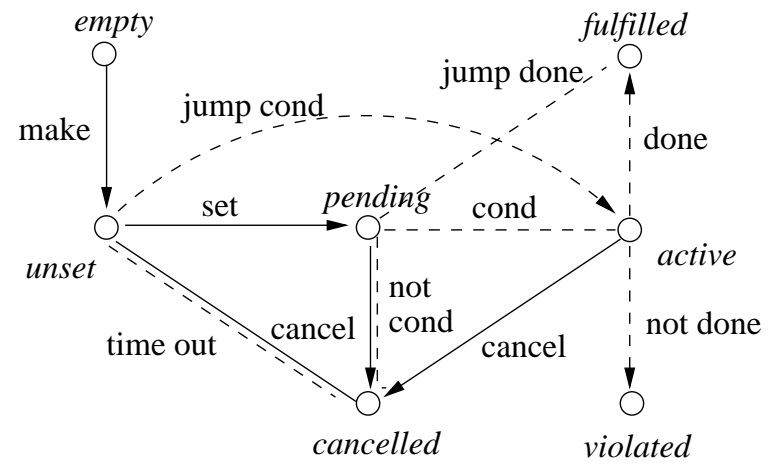

Fig. 4. Commitment State Automaton (Fornara and Colombetti, 2004).

\begin{tabular}{lrll} 
Action & Change & & \\
\hline make & empty & $\rightarrow$ & $C($ unset $, i, j, \varphi \mid \psi)$ \\
set & $C($ unset $, i, j, \varphi \mid \psi)$ & $\rightarrow$ & $C($ pending, $, i, j, \varphi \mid \psi)$ \\
cancel & $C(-, i, j, \varphi \mid \psi)$ & $\rightarrow$ & $C($ canceled $, i, j, \varphi \mid \psi)$
\end{tabular}

Table 7

Commitment Change Rules, solid lines in Figure 4 (Fornara and Colombetti, 2004).

The example shows the interplay between mental attitudes and how the public character of roles' mental attitudes is used to make inferences in the interpretation process. Note that from the example nothing can be inferred about the private mental states of agents $x$ and $y$. To make these inferences it is necessary to assume explicitly the sincerity of both agents.

\section{From commitments to roles}

In this section we show how to define a social semantics in the role-based model. We selected the version of Fornara and Colombetti (2004), because it is worked out in detail.

\subsection{Social Commitment Semantics}

The social commitment approach uses an operational semantics, meaning that the semantics of the speech acts of an agent communication language are expressed as changes in the state a commitment can be in. A social commitment is a relationship that gets established between two agents: the debtor, i.e., the agent who makes the commitment, and a creditor, i.e., the agent to which the commitment is made. The commitment is to achieve or maintain some state of affairs, expressed by a proposition. In many applications, commitments are made under certain conditions. Therefore, the conditions under which commitments are made are also expressed. Representation $C($ state, $i, j, \varphi \mid \psi)$ expresses a social commitment of status state, between debtor $i$ and creditor $j$, to accomplish or maintain $\varphi$, under condition that $\psi$.

A commitment can have different states: unset (i.e., to be confirmed), pending (i.e., confirmed, but its condition is not true), active (i.e., confirmed and its condition is true), fulfilled (i.e., its content is true), violated (i.e., the content is false even if the commitment was active), or canceled (e.g., the debtor does not want to be committed to the action). This state can be modified by speech acts of the participants or by external events, like the execution of an action.

The state changes can be depicted in the form of a finite state automaton (Figure 4). The effects of external events and of speech acts on the status of a commitment are represented in Table 7 and Table 8 respectively. Using these semantics, Fornara and Colombetti define speech acts as given in Table 9. They 


\begin{tabular}{|c|c|c|c|c|c|}
\hline Rule & Label & Event & Status Change & & \\
\hline 1. & done & $v(\varphi):=1$ & $C($ active $, i, j, \varphi \mid \top)$ & $\rightarrow$ & $C($ fulfilled $, i, j, \varphi \mid \top)$ \\
\hline 2. & not done & $v(\varphi):=0$ & $C($ active $, i, j, \varphi \mid \top)$ & $\rightarrow$ & $C($ violated $, i, j, \varphi \mid \top)$ \\
\hline 3. & condition & $v(\psi):=1$ & $C($ pending $, i, j, \varphi \mid \psi)$ & $\rightarrow$ & $C($ active $, i, j, \varphi \mid \top)$ \\
\hline 4. & not condition & $v(\psi):=0$ & $C($ pending $, i, j, \varphi \mid \psi)$ & $\rightarrow$ & $C($ canceled $, i, j, \varphi \mid \psi)$ \\
\hline 5. & jump condition & $v(\psi):=1$ & $C($ unset $, i, j, \varphi \mid \top)$ & $\rightarrow$ & $C($ active $, i, j, \varphi \mid \top)$ \\
\hline 6. & jump done & $v(\varphi):=1$ & $C($ pending $, i, j, \varphi \mid \psi)$ & $\rightarrow$ & $C($ fulfilled $, i, j, \varphi \mid \psi)$ \\
\hline 7. & time out & & $C($ unset $, i, j, \varphi \mid \psi)$ & $\rightarrow$ & $C($ canceled $, i, j, \varphi \mid \psi)$ \\
\hline
\end{tabular}

Table 8

Update Rules: dashed lines in Figure 4 (Fornara and Colombetti, 2004).

\begin{tabular}{|l|l|}
\hline Precondition & Definition \\
\hline & inform $(i, j, P):=\left\{C_{i}() \cdot\right.$ make $(i, j, P, \top) . \operatorname{set}($ pending $\left.)\right\}$ \\
\hline & request $(i, j, P, Q):=\left\{C_{i}() \cdot \operatorname{make}(j, i, P, Q)\right\}$ \\
\hline & $\operatorname{promise}(i, j, P, Q):=\left\{C_{i}() \cdot \operatorname{make}(i, j, P, Q) \cdot \operatorname{set}(\right.$ pending $\left.)\right\}$ \\
\hline$\exists C_{i}($ unset $, i, j, P \mid Q)$ & accept $\left(i, j, C_{i}(\right.$ unset $\left., i, j, P \mid Q)\right):=\left\{C_{i}(\right.$ unset $, i, j, P \mid Q)$. set $($ pending $\left.)\right\}$ \\
\hline$\exists C_{i}$ (unset $\left., i, j, P \mid Q\right)$ & reject $\left(i, j, C_{i}(\right.$ unset $\left., i, j, P \mid Q)\right):=\left\{C_{i}(\right.$ unset $, i, j, P \mid Q)$. set $($ cancelled $\left.)\right\}$ \\
\hline
\end{tabular}

Table 9

Speech acts of Fornara and Colombetti

use unset for the state resulting from a request and pending for conditional commitments, whose condition is not yet true.

Our mapping is only partial. For example, we do not have timeouts. There is no translation of the update rules, because we model them by means of the logic, e.g., using modus ponens in case of conditionals.

\subsection{Translating Commitment States}

In order to perform the translation, we adopt the following methodology: first, we map each commitment state to certain beliefs and intentions of the roles. Second, according to the constitutive rules $(C R)$ of the role model, a speech act directly changes those beliefs and intentions in such a way to reflect the commitment introduction or change of state.

In a social commitments semantics in general, the difference between propositional and action commitment lies only in the type of their content: an action or a proposition. Fornara and Colombetti (2004) do not have a separate type of action in their language. They can only distinguish the kind of proposition: an informative proposition, or a proposition that represents a desired state of affairs. Therefore there is no substantial difference between an inform and a promise in Table 9. By contrast, according to Walton and Krabbe (1995), a propositional commitment can be seen as a kind of action commitment to defend it. In the mapping between social commitments and the role model a new distinction emerges: rather than having commitment stores, we model propositional commitments as beliefs of the role and action commitments as intentions. How to capture the idea of a commitment to defend one's position is discussed in Section 5. In this section we focus on action commitment only.

Here, we represent conditional commitments $C($ state, $i, j, p \mid q)$ as a conditional intention $p$, for example, of role $i$ in case $q$ is true: $B_{i}\left(q \rightarrow I_{i}(p)\right)$. Note that since the condition $q$ is outside the intention operator, the whole implication is nested in a (public) belief of the role, as we did with the translation of FIPA. We believe that conditional attitudes are better accounted for in a conditional logic, like Input/Output logic (Makinson and van der Torre (2000)). As in Section 3, we stick to FIPA's solution for the sake of clarity, while being aware of its limitations. Moreover, like in Section 3, we use a simplified notation Done $(i, a c t)$ to indicate that agent $i$ is supposed to perform the action act. The Done() construct turns an action into a proposition, expressing a state of affairs. 
Given these preliminaries, the state translations are as follows:

- An unset commitment corresponds to a conditional intention believed by the creditor.

$$
C \text { (unset, } i, j, \text { Done }(i, \text { act }) \mid q) \equiv B_{j}\left(q \rightarrow I_{j} I_{i} \text { Done }(i, \text { act })\right)
$$

In the antecedent of this rule, the commitment condition $q$ becomes a condition on the intention assumed by the creditor. At this stage, no mental attitude is attributed to the debtor: it has not publicly assumed any intention, but has only been publicly requested to.

- A commitment is pending when it is a conditional intention of the creditor and the debtor of the commitment conditionally wants to perform the action provided the associated condition $q$ is true, and the creditor has this as a belief:

$$
\begin{aligned}
& C(\text { pending }, i, j, \text { Done }(i, \text { act }) \mid q) \equiv B_{j}\left(q \rightarrow I_{j} I_{i} \text { Done }(i, \text { act })\right) \wedge \\
& \quad B_{i}\left(q \rightarrow I_{i} \text { Done }(i, \text { act })\right) \wedge B_{j}\left(q \rightarrow I_{i} \text { Done }(i, \text { act })\right)
\end{aligned}
$$

In the automaton of Figure 4, the unset and pending states are disjoint. In the above formulation however, pending implies unset. So if it is necessary to mirror the exact definition, the unset state must be augmented, e.g.:

$$
\begin{gathered}
C \text { (unset }, i, j, \text { Done }(i, \text { act }) \mid q) \equiv B_{j}\left(q \rightarrow I_{j} I_{i} \text { Done }(i, \text { act })\right) \wedge \\
\left(\neg B_{i}\left(q \rightarrow I_{i} \text { Done }(i, \text { act })\right) \vee \neg B_{j}\left(q \rightarrow I_{i} \text { Done }(i, \text { act })\right)\right)
\end{gathered}
$$

- A commitment is active when it is both an intention of the debtor and of the creditor, and the pending condition is true:

$$
C \text { (active, } i, j, \text { Done }(j, \text { act }) \mid \top) \equiv I_{i} \text { Done }(i, \text { act }) \wedge I_{j} \text { Done }(i, \text { act })
$$

Note that to make a pending commitment active, it is sufficient that the condition $q$ is believed to be true, since from

$$
B_{i}\left(q \wedge\left(q \rightarrow I_{i} \operatorname{Done}(i, a c t)\right)\right)
$$

we can derive $I_{i}($ Done $(i, a c t))$ with rule (RL1).

- Commitments are violated or fulfilled when they are intentions of the creditor and the content of the commitment is respectively true or false according to the beliefs of the creditor (abstracting here from temporal issues):

$$
\begin{aligned}
& C \text { (fulfilled }, i, j, \text { Done }(i, \text { act }) \mid \top) \equiv B_{j} \text { Done }(i, \text { act }) \wedge I_{j} \text { Done }(i, \text { act }) \\
& C \text { (violated }, i, j, \text { Done }(i, \text { act }) \mid \top) \equiv B_{j} \neg \text { Done }(i, \text { act }) \wedge I_{j} \text { Done }(i, \text { act })
\end{aligned}
$$

Since roles are public, fulfilment and violation are not dependent on what the agents subjectively believe about the truth value of the content of the commitment, but on roles' public beliefs.

- Finally, a commitment is canceled if the creditor does not want the intention to be achieved anymore, regardless if the debtor still wants it:

$$
C \text { (canceled }, i, j, \text { Done }(i, \text { act }) \mid q) \equiv \neg I_{j} \text { Done }(i, \text { act })
$$

\subsection{Translating Speech Acts}

Given the definition of the commitment state in terms of the mental states of the roles, we can provide the following translation of the speech acts semantics defined by Fornara and Colombetti (2004). In this section, we deal only with speech acts introducing action commitments, like request, propose, promise, accept and reject, while speech acts introducing propositional commitments, such as assertions, are discussed in Section 5.

The translation of the speech act can be directly done using the translation of commitments above and applying it to the definition of speech acts in Fornara and Colombetti (2004). However, as in the case of 


\begin{tabular}{|c|c|c|}
\hline promise & $\begin{aligned} \text { Done }(\text { promise }(i, j, \text { Done }(i, \text { act }), q)) \rightarrow & \left(q \rightarrow I_{j} I_{i} \text { Done }(i, \text { act })\right) \wedge \\
& B_{i}\left(q \rightarrow I_{i} \text { Done }(i, \text { act })\right) \wedge \\
& B_{j}\left(q \rightarrow I_{i} \text { Done }(i, a c t)\right)\end{aligned}$ & (CR46) \\
\hline request & Done $($ request $(i, j$, Done $(j$, act $), q)) \rightarrow\left(q \rightarrow I_{i} I_{j}\right.$ Done $(j$, act $\left.)\right)$ & (CR47) \\
\hline accept & $\begin{array}{r}\text { Done }(\text { accept }(i, j, \text { Done }(i, \text { act }), q)) \rightarrow\left(q \rightarrow I_{j} I_{i} \text { Done }(i, \text { act })\right) \rightarrow \\
\left(B_{i}\left(q \rightarrow I_{i} \text { Done }(i, \text { act })\right) \wedge\right. \\
\left.B_{j}\left(q \rightarrow I_{i} \text { Done }(i, \text { act })\right)\right)\end{array}$ & (CR48) \\
\hline reject & $\begin{aligned} \text { Done }(\operatorname{reject}(i, j, \text { Done }(j, \text { act }), q)) \rightarrow & \left(q \rightarrow I_{j} I_{i} \text { Done }(i, \text { act })\right) \rightarrow \\
& \left(B_{i} \neg I_{i} \text { Done }(i, \text { act }) \wedge B_{j} \neg I_{i} \text { Done }(i, \text { act })\right)\end{aligned}$ & (CR49) \\
\hline propose & $\begin{aligned} & \text { Done }(\operatorname{propose}(i, j, \text { Done }(j, p), q)) \rightarrow\left(\text { Done }(i, q) \rightarrow I_{i} I_{j} \text { Done }(j, p)\right) \wedge \\
&\left(B_{i}\left(s \rightarrow I_{i} \text { Done }(i, q)\right) \wedge\right. \\
& B_{j}\left(s \rightarrow I_{i} \text { Done }(i, q)\right) \\
& \text { where } s \equiv B_{i}\left(\operatorname{Done}(i, q) \rightarrow I_{j}(\operatorname{Done}(j, p))\right) \wedge B_{j}\left(\operatorname{Done}(i, q) \rightarrow I_{j}(\operatorname{Done}(j, p))\right)\end{aligned}$ & $($ CR50) \\
\hline
\end{tabular}

Table 10

Speech acts and the translation of commitments

FIPA, we also provide the rules for translating speech acts into mental attitudes; they are summarized in Table 10.

Even if the translation is straightforward, some consideration are needed:

- First of all, in contrast with the FIPA translation, speech acts affect both the beliefs and intentions of speaker and hearer and not only of the speaker. This represents the fact that a commitment is seen as a relationship, which is publicly established.

- Second, the interpretation of promise and accept in Fornara and Colombetti (2004) needs some discussion. According to Guerini and Castelfranchi (2007) the definition above is too weak: a promise needs to be explicitly accepted, and then kept. It is not enough that $j$ has indicated to prefer $i$ to do the action, and that $i$ and $j$ believe that $i$ has an intention to do the action; what is missing is $j$ 's belief, as a result of the acceptance, that $i$ will actually do it. Nevertheless, we think that the conditional nature of a promise, i.e., that it requires explicit acceptance, is well covered by the combination of rules (CR46) and (CR48). Rule (CR46) covers only an initiative to make a promise; the promise is only complete when accepted, and fulfilled when successful.

- A request introduces an unset commitment with the receiver as debtor, i.e., to perform the requested action (Rule CR40). It does not create any intention for the addressee until he has accepted it.

- Accept and reject change the state of an existing unset commitment to pending and canceled respectively. In order to account for this fact, we insert in the antecedent of the rules for accept and reject the reference to the configuration of beliefs and intentions that represent an existing commitment.

- A propose is a complex speech act composed by a request and a conditional promise; it introduces an unset commitment with the receiver as debtor and a pending commitment with the speaker as debtor. Since a propose is used in a negotiation, $q$ and $p$ refer respectively to an action of the speaker and of the receiver.

$$
\begin{aligned}
& \operatorname{propose}(i, j, \text { Done }(j, p), \text { Done }(i, q)) \equiv \\
& \quad \operatorname{request}(i, j, \operatorname{Done}(j, p), \text { Done }(i, q)) ; \operatorname{promise}(i, j, \operatorname{Done}(i, q), s)
\end{aligned}
$$

where $s \equiv B_{i}\left(\operatorname{Done}(i, q) \rightarrow I_{j} \operatorname{Done}(j, p)\right) \wedge B_{j}\left(\operatorname{Done}(i, q) \rightarrow I_{j} \operatorname{Done}(j, p)\right)$, i.e., $p$ is a pending commitment of the receiver.

\subsection{Example}

We now return to the running example, to illustrate some of the details of our interpretation of the social commitment approach. We now use notation $\varphi \mid \psi$ for conditional commitments, as in the original. The result is shown in Table 11. 


\begin{tabular}{|c|c|c|}
\hline $\mathrm{x}-1:$ & request $(i, j$, Done $(j$, dishes $) \mid \top)$ & $C$ (unset $, j, i$, Done $(j$, dishes $) \mid \top)$ \\
\hline $\mathrm{y}-1:$ & $\operatorname{reject}(j, i$, Done $(j$, dishes $) \mid \top)$ & $C($ canceled $, j, i$, Done $(j$, dishes $) \mid \top)$ \\
\hline & propose $(j, i$, Done $(i$, garbage $) \mid$ Done $(j$, dishes $))$ & $\begin{array}{l}C(\text { unset }, i, j, \text { Done }(i, \text { garbage }) \mid \text { Done }(j, \text { dishes })) \\
C(\text { pending }, j, i, \text { Done }(j, \text { dishes }) \mid \sigma) \\
\sigma \equiv C(\text { pending }, i, j, \text { Done }(i, \text { garbage }) \mid \text { Done }(j, \text { dishes }))\end{array}$ \\
\hline $\mathrm{x}-2:$ & accept $(i, j$, Done $(i$, garbage $) \mid$ Done $(j$, dishes $))$ & $\begin{array}{l}C(\text { pending }, i, j, \text { Done }(i, \text { garbage }) \mid \text { Done }(j, \text { dishes })) \\
C(\text { pending }, j, i, \text { Done }(j, \text { dishes }) \mid \top) \quad(\text { resolving } \sigma)\end{array}$ \\
\hline
\end{tabular}

Table 11

The running example, in the social commitments approach

$\mathrm{x}-1$ : Could you do the dishes?

$\mathrm{y}-1$ : Only if you put the garbage out

$\mathrm{x}-2$ : OK.

We start from state empty. According to Fornara and Colombetti (2004), the request from $x$ in role $r_{1}$, $\left(x r_{1}=i\right)$, creates an unset commitment waiting for acceptance. Now $y$ in role $r_{2},\left(y r_{2}=j\right)$ responds with a conditional acceptance, which could be seen as a kind of counter proposal. However, there are two issues for discussion.

First, a request can only be accepted or rejected, but not be adjusted by a counter proposal. So just like in Section 3 we must interpret $j$ 's response here as rejection, followed by a conditional proposal.

Second, in Fornara and Colombetti's account, rendered in rule (CR50), conditional proposals for $\varphi \mid \psi$ are seen as a combination of a request for $\varphi$ with a conditional promise to make sure that $\psi{ }^{2}$ The promise is again conditional on a statement $\sigma$ which implies the conditional commitment $\varphi \mid \psi$ under discussion. A promise is in turn interpreted as combination of a make and a set action, resulting in the pending state. So under this interpretation a promise does not need acceptance ${ }^{3}$.

Our interpretation of the dialogue above, would be as in Table 11. Remember that $i=x r_{1}, j=y r_{2}$ for some roles $r_{1}, r_{2}$. So we end up with the following set of commitments.

$$
\{C \text { (pending, } i, j, \text { Done }(i, \text { garbage }) \mid \text { Done }(j \text {,dishes })), C \text { (pending, } j, i, \text { Done }(j, \text { dishes }) \mid \top)\}
$$

This is a conditional commitment of $i$ to put the garbage out, provided $j$ does the dishes, and a commitment of $j$ to indeed do to the dishes, given the context of this other commitment. Note that once we have resolved $\sigma$ the conditional nature of the commitment of $j$ to do the dishes, is lost.

Using the translation rules, in particular (CR41), we get the situation described in Table 12. Note that due to the public nature of commitments, as defined in terms of intentions and beliefs, it is not necessary to resort to the reliability rule like in Section 3.3. Consider for example the case of the reject, which is defined in terms of beliefs of both debtor and creditor.

\footnotetext{
${ }^{2}$ Thanks to the formalization of conditional proposal, it is possible to write propose $(j, i$, Done $(i$, garbage $)$ Done $(j$, dishes $)$ ); by contrast, in FIPA, we had to resort to the notion of joint plan to approximate the same notion, establishing an equivalence between kitchen $(i, j)$ and $\operatorname{dishes}(j)$; garbage $(i)$.

${ }^{3}$ We believe this interpretation of proposals is conceptually mistaken. First, proposals are concerned with actions to be done by the speaker; requests deal with actions to be done by the hearer. Second, implicit requests are better made explicit in the agent communication protocol. Third, promises do need acceptance. Nevertheless we continue with Fornara and Colombetti's interpretation here for the sake of the argument
} 


\begin{tabular}{|c|c|c|}
\hline & Role $i$ & Role $j$ \\
\hline \multirow[t]{2}{*}{$\begin{array}{l}\mathrm{x}-1: \\
\text { request }\end{array}$} & $\begin{array}{l}\text { BELIEFS } \\
\top \rightarrow I_{i}\left(I_{j}(\text { Done }(j, \text { dishes }))\right)\end{array}$ & BELIEFS \\
\hline & $\begin{array}{l}\text { INTENTIONS } \\
I_{j}(\text { Done }(j, \text { dishes }))\end{array}$ & INTENTIONS \\
\hline \multirow[t]{2}{*}{$\begin{array}{l}\mathrm{y}-1: \\
\text { reject }\end{array}$} & $\begin{array}{l}\text { BELIEFS } \\
\top \rightarrow I_{i}\left(I_{j}(\text { Done }(j, \text { dishes }))\right) \\
\neg I_{j}(\text { Done }(j, \text { dishes }))\end{array}$ & $\begin{array}{l}\text { BELIEFS } \\
\neg I_{j}(\text { Done }(j, \text { dishes }))\end{array}$ \\
\hline & INTENTIONS & $\begin{array}{l}\text { INTENTIONS } \\
\neg I_{j}(\text { Done }(j, \text { dishes }))\end{array}$ \\
\hline \multirow[t]{2}{*}{$\begin{array}{l}\mathrm{y}-1: \\
\text { propose }\end{array}$} & $\begin{array}{l}\text { BELIEFS } \\
\top \rightarrow I_{i}\left(I_{j}(\text { Done }(j, \text { dishes }))\right) \\
\neg I_{j}(\text { Done }(j, \text { dishes })) \\
\begin{array}{r}s \rightarrow I_{j}(\text { Done }(j, \text { dishes })) \\
\text { where } s \equiv B_{j}\left(\text { Done }(j, \text { dishes }) \rightarrow I_{i}(\text { Done }(i, \text { garbage }))\right) \wedge \\
\quad B_{i}\left(\text { Done }(j, \text { dishes }) \rightarrow I_{i}(\text { Done }(i, \text { garbage }))\right)\end{array}\end{array}$ & $\begin{array}{l}\text { BELIEFS } \\
\qquad I_{j}(\text { Done }(j, \text { dishes })) \\
\text { Done }(j, \text { dishes }) \rightarrow \\
\quad I_{j}\left(I_{i}(\text { Done }(i, \text { garbage }))\right) \\
s \rightarrow I_{j}(\text { Done }(j, \text { dishes }))\end{array}$ \\
\hline & INTENTIONS & $\begin{array}{l}\text { INTENTIONS } \\
\neg I_{j}(\text { Done }(j, \text { dishes }))\end{array}$ \\
\hline \multirow[t]{2}{*}{$\begin{array}{l}\mathrm{x}-2: \\
\text { accept }\end{array}$} & $\begin{array}{l}\text { BELIEFS } \\
\top \rightarrow I_{i}\left(I_{j}(\text { Done }(j, \text { dishes }))\right) \\
s \rightarrow I_{j}(\text { Done }(j, \text { dishes })) \\
\text { Done }(j, \text { dishes }) \rightarrow I_{i}(\text { Done }(i, \text { garbage })) \\
s \\
I_{j}(\text { Done }(j, \text { dishes }))\end{array}$ & $\begin{array}{l}\text { BELIEFS } \\
s \rightarrow I_{j}(\text { Done }(j, \text { dishes })) \\
\text { Done }(j, \text { dishes }) \rightarrow I_{i}(\text { Done }(i, \text { garbage })) \\
s \\
I_{j}(\text { Done }(j, \text { dishes }))\end{array}$ \\
\hline & INTENTIONS & $\begin{array}{l}\text { INTENTIONS } \\
\text { Done }(j, \text { dishes })\end{array}$ \\
\hline
\end{tabular}

Table 12

The running example in the translated social commitments approach

\section{Persuasion dialogues in the social commitments approach}

The purpose of this section is to extend social commitment approaches to persuasion dialogues to show that our role-based semantics is general enough. A similar extension could be made for FIPA, but this extension is more interesting, due to the ambiguity of the term commitment.

We distinguish action commitments from propositional commitments, because an action commitment is fulfilled when the hearer believes that the action is performed, whereas a propositional commitment is fulfilled only when the hearer concedes to the proposition. Using the role-based semantics, we will show that propositional commitments are related to public beliefs, and action commitments to public intentions.

Consider for example the following two sentences:

(1) $A$ promises $B$ to deliver the goods before Friday.

(2) $A$ informs $B$ that $\mathrm{Al}$ Gore would have been a better president than Bush.

The first sentence commits agent $A$ to the delivery of goods before Friday and the second sentence commits agent $A$ to defend the argument that $\mathrm{Al}$ Gore would have been a better president than Bush. We say that the first sentence leads to an action commitment and the second sentence leads to a propositional commitment. Researchers in the social commitment approach to agent communication (Castelfranchi, 1995; Singh, 2000; Fornara and Colombetti, 2004) focus on the former, because they are interested in task oriented dialogues and negotiation. Researchers in the argumentation tradition on the other hand (Hamblin, 1970; Walton and Krabbe, 1995), focus on the latter: 
"to assert a proposition may amount to becoming committed to subsequently defending the proposition, if one is challenged to do so by another speaker" (Walton and Krabbe, 1995).

Despite these differences, someone could argue that a promise and an assertion have the same effect: they create a commitment of the speaker, respectively an action commitment or a propositional commitment. This is coherent with the idea that the meaning of a speech act is defined in terms of the attitudes of the speaker, without any effects on the hearer. But according to Kibble (2005), the meaning of a speech act must also be defined in terms of the effects on the hearer. In particular, an assertion that goes unchallenged may count as a concession for the hearer. This corresponds to the 'silence means consent' principle, already studied by Mackenzie (1979). Walton and Krabbe argue that in case of a concession, the hearer becomes weakly committed to the proposition: the hearer can no longer make the speaker defend the proposition by challenging him, albeit the hearer does not have to defend the proposition himself if challenged. After an assertion the hearer can make a concession explicitly, or implicitly by not challenging the assertion. So we reinforce Kibble's claim that speech acts also have an effect on the attitudes of the hearer. To show the feasibility of the semantics, we model persuasion dialogues loosely inspired by the $P P D_{0}$ protocol of Walton and Krabbe (1995). We illustrate the approach by a dialogue that involves a mixture of propositional and action commitments. Despite the differences, action commitment and propositional commitment have more than accidental similarities too. This needs some argument, because

"... the word commitment and the corresponding verb commit are used in many different ways, and there is no reason to suspect that there is a common core meaning in all of them." (Walton and Krabbe, 1995, p 13).

Our general intuition is that through making a commitment, the number of future options becomes restricted. For action commitment, this can be explained by referring to the well-known slogan that "intention is choice with commitment" (Cohen and Levesque, 1990). Having the stability provided by commitments makes sense when re-planning is costly, and when certain resources must to be reserved in advance, e.g. time slots in an agenda (Bratman, 1987). The same holds for commitments made to other agents. For example, by agreeing to meet on Friday at noon, future options to do other things on Friday become restricted. Analogously, conceding to an action of another agent means agreeing not to prevent him from doing the action by making other conflicting commitments. For example, a concession to do an action which requires a car, implies that the conceder will not use the car for other purposes. The relation between commitment and concession is similar to the one between obligation and permission.

If we view argumentation or persuasion as a kind of game in which players make moves, a propositional commitment can also be said to limit the future possible moves a player can make. In particular, whenever an agent, the proponent, makes an assertion, he is committed to uphold that proposition. This means that all moves which would enable the other player, the opponent, to force the proponent to retract the proposition, must be avoided. Here too, commitments restrict the set of future options.

Social commitments are contrasted with the notion of commitment of Cohen and Levesque (1990) used in the definition of intention, due to the interactional character of social commitments. Thus, at first sight, our argument above seems to confuse the two levels. However, when we equate social commitment with either intention (for action commitment) or belief (for propositional commitment) it must be noted that we refer to public intentions and public beliefs, i.e., to the kind of mental attitudes attributed to roles. In this way, we capture both the traditional view of commitment as limiting options and the interactional nature of social commitments.

Just like one needs a specific logic of practical reasoning to explain action commitment, we need a specific persuasion protocol to explain propositional commitment. Here we will take a simple persuasion protocol. Although this protocol is simplified, we believe it is sufficient to illustrate the notion of propositional commitment. The protocol is depicted in Figure 5. The idea behind the protocol corresponds to the following quote, which is often cited to relate propositional commitment to a kind of action commitment:

"Suppose $X$ asserts that $P$. Depending on context, $X$ may then become committed to a number of things, for example, holding that $P$, defending that $P$ (if challenged), not denying that $P$, giving 


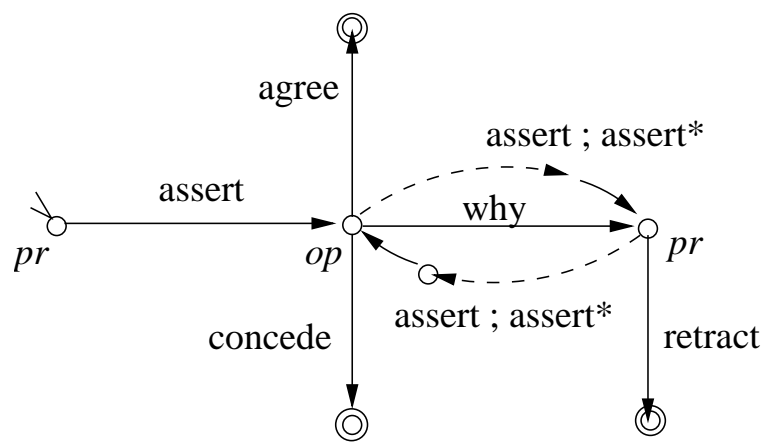

Fig. 5. A protocol for persuasion dialogues.

evidence that $P$, arguing that $P$, proving or establishing that $P$ and so on" (Walton and Krabbe, 1995, p23).

The protocol is defined as follows. For each instantiation of the protocol, there are participants in two roles: the proponent ( $p r)$, and the opponent (op). By definition, the proponent is the agent who makes the initial assertion. Proponent and opponent have different burden of proof. Note that the agent who is the opponent of some proposition $p$, may very well become the proponent of another proposition $q$ later in the dialogue. For this reason, it is crucial that we have an explicit representation of roles.

Following an assertion by the proponent, the opponent can respond by a challenge like a why-question, which essentially requests the proponent to come up with some arguments to support the assertion, or by a rebutting argument. The opponent may also agree with the asserted proposition, or concede the asserted proposition. Agreeing means not only relieving the proponent from the burden of proof, but also adapting the burden of proof towards third parties. Conceding means only that the opponent has given up the right to challenge the proposition, and has relieved the proponent of the burden of proof. Thus agreement implies concession. Alternatively, we can say that not challenging means a concession. In this way we capture Kibble's (2005) idea that entitlement to a commitment comes by default after an assertion.

In response to a challenge, the proponent must give some argument in support of the proposition, which itself consists of one or more assertions, or retract the original assertion. Following the assertion in response to a challenge, the opponent may either concede the proposition, or challenge or concede any of the assertions made by the proponent during the argument in support.

A why-challenge does not add any new material. But an opponent can also challenge propositions by so called rebutting arguments, that provide an independent argument for the opposite assertion. Rebutting arguments can be handled as assertions by the opponent, which trigger another instantiation of the same protocol, with a role reversal. So for rebutting arguments, the burden of proof lies with the rebutter.

The dialogue ends either when the proponent has run out of arguments to support his assertion; in this case he is forced to retract the proposition, or when the opponent has run out of challenges; in this case he is forced to concede. An agreement of the opponent, is essentially an assertion by the opponent. This end condition corresponds to Walton and Krabbe's win and loss rules of $\mathrm{PPD}_{0}$ persuasion dialogues (Walton and Krabbe, 1995, p.152).

\subsection{Propositional commitment}

In our formalization of the persuasion protocol, propositional commitments of the proponent are modeled as public beliefs. Open challenges are modeled as public negated belief of the opponent. In the definition of the update rules in Table 13, the Before and After fields indicate respectively the condition necessary for the utterance to have an effect on the public beliefs, and the effect that results. They should not be confused with the FP and RE conditions of FIPA.

In the translation, we use the principle that a concession $P P$ is represented by the fact that the debtor $i$ does not believe that $\neg p$. This makes it impossible for the debtor to raise further challenges. 


\begin{tabular}{lll} 
Before & speech act & After \\
\hline \hline-- & assert $(p r, o p, p)$ & $B_{p r} p$ \\
\hline$B_{p r} p$ & why $(o p, p r, p)$ & $\neg B_{o p} p$ \\
$B_{p r} p$ & concede $(o p, p r, p)$ & $\neg B_{o p} \neg p$ \\
$B_{p r} p$ & agree $(o p, p r, p)$ & $B_{o p} p$ \\
\hline$B_{p r} p, \neg B_{o p} p$ & retract $(p r, o p, p)$ & $\neg B_{p r} p$ \\
\hline \multicolumn{3}{c}{ Table 13}
\end{tabular}

Updates rules in Persuasion protocol; $p r$ is the proponent and $o p$ the opponent, for $x, y \in A$ and $s \in S$.

$$
P P(i, j, p) \equiv \neg B_{i} \neg p
$$

As usual, $i$ and $j$ are the role instances associated with the two interacting agents.

A propositional commitment $P C$ is active when the debtor $i$ believes the proposition, while nothing is required of the creditor $j$ :

$$
P C(\text { active }, i, j, p) \equiv B_{i} p
$$

A propositional commitment $P C$ is fulfilled when the creditor concedes the proposition, and therefore cannot challenge it anymore:

$$
P C(\text { fulfilled }, i, j, p) \equiv \neg B_{j} \neg p
$$

A propositional commitment $P C$ is violated when the debtor's beliefs are in contradiction, due to the failure of defending some previous commitment.

$$
P C(\text { violated }, i, j, p) \equiv \neg B_{i} p \wedge B_{i} p
$$

Note that a proper treatment of this issue requires a detailed mechanism for dealing with temporal issues which is missing in FIPA-SL. It is not clear whether a conditional propositional commitment is different from a propositional commitment about a conditional, and it is also unclear what it means for a propositional commitment to be unset or pending. Thus we do not define such states here, nor do we define cancelation, which in addition requires to introduce time.

\subsection{Speech acts}

Given the definition of the commitment states in terms of the mental states of the roles, we can provide the following translation of the speech acts semantics. Speech acts affect both the beliefs and intentions of speaker and hearer and not only of the speaker. This represents the fact that in social commitments agents are publicly committed to the mental attitudes attributed by the constitutive rules to the roles they play. No cooperativity or sincerity assumptions are necessary, by contrast to FIPA.

In Table 14 we report the constitutive rules of the speech acts for persuasion:

- An assertion introduces an active propositional commitment of the speaker and, if it is not challenged, it also introduces a concession of the hearer: CR55, CR56.

- Both implicit and explicit concessions are modeled as the absence of the belief to the contrary. This is similar to weak commitment (Gaudou et al., 2006a, eq 17 p.128): CR56, CR59.

- Agreement simply means that the hearer becomes committed too. So agreement implies concession: CR60.

- Asserting an argument against $p$ counts as a rebut-challenge. We simplify here for space reasons the notion of argument: CR57

- A why-challenge asks arguments to support the assertion. It indicates that the opponent is not yet convinced: CR61.

- Not replying to a why challenge with a supporting argument, counts as a retraction: CR62.

- Putting forward an argument in support of the original assertion is a way to reply to a why challenge: CR58. 


\begin{tabular}{|l|l|l|}
\hline assert & Done $($ assert $(i, j, p)) \rightarrow B_{i} p$ & (CR55) \\
& Done $\left(\right.$ assert $(i, j, p) \wedge(\neg$ Done $(w h y(j, i, p)) \vee \neg$ Done $($ rebut $(j, i, p))) \rightarrow \neg B_{j}(\neg p)$ & (CR56) \\
& $B_{j} p \wedge$ Done $($ assert $(i, j,(q \rightarrow \neg p) \wedge q) \rightarrow$ Done $(\operatorname{rebut}(i, j, p))$ & (CR57) \\
& $\neg B_{j} p \wedge$ Done $(\operatorname{assert}(i, j,(q \rightarrow \neg p) \wedge q) \rightarrow$ Done $(\operatorname{support}(i, j, p))$ & (CR58) \\
\hline concede & $B_{j} p \wedge$ Done $($ concede $(i, j, p)) \rightarrow \neg B_{i}(\neg p)$ & (CR59) \\
\hline agree & $B_{j} p \wedge$ Done $($ agree $(i, j, p)) \rightarrow B_{i}(p)$ & (CR60) \\
\hline why & $B_{j} p \wedge$ Done $($ why $(i, j, p)) \rightarrow \neg B_{i} p$ & (CR61) \\
& Done $($ why $(i, j, p)) \wedge \neg$ Done $(\operatorname{support}(j, i, p)) \rightarrow \neg B_{j}(p)$ & (CR62) \\
\hline
\end{tabular}

Table 14

Speech acts for persuasion

Replying to a rebut challenge with a counter argument is also compulsory, but because the rebut challenge is performed by means of a set of assertions, this is already accounted for by rule CR58.

Once a concession has been introduced it prevents the agent from committing itself to the opposite proposition, since this would lead to a contradiction: $\neg B_{i}(\neg p) \wedge B_{i} p$. So now $i$ cannot assert $\neg p$ nor challenge $p$, since a challenge is performed by informing about an argument for $\neg p$. Avoiding a contradiction explains also why an agent would challenge an assertion, if he previously committed himself to the contrary.

\subsection{Example}

In Table 15 we show the interpretation of the following dialogue. For each turn, we report the beliefs and intentions which are created and those which persist from the previous turn.

\section{Example 2}

$\mathrm{x}-1$ : Tomorrow the University is open. $\quad$ Done $(\operatorname{assert}(i, j$, open $))$

$\mathrm{x}-2$ : Can you give the exams for me? Done $($ request $(i, j$, Done $(j$, give-exam $)))$

y-1: Isn't it closed for the Olympic games? Done $(\operatorname{assert}(j, i,($ games $\rightarrow \neg$ open $) \wedge$ games $))$

Done $(\operatorname{rebut}(j, i$, open $))$

x-3: Not for exams. $\quad$ Done $($ assert $(i, j,($ exam $\wedge$ games $\rightarrow$ open $) \wedge$ exam $))$

y-2: I see. $\quad$ Done $(\operatorname{agree}(j, i$, open $))$

y-3: OK Done $(\operatorname{accept}(j, i$, Done $(j$, give-exam $)))$

$\mathrm{y}-4: \quad[j$ gives the exam] Done $(j$, give-exam $)$

After $i$ 's assertion, if $j$ would not reply, $j$ would be publicly considered to concede that the university is open, by rule CR56:

$$
\neg(\text { Done }(\text { why }(j, i, \text { open })) \vee \operatorname{Done}(\operatorname{rebut}(j, i, \text { open }))) \rightarrow \neg B_{j}(\neg \text { open })
$$

So $j$ must reply by doing a rebut challenge, after which $i$ will get stuck in the contradiction, if he does not defend $B_{i}($ open $\wedge \neg$ open $)$, due to rule CR56:

$$
\begin{aligned}
& \neg(\text { Done }(\text { why }(i, j,(\text { games } \rightarrow \neg \text { open }) \wedge \text { games })) \vee \\
& \quad \text { Done }(\text { rebut }(i, j,(\text { games } \rightarrow \neg \text { open }) \wedge \text { games }))) \rightarrow \neg B_{i}(\text { open })
\end{aligned}
$$

Since $i$ replies with a rebutting argument, $j$ will implicitly concedes if he does not reply (CR56):

$$
\begin{aligned}
& \neg(\text { Done }(\text { why }(j, i,(\text { exam } \wedge \text { games } \rightarrow \text { open }) \wedge \text { exam })) \vee \\
& \quad \text { Done }(\operatorname{rebut}(j, i,(\text { exam } \wedge \text { games } \rightarrow \text { open }) \wedge \text { exam }))) \rightarrow \neg B_{j}(\neg \text { open })
\end{aligned}
$$

However, in $\mathrm{y}-2$, he agrees with $i$ and adopts his belief. 


\begin{tabular}{|c|c|c|}
\hline & Proponent $i$ & Opponent $j$ \\
\hline \multirow[t]{2}{*}{$\begin{array}{l}\mathrm{x}-1: \text { assert } \\
\mathrm{x}-2: \text { request }\end{array}$} & $\begin{array}{l}\text { BELIEFS } \\
\text { open }\end{array}$ & BELIEFS \\
\hline & $\begin{array}{l}\text { INTENTIONS } \\
I_{j} \text { Done }(j, \text { give-exam })\end{array}$ & INTENTIONS \\
\hline \multirow[t]{2}{*}{$\begin{array}{c}\mathrm{y}-1: \text { assert } \\
\text { rebut }\end{array}$} & $\begin{array}{l}\text { BELIEFS } \\
\text { open }\end{array}$ & $\begin{array}{l}\text { BELIEFS } \\
(\text { games } \rightarrow \neg \text { open }) \wedge \text { games }) \\
\neg \text { open }\end{array}$ \\
\hline & $\begin{array}{l}\text { INTENTIONS } \\
\text { Done }(j, \text { give-exam })\end{array}$ & INTENTIONS \\
\hline \multirow[t]{2}{*}{$\begin{array}{c}\mathrm{x}-3 \text { : } \\
\text { assert } \\
\text { rebut }\end{array}$} & $\begin{array}{l}\text { BELIEFS } \\
(\text { exam } \wedge \text { games } \rightarrow \text { open }) \wedge \text { exam }) \\
\text { open }\end{array}$ & $\begin{array}{l}\text { BELIEFS } \\
\neg \text { open }\end{array}$ \\
\hline & $\begin{array}{l}\text { INTENTIONS } \\
\text { Done }(j, \text { give-exam })\end{array}$ & INTENTIONS \\
\hline \multirow[t]{2}{*}{$\begin{array}{l}\mathrm{y}-2: \text { agree } \\
\mathrm{y}-3: \text { accept }\end{array}$} & $\begin{array}{l}\text { BELIEFS } \\
\text { open }\end{array}$ & $\begin{array}{l}\text { BELIEFS } \\
\text { open }\end{array}$ \\
\hline & $\begin{array}{l}\text { INTENTIONS } \\
\text { Done }(j, \text { give-exam })\end{array}$ & $\begin{array}{l}\text { INTENTIONS } \\
\text { Done }(j, \text { give-exam })\end{array}$ \\
\hline $\mathrm{y}-4:$ give-exam & $\begin{array}{l}\text { BELIEFS } \\
\text { Done }(j, \text { give-exam })\end{array}$ & $\begin{array}{l}\text { BELIEFS } \\
\text { Done }(j, \text { give-exam })\end{array}$ \\
\hline
\end{tabular}

Table 15

The interpretation of the dialogue in Example 2.

\section{Comparisons}

In this section we give two examples of a comparison between FIPA and social commitments using our role semantics, to assess the feasibility of the role semantics as an intermediate language. We want to illustrate that no information gets lost in the translation from the social commitment approach to the beliefs and intentions of roles.

\subsection{Comparing Inform}

We first consider the case of inform. We show that by first translating a state described in terms of commitments into public beliefs and intentions, and then applying a speech act as defined in Role SL, we get the same results as when we first apply the social commitments version of a speech act, and then translate the resulting state in public beliefs and intentions. The general idea is shown in Figure 6.

Suppose we have a state $s_{1}$ with no commitments: state empty. According to Fornara and Colombetti (2004), the meaning of an inform is the combination of a make and a set of an unconditional commitment. The result is:

$$
C(\text { pending }, i, j, \varphi \mid \top)
$$

Because it concerns an unconditional commitment, there is no difference between active and pending commitments. So we use 'jump condition' rule number 5 (See Table 4 in Section 4). We also leave the condition ' $T$ ' out of the notation. That produces:

$$
C(\text { active }, i, j, \varphi)
$$

Now we translate according to rule (CR42), which produces

$$
B_{i} \varphi
$$

So the translation of $s_{2}$ becomes $\left\{B_{i} \varphi\right\}$. That completes the first half of the diagram. 


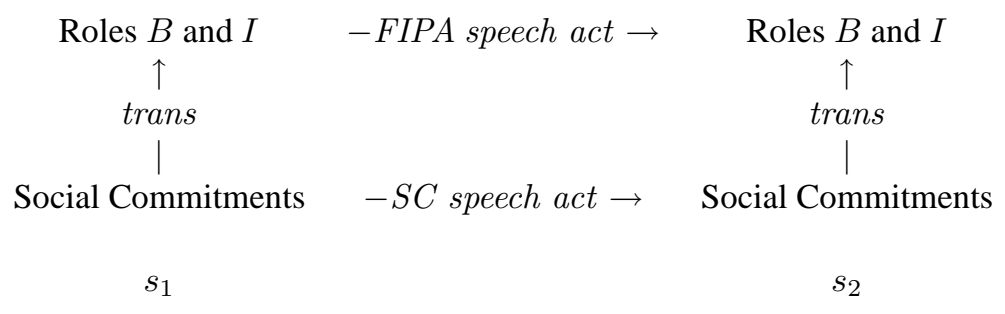

Fig. 6. Comparison diagram.

For the role translation, the empty state corresponds to a set of no beliefs or intentions: \{\} , Now, the meaning of the inform speech act, is captured by rules (CR17) - (CR19). So, in the role version of FIPA, $s_{2}$ becomes

$$
\left\{B_{i} \varphi, \neg B_{i}\left(B_{j} \varphi \vee B_{j} \neg \varphi\right), I_{i} B_{j} \varphi\right\}
$$

Clearly, this is more detailed than the translation of the social commitments approach. The FIPA version contains presupposed information about what the speaker does not know beforehand, and about hist intentions. However, we can say that going from social commitments to roles, no information is lost.

The difference in the amount of detail between the approaches, becomes even more clear when we compare the outcomes of the garbage example, discussed at the end of Section 3 and Section 4 . Here too the social commitments approach is less elaborate.

But in addition to the different level of detail (feasibility preconditions vs operational semantics), there are also important conceptual differences in the way proposals are modeled.

\subsection{Propose Interaction Protocol}

As a second example, we have chosen the Propose interaction protocol of FIPA (2002a). This protocol consists of a propose followed by an acceptance or a refusal.

For a specific instantiation of the protocol, we compare the set of beliefs and intentions produced by the translation of FIPA and social commitments into the role-based semantics to assess whether the intentions concerning executable actions are the same in the two approaches - the agents would act in a similar fashion - and whether it is possible to find in FIPA the same commitments as in the social commitments approach.

The main difficulty in mapping FIPA onto social commitments concerns the communicative act propose. In social commitments approaches, it is viewed as a way to negotiate a joint plan: "If I do $q$, then you do $p$ ". This can model auctions for example (Fornara and Colombetti, 2004). Instead, the FIPA definition of propose refers to one action only.

\section{Example 3}

$\begin{array}{lll}\mathrm{x}-1: & \text { propose } & \text { This bike costs only } \$ 50 ! \\ \mathrm{y}-1: & \text { accept } & \text { All right. } \\ \mathrm{x}-2: & \text { give } & \text { [ gives bike ] } \\ \mathrm{y}-2: & \text { pay } & \text { [ pays } \$ 50 \text { ] } \\ & & \\ \mathrm{y}-1^{\prime}: & \text { reject } & \text { No, that is too much. }\end{array}$

We are inspired by the example in FIPA (2002a), which reports the action of selling an item for a given amount of money. Note that the action of selling is conceived of as a joint action composed of giving the item and paying the money, as in Section 3.3: $\operatorname{sell}(i, j) \equiv \operatorname{give}(i) ; \operatorname{pay}(j)$.

We could say that an accepted propose act forms a plan to be performed by both agents. Once the intentions of both agents to perform the plan have been formed, the plan is distributed between the agents 


\begin{tabular}{|c|c|c|}
\hline & Sender $i$ & Receiver $j$ \\
\hline \multirow[t]{2}{*}{$\mathrm{x}-1$ : propose } & $\begin{array}{l}\text { BELIEFS } \\
I_{j} \text { Done }(\operatorname{sell}(i, j)) \rightarrow I_{i} \operatorname{Done}(\operatorname{sell}(i, j))\end{array}$ & $\begin{array}{l}\text { BELIEFS } \\
I_{j} \text { Done }(\operatorname{sell}(i, j)) \rightarrow I_{i} \operatorname{Done}(\operatorname{sell}(i, j))\end{array}$ \\
\hline & $\begin{array}{l}\text { INTENTIONS } \\
B_{j}\left(I_{j} \text { Done }(\operatorname{sell}(i, j)) \rightarrow I_{i} \text { Done }(\operatorname{sell}(i, j))\right)\end{array}$ & INTENTIONS \\
\hline \multirow[t]{2}{*}{$\mathrm{y}-1:$ accept } & $\begin{array}{l}\text { BELIEFS } \\
I_{j} \text { Done }(\operatorname{sell}(i, j)) \rightarrow I_{i} \text { Done }(\operatorname{sell}(i, j)) \\
I_{j} \text { Done }(\operatorname{sell}(i, j)) \\
I_{i} \text { Done }(\operatorname{sell}(i, j)) \\
I_{i} \text { Done }(\operatorname{give}(i)) \\
\text { Done }(\operatorname{give}(i)) \rightarrow I_{i} \text { Done }(\operatorname{pay}(j)) \\
\text { Done }(\operatorname{give}(i)) \rightarrow I_{j} \text { Done }(\operatorname{pay}(j))\end{array}$ & $\begin{array}{l}\text { BELIEFS } \\
I_{j} \text { Done }(\operatorname{sell}(i, j)) \rightarrow I_{i} \text { Done }(\operatorname{sell}(i, j)) \\
I_{j} \text { Done }(\operatorname{sell}(i, j)) \\
I_{j} \text { Done }(\operatorname{give}(i)) \\
\text { Done }(\operatorname{give}(i)) \rightarrow I_{j} \text { Done }(\operatorname{pay}(j))\end{array}$ \\
\hline & $\begin{array}{l}\text { INTENTIONS } \\
\text { Done }(\text { give }(i))\end{array}$ & $\begin{array}{l}\text { INTENTIONS } \\
B_{i} I_{j} \text { Done }(\operatorname{sell}(i, j))\end{array}$ \\
\hline \multirow[t]{2}{*}{$\mathrm{x}-2$ : give } & $\begin{array}{l}\text { BELIEFS } \\
I_{j} \text { Done }(\operatorname{sell}(i, j)) \rightarrow I_{i} \text { Done }(\operatorname{sell}(i, j)) \\
\text { Done }(\operatorname{give}(i)) \rightarrow I_{j} \text { Done }(\operatorname{pay}(j)) \\
\text { Done }(\operatorname{give}(i)) \rightarrow I_{i} \text { Done }(\operatorname{pay}(j)) \\
I_{i} \text { Done }(\operatorname{give}(i)) \\
\text { Done }(\operatorname{give}(i)) \\
I_{j} \text { Done }(\operatorname{pay}(j)) \\
I_{i} \text { Done }(\operatorname{pay}(j))\end{array}$ & $\begin{array}{l}\text { BELIEFS } \\
I_{j} \text { Done }(\operatorname{sell}(i, j)) \rightarrow I_{i} \text { Done }(\operatorname{sell}(i, j)) \\
\text { Done }(\operatorname{give}(i)) \rightarrow I_{j} \text { Done }(\operatorname{pay}(j)) \\
I_{j} \text { Done }(\operatorname{give}(i)) \\
\text { Done }(\operatorname{give}(i)) \\
I_{j} \text { Done }(\operatorname{pay}(j))\end{array}$ \\
\hline & INTENTIONS & $\begin{array}{l}\text { INTENTIONS } \\
\text { Done }(\operatorname{pay}(j))\end{array}$ \\
\hline \multirow[t]{2}{*}{$\mathrm{y}-2: p a y$} & $\begin{array}{l}\text { BELIEFS } \\
I_{j} \text { Done }(\operatorname{sell}(i, j)) \rightarrow I_{i} \text { Done }(\operatorname{sell}(i, j)) \\
\text { Done }(\operatorname{give}(i)) \rightarrow I_{j} \text { Done }(\operatorname{pay}(j)) \\
\text { Done }(\operatorname{give}(i)) \rightarrow I_{i} \text { Done }\left(I_{j} \operatorname{Done}(\operatorname{pay}(j))\right) \\
I_{j} \operatorname{Done}(\operatorname{pay}(j)) \\
\text { Done }(\operatorname{pay}(j))\end{array}$ & $\begin{array}{l}\text { BELIEFS } \\
\left.I_{j} \text { Done }(\operatorname{sell}(i, j)) \rightarrow I_{i} \operatorname{Done}(\operatorname{sell}(i, j))\right) \\
\text { Done }(\operatorname{give}(i)) \rightarrow I_{j} \text { Done }(\operatorname{pay}(j)) \\
I_{j} \operatorname{Done}(\operatorname{pay}(j)) \\
\text { Done }(\operatorname{pay}(j))\end{array}$ \\
\hline & INTENTIONS & INTENTIONS \\
\hline \multirow[t]{2}{*}{$\mathrm{y}-1^{\prime}:$ reject } & $\begin{array}{l}\text { BELIEFS } \\
I_{j} \text { Done }(\operatorname{sell}(i, j)) \rightarrow I_{i} \operatorname{Done}(\operatorname{sell}(i, j)) \\
\neg I_{j} \operatorname{Done}(\operatorname{sell}(i, j))\end{array}$ & $\begin{array}{l}\text { BELIEFS } \\
I_{j} \text { Done }(\operatorname{sell}(i, j)) \rightarrow I_{i} \text { Done }(\operatorname{sell}(i, j)) \\
\neg I_{j} \text { Done }(\operatorname{sell}(i, j)) \\
\end{array}$ \\
\hline & INTENTIONS & $\begin{array}{l}\text { INTENTIONS } \\
B_{i} \neg I_{j} \text { Done }(\operatorname{sell}(i, j))\end{array}$ \\
\hline
\end{tabular}

Table 16

Example 3 with FIPA.

according to rules RL38 and RL39 which we introduced in Section 3.3, and the intentions concerning the steps of the plan are formed. By contrast, if the proposal is not accepted, as in $y-1^{\prime}$, no intentions are formed according to the plan. The remainder of the translation is straightforward: at each turn, the rules for translating the semantics of FIPA and social commitments into the role-based semantics are applied (see Sections 3 and 4). Then, modus ponens and the rules are applied. We assume here that beliefs and intentions which are not affected by subsequent speech acts persist.

\subsection{Example in FIPA}

In FIPA (see Table 16), the proposal to sell propose $(i, j$, Done $(\operatorname{sell}(i, j))$ is an inform that introduces in the role $i$ the belief that the precondition $I_{j} \operatorname{Done}(\operatorname{sell}(i, j)) \rightarrow I_{i} \operatorname{Done}(\operatorname{sell}(i, j))$ is true. We skip for space reasons the other feasibility precondition, but the reader can easily check that it is true and consistent 
with the state of the dialogue. The rational effect is an intention of the speaker, but since the speaker is reliable (it has correct beliefs about its own mental states), after the proposal, the receiver believes $I_{j}$ Done $(\operatorname{sell}(i, j)) \rightarrow I_{i}$ Done $(\operatorname{sell}(i, j))$ too.

The acceptance of the proposal by $j$ in FIPA is an inform that $j$ has the intention $I_{j}$ Done $(\operatorname{sell}(i, j))$. Again, the receiver believes the content of the accept proposal speech act because an agent in a role is reliable about its own mental states. Since the speaker believes $I_{j} \operatorname{Done}(\operatorname{sell}(i, j)) \rightarrow I_{i} \operatorname{Done}(\operatorname{sell}(i, j))$ and $I_{j} \operatorname{Done}(\operatorname{sell}(i, j))$, it believes also to have Done $(\operatorname{sell}(i, j))$ as a intention (by modus ponens) and, by rule (RL1), it actually has the intention to sell. Most importantly, if an agent in a role has the intention to make a joint plan, by the rule RL38, then it has the intention to do its part at the right moment (the others know this) and the intention that the others do their part. The result of the distribution is: $B_{i}\left(\right.$ Done $($ give $(i)) \rightarrow I_{i}$ Done $($ pay $\left.(j))\right) \wedge B_{j}\left(\right.$ Done $($ give $(i)) \rightarrow I_{j}$ Done $($ pay $\left.(j))\right)$. Thus, when $\operatorname{Done}($ give $(i))$ is true, we derive that both $i$ and $j$ intend $j$ to do its part: $I_{i} \operatorname{Done}(\operatorname{pay}(j))$. Subsequently, this intention is carried out.

However, if the proposal is rejected, as in $\mathrm{y}-1^{\prime}$, this means that $j$ has no intention for the joint plan $\operatorname{sell}(i, j): \neg I_{j} \operatorname{Done}(\operatorname{sell}(i, j))$. Consequently, no distribution of intentions is possible, and no individual intention to give is formed by $i$.

\subsection{Example in Social Commitments}

The translation from social commitments ACL semantics to the role-based semantics is accomplished by applying the rules defined in the previous section (see Figure 17). Given the FIPA propose speech act, the corresponding speech act in social commitments ACL is propose $(i, j$, Done $($ give $(i))$, Done $($ pay $(j)))$. By applying the rule that translates this speech act in the role-based ACL semantics, we get to the state in which both $i$ and $j$ have the belief that Done $($ give $(i)) \rightarrow I_{i} I_{j}$ Done $($ pay $(j))$ representing an unset commitment of $j$. Moreover, a pending commitment by $i$ is represented by $s \rightarrow I_{i}$ Done $($ give $(i))$, where $s \equiv B_{i}\left(\right.$ Done $($ give $(i)) \rightarrow I_{j}$ Done $($ pay $\left.(j))\right) \wedge B_{j}\left(\right.$ Done $($ give $(i)) \rightarrow I_{j}$ Done $($ pay $\left.(j))\right)$.

The accept proposal is modeled by accept $\left(j, i, C_{i}\right.$ (unset, $j, i$, Done $($ pay $(j)) \mid \operatorname{Done}($ give $\left.(i))\right)$ in social commitments semantics. This speech act, whose precondition is true, results in $j$ 's act of creating the belief of $i$ that $j$ believes Done $($ give $(i)) \rightarrow I_{j}$ Done $(\operatorname{pay}(j))$, thus making the condition $s$ true. The application of modus ponens to the belief $s \rightarrow I_{i}$ Done $($ give $\left.(i))\right)$ and this new belief results in the introduction of an active commitment whose debtor is role $i$ : $I_{i}$ Done $($ give $(i)) \wedge I_{j}$ Done $($ give $(i))$ When give $(i)$ is executed, the commitment of $i$ to do give $(i)$ is fulfilled and the commitment of $j$ to do pay $(j)$ is active: its condition Done $($ give $(i))$ is satisfied.

The reject proposal communicative act in FIPA in social commitments semantics corresponds to the speech act $\operatorname{reject}\left(j, i, C_{i}\right.$ (unset, $j, i$, Done $(\operatorname{pay}(j)) \mid \operatorname{Done}($ give $\left.(i))\right)$. The reject speech act attributes to both $i$ and $j$ the belief that $j$ does not have the intention Done $($ pay $(i))$, thus retaining $i$ 's pending commitment from becoming active and canceling the unset commitment from $j$.

\subsection{Comparison}

What are the main differences between these approaches? Table 16 and 17 show that, once translated into the role-based semantics, the resulting intentions of the two approaches coincide, with one significant exception.

The difference can be observed in the first row. After the propose act there is no equivalent in FIPA of the belief - publicly attributed to the proponent - that it has the intention that the addressee forms a conditional intention to pay the requested amount of money for the sold item, where the condition consists of the proponent giving the item. Such a belief can be found in the social commitments approach.

This difference is due to the definition of proposing in FIPA. FIPA does not express the advantage of the proposer in proposing the plan. For example, in the selling case, there is no clue of the reason why the joint plan is proposed, namely to receive the specified money. This is left implicit in the definition of the selling act. In social commitments approaches, reciprocity is expressed explicitly by the fact that a propose 


\begin{tabular}{|c|c|c|}
\hline & Sender $i$ & Receiver $j$ \\
\hline \multirow[t]{2}{*}{$\mathrm{x}-1$ : propose } & 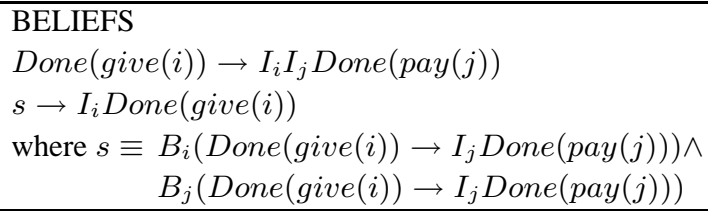 & $\begin{array}{l}\text { BELIEFS } \\
s \rightarrow I_{i} \text { Done }(\text { give }(i))\end{array}$ \\
\hline & INTENTIONS & INTENTIONS \\
\hline \multirow[t]{2}{*}{$\mathrm{y}-1:$ accept } & $\begin{array}{l}\text { BELIEFS } \\
\text { Done }(\text { give }(i)) \rightarrow I_{i} I_{j}(\operatorname{pay}(j)) \\
s \rightarrow I_{i} \text { Done }(\text { give }(i)) \\
\text { Done }(\operatorname{give}(i)) \rightarrow I_{j} \text { Done }(\text { pay }(j)) \\
s \\
I_{i} \text { Done }(\operatorname{give}(i))\end{array}$ & $\begin{array}{l}\text { BELIEFS } \\
s \rightarrow I_{i} \text { Done }(\text { give }(i)) \\
\text { Done }(\text { give }(i)) \rightarrow I_{j} \text { Done }(\text { pay }(j)) \\
s \\
I_{i} \text { Done }(\operatorname{give}(i))\end{array}$ \\
\hline & $\begin{array}{l}\text { INTENTIONS } \\
\text { Done }(\text { give }(i))\end{array}$ & INTENTIONS \\
\hline \multirow[t]{2}{*}{$\mathrm{x}-2$ : give } & $\begin{array}{l}\text { BELIEFS } \\
\text { Done }(\text { give }(i)) \rightarrow I_{i} I_{j} \text { Done }(\text { pay }(j)) \\
s \rightarrow I_{i} \text { Done }(\text { give }(i)) \\
\text { Done }(\text { give }(i)) \rightarrow I_{j} \text { Done }(\text { pay }(j)) \\
I_{i} \text { Done }(\operatorname{give}(i)) \\
\text { Done }(\operatorname{give}(i)) \\
I_{j} \text { Done }(\operatorname{pay}(j))[\mathrm{MP}]\end{array}$ & $\begin{array}{l}\text { BELIEFS } \\
s \rightarrow I_{i} \text { Done }(\text { give }(i)) \\
\text { Done }(\text { give }(i)) \rightarrow I_{j} \text { Done }(\text { pay }(j)) \\
I_{i} \text { Done }(\text { give }(i)) \\
\text { give }(i) \\
I_{j} \text { Done }(\text { pay }(j))\end{array}$ \\
\hline & INTENTIONS & $\begin{array}{l}\text { INTENTIONS } \\
\text { Done }(\operatorname{pay}(j))\end{array}$ \\
\hline \multirow[t]{2}{*}{$\mathrm{y}-2: p a y$} & $\begin{array}{l}\text { BELIEFS } \\
I_{j} \text { Done }(\operatorname{pay}(j)) \\
\text { Done }(\text { pay }(j))\end{array}$ & $\begin{array}{l}\text { BELIEFS } \\
I_{j} \text { Done }(\operatorname{pay}(j)) \\
\text { Done }(\text { pay }(j))\end{array}$ \\
\hline & INTENTIONS & INTENTIONS \\
\hline \multirow[t]{2}{*}{$\mathrm{y}-1^{\prime}:$ reject } & $\begin{array}{l}\text { BELIEFS } \\
\text { Done }(\text { give }(i)) \rightarrow I_{i} I_{j} \text { Done }(\text { pay }(j)) \\
s \rightarrow I_{i} \text { Done }(\text { give }(i)) \\
\neg I_{j} \text { Done }(\operatorname{pay}(j))\end{array}$ & $\begin{array}{l}\text { BELIEFS } \\
s \rightarrow I_{i} \text { Done }(\text { give }(i)) \\
\neg I_{j} \text { Done }(\operatorname{pay}(j))\end{array}$ \\
\hline & INTENTIONS & INTENTIONS \\
\hline
\end{tabular}

Table 17

Example 3 with social commitments.

is composed of a conditional promise together with a request (see also the model in (Yolum and Singh, 2002)). This provides a way to express any kind of arrangement, even non conventional ones. In social commitments approaches, the subsequent acceptance presupposes the existence of an unset commitment having as debtor the participant to which the proposal was addressed. However, FIPA's accept proposal in the second turn fills the gap: when the addressee displays the intention to take part in the joint plan, the distribution of the tasks of giving and paying takes place, generating the appropriate intentions for the two roles.

\section{Related work}

Here we discuss the historical development of work on the semantics of agent communication languages, work on roles, as well as our own related work.

\subsection{Agent communication languages}

The linguistic and philosophical theory of speech acts has inspired a number of models of agent communication languages. Most models of communicative acts can be classified into two types, depending 
on whether they rely on mental attitudes or social commitment, including both action commitment and propositional commitment.

\subsubsection{Mental attitudes}

The semantics of agent communication languages provided by the Foundation for Intelligent Physical Agents (FIPA, 2002a), is paradigmatic of the models based on mental attitudes. The FIPA standards and the work at France Telecom on which it is based (Sadek, 1992; Bretier and Sadek, 1997; Sadek et al., 1997), are based on earlier models for natural language interpretation (Cohen and Perrault, 1979; Allen and Perrault, 1980). FIPA has concentrated most on collaborative dialogue types, like information seeking. The sincerity condition assumed by FIPA makes sense for natural language dialogue, because such dialogue agents are designed to be cooperative. However, the generalization of the FIPA language to noncooperative settings has proven to be problematic. The combination of speech act theory (Searle, 1969), with a general theory of planning and action, e.g. (Pollack, 1990), provides a general model of rational interaction. Essentially, the speech acts are treated as any other action, which can be combined into plans by means of their preconditions and intended effects. In FIPA, communicative acts are defined in terms of the mental states of the agent who issues them. Mental states are represented according to the well known Belief-Desire-Intention (BDI) framework (Bratman, 1987; Cohen and Levesque, 1990). The bridge between the communicative acts and the behavior of agents is provided by the notions of rational effect (RE) and feasibility preconditions (FP). The rational effect is the mental state that the speaker intends to bring about in the hearer by issuing a communicative act, and the feasibility preconditions encode the appropriate mental states for issuing a communicative act. To guarantee communication, the framework relies on intention recognition on the part of the hearer.

Despite the fact that FIPA standards are well known, they are not yet widely used in agent applications. One of the reasons might be that it is counterintuitive to generalize the FIPA approach to non-cooperative settings. By definition, the private mental states of agents cannot be inspected, and can therefore not be verified (Pitt and Mamdani, 1999; Wooldridge, 2000). Such an approach is not appropriate in situations in which agents may be insincere or non-cooperative, as in argumentation or negotiation settings. Moreover, a typical agent application involves stake holders with diverse or opposed interests. Typical agent applications will therefore often involve non-cooperative settings.

\subsubsection{Social commitments: action commitment in negotiation}

Agent communication languages based on social commitment constitute an attempt to overcome the mentalistic assumptions of FIPA by restricting the analysis to public communication (Castelfranchi, 1995; Singh, 2000; Colombetti et al., 2004; Fornara and Colombetti, 2004; Bentahar et al., 2004). Communicative acts are defined in terms of the social commitments they publicly determine for speaker and the hearer. According to Fornara and Colombetti (2004) commitment is "a social relationship between the speaker and the hearer". The notion of social commitment does not refer to intentionality; it is a primitive social notion, developed for modeling mutual commitments in contracts and electronic commerce, for example. A commitment in Fornara and Colombetti (2004) has a debtor and creditor, i.e., respectively, the agent who makes the commitment, and the agent to which the commitment is made. We could say that the creditor is interested in the content of the commitment. The social commitments approach has focussed on non-cooperative types of dialogue, like negotiation, but not on persuasion. Researchers working in the tradition of Winograd and Flores (1986), such as Singh (2000), are interested in cooperative dialogue and negotiation. Hence they tend to investigate the action commitments, that typically result from directives like request and propose.

The social commitment approach also has a number of drawbacks. A social commitment describes the status of an agreement about future action. Unlike intentions, as usually conceived, social commitments do not have a causal relation to action. So the use of social commitments to model the effects of communication relies on the notion of obligation to explain how commitments affect the behavior of the individual agents. As it appears, commitments require enforcement mechanisms of obligations, like sanctions (Pasquier et al., 2004). This makes sense for competitive environments, like argumentation dialogue or negotiation, but it does not make sense in cooperative environments, like information seeking or inquiry, 
where a commitment can simply be interpreted as an expectation. Even though social commitments may be useful in the analysis and design of communication protocols for applications like electronic commerce, this does not mean that they provide an adequate semantics of the speech acts involved. ${ }^{4}$ The social semantics is an operational semantics, which translates speech acts to transitions in a data-structure, the commitment automata.

\subsubsection{Social commitments: propositional commitment in persuasion}

The argumentation tradition originating with Hamblin (1970) and Walton and Krabbe (1995), focuses on propositional commitments: "to assert a proposition may amount to becoming committed to subsequently defending the proposition, if one is challenged to do so by another speaker" (Walton and Krabbe, 1995). However, this kind of propositional commitment is biased towards argumentation dialogue, with assertive speech acts like 'assert' or 'challenge', failing to be general enough for cooperative dialogues.

In many social semantics the distinction between the commitment to an action and the propositional commitments of assertives becomes blurred. This is a problem when dealing with argumentation and persuasion dialogues where the notion of propositional commitment is basic. According to Bentahar et al. (2004), the difference between an action commitment and a propositional commitment lies only in the type of content, and both kinds of commitment are fulfilled (or violated) if the content is true (false) in the world, albeit the debtor cannot do anything to make a propositional commitment true, whereas he can perform the action object of a commitment. At a closer analysis the definition of fulfillment is not correct for action commitment. According to these authors, a commitment is fulfilled if (at the deadline time) its content is true in the world. This objectivistic solution is too weak: fulfillment does not only depend on what is true in the world, but also on what is believed by the creditor. Thus the creditor can still claim to be entitled to the commitment, until he is convinced and the evidence is shared by both agents. Moreover, this view of fulfillment is not realistic for propositional commitment. For propositional commitments the problem is made worse by the fact that their content is not restricted to actions whose execution can be monitored in the world. Consider, e.g., a commitment towards the fact that Al Gore would have been a better president than Bush. There is no way to fulfill such a hypothetical commitment, unless one of the agents concedes on the basis of arguments. This is the general case in persuasion and argumentation, e.g., in a political debate or in a trial. An alternative solution is to define that a propositional commitment it fulfilled when the creditor also becomes committed to the proposition. But this is too strong, since not all assertions and informs aim to make the hearer believe them, and viceversa, not all informs and assertions aim to satisfy a goal of the creditor to know information. For example, in information seeking, the creditor wants to have reliable information but, in a dispute, he wants to win. In general, and in contrast with action commitment, there is not always a creditor who has the goal to have some information. In our approach, a propositional commitment is fulfilled when the hearer concedes, and, thus, cannot challenge the proposition anymore. Other possible goals, like that the hearer must come to believe the proposition, depend on particular types of dialogues and have their own fulfillment conditions. Our rolebased semantics allows to associate different fulfillment conditions to the roles that belong to different dialogue games.

The social semantics approach - with the noticeable exception of Kibble (2005) - generally only makes the speaker committed to the propositional content of an assertive. It do not express the notion of communicative intention: an inform makes mutually believed the intention of the speaker that the addressee believes the proposition. Social semantics delegate to the protocol level the coherence of a dialogue, by dropping communicative intentions from the speech act level. Concerning concessions, inspired by Kibble (2005), we argue that an assertion creates by default a concession of the hearer, which can be in contradiction with his beliefs, unless he challenges the assertion. When the hearer challenges the assertion, the contradiction is passed to the speaker, and forces a retraction of the speaker, if not challenged in turn; and so on, until one of them does not have any arguments left to put forward, and concedes or retracts explic-

\footnotetext{
${ }^{4} \mathrm{We}$ are aware of the difference between semantics in computer science, and in linguistics. In linguistics, a semantics of an agent communication language would rather be called a pragmatics, because it mainly concerns the correct use of these acts. See the insightful discussion in Coleman et al. (2006).
} 
itly. Finally, considering the creation of a commitment as a result of an assertion, as social commitments semantics does, is different from the traditional interpretation, which sees propositional commitments as a kind of action commitment to defend the proposition. Instead, a commitment to defend, is typically created by the challenge.

\subsubsection{Bridges}

Recently, some other papers went in the same direction of redefining FIPA semantics, using public mental states e.g., (Nickles et al., 2006; Verdicchio and Colombetti, 2006; Gaudou et al., 2006a). However, these other solutions need to add to dialogue new theoretical concepts which are not always completely clear or diverge from existing work. In particular, Gaudou et al. (2006a) use an explicit grounding operator, which only partially overlaps with the tradition of grounding in theories of natural language dialogue. Opinions (Nickles et al., 2006) are introduced specifically for modeling dialogue, but with no relation with persuasion and argumentation. Finally, commitments in Verdicchio and Colombetti (2006) roughly overlap with obligations. Moreover, these other approaches relate to the well known FIPA semantics in different degrees: Gaudou et al. (2006a) and Nickles et al. (2006) try to stay close to the original semantics, as we do, while Verdicchio and Colombetti (2006) substitute it entirely with a new semantics, which, among other things, does not consider preconditions of actions.

\subsubsection{Other work in agent communication}

In this paper we do not consider the construction of communicative protocols out of individual speech acts. There exist first principle methodologies, in which the semantics of a protocol is defined completely in terms of the semantics of the individual speech acts. Alternatively, larger protocols may be construed from basic protocols consisting of speech acts that naturally belong together. For example, propose, accept and reject form such a basic interaction protocol, see Section 4.

\subsection{Roles}

The distinguishing feature of our approach is that the public mental attitudes attributed to agents during the dialogue are associated with roles. he importance of roles is recognized in multiagent systems and their function ranges from attributing responsibilities to assigning powers to agents in organizations. In this paper we exploit the notion of roles, as introduced in agent-oriented software engineering, e.g., GAIA (Wooldridge et al., 2000), TROPOS (Bresciani et al., 2004). We distinguish interactive roles, such as speaker, (over)hearer and addressee. Clearly, different constitutive rules apply to speaker and hearer. Further, we could add rules so that the effects of implicit acknowledgement differ between the addressee of a message, and a mere overhearer (Gaudou et al., 2006b). We also distinguish social roles, that belong to a particular type of dialogue. E.g., in argumentation, the 'burden of proof' is different for the proponent or the opponent of a claim. Or consider the fact that a request cannot be refused, if there is some authority relation between speaker and addressee. In fact, such a relationship turns a request into an order. Note that one agent can play different roles in different interactions with the same or different interlocutors. For example, the buyer of a good may become a seller in a second transaction. Because social roles are associated with dialogue types, each with a specific set of dialogue rules, roles allow us to reason about assumptions in different kinds of dialogues. Thus, sincerity could be assumed in cooperative dialogues, such as information exchange, but not in non-cooperative dialogues, such as persuasion or negotiation. Ostensible beliefs and the grounding operator can only distinguish interactive roles like speaker and addressee, and the effect on different groups of agents.

\subsubsection{Roles as prescriptions}

Roles in a social institution are traditionally used to determine the obligations, permissions and institutional powers of an agent. Roles prescribe which possible speech acts are allowed for an agent, and possibly which acts must be used to respond. This idea is most prominent in the ISLANDER system (Esteva et al., 2002), in which the roles of agents determine the interface with the environment. In our role model (Boella and van der Torre, 2004) roles are always associated to some kind of institution, see also e.g. 
Kagal and Finin (2005), which is described by constitutive rules. In the case of dialogue, the institution is represented by the type of dialogue game played by the participants.

In the role-based semantics introduced in this paper, the moves available to the dialogue participants are based on the mental attributes of the roles only, not on the mental attitudes of the agent. Since an agent enters in a dialogue only in a certain role, the communicative actions at his disposal, depend on the role. Thus, agents participating in a dialogue in different roles can perform different kinds of communicative actions. This is due to the fact that the communicative action performed depends on the constitutive rules of the dialogue game: if an agent utters a sentence which is not recognized as a communicative action since the agent is not playing the right role, the communicative action is considered not to be performed.

A typical situation is represented by the Contract Net Protocol (Smith, 1980) where the initiator role and the participant role can perform different actions, e.g., call for proposal and proposal respectively. The initiator and participant role are already present in the Contract Net Protocol. The distinguishing property of our approach is that roles are not simply labels, but they are associated with instances representing the state of the participant in the interaction. The state is represented as a set of beliefs and intentions attributed to the role enacting agent. Such a state is modified by the communicative actions performed during the dialogue according to the constitutive rules of the dialogue game.

Each instance of a dialogue game is associated with instances of the roles played by the agents; so each agent is associated with a different state of the interaction in each dialogue he is participating in. For example, an agent who is playing the role of participant in a Contract Net negotiation, can at the same time participate as initiator in another negotiation to subcontract part of the task. In each of his roles the agent is associated with a set of beliefs and intentions representing the situation of the conversation thus far. Moreover, the price the agent as initiator can pay to a sub-contractor, depends on the price for which it undertook the task, as participant. So all the roles must be related to a common agent, who has to direct the different negotiations according to his private reservation price and to the outcome of the other interactions.

\subsection{Roles as expectations}

Agents can make predictions, and use these predictions to coordinate their behavior with an agent, due to the fact that the agent enacts a particular role. This predictive aspect is common in the social sciences, made famous by the restaurant script (Schank and Abelson, 1977) and emphasized in agent theory by Castelfranchi (1998). An example from human life, is the fact that the car of someone taking driving lessons, is clearly marked with a sign, like ' $\mathrm{L}$ ' or ' $\mathrm{E}$ '. This sign does not change the prescriptive status the traffic code applies just as much - but it signals to other drivers to be careful and more considerate.

In our role-based semantics, expectations are based both on the mental attitudes ascribed to the agent and to the role. To play a role, an agent is expected to act as if the beliefs and intentions of the role were his own, and to keep them coherent, as he does for his own mental attitudes. An agent should adopt the role's intentions and carry them out according to the beliefs of the role. This holds despite the fact that the model remains neutral with respect to the motivations that agents may have when playing a role; agents can adopt the mental attitudes attributed to their roles as a form of cooperation, or they can be publicly committed to their roles. The roles' attitudes represent what the agent is publicly held responsible for: if the agent does not adhere to his role, he can be sanctioned or blamed.

Expectations follow also from the objectives of the agents. For agent communication languages, the most convincing example has to do with bargaining. In protocols like the Contract Net, listed above, there are no constraints on the content of proposals. However, purely based on the apparent objectives of agents when entering a conversation in a particular role, we can infer a number of preferences:

- the initiator wants to have some task achieved, otherwise he would not send the call for proposals

- the initiator wants to give up as little as possible, in return for the accomplishment of the task

- a participant may either want to achieve the task, or not. In the first case, he will send some offer. In the last case he will send a reject, or fail to reply. 
- if a participant is interested, he will want as much as possible in return for doing the task

From such preferences, we can infer some coherence conditions on the content of proposals. For example, it will be very unlikely that a participant will first offer to do the task for 40 , and later to do the task for 50, because the participant does not expect the initiator to accept the higher offer, after the lower offer was declined. More about coherence conditions can be found in Hulstijn et al. (2004).

\subsection{Our own earlier work}

This journal paper is a revised and extended version of various workshop and conference papers. Boella et al. $(2005,2006 \mathrm{~b})$ discuss a role-based semantics, which can deal with both mentalistic approaches and social commitments. Of this work, Boella et al. (2005) is based on our normative multi-agent systems framework (Boella and van der Torre, 2006a,b), which describes roles by means of the agent metaphor, and is formalized in Input/Output logic (Makinson and van der Torre, 2000). This work only addresses part of the problem; it focuses on persuasion dialogues. Boella et al. (2006a) provide a common rolebased semantics for both approaches, using a representation format based on the FIPA semantic language. We model additional categories of speech acts, like commissives and directives, which can be applied in negotiation dialogues between cooperative agents.

\section{Conclusions}

Public mental attitudes have been proposed to bridge the gap between the two main traditions in defining a semantics for agent communication languages,: mental attitudes and social commitments. These traditions, even if they share the idea of speech acts as operators with preconditions and effects, and agents playing roles like speaker and hearer, rely on completely distinct ontologies. Not only does the mental attitudes approach refer to concepts like belief, desire, or intention and is the social commitment approach based on the notion of commitment, but the two approaches also refer to distinct speech acts, distinct types of dialogue games, and so on. Public mental attitudes avoid the problems of mentalistic semantics, such as the unverifiability of private mental states, and they allow the reuse of the logics and implementations developed for FIPA compliant approaches. The distinction between private and public mental attitudes allows us to model multiple communication sessions, as well as bluffing and lying agents in persuasion and negotiation.

We associate public beliefs and intentions, representing what the agent is publicly held responsible for, with a role instance. If the agent does not adhere to his role, then he can be sanctioned or blamed. The mental attitudes contain precisely the public beliefs and intentions which follow from speech acts being uttered - requirements as well as consequences - together with commonly held beliefs about the attitudes of roles.

The existence of such public mental attitudes is the only property of role instances we require, and this property therefore characterizes our notion of role in agent communication. This characteristic also forms the main distinction with most other role-based models, which typically allow private mental attitudes like secrets for role-enacting-agents or agents-in-a-role too.

The final constituent of our model is the set of constitutive rules which defines the effects of speech acts on the mental attitudes of role instances, for dialogue types like information seeking, negotiation and persuasion. Communication is public for every agent who overhears the speech acts, and has same prior knowledge of roles. Consequently, for public communication, the mental attitudes of a role are public. The challenge of agent communication languages whose semantics is based on public mental attitudes, such as our role-based semantics, is to define mappings from existing languages to the new one. In this paper we show how to map the two existing traditions to our role-based ontology of public mental attitudes and we show how it allows for a comparison.

The mapping from FIPA semantics to our role-based semantics interprets the feasibility precondition of FIPA as a kind of presupposition. For example, if an agent $i$ informs another agent that $\varphi$ with 
$\operatorname{inform}(i, j, \varphi)$, then in the original FIPA semantics, agent $i$ should already believe the proposition $\varphi$. Otherwise, $i$ would be insincere. With public mental attitudes, however, it is not the case that an agent must already publicly believe $\varphi$. Instead, it is presupposed that the agent believes $\varphi$, and therefore, the agent is publicly held responsible for the fact that $\varphi$ holds. If $\varphi$ does not hold, then the agent can be sanctioned or blamed. Moreover, FIPA notions like reliability and sincerity are no longer implicitly built into the formal system. By making such notions explicit for different contexts of use, we can integrate the social commitment approach, and deal both with cooperative and non-cooperative types of dialogue.

The first mapping from the social commitment semantics focuses on action commitments (Singh, 2000; Fornara and Colombetti, 2004), with an operational semantics. We introduce six update rules mirroring the commitment update rules of Fornara and Colombetti: an unset commitment corresponds to an intention of the creditor, a commitment is pending when it is an intention of the creditor and the debtor of the commitment conditionally wants to perform the action if the associated condition $q$ is true, and the creditor has this as a belief, and so on. Then:

A promise introduces a pending commitment of the speaker;

A request introduces an unset commitment with the receiver as debtor, i.e., the agent of the requested action;

Accept and reject change the state of an existing unset commitment to pending and canceled respectively. In order to account for this fact, we insert in the antecedent of the rules for accept and reject the reference to the configuration of beliefs and intentions that represent an existing commitment.

A propose is a complex speech act composed by a request and a conditional promise; it introduces an unset commitment with the receiver as debtor and a pending commitment with the speaker as debtor. Since a propose is used in a negotiation, $q$ and $p$ refer respectively to an action of the speaker and of the receiver.

The second mapping from the social commitment semantics focuses on propositional commitments. We distinguish propositional and action commitment by means of the notion of concession, inspired by Walton and Krabbe (1995) and Kibble (2005). We map propositional commitments to the roles' beliefs, and action commitments to their intentions. The constitutive rules of a dialogue game represent the effects that speech acts have on the roles' mental attitudes, and, thus, indirectly on commitments. Concessions are introduced as the absence of a belief to the contrary, and prevent further challenges. This is analogous to action commitments, which prevent future actions that require the same resources. We illustrate propositional commitments by modeling a particular persuasion protocol, inspired by Walton and Krabbe. This protocol endorses the 'silence means consent' principle. Since under this principle concessions of the hearer can be made by default, i.e., by not challenging an assertion, we demonstrate that the semantics of speech acts should not only be expressed in terms of the effects on the attitudes of the speaker, but also on those of the hearer.

The research reported in this paper does have some limitations. One limitation is that we have chosen to stay relatively close to the original FIPA-SL language, so we inherit some of its well known problems. In Section 7 we have discussed various alternative representation languages for agent communication to overcome these problems. A limitation that our approach has in common with many other approaches, is the absence of explicit time variables in the representation language. This may become a problem, when we are modeling the feasibility preconditions of a speech act. For example, an inform only makes sense when the hearer does not already know the information. So, from the utterance of an inform act, we may infer that the speaker believes the hearer does not know the information. But this is immediately overruled by the rational effect of the inform act. Similar problems exist for the retraction of claims, as we discussed in Section 5. We believe that these problems may be overcome by the use of a defeasible logic with explicit time variables. An initial proposal in this direction is made by Boella et al. (2007).

The first issue for further research is the use of our role-based ontology for descriptions of expected behavior. To play a role, an agent is expected to act as if the beliefs and intentions of the role were his own, and to keep them coherent, as he does for his own mental attitudes, see also (Goffman, 1959). An agent should adopt to the intentions of its role, and carry them out according to his role's beliefs. The only 
thing agents have to do when communicating is to respect the rules of the dialogue; otherwise they are out of the game. Agents thus have to respect the cognitive coherence of the roles they play or they get into a contradictory position. We may adopt Pasquier and Chaib-draa's (2003) view that dialogue arises from the need to maintain coherence of mental states: "two agents communicate if an incoherence forces them to do so. [...] Conversation might be seen [...] as a generic procedure for attempting to reduce incoherence". An agent engaged in a dialogue tries to avoid contradictions, not with his private mental states, but with the public image which his role constitutes. Here we do not consider the coherence of the private mental states of the agents, but the coherence of the mental attitudes of the roles. Referring only to the agents' beliefs would not be realistic, since there is no way to ensure that the addressee of an inform act, for example, accepts to believe a proposition. After all, agents are autonomous. This is one of the limitations of the traditional mentalistic approach. We overcome it using rules referring to public mental attitudes, but those of the roles, rather than the agents'. According to Goffman (1959), the pretense that roles and individual agents are identical, is very common in everyday practice. Although we suspect that others have beliefs and strategic goals different from the ones publicly expressed, we pretend not to see this. If we would, we would have to accuse the other openly of insincerity, often without proper evidence. The resulting conflict would be unwanted for all participants.

The second issue for further research is a formalization of the notion of responsibility, and the way in which the obligation to play a role consistently can be enforced. The introduction of obligations requires the reference to an explicit multi-agent normative system, as described in (Boella and van der Torre, 2004). Formalizing responsibility and enforcement starts with formalizing violations, which should be based on explicit evidence. Consider again the example of a liar, who once he starts lying, has to continue the dialogue consistently with what he said before, independently of his real beliefs. To formalize the consistent liar, we also have to define what it means that he no longer is able to lie, for example due to explicit evidence to the contrary.

\section{References}

Allen, J. F. and Perrault, C. R. (1980). Analyzing intention in utterances. Artificial Intelligence, 15(3):143-178.

Austin, J. (1962). How to do things with words. Harvard University Press.

Baldoni, M., Boella, G., and van der Torre, L. (2007). Interaction between objects in powerJava. Journal of Object Technology, 6(2):7-12.

Bentahar, J., Moulin, B., Meyer, J., and Chaib-draa, B. (2004). A modal semantics for an argumentation-based pragmatics for agent communication. In Argumentation in Multi-Agent Systems (ArgMAS'04), LNCS 3366, pages 44-63, Berlin. Springer.

Boella, G., Damiano, R., Hulstijn, J., and van der Torre, L. (2006a). ACL semantics between social commitments and mental attitudes. In Agent Communication II (AC'05 and AC'06), LNCS 3859, pages 30-44. Springer, Berlin.

Boella, G., Damiano, R., Hulstijn, J., and van der Torre, L. (2006b). Role-based semantics for agent communication: Embedding of the 'mental attitudes' and 'social commitments' semantics. In Proceedings of the 5th International Joint Conference on Autonomous Agents and Multiagent Systems (AAMAS'06), pages 688-690. ACM Press.

Boella, G., Hulstijn, J., Governatori, G., Riveret, R., Rotolo, A., and van der Torre, L. (2007). FIPA communicative acts in defeasible logic. In Proceedings of 7th IJCAI International Workshop on Nonmonotonic Reasoning, Action and Change (NRAC'07), pages 9-16. University of Technology.

Boella, G., Hulstijn, J., and van der Torre, L. (2005). A synthesis between mental attitudes and social commitments in agent communication languages. In Proceedings of the 2005 IEEE/WIC/ACM International Conference on Intelligent Agent Technology (IAT'05), pages 358-364, Piscataway, NJ. IEEE Computer Society.

Boella, G. and van der Torre, L. (2004). Organizations as socially constructed agents in the agent oriented paradigm. In Engineering Societies in the Agent World (ESAW'04), LNAI 3451, pages 1-13, Berlin. Springer.

Boella, G. and van der Torre, L. (2006a). A game theoretic approach to contracts in multiagent systems. IEEE Transactions on Systems, Man and Cybernetics - Part C: Applications and Reviews, 36(1):68-79.

Boella, G. and van der Torre, L. (2006b). Security policies for sharing knowledge in virtual communities. IEEE Transactions on Systems, Man and Cybernetics - Part A: Systems and Humans, 36(3):439-450.

Bratman, M. (1987). Intention, plans, and practical reason. Harvard University Press, Cambridge Mass.

Bresciani, P., Giorgini, P., Giunchiglia, F., Mylopoulos, J., and Perini, A. (2004). TROPOS: An agent-oriented software development methodology. Autonomous Agents and Multi-Agent Systems, 8(3):203-236.

Bretier, P. and Sadek, D. (1997). A rational agent as the kernel of a cooperative spoken dialogue system: implementing a logical theory of interaction. In Muller, J. P., Wooldridge, M. J., and Jennings, N. R., editors, Intelligent Agents III, LNAI 1193, pages 189-203. Springer, Berlin.

Caminada, M. W. A. (2004). For the Sake of the Argument; explorations into argument-based reasoning. Phd. thesis, Vrije Universiteit, Amsterdam. 
Castelfranchi, C. (1995). Commitment: from intentions to groups and organizations. In Proceedings of the First International Confernce on Multi-Agent System (ICMAS'95), pages 41-48. AAAI/MIT Press.

Castelfranchi, C. (1998). Modeling social action for AI agents. Artificial Intelligence, 103(1-2):157-182.

Cohen, P. R. and Levesque, H. J. (1990). Intention is choice with commitment. Artificial Intelligence, 42(2-3):213-261.

Cohen, P. R. and Perrault, C. R. (1979). Elements of a plan-based theory of speech acts. Cognitive Science, 3:177-212.

Coleman, J., O'Rourke, M., and Edwards, D. B. (2006). Pragmatics and agent communication languages. In Proceedings of the ESSLLI Workshop on Formal Ontologies for Communicating Agents. ESSLLI.

Colombetti, M., Fornara, N., and Verdicchio, M. (2004). A social approach to communication in multiagent systems. In Declarative Agent Languages and Technologies (DALT'03), LNCS 2990, pages 191-220. Springer, Berlin.

Dastani, M., Dignum, V., and Dignum, F. (2003). Role-assignment in open agent societies. In Proceedings of the 2nd International Joint Conference on Autonomous Agents and Multiagent Systems (AAMAS'03), pages 489-496. ACM Press.

Dastani, M., Herzig, A., Hulstijn, J., and Van der Torre, L. (2004). Inferring trust. In Proceedings of 5th Workshop on Computational Logic in Multi-agent Systems (CLIMA V), LNAI 3487, pages 144- 160, Berlin. Springer.

Demolombe, R. (2001). To trust information sources: a proposal for a modal logical framework. In Trust and Deception in Virtual Societies, pages $111-124$. Kluwer.

Esteva, M., de la Cruz, D., and Sierra, C. (2002). ISLANDER: an electronic institutions editor. In Proceedings of the 1st International Joint Conference on Autonoumous Agents and Multiagent Systems (AAMAS'02), pages 1045 - 1052 . ACM Press.

Ferber, J., Gutknecht, O., and Michel, F. (2003). From agents to organizations: an organizational view of multiagent systems. In Agent-Oriented Software Engineering IV (AOSE'03), LNCS 2935, pages 214-230, Berlin. Springer.

FIPA (2002a). FIPA ACL communicative act library specification. Technical Report SC00037J, Foundation for Intelligent Physical Agents.

FIPA (2002b). FIPA ACL message structure specification. Technical Report SC00061G, Foundation for Intelligent Physical Agents.

FIPA (2002c). FIPA Propose interaction protocol specification. Technical Report SC00036H, Foundation for Intelligent Physical Agents.

Fornara, N. and Colombetti, M. (2004). A commitment-based approach to agent communication. Applied Artificial Intelligence, 18(9-10):853-866.

Garson, J. W. (1984). Quantification in modal logic. In Handbook of Philosophical Logic, volume II, pages 249-307. Reidel.

Gaudou, B., Herzig, A., and Longin, D. (2006a). A logical framework for grounding-based dialogue analysis. Electronic Notes in Theoretical Computer Science, 157(4):117-137.

Gaudou, B., Herzig, A., Longin, D., and Nickles, M. (2006b). A new semantics for the fipa agent communication language based on social attitudes. In Proceedings of the 17th European Conference on Artificial Intelligence (ECAI'06), pages 245-249. IOS Press.

Goffman, E. (1959). The Presentation of Self in Everyday Life. Doubleday.

Guerini, M. and Castelfranchi, C. (2007). Promises and threats in persuasion. Pragmatics and Cognition, 15(2):277-311).

Halpern, J. Y. and Moses, Y. (1985). A guide to the modal logics of knowledge and belief: Preliminary draft. In Proceedings of the 9th International Joint Conference on Artificial Intelligence (IJCAI'85), pages 480-490. Morgan Kaufmann.

Hamblin, C. L. (1970). Fallacies. Methuen \& Co.

Herrmann, S. (2007). A precise model for contextual roles: The programming language ObjectTeams/Java. Applied Ontology, 2(2):181-207.

Herzig, A. and Longin, D. (2000). Belief dynamics in cooperative dialogues. Journal of Semantics, 17(2):91-118.

Hulstijn, J., Dignum, F., and Dastani, M. (2004). Coherence in multi-agent interaction. In Agent Communication (AC'2004), LNCS 3396, pages 134-152. Springer, Berlin.

Kagal, L. and Finin, T. (2005). Modeling conversation policies using permissions and obligations. In Developments in Agent Communication (AC'04), LNCS 3396, pages 120-133, Berlin. Springer.

Kibble, R. (2005). Speech acts, commitment and multi-agent communication. Computational \& Mathematical Organization Theory, 12(2-3):127-145.

Lewis, D. (1969). Convention: A Philosophical Study. Harvard University Press.

Liau, C. (2003). Belief, information acquisition, and trust in multi-agent systems - a modal formulation. Artificial Intelligence, $149: 31-60$.

Lochbaum, K. E. (1998). A collaborative planning model of intentional structure. Computational Linguistics, 24(4):525-572.

Loebe, F. (2007). Abstract vs. social roles - towards a general theoretical account of roles. Applied Ontology, 2(2):127-158.

Mackenzie, J. (1979). Question begging in non-cumulative systems. Journal of Philosophical Logic, 8:117-133.

Makinson, D. and van der Torre, L. (2000). Input-output logics. Journal of Philosophical Logic, 29(4):383-408.

Masolo, C., Guizzardi, G., Vieu, L., Bottazzi, E., and Ferrario, R. (2005). Relational roles and qua-individuals. In Proceedings of the AAAI Fall Symposium on 'Roles, an Interdisciplinary Perspective', pages 103-112. AAAI Press, Menlo Park.

Nickles, M., Fischer, F., and Weiss, G. (2006). Communication attitudes: A formal approach to ostensible intentions, and individual and group opinions. Electronic Notes in Theoretical Computer Science, 157(4):95-115.

Pasquier, P. and Chaib-draa, B. (2003). The cognitive coherence approach for agent communication pragmatics. In Proceedings of the 2nd International Joint Conference on Autonomous Agents and Multiagent Systems (AAMAS'03), pages 544-551, New York. ACM Press.

Pasquier, P., Flores, R., and Chaib-draa, B. (2004). Modelling flexible social commitments and their enforcement. In Engineering Societies in the Agent World (ESAW'04), LNCS 3451, pages 139-151, Berlin. Springer.

Pitt, J. and Mamdani, A. (1999). Some remarks on the semantics of FIPA's agent communication language. Autonomous Agents and Multi-Agent Systems, 2(4):333-356. 
Pollack, M. E. (1990). Plans as complex mental attitudes. In Intentions in Communication, pages 77-103. MIT Press.

Sadek, D. M. (1991). Attitudes Mentales et Interaction Rationnelle: Vers une Théorie Formelle de la Communication. PhD thesis, Université de Rennes I.

Sadek, D. M. (1992). A study in the logic of intention. In Proceedings of the 3rd International Conference on Principles of Knowledge Representation and Reasoning (KR'92), pages 462-473. Morgan Kaufmann.

Sadek, D. M. (1999). Design considerations on dialogue systems: From theory to technology - the case of ARTIMIS. In Proceedings of the ESCA Tutorial and Research Workshop on Interactive Dialogue in Multi-Modal Systems, Kloster Irsee, pages 173- 188. ISCA Archive.

Sadek, D. M., Bretier, P., and Panaget, E. (1997). ARTIMIS: Natural dialogue meets rational agency. In Proceedings of the 15th International Joint Conference on Artificial Intelligence (IJCAI'97), pages 1030-1035. Morgan Kaufmann.

Schank, R. C. and Abelson, R. P. (1977). Scripts, Plans, Goals and Understanding: an Inquiry into Human Knowledge Structures. Erlbaum.

Searle, J. (1969). Speech Acts: an Essay in the Philosophy of Language. Cambridge University Press, Cambridge, UK.

Singh, M. P. (2000). A social semantics for agent communication languages. In Issues in Agent Communication (AC'O0), LNCS 1916, pages $31-45$. Springer, Berlin.

Smith, R. G. (1980). The contract net protocol: High-level communication and control in a distributed problem solver. IEEE Transactions on Computers, 29(12):1104-1113.

Stalnaker, R. C. (2002). Common ground. Linguistics and Philosophy, 25:701-721.

Verdicchio, M. and Colombetti, M. (2006). From message exchanges to communicative acts to commitments. Electronic Notes in Theoretical Computer Science, 157(4):75-94.

Walton, D. N. and Krabbe, E. C. (1995). Commitment in Dialogue: Basic Concepts of Interpersonal Reasoning. State University of New York Press.

Winograd, T. and Flores, F. (1986). Understanding Computers and Cognition. Addison-Wesley.

Wooldridge, M. (2000). Semantic issues in the verification of agent communication languages. Autonomous Agents and MultiAgent Systems, 3(1):9-31.

Wooldridge, M., Jennings, N., and Kinny, D. (2000). The Gaia methodology for agent-oriented analysis and design. Autonomous Agents and Multi-Agent Systems, 3(3):285-312.

Yolum, P. and Singh, M. P. (2002). Flexible protocol specification and execution: Applying event calculus planning using commitments. In Proceedings of the 1st International Joint Conference on Autonomous Agents and MultiAgent Systems (AAMAS'02), pages 527-534. ACM Press.

Zambonelli, F., Jennings, N., and Wooldridge, M. (2003). Developing multiagent systems: The Gaia methodology. IEEE Transactions of Software Engineering and Methodology, 12(3):317-370. 


\title{
Roles, an Interdisciplinary Perspective
}

\author{
Guido Boella ${ }^{\mathrm{a}, *}$, Leendert van der Torre ${ }^{\mathrm{b}}$ and Harko Verhagen ${ }^{\mathrm{c}}$ \\ ${ }^{a}$ University of Torino, Italy - email: guido@di.unito.it \\ ${ }^{\mathrm{b}}$ University of Luxembourg, Luxembourg - email: leendert@vandertorre.com \\ c Stockholm University, Sweden - email:verhagen@dsv.su.se
}

\section{Introduction}

The notion of role is ubiquitous in many fields of computer science, like programming languages, software engineering, coordination languages, databases, multiagent systems, knowledge representation, formal ontology, computational linguistics, security, and conceptual modelling, and also outside computer science, like in cognitive science, organizational science and linguistics.

In computer science, the discussion about roles started in the '70s with Bachman and Daya (1977), and, with a recurring trend, it comes back to the attention of the research community. Recently, roles have been used in many areas to handle interaction, for example, role based access control in security with the RBAC model (Sandhu et al. (1996)), collaboration roles in UML to describe the interaction among classes (Rumbaugh et al. (2004)), channels connecting components in coordination languages (Arbab (2003)), the separation of concerns to describe the interaction properties of objects in new contexts in programming languages, etc. With the rise of the internet, new communication possibilities and interactive computing created a new demand of research about roles, for example, in organizations in open multiagent systems, in role based programming languages, in using roles for the composition of web services, and in defining roles in standards for interoperability.

Notwithstanding this revival of the research about the notion of role, little agreement seems possible among the proposals in the different fields. This lack of agreement leads to the impossibility of transferring the results from one area to the other, and even inside a single area, a consequence which is quite unpleasant in a moment where the sharing of knowledge and standardization represent an added value in many fields. For example, in ontology, the lack of a common definition of role constitutes a problem for the interconnection of different knowledge bases: the result is that a widely used ontology language like OWL does not consider roles as a primitive. In multiagent systems, the openness of systems highlights the need of commonly accepted definitions, but again without a common notion of role it is not possible for a new agent to become part of an organization to interact with other agents; and in programming languages, software reuse can be improved only by a more developed theory of how objects interact with each other basing on the roles they play.

The likely reasons of these divergences are that many papers on the notion of role fail to have an interdisciplinary character, that much work proposes new definitions of roles to deal with particular practical problems, and that role seems an intuitive notion which can be grasped in its prototypical characters, but it is instead a deceptive one when details must be clarified. Few proposals, like Steimann (2000) or Masolo et al. (2004), have a more general attitude, and try to find a problem independent definition of role and to formalize it.

\footnotetext{
*Corresponding author: Guido Boella, Dipartimento di Informatica, Università di Torino, Cso Svizzera 18510149 Torino, Italy - email: guido@di.unito.it
} 


\section{The contributions collected in this issue}

The recognition of the need of a wider agreement on roles lead us to organize with James Odell the first Roles event: "Roles, an Interdisciplinary Perspective - Roles'05" (Boella et al. (2005)), an American Association for Artificial Intelligence (AAAI) Fall Symposium, held on November 3-6, 2005 at Hyatt Crystal City in Arlington, Virginia (see the website http://normas.di.unito.it/zope/roles 05). Despite the event was organized in the area of artificial intelligence, there was a good response also from the community of programming languages and even from sociology. No previous event focussed on roles, even if some other workshops offered the environment for discussing roles, like CorOrg, NorMas, AOSE, COIN, VAR. However, they either don't have an interdisciplinary character, or they discuss roles from a specific perspective, for example, NorMas is focused on normative systems (Boella et al. $(2006,2007)$ ).

The call for papers of Roles'05 produced 30 submissions of which 22 presented at the workshop. The peculiar environment of the AAAI Symposia offered the opportunity for a lively discussion among researchers from different fields. From the presented papers five were selected for this special issue, representing the different areas involved in the workshop: ontology, programming languages and multiagent systems. Moreover, the article of Friedrich Steimann, the invited speaker of the workshop, complements the other ones by presenting an historical perspective on the subject, analysing the seminal work of Bachman and Daya (1977) on the Role data model for databases.

Roles'05 is followed after two years by the Roles'07 workshop which will be held on July 30-31, 2007 at Berlin inside the 21th European Conference on Object-Oriented Programming (ECOOP'07) on the topic "Roles and Relationships in Object Oriented Programming, Multiagent Systems, and Ontologies", in cooperation with Friedrich Steimann, Steffen Göbel and Steffen Zschaler (see the website http://normas.di.unito.it/zope/roles07).

Roles, like objects, classes and relationships, pervade the vocabulary of all sciences that deal with the nature of things and how these things relate to each other. Ontology is at the core of sciences analysing the nature of things. Given their widespread presence in all fields, it is possible to argue that roles are so fundamental a notion that they must be granted the status of an ontological primitive, besides objects, classes, and relationships. We choose the Applied Ontology Journal for publishing the best papers of Roles'05 also because we believe that, on the one hand, the area of ontology constitutes the right area for finding an agreement between the different positions on roles: it provides a formal setting which can be neutral with respect to different applications. On the other hand, ontologies can benefit from looking at other formalisms, in particular for what concerns behavioral aspects of roles, as discussed in the article of Stephan Herrmann in this issue (Herrmann (2007)).

This special issue includes five articles: an historical perspective from the field of databases, a contribution from multiagent systems which propose an organization centered view of roles, an ontological classification of roles, a description of a tool for building ontologies which include roles, and a discussion on the properties of roles in object oriented programming languages.

- In "The Role Data Model Revisited" Friedrich Steimann shows how roles have been reinvented many times in computer science. This effort would have been avoided if the work of Charles Bachman in 1977 on the introduction of roles in database models (Bachman and Daya (1977)) were not ignored due to the success of the alternative of relational data base management systems and of the entity-relationship model. As a further negative effect, the success of these solutions lead with them the limiting idea that roles are just names used to distinguish places of a relationship (or columns of a table) that happen to have the same type - and, thus, could not be distinguished by their type names. Bachman's innovation starts from the recognition that most conventional records in databases are defined on roles: e.g., employees, customers, patients, or students. Roles like employers and employees are connected by (1:n) relationships. These roles can be played by different kinds of entities, e.g., persons and organizations, which in turn can play more than one role at the same time. Thus, Bachman separated the existence of an entity from its appearance in a relationship, where it exhibits rolespecific behavior: roles can be acquired and released dynamically. The properties which Steimann 
recognizes in Bachman's model are quite general and can be found also in other disciplines. Thus, if object, class, and relationship are ontological primitives, the notion of role deserves the same status. Bachman seems to anticipate also recent works in the ontology field, like Guarino and Welty (2002), where roles are defined as founded, since roles are defined in the context of relationships, and as not semantically rigid, since entities can assume and drop roles without losing identity. The most evident limitation of Bachman's model is that the same entity cannot play the same role more than once at the same time. This limitation has been solved in subsequent approaches by considering roles as adjunct instances associated (without an identity) to their players. Instead, the only solution coherent with the original role data model, according to Steimann, would have been to use the relationship as the place where to add the properties assigned to roles.

- "Roles, Players and Adaptable Organizations" by Alan W. Colman and Jun Han represents a contribution from the area of multiagent systems. The article focuses on building software systems on the basis of social contexts which are intentionally designed and structured in the systems. The social contexts give to the systems an organizational structure composed of a network of relationships among roles, and of the processes that maintain the viability of these relationships in response to changing goals and changing environments. The possibility to restructure itself distinguishes an organization from a mere structure. The restructuring processes can be regulated by some of the roles of the organization by means of interaction protocols and authority relationships (powers, expectations and obligations) which regulate the interaction among roles. Complex organizational structures are built by allowing organizations to play roles in other organizations.

In an open system it is difficult to make assumptions on the possible players of roles. Different kind of players, with different reasoning and acting capabilities, may require different degrees of autonomy. In this regard, the article discusses the shifting boundary between role and role-player: for example, a role for an inexperienced player can give to it more details about the procedures to achieve the role's goals with respect to a role for an experienced player.

The authors contest approaches where roles are used in the modelling and to inform the design, but disappear as entities during implementation. They claim that roles should be first class entities also during the runtime phase of a system. Moreover, the authors contest the view of the dependence of roles from players, as deriving from an object-centric or player-centric perspective. In this perspective, the identity of the role is an adjunct to the identity of the object. Instead, the role should be seen as the stable entity (thanks to its link with the organization) to which transient players are attached. Their perspective looks at roles from an organization-centric viewpoint. From this perspective, a role's identity and existence derives from the organization that defines the roles and associations - not from the player itself. This view contrasts with ontological analyses where roles are founded mainly on players, or on other concepts, but not on the organizations offering the roles (Masolo et al. (2004)).

- In "Abstract vs. Social Roles - Towards a General Theoretical Account of Roles" of Frank Loebe, an ontology of roles is proposed. Roles are associated both with a player and a context, in an extension of the top-level ontology General Formal Ontology - GFO - (Herre et al. (2006)). The author classifies three kinds of roles according to the different kinds of contexts they belong to. Roles are parts of contexts (in some sense of the term) and the contexts emerge from the existence of the roles, in a mutual existential dependence. Three kinds of contexts are considered in the classification of role, which determine different playing relations:

* Relational role: corresponds to the way in which an argument participates in the context of some relationship; e.g., two as a factor of four refers to a relationship. Relational roles are special properties, and the "plays" relationship between entities and relational roles is thus subsumed by the "has-property" relation.

* Processual role: corresponds to the manner in which a player behaves in the context of some process (i.e., it participates in the process): e.g., John as the mover of some pen is categorized as a processual role. Processual roles are parts of processes, and, therefore, processes themselves. 
* Social role: corresponds to the involvement of a social object within the context of some society; e.g., a student in a university. A genuine notion of playing a social role is required based on the notion of counts as (Searle (1995)), which is vaguely similar to inherence. However, inherence in the case of social roles is considered with respect to roles as "complex properties", since social roles are often defined with their own properties, relations and processes in which they (may) participate (confirming the view of roles as "patterns of behavior"). This means that social roles are considered as objects.

- "A Model of Roles in Ontology Development Tool: Hozo" by Riichiro Mizoguchi, Eiichi Sunagawa, Kouji Kozaki and Yoshinobu Kitamura merges an ontological analysis of roles with practical considerations about designing tools for building ontologies. The most novel element of their model is the notion of role holder, an abstraction of a composition of a role-playing entity with an instance of a role concept. In turn, the role concept instance can exist only in presence of an instance of the context the role is associated with. The introduction of the notion of role holder allows the authors to explain in which sense roles can play roles, and there can be instances of role concepts without a corresponding player. When we say that a citizen becomes a minister, in the Hozo model the situation is represented by making the role of minister played by the role holder of the citizen role, rather then by the instance of the role concept. In this way, it is possible also to express the requirement that to play a role it is necessary to be the player of another role.

As in Colman and Han (2007), roles (in the sense of role concept instances) can exist without a player, due to their relation with a context. Conversely, some contexts exist only in presence of the roles which compose the context. For example, a marriage exists only as far as both the roles of wife and husband exist. As Loebe (2007) does, the authors provide a classification of roles basing on the type of context they are related to. Besides relational roles, processual roles and social roles, Hozo distinguishes action related roles, attribute roles and composite roles. Composite roles allow to model a role like teacher, which includes both the role of staff member of a school and of agent of a teaching activity, and, thus, they depend on multiple contexts at a time.

- "A Precise Model for Contextual Roles: The Programming Language ObjectTeams/Java" by Stephan Herrmann describes the properties of roles in a modern approach which introduces roles in object oriented programming languages. According to a recent trend, witnessed also by Tamai (2002) and by Baldoni et al. (2007a), roles are considered as existing only in a context, where they explain new possibilities of interaction given to their players. This is another example of what Colman and Han (2007) in this issue call the organizational-centric view of roles. Contexts, called object teams, are defined at the universal level as classes which include other classes. These "inner" classes describe roles played by other objects, and they specify the properties and the behavior of the playing objects in the context of the team. When an object plays a role in a context, its behavior is changed according to what specified in the role: its methods, when they are invoked, can be substituted by the methods specified in the role, by means of aspect programming techniques (Kiczales et al. (1997)). Including roles in contexts promote coordination, as the roles, contrary to the usual encapsulation principle, can access the private state of each other and of the context, since they belong to the same namespace. Contexts partly correspond in multiagent systems to the concept of organization of Colman and Han (2007).

In this article, on the one hand, it is possible to find many of the properties associated in ontological analysis of roles, among which Loebe (2007) in this issue: roles are acquired and relinquished dynamically; roles do not exist independently of their players and contexts, even if they have instances to represent context dependent attributes and behavior; and an object can play several roles simultaneously, also of the same type, if the role instances belong to different instances of the same context. On the other hand, providing a programming language approach allows to clarify some issues which are less easy to detail in a more static approach like ontology. In particular, roles are often associated with behaviors, but the relation between the behavior of the role and the behavior of the player is not clear if the technical details of method invocation are not analysed. 


\section{What is a role?}

This special issue does not propose an overall definition of roles. In this editorial, however, we want to highlight some features of roles acknowledged by the articles collected here, which are often disregarded in the literature. Undisputed distinguishing features of roles seem to be their dependence on some other entities and their dynamic character. These properties contrast roles with the notion of natural type. Its natural type is essential to an entity: if an entity changes its natural type, it loses its identity; instead, in Guarino and Welty (2002)'s terms, roles lack the rigidity which natural types possess.

The first conclusion we can draw from the articles of this special issue is that roles have a relational nature, and, for this reason, they are linked with the notion of interaction. Already Masolo et al. (2005) elaborate on the relational nature of roles, highlighting their definitional dependence on relationships, but this nature is not always accepted. The connection between roles and relationships has been disregarded since relationship itself is a notion whose importance is not fully recognized yet, for example, in programming languages. With the advent of the object orientation paradigm relationships have moved out of the focus of attention at some levels of representation, like programming languages, giving way to the more restricted concept of attributes or, more technically, references to other objects. A reference is tied to the object holding it and as such is asymmetric - at most the target of the reference can be associated with a role. This is in contrast with the intuition that every role should have at least one counter-role (e.g., student and professor, buyer and seller, initiator and bidder, etc.). The existence of a counter role, in turn, highlights the function of roles in describing the interaction of objects in the scope of a relationship (e.g., a negotiation process in the case of the pair of roles like initiator and bidder): each role is associated with another role, namely the one it interacts with. In other disciplines, like ontology and multiagent systems this connection with interaction already emerged. In the ontology of Loebe (2007), social roles participate in processes, to model the interactions among roles, and play roles in relationships associating them to their counter roles. The relational and interactional nature of roles emerge clearly in the area of multiagent systems (see Colman and Han (2007)), since roles specify the interaction among agents and the communication protocols.

The second conclusion is strictly related to the first one. As Loebe (2007) recognizes, often, relational roles, at a deeper analysis, can turn into social roles. Thus, roles are associated to a social context they belong to. This view is directly inspired by the organizational metaphor, where roles are a way to structure an organization and to distribute responsibilities. The relationships connecting roles and counter-roles span across an organization. This metaphor is particularly effective in the field of multiagent systems, where organizational structures are used to coordinate agents in open systems by assigning them roles specifying their responsibilities. The organizational-centric point of view theorized by Colman and Han (2007) in this issue sharply contrasts with past work on roles, which still constitutes the prototypical view in many communities (see, e.g., Bäumer et al. (1997)): roles as extensions of the behavior of objects or agents, disregarding the context the roles belong to and the interactions they allow.

The third conclusion is that behavior should not be disregarded as a main feature of roles. In sociology, often, roles have been defined as "patterns of behavior". In computer science, on the one hand, programming languages associate methods to roles, and, sometimes, like in Herrmann (2007), the methods of the roles even substitute the methods of their players. On the other hand, in multiagent systems (like in Colman and Han (2007) and Boella and van der Torre (2007)), the behavior associated with roles has a different nature with respect to the behavior associated with the agents playing them. While the players can perform physical actions, roles exercise powers. The notion of power is derived from the organizational (or institutional) metaphor: an action of the player of a role counts as an institutional action which affects the obligations, permissions, responsibilities and powers of the organizations and of the other roles involved. These two kinds of actions seem to have a different ontological status which is partly unexplored. 


\section{Open questions}

Even if this special issue clarifies some problems about the notion of role, many questions remain open. Among them, we identify the following ones:

1. How the notion of role in computer science can benefit from the analyses made in other disciplines? For example, organizational theory and the construction of social reality proposed in philosophy by Searle (1995) and Tuomela (1995) have been recently considered to model roles in ontologies (Bottazzi and Ferrario (2005)). Notions like sociotechnical systems developed in sociology are introduced by Jahnke et al. (2005).

2. The notion of role emerges as a likely candidate to be added besides the other ontological primitives, like object, class and relationship. Often, ontologies have a cognitive bias, like DOLCE has (Gangemi et al. (2002)). Thus, the question becomes: is the notion of role also a cognitive primitive or can it be related to other primitives? For example, recent work (Baldoni et al. (2006)) suggests that the notion of role is related to the notion of affordance developed in cognitive science by Gibson (1979). Affordances are the interaction possibilities offered by an environment to an animal living in it. Roles can be explained as sets of affordances (niches in Gibson (1979)'s terminology) and can be played by different entities according to their capabilities. the connection between the notion of role and the cognitive notion of affordance, thus, explains also the interactional character of roles discussed above.

3. Roles and relationships seem to be two sides of the same coin and, thus, are strictly interconnected. Recent work on relationships in object oriented languages witnesses the existing interest for relationships (Balzer et al. (2007)). However, roles are rarely connected with relationships. Thus, the following one remains an open question: when relationships are introduced in object oriented languages, how can roles be added too? The solution supported by Steimann (2007) is to overload the notion of relationship with the properties and behaviors which are associated to the roles a relationship identifies. Baldoni et al. (2007b) propose the opposite solution: relations should be reduced to roles, rather than to attributes or references, and the roles should be overloaded with the properties specific of the relationship. Can the two views be merged or are they incompatible?

4. Is a single definition of role possible? And, if it is not useful to have one or, if it does not exist, how many different notions of role exist? Loebe (2007) in this issue distinguishes between relational, processual and social roles on the basis of the context a role belongs to, and Mizoguchi et al. (2007) introduce further distinctions. A new challenge is to define a metalevel framework to cover different definitions of roles by specifying it by means of different parameterizations, to understand which are all the variables at issue. A preliminary tentative is given by Genovese (2007) where a metamodel is defined and different models of roles, like RBAC, are described with it.

5. Which are the criteria for evaluating role models? Which are the benchmark examples to be covered? Most works on roles use the student-course example, but it is rather simple and it is not clear whether it is general enough to cover most features attributed to the different types of roles. Steimann (2007) proposes as a criterion the possibility to use a model of roles as a metamodel of itself. Herrmann (2007) shows how Object Teams can provide a metamodel of itself: which other proposals can pass this test?

6. Mizoguchi et al. (2007) propose a first tentative to model roles in OWL. However, a general agreement must still be found. How can the notion of role be introduced as an ontological primitive in the standards for the semantic web? How and in which setting is it possible to formulate a proposal and find the necessary political agreement on it?

We hope that the discussion at the next edition of Roles at ECOOP'07, which aims at proposing a "Role Manifesto", will be able to answer these questions and to identify the general properties of roles which can be accepted in different research communities. 


\section{References}

Arbab, F. (2003). Abstract behavior types: A foundation model for components and their composition. In Formal Methods for Components and Objects, volume 2852 of LNCS, pages 33-70. Springer, Berlin.

Bachman, C. and Daya, M. (1977). The role concept in data models. In Procs. of Very Large Databases (VLDB'77), pages 464-476.

Baldoni, M., Boella, G., and van der Torre, L. (2006). Modelling the interaction between objects: Roles as affordances. In Procs. of Knowledge Science, Engineering and Management, KSEM'06, volume 4092 of LNCS, pages 42-54, Berlin. Springer.

Baldoni, M., Boella, G., and van der Torre, L. (2007a). Interaction between objects in powerJava. Journal of Object Technology, $6(2): 7-12$.

Baldoni, M., Boella, G., and van der Torre, L. (2007b). Relationships meet their roles in object oriented programming. In Procs. of the 2nd International Symposium on Fundamentals of Software Engineering 2007 Theory and Practice (FSEN '07), LNCS, Berlin. Springer.

Balzer, S., Gross, T., and Eugster, P. (2007). A relational model of object collaborations and its use in reasoning about relationships. In Procs. of the 21 th European Conference on Object-Oriented Programming (ECOOP'07), Berlin. Springer.

Bäumer, D., Riehle, D., Siberski, W., and Wulf, M. (1997). The role object pattern. In Procs. of Conference on Pattern Languages of Programs (PLOP'97).

Boella, G., Odell, J., van der Torre, L., and Verhagen, H., editors (2005). AAAI 2005 Fall Symposium on Roles, an interdisciplinary perspective (Roles'05), Arlington, VA, 03/11/05-06/11/05, volume FS-05-08 of AAAI Technical Report, Menlo Park (CA). AAAI.

Boella, G. and van der Torre, L. (2007). The ontological properties of social roles in multi-agent systems: Definitional dependence, powers and roles playing roles. Artificial Intelligence and Law Journal (AILaw).

Boella, G., van der Torre, L., and Verhagen, H. (2006). Introduction to normative multiagent systems. Computation and Mathematical Organizational Theory, special issue on normative multiagent systems, 12(2-3):71-79.

Boella, G., van der Torre, L., and Verhagen, H., editors (2007). Normative Multiagent Systems, number 07122 in Dagstuhl Seminar Proceedings. Internationales Begegnungs- und Forschungszentrum fuer Informatik (IBFI), Schloss Dagstuhl, Germany.

Bottazzi, E. and Ferrario, R. (2005). A path to an ontology of organizations. In Procs. of EDOC Int. Workshop on Vocabularies, Ontologies and Rules for The Enterprise (VORTE 2005).

Colman, A. and Han, J. (2007). Roles, players and adaptable organizations. Applied Ontology, this issue.

Gangemi, A., Guarino, N., Masolo, C., Oltramari, A., and Schneider, L. (2002). Sweetening ontologies with DOLCE. In Procs. of 13th International Conference on Knowledge Engineering and Knowledge Management. Ontologies and the Semantic Web (EKAW 2002), volume 2473 of LNCS, pages 166-181, Berlin. Springer.

Genovese, V. (2007). Towards a general framework for modelling roles. In Boella, G., van der Torre, L., and Verhagen, H., editors, Normative Multiagent Systems, number 07122 in Dagstuhl Seminar Proceedings. Internationales Begegnungs- und Forschungszentrum fuer Informatik (IBFI), Schloss Dagstuhl, Germany.

Gibson, J. (1979). The Ecological Approach to Visual Perception. Lawrence Erlabum Associates, New Jersey.

Guarino, N. and Welty, C. (2002). Evaluating ontological decisions with ontoclean. Communications of ACM, 45(2):61-65

Herre, H., Heller, B., Burek, P., Hoehndorf, R., Loebe, F., and Michalek, H. (2006). General formal ontology (GFO) - A foundational ontology integrating objects and processes. Onto-Med Report 8, Research Group Ontologies in Medicine, Institute of Medical Informatics, Statistics and Epidemiology, University of Leipzig.

Herrmann, S. (2007). A precise model for contextual roles: The programming language ObjectTeams/Java. Applied Ontology, this issue.

Jahnke, I., Ritterskamp, C., and Herrmann, T. (2005). Sociotechnical roles for sociotechnical systems: a perspective from social and computer science. In AAAI 2005 Fall Symposium on Roles, an interdisciplinary perspective (Roles'05), volume FS-05-08 of AAAI Technical Report, Menlo Park (CA). AAAI Press.

Kiczales, G., Lamping, J., Mendhekar, A., Maeda, C., Lopes, C., Loingtier, J., and Irwin, J. (1997). Aspect-oriented programming. In Procs. of the 11th European Conference on Object-Oriented Programming (ECOOP'97), volume 1241 of LNCS, pages 220-242, Berlin. Springer.

Loebe, F. (2007). Abstract vs. social roles - towards a general theoretical account of roles. Applied Ontology, this issue.

Masolo, C., Guizzardi, G., Vieu, L., Bottazzi, E., and Ferrario, R. (2005). Relational roles and qua-individuals. In AAAI 2005 Fall Symposium on Roles, an interdisciplinary perspective (Roles'05), volume FS-05-08 of AAAI Technical Report, Menlo Park (CA). AAAI.

Masolo, C., Vieu, L., Bottazzi, E., Catenacci, C., Ferrario, R., Gangemi, A., and Guarino, N. (2004). Social roles and their descriptions. In Procs. of Conference on the Principles of Knowledge Representation and Reasoning (KR'04), pages 267277. AAAI Press.

Mizoguchi, R., Sunagawa, E., Kozaki, K., and Kitamura, Y. (2007). A model of roles in ontology development tool: Hozo. Applied Ontology, this issue.

Rumbaugh, J., Jacobson, I., and Booch, G. (2004). Unified Modeling Language Reference Manual, The (2nd Edition). Pearson Higher Education.

Sandhu, R., Coyne, E., Feinstein, H., and Youman, C. (1996). Role-based access control models. IEEE Computer, 2:38-47.

Searle, J. (1995). The Construction of Social Reality. The Free Press, New York.

Steimann, F. (2000). On the representation of roles in object-oriented and conceptual modelling. Data and Knowledge Engineering, 35:83-848.

Steimann, F. (2007). The role data model revisited. Applied Ontology, this issue.

Tamai, T. (2002). Evolvable programming based on collaboration-field and role model. In Procs. of International Workshop on Principles of Software Evolution (IWPSE'02), pages 1-5, New York. ACM.

Tuomela, R. (1995). The Importance of Us: A Philosophical Study of Basic Social Notions. Stanford University Press, Standford (CA). 


\title{
The Ontological Properties of Social Roles: Definitional Dependence, Powers and Roles Playing Roles
}

\author{
Guido Boella (guido@di . unito.it) \\ Dipartimento di Informatica - Università di Torino - Italy
}

Leendert van der Torre (torre@ cwi.nl)

CWI - Amsterdam and TU Delft The Netherlands

\begin{abstract}
In this paper we address the problem of defining social roles in MAS. Social roles provide the basic structure of social institutions and organizations. We start from the properties attributed to roles both in the MAS and the Object Oriented community, and we use them in an ontological analysis of the notion of social role. We thus identify as the main properties of social roles being definitionally dependent on the institution they belong to, attributing powers to the agents playing them, and allowing roles to play roles. The methodology we use to model roles is the agent metaphor: social roles, in the same way as social institutions, like normative systems and organizations, are attributed mental attitudes to explain their behavior.
\end{abstract}

\section{Introduction}

The social structures developed in multiagent systems are often proposed in the design of open systems as a solution for controlling the autonomy of the different participants (Artikis et al., 2002). A key notion in the social structure of a MAS is that of social role. Social roles allow to specify the activities delegated by a social institution to individuals to achieve its purpose, while abstracting from the individuals which will eventually play them. The description of a social role is given, e.g., in terms of rights, permissions and obligations (i.e., normative descriptions) (Pacheco and Carmo, 2003), expectations, standardised patterns of behavior (Esteva et al., 2001), social commitments (Cavedon and Sonenberg, 1998; Fasli, 2001), goals and planning rules (Dastani et al., 2003).

Even if social roles have such a central position in MAS coordination, there are still some problems. First, it is not clear which are the desired properties and how to realize them. Second, normative descriptions are mostly limited to rights, while the notion of power seems relevant as well.

We want to extend the notion of social role in Agent Oriented systems (AO), and to make it more concrete we use ideas and concepts from the properties of roles discussed in the Object Oriented paradigm (OO). A sideeffect is that a unified model of roles in AO and OO not only impacts in $\mathrm{AO}$, but also in OO. Roles are central not only in MAS, but also in Object Oriented modelling and programming. Roles in $\mathrm{OO}$ are used to dynamically add behaviors to objects, to factorize features of objects like methods or ac- 
cess rights, to separate the interactional properties of objects from their core behavior, and to allow exogenous coordination (Baldoni et al., 2005).

This paper addresses the following questions:

- What are the desirable properties of social roles in MAS?

- How to build a model satisfying these properties?

To extend the existing ideas in $\mathrm{AO}$ and to use the ideas in $\mathrm{OO}$, we refer to social theory, which suggests:

- Roles are always involved in a relationship with another entity, which seems to come first: roles belong to organizations and institutions which define them; hence, they are social roles.

- Concerning the normative positions, besides rights and permissions, social roles are associated to powers in the institution they belong to.

- If roles can play roles as any other agent, then social roles should be considered as a kind of agent.

Besides treating roles as usual in both $\mathrm{AO}$ and $\mathrm{OO}$ as first class citizens of the theory, here social roles are treated as agents. However, social roles are not autonomous, and they should therefore be treated as agents of a special kind.

We call this methodology the agent metaphor. Though at first sight social roles are anything but agents we treat social roles as agents because we attribute mental attitudes to agents, as done by (Boella and van der Torre, 2004a; Boella and van der Torre, 2004b); this has as additional benefit that we can reuse for social roles existing theories, models and tools developed for agents. Analogously, social institutions can be described in the agent metaphor as agents to which mental attitudes are attributed. We apply the methodology used by (Boella and van der Torre, 2006) to describe and reason about other social entities like groups, virtual communities, contracts, and normative multiagent systems.

In the next section we analyse the properties which are commonly attributed to roles in $\mathrm{AO}$ and $\mathrm{OO}$. Then, we present their basic properties in our model: the definitional dependence in Section 3, the powers of roles in Section 4 and roles playing roles in Section 5, where the agent metaphor is further discussed. Then, in Section 6 we present our formal model of roles in MAS. Conclusions close the paper. 
1. Agents can play multiple roles, and in some approaches they can even have to play a role.

2. Roles are views on agents.

3. Individual are uncoupled from roles. E.g., attributes like wage are associated to the employee role rather than to individuals.

4. Roles enhance reusability: the same role can be used by different agents.

5. Roles define expected behavior and obligations. E.g., a program chair is expected and obliged to select the papers of a conference.

6. Roles define sets of rights and permissions. E.g., the access rights.

7. Roles embed behavior specific to a context, like a group, which forms a subenvironment of coherent roles.

8. Roles define common interactions and embed information and capabilities needed to communication and coordination. E.g., the roles of auctioneer and bidder in an auction, each with their possible moves.

9. Promote an organizational view of the system, where roles are coordinating rather than coordinated entities.

Figure 1. The properties of roles in AO.

\section{Properties of roles}

In their survey about roles in MAS, (Cabri et al., 2004) identify several properties attributed to roles, which are illustrated in Figure 1. There are two problems. First, it is not clear which model of role can support all these properties. The second problem is that rights are a too limited notion. It suffices for role-based access, but in general we also need powers to specify normative positions.

The properties attributed to roles in the Object Oriented community are summarized by (Steimann, 2000). In Figure 2, we show here how these properties are also relevant for agents by giving some examples. These properties only partially overlap with the previous list. In particular, properties 5-9 of Figure 1 assume that agents are autonomous, they can violate obligations, they interact with each other, and they form social institutions like organizations and groups. The properties discussed in $\mathrm{OO}$ are more concrete and talk about roles as adjunct instances to objects (11), states of roles (7), sequences of acquisitions (6), identity (14), polymorphism (7) and other phenomena, and thus address the first problem discussed in the paragraph above. However, they do not help with the generalization of rights to other powers. Moreover, this more concrete descriptions also give rise to two new questions. First, the fact that roles depend on relationships with other entities implies that first come these other entities, then the roles. Second, roles playing roles imply a kind of role hierarchy. Groups and contexts are not sufficient to model all aspects of this. We need to model role as a non autonomous notion of agent. 
1. A role comes with its own properties and behavior. Hence, it is a type. E.g., a director of a department commands other members and makes buy-orders.

2. Roles depend on relationships: e.g., a student is related to a school, etc.

3. An object may play different roles. E.g., a person can be both a student and an employee.

4. An object may play the same role several times. E.g., a person can hold several employments.

5. An object may acquire and abandon roles dynamically. E.g., a person can acquire the role of student or of employee.

6. The sequence in which roles may be acquired and relinquished can be subject to restrictions. E.g., a person becomes a teaching assistant only if it is a student.

7. Objects of unrelated types can play the same role. E.g., both a person and an organization are customers.

8. Roles can play roles. E.g., an employee can be a project leader: a role of the employee.

9. A role can be transferred from one object to another. E.g., the salary of an open position may be specified independently of the person that will be employed.

10. The state of an object can vary depending on the role in which it is being addressed: this should be viewed as a separate instance of the object. E.g., an employee has an address per job and also a private one.

11. If an object plays several of roles simultaneously, it responds according to the role in which it is being addressed. E.g., an person gives the address of the employee it is playing.

12. Roles restrict access. This corresponds to an object having different perspectives, facets, or aspects. E.g., the private phone number of an employee can be invisible when the person is playing the employee role.

13. Different roles may share structure and behavior. This usually means that role definitions inherit from each other. E.g., the role student can have associated the behavior of giving exams, and more specific roles (like first year student) inherit this behavior.

14. An object and its roles share identity. Since roles do not exist by themselves they cannot have an identity.

15. An object and its roles have different identities. This view solves the so-called counting problem. E.g., the number of passengers of an airline can be greater than the number of person who travelled with it.

Figure 2. The properties of roles in OO.

Thus, there are three open problems: how to define dependencies of social roles on relationships, how to extend normative positions from rights to powers, and how to model social roles as agents that play roles. These issues are discussed in the following three sections. We support these properties by means of an ontological analysis of the notion of social role. Roles deserve an ontological analysis in that they are among the basic notions of an ontology besides the notion of natural type, substance, property, and relation. Ontological analysis aims at identifying the metaproperties distinguishing roles from those other notions, as done by (Masolo et al., 2004). 


\section{Definitional dependence}

Social theory considers social roles as a way to structure organizations so to distribute responsibilities. Thus, for social theory, roles exist only in function of the organization they belong to. This feature has been recognized also by ontological analysis of roles. (Guarino and Welty, 2002) notice two characteristic properties of roles distinguishing them from natural types: roles are non-rigid entities and do not exist independently from other entities. Rigidity means that an entity can stop to play a role without losing its identity. E.g., a person can stop being a student, but not a person.

The dependence of a role, as suggested by the work of (Sowa, 2000) and (Guarino and Welty, 2002), is a consequence of the fact that a role is meaningful only in the context of a relationship with another entity. This property is also called foundation: a role must always be associated with another entity through some relationship. Some hints of this ontological property of roles could already be found in the literature. In the traditional approach to roles in linguistics, words are always related to other words: every word in a sentence has slots to be filled by others; e.g., a verb like eating has an agent and patient role. Concerning conceptual modelling, in UML a role is correlated by an association to other roles. In Agent-UML a role is related to a group (Bauer et al., 2001)

The dependence of a role from another entity is not contingent, but it rests in the definition itself of the role. For this reason, (Fine, 1995) introduces the following notion of dependence: "to say that an object $x$ depends upon an $F$ is to say that an $F$ will be ineliminably involved in any definition of $x$ ". This notion is elaborated by (Masolo et al., 2004) into the notion of definitional dependence: e.g., the definition of the concept of student makes reference not to a specific school but to the concept of school, the employee to the concept of organization, the director to the concept of department, the president to the concept of state, etc.

We believe, however, that this definitional dependence should be interpreted in an even stronger way. First of all, not only social roles all depend on other entities, but the entities they depend on all belong to a common category; they all are social entities: groups, organizations, departments, states, etc. In a word, social institutions. Secondly, not only social roles do not exist without social entities, but, in turn, roles are essential to them: there is no state without a president, no school without a student. Hence, we adopt a stronger notion of definitional dependence. We say that the definition of the social institution $(F)$ the social role $x$ belongs to contains the definition of the role $(x)$. E.g., the social role of president of a state is defined in the constitution of that state. The role president does not exist without the state and its definition, but also the state itself is not the same without the role of president: its definition would be different. 


\section{Roles, powers and institutions}

According to Property 6 of Figure 1 rights and permissions are a fundamental feature of normative positions of roles. Rights are used to regulate access to resources by agents playing roles, e.g., in role based access control (RBAC). However, as, amongst others, (Makinson, 1986) has noticed, the terms right and permission often should be intended in the sense of institutional power. The notion of power is certainly relevant here, since, e.g., a director of a department has not only the right to give commands to the employee, but, above all, it has the power to do so. But, as witnessed also by (Dastani et al., 2004)'s survey, the MAS model of role is mostly limited to rights. Moreover, in Figure 1, roles are associated to new capabilities. In Figure 2, roles are associated with behaviors (1). Roles as a way of grouping context dependent behavior do not explain why we need roles to do this grouping and not simply the notion of class, albeit a dynamic one. We claim that the reason is because these capabilities have a peculiar character: they are powers.

Again, some insights can be gained by considering which capabilities are added to a social role. They can be grouped in three categories:

- Actions of the role that are recognized as actions of the institution: e.g., a director's signature on a buy-order is considered as a commitment of its department to pay for the requested item.

- Actions of the agent playing the role that can modify the state of the role itself. E.g., a director can commit itself to new responsibilities.

- Interaction capabilities with other roles in the same institution. An agent in a role can send a message to another role, e.g., a director can give a command to an employee.

Not only social roles do not exist without social entities, but they cannot do anything without their consent. The reason is that social entities are not material entities: agents playing roles cannot do anything for affecting them.

Social institutions are socially constructed entities which exist thanks to the collective acceptance by agents of the regulative and constitutive rules regulating them. In particular, they are created by means of the notion of constitutive rule introduced by (Searle, 1995). Searle argues that there is a distinction between two types of rules:

Some rules regulate antecedently existing forms of behaviour. [...] Some rules, on the other hand, [...] create the possibility of or define that activity. The activity of playing chess is constituted by action in accordance with these rules. The institutions of marriage, promising [...] are systems of such constitutive rules or conventions. 
Constitutive rules have of the form "such and such an $\mathrm{X}$ counts as $\mathrm{Y}$ in context $\mathrm{C}$ " where $\mathrm{X}$ is any object satisfying certain conditions and $\mathrm{Y}$ is a label that qualifies $\mathrm{X}$ as being something of an entirely new sort: an institutional fact. Examples of constitutive rules are " $\mathrm{X}$ counts as a presiding official in a wedding ceremony", "this bit of paper counts as a five euro bill" and "this piece of land counts as somebody's private property".

Thus, institutions are composed of regulative and constitutive rules. But since social roles are defined by the institution they are defined in turn in terms of constitutive rules and regulative rules attributed to them by the institution. Since constitutive rules are at the basis of an institution and of roles, an agent can act in the institution only if for the institution the agent's actions "count as" some institutional fact. In this sense, the new capabilities added by the role are given by the institution; the role is empowered by the institution: the actions which it performs in its role "count as" (Searle, 1995) actions of the institution itself.

We can explain the three different kinds of powers discussed above as different kinds of constitutive rules. First of all, actions of the player of the role "count as" institutional facts according to some constitutive rule of the institution. So it can affect the institution. Secondly, if the constitutive rules creating an institutional fact belong to the role the agent it is playing, the agent can affect its role. Thirdly, if the constitutive rule belongs to some other role of the institution, the agent in playing its role can affect this other role.

The effects of the action of a player of the role are not limited to making true institutional facts. Institutional facts can have, in turn, an effect on the institution and on the roles, via other constitutive rules introducing new constitutive and regulative rules. For example, the signature of the director "counts as" the commitment of the department (i.e., a new obligation) to pay for the delivered goods. And the command of the director "counts as" an obligation for the members of the department.

Finally, note that if we consider the possibility that a role is changed by the exercise of a power from another role we admit implicitly that a role is not only a type specifying the behavior expected by the player. Rather, a role is an instance which its own state. This state specifies the expected behavior of an agent, and this specification can vary over time according to the exercise of power by the player, the institution and by other roles.

The counterpart of strong definitional dependence and its ability to make roles access the institution's state, in the Object Oriented world, is that roles should be defined in the definition of an object, i.e., a class, that determines its scope: all roles should be encapsulated in other classes. 


\section{Roles playing roles}

Another important feature of roles is that roles can play roles as well. For example, an empolyee can be a project leader, a member of the board can be the CEO of an enterprise, etc.

Roles are usually played by agents: a person can be an employee, a member of a club, of a board or an MP. But how can a role play a role? This is possible only if an agent and a role share some properties. As we will see in the next section, this is possible in our model since roles are described as agents, i.e., they are attributed mental attitudes as well. Note that in many models, e.g., (Dahchour et al., 2002), roles do no play roles, and a role like project leader is modelled simply as specification of the employee role. However, this solution relies on a type specification hierarchy of roles and requires introducing dynamic reclassification. Instead, our approach does not require this feature, but it allows anyway to create a hierarchy among roles: the hierarchy is based on the inherently dynamic played-by relation between roles and agents, rather than on a specification relation.

The methodology of our work is inspired to the agent metaphor of (Boella and van der Torre, 2006). They model entities of social reality like groups, normative systems, organizations and roles as agents. Their ontological claim is that social reality is difficult to understand for humans, even if humans themselves create it. Hence, to understand social reality humans resort to metaphorically mapping the social domain in a better known domain: the domain of agents. Social entities exist because they are collectively accepted by agents (Searle, 1995). To define the behavior of social entities, they are collectively attributed by the agents' mental attitudes.

This metaphorical mapping allows to explain the features of social entities in terms of the features of agents. In particular, in this mapping a social institution can be considered as an agent where the regulative norms, like obligations and permissions, are mapped into the goals of an agent; the constitutive norms creating powers are mapped into the beliefs of the agent.

Moreover, the institution, as a normative system, is supposed to have an autonomous behavior as an agent has: it aims at restoring the regularities prescribed by norms by means of monitoring violations and sanctioning them. The metaphor, however, stops here since social entities cannot act in the world. Monitoring and sanctioning are carried out by real agents working in the institution.

Roles in sociology are often described as expected behavior. To describe behavior, agent theory uses beliefs, desires and goals. Hence, roles can be considered as agent descriptions. This is different from the fact that roles are also played by agents, their actors. Since roles are considered as agents, they can play roles in turn. In the metaphorical mapping (Boella and van der Torre, 2004a; Boella and van der Torre, 2004b) of the role's expertise are represented 
by beliefs of the agent and its responsibilities as the goals of the agent. To play a role an agent has to adopt the goals representing its responsibilities and to carry out them according to the beliefs representing its expertise: the player has to act $a s$ if it had the beliefs and goals of the role.

In the same way as social entities are constructed by the collective attribution of mental entities by agents, roles exist only because they are attributed mental attitudes by the institution they belongs to. The institution is thus defined by its beliefs and goals representing constitutive and regulative rules and by the beliefs and goals it attributes to its roles. While (Boella and van der Torre, 2004a; Boella and van der Torre, 2004b) focus on responsibilities of roles, in this paper we focus on their powers.

\section{Formalization of roles}

In this section we introduce our model of roles and institutions.

First of all, a set of propositional variables $X$ describes the different aspects of the world, and rules $\operatorname{Rul}(X)$ are used to represent mental attitudes.

Secondly, we consider different sorts of agents $A$. Besides real agents $R A$ (either human or artificial) we consider as agents in the model also social institutions $S A$, like groups, normative systems and organizations, and roles $R O$ composing the structure of agents in $S A$.

By mental attitudes we mean beliefs $B$, desires $D$ and goals $G$. Mental attitudes are described by rules. Moreover, different mental attitudes are attributed to the agents by the agent description relation $A D$. It associates to each agent a set of beliefs, desires and goals. Moreover, $A D$ associates also agents to agents, because groups, normative systems, organizations, and roles as agents exist only as profiles attributed to them by real agents. So social institutions and roles exist only as they are described as agents by real agents, according to the agent description relation.

\section{DEFINITION 1 (MAS).}

Let $X$ be a set of variables. The set of literals built from $X$, written as $\operatorname{Lit}(X)$, is $X \cup\{\neg x \mid x \in X\}$, and the set of rules built from $X$, written as $\operatorname{Rul}(X)=2^{\operatorname{Lit}(X)} \times \operatorname{Lit}(X)$, is the set of pairs of a set of literals built from $X$ and a literal built from $X$, written as $\{l 1, \ldots, \ln \} \rightarrow l$. We also write $l_{1} \wedge \ldots \wedge l_{n} \rightarrow l$ and when $n=0$ we write $T \rightarrow l$.

A multiagent system is a tuple

$\langle R A, S A, R O, X, B, D, G, A D, M D, \geq, I, P L\rangle$ where:

- The real agents $R A$, social institutions $S A$ and roles $R O$, propositional variables $X$, beliefs $B$, desires $D$, and goals $G$ are all finite disjoint sets. We write $R A \cup S A \cup R O=A$ for the set of all agents and $M=D \cup G$ for their motivations. 
- An agent description $A D: A \rightarrow 2^{A \cup X \cup B \cup D \cup G}$ is a complete function that maps each agent to other agents that exist in its profile, sets of variables (its decision variables), and its beliefs, desires and goals. For each agent $a \in A$, we write $A_{a}$ for $A \cap A D(a)$, and $B_{a}$ for $B \cap A D(a)$, et cetera. We write parameters $P=X \cup_{a \in A} X_{a}$.

- The mental description $M D: B \cup D \cup G \rightarrow \operatorname{Rul}(X)$ is a complete function from the sets of beliefs, desires and goals to the set of rules built from $X$. We write $m x \rightarrow y$ for: $m$ such that $M D(m)=x \rightarrow y$.

- A priority relation is used to resolve conflicts among motivational attitudes: $\geq: A \rightarrow 2^{M} \times 2^{M}$ is a function from agents to a transitive and reflexive relation on the powerset of the motivations containing at least the subset relation. We write $\geq_{a}$ for $\geq(a)$.

- The institutional facts $I \subseteq P$ are parameters.

- The role playing function $P L: R O \rightarrow R A$ associates a role to its player.

The set of variables whose truth value is determined by an agent (decision variables representing actions) are distinguished from those which are not directly determined by the agent ( $P$, the parameters). Only real agents act in the world, while social institutions act only through the agents playing roles in them. For this reason, social institutions are not associated with decision variables $\left(\cup_{\{a \in S A \cup R O\}} X_{a}=\emptyset\right)$.

Besides, "institutional facts" $I$ are states of affairs which exist only inside normative systems and organizations. As discussed in Section 4, (Searle, 1995) suggests that money, properties, marriages exist only as part of social reality; since we model social reality by means of the attribution of mental attitudes to social entities, institutional facts are just in the beliefs of these agents.

EXAMPLE 1. $M A S=\langle R A, S A, R O, X, B, D, G, A D, M D, \geq, I, P L\rangle$ with $R A=\{\mathbf{A}\}, S A=\{\mathbf{O}\}, R O=\{\mathbf{B}\}, P=\{p, q, r, s\}$, and $X P$, $B, D, G, A D, M D$ and $\geq$ are implicitly given by the following table:

A

$$
\mathrm{O}
$$

\begin{tabular}{|c|c|c|c|c|c|c|}
\hline$A$ & $\mathrm{O}$ & & B & & & - \\
\hline$B$ & & & $b_{1}$ & $x_{2} \rightarrow p$ & & \\
\hline$D$ & $d_{1}$ & $T \rightarrow \neg p$ & & & & \\
\hline$G$ & $g_{1}$ & $T \rightarrow x_{1}$ & $g_{2}$ & $T \rightarrow p$ & $g_{3}$ & $T \rightarrow x_{2}$ \\
\hline$X$ & $x_{1}, x_{2}$ & & & - & & - \\
\hline$\geq$ & $d_{1}>$ & $>g_{2}>g_{3}$ & $g_{2}$ & $3>d_{1}>g_{1}$ & & $g_{2}>d_{1}=g_{1}$ \\
\hline
\end{tabular}


This table should be read as follows. There are three agents, one real agent $\mathbf{A}$, a social institution $\mathbf{O}$ and a role $\mathbf{B}$ of the institution, played by $\mathbf{A}$. The $A$ row specifies which profiles are attributed by each agent: agent $\mathbf{A}$ attributes profile $\mathbf{O}$ to the institution, and the institution in turn defines role $\mathbf{B}$ by attributing to it the mental attitudes specified in the last column. The long dashes in a cell represent that the field cannot have a value.

Agent $\mathbf{A}$ has, among others, a desire $d_{1}\left(M D\left(d_{1}\right)=T \rightarrow \neg p\right)$, and the institution has a goal $g_{2}$ which can be realized by an action of agent $\mathbf{A} x_{2}$ since $M D\left(b_{1}\right)=x_{2} \rightarrow p$.

Finally, only a fragment of the priority relation is given, because it is only given for singleton motivations, whereas it is defined over sets of motivations. It says that each agent gives highest priority to its own motivations. The table can be extended to deal with more detailed motivations in the obvious way.

Social institutions like normative systems and organizations are able to change themselves. E.g., they specify how their norms can be modified. Since social institution depend on the attribution of mental attitudes which define both the regulative and constitutive norms, we represent their modification by means of the modification of their mental attitudes expressed as rules.

We adopt here a relatively simple solution for adding, revising and removing rules from a rule base; it is based on the assumption that all relevant beliefs, desires and goals are already present in the system, such that we only have to adapt the agent description $A D$. An advantage of this construction is that the priorities of the desires and goals are also already defined in the multiagent system, and we do not have to introduce an update mechanism.

Additions (a.k.a. expansions) to the agent description are defined as + : $A \times(B \cup D \cup G) \rightarrow I$, i.e., as for each agent mappings from mental attitudes to institutional facts. Since institutional facts $I$ like the additions exist only in the beliefs of a normative system or an organization, we need a way to express how these beliefs can be made true. The relations among propositional variables are expressed as belief rules. Rules concerning beliefs about institutional facts are called constitutive rules and represent the "counts-as" relations introduced by (Searle, 1995).

DEFINITION 2 (Counts-as). Given $M A S=\langle R A, S A, R O, X, B, D, G, A D, M D, \geq, I, P L\rangle$

Counts-as conditionals $C A \subseteq B_{o}$ of constitutive norms are beliefs of a social institution $o \in S A$, such that constitutive rules $C R=M D(C A)$ are the set of rules whose heads are elements of literals built out of institutional facts Lit $(I)$. We write counts-as ${ }_{o}(Y, p)$ where $Y \subseteq \operatorname{Lit}(X)$ and $p \in I$ if $\exists m \in C A$ such that $M D(m)=Y \rightarrow p$.

EXAMPLE 2 (continued). Given $I=\{r, s\}$. 
A

O B

\begin{tabular}{|c|c|c|c|c|c|c|}
\hline$A$ & $\mathrm{O}$ & & B & & & - \\
\hline \multirow[t]{2}{*}{$B$} & & & $b_{1}$ & $x_{s} \rightarrow p$ & $b_{3}$ & $x_{2} \rightarrow s$ \\
\hline & & & $b_{2}$ & $x_{1} \rightarrow r$ & & \\
\hline$D$ & $d_{1}$ & $T \rightarrow \neg p$ & & & & \\
\hline$G$ & $g_{1}$ & $T \rightarrow x_{1}$ & $g_{2}$ & $T \rightarrow p$ & $g_{3}$ & $T \rightarrow x_{2}$ \\
\hline$X$ & $x_{1}, x$ & & & - & & - \\
\hline$\geq$ & $d_{1}>$ & $>g_{2}>g_{3}$ & $g_{2}$ & $g_{3}>d_{1}>g_{1}$ & & $g_{2}>d_{1}=g_{1}$ \\
\hline$P L$ & & B & & - & & \\
\hline
\end{tabular}

Belief rules $b_{2}$ and $b_{3}$ are constitutive rules establishing institutional facts $r$ and $s$ via action $x_{1}$ and $x_{2}$ of agent $\mathbf{A}$. Note that $b_{3}$ is a constitutive rule of the role: action $x_{2}$ of the player of the role makes it true.

DEFINITION 3 (SMAS). A self modifying MAS is defined as $\langle R A, S A, R O, X, B, D, G, A D, M D, \geq, I, P L,+, C A\rangle$ with

- Additions $+: A \times(B \cup D \cup G) \rightarrow I$. We write $+{ }_{a}(m)$ for $+(a, m)$.

The update of a SMAS by a set of literals $L \subseteq \operatorname{Lit}(I)$ is $A D_{a}^{\prime}=A D_{a} \cup\{m \mid$ $\left.+_{a}(m) \in L\right\}$.

EXAMPLE 3 (continued). We introduce additions:

A

O B

\begin{tabular}{lcccccc}
\hline$A$ & $\mathbf{O}$ & & $\mathbf{B}$ & & & - \\
$B$ & & & $b_{1}$ & $x_{2} \rightarrow p$ & $b_{3}$ & $x_{2} \rightarrow s$ \\
& & & $b_{2}$ & $x_{1} \rightarrow r$ & $b_{6}$ & $s \rightarrow+_{\mathbf{B}}\left(g_{4}\right)$ \\
& & & $b_{4}$ & $r \rightarrow+\mathbf{O}\left(b_{5}\right)$ & & \\
$D$ & $d_{1}$ & $T \rightarrow \neg p$ & & & & \\
$G$ & $g_{1}$ & $T \rightarrow x_{1}$ & $g_{2}$ & $T \rightarrow p$ & $g_{3}$ & $T \rightarrow x_{2}$ \\
$X$ & $x_{1}, x_{2}$ & & & - & & - \\
$\geq$ & $d_{1}>g_{1}>g_{2}$ & $g_{1}>d_{1}>g_{2}$ & $g_{2}>d_{1}=g_{1}$ \\
$P L$ & \multicolumn{1}{c}{} & & &
\end{tabular}

The institutional fact $r$ (performed via A's $x_{1}$ ) "counts as" $\left(b_{4}\right)$ adding $b_{5}$ of the beliefs of the institution: this means that the agent $\mathbf{A}$ has the power to express the opinion of the institution it belongs to. Moreover, the institutional fact $s$ (performed via A's $x_{2}$ ) "counts as" $\left(b_{6}\right)$ the introduction of a goal $g_{4}$ in the state of the role $\mathbf{B}$ : $\mathbf{A}$ has the power to commit the role $\mathbf{B}$ to a certain goal by means of its actions.

The consequences of belief rules are incorporated via a logic of rules called out. It takes the transitive closure of a set of rules, which can be extended in the process, and it is an extension of reusable throughput in input/output logic (Makinson and van der Torre, 2000) with generator revision. 
5 Roles define expected behavior and obligations: a role's goals are its responsibilities.

6 Roles define sets of rights: since institutions are modelled as normative systems they can associate not only obligations but also rights and authorizations to roles.

7 Roles embed behavior specific to a context; roles exist only and because of the institution they belong to: the institution is the context of a role defining its specific behavior.

8 Define common interactions: constitutive rules define also how an action of a player of a role affects the beliefs and goals of another role, thus allowing communication.

9 Promote an organizational view of the system: roles compose the organizational structure of an institution and the institution gives them the power to exogenously coordinate its own behavior.

Figure 3. Some properties of roles in AO.

DEFINITION 4 (Consequences). $U=2^{\operatorname{Lit}(X)} \rightarrow 2^{R u l(X)}$ are the possible add lists of sets of rules in situations. out is a function from set of rules, sets of formulas and add lists to new sets of rules and sets of formulas: out : $2^{\text {Rul }(X)} \times 2^{\text {Lit }(X)} \times 2^{U} \rightarrow 2^{\text {Lit }(X)}$. Let out $(E, S, R)$ be the closure of $S$ under the rules $E$ updated by added rules $R$, defined as follows.

- out $E^{0}(E, S, R)=E$

- out ${ }^{0}(E, S, R)=S$

- out $E^{i+1}(E, S, R)=$ out $E^{i} \cup R\left(\right.$ out $\left.^{i}(E, S, R)\right)$

- out ${ }^{i+1}(E, S, R)=$ out $^{i}(E, S, R) \cup$

$\left\{l \mid L \rightarrow l \in\right.$ out $^{i}(E, S, R), L \subseteq$ out $\left.^{i}(E, S, R)\right\}$

- out $(E, S, R)=\cup_{o}^{\infty}$ out $^{i}(E, S, R)$

Here we are interested in the closure of a decision under a set of belief rules. The new belief rules of an agent $a$ in situation $S$ is $R_{a}^{+}$, defined by $R_{a}^{+}(S)=\left\{M D(b) \mid b \in B,+{ }_{a}(b) \in S\right\}$.

We finally introduce decisions of agents; they must be consistent with the consequences of beliefs according to the two agents $\mathbf{A}\left(\operatorname{out}\left(B_{\mathbf{A}}, \delta, R_{\mathbf{A}}^{+}\right)\right)$. The set of decisions $\Delta$ is the set of sets $\delta_{\mathbf{A}} \subseteq \operatorname{Lit}(X)$ such that their closures under the beliefs $\operatorname{out}\left(B_{\mathbf{A}}, \delta, R_{\mathbf{A}}^{+}\right)$do not contain a variable and its negation.

EXAMPLE 4 (Continued). out $\left(B_{\mathbf{B}},\left\{x_{1}, x_{2}\right\}, R_{\mathbf{B}}^{+}\right)=\left\{x_{1}, x_{2}, s,+_{\mathbf{B}}\left(g_{4}\right)\right\}$. According to role $\mathbf{B}, A^{\prime}$ s decision $x_{2}$ leads to $s$ and adds goal $b_{4}$ 
1. Roles have properties, for example, each role has its own beliefs and goals. Moreover, roles have behavior in the sense that they can execute institutional actions via the actions of their players.

2. Dependence of roles from relationships is implied by the stronger notion of definitional dependence: the relation they depend on is the relation between the role and the social institution which defines it.

3. An agent may play different roles simultaneously: the role playing function is not surjective.

4. An agent may play the same role several times: a role is not defined by its beliefs and goals only, but also by the institution attributing them to the role. The same roles in two different institutions are different roles and nothing prevents to play both.

5. An agent may acquire and abandon roles dynamically: to play a role it is sufficient to know which beliefs and to adopt which goals the player is expected to have. The model can be extended with constitutive rules which affect the role playing relation.

6. The sequence in which roles may be acquired and relinquished is subject to restrictions which are specified in the constitutive rules of the social institution.

7. Objects of unrelated types can play the same role: to play a role it is necessary to be able to perform the actions which "count as" actions of the institution. A different issue is if the agent is suited to play a role, i.e., which are its beliefs and motivations.

8. Roles can play roles since roles are defined as agents and agents can play roles.

9. A role can be transferred from one agent to another: the new player is expected to behave as if it has the current beliefs and goals attributed to the role.

10. The state of an agent is role-specific: the agent's powers change with the role it is playing.

11. Features of an agent can be role-specific: according to its role, the agent has to act as if it has the beliefs and goals of the role.

12. Roles restrict access. Roles are accessed only via powers.

13. Different roles may share structure and behavior: role definitions can be organized in a hierarchical way.

14. An agent and its roles share identity. Roles are not real agents, but only descriptions of agents. So they have no identity as agents.

15. An agent and its roles have different identities and role are instances.

Figure 4. The properties of roles in $\mathrm{OO}$ revisited.

\section{Conclusion}

In this paper, we analyse the properties of social roles and we provide a simple formal model of social institutions with roles. The main properties we attribute to roles are three. First their definitional dependence: social roles exist only as they are defined by some social institution; second, besides rights and permissions, social roles are associated to powers in the institution they belong to. Finally roles can play roles as any other agent, since in our model social roles should be considered as an agent. 
For this reason as methodology we use the agent metaphor: both social institutions and social roles are modelled as a kind of agent, since they are attributed mental attitudes to describe their behavior. When attributing mental attitudes to social entities, we show that regulative rules can be defined as goals of the social institution and constitutive rules as its beliefs, like in (Boella and van der Torre, 2006).

In Figure 3 and 4, we reconsider the properties attributed in Section 2 to roles by, respectively, $\mathrm{AO}$ and $\mathrm{OO}$, and we show how they are dealt with in our model. Since the two lists of properties overlap, in Table 3 we focus only on the properties which require the autonomy of the agent playing the role.

Future work is how to use the current model to propose social roles in agent communication languages. Finally, our model of roles is being used as the basis to introduce roles in Object Oriented programming languages like Java. In this way we offer a unified notion of roles in both Agent Oriented systems and Object Oriented ones. The integration between agent systems and more traditional $\mathrm{OO}$ systems can be fostered, e.g., agents can be used to play roles in $\mathrm{OO}$ systems. Moreover, agent systems implemented using $\mathrm{OO}$ architectures and languages can have already at disposal roles as a primitive.

\section{References}

Artikis, A., J. Pitt, and M. Sergot: 2002, ‘Animated specifications of computational societies'. In: Procs. of AAMAS'02. pp. 1053-1061, ACM press.

Baldoni, M., G. Boella, and L. van der Torre: 2005, 'Roles as a Coordination Construct: Introducing powerJava'. In: Procs. of MTCoord'05 workshop at COORDINATION'05.

Bauer, B., J. Muller, and J. Odell: 2001, 'Agent UML: A formalism for specifying multiagent software systems'. Int. Journal of Software Engineering and Knowledge Engineering 11(3), 207-230.

Boella, G. and L. van der Torre: 2004a, 'Attributing Mental Attitudes to Roles: The Agent Metaphor Applied to Organizational Design'. In: Procs. of ICEC'04. IEEE Press.

Boella, G. and L. van der Torre: 2004b, 'Organizations as Socially Constructed Agents in the Agent Oriented Paradigm'. In: LNAI n. 3451: Procs. of ESAW'04. Berlin, pp. 1-13, Springer Verlag.

Boella, G. and L. van der Torre: 2006, 'A Game Theoretic Approach to Contracts in Multiagent Systems'. IEEE Transactions on Systems, Man and Cybernetics - Part C.

Cabri, G., L. Ferrari, and L. Leonardi: 2004, 'Agent Role-based Collaboration and Coordination: a Survey About Existing Approaches'. In: IEEE Systems, Man and Cybernetics Conference.

Cavedon, L. and L. Sonenberg: 1998, 'On social commitments, roles and preferred goals'. In: Procs. of ICMAS'98. Berlin, pp. 80-87, Springer Verlag.

Dahchour, M., A. Pirotte, and E. Zimanyi: 2002, 'A Generic Role Model for Dynamic Objects'. In: Procs. of CAiSE'02, Vol. 2348 of LNCS. pp. 643-658, Springer.

Dastani, M., V. Dignum, and F. Dignum: 2003, 'Role-Assignment in Open Agent Societies'. In: Procs. of AAMAS'03. Melbourne, pp. 489-496, ACM Press.

Dastani, M., B. van Riemsdijk, J. Hulstijn, F. Dignum, and J.-J. Meyer: 2004, 'Enacting and Deacting Roles in Agent Programming'. In: Procs. of AOSE'04. New York. 
Esteva, M., J. Padget, and C. Sierra: 2001, 'Formalizing a language for institutions and norms'. In: Intelligent Agents VIII: Procs. of ATAL'01. Berlin, pp. 348-366, Springer Verlag.

Fasli, M.: 2001, 'On Commitments, Roles and Obligations'. In: From Theory to Practice in Multi-Agent Systems, LNAI 2296. Berlin, pp. 93-102, Springer-Verlag.

Fine, K.: 1995, 'Ontological Dependence'. Proceedings of the Aristotelian Society 95, 269 290.

Guarino, N. and C. Welty: 2002, 'Evaluating ontological decisions with ontoclean'. Сотmunications of ACM 45(2), 61-65.

Makinson, D.: 1986, 'On the Formal Representation of Rights Relations'. Journal of Philosophical Logic 15, 403-425.

Makinson, D. and L. van der Torre: 2000, 'Input-output logics'. Journal of Philosophical Logic 29, 383-408.

Masolo, C., L. Vieu, E. Bottazzi, C. Catenacci, R. Ferrario, A. Gangemi, and N. Guarino: 2004, 'Social Roles and their Descriptions'. In: Procs. of KR'04. pp. 267-277, AAAI Press.

Pacheco, O. and J. Carmo: 2003, 'A role based model of normative specification of organized collective agency and agents interaction'. Autonomous Agents and Multiagent Systems 6, $145-184$.

Searle, J.: 1995, The Construction of Social Reality. New York: The Free Press.

Sowa, J.: 2000, Knowledge Representation: Logical, Philosophical, and Computational Foundations. Pacific Growe (CA): Brooks/Cole.

Steimann, F.: 2000, 'On the representation of roles in object-oriented and conceptual modelling'. Data and Knowledge Engineering 35, 83-848. 


\title{
From Social Power to Social Importance
}

\author{
Guido Boella \\ Dipartimento di Informatica \\ Università di Torino - Italy \\ guido@di.unito.it \\ Luigi Sauro \\ Dipartimento di Scienze Fisiche \\ Università "Federico II" di Napoli - Italy \\ sauro@na.infn.it \\ Leendert van der Torre \\ Computer Science and Communications \\ University of Luxembourg \\ leendert@vandertorre.com
}

November 25, 2006

\begin{abstract}
In this paper we introduce a method to measure the social importance of an agent in a multiagent system, using a directed graph representing dependencies among agents to achieve their goals, so-called dependence graphs pioneered by Castelfranchi, Conte and Sichman. Our measure is derived from van den Brink and Gilles' $\beta$-measure to rank agents, using a directed graph representing an abstract dominance relation among agents. In particular, we show how to define power structures and dependence networks from the goals and skills of individual agents, and how to adapt the $\beta$-measure for such dependence networks based on their topological properties. Moreover, we show that our notion of social importance has a simple and intuitive meaning: it measures the discontent of the other agents in case the agent would leave the society.
\end{abstract}

keywords: Cooperation, dependence networks, social importance. 


\section{Introduction}

Heterogeneous agents help other agents to achieve their goals in various ways and to various degrees, raising the problem how to measure the social importance of an agent inside a community. Social importance plays a key role in the explanation of several so-called emergence and micro-macro link problems. For example, it can be used to estimate the influence of an agent in a collaboration process, to justify the emergence of various social positions and hierarchical organizations in a community of peers, to distribute obligations [5] or to analyze to which extent a community tends to one of its members in order to keep it inside. Castelfranchi [7] argues that some aspects of the emergence and micro-macro link problems arise from and can be modeled with a description of the power of groups of agents and their inter-dependencies.

In this paper, we consider the formalization of the social importance of agents using a directed graph representing dependencies among agents to achieve their goals, so-called dependence graphs pioneered by Castelfranchi [7], as well as Conte and Sichman [8]. To address the social importance we use the $\beta$-measure developed by van den Brink and Gilles [18] to rank agents inside a dominance structure, i.e., a weighted directed graph where each node represents an agent and a weighted arc represents the strength of a dominance relation between two agents. We focus on the $\beta$-measure, because it satisfies some properties which should also hold for dependence. For example, if an agent does not dominate any other agent, then its $\beta$-measure is equal to zero, and analogously, if no agent depends on a given agent, then the social importance of the latter should be equal to zero.

The problem we address is therefore how to adapt the $\beta$-measure in order to capture the strength of an agent in the satisfaction of the goals of other agents. Our problem breaks down in the following two related questions:

1. How to define a dependence network for agents based on their abilities and desires?

2. How to adapt van den Brink and Gilles' $\beta$-measure to measure social importance in these dependence networks?

To answer the first question, we adapt the dependence networks we have developed in previous work to define admissible criteria for coalition formation (see [15] for a detailed description), introducing the minimization on the action assignment in the definition of minimal power. In particular, a possible notion of power is the so-called $\alpha$-ability: the capability of a group of agents to ensure a state of affairs, no matter what the other agents do [13, 1, 19]. This notion originates from classical game theory [14], where it has been shown that it satisfies the property 
of coalitional monotonicity, i.e., adding new members to a group of agents does not decrease its power. Though this property seems natural in several contexts, it is not well suited to define the notion of dependence among agents as developed by Castelfranchi and others, because it admits in a group which has the power to achieve a certain goal the presence of useless agents. Therefore, we study also a notion of power implying $\alpha$-ability, but requiring also that if a group of agents $Q$ has the power to achieve a goal $g$, then no subsets $Q^{\prime}$ of $Q$ can achieve $g$ with less efforts. This means that all the agents in $Q$ play a profitable role in the achievement of $g$. Starting from formalization of the notion of power we represent dependencies among group of agents by means of graphs following the approach of Conte and Sichman [8].

The second question addresses the primary aim of this paper, i.e. the definition of our measure of the social importance of an agent inside a community. This issue, as far as we know, is novel in the multiagent system community. Intuitively, our notion of social importance measures the dissatisfaction of a community in case one of its members decides to leave it. This measure depends essentially on three factors, the goals in which achievement that agent is involved, the number of agents that desire these goals and the number of alternatives which subsist in order to achieve these goals. More precisely, we adapt the notion of $\beta$-measure, because dominance concerns only two agents. First, the notion of dependence is relative to the achievement of a goal, and since a priori goals cannot be assumed equivalent, we have to consider separately the importance that any single goal provides to an agent. Second, dependence concerns one agent with respect to a group of agents which has the power to achieve one of its goals, and not only with respect to one other agent only.

There are several related issues we do not address in this paper. We use a level of abstraction which enables to model all the relevant aspects of our problem and to reveal differences with other works to a limited extent concerning our problem. Several natural improvements concerning non-deterministic or more sophisticated models of the environment (see [12]) are beyond the scope of this paper. Similarly, the description of temporal aspects of goals, as addressed in ATL [1], for example, or the study of the complex plans involving concatenation of actions, conditionals, iteration can be subject of future research.

The layout of this paper is as follows. In Section 2 we formalize a Multiagent System, providing the definition of the single agents, the actions they can execute and their effects. In Section 3, the notions of the power and lack of power for groups of agents are formalized. Section 4 is dedicated to dependencies, relating them to the previous definitions of power and lack of power, and in Section 5 a graph representation of the dependencies among agents is provided. Section 6 concerns the formalization of the social importance of an agent in a Multiagent 
System, which extends the notion of $\beta$-measure of van den Brink and Gilles [18]. Related work and conclusions end the paper.

\section{Multiagent System}

Given a set of Boolean variables $V$, in the following, we denote with $\mathcal{L}_{V}$ the set of all Boolean formulae on the variables $V$. If $\nu \in \mathcal{L}_{V}$, then $V[\nu]$ is the set of all the variables in $V$ that occurs in $\nu$. Given a formula $\nu \in \mathcal{L}_{V}$ and a truth assignment $\mu$ of $V$, we write $=_{\mu} \nu$ to indicate that $\mu$ satisfies the formula $\nu$ in the usual propositional semantics. As usual a formula $\nu \in \mathcal{L}_{V}$ is consistent if there exists a truth assignment $\mu$ such that $=_{\mu} \phi$, we denote with $\mathcal{L}_{P}^{*} \subset \mathcal{L}_{P}$ the subset of all consistent formulae.

A multiagent system can be viewed as a set of agents populating an environment. Agents can affect the environment by executing actions. In this work we consider a simple model where the evolution of the environment consists of a single step from an initial state to its next state. In this way, we abstract from a detailed description of an action - whether, for example, it can be decomposed in a sequence of more elementary actions - as well as from low-level coordination issues. Mainly, this choice is due to simplicity reasons. However, several studies on strategic reasoning and collaborative behaviors are used to restrict the complexity of the agent interaction. For example in classical game theory the strategic form of a game abstracts from the detailed description of what a strategy consists of and directly describes the result of strategy profiles by means of an outcome function [13].

The evolution of the environment is described by means of a pair of states, the initial state and the next state. The initial state consists of a truth assignment $\theta$ on a set of propositional variables $P$. The next state is represented by means of a truth assignment $\theta^{\prime}$ on a set of propositional variables $P^{\prime}=\left\{p^{\prime} \mid p \in P\right\}$.

The environment evolves according to the actions that agents execute in the initial state, and is characterized by effect rules. We introduce a finite set of Boolean variables $A c$ denoting all the actions that can be performed by an agent. An effect rule is conditional both on the initial state and on the actions performed by the agents. In order to express the relation between actions and their effects we formalize not only the fact that some actions should be performed to have some effects, but also that some other actions should not be performed. For example to open a door one has to push it, but at the same time no other person should be pushing it on the other side.

Definition 1 (Effect rule) An effect rule is a formula $\phi \wedge \psi \rightarrow \phi^{\prime}$, where $\psi \in$ 
$\mathcal{L}_{A c}^{*}, \phi \in \mathcal{L}_{P}^{*}$ and $\phi^{\prime} \in \mathcal{L}_{P^{\prime}}^{*}$ is a conjunction of literals over $P^{\prime}$. We denote with $\mathcal{E}_{P, A c, P^{\prime}} \subset \mathcal{L}_{P \cup P^{\prime} \cup A c}^{*}$ the set of all possible effect rules.

Given an effect rule $r=\phi \wedge \psi \rightarrow \phi^{\prime}$, we denote with effects $(r) \phi^{\prime}$ and, given a set of effect rules $R$ we denote with effects $(R)=\bigwedge_{r \in R}$ effects $(r)$. In the same manner, we denote with preconditions $(r)$ the formula $\phi \wedge \psi$ and with preconditions $(R)=\bigwedge_{r \in R}$ preconditions $(r)$.

Example 1 A possible rule is: $s_{1} \wedge s_{2} \wedge a \rightarrow \neg s_{1}^{\prime}$. Informally, this rule tells $u s$ that in every state where both $s_{1}$ and $s_{2}$ are true, if the action a is performed, then in the next state $s_{1}$ is false.

The environment is regulated by a finite set of effect rules $R l \subset \mathcal{E}_{P, A c, P^{\prime}}$. Given an initial state $\theta$ and a truth assignment $\alpha$ on the action variables $A c$, if $R \subseteq R l$ is the set of effect rules such that $=_{\theta \cup \alpha}$ preconditions $(R)$, then next state $\theta^{\prime}$ satisfies the effects of all the effect rules in $R,=_{\theta^{\prime}}$ effects $(R)$. However, there could be more than one truth assignment $\theta^{\prime}$ that satisfies effects $(R)$, therefore which of them is the next state? We assume that the effect rules operate like STRIPS-operators [10], i.e., the values of the attributes not involved in the effects of the activated rules remain unchanged. We call this behavior the inertia assumption and we formalize it as follows:

Definition 2 (Next state) Given a set of effect rules $R l$, an initial state $\theta$, a truth assignment of the actions $\alpha$. Denoted with $R \subseteq R l$ the set of all the rules $r \in R l$ such that $\models_{\theta \cup \alpha}$ preconditions $(r)$. The next state resulting from $\theta$ and $\alpha$ under $R l$ is the truth assignment $\theta^{\prime}$ on the variables $P^{\prime}$ such that $=_{\theta^{\prime}}$ effects $(R)$ and for all the variables $p^{\prime} \in P^{\prime} \backslash P^{\prime}($ effects $(R)), \theta^{\prime}\left(p^{\prime}\right)=\theta(p)$. In the following we denote the next state corresponding to $\theta, \alpha$ and $R l$ as $N \operatorname{ext}(\theta, \alpha, R l)$.

Example 2 Let $\theta$ be the state $\left\{\left(s_{1}, 1\right),\left(s_{2}, 1\right)\right\}$ and $R$ the set of activated rules consisting of the single rule $s_{1} \wedge s_{2} \wedge a \rightarrow \neg s_{1}^{\prime}$ as in Example 1, effects $(R)$ is the formula $\neg s_{1}^{\prime}$, therefore $P^{\prime}($ effects $(R))$ is the variable $s_{1}^{\prime}$. Due to Definition 2 , the next state $\theta^{\prime}$ satisfies the formula $\neg s_{1}^{\prime}$, so $\theta^{\prime}\left(s_{1}^{\prime}\right)=0$, whereas $\theta^{\prime}\left(s_{2}^{\prime}\right)=\theta\left(s_{2}\right)$, i.e., it is equal to 1.

Given a generic set of rules $R l$, describing the evolution of the environment, it could be that two or more effect rules in $R l$ have inconsistent effects even if their preconditions are consistent.

Example 3 Consider the two rules $r_{1}=f \rightarrow \neg s_{1}^{\prime}$ and $r_{2}=\neg s_{1} \wedge a_{1} \rightarrow s_{1}^{\prime}$. Let $\theta$ be an initial state such that $\theta(f)=1$ and $\theta\left(s_{1}\right)=0$ and let $\alpha$ be an assignment on the action variables such that $\alpha\left(a_{1}\right)=1$. Then, $\theta$ and $\alpha$ satisfy the preconditions 
of both $r_{1}$ and $r_{2}, \models_{\theta \cup \alpha}$ preconditions $\left(\left\{r_{1}, r_{2}\right\}\right)$, nevertheless there does not exist a next state $\theta^{\prime}$ such that $\models_{\theta^{\prime}} s_{1}$ and $\models_{\theta^{\prime}} \neg s_{1}$.

For this reason we explicitly require that activated rules do not lead to inconsistent effects. We call such sets of rules feasible.

Definition 3 (Feasible rules) A set of rules $R l$ is feasible if and only if for all the initial states $\theta$ and for all truth assignment $\alpha$ of Ac, there exists a next state $\theta^{\prime}$ such that $\theta^{\prime}=\operatorname{Next}(\theta, \alpha, R l)$.

The following example illustrates the distinction between feasible and not feasible rules.

Example 4 (Continued) Consider the set of rules $R l=\left\{r_{1}, r_{2}\right\}$ of the Example 3. To make this set of rules feasible we modify the rule $\neg s_{1} \wedge a_{1} \rightarrow s_{1}^{\prime}$ with the rule $\neg s_{1} \wedge \neg f \wedge a_{1} \rightarrow s_{1}^{\prime} . . R l$ is feasible because the formulae $f$ and $\neg s_{1} \wedge \neg f$ are inconsistent, therefore, the preconditions of $r_{1}$ and $r_{2}$ cannot be satisfied at the same time.

Finally, we introduce agents. An agent is represented by means of its abilities and motivations. The abilities of an agent $a g_{i}$ are the actions it can perform $A c_{i} \subseteq$ Ac.

Motivations are formalized as goals. Informally, a goal is a generic formula $\gamma$ involving variables from $P, A c$ and $P^{\prime}$ which describe respectively in which initial states it is activated, which actions are desired to be executed and which final state is desires.

Definition 4 (Goals) A goal is a formula $\gamma$ of $\mathcal{L}_{P \cup A c \cup P^{\prime}}$. A set of effect rules $R l$, an initial state $\theta$ and an action assignment $\alpha$ satisfy a goal $\gamma, \operatorname{Sat}(\theta, \alpha, \gamma)$, if and only if $\models_{\theta \cup \alpha \cup N \operatorname{ext}(\theta, \alpha, R l)} \gamma$.

Example 5 Consider the conditional goal $s \wedge \neg a \wedge \neg s^{\prime}$, this goal states that whenever the initial state satisfies $s$, then the agent desires that the action a is not executed and the environment evolves in a next state which does not satisfy $s^{\prime}$.

A Multiagent System consists of a set of agents together with their actions and goals.

Definition 5 (Multiagent System) A Multiagent System, MaS, is tuple

$$
\left\langle A g, P, A c_{1}, \ldots, A c_{|A g|}, R l, G_{1}, \ldots, G_{|A g|}\right\rangle
$$

where $A g$ is a set of agents. $P$ is a set of propositional variables. Ac $c_{i}$ is the set of actions the agent $i$ can perform, where Ac denotes the union of all the $A c_{i}$. Rl is a 
feasible set of rules on the propositional variables $P$ and $A$ c. $G_{i} \subset \mathcal{L}_{P}^{*} \times \mathcal{L}_{A c}^{*} \times \mathcal{L}_{P^{\prime}}^{*}$ is a finite set of goals associated with agent $i$, where Gl denotes the union of the $G_{i}$, i.e., the set of all the goals.

To illustrate our model of a multiagent system, we consider an example regarding the safety of a prison. The prison is composed by two interiors, a dormitory and a courtyard. A gate separates the dormitory from the courtyard, whereas another gate separates the courtyard from the outside. $s_{1}$ and $s_{2}$ denote the attributes corresponding respectively to the first and the second gate, in particular $s_{i}$ represents that the corresponding gate is closed and $\neg s_{i}$ represents that it is open. The two gates are ruled by pressing the two buttons, denoted with the actions $a_{1}$ and $a_{2}$, and a fire alarm. The fire alarm is described by the attribute variable $f$, if the value of $f$ is true, then a fire is spreading through the dormitory. In this case, independently from the states of the gates and the activation of the buttons, the gate $s_{1}$ is opened to assure the prisoners to leave the dormitory. Button $a_{1}$ closes the gate $s_{1}$ in the case it is open and it opens $s_{1}$ only if both $s_{1}$ and $s_{2}$ are closed. Button $a_{2}$ has the same behavior as button $a_{1}$, but it governs gate $s_{2}$. The agents are two guards $g d_{1}$, $g d_{2}$ and a prisoner $p r$. Guard $g d_{1}$ handles the controller $a_{1}$, whereas the guard $g d_{2}$ handles the controller $a_{2}$. The prisoner $p r$ can perform an action $b$ that makes $f$ true. We are not interested in the goals of the guards whereas we know that the goal of the prisoner is to escape. We assume that whenever a gate is open the prisoner can pass through it, therefore, the goal to escape is formalized as follows: if the gate $s_{1}$ is closed, then $p r$ wants that in next state both $s_{1}$ and $s_{2}$ are open. In the case that $s_{1}$ is open and the gate $s_{2}$ is closed, then $p r$ has already the possibility to be over $s_{1}$, therefore it only desires that in the next state the gate $s_{2}$ is open. Finally, if both the gates $s_{1}$ and $s_{2}$ are open, then he can escape, and hence its goal is already satisfied.

Example 6 The multiagent system relative to this scenario is described in Figure 1 .

\section{Power of groups of agents}

In this section we define a notion of power which is based on two facts. First, we consider that agents can not only help, but also obstruct each other. For this reason we consider a notion of power which is related to the game theoretic notion of $\alpha$ ability [13, 19], i.e., a group of agents $Q$ has the power to achieve a state of affairs if there exists a joint behavior ensuring that state of affairs and the other agents cannot obstruct $Q$. 


\begin{tabular}{|c|c|c|}
\hline Agents & actions & Goals \\
\hline$g d_{1}$ & $\overline{a_{1}}$ & $\emptyset$ \\
\hline$g d_{2}$ & $a_{2}$ & $\emptyset$ \\
\hline$p r$ & $b$ & $g_{1}=\left(s_{1} \wedge \neg s_{1}^{\prime} \wedge \neg s_{2}^{\prime}\right) \vee\left(\neg s_{1} \wedge s_{2} \wedge \neg s_{2}^{\prime}\right) \vee\left(\neg s_{1} \wedge \neg s_{2}\right)$ \\
\hline \multicolumn{3}{|c|}{ Effect Rules } \\
\hline \multicolumn{3}{|l|}{$b \rightarrow f^{\prime}$} \\
\hline \multicolumn{3}{|c|}{$f \rightarrow \neg s_{1}^{\prime}$} \\
\hline \multicolumn{3}{|c|}{$s_{1} \wedge s_{2} \wedge a_{1} \rightarrow \neg s_{1}^{\prime}$} \\
\hline \multicolumn{3}{|c|}{$\neg s_{1} \wedge \neg f \wedge a_{1} \rightarrow s_{1}^{\prime}$} \\
\hline \multicolumn{3}{|c|}{$s_{1} \wedge s_{2} \wedge a_{2} \rightarrow \neg s_{2}^{\prime}$} \\
\hline & & \\
\hline
\end{tabular}

Figure 1: The Multiagent system of the prison example.

Second, inspired by Castelfranchi [7], by power we mean the capability of a group of agents to achieve goals. Therefore, power does not consist only of the group's abilities to achieve effects. There should also be at least one agent that desires those effects.

Given a multiagent system $M a S$, an action assignment is a function which specifies, for each agent, the actions it executes in the initial state.

Definition 6 (Action assignment) Given a set of agents $Q \subseteq A g$, we denote with $K_{Q}$ an action assignment over the agents in $Q$, i.e., a function that associates, for all the agents $a g_{i} \in Q$, a non-empty subset of $A c_{i}$ and, for all $a g_{j} \notin Q$, the empty set. We denote with $\mathcal{U}\left(K_{Q}\right)$ the union of all the actions assigned in $K_{Q}$ :

$$
\mathcal{U}\left(K_{Q}\right)=\bigcup_{a g \in Q} K_{Q}(a g)
$$

Given two action assignments $K_{Q}$ and $K_{Q^{\prime}}$, we denote with $K_{Q} \sqcup K_{Q^{\prime}}$ the action assignment such that:

- for all ag $\notin Q \cup Q^{\prime}, K_{Q} \sqcup K_{Q^{\prime}}(a g)=\emptyset$

- for all ag $\in Q \cup Q^{\prime}, K_{Q} \sqcup K_{Q^{\prime}}(a g)=K_{Q}(a g) \cup K_{Q^{\prime}}(a g)$

Finally, we write $K_{Q^{\prime}} \sqsubseteq K_{Q}$, when $Q^{\prime} \subseteq Q$ and for all ag $\in Q^{\prime}, K_{Q^{\prime}}(a g) \subseteq$ $K_{Q}(a g)$. As usual, $K_{Q^{\prime}} \sqsubset K_{Q}$ means that $K_{Q^{\prime}} \sqsubseteq K_{Q}$ and $K_{Q^{\prime}} \nsubseteq K_{Q}$.

A set of agents $Q$ has the power to achieve a goal $\gamma$ by means of an action assignment $K_{Q}$ in an initial state $\theta$ if and only if $\gamma$ is satisfied no matter which actions the other agents execute. 
Definition 7 (Agents' power) Given a goal $\gamma \in G l$, a set of agents $Q \subseteq A g$ has the power to achieve $\gamma$ according to the action assignment $K_{Q}$ in the state $\theta$, $\operatorname{Pow}\left(Q, \gamma, K_{Q}, \theta\right)$, if and only if for all $K_{A g \backslash Q}$, Sat $\left(\theta, \mathcal{U}\left(K_{Q} \cup K_{A g \backslash Q}\right), \gamma\right)$.

We generalize the notion of power for sets of goals $G \subseteq G l: \operatorname{Pow}\left(Q, G, K_{Q}, \theta\right)$ if and only if for all $\gamma \in G, \operatorname{Pow}\left(Q, \gamma, K_{Q}, \theta\right)$.

Definition 7 formulates the notion of $\alpha$-ability with respect to the possibility to achieve one or more goals at the same time. $\alpha$-ability has been proved to satisfy several relevant properties such as coalition monotonicity or super-additivity [13]. However, $\alpha$-ability has been defined as the capability for a group of agents to ensure a state of affairs and not to collect goals as in our case. For this reason we re-define these properties in our framework and we prove that Definition 7 satisfies them. Informally, these properties are:

goal monotonicity: if a set of agents $Q$ can achieve a set of goals $G$ by means of the action assignment $K_{Q}$, then it can achieve, by means of the same $K_{Q}$, every subset $G^{\prime}$ of $G$.

coalition monotonicity: if the set of agents $Q$ has the power to achieve the set of goals $G$ by means of $K_{Q}$, then every set of agents $Q^{\prime}$ that contains $Q$ can achieve $G$ by means of an action assignment $K_{Q^{\prime}}$ such that $K_{Q} \sqsubseteq K_{Q^{\prime}}$.

super-additivity: if two disjoint sets of agents $Q_{1}$ and $Q_{2}$ can respectively achieve two sets of goals $G_{1}$ and $G_{2}$ by means of the actions assignments $K_{Q_{1}}$ and $K_{Q_{2}}$, then joined they can achieve both $G_{1}$ and $G_{2}$ by means of the action assignment $K_{Q_{1}} \sqcup K_{Q_{2}}$.

Fact 1 The following properties hold:

1. If Pow $\left(Q, G, K_{Q}, \theta\right)$, then for all $G^{\prime} \subset G$, Pow $\left(Q, G^{\prime}, K_{Q}, \theta\right)$.

2. If Pow $\left(Q, G, K_{Q}, \theta\right)$, then for all $Q^{\prime} \supseteq Q$ and $K_{Q^{\prime}} \sqsupseteq K_{Q}$,Pow $\left(Q^{\prime}, G, K_{Q^{\prime}}, \theta\right)$.

3. If Pow $\left(Q_{1}, G_{1}, K_{Q_{1}}, \theta\right)$, Pow $\left(Q_{2}, G_{2}, K_{Q_{2}}, \theta\right)$ and $Q_{1} \cap Q_{2}=\emptyset$, then $\operatorname{Pow}\left(Q_{1} \cup Q_{2}, G_{1} \cup G_{2}, K_{Q_{1}} \sqcup K_{Q_{2}}, \theta\right)$.

proof:

1) It directly derives from Definition 7.

2) Due to Definition 7, for all $A \subseteq \bigcup_{a g_{i} \in A g \backslash Q^{\prime}} A c_{i}$, it is the case that for all $\gamma \in G$ :

$$
\operatorname{Sat}\left(\theta, \mathcal{U}\left(K_{Q}\right) \cup \mathcal{U}\left(K_{Q^{\prime}} \backslash K_{Q}\right) \cup T^{\prime}, g\right)
$$

But since $\mathcal{U}\left(K_{Q}\right) \cup \mathcal{U}\left(K_{Q^{\prime}} \backslash K_{Q}\right)=\mathcal{U}\left(K_{Q^{\prime}}\right)$, then $\operatorname{Pow}\left(Q^{\prime}, G, K_{Q^{\prime}}, \sigma\right)$ holds. 
3) Let $K_{Q_{1} \cup Q_{2}}=K_{Q_{1}} \sqcup K_{Q_{2}}$. Since $Q_{1} \cap Q_{2}=\emptyset$, then $K_{Q_{i}} \subseteq K_{Q_{1} \cup Q_{2}}$ with $i \in\{1,2\}$. By hypothesis $\operatorname{Pow}\left(Q_{1}, G_{1}, K_{Q_{1}}, \sigma\right)$, therefore for the coalition monotonicity property $\operatorname{Pow}\left(Q_{1} \cup Q_{2}, G_{1}, K_{Q_{1} \cup Q_{2}}, \sigma\right)$. Analogously it can be proved that $\operatorname{Pow}\left(Q_{1} \cup Q_{2}, G_{2}, K_{Q_{1} \cup Q_{2}}, \sigma\right)$ and hence, by Definition 7, $\operatorname{Pow}\left(Q_{1} \cup\right.$ $\left.Q_{2}, G_{1} \cup G_{2}, K_{Q_{1} \cup Q_{2}}, \sigma\right)$.

An important issue regarding the formalization of dependencies among agents is the minimality of the set of actions a set of agents has to perform to achieve a goal. Roughly, an agent $a g$ is dependent for a goal $g$ on an action assignment $K_{Q}$ of the set of agents $Q$ only if all the activities in $K_{Q}$ play a role in the achievement of $g$. Due to the coalition monotonicity property the notion of Pow does not satisfy this minimality, therefore we define a notion of minimal power corresponding to the fact that $g$ can be achieved with an activity distribution $K_{Q}$ such that there does not exist a $K_{Q^{\prime}}$ such that for each agent assigns less actions of $K_{Q}$, formally $K_{Q^{\prime}} \sqsubset K_{Q}$.

Definition 8 (Power with minimality) A set of agents $Q \subseteq A g$ has the minimal power to achieve the goal formula $\gamma$ by means of the action assignment $K_{Q}$ in the state $\theta$, Min_Pow $\left(Q, \gamma, K_{Q}, \theta\right)$, if and only if $\operatorname{Pow}\left(Q, \gamma, K_{Q}, \theta\right)$ and for all $Q^{\prime} \subseteq Q$ it does not exist a $K_{Q^{\prime}} \sqsubset K_{Q}$ such that Pow $\left(Q^{\prime}, \gamma, K_{Q^{\prime}}, \theta\right)$.

Given a set of goals $G \subseteq G$, Min_Pow $\left(Q, G, K_{Q}, \theta\right)$ holds if and only if $\operatorname{Pow}\left(Q, G, K_{Q}, \theta\right)$ and there exists at least a $\gamma \in G$ such that Min_Pow $\left(Q, \gamma, K_{Q}, \theta\right)$.

Min_Pow satisfies the following properties:

Goals maximality: If a set of agents $Q$ has the minimal power to achieve the goals in $G$ by means of an action assignment $K_{Q}$, then, given a set of goals $G^{\prime}$ that contains $G$, it does not exist a $Q^{\prime} \subseteq Q$ and a $K_{Q^{\prime}} \subset K_{Q}$ such that $Q^{\prime}$ has the minimal power to achieve the goals in $G^{\prime}$ by means of $K_{Q^{\prime}}$.

Goals monotonicity with minimality: If $Q$ has the min-power to achieve a set of goals $G$, then for every subset $G^{\prime}$ of $G$ there exists a subset $Q^{\prime}$ of $Q$ such that it has the min-power to achieve $G^{\prime}$.

Super-additivity with minimality: If two disjoint sets of agents $Q_{1}$ and $Q_{2}$ can respectively achieve to sets of goals $G_{1}$ and $G_{2}$ by means of the action assignments $K_{Q_{1}}$ and $K_{Q_{2}}$, then there exists a subset of $Q$ of $Q_{1} \cup Q_{2}$ that can achieve both $G_{1}$ and $G_{2}$ by means of an action assignment $K_{Q}$ such that $K_{Q} \sqsubseteq K_{Q_{1}} \sqcup K_{Q_{2}}$.

Fact 2 The following properties hold: 
1. If Min_Pow $\left(Q, G, K_{Q}, \theta\right)$, then for all $G^{\prime}(\supset G)$ there not exists $Q^{\prime} \subset Q$, and a $K_{Q^{\prime}} \sqsubset K_{Q}$ such that Min_Pow $\left(Q^{\prime}, G^{\prime}, K_{Q^{\prime}}, \theta\right)$.

2. If Min_Pow $\left(Q, G, K_{Q}, \theta\right)$, then for all $G^{\prime} \subseteq G$ there exits $a Q^{\prime} \subseteq Q$ and $a$ $K_{Q^{\prime}} \sqsubseteq K_{Q}$ such that Min_Pow $\left(Q^{\prime}, G^{\prime}, K_{Q^{\prime}}, \theta\right)$.

3. If it is the case that Min_Pow $\left(Q_{1}, G_{1}, K_{Q_{1}}, \theta\right)$, Min_Pow $\left(Q_{2}, G_{2}, K_{Q_{2}}, \theta\right)$ and $Q_{1} \cap Q_{2}=\emptyset$, then there exists $a Q^{\prime} \subseteq Q_{1} \cup Q_{2}$ and $a K_{Q^{\prime}} \sqsubseteq K_{Q_{1}} \sqcup K_{Q_{2}}$ such that Min_Pow $\left(Q^{\prime}, G_{1} \cup G_{2}, K_{Q^{\prime}}, \theta\right)$.

proof:

1) Proof by contradiction. Assume that there exists a $Q^{\prime} \subset Q$ and an action assignment $K_{Q^{\prime}} \sqsubset Q^{\prime}$ such that Min_Pow $\left(Q^{\prime}, G^{\prime}, K_{Q^{\prime}}, \theta\right)$. Then also Pow $\left(Q^{\prime}, G^{\prime}, K_{Q^{\prime}}, \theta\right)$ holds and for the goals monotonicity property $\operatorname{Pow}\left(Q^{\prime}, G, K_{Q^{\prime}}, \theta\right)$. But this is against the hypothesis that $\operatorname{Min}_{-} \operatorname{Pow}\left(Q, G, K_{Q}, \theta\right)$.

2) Let $G^{\prime} \subseteq G$. Since $\operatorname{Min}_{-} \operatorname{Pow}\left(Q, G, K_{Q}, \theta\right)$, then also $\operatorname{Pow}\left(Q, G, K_{Q}, \theta\right)$ and hence, due to the goals monotonicity property, $\operatorname{Pow}\left(Q, G^{\prime}, K_{Q}, \theta\right)$. Let

$$
\mathcal{K}=\left\{K_{Q^{\prime}} \sqsubseteq K_{Q} \mid \operatorname{Pow}\left(Q^{\prime}, G^{\prime}, K_{Q^{\prime}}, \theta\right)\right.
$$

$\mathcal{K}$ is not empty since $K_{Q} \in \mathcal{K}$. Now let $K_{\bar{Q}}$ be the actions allocation minimal with respect to the inclusion relation. By construction $\operatorname{Pow}\left(\bar{Q}, G^{\prime}, K_{\bar{Q}}, \theta\right)$.

3) Since Min_Pow $\left(Q_{1}, G_{1}, K_{Q_{1}}, \theta\right)$ and $\operatorname{Min}_{-} \operatorname{Pow}\left(Q_{2}, G_{2}, K_{Q_{2}}, \theta\right)$, then also $\operatorname{Pow}\left(Q_{1}, G_{1}, K_{Q_{1}}, \theta\right)$ and $\operatorname{Pow}\left(Q_{2}, G_{2}, K_{Q_{2}}, \theta\right)$ hold. This means for the superadditive property of the relation $P$ ow that $P o w\left(Q_{1} \cup Q_{2}, G_{1} \cup G_{2}, K_{Q_{1}} \sqcup K_{Q_{2}}, \theta\right)$, hence for the previous property there exists a $Q^{\prime} \subseteq Q_{1} \cup Q_{2}$ and a $K_{Q^{\prime}} \sqsubseteq K_{Q_{1}} \sqcup$ $K_{Q_{2}}$ such that Min_Pow $\left(Q^{\prime}, G_{1} \cup G_{2}, K_{Q^{\prime}}, \theta\right)$.

Some definitions of dependence require that if an agent is dependent for a goal, then it is has not the power itself to achieve that goal. For this reason we also define the notion of lack of power, $\operatorname{Lack}_{-} P o w(Q, G, \theta)$, when a group of agents $Q$ desires some set of goals $G$, but it has not the power to achieve it in a state $\theta$.

Definition 9 (Lack of power) An agent ag $g_{i}$ lacks the power to achieve a goal $\mathrm{g}$, in a state $\theta, L_{a c k}{ }_{-} P o w\left(a g_{i}, g, \theta\right)$, if and only if $g \in G_{i}$ and there does not exist an action assignment $K_{\left\{a g_{i}\right\}}$ such that Pow $\left(\left\{a g_{i}\right\}, g, K_{\left\{a g_{i}\right\}}, \theta\right)$.

Example 7 Consider the Multiagent system described in Example 6. We want to analyze security issues. The prison can be in one of eight states corresponding to the different values of the variables $s_{1}, s_{2}$ and $f$.

The first issue regards the security of the prisoner in the case of fire. Pow $\left(\emptyset,\left\{s_{1}\right\}, \emptyset, f\right)$ holds, and therefore the prisoner is not dependent on any other agent for his safety. Now we want to analyze whether the prisoner has an opportunity to escape. We 
provide a classification of the danger of a state of the prison with respect to the possibility in the next state to reach the goal of the prison to escape.

1. A state is fatal if it satisfies the goal of the prisoner.

2. It is an alarm state if the prisoner alone has the ability to force the next state in a fatal state.

3. It is a risky state if there is a guard that alone has the power to achieve a fatal state.

4. It is suspicious if both the guards are necessary to achieve a fatal state.

5. A state is safe if no a set of agents has the power to force the next state in a fatal state.

In Figure 2 all the possible states of the prison are listed with the action assignment that assure a set of agents to achieve the goal of the prisoner. The states 4 and 8 are the fatal states, whereas the states 3,6 and 7 are safe. There is one suspicious state and two risky states. Fortunately there are not alarm states. This example shows that the controller mechanism is not so good. Indeed we would like that the most safe state would be the state in which all the gates are closed and there is no fire. Instead this state is suspicious, whereas the state $\neg s_{1} \wedge s_{2} \wedge f$ is safe. Moreover, there are two risky states and if we consider that the prison is normally in the state $s_{1} \wedge s_{2} \wedge \neg f$ and that the prisoner has the power of start a fire, then the prisoner has the power to reach a risky state, i.e., the state 1 in Figure 2.

A possible improvement is to update the effect rule $s_{1} \wedge s_{2} \wedge a_{2} \rightarrow \neg s_{2}^{\prime}$, with $s_{1} \wedge s_{2} \wedge a_{2} \wedge \neg a_{1} \rightarrow \neg s_{2}^{\prime}$. In this case also the state 5 would be safe and the state 1 is the only suspicious one.

\section{Formalization of Dependence}

In this section we formalize the notion of dependence. In general, dependence concerns the possibility of a group $Q$ of agents to satisfy goals of agents. Obviously $Q$ has to be able to achieve those goals, but a further condition is that the agents in the group $Q$ should be all useful for the fulfillment of the goals, as we do not want to formalize the dependence on useless agents. As said in the previous section the definition of Pow allows the presence of useless members, as represented by coalition monotonicity. To avoid this problem we have defined in the previous section a notion of Min_Pow that, satisfying the minimality condition, does not allow the presence of members that do not contribute to goals of other members. 


\begin{tabular}{|c|c|c|c|c|}
\hline \multicolumn{4}{|c|}{ States } & Minimal power \\
\hline 1 & $s_{1}$ & $s_{2}$ & $f$ & $\left\{\left(g d_{2}, a_{2}\right)\right\}$ \\
\hline 2 & $s_{1}$ & $\neg s_{2}$ & $f$ & $\left\{\left(g d_{2}, \emptyset\right)\right\}$ \\
\hline 3 & $\neg s_{1}$ & $s_{2}$ & $f$ & - \\
\hline 4 & $\neg s_{1}$ & $\neg s_{2}$ & $f$ & $\{(\emptyset, \emptyset)\}$ \\
\hline 5 & $s_{1}$ & $s_{2}$ & $\neg f$ & $\left\{\left(g d_{1}, a_{1}\right) ;\left(g d_{2}, a_{2}\right)\right\}$ \\
\hline 6 & $s_{1}$ & $\neg s_{2}$ & $\neg f$ & - \\
\hline 7 & $\neg s_{1}$ & $s_{2}$ & $\neg f$ & - \\
\hline 8 & $\neg s_{1}$ & $\neg s_{2}$ & $\neg f$ & $\{(\emptyset, \emptyset)\}$ \\
\hline
\end{tabular}

Figure 2: The minimal power in achieving the goal of the prisoner for each state of the prison.

We define three degrees of dependence: weak dependence, dependence and strong dependence. Roughly, weak dependence means that a set $Q$ of agent is able to achieve a goal $g$ desired by an agent, so there is the possibility to ask them to achieve that goal. Dependence adds to the weak dependence also the condition that the agent does not have the power to achieve $g$. Strong dependence adds to dependence the condition that $Q$ is the only set of agents that is able to achieve $g$ and, hence, the agent can resort only on them if the agent wants to see $g$ satisfied.

Definition 10 (Weak dependence) An agent $a g_{i}$ weak depends on the group of agents $Q$, in the state $\theta$, for the goal $g, W_{-} D e p\left(a g_{i}, Q, g, K_{Q}, \theta\right)$, if and only if $g \in G_{i}$ and Min_Pow $\left(Q, g, K_{Q}, \theta\right)$

We define the dependence of an agent $a g_{i}$ on a set of agents $Q$ to achieve the goal $g$ by means of $K_{Q}$ as: $a g_{i}$ desires $g$, but it lacks the power to achieve $g$, whereas $Q$ is a minimal group which has the power to achieve $g$.

Definition 11 (Agent dependence) An agent $a g_{i}$ depends on the group of agents $Q$, in the state $\theta$, for the goal $g, \operatorname{Dep}\left(a g_{i}, Q, g, K_{Q}, \theta\right)$, if and only if $W \_D e p\left(a g_{i}, Q, g, K_{Q}, \theta\right)$ and Lack_Pow $\left(\left\{a g_{i}\right\},\{g\}, \theta\right)$.

Besides the previous definitions we also define the notion of strong dependence when $Q$ is the only set of agents that is able to achieve any of the goals in $G$.

Definition 12 (Strong dependence) An agent $a g_{i}$ strongly depends on the set of agents $Q$, in the state $\theta$, for the goal $g, S_{-} D e p\left(a g_{i}, Q, g, K_{Q}, \theta\right)$, if and only if it depends on $Q$ for $g$, Dep $\left(a g_{i}, Q, g, K_{Q}, \theta\right)$, and it does not exist a set of agents $Q^{\prime} \neq Q$ and an action assignment $K_{Q^{\prime}}$ such that Min_Pow $\left(Q^{\prime}, g, K_{Q^{\prime}}, \theta\right)$. 


\section{Topological representation of dependencies}

Weak and normal dependence describe the structure underlying possible cooperations and exchanges. The topological properties of this structure, as shown in [8], are crucial for an analysis of the cohesion of these phenomena. Following [8] we represent the dependencies among agents as a graph. In particular, we use taggedand-graphs to describe the fact that a single agent may (weakly/strongly) depend on another set of agents for the achievement of a goal. First of all we provide the definition of a tagged-and-graph:

Definition 13 A tagged-and-graph is a structure $\langle N, T, E\rangle$, where

- $N$ is a finite set of nodes.

- $T$ is a finite set of tags.

- $E: N \times\left(2^{N} \backslash \emptyset\right) \times T$ is a set of tagged-and arcs, i.e., a set of triples $(v, V, t)$, where $v$ is a node, $V$ is a non-empty set of nodes and $t$ is a tag.

In the following, given a tagged-and-graph $\langle N, T, E\rangle$ and a tag $\bar{t} \in T$, we denote with $\left.E\right|_{\bar{t}}$ the subset of $E$ containing only the arcs tagged with $\bar{t}$, i.e., $\left.E\right|_{\bar{t}}=$ $\{(v, V, \bar{t}) \in E\}$.

The idea of relating dependencies among agents to tagged-and-graphs is the following. If an agent $a g$ (weakly/strongly) depends on a set of agents $Q$ for the fulfilment of a goal $g$, then we consider and tagged-and-arc $e=(a g, Q, t)$ that starts from $a g$ and reaches $Q$. The tag $t$ of $e$ is itself a structure that provide further information about the dependence represented by $e$, such as the goal that is achieved or the action assignment necessary to achieve that goal. Which kind of information $t$ represents may depend on the type of analysis it is intended to do on the graph or on some aspects that can extend the expressiveness of the Multiagent System, as for example the resources needed to execute a given action and the relative costs [16]. Here, since we have not formalized resources and the relative costs, a tag represents only the goal achieved in a dependence relation.

Therefore, we provide a definition of the tagged-and-graph describing the dependence relations occurring in a Multiagent System.

Definition 14 (Dependence graph) Given a Multiagent system $M a S$

$$
\left\langle A g, G_{1}, \ldots, G_{|A g|}, A c_{1}, \ldots, A c_{|A g|}, R l\right\rangle
$$

the dependence graph relative to $M a S$ in a state $\theta$ is the tagged-and-graph $\langle A g, G l, E\rangle$ such that $\left(a g_{i}, Q, g\right) \in E$ if and only if $\exists K_{Q} \operatorname{Dep}\left(a g_{i}, Q, g, K_{Q}, \theta\right)$ 
Analogously, the weak- and strong-dependence graph can be defined relative to a $M a S$ in a given state $\theta$.

A dependence graph describes the set of all possible dependencies among agents. However, not all of them can occur at the same time, because some goals may require incompatible action assignments. For this reason it is useful to define dependence graphs that describes the maximal sets of compatible dependencies.

Definition 15 (Compatible dependence graph) Given a Multiagent system MaS

$$
\left\langle A g, G_{1}, \ldots, G_{|A g|}, A c_{1}, \ldots, A c_{|A g|}, R l\right\rangle
$$

a tagged-and-graph $\mathcal{G}=\langle A g, G l, E\rangle$ is a compatible dependence graph if and only if for all $\left(a g_{i}, Q, g\right) \in E$ there exists an action assignment $K_{Q}$ such that $\operatorname{Dep}\left(a g_{i}, Q, g, K_{Q}, \theta\right)$ and Pow $\left(\bigcup Q, \bigcup\{g\}, \bigsqcup_{Q} K_{Q}, \theta\right)$.

A compatible dependence graph $\mathcal{G}$ is maximal if and only if it does not exist a compatible dependence graph $\mathcal{G}^{\prime}$ such that $\mathcal{G}$ is a subgraph of $\mathcal{G}^{\prime}$.

\section{Importance measurement}

We formalize the social importance of an agent, $s_{a g}(M a S)$, in a state $\theta$. This measure is based on the notion of $\beta$-measure defined in van den Brink and Gilles [18] for directed graphs $\langle N, E\rangle$, where $N$ is a set of nodes, and $E \subseteq N \times N$ is an irreflexive set of arcs that describes a dominance relation among the nodes, i.e., $(v, w) \in E$ means that $v$ dominates $w$ in some sense. The $\beta$-measure of a node $v$ measures the importance of $v$ inside the graph:

$$
\beta_{v}(\langle N, E\rangle)=\sum_{w \in S(v)} \frac{1}{p(w)}
$$

Where $S(v)$ is the set of successors of $v, S(v)=\{w \in N \mid(v, w) \in E\}$, and $p(w)$ is the cardinality of the set of predecessors of $w, p(w)=|\{h \in N \mid(h, w) \in E\}|$. Informally, in order to define an analogy of the $\beta$-measure, an agent $a g_{1}$ dominates an agent $a g_{2}$ if $a g_{2}$ depends on $a g_{1}$ for the achievement of some goals. However, since in our case not all the dependencies can be satisfied at the same time, we do not measure importance directly on the dependence graph relative to a $M a S$, but we first measure it for each maximal compatible dependence graph and then we consider $s_{a g}(M a S)$ as a function of these values. In this work, we assume that it is not known which particular maximal compatible dependence graph will actually come out, so if $\mathcal{G}_{1}, \ldots, \mathcal{G}_{n}$ are the set of maximal compatible dependence graphs 
and $s_{a g}\left(\mathcal{G}_{i}\right)$ is the importance measure of $a g$ in $\mathcal{G}_{i}$, then $s_{a g}(M a S)$ is given by the average of the $s_{a g}\left(\mathcal{G}_{i}\right)$ :

$$
s_{a g}(M a S)=\frac{1}{n} \sum_{i=1}^{n} s_{a g}\left(\mathcal{G}_{i}\right)
$$

To calculate the importance $s_{a g}(\mathcal{G})$ of an agent $a g$ in a compatible dependence graph $\mathcal{G}=\langle A g, G l, E\rangle$ we have to consider that the dependence relations in it are parameterized with respect to the goals. We separately measure the importance that each goal $g$ provides to an agent $a g$ in $\mathcal{G}, s_{a g}(g, \mathcal{G})$, and after we define the overall measure as the sum of these measures.

$$
s_{a g}(\mathcal{G})=\sum_{g \mid\left(a g^{\prime}, Q, g\right) \in E} s_{a g}(g, \mathcal{G})
$$

Finally, we define the importance that a goal $g$ provides to an agent $a g$ in a dependence graph $\mathcal{G}, s_{a g}(g, \mathcal{G})$, by reformulating Formula 1 . There, $S(v)$ is the set of successors of the node $v$, i.e., the set of nodes that $v$ dominates. Here, domination is provided by the dependence relation, where we take into account, with respect to the case of directed graph, that an agent $a g^{\prime}$ may depend for $g$ on different sets of agents $Q$. Furthermore, arcs in [18] are supposed to be irreflexive, i.e., a node cannot dominate itself. Similarly, we assume that the importance of an agent stems on its capability to satisfy the goals of the other agents and not of its owns, so we assume that $a g^{\prime} \neq a g$. Therefore, the successors of $a g$ with respect to the goal $g$ are is defined as the set of all dependence $\operatorname{arcs}\left(a g^{\prime}, Q, g\right)$ such that $a g \in Q$ and $a g^{\prime} \neq a g$.

In Formula $1 p(w)$ is the cardinality of the predecessor of the node $w$, i.e. the set of nodes that dominates $w$. In our case, given an agent $a g, p(a g, g)$ is the set of agents $Q$ on which $a g$ depends for the achievement of the goal $g$, formally:

$$
p(a g, g)=|\{Q \mid(a g, Q, g) \in E\}|
$$

By construction this number is equal to 0 if $a g$ do not desires $g$ or equal to the cardinality $p(g)$ of the set of agents $Q$ that has the power to achieve $g$. Since in the formula $s_{a g}(g, \mathcal{G})$ we consider the predecessors of agents that desires $g$, because they depends for $g$ on some set $Q$ that contains $a g$, then we have that their cardinality is always equal to $p(g)$. Finally, the formula of $s_{a g}(g, \mathcal{G})$ is given by:

$$
s_{a g}(g, \mathcal{G})=\sum_{\left(a g^{\prime}, Q, g\right) \in E \mid a g^{\prime} \neq a g \wedge a g \in Q} \frac{1}{p(g)}
$$




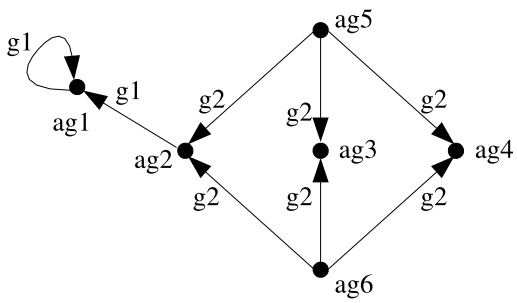

Figure 3: An example of Dependence Graph.

We note that the previous formula is given also by the number $r(g)$ of agents that desires $g$, eventually minus 1 if $a g$ itself desires $g$, times the rate between the number of the sets $Q$ containing $a g$ having the power to achieve $g, a(g, a g)$, and the number of all the sets of agents having the power to achieve $g, p(g)$.

$$
s_{a g}(g, \mathcal{G})=(r(g)-d(g, a g)) \frac{a(a g, g)}{p(g)}
$$

Where $d(g, a g)$ is equal to 1 if $a g$ desires $g, 0$ otherwise.

In Figure 3 we show an example of a compatible dependence graph $\mathcal{G}$ to explain the idea underlining the function $s_{a g}(g, \mathcal{G})$. In particular we consider the importance that the goal $g_{1}$ provides to $a g_{1}$ and the importance that $g_{2}$ provides to $a g_{2}$. Considering the Formula 5 we have that

$$
\begin{aligned}
s_{a g_{1}}\left(g_{1}, \mathcal{G}\right) & =1 \\
s_{a g_{2}}\left(g_{2}, \mathcal{G}\right) & =\frac{2}{3}
\end{aligned}
$$

Suppose now to eliminate the agent $a g_{1}$ from the Multiagent System. In $\mathcal{G}$ this means to remove the node $a g_{1}$ and also all the arcs that have $a g_{1}$ has an end or start point. In this new graph $\mathcal{G}^{\prime}$ the agent $a g_{2}$ does not have the possibility to satisfy the goal $g_{1}$, instead for all the other agents removing $a g_{1}$ does not affect the chances to achieve any of their goals. Since we consider that all the goals have the same relevance, we can estimate that $a g_{2}$, passing from $\mathcal{G}$ to $\mathcal{G}^{\prime}$, shows a displeasure equal to 1 , since one of its goals cannot be achieved anymore. Analogously, all the other agents in $\mathcal{G}^{\prime}$ show a displeasure equal to 0 .

In the case $a g_{2}$ is removed from $\mathcal{G}$, then $a g_{5}$ loses one of three opportunities on which it can resort for the achievement of $g_{2}$ - namely the agents $a g_{2}, a g_{3}$ and $a g_{4}$. Therefore, its displeasure is equal to $\frac{1}{3}$. For the same reason also $a g_{6}$ shows the same displeasure, whereas all the other agents shows a displeasure equal to 0 . 


\begin{tabular}{|c|c|c|}
\hline States & $g d_{\mathbf{1}}$ & $g d_{\mathbf{2}}$ \\
\hline 1 & 0 & 1 \\
2 & 0 & 1 \\
3 & 0 & 0 \\
4 & 0 & 0 \\
5 & 1 & 1 \\
6 & 0 & 0 \\
7 & 0 & 0 \\
8 & 0 & 0 \\
\hline tot & $\frac{1}{8}$ & $\frac{3}{8}$ \\
\hline
\end{tabular}

Figure 4: Importance of the agents $g d_{1}$ and $g d_{2}$ in the prison example.

Therefore, the total displeasure is equal to $\frac{2}{3}$. These values are the ones calculated in the Formulas 7 and 8.

Example 8 In Figure 2 it is shown for each state the minimal group of agents which is necessary for the satisfaction of the goal of the prisoner. Figure 2 shows that, for each state, the prisoner has not the power to achieve his own goal and also there exists only one set of agents the prisoner weak depends, depends and strong depends on. So, for each state, there exists only one compatible dependence graph and this dependence graph contains only one dependence arc.

The importance of each guard in a given state corresponds to importance calculated by the Formula 5 to the relative dependence arc. For example the importance of the guard $g d_{2}$ in the state 1 is equal to 1 , because he takes part in the satisfaction of the goal of the prisoner and there exists only a set of agents, $g d_{2}$ himself, that is able to satisfy that goal. Analogously, the importance of $g d_{1}$ in the state 1 is equal to zero since he does not take a part in the satisfaction of the goal of the prisoner.

In Figure 4 the importance of the guards $g d_{1}$ and $g d_{2}$ is shown for each state. The last row shows the total power of the two guards. As you can see the guard $g d_{2}$ has a bigger importance, according with the intuition that the prisoner in each state desires that the second gate is open in the next state, whereas it desires that the first gate is open only in the case that he is in the dormitory.

If the effect rule $s_{1} \wedge s_{2} \wedge a_{2} \rightarrow \neg s_{2}^{\prime}$ would be replaced with the rule $s_{1} \wedge s_{2} \wedge$ $a_{2} \wedge \neg a_{1} \rightarrow \neg s_{2}^{\prime}$ as discussed in Section 3 (an hence the state 5 is safe and the state 1 suspicious) we would have that the importance of $g d_{1}$ is $\frac{1}{8}$ and the importance of the guard $g d_{2}$ is $\frac{2}{8}$. 


\section{Related work}

In the field of Multiagent Systems, Social Reasoning Mechanism [17] provides a way to reason about the potential for cooperation and social exchange under the assumption that agents are self-interested. The core of this approach is given by the notion of social dependence defined by Castelfranchi [6,9] and this work presents a formal definition of the notion of social power and dependence among agents.

With respect to [17], where the possible plans of the agents cannot interfere with each other, our formalization is an attempt to introduce a more rich description in which a dependence may arise not only for the lack of a resource or a particular skill, but also because agents can obstruct the execution of a plan. We assumed that agents cannot look after with each other, therefore, as suggested in [19], the notion of power has been formalized inspired by the game theoretical notion of $\alpha$-ability [2]. Informally, $\alpha$-ability means that a set of agents $Q$ has the power to force an outcome to be into a set of possible of outcomes $O$ if there exists a common strategy of $Q$ such that for all the strategies of the other agents the outcome will be in $O$.

Properties of $\alpha$-ability, such as coalitional monotonicity or super-additivity, have been logically formalized in [13]. However, we do not consider the notion of power as a mere coordination property, so we reformulate $\alpha$-ability as the capability to reach one or more goals of the agents and not to force the system to be in generic set of states.

As in [19], we provide a simple model of a Multiagent System which evolves of a single step ahead from an initial state to a final state. Since it was not the purpose of this paper to provide an expressive framework to address coordination issues, the paper abstracts away from a detailed description of plans as sequences of actions, as done for example in ATL [1], but it simply describes which goals the concurrent execution of different actions involves [11]. However, if our approach is less expressive than ATL in this sense, we have the advantage to explicitly describe in our language the action assignment that a set of agents have to execute in order to achieve a goal. This gives us the possibility to refine the notion of power, intended as $\alpha$-ability, with a the notion of power with minimality which is more suited to correctly formalize dependencies among the agents. Since this notion explicitly concerns the action assignments, it cannot be formulated in ATL.

\section{Summary}

In this paper, we consider the formalization of the social importance of agents using a tagged-and-graph representing dependencies among agents to achieve their 
goals. The social importance of agents cannot be reduced to a property of a single agent, but it depends on an entire community in which that agent lives and in particular on its capability and exclusiveness with respect to the other agents to achieve their goals. A running example shows how this notion is able to reveal these asymmetries inside a community.

The contributions of this work can be summarized as follows. First, we define a dependence network for agents based on their abilities and desires, which can be used to measure their social importance. This formalization is derived from the one used in previous works $[15,3,4]$ to study qualitative coalition formation. However, some improvements have been made.

- The formal definition of a multiagent system allows a more expressive specification of goals, for example a goal can specify that a given action has not to be executed.

- The definition of power is based on goal monotonicity, coalition monotonicity and super-additivity. The minimization of power is done on action assignment and not only on the number of agents.

- Three kinds of dependence are defined, called weak, agent and strong dependence.

- A compatible dependence graph is defined describing maximal sets of compatible dependencies.

Second, we adapt van den Brink and Gilles' $\beta$-measure such that it can be used for these dependence networks. The $\beta$-measure is used to rank agents inside a dominance structure, i.e., a weighted directed graph where each node represents an agent and a weighted arc represents the strength of a dominance relation between two agents. We extend the $\beta$-measure in order to capture the strength of an agent in the satisfaction of the goals of other agents or, in other words, the discontent of the other agents in the case an agent would leave the society. We formalize the social importance of an agent, $s_{a g}(M a S)$, in a state $\theta$. If $\mathcal{G}_{1}, \ldots, \mathcal{G}_{n}$ are the set of maximal compatible dependence graphs and $s_{a g}\left(\mathcal{G}_{i}\right)$ is the importance measure of $a g$ in $\mathcal{G}_{i}$, then $s_{a g}(M a S)$ is given by the average of the $s_{a g}\left(\mathcal{G}_{i}\right)$ :

$$
s_{a g}(M a S)=\frac{1}{n} \sum_{i=1}^{n} s_{a g}\left(\mathcal{G}_{i}\right)
$$

The overall measure is the sum of the importance that each goal $g$ provides to an agent $a g$ in $\mathcal{G}, s_{a g}(g, \mathcal{G})$.

$$
s_{a g}(\mathcal{G})=\sum_{g \mid\left(a g^{\prime}, Q, g\right) \in E} s_{a g}(g, \mathcal{G})
$$


$s_{a g}(g, \mathcal{G})$ is given by the number $r(g)$ of agents that desires $g$, eventually minus 1 if $a g$ itself desires $g$, times the rate between the number of the sets $Q$ containing $a g$ having the power to achieve $g, a(g, a g)$, and the number of all the sets of agents having the power to achieve $g, p(g)$. Let $d(g, a g)$ be equal to 1 if $a g$ desires $g, 0$ otherwise.

$$
s_{a g}(g, \mathcal{G})=(r(g)-d(g, a g)) \frac{a(a g, g)}{p(g)}
$$

This qualitative measure does not make any particular assumption on the internal preferences of an agent with respect its own goals and hence it can be applied in contexts where this private information is not available.

There are two directions for further research. First, the underlying framework can be improved in order to capture more complex scenarios. In particular, the notion of power can be defined in a more expressive structure in which complex plans and goals can be described. Secondly, the notion of social importance can be extended to better understand the bargaining power in a trade process. Intuitively, an agent which has no interest, or a marginal interested, to be a member of a community cannot play a crucial role in it. For this reason, the notion of social importance should not only take into account the goals an agent can offer to the others, but also the ones it asks in exchange.

\section{Acknowledgements}

We acknowledge the contributions of Luca Tummolini and Jelle Gerbrandy in the development of this paper.

\section{References}

[1] R. Alur, T.A. Henzinger, and O. Kupferman. Alternating-time temporal logic. Journal of ACM, 49(5):672-713, 2002.

[2] R.J. Aumann. The core of a cooperative game without side payments. Transaction of the American Mathematical Society, 98:539-552, 1961.

[3] G. Boella, L. Sauro, and L. van der Torre. Admissible agreements among goal-directed agents. In Procs. of 2005 IEEE/WIC/ACM International Conference on Intelligent Agent Technology (IAT'05), Paris, France, 2005.

[4] G. Boella, L. Sauro, and L. W. N. van der Torre. Strengthening admissible coalitions. In Proceedings of the 17th European Conference on Artificial Intelligence (Ecai 2006), pages 195-199, Riva del Garda, Italy, 2006. 
[5] G. Boella and L. van der Torre. The distribution of obligations by negotiation among autonomous agents. In Procs. of 16th European Conference on Artificial Intelligence (ECAI'04), pages 13-17, Amsterdam, 2004. IOS Press.

[6] C. Castelfranchi. Founding agents' "autonomy" on dependence theory. In Proceedings of the 14th European Conference on Artificial Intelligence (ECAI'00), pages 353-357, Berlin, Germany, 2000.

[7] C. Castelfranchi. The Micro-Macro Constitution of Power. ProtoSociology, 18-19, 2003.

[8] R. Conte and J. Sichman. Multi-Agent Dependence by Dependence Graphs. In Proceedings of The First International Joint Conference on Autonomous Agents \& Multiagent Systems (AAMAS'02), pages 483-490, Bologna, Italy, 2002.

[9] M. d'Inverno and M. Luck. A formal view of social dependence networks. In Proceedings of First Australian Workshop on Distributed Artificial Intelligence (DAI'95), volume 1087 of Lecture Notes in Computer Science, Camberra, Australia, 1996.

[10] R. Fikes and N. Nilsson. Strips: A new approach to the application of theorem proving to problem solving. Artificial Intelligence, 2(3-4):189-208, 1971.

[11] S. Kraus, O. Shehory, and G. Taase. The advantages of compromising in coalition formation with incomplete information. In Procs. of AAMAS'04, pages 588-595, 2004.

[12] J. Odell, H. Parunak, M. Fleischer, and S. Brueckner. Modeling agents and their environment. In Proceedings of AOSE 2002, Bologna, Italy, 2002.

[13] M. Pauly. A Modal Logic for Coalitional Power in Games. Journal of Logic and Computation, 12:146-166, 2002.

[14] B. Peleg. Effectivity functions, game forms, games, and rights. Social Choice Theory, 15:67-80, 1998.

[15] L. Sauro. Formalizing Admissibility Criteria in Coalition Formation among Goal Directed Agents. PhD thesis, Dipartimento di Informatica, Università di Torino, 2005.

[16] O. Shehory and S. Kraus. Methods for Task Allocation via Agent Coalition Formation. Artificial Intelligence, 101(1-2):165-200, 1998. 
[17] J. S. Sichman and Y. Demazeau. On social reasoning in multi-agent systems. Revista Iberoamericana de Inteligencia Artificial, 13:68-84, 2001.

[18] R. van den Brink and R.P. Gilles. Measuring domination in directed graphs. Social Networks, 22(2):141-157, 2000.

[19] W. van der Hoek and M. Wooldridge. On the logic of cooperation and propositional control. Artificial Intelligence, 64:1-2, 64(1-2):81-119, 2005. 


\title{
Introduction to Normative Multiagent Systems
}

\author{
Guido Boella \\ Dipartimento di Informatica \\ Università di Torino \\ Italy \\ guido@di.unito.it
}

Leendert van der Torre

Department of Computer Science

University of Luxembourg

Luxembourg

leendert@vandertorre.com

\author{
Harko Verhagen \\ Dept. of Computer and Systems Sciences \\ Stockholm University / KTH, \\ Forum 100, SE-16440 Kista, Sweden \\ verhagen@dsv.su.se \\ May 29, 2006
}

Normative multiagent systems as a research area can be defined as the intersection of normative systems and multiagent systems. Since the use of norms is a key element of human social intelligence, norms may be essential too for artificial agents that collaborate with humans, or that are to display behavior comparable to human intelligent behavior. By integrating norms and individual intelligence normative multiagent systems provide a promising model for human and artificial agent cooperation and co-ordination, group de- 
cision making, multiagent organizations, regulated societies, electronic institutions, secure multiagent systems, and so on.

With 'normative' we mean 'conforming to or based on norms', as in normative behavior or normative judgments. According to the Merriam-Webster Online (2005) Dictionary, other meanings of normative not considered here are 'of, relating to, or determining norms or standards', as in normative tests, or 'prescribing norms', as in normative rules of ethics or normative grammar. With 'norm' we mean 'a principle of right action binding upon the members of a group and serving to guide, control, or regulate proper and acceptable behavior'. Other meanings of 'norm' given by the Merriam-Webster Online Dictionary but not considered here are 'an authoritative standard or model', 'an average like a standard, typical pattern, widespread practice or rule in a group', and various definitions used in mathematics.

Normative multiagent systems are an example of the use of sociological theories in multiagent systems, and more generally of the relation between agent theory and the social sciences such as sociology, philosophy, economics, and legal science. The need for social science theories and concepts like norms in multiagent systems is now well established. For example, Wooldridge's weak notion of agency is based on flexible autonomous action (Wooldridge, 2002), and social ability as the interaction with other agents and co-operation is one of the three meanings of flexibility; the other two are reactivity as interaction with the environment, and pro-activeness as taking the initiative. In this definition autonomy refers to non-social aspects, such as operating without the direct intervention of humans or others, and have some kind of control over their actions and internal state. For some other arguments for the need for social theory in multiagent systems, see, for example, (Bond and Gasser, 1988; Conte and Gilbert, 1995; Verhagen and Smit, 1996). For a more complete discussion on the need of social theory in general, and norms in particular, see the AgentLink roadmap (roa, 2005). 
Social concepts like norms are important for multiagent systems, because multiagent system research and sociology share the interest in the relation between micro-level agent behaviour and macro-level system effects. In sociology this is the (in)famous micro-macro link (Alexander et al., 1987) that focuses on the relation between individual agent behaviour and characteristics at the level of the social system. In multiagent system research, this boils down to the question "How to ensure efficiency at the level of the multiagent system whilst respecting individual autonomy?". According to Verhagen (2000) three possible solutions to this problem comprise of the use of central control which gravely jeopardizes the agent's autonomy, internalized control like the use of social laws (Shoham and Tennenholtz, 1992), and structural coordination (Ossowski, 1999) including learning norms.

Before we discuss normative multiagent systems, we consider some discussions on norms in the social sciences.

\section{Norms and normative systems}

In the 1960's, the sociologist Gibbs (1965) wrote an influential article on the problems concerning the definition and classification of norms, and observes that the various types of norms involve "a collective evaluation of behavior in terms of what it ought to be; a collective expectation as to what behavior will be; and/or particular reactions to behavior, including attempts to apply sanctions or otherwise induce a particular kind of conduct." (Gibbs, 1965, p. 589, original emphasis)

More recently, Therborn (2002) presented an overview of the role of norms for social theory and analysis. Normative action is based upon wanting to do the right thing rather than the thing that leads to ends or goals, which he calls teleological action, or the thing that leads to, expresses, or is caused by an emotion, called emotional action.

Therborn distinguishes among three kinds of norms. Constitutive norms define a sys- 
tem of action and an agent's membership in it, regulative norms describe the expected contributions to the social system, and distributive norms defining how rewards, costs, and risks are allocated within a social system. Furthermore, he distinguishes between noninstitutionalized normative order, made up by personal and moral norms in day-to-day social traffic, and institutions, an example of a social system defined as a closed system of norms. Institutional normative action is equaled with role plays, i.e., roles find their expressions in expectations, obligations, and rights vis-a-vis the role holder's behaviour.

Therborn also addresses the dynamics and changing of norms. The dynamics of norms at the level of the individual agent is how norms are learned or propagated in a population. Socialization is based on identification, perceiving the compliance with the norms by other agents, or the entering of an institution. Norms are (re)enforced by the presence of incentives or sanctions. Changes in either of these three three socialization mechanisms lead to changes in the set of norms of the individual agent. These changes may be inhibited either by changes in the social system or changed circumstances, or by changes in the interpretation of the norms by the agents within the system.

Within philosophy normative systems have traditionally been studied by moral and legal philosophers. Alchourròn and Bulygin (1971) argue that a normative system should not be defined as a set of norms, as is commonly done, but in terms of consequences:

"When a deductive correlation is such that the first sentence of the ordered pair is a case and the second is a solution, it will be called normative. If among the deductive correlations of the set $\alpha$ there is at least one normative correlation, we shall say that the set $\alpha$ has normative consequences. A system of sentences which has some normative consequences will be called a normative system." (Alchourròn and Bulygin, 1971, p.55).

In computer science, Meyer and Wieringa define normative systems as "systems in the behavior of which norms play a role and which need normative concepts in order to 
be described or specified" (Meyer and Wieringa, 1993, preface). They also explain why normative systems are intimately related with deontic logic.

"Until recently in specifications of systems in computational environments the distinction between normative behavior (as it should be) and actual behavior (as it is) has been disregarded: mostly it is not possible to specify that some system behavior is non-normative (illegal) but nevertheless possible. Often illegal behavior is just ruled out by specification, although it is very important to be able to specify what should happen if such illegal but possible behaviors occurs! Deontic logic provides a means to do just this by using special modal operators that indicate the status of behavior: that is whether it is legal (normative) or not" (Meyer and Wieringa, 1993, preface).

\section{Normative multiagent systems}

The agents in the environment of a normative system interact with the normative system in various ways. First, from the perspective of the agents, agents can create new norms, update or maintain norms, and enforce norms, using roles defined in the normative system such as legislators or policemen. Secondly, from the perspective of social order, we can also look at the interaction between the normative system and its environment from the viewpoint of the normative system. In this viewpoint, the normative system uses the agents playing a role in it - the legislators, policemen and the like - to maintain an equilibrium in the normative multiagent system. In this perspective, we can distinguish at least two levels of equilibrium. First, norms are used to maintain social order in a normative multiagent system. Second, normative system contain a mechanism for updating themselves, to adapt to changing circumstances in its environment.

Jones and Carmo (2001) define a normative system as "Sets of agents whose interactions 
are norm-governed; the norms prescribe how the agents ideally should and should not behave. [...] Importantly, the norms allow for the possibility that actual behavior may at times deviate from the ideal, i.e., that violations of obligations, or of agents' rights, may occur." In our opinion, this is too general, as a normative system does not contain the agents themselves. It also is not a satisfactory definition of normative multiagent system, because it precludes the agents' control over the set of norms. We therefore use the following definition in this paper.

A normative multiagent system is a multiagent system together with normative systems in which agents on the one hand can decide whether to follow the explicitly represented norms, and on the other the normative systems specify how and in which extent the agents can modify the norms.

Note that this definition makes no presumptions about the internal workings of an agent nor of the way norms find their expression in agent's behaviour.

Since norms are explicitly represented, according to our definition of a normative multiagent system, the question should be raised how norms are represented. Norms can be interpreted as a special kind of constraint, and represented depending on the domain in which they occur. However, the representation of norms by domain dependent constraints runs into the question what happens when norms are violated. Not all agents behave according to the norm, and the system has to deal with it. In other words, norms are not hard constraints, but soft constraints. For example, the system may sanction violations or reward good behavior. Thus, the normative system has to monitor the behavior of agents and enforce the sanctions. Also, when norms are represented as domain dependent constraints, the question will be raised how to represent permissive norms, and how they relate to obligations. Whereas obligations and prohibitions can be represented as constraints, this does not seem to hold for permissions. For example, how to represent the permission to 
access a resource under an access control system? Finally, when norms are represented as domain dependent constraints, the question can be raised how norms evolve.

We therefore believe that norms should be represented as a domain independent theory, for example in deontic logic (von Wright, 1951; van der Torre and Tan, 1999; van der Torre, 2003; Makinson and van der Torre, 2000; Makinson and van der Torre, 2001; Makinson and van der Torre, 2003). Deontic logic studies logical relations among obligations and permissions, and more in particular violations and contrary-to-duty obligations, permissions and their relation to obligations, and the dynamics of obligations over time. Therefore, insights from deontic logic can be used to represent and reason with norms. Deontic logic also offers representations of norms as rules or conditionals. However, there are several aspects of norms which are not covered by constraints nor by deontic logic, such as the relation between the cognitive abilities of agents and the global properties of norms.

Conte, Falconi and Sartor (1999) say that normative multiagent systems research focuses on two different sets of problems. On the one hand, they claim that legal theory and deontic logic supply a theory for of norm-governed interaction of autonomous agents while at the same time lacking a model that integrates the different social and normative concepts of this theory. On the other hand, they claim that three other problems are of interest in multiagents systems research on norms: how agents can acquire norms, how agents can violate norms, and how an agent can be autonomous. For artificial agents, norms can be designed as in legal human systems, forced upon, for example when joining an institution, or they can emerge from the agents making them norm autonomous (Verhagen, 2000). Agent decision making in normative systems and the relation between desires and obligations has been studied in agent architectures (Broersen et al., 2002), which thus explain how norms and obligations influence agent behavior.

An important question is where norms come from. Norms are not necessarily created by a single legislator, they can also emerge spontaneously, or be negotiated among the agents. 
In electronic commerce research, for example, cognitive foundations of social norms and contracts are studied (Boella and van der Torre, 2006a). Protocols and social mechanisms are now being developed to support such creations of norms in multiagent systems. When norms are created, the question how they are enforced can be raised. For example, when a contract is violated, the violator may have to pay a penalty. But then there has to be a monitoring and sanctioning system, for example police agents in an electronic institution. Such protocols or roles in a multiagent system are part of the construction of social reality, and Searle (1995) has argued that such social realities are constructed by constitutive norms. This again raises the question how to represent such constitutive or counts-as norms, and how they are related to regulative norms like obligations and permissions (Boella and van der Torre, 2006a).

Not only the relation between norms and agents must be studied, but also the relation between norms and other social and legal concepts. How do norms structure organizations? How do norms coordinate groups and societies? How about the contract frames in which contracts live? How about the legal contexts in which contract frames live? How about the relation between legal courts? Though in some normative multiagent systems there is only a single normative system, there can also be several of them, raising the question how normative systems interact. For example, in a virtual community of resource providers each provider may have its own normative system, which raises the question how one system can authorize access in another system, or how global policies can be defined to regulate these local policies (Boella and van der Torre, 2006b).

Summarizing, normative multiagent systems study general and domain independent properties of norms. It builds on results obtained in deontic logic, the logic of obligations and permissions, for the representation of norms as rules, the application of such rules, contrary-to-duty reasoning and the relation to permissions. However, it goes beyond logical relations among obligations and permissions by explaining the relation among social norms 
and obligations, relating regulative norms to constitutive norms, explaining the evolution of normative systems, and much more.

The papers in this double special issue on normative multiagent systems address some of these issues, but they also address new research issues that are of central importance for the whole field of normative multiagent systems. These include how to combine theories of teleological action (e.g., the BDI model of agency) with models of normative action, how to model the dynamics of norms when institutions' norm sets are to be combined, the development and testing of logics of normative reasoning and dynamics, and the formalization descriptive social theories of normative action into implementable formal models.

\section{NorMAS 2005}

NorMAS05 was an international symposium on normative multiagent systems, organized in April 2005 by the authors of this article as part of the 2005 AISB convention (AISB standing for the Society for the Study of Artificial Intelligence and the Simulation of Behaviour). The symposium attracted papers from a variety of areas, such as the social sciences (and computational sociology in particular), computer science, and formal logics. A number of these papers representing these areas were selected for this double special issue on normative multiagent systems. Four general themes are addressed in these papers, namely intra-agent aspects of norms, interagent aspects of norms, normative systems and their borders, and combining normative systems.

\subsection{Intra-agent aspects of norms}

The paper "My Agents Love to Conform: Norms and Emotion in the Micro-Macro Link" by von Scheve et al. investigates the function of emotion in relation to norms in natural and artificial societies. It shows that unintentional behavior can be normative and socially 
functional at the same time, thereby highlighting the role of emotion. By defining norms as mental objects, the role of emotion in maintaining and enforcing norms is studied, relates these findings social structural dynamics in natural and societies,and outlines the possibilities of an application to a multi-agent architecture.

Sadri, Stati, and Toni's "Normative KGP Agents" extends the logical model of agency known as the KGP model to support agents with normative concepts, based on the roles an agent plays and the obligations and prohibitions that result from playing these roles. The proposed framework illustrates how the resulting normative concepts, including the roles, can evolve dynamically during the lifetime of the agent. It also illustrates how these concepts can be combined with the existing capabilities of KGP agents in order to plan for their goals, react to changes in the environment, and interact with other agents. Finally, the paper gives an executable specification of normative concepts that can be used directly for prototyping applications.

\subsection{Interagent aspects of norms}

Kibble's paper "Speech acts, commitment and multiagent communication" aims to reconsider the suitability of speech act theory as a basis for agent communication languages. It models dialogue states as deontic scoreboards which keep track of commitments and entitlements that speakers acknowledge and hearers attribute to other interlocutors and outlines an update semantics and protocol for selected locutions.

Sauro's paper "Qualitative Criteria of Admissibility for Enforced Agreements" focuses on the desirablility of artificial agents to help each other when they cannot achieve their goals, or when they profit from social exchanges. It studies the coalition formation processes supported by enforced agreements and defines two qualitative criteria that establish when a coalition is admissible to be formed. These two properties can be used when the space of possible coalitions is unknown. 


\subsection{Normative systems and their borders}

Davidsson and Johansson classify artificial societies and identify four different types of stakeholders in their paper "On the Potential of Norm-Governed Behavior in Different Categories of Artificial Societies". The potential of norm-governed behavior in different types of artificial societies is investigated based on the preferences of the stakeholders and how they influence the state of the society. The paper concludes that the more open a society is the more it has to rely on agent owners and designers to achieve norm-governed behavior, whereas in more closed societies the environment designers and owners may control the degree of norm-governed behavior.

Hahn, Fley, and Florian argue in "A Framework for the Design of Self-Regulation of Open Agent-based Electronic Marketplaces" that allowing self-interested agents to activate social institutions during run-time can improve the robustness of open multiagent systems. Based on sociological theory, institutions are seen as rules which have to be activated and adopted by the agent population. A framework for self-regulation of multiagent system for the domain of electronic marketplaces is developed, consisting of three different institutional forms that are defined by the mechanisms and instances that generate, change, or safeguard them. The paper shows that allowing autonomous agents both the reasoning about their compliance with a rule and the selection of the form of an institution helps to balance the trade-off between the autonomy of self-interested agents and the maintenance of social order in an open multiagent system and to ensure almost the same qualities as in closed environments.

In "Mapping Deontic Operators to Abductive Expectations", Alberti et al. propose a mapping of deontic operators (obligations, prohibition, permission) to language entities (expectations) available within the an agent framework developed for agent interaction in open agent societies. The mapping is supported by showing a similarity between the abductive semantics for expectations and the Kripke semantics that can be given to deontic 
operators.

In "A Normative Framework for Agent-Based Systems", López y López, Luck, and d'Inverno present a formal normative framework for agent-based systems that adresses two omissions of previous research on the use of norms in computational models of open societies to help to cope with the heterogeneity, the autonomy and the diversity of interests among their members. These are the lack of a canonical model of norms that facilitates their implementation and enables the description of the processes of reasoning about norms, and secondly the perspective of individual agents and what they might need to effectively reason about the society in which they participate.

\subsection{Combining normative systems}

Grossi et al. introduce the notion of contextual ontologies in their paper "Ontological Aspects of the Implementation of Norms in Agent-Based Electronic Institutions" and also provide a formal machinery to characterise this notion. This notion solves the problem of different institutions implementing the same set of norms in different ways presupposing divergent ontologies of the concepts in which that set of norms is formulated.

\section{References}

2005. Agent Technology Roadmap: A Roadmap for Agent-Based Computing.

Alchourròn, C.E. and E. Bulygin. 1971. Normative Systems. Springer.

Alexander, J.C., B. Giesen, R. Münch, and N.J. Smelser, editors. 1987. The Micro-Macro Link. University of California Press.

Boella, G. and L. van der Torre. 2006a. A game theoretic approach to contracts in multiagent systems. IEEE Trans. SMC, Part $C$. 
Boella, G. and L. van der Torre. 2006b. Security policies for sharing knowledge in virtual communities. IEEE Trans. SMC, Part A.

Bond, A. H. and L. Gasser. 1988. An Analysis of Problems and Research in DAI. In A. H. Bond and L. Gasser, editors, Readings in Distributed Artificial Intelligence, pages 3-35. Morgan Kaufmann.

Broersen, J., M. Dastani, J. Hulstijn, and L. van der Torre. 2002. Goal generation in the BOID architecture. Cognitive Science Quarterly, 2(3-4):428-447.

Conte, R., R. Falcone, and G. Sartor. 1999. Introduction: Agents and Norms: How to Fill the Gap? Artificial Intelligence and Law, pages $1-15$.

Conte, R. and N. Gilbert, 1995. "Computer Simulation for Social Theory". In R. Conte and N. Gilbert, editors, Artificial Societies: The Computer Simulation of Social Life, chapter Computer Simulation for Social Theory, pages 1 - 18. UCL Press.

Gibbs, J. P. 1965. Norms: The Problem of Definition and Classification. The American Journal of Sociology, 70(5):586 - 594 .

Jones, A. and J. Carmo. 2001. Deontic Logic and Contrary-to-Duties. In D. Gabbay, editor, Handbook of Philosophical Logic. Kluwer, page 203279.

Makinson, D. and L. van der Torre. 2000. Input-output logics. Journal of Philosophical Logic, 29:383-408.

Makinson, D. and L. van der Torre. 2001. Constraints for input-output logics. Journal of Philosophical Logic, 30(2):155-185.

Makinson, D. and L. van der Torre. 2003. Permissions from an input/output perspective. Journal of Philosophical Logic, 32(4):391-416.

Merriam-Webster OnLine. 2005. The Language Center. www.m-w.com/.

Meyer, J-J. and R. Wieringa, editors. 1993. Deontic Logic in Computer Science: Normative System Specification. Wiley. 
Ossowski, S. 1999. Co-ordination in Artificial Agent Societies. Springer.

Searle, J. R. 1995. The Construction of Social Reality. The Free Press.

Shoham, Y. and M. Tennenholtz. 1992. On the Synthesis of Useful Social Laws for Artificial Agent Societies (Preliminary Report). In Proceedings of the National Conference on Artificial Intelligence, pages 276-281, San Jose, CA.

Therborn, G. 2002. Back to Norms! On the Scope and Dynamics of Norms and Normative Action. Current Sociology, 50(6):863 - 880.

van der Torre, L. 2003. Contextual deontic logic: Normative agents, violations and independence. Annals of Mathematics and Artificial Intelligence, 37(1-2):33-63.

van der Torre, L. and Y. Tan. 1999. Contrary-to-duty reasoning with preference-based dyadic obligations. Annals of Mathematics and Artificial Intelligence, 27:49-78.

Verhagen, H. 2000. Norm Autonomous Agents. Ph.D. thesis, Department of System and Computer Sciences, The Royal Institute of Technology and Stockholm University, Sweden.

Verhagen, H. and R. Smit. 1996. Modelling Social Agents in a Multiagent World. In W. Van de Velde and J. W. Perram, editors, , Position Papers MAAMAW 1996, Technical Report 96-1. Vrije Universiteit Brussel - Artificial Intelligence Laboratory.

von Wright, G.H. 1951. Deontic logic. 60:1-15.

Wooldridge, M. 2002. An Introduction to MultiAgent Systems. Wiley.

\section{Bibliography}

\section{Guido Boella}

Guido Boella received the $\mathrm{PhD}$ degree at the University of Torino in 2000. He is currently professor at the Department of Computer Science of the University of Torino. His research interests include multi-agent systems, in particular, normative systems, institutions and 
roles using qualitative decision theory. He is the co-chair of the first workshops on normative multi-agent systems (NorMas05), on coordination and organization (CoOrg05), and the AAAI Fall Symposium on roles (Roles05).

\section{Leendert van der Torre}

Leendert van der Torre received the Ph.D. degree in computer science from Erasmus University Rotterdam, The Netherlands, in 1997. He is currently a Full Professor at the University of Luxembourg. He has developed the so-called input/output logics and the BOID agent architecture. His current research interests include deontic logic, qualitative game theory, and security and coordination in normative multiagent systems.

\section{Harko Verhagen}

Harko Verhagen received his Ph.D. degree in computer and systems sciences from Stockholm University (Sweden) in 2000 and is currently an associate professor at the department. His research has focussed on simulation of organizational behaviour, simulation as a scientific method, the use of sociological theories in multiagent systems research and more in particular theories on norms and autonomy. 


\title{
Security Policies for Sharing Knowledge in Virtual Communities
}

\author{
Guido Boella and Leendert van der Torre
}

\begin{abstract}
Knowledge management exploits the new opportunities of sharing knowledge among members of virtual communities in distributed computer networks, and knowledge-management systems are therefore modeled and designed as multiagent systems. In this paper, normative multiagent systems for secure knowledge management based on access-control policies are studied. It is shown how distributed access control is realized by means of local policies of access-control systems for documents of knowledge providers, and by means of global community policies regulating these local policies. Moreover, it is shown how such a virtual community of multiple knowledge providers respects the autonomy of the knowledge providers.
\end{abstract}

Index Terms-Knowledge management, multiagent systems, normative systems, policies, virtual communities.

\section{INTRODUCTION}

$\mathbf{M}$ ANY multiagent systems for knowledge management have been proposed. For example, KAoS[1] is a system for the management of technical information contained in documents, KRAFT [2] aims at fusing information from different sources, FRODO [3] represents distributed organizational memories, and MARS [4] is an adaptive social network for information access. Van Elst et al. [5] observe several reasons why multiagent systems are useful for knowledge management. The autonomy as well as proactiveness of agents help accommodating to the reality that knowledge workers typically adopt knowledge-management goals with a low priority. The flexibility to adapt to unforeseen situations of agents and multiagent systems helps knowledge management to deal with changing environments, such as the addition of new members to the knowledge-management system [6]. Moreover, agent technology provides tools and models to develop knowledgemanagement systems, for example, to incorporate legacy systems into modern distributed information systems.

However, there is no consensus on how multiagent systems can be used to deal with security considerations in knowledge management. In this paper, we address two requirements of secure distributed knowledge management.

1) Knowledge providers should not give up their autonomy to prohibit access to users they do not trust, even when they satisfy the security rules of the virtual community.

Manuscript revised January 9, 2006. This paper was recommended by Associate Editors H. R. Rao and S. J. Upadhyaya.

G. Boella is with the Dipartimento di Informatica, Università di Torino, Torino 10149, Italy (e-mail: guido@di.unito.it).

L. van der Torre is with the University of Luxembourg, Luxembourg L-1359, Luxembourg (e-mail: leendert@vandertorre.com).

Digital Object Identifier 10.1109/TSMCA.2006.871793
2) The policy rules for managing knowledge in a secure way concern not only which knowledge the users are prohibited or permitted to access, but also which regulations the community members are allowed or obliged to enforce.

Traditionally, multiagent systems are developed without security considerations in mind, by focussing on the coordination of cooperative agents. Likewise, knowledge-management systems are traditionally developed without any security considerations, for example, because the users of the systems know each other, share the same goal, and share a fixed set of administrative rules. When passing from knowledge management in teams to knowledge management in virtual communities, the flow of knowledge must be subject to restrictions [7], [8].

We introduce a model of policies for secure knowledge management in virtual communities satisfying the above requirements by modeling distributed knowledge management by means of normative multiagent systems (NMAS). Knowledge providers are modeled as normative systems creating their own norms. Global policies are enforced by detective rather than preventative control, such that global policies can be violated by knowledge providers if they believe this is necessary. Various types of agent types can be modeled to reflect ways in which agents can be motivated, such as norm internalizing agents, respectful agents, and selfish agents. Games among agents are used to analyze interaction in secure knowledge management.

We illustrate our model of secure knowledge management by highlighting an important subtlety involved in global policies regulating local policies. It is concerned with the notion of entitlement, which distinguishes between users that are only permitted to access knowledge, and users that are also entitled to it in the sense that knowledge providers are obliged to permit them access [9]. The additional issue discussed, formalized, and analyzed in this paper is that global policies do not only describe who is entitled to access resources, because the gametheoretic analysis shows that this is too weak to obtain the desired system behavior. Global policies do not only refer to the existence of local norms, but they have to refer also to the enforcement of the norms by the local provider recognizing and sanctioning violations.

This paper is organized as follows. In Section II, we discuss decentralized control in secure knowledge management. In Section III, we discuss our two requirements, which are detailed in Sections IV and V. In Sections VI and VII, we present the logical model, and in Section VIII, we apply it to an example of global policies concerned with entitlement. 


\section{Secure Knowledge Management}

Knowledge management is a management discipline taking advantage of new technologies like peer-to-peer, multiagent, and normative systems respecting the distributed nature of knowledge in organizations.

\section{A. Distributed Knowledge Management}

Van Elst et al. define knowledge management as "the systematic, holistic approach for sustainably improving the handling of knowledge on all levels of an organization (individual, group, organizational and inter-organizational level) in order to support the organization's business goals, such as innovation, quality, cost effectiveness, etc." [5].

Knowledge is distributed in organizations, since the division of labor in modern companies leads to a distribution of resources, and knowledge management has to respect the distributed nature of knowledge in organizations. Bonifacio et al. explain that knowledge is the result of different perspectives and partial interpretations of world portions or domains, called the subjectivity and sociality of knowledge, and therefore, it should not be viewed as an absolute monolithic matter, but as a "system of local 'knowledges' continuously negotiated by communities of 'knowers"' [10].

Van Elst et al. observe that the distributed nature of knowledge in organizations "is not a 'bug' but rather a feature which is not only a matter of physical or technical location of some file" [5]. For example, knowledge management typically resides in environments subject to frequent changes, either in the organizational structure, in business processes, or in the information-technology infrastructure, and centralized solutions are often ill suited to deal with continuous modification in the enterprise.

\section{B. Multiagent Systems}

Due to technological innovations knowledge-management systems are no longer limited to a single organization [10]. Van Elst et al. observe that "the role of information technology as an enabling factor is also widely recognized and ... a variety of proposals exist showing how to support knowledge management with specialized information systems" [5].

For example, Bonifacio et al. argue that a member of a distributed knowledge-management system can be seen "as a peer [of a peer-to-peer system] that manages and has control over a set of local technologies, applications and services. ... In order to join the system, every member must provide resources or services to the others" [11].

As another example, Tacla and Barthes develop a multiagentsystem architecture for knowledge management, because "knowledge is contained in the information produced, retrieved and exchanged among members of the projects," which results in "a distributed group memory where each member keeps part of the knowledge of the team." They introduce a multiagent-system architecture for knowledge-management systems, because, "like in a team, a multiagent system is composed by a group of possibly heterogeneous and autonomous agents" [12].

\section{Secure Knowledge Management}

Secure knowledge management is an important issue, because an organization's knowledge is easy to view, steal, manipulate, and delete. For example, Mundy and Chadwick discuss the need of secure knowledge management in the health-care industry. As this industry "enters the era of knowledge management it must place security at the foundation of the transition. Risks are pervasive to every aspect of information and knowledge management. ... In an age where risks and security threats are ever-increasing, secure knowledge management is an essential business practice" [13].

The national information systems security glossary (NSTISSI 4009) distinguishes the following five components of secure knowledge management. Authentication is a security measure to establish the validity of a message or verifying the eligibility of an individual to receive information. Authorization is the set of rights granted to a user to access, read, insert, or delete data. Data security or privacy is the protection from unauthorized disclosure. Data integrity is the requirement that data are unchanged from their source. Information security policy is the set of organizational guidelines, rules, regulations, and procedures that are used to protect an organization. It emphasizes that all components are essential and mutually supportive. For example, authentication without authorization would mean that only valid users could gain access but could execute any operation.

It also observes that, without an information security policy, there would be no apparent requirement for secure practices, and security would either be ignored or implemented in an ad hoc manner, and thus, control could be either too rigid or completely missing.

\section{Normative Multiagent Systems and Access Control}

Secure knowledge management takes advantage of new technologies like normative multiagent systems, such as access-control techniques, languages, and models for policy requirements, etc. Normative multiagent systems can be used as a software engineering method to develop secure knowledgemanagement systems, both providing high-level specifications as well as system realizations. Moreover, normative multiagentsystem models can be used to analyze the system, or simulate solutions before they are built.

The NSTISSI acknowledges the importance of access control, and mentions various models. Discretionary access control can be viewed as an owner-based administration of users' rights, where the owner has authority over which other users can access the object. Discretionary access control has some limitations, in particular, in the way the owner can delegate his discretionary power to other people. Mandatory access control is based on security labels given to objects (security classification) and users (security clearance). Role-based access control has been developed to enhance maintenance and scalability. Permissions are granted to roles that are assigned to users. A security officer may have the right to assign roles to users without having an access right to the resources. 


\section{Two Requirements And Some Consequences}

In this section, we discuss two requirements for the development of an integrated approach to distributed knowledge management and security, which we use to motivate and evaluate our multiagent model for secure knowledge management.

\section{A. Autonomy of Local Knowledge Providers}

A multiinstitutional or multiorganizational set of agents is a virtual community [14] if they use a set of rules, a policy, to specify how to share their resources, like documents and knowledge. A virtual community can be modeled as a system containing role-playing agents. Every agent in the community can play both the role of a document user as well as that of a document provider, because agents do not only use documents, but they also put the documents they own at disposal to the other participants of the community. Document providers retain the control of their documents and they specify in local policies the conditions of use of their documents.

The knowledge providers prefer not to give up their own power to enforce local policies for the access to the documents they control. For example, as Sadighi Firozabadi et al. [15] argue, there are "cases where security administrators are not fully trustworthy," for example, when multinational virtual communities are headed by foreign countries with varying security standards. The agents participating in the community are heterogeneous and change frequently, so they cannot be assumed to be always cooperative and to act in accordance to the system policies, both when requesting access to documents and when providing access to their documents. Decentralized authorities can cope in a better way with local idiosyncratic situations: "each party of the network can decide in each circumstance whether to accept credentials presented by a second party" [16]. Bonifacio et al. call it the principle of autonomy: "each community has a high degree of autonomy to manage its local knowledge" [10].

\section{B. Local and Global Security Policies}

However, the need of leaving autonomy to the knowledge providers must be balanced with the requirement that their local access policies should be organized according to a global policy defining how the knowledge should be shared among participants. The distinction between local and global policies is analogous to the distinction between local and global knowledge. As van Elst et al. [5] observe, "departments, groups and individual experts develop their particular views on a given subject. These views are motivated and justified by the particularities of the actual work, goals and situation. Obtaining a single, globally agreed upon vocabulary (or ontologies) within a level of detail which is sufficient for all participants, may incur high costs (e.g., for negotiation)" [5]. In virtual communities, global access control cannot be directly implemented, since nobody owns all the documents. There is no central manager of a system permitting agents to access the resources it owns and controls, according to the policies defined by itself. Such a centralized administration could be a too heavy burden and it can affect the core business activities of the system.
The centralized management of resources owned by the single resource providers is only partially performed by a community agent [7], which is in charge for maintaining the list of registered members and offering brokerage services for available information sources, but not for policing accesses to resources. The global policies are issued and enforced, for example, by a community authorization service (CAS): "A community runs a CAS server to keep track of its membership and fine-grained access-control policies. A user wishing to access community resources contacts the CAS server, which delegates rights to the user based on the request and the user's role within the community. These rights are in the form of capabilities which users can present at a resource to gain access on behalf of the community" [14].

Representations of local access-control policies can be obtained from existing theories of access control. Therefore, as observed by Pearlman et al. [14], "a key problem associated with the formation and operation of distributed virtual communities is that of how to specify and enforce community policies." In the remainder of this paper, this key problem is discussed and analyzed in our model of normative multiagent systems, which contains various incentives to motivate knowledge providers.

\section{Incentives for Knowledge Providers}

Since knowledge providers are on the one hand autonomous, but on the other hand cannot be coerced to provide their services or to deny them to users, it is necessary that these local providers are provided with incentives to implement the global policies by means of local ones. In other words, knowledge providers must be motivated by rewards and sanctions, such as the exclusion from the community. Moreover, there also has to be a monitoring system detecting violations and enforcing sanctions.

Sanction-based control implies also that an agent may influence negatively the behavior of other agents. In the terminology of Sichman et al. [17], other agents depend on it. In our model, this dependence is the essential precondition for the ability to issue policies. In this sense, the control of resources does not mean only that a given service is not provided if the provider does not want to, for example, the files of a web server cannot be accessed if it does not provide an answer to a request. Sanction-based control implies, for example, that agents depend on the global authority for their membership of the community. If knowledge providers do not behave according to its policies, then they are denied citizenship. At the local level, agents depend on the provider for the current and future access to the local resource.

Summarizing, we have to deal with the autonomy of local providers by modeling the security policies of the system, but also to limit the autonomy of agents, defining their responsibilities and roles. In particular, the respect of norms must be motivated in two distinct ways. Knowledge users must be provided with an incentive to respect the norms, and local knowledge providers must be motivated to issue policies respecting the global ones, and to enforce them. 


\section{Normative Multiagent Systems}

To deal with the requirement that local knowledge providers are autonomous, we model each knowledge provider as a normative system. We thus say that an agent is autonomous in the literal sense of "making its own norms." Consequently, in a virtual community, we have to deal with the interaction among normative systems.

\section{A. Norms and Control}

The role of norms in local and global policies is a major aspect of secure knowledge-management systems. We model a secure knowledge-management system as a normative multiagent system, which are "sets of agents ... whose interactions can be regarded as norm-governed; the norms prescribe how the agents ideally should and should not behave. ... Importantly, the norms allow for the possibility that actual behavior may at times deviate from the ideal, i.e., that violations of obligations, or of agents' rights, may occur" [18], where the norms are used to address security concerns of knowledgemanagement systems. Note that the notion of a normative system regulating an agent society has also fruitfully been employed elsewhere, such as electronic commerce, theories of fraud and deception, of trust dynamics and reputation, etc. [19].

Since there is no plausible way to enforce the respect of global policies by constraining the architecture, it is necessary to have a normative control mechanism to specify global policies about local policies. Normative systems contain control procedures, which are policies and procedures that help to ensure that management directives are carried out [20], because, intentionally or not, an agent may fail to comply with the policy. Moreover, the norms of global policies must be represented as soft constraints, which are used in detective control systems where violations can be detected, instead of hard constraints restricted to preventative control systems, in which violations are impossible [21]. A typical example of the former is that you can enter a train without a ticket, but you may be checked and sanctioned, and an example of the latter is that you cannot enter a metro station without a ticket. Detective control is the result of actions of agents and therefore subject to errors and influenceable by actions of other agents.

\section{B. Secure Agent Interaction}

The conceptual machinery of agents, obligations, norms, control, roles, policies, organizations, contracts, etc., offered by normative multiagent systems, is used to describe secure interaction among humans and systems. Agents must reason about the fulfillment of norms, the possible violations, and also what to do to repair such violations. A crucial point in secure knowledge management is the careful planning of moves during access control. Accordingly, agents must evaluate the effects of accessing documents both when making and evaluating an access request. In particular, since the compliance of the providers to the policies cannot be taken for granted, a user must consider whether they will fulfill their commit- ments or not. To make a prediction about the behavior of a local provider, it is necessary to consider also the reaction of the global authority enforcing control by monitoring and sanctioning violations.

\section{Game-Theoretic Approach to Norms}

Boella and Lesmo [22] analyze the motivational aspects of norms in the context of multiagent systems composed of heterogeneous agents, on the assumption that norms are useless unless supported by sanctions. They argue that sanctions must be modeled as actions of the normative system, since it is not possible to presuppose that they are mere consequences of violations. Hence, Boella and Lesmo attribute to the normative system the status of an agent deciding whether the behavior of agents counts as a violation, and thus deserves to be sanctioned by it.

Boella and Lesmo's model of agent interaction in normative systems is based on the philosophical foundations on strategic interaction in the work of sociologist Goffman [23]. "Strategic interaction" here means, according to Goffman, taking into consideration the actions of other agents. Boella and Lesmo call it the game-theoretic approach to norms.

Inspired by Boella and Lesmo's game-theoretic approach to norms, in earlier work [19], we propose a logical framework for reasoning about obligations and norms. It does not use a preventative control system, because agents are not constrained to respect norms. They can decide whether to respect norms or not based on a rational balance between the advantage of not respecting a norm and the disadvantage of being sanctioned. We use goal-based theories developed in artificial intelligence [24] and agent theory replacing probabilities and utilities by informational (knowledge, belief) and motivational attitudes (goal, desire), respectively, and the decision rule by a process of deliberation, in particular, based on the belief-obligation-intention-desire architecture (BOID) [25]. We replace the equilibria analysis in classical game theory by Gmytrasiewitcz and Durfee's recursive modeling [26].

\section{New Challenges for Our Game-Theoretic Model}

Distributed access control poses new challenges to the gametheoretic model of normative systems presented in [19]. The model must be extended with priorities among beliefs to model the defeasible effects of actions, with permissions as exceptions to model access rights, and, most importantly, with global policies regulating local ones. In [19], we consider agents modeling the contract partner recursively modeling the normative system. In distributed access control, however, there is no longer a single normative system, but each knowledge provider acts as one. In our games, we therefore have to deal with the interaction among normative systems. Agents recursively model the local provider, which in turn recursively model the global authority. Consequently, the relation between the local and global level, and thus the relation between local and global policies, plays a central role in the interaction among normative systems. 


\section{Global Policies Regulating Local Policies}

To deal with the requirement to model global policies regulating local ones, we consider the rationale of global policies, called the transmission of will.

\section{A. Transmission of Will}

According to von Wright's principle of transmission of will, "an authority who orders that something be made obligatory wants the obligation satisfied. He, as it were, 'transmits' his will through the intermediary of a lower authority. Therefore, his will is not fulfilled unless the norms which are its immediate objects are themselves satisfied" [27, p.93].

For example, consider the notion of entitlement. Sadighi Firozabadi and Sergot [9] define entitlement to a resource by the obligation of a resource provider to permit access, and distinguish it from a mere permission of an agent to access the resource. The following scenario considers entitlement from the perspective of a local knowledge provider. An agent $p$ has joined a virtual community $n$. Its contract for the participation prescribes that it should provide access to all the members of the community. Another participating agent, say agent $a$, tries to access agent $p$ 's system. However, previous experiences before joining the community advice agent $p$ that agent $a$ could damage its resources. Should agent $p$ grant agent $a$ access to its resources?

The fact that the agent $a$ is entitled to access the resource, in the sense that the knowledge provider is obliged to permit him access, is not enough to ensure that he is given access to the resource, and therefore is not enough for the transmission of will. Global policies therefore do not refer only to the fact that a local norm exists, but also to the fact that the local provider enforces it by recognizing and sanctioning violations.

\section{B. Policies for the Transmission of Will}

In the above scenario for entitlement, the management of the community is organized in at least two levels: the global level (agent $n$ ) and the local one (agent $p$ ). Agent $n$ is a distinguished authority, like the community authorization service playing the role of a global authority issuing global policies and negotiating the conditions for the participation of agents to the virtual community. Agent $p$ is a provider of a document it controls. Moreover, all the agents $(n, p$, and $a$ ) can also play the role of users of the resources of the community.

Policies concern the behavior of participants. For example, at the global level, participants should not communicate their passwords, or distribute copyrighted files by means of the system. Otherwise, they are banned from the community, since the membership to the system is under the control of the global authority. At the local level, policies forbid agents to store files exceeding $1 \mathrm{~GB}$ on a file-sharing service, or they permit participants of the community to download copyrighted files from the web server.

Moreover, there are policies that apply to other policies, such as global policies that constrain or permit local policies [28]. In our scenario, agent $n$ obliges agent $p$ to permit members of the community to access its resources. Analogously, the global authority could oblige local ones to forbid access, permitto-permit access, or permit-to-forbid access. However, it is not sufficient that the global obligation to permit or oblige access is satisfied by the fact that the local provider issues a permission or an obligation. Norms are ineffective if they are not enforced by the normative system who issued them: violations of norms should be recognized as such and sanctioned.

Reconsider the notion of entitlement. An agent $n$ obliges agent $p$ to permit agent $a$ to do $x$ if agent $n$ obliges agent $p$ not to consider $\neg x$ as a violation. The local normative system, however, can still violate this global policy and prevent access to users if it prefers to face the sanction with respect to permitting access. It is possible that agent $p$ does not grant agent $a$ the resource it is entitled to by the global policy, and this may be considered rational. Facing a sanction by the global authority, e.g., being excluded by the community for a certain period of time, is preferred to the possibility that agent $a$ damages the system, e.g., agent $a$ could create a denial-of-service attack. Thus, the autonomy of the provider is guaranteed, even if a provider violating a global policy must take the consequences of its actions into account. The example is formalized in Section VIII.

\section{Transmission of Will in Our Game-Theoretic Model}

In our model, obligations are defined in terms of goals of a normative system. The attribution of goals and beliefs to normative systems is an instance of Dennett's intentional stance [29]: Agents behave as if they are endowed with such motivational attitudes. A global obligation by agent $n$ that agent $p$ obliges agent $a$ to do $x$ implies an obligation that agent $p$ considers $\neg x$ as a violation and sanctions it. Since, in turn, the obligation of agent $n$ is expressed in terms of goals that something counts as a violation, the global obligation by agent $n$ is defined as the goal that agent $p$ considers $\neg x$ as a violation and the goal that if $p$ does not do so, then its behavior is considered a violation by agent $n$. Analogously, a permission by agent $n$ that $p$ obliges that agent $a$ does $x$ is expressed as a permission by agent $n$ to consider $\neg x$ as a violation: Agent $n$ has the goal that agent $p$ is not considered a violator by agent $n$ if it considers agent $a$ as a violator.

Our game-theoretic model highlights two important properties of the transmission of will. First, our game-theoretic model can be used to show that it is not sufficient that global norms refer only to the existence of local norms, by illustrating scenarios in which this is the case, but the local norm is not enforced.

Second, it gives an additional argument supporting the reduction of policies about policies to obligations and permissions about considering something as a violation or not. The agent does not have to be implemented in terms of explicit beliefs and goals. The basis for judging an agent cannot be its implementation, but the basis can be only its behavior. Analogously, the basis to say that an obligation is satisfied cannot be based on whether a knowledge provider has or does not have a goal. The only clue we have is its behavior, and in particular, whether it sanctions or not. 


\section{LOGICAL FRAMEWORK}

In this section, we present a simplified version of our logical model of normative multiagent systems introduced in [19]. We do not discuss constitutive norms, but we add priorities among beliefs, and undercutters to goals. In the following section, we discuss the extended games for modeling global policies regulating local ones.

\section{A. Multiagent Systems}

We first introduce the structural concepts and their relations. A set of propositional variables $X$ describes the aspects of the world, and we extend it to literals built out of $X$ $(\operatorname{Lit}(X))$ to consider also the absence of states of affairs. Rules built out of the literals $(\operatorname{Rul}(X))$ describe the relations among the propositional variables. A rule $l_{1} \wedge \ldots \wedge l_{n} \rightarrow l$ is a pair of a set of literals built from $X$ and a literal built from $X$ : Rules represent the relations among literals existing in the agent's mental attitudes.

Definition 1: Let $X$ be a set of propositional variables. The set of literals built from $X, \operatorname{Lit}(X)$ is $X \cup\{\neg x \mid x \in X\}$, and the set of rules built from $X$, written as $\operatorname{Rul}(X)$, is defined by $2^{\operatorname{Lit}(X)} \times \operatorname{Lit}(X)$, the set of pairs of a set of literals built from $X$ and a literal built from $X$. A rule is written as $\left\{l_{1}, \ldots, l_{n}\right\} \rightarrow l$; we also write $l_{1} \wedge \ldots \wedge l_{n} \rightarrow l$, and when $n=0$, we write $\top \rightarrow l$. Moreover, for $x \in X$, we write $\sim x$ for $\neg x$ and $\sim \neg x$ for $x$.

The mental attitudes attributed to agents consist of beliefs $B$, desires $D$, goals $G$, and undercutters $H$. An undercutter is a mental attitude expressing the absence of a desire or goal, which we use in the following section to model permissions as exceptions [30]. A mental description function $M D$ associates a rule in $\operatorname{Rul}(X)$ with each belief, desire, goal, and undercutter. We introduce priority relations to resolve conflicts among mental attitudes. A function $\geq$ associates with an agent a transitive and reflexive relation on the powerset of the motivations and beliefs containing at least the subset relation. Moreover, various mental attitudes are attributed to agents by the agent description relation $A D$. It associates with each agent a set of beliefs, desires, goals, and undercutters.

Multiagent systems also contain concepts concerning informational aspects. First of all, the set of variables whose truth value is determined by an agent (decision variables) are distinguished from those which are not directly determined by the agent $(P$, the parameters using the terminology of Lang et al. [31]). Concerning the relations among these concepts, we have that parameters $P$ are a subset of the propositional variables $X$. The complement of $P$ represents the decision variables controlled by the agents. Hence, we associate to each agent a subset of $X \backslash P$ by extending the agent description $A D$.

Definition $2(M A S)$ : A multiagent system $(M A S)$ is a tuple $\langle A, X, B, D, G, H, A D, M D, \geq\rangle$, where:

1) The agents $A$, propositional variables $X$, beliefs $B$, desires $D$, goals $G$, and undercutters $H$ are six finite disjoint sets. We write $M=D \cup G$ for motivations.

2) An agent description $A D: A \rightarrow 2^{X \cup B \cup D \cup G \cup H}$ is a complete function that maps each agent to a set of variables (its decision variables), and to its beliefs, desires, goals, and undercutters. For each agent $a \in A$, we write $X_{a}$ for $X \cap A D(a), B_{a}$ for $B \cap A D(a)$, etc. We write also $P=X \backslash \cup_{a \in A} X_{a}$ for the parameters.

3) A mental description $M D: B \cup D \cup G \cup H \rightarrow \operatorname{Rul}(X)$ is a complete function from the sets of beliefs, desires, goals, and undercutters to the set of rules built from $X$. For $S \subseteq B \cup D \cup G \cup H$, we write also $M D(S)=\{M D(s) \mid s \in S\}$. Moreover, we write $s x \rightarrow y$ for denoting: $s$ such that $M D(s)=x \rightarrow y$.

4) A priority relation $\geq: A \rightarrow\left(\left(2^{B} \times 2^{B}\right) \cup\left(2^{M} \times 2^{M}\right)\right)$ is a function from agents to a transitive and reflexive relation on the powerset of the mental attitudes containing at least the subset relation. We write $\geq_{a}$ for $\geq(a)$.

Example 1 illustrates the running example as a multiagent system. In conceptual models used in practice as well as in the more detailed examples in the following sections, we use meaningful names; here, we use single letters to save space.

Example 1: Let $M A S=\langle A, X, B, D, G, H, A D, M D, \geq\rangle$ with $A=\{\mathbf{a}, \mathbf{p}, \mathbf{n}\}, P=\{q, r\}, H=\emptyset$, and $X \backslash P, B, D, G$, $A D, M D$, and $\geq$ are given by the following table:

\begin{tabular}{|c|c|c|c|c|c|c|}
\hline & \multicolumn{2}{|r|}{ a } & \multicolumn{2}{|r|}{$\mathrm{p}$} & \multicolumn{2}{|r|}{$\mathbf{n}$} \\
\hline $\bar{X}$ & & $x_{1}, x_{2}$ & & $x_{3}, x_{4}$ & & $x_{5}$ \\
\hline$B$ & $b_{1}$ & $x_{1} \rightarrow q$ & $b_{2}$ & $x_{1} \rightarrow q$ & & \\
\hline$B$ & $b_{3}$ & $x_{3} \rightarrow r$ & $b_{4}$ & $x_{3} \rightarrow r$ & & \\
\hline$B$ & $b_{5}$ & $x_{4} \rightarrow \neg q$ & $b_{6}$ & $x_{4} \rightarrow \neg q$ & & \\
\hline$B$ & $b_{7}$ & $x_{2} \rightarrow \neg r$ & $b_{8}$ & $x_{2} \rightarrow \neg r$ & & \\
\hline$D$ & $d_{1}$ & $\top \rightarrow q$ & $d_{2}$ & $\top \rightarrow r$ & & \\
\hline$D$ & $d_{3}$ & $\top \rightarrow \neg x_{4}$ & $d_{4}$ & $\top \rightarrow \neg x_{2}$ & & \\
\hline$D$ & & & $d_{5}$ & $\top \rightarrow \neg x_{5}$ & & \\
\hline$G$ & & $x_{3} \rightarrow x_{2}$ & $g_{2}$ & $x_{1} \rightarrow x_{4}$ & $g_{3}$ & $x_{1} \wedge x_{4} \rightarrow x_{5}$ \\
\hline $\begin{array}{l}\geq \\
\geq\end{array}$ & $g_{1}$ & $\begin{array}{l}>d_{3}>d_{1} \\
b_{5}>b_{1} \\
b_{7}>b_{3}\end{array}$ & & $\begin{aligned} & d_{5}>d_{4}>d_{2} \\
& b_{6}>b_{2} \\
& b_{8}>b_{4}\end{aligned}$ & & $g_{3}>g_{1}=g_{2}$ \\
\hline
\end{tabular}

Agent a desires $\left(d_{1}\right)$ to get information $q$ to increase its knowledge by doing $x_{1}\left(b_{1}\right)$, i.e., by making a request to agent $\mathbf{p}$. Vice versa, agent $\mathbf{p}$ desires $\left(d_{2}\right)$ to know $r$ by requesting it to agent $\mathbf{a}$ with action $x_{3}$. Therefore, both agents $\mathbf{a}$ and $\mathbf{p}$ share resources and request them. Moreover, they want to react to the request of the other one by respectively doing actions $x_{2}$ and $x_{4}\left(g_{1}\right.$ and $\left.g_{2}\right)$, which are not desired by the other agent $\left(d_{3}\right.$ and $d_{4}$ ). These actions are exceptions to the effect of, respectively, requests $x_{1}$ and $x_{3}$, and thus, they prevent their results ( $q$ and $r$ ). Agent $\mathbf{n}$ is monitoring the behavior of agent $\mathbf{p}$ and, if agent $\mathbf{p}$ achieves its goal $x_{4}$ when $x_{1}$ is true, then it wants $\left(g_{3}\right)$ to perform action $x_{5}$, which is disliked by agent $\mathbf{p}\left(d_{5}\right)$.

Finally, only a fragment of the priority relation is given, because it is only given for singleton motivations and beliefs, whereas it is defined over sets of motivations.

The example already illustrates a drawback of using only multiagent systems to describe the example, because there is no notion of obligation, violation, or sanction. We therefore introduce normative multiagent systems.

\section{B. Normative Multiagent Systems}

A normative multiagent system contains a norm (violation) description $V$, a function from agents and literals to the decision variables of the normative system together with the parameters. We write $V_{a}(x)$ for the decision variable representing that there is a violation $x \in \operatorname{Lit}\left(X_{a} \cup P\right)$ by agent $a \in A$. 
Definition 3: A normative multiagent system NMAS is a tuple $\langle A, X, B, D, G, H, A D, M D, \geq, V\rangle$ that extends a multiagent system with a partial (violation) function $V: A \times \operatorname{Lit}(X) \rightarrow X \backslash P$ from agents and literals to decision variables. We write $V_{a}(x)$ for $V(a, x)$.

We can introduce decision variables like $V_{b}\left(V_{a}(x)\right)$, where agent $b$ is considered as a violator if it considers $x \in \operatorname{Lit}(X)$ as a violation done by agent $a$. Analogously, $V_{b}\left(\neg V_{a}(x)\right)$ means that agent $b$ is considered as a violator, because agent $b$ does not consider $x$ as a violation done by agent $a$.

Example 2 (Continued): The agents $\mathbf{a}, \mathbf{p}, \mathbf{n}$ in Example 1 are now interpreted as a virtual community where $\mathbf{a}$ is considered only a user of the system, and $\mathbf{p}$ only as a local provider owning a document, and $\mathbf{n}$ is a global authority. Assume that $V$ is defined as: $V_{\mathbf{a}}\left(x_{1}\right)=x_{4}, V_{\mathbf{p}}\left(x_{4}\right)=V_{\mathbf{p}}\left(V_{\mathbf{a}}(q)\right)=x_{5}$, and $V_{c}(y)=$ undefined for all other values of $c \in A$ and $y \in \operatorname{Lit}(X)$. This means that if $x_{4}$ is the case, then $x_{1}$ is recognized as a violation of agent $\mathbf{a}$, and if $x_{5}$ is the case, then doing $V_{\mathbf{a}}\left(x_{1}\right)$ is recognized as a violation of agent $\mathbf{p}$.

\section{Obligation}

The definition of obligation contains several clauses. The first clause says that the obligation is in the desires and in the goals of a normative system $b$ ("your wish is my command"). The second and third clauses can be read as "the absence of $\sim x$ is considered as a violation." The association of obligations with violations is inspired by Anderson's reduction of deontic logic to alethic modal logic [32]. The third clause says that the normative system desires that there are no violations. The fourth and fifth clauses relate violations to sanctions and assume that normative system $b$ is motivated to apply sanctions only as long as there is a violation; otherwise, the norm would have no effect. Finally, for the same reason, we assume in the last clause that the agent does not like the sanction.

Definition 4 (Obligation): Let a normative multiagent system $N M A S$ be $\langle A, X, B, D, G, H, A D, M D, \geq, V\rangle$. Agent $a \in A$ is obliged in NMAS to decide to do $x \in \operatorname{Lit}\left(X_{a} \cup P\right)$ with sanction $s \in \operatorname{Lit}\left(X_{b} \cup P\right)$ if $Y \subseteq \operatorname{Lit}(X)$ by normative system $b \in A$, written as $N M A S=O_{a, b}(x, s \mid Y)$, if and only if the following conditions are met.

1) $Y \rightarrow x \in M D\left(D_{b}\right) \cap M D\left(G_{b}\right)$ : If normative system $b$ believes $Y$, then it desires $x$ and has $x$ as a goal.

2) $Y \cup\{\sim x\} \rightarrow V_{a}(\sim x) \in M D\left(D_{b}\right) \cap M D\left(G_{b}\right)$ : If normative system $b$ believes $Y$ and $\sim x$, then it has the goal and the desire $V_{a}(\sim x)$ : to recognize $\sim x$ as a violation by agent $a$.

3) $\top \rightarrow \neg V_{a}(\sim x) \in M D\left(D_{b}\right)$ : Normative system $b$ desires that there are no violations.

4) $Y \cup\left\{V_{a}(\sim x)\right\} \rightarrow s \in M D\left(D_{b}\right) \cap M D\left(G_{b}\right)$ : if normative system $b$ believes $Y$ and decides $V_{a}(\sim x)$, then it desires and has as a goal that it sanctions agent $a$ with $s$.

5) $Y \rightarrow \sim s \in M D\left(D_{b}\right)$ : If normative system $b$ believes $Y$, then it desires not to sanction, $\sim s$. The normative system only sanctions in case of violation.

6) $Y \rightarrow \sim s \in M D\left(D_{a}\right)$ : If agent $a$ believes $Y$, then it desires $\sim s$, which expresses that it does not like to be sanctioned.

\section{Games With Global Policies}

We first introduce documents and permissions, then we define global policies, and finally, we introduce the extended game theory.

\section{A. Documents}

We introduce some syntactic sugar. Management of knowledge in the multiagent system is represented by access of documents $D C$. We are inspired by Lee [33]: "we use the term 'document' since most information parcels in business practice are mapped on paper documents." Of course, knowledge is distinct from information. In the context of knowledge management, knowledge is described as information that has a use or purpose. Whereas information can be placed onto a computer, knowledge is emergent and socially constructed, in the sense that it exists in the heads of people: Knowledge is information to which an intent has been attached. The distinction between the two can be reflected by the internal structure of the documents, and how agents update their mental states once they receive documents. For example, receiving information only updates the agent's beliefs, whereas receiving knowledge can extend the agent's capabilities. In this paper, we do not consider the internal structure of knowledge documents, but we focus on operations agents can perform on documents.

Definition 5 (Documents): Let $D C$ be a set of documents. Let $D A_{a}=\{f(a, d) \mid d \in D C\}$ be a set of actions of the agent $a \in A . f(a, d) \in D A_{a}$ can belong to the decision variables $X_{a}$ or it can be a parameter such that there exists a decision variable $x \in X_{a}$ such that $x \rightarrow f(a, d) \in B_{a}: f(a, d)$ is an effect of a decision variable $x$ of agent $a$ representing agent $a$ 's beliefs that $x$ is a fallible tentative of accessing document $d$.

The following example illustrates that similar syntactic sugar is also used for the description of the state of the world.

Example 3 (Continued): Possible actions on the documents are the create, read, update, and delete (CRUD) actions of the CRUD security model in databases: e.g., $x_{1}=\operatorname{read}(\mathbf{a}, d)$ and $q=\operatorname{info}(\mathbf{a})$. To have an information info(a), agent a believes it has to perform action $\operatorname{read}(\mathbf{a}, d)\left(b_{1}\right)$ as an attempt to get it.

$N M A S \models O_{\text {ap }}(\neg \operatorname{read}(\mathbf{a}, d), \operatorname{san} \mid \top)$, agent a is obliged not to read document $d \in D C\left(\operatorname{read}(\mathbf{a}, d) \in X_{\mathbf{a}}\right)$, or else it is sanctioned with $\operatorname{san} \in \operatorname{Lit}\left(X_{\mathbf{p}} \cup P\right)$, if

$$
\begin{array}{ccl}
g_{4} & d_{6} & \top \rightarrow \neg \operatorname{read}(\mathbf{a}, d) \in G_{\mathbf{p}}, D_{\mathbf{p}} \\
g_{2} & d_{7} & \operatorname{read}(\mathbf{a}, d) \rightarrow V_{\mathbf{a}}(\operatorname{read}(\mathbf{a}, d)) \in G_{\mathbf{p}}, D_{\mathbf{p}} \\
& d_{8} & \top \rightarrow \neg V_{\mathbf{a}}(\operatorname{read}(\mathbf{a}, d)) \in D_{\mathbf{p}} \\
g_{5} & d_{9} & V_{\mathbf{a}}(\operatorname{read}(\mathbf{a}, d)) \rightarrow \operatorname{san} \in G_{\mathbf{p}}, D_{\mathbf{p}} \\
& d_{10} & \top \rightarrow \neg \operatorname{san} \in D_{\mathbf{p}} \\
& d_{3} & \top \rightarrow \neg \operatorname{san} \in D_{\mathbf{a}}
\end{array}
$$

\section{B. Conditional Permission}

To model permission, we consider clauses analogous to the ones for obligation. Since most clauses have to do with sanctions, we have to consider only clauses analogous to the first two clauses of obligation. Whereas obligation corresponds to a kind of normative goal in our model, permission corresponds 
to a kind of undercutter to a normative goal. Moreover, whereas obligations imply, under some conditions, a violation, permissions may imply the absence of a violation.

Definition 6 (Permission): Let a normative multiagent system $N M A S$ be $\langle A, X, B, D, G, H, A D, M D, \geq, V\rangle$. Agent $a \in A$ is permitted to decide to do $x \in \operatorname{Lit}\left(X_{a} \cup P\right)$ if $Y \subseteq \operatorname{Lit}(X)$ in $N M A S$ by normative system $b \in A$, written as $N M A S \models P_{a, b}(x \mid Y)$, if and only if

1) $Y \rightarrow x \in M D\left(H_{b}\right)$ : If normative system $b$ believes $Y$, then it does not have a desire or goal $x$.

2) $Y \cup\{x\} \rightarrow \neg V_{a}(x) \in M D\left(D_{b}\right) \cap M D\left(G_{b}\right)$ : If normative system $b$ believes $Y$ and $x$, then it does not want to count $x$ as a violation.

\section{Policies}

Consider now the notion of entitlement: An agent is obliged to permit another agent. If we would add nested obligations and permissions as in standard modal logic, then an obligation to permit $O_{b, c}\left(P_{a, b}(x \mid Y), s \mid W\right)$ could be represented by $O_{b, c}(\Psi, s \mid W)$, where $\Psi$ is, respectively, $Y \rightarrow x \in M D\left(H_{b}\right)$ and $Y \wedge x \rightarrow \neg V_{a}(x) \in M D\left(G_{b}\right)$. Note that in the latter formula, as well as the other formulas in the subsection, we write conjunction $\wedge$ for the union $\cup$ to facilitate the reading of these formulas. This reduces to 12 clauses, since each of the two obligations is made of the following six clauses:

1) $W \rightarrow \Psi \in M D\left(D_{c}\right) \cap M D\left(G_{c}\right)$;

2) $W \wedge \neg \Psi \rightarrow V_{b}(\neg \Psi) \in M D\left(D_{c}\right) \cap M D\left(G_{c}\right)$;

3) $\top \rightarrow \neg V_{b}(\neg \Psi) \in M D\left(D_{c}\right)$;

4) $W \wedge V_{b}(\neg \Psi) \rightarrow s \in M D\left(D_{c}\right) \cap M D\left(G_{c}\right)$;

5) $W \rightarrow \sim s \in M D\left(D_{c}\right)$;

6) $W \rightarrow \sim s \in M D\left(D_{b}\right)$.

Our reduction, instead, claims that, for global policies, a nested modality is too weak, and we therefore remove the inner modalities. For example, the second clause reduces to $O_{b, c}\left(\neg V_{a}(x), s \mid Y \wedge W \wedge x\right)$. This removal, however, does not have to lead to something making sense. For example, for the first clause $O_{b, c}(x, s \mid Y \wedge W)$ is too strong, as $x$ is not obligatory, but only permitted. Thus, we define the policy concerned with obligation to permit as only the second clause.

Definition 7: A global policy of global authority $c \in A$ in context $W \subseteq \operatorname{Lit}(X)$ with sanction $s \in \operatorname{Lit}\left(X_{c} \cup P\right)$ for the entitlement of agent $a \in A$ to $x \in \operatorname{Lit}\left(X_{a} \cup P\right)$ from knowledge provider $b \in A$ if $Y \subseteq \operatorname{Lit}(X)$ is

$$
O_{b, c}\left(\neg V_{a}(x), s \mid Y \wedge W \wedge x\right) .
$$

Consequently, the global policy implies the following six clauses, which illustrate that removal of nested obligations and permissions has left a nested violation predicate:

1) $W \wedge Y \wedge x \rightarrow \neg V_{a}(x) \in M D\left(D_{c}\right) \cap M D\left(G_{c}\right)$;

2) $W \wedge Y \wedge x \wedge V_{a}(x) \rightarrow V_{b}\left(V_{a}(x)\right) \in M D\left(D_{c}\right) \cap M D\left(G_{c}\right)$;

3) $\top \rightarrow \neg V_{b}\left(V_{a}(x)\right) \in M D\left(D_{c}\right)$;

4) $W \wedge Y \wedge V_{b}\left(V_{a}(x)\right) \rightarrow s \in M D\left(D_{c}\right) \cap M D\left(G_{c}\right)$;

5) $W \wedge Y \rightarrow \sim s \in M D\left(D_{c}\right)$;

6) $W \wedge Y \rightarrow \sim s \in M D\left(D_{b}\right)$.

Analogously, policies concerned with an obligation to oblige contain clauses like $O_{b, c}\left(V_{a}(\sim x), s \mid Y \wedge W \wedge \sim x\right)$, a per- mission to permit contains $P_{b, c}\left(\neg V_{a}(x) \mid Y \wedge W \wedge x\right)$, and a permission to oblige $P_{b, c}\left(V_{a}(\sim x) \mid Y \wedge W \wedge \sim x\right)$. Moreover, they may also contain additional clauses. For example, if the global authority explicitly states the sanctions for the local providers, then we may have several clauses such as $O_{b, c}\left(s^{\prime}, s \mid Y \wedge W \wedge \sim x \wedge V_{a}(\sim x)\right)$ for the obligation to oblige and $P_{b, c}\left(s^{\prime} \mid Y \wedge W \wedge \sim x \wedge V_{a}(\sim x)\right)$ for the permission to oblige.

\section{Games}

The agents believe to be in a state that is the result of the application of the prioritized belief rules.

Definition 8 (Consequences of Beliefs): Let $R$ be a set of rules in $\operatorname{Rul}(X), Q$ a set of literals in $\operatorname{Lit}(X)$, and $\geq$ a transitive and reflexive relation on the powerset of $R$ containing at least the superset relation.

1) $\operatorname{out}(R, Q)=\cup_{0}^{\infty}$ out $^{i}(R, Q)$ is the state obtained by the sequence out ${ }^{0}(R, Q)=\emptyset$ and out $^{i+1}(R, Q)=$ out $^{i}(R, Q) \cup\left\{l \mid L \rightarrow l \in R\right.$ and $L \subseteq Q \cup$ out $\left.^{i}(R, Q)\right\}$;

2) $\max f \operatorname{amily}(R, Q, \geq)$ is the set of maximal subsets $R^{\prime}$ of $R$ with respect to set inclusion such that $Q \cup \operatorname{out}\left(R^{\prime}, Q\right)$ is consistent (does not contain a literal and its negation);

3) preffamily $(R, Q, \geq)$ is the set of maximal elements of maxfamily with respect to the $\geq$ ordering;

4) outfamily $(R, Q, \geq)$ is the output under the elements of preffamily, $\left\{\right.$ out $\left(R^{\prime}, Q\right) \mid R^{\prime} \in$ preffamily $\left.(R, Q, \geq)\right\}$;

5) $x \in \operatorname{out}(R, Q, \geq)$ iff $x \in \operatorname{\cap outfamily}(R, Q, \geq)$.

Decisions of agents are consistent sets of literals built from decision variables.

Definition 9 (Decisions): The set of decisions $\Delta$ of a set of agents $A S=\left\{a_{1}, \ldots, a_{n}\right\} \subseteq A$ is the set of consistent sets $\delta=\bigcup_{a_{i} \in A S} \delta_{i} \subseteq \operatorname{Lit}(X)$.

To define the optimal decisions, we consider the expected effects of decisions by applying belief rules and by recursively modeling the decisions of other agents. These effects are used to order the decisions using the desire and goal rules. The unfulfilled motivations of decision $\delta$ according to agent $a \in A$ are the set of motivations whose body is part of the closure of the decision under the belief rules but whose head is not, and which are not undercut by any rule in $H$.

Definition 10 (Recursive Modeling): Given the set of agents $A S=\left\{a_{1}, \ldots, a_{n}\right\} \subseteq A$ :

1) Given $S_{i}=\operatorname{out}\left(B_{i}, \bigcup_{0<j<n} \delta_{j}, \geq_{i}\right) \cup \bigcup_{0<j<n} \delta_{j}$, $U\left(\delta, a_{i}\right)$ is the set of $l_{1} \wedge \ldots \wedge l_{n} \rightarrow l \in M$ such that:

1) $\left\{l_{1}, \ldots, l_{n}\right\} \subseteq S_{i}$ and $l \notin S_{i}$;

2) there does not exist $l_{1} \wedge \ldots \wedge l_{n} \rightarrow l \in H_{i}$ such that $\left\{l_{1}, \ldots, l_{n}\right\} \subseteq S_{i}$.

2) A decision $\delta$ is optimal for agent $a_{i}$ if and only if it is optimal for agents $a_{i+1}, \ldots, a_{n}$ and there is no decision $\delta_{i}^{\prime}$ such that for all decisions $\delta^{\prime}=\delta_{0} \cup \ldots \cup \delta_{i}^{\prime} \cup \ldots \cup \delta_{n}^{\prime}$ and $\delta^{\prime \prime}=\delta_{0} \cup \ldots \cup \delta_{i} \cup \ldots \cup \delta_{n}^{\prime \prime}$ optimal for agents $a_{i+1}, \ldots, a_{n}$, we have that $U\left(\delta^{\prime}, a_{i}\right)>_{i} U\left(\delta^{\prime \prime}, a_{i}\right)$.

Example 4 (Continued): When $\delta=\left\{x_{1}, x_{4}\right\}$, then $\operatorname{out}\left(B_{\mathbf{a}}, \delta, \geq_{\mathbf{a}}\right)=\left\{x_{1}, x_{4}, q\right\}$ and $U(\delta, \mathbf{a})=\left\{d_{3} \top \rightarrow \neg x_{4}\right\}$, $U(\delta, \mathbf{p})=\left\{d_{2} \top \rightarrow r\right\}$, and $U(\delta, \mathbf{n})=\left\{g_{3} x_{1} \wedge x_{4} \rightarrow x_{5}\right\}$. When $\delta=\left\{x_{1}, x_{3}\right\}$, then $\operatorname{out}\left(B_{\mathbf{a}}, \delta, \geq_{\mathbf{a}}\right)=\left\{x_{1}, x_{3}, q, r\right\}$ and $U(\delta, \mathbf{a})=\left\{g_{1} x_{3} \rightarrow x_{2}\right\}, U(\delta, \mathbf{p})=\left\{g_{2} x_{1} \rightarrow x_{4}\right\}, U(\delta, \mathbf{n})=\emptyset$. 
TABLE I

User a, KNowledge Provider p, AND Global Authority n

\begin{tabular}{|c|c|c|c|}
\hline & $\mathbf{a}$ & p & $\mathbf{n}$ \\
\hline $\bar{X}$ & $\operatorname{read}(\mathbf{a}, d)$ & $V_{\mathbf{a}}(\operatorname{read}(\mathbf{a}, d))$, san & $V_{\mathbf{p}}\left(V_{\mathbf{a}}(\operatorname{read}(\mathbf{a}, d))\right)$ \\
\hline$B$ & $b_{1} \operatorname{read}(\mathbf{a}, d) \rightarrow \operatorname{info}(\mathbf{a})$ & $b_{4} \operatorname{read}(\mathbf{a}, d) \rightarrow \operatorname{info}(\mathbf{a})$ & \\
\hline$B$ & $b_{2} \operatorname{san} \rightarrow \neg \operatorname{info}(\mathbf{a})$ & $b_{5}$ san $\rightarrow \neg$ info $(\mathbf{a})$ & \\
\hline$B$ & $b_{3} \top \rightarrow \operatorname{project}(\mathbf{a})$ & $b_{6} \top \rightarrow \operatorname{project}(\mathbf{a})$ & $b_{7} \top \rightarrow \operatorname{project}(\mathbf{a})$ \\
\hline$D G$ & $d_{1} \top \rightarrow \operatorname{info}(\mathbf{a})$ & $d_{3} \top \rightarrow \neg \operatorname{read}(\mathbf{a}, d)$ & $d_{10} \operatorname{project}(\mathbf{a}) \wedge \operatorname{read}(\mathbf{a}, d) \rightarrow \neg V_{\mathbf{a}}(\operatorname{read}(\mathbf{a}, d)$ \\
\hline$D G$ & $d_{2} \top \rightarrow \neg$ san & $d_{4} \operatorname{read}(\mathbf{a}, d) \rightarrow V_{\mathbf{a}}(\operatorname{read}(\mathbf{a}, d))$ & $d_{11} V_{\mathbf{a}}(\operatorname{read}(\mathbf{a}, d)) \rightarrow V_{\mathbf{p}}\left(V_{\mathbf{a}}(\operatorname{read}(\mathbf{a}, d))\right)$ \\
\hline$D G$ & & $d_{5} V_{\mathbf{a}}(\operatorname{read}(\mathbf{a}, d)) \rightarrow$ san & $d_{12} \top \rightarrow \neg V_{\mathbf{p}}\left(V_{\mathbf{a}}(\operatorname{read}(\mathbf{a}, d))\right)$ \\
\hline$D G$ & & $d_{6} \top \rightarrow \neg V_{\mathbf{p}}\left(V_{\mathbf{a}}(\operatorname{read}(\mathbf{a}, d))\right)$ & \\
\hline$D G$ & & $d_{7} \operatorname{hack}(\mathbf{a}) \rightarrow \neg \operatorname{info}(\mathbf{a})$ & \\
\hline$D G$ & & $d_{8} \top \rightarrow \neg V_{\mathbf{a}}(\operatorname{read}(\mathbf{a}, d))$ & \\
\hline$D G$ & & $d_{9} \top \rightarrow \neg$ san & \\
\hline$\geq$ & $b_{2}>b_{1}$ & $b_{4}>b_{5}$ & \\
\hline$\geq$ & $d_{2}>d_{1}$ & $d_{4}>d_{8}, d_{5}>d_{9}, d_{7}>d_{6}$ & \\
\hline $\bar{\delta}$ & $\operatorname{read}(\mathbf{a}, d)$ & & \\
\hline out & $\operatorname{read}(\mathbf{a}, d), \operatorname{info}(\mathbf{a}), \operatorname{project}(\mathbf{a})$ & $\operatorname{read}(\mathbf{a}, d), \operatorname{info}(\mathbf{a}), \operatorname{project}(\mathbf{a})$ & $\operatorname{read}(\mathbf{a}, d), \operatorname{project}(\mathbf{a})$ \\
\hline$U$ & & $\top \rightarrow \neg \operatorname{read}(\mathbf{a}, d)$ & \\
\hline$U$ & & $\operatorname{read}(\mathbf{a}, d) \rightarrow V_{\mathbf{a}}(\operatorname{read}(\mathbf{a}, d))$ & \\
\hline
\end{tabular}

\section{EXAMPLE}

Table I illustrates an example of a virtual community composed of a user $\mathbf{a}$, a knowledge provider $\mathbf{p}$, and a CAS agent $\mathbf{n}$, which establishes the global policy of the community. When agent a takes its decision $\delta_{\mathbf{a}}$, it has to minimize its unfulfilled motivational attitudes. However, when it considers these attitudes, it must not only consider its decision $\delta_{\mathbf{a}}$ and its consequences, it must consider also the decision $\delta_{\mathbf{p}}$ of the local provider $\mathbf{p}$ and its consequences, for example, that it is sanctioned by it. Therefore, agent a recursively considers which decision the normative system $\mathbf{p}$ will take depending on its different decisions $\delta_{\mathbf{a}}$. In turn, agent $\mathbf{p}$ takes its decision $\delta_{\mathbf{p}}$ under the light of what will do the authority $\mathbf{n}$ it is subject to. The optimal decision of agent $\mathbf{p}$ depends on each decision $\delta_{\mathbf{a}}$ and also on the optimal decision $\delta_{\mathbf{n}}$ for normative system $\mathbf{n}$ for each decision $\delta_{\mathbf{a}} \cup \delta_{\mathbf{p}}$. Instead, given a decision $\delta_{\mathbf{a}} \cup \delta_{\mathbf{p}}$, a decision $\delta_{\mathbf{n}}$ is optimal for agent $\mathbf{n}$ if it minimizes the unfulfilled motivational attitudes in $D_{\mathbf{n}}$ and $G_{\mathbf{n}}$ according to the $\geq_{\mathbf{n}}$ relation, without further recursive modeling.

Agent a reads a document $d\left(\operatorname{read}(\mathbf{a}, d) \in X_{\mathbf{a}}\right)$, which is under the control of agent $\mathbf{p}$ to get an information info $(\mathbf{a}) \in P$ $\left(\operatorname{read}(\mathbf{a}, d) \rightarrow \operatorname{info}(\mathbf{a}) \in B_{\mathbf{a}}\right)$. Moreover, The CAS $\mathbf{n}$ issues global policies addressed to normative system $\mathbf{p}$ and $\mathbf{p}$ issues local policies addressed to user $\mathbf{a}$.

The example shows a case of entitlement. Even if the provider $\mathbf{p}$ locally forbids access to the information, agent $\mathbf{a}$ is entitled to do so by the global policy in the context of some project $(\operatorname{project}(\mathbf{a}) \in P)$. The local policy is represented by $O_{\mathbf{a}, \mathbf{p}}(\neg \operatorname{read}(\mathbf{a}, d), \operatorname{san} \mid \top)$, while the global one is $O_{\mathbf{p}, \mathbf{n}}\left(\neg V_{\mathbf{p}}(\operatorname{read}(\mathbf{a}, d)) \mid \operatorname{project}(\mathbf{a}) \wedge \operatorname{read}(\mathbf{a}, d)\right)$. The sanction san makes false the effect info(a) of the access to the resource $d$ by $\operatorname{read}(\mathbf{a}, d)$. However, if agent $\mathbf{p}$ believes that agent $\mathbf{a}$ is a hacker $(\operatorname{hack}(\mathbf{a}) \in P)$, it prefers to violate the global policy with respect to letting agent a access the document.

The bottom three lines of Table I represent the output and unfulfilled motivations when agent a decides to do $\delta_{\mathbf{a}}=\{\operatorname{read}(\mathbf{a}, d)\}$ and agents $\mathbf{p}$ and $\mathbf{n}$ decide to do nothing $\left(\delta_{\mathbf{p}}=\delta_{\mathbf{n}}=\emptyset\right)$. Had agent $\mathbf{p}$ 's decision been $\delta_{\mathbf{p}}^{\prime}=$ $\left\{V_{\mathbf{a}}(r e a d(\mathbf{a}, d)), \operatorname{san}\right\}$, then info(a) would not have been true anymore in $\operatorname{out}\left(B_{\mathbf{a}}, \delta, \geq_{\mathbf{a}}\right)$, due to the sanction applied by agent $\mathbf{p}$ : the rule $\operatorname{san} \rightarrow \neg$ info $(\mathbf{a}) \in B_{\mathbf{a}}$ has priority over the rule $\operatorname{read}(\mathbf{a}, d) \rightarrow \operatorname{info}(\mathbf{a}) \in B_{\mathbf{a}}\left(b_{2}>b_{1}\right)$. Thus, agent a's unfulfilled desires would have been

$$
U\left(\delta^{\prime}=\delta_{\mathbf{a}} \cup \delta_{\mathbf{p}}^{\prime}, \mathbf{a}\right)=\{\top \rightarrow \operatorname{info}(\mathbf{a}), \top \rightarrow \neg \text { san }\}
$$

To take a decision between $\delta_{\mathbf{p}}$ and $\delta_{\mathbf{p}}^{\prime}$, agent $\mathbf{p}$ compares which of its goals and desires remain unsatisfied under the light of agent $\mathbf{n}$ 's reaction: In fact, if agent $\mathbf{p}$ decides for $\delta_{\mathbf{p}}^{\prime}, U \delta_{\mathbf{n}}^{\prime}$ would be $\left\{V_{\mathbf{p}}\left(V_{\mathbf{a}}(\operatorname{read}(\mathbf{a}, d))\right)\right\}$.

$$
U\left(\delta^{\prime}, \mathbf{n}\right)=\left\{\top \rightarrow \neg V_{\mathbf{p}}\left(V_{\mathbf{a}}(\operatorname{read}(\mathbf{a}, d))\right)\right\}
$$

However, $U\left(\delta^{\prime}, \mathbf{p}\right) \geq_{\mathbf{p}} U(\delta, \mathbf{p})$ and thus agent $\mathbf{p}$ decides $\delta_{\mathbf{p}}$

$$
\begin{aligned}
U\left(\delta^{\prime}, \mathbf{p}\right) & =\left\{\top \rightarrow \neg V_{\mathbf{p}}\left(V_{\mathbf{a}}(\operatorname{read}(\mathbf{a}, d))\right)\right\} \\
U(\delta, \mathbf{p}) & =\left\{\operatorname{read}(\mathbf{a}, d) \rightarrow V_{\mathbf{a}}(\operatorname{read}(\mathbf{a}, d))\right\}
\end{aligned}
$$

If agent $\mathbf{p}$ believes that agent $\mathbf{a}$ is $\mathbf{a}$ hacker, $\top \rightarrow \operatorname{hack}(\mathbf{a}) \in B_{\mathbf{p}}^{\prime}$, i.e., if $\operatorname{hack}(\mathbf{a})$ is true in $\operatorname{out}\left(B_{\mathbf{p}}^{\prime}, \delta, \geq_{\mathbf{p}}\right)$, then $\mathbf{p}$ believes that agent $\mathbf{a}$, by knowing the content of the document $($ info $(\mathbf{a}))$, will damage the system, so it prefers that it does not access it $\left(\operatorname{hack}(\mathbf{a}) \rightarrow \neg i n f o(\mathbf{a}) \in D_{\mathbf{p}} \cap G_{\mathbf{p}}\right)$. In this case, agent $\mathbf{p}$ prefers to be considered a violator by agent $\mathbf{n}$ with respect to allowing agent $\mathbf{p}$ trying to access the document, since its priority is

$$
h a c k(\mathbf{a}) \rightarrow \neg \operatorname{info}(\mathbf{a}) \geq_{\mathbf{p}} \top \rightarrow \neg V_{\mathbf{p}}\left(V_{\mathbf{a}}(\operatorname{read}(\mathbf{a}, d))\right) .
$$

Thus, decision $\delta_{\mathbf{p}}^{\prime \prime}$ would be $\left\{V_{\mathbf{a}}(\operatorname{read}(\mathbf{a}, d))\right.$, san $\}$.

The example can be extended with sanctions toward agent $\mathbf{p}$ in the obvious way. Moreover, obligations to oblige or to prohibit can be defined analogously. The number of clauses increases, as discussed in Section VII-C, but the analysis can be made in the same way. 


\section{RELATED WORK}

The problem of striking some balance between local versus global policies has been studied in other contexts too, as part of the development of distributed computing systems. Numerous solutions have been proposed, including that of security in a grid computing environment [14] as well as in web-services security architectures [34]. An advantage of the formalization of the balance introduced in this paper is that it is characterized using an abstract framework. Another advantage is that normative multiagent systems offer the formal framework to analyze or simulate complex scenarios, using the underlying theories of normative systems and multiagent systems, including ones of fraud and deception [35].

In particular, the problem has been addressed in the context of trust-management systems, trust networks, and in reputationbased systems [36]. These theories and systems originate from distinct but related traditions (see, e.g., [37] for a discussion of how trust and norms may be related in a game-theoretic setting). However, they also have some remarkable similarities. We believe that further study is needed to investigate the assumptions of the two approaches. For example, the way in which agents anticipate the behavior of other agents in normative systems seems distinct from the way they anticipate behavior in trust and reputation-based systems.

Moreover, while here we study the decentralization of control, our framework is used also to cope with the symmetric issue of how to centralize those aspects of security in distributed environments that cannot be dealt with at the local level, such as which are the members of the community and which ones should be authorized to access a resource. In particular, in [38], we explore the problem of how local providers can delegate to other agents the power to authorize access without giving up their autonomy. We argue that the problem can be solved by means of counts-as relations [39]: An agent empowered to authorize can issue declarations that are considered as authorizations by the local provider. Moreover, these authorizations appear in the conditions of permissions, which act as exceptions to the prohibitions concerning general users. In this way, on the one hand, a CAS agent can regulate at the central level which agents are allowed to access resources on the basis of the up-to-date list of members and policies; on the other hand, the local providers are not overburdened by the management of information about memberships and maintain their autonomy to issue obligations, prohibitions, and permissions to regulate access to their resources.

We believe that a further study into the similarities and distinctions between these areas can lead to some fruitful exchanges of ideas. A contribution of the normative systems' framework is the logical analysis methods, the interaction among the logical framework and the game-theoretic elements, and the possibilities to simulate fraud and deception. Also, trust and reputation mechanisms can be used to enrich existing normative multiagent systems. For example, if one agent believes that another one is going to harm resources under its control, this information should be propagated to the global level. There should be some form of mechanism to capture such "reputation" information in the normative architecture.

Normative systems have traditionally been concerned with more static systems such as bureaucratic and legal systems. Recent work on normative multiagent systems is concerned with applications in modern electronic networks, which are much more dynamic and uncertain. It therefore takes its inspiration from social theories such as Searle's work on the construction of social reality, and is concerned with the powers of agents to change the normative system [19].

The formal framework presented in this paper builds on deontic logic and BOID agent architecture. It extends our previous work on normative multiagent systems, e.g., in [19], in various ways. For example, it introduces the notion of prioritized beliefs, documents, and most importantly, it studies more complex games involving an arbitrary number of agents, within a realistic setting. Whereas arbitrary games among a large number of agents get very complex, in particular in the context of uncertainty and observations, and introduce new conceptual problems, see, e.g., [40] for an example, the games discussed in this paper are relatively simple due to the fact that each agent has to consider only the decision of the next agent who can consider it as a violator.

We do not define permissions as the absence of obligation, so-called negative permission, but as exceptions to obligations, a kind of positive permission. For a discussion on the issues involved in modeling permission, see [41]. Permission is simpler than obligation, since permissions cannot lead to violations and sanctions. It is only due to entitlement that knowledge providers may be sanctioned when they do not permit a user to access documents, but the user itself cannot be a violator and be sanctioned due to its permissions to access a document. The various clauses of obligation have been motivated by a gametheoretic analysis [19]. The first clause ensures that "respectful" agents internalizing the goals of the normative system will fulfill their obligation under typical circumstances; the second and third clauses do so for "respectful" agents that do not want to be considered as violators even if they do not internalize the norm as one of their goals. The other clauses do so for "selfish" types of agents, which care only about not being sanctioned. Similar games can be played to show that the clauses of permission are necessary, again for norm internalizing agents and other types of agents, respectively. Since permissions are exceptions to obligations, a "respectful" internalizing agent would still adopt the content of the obligation as one of its goals. For this reason, we added the first clause containing an undercutter to the goal of the obligation the permission is an exception of.

Here, we only discuss regulative norms and we do not discuss constitutive ones, though the new models introduced in this paper can also be extended with constitutive norms along the lines discussed in [19]. For constitutive rules, we adopt the same strategy of attributing mental attitudes to normative systems. Whereas regulative norms are defined in terms of goals of the normative systems, constitutive norms establishing what counts as institutional facts are defined in terms of the beliefs of the normative system. 


\section{SUmmary}

Any scalable solution for secure knowledge management has to be able to distribute not only the knowledge-management system, but also the security system. Once knowledge management becomes distributed, security becomes distributed too. A distributed security system assumes that security concerns are incorporated from the first design of the distributed knowledgemanagement system. For example, Kolp [7] analyzes the development of knowledge-management systems and argues that "existing proprietary information management tools block the exchange of information between applications and users," and therefore "a holistic approach is required in the design of information technology infrastructures as well as the actual business process reengineering for a successful knowledge management effort."

We develop an integrated approach to distributed knowledge management and security, using multiagent systems and accesscontrol policies in virtual communities. Distributed access control is realized by a virtual community of multiple knowledge providers with their own local access-control system to their documents and local policies, and by global community policies regulating these local policies. The issue at stake is the rational balance of global versus local control in virtual communities. Knowledge providers must be autonomous, but not unconstrained.

Autonomy of local knowledge providers is ensured by modeling each member of the virtual community as a normative system interacting with other members and posing prohibitions and permissions about access to its knowledge. Participants do not give up their autonomy to prohibit access to knowledge to users they do not trust, even when the users satisfy the security rules of the virtual community. Resource providers are therefore not forced, but motivated by the global authority to behave as required. Local providers consider whether to violate global policies in some situations. Games among the three agents involved in knowledge access-the user, the knowledge provider, and the global authority-explain how these motivations work. The games can be used also to analyze the knowledge-management system, or to simulate it.

Global community policies regulate local policies based on von Wright's notion of transmission of will. The rules of policies for secure knowledge management do not concern only what knowledge the users are prohibited or permitted to access, but they also concern which regulations the knowledge providers are allowed or obliged to enforce. This is a challenge, because rules refer usually to the actions of users and not to other rules, which are obligatory or permitted to adopt. In our model, we incorporate a mechanism for interaction among normative systems. We generalize the framework with decentralized systems composed of global authorities and local providers; they are both normative systems, but global authorities can specify duties and permissions of local providers concerning the local policies.

A game-theoretic analysis is used to define global policies. These games describe interactions among the agents in the normative multiagent systems. Agents must evaluate the effects of accessing documents both when making and evaluating an access request. In particular, since the compliance of the providers to the policies cannot be taken for granted, a user must consider whether they will fulfill their commitments or not. To make a prediction about the behavior of a local provider, it is necessary to consider also the reaction of the global authority enforcing control by monitoring and sanctioning violations.

For example, the notion of entitlement, i.e., when a knowledge provider is obliged to permit access to a user, could be interpreted as the obligation of the provider to create a permission. However, the knowledge provider can create the permission, but still does not let the user access the document. In such a case, the knowledge provider does not act according to the permissions it created itself. This scenario shows that such definition of global policies is ineffective. The game-theoretic analysis of the notion of entitlement in normative multiagent systems illustrates that this definition of global policy is ineffective and may not change the behavior of the providers.

Our solution is to define global policies concerning the behavior of knowledge providers: considering specified behaviors as violations and sanctioning them. In the case of entitlement, this amounts to being obliged not to consider the specified behavior as a violation. For the case in which users are motivated by sanctions only, in the sense that they are not respectful and act according to the norm simply due to the existence of the norm, our game-theoretic analysis shows that the existence of nested obligations and permissions is not only too weak, it is even superfluous. The only clause that is important is that knowledge providers are sanctioned in the case that the user is not acting as desired.

Similar scenarios occur when the global policies dictate that the knowledge provider should oblige the user. In that case, the knowledge provider can simply not create the obligation, or it can create the obligation but not enforce it. Again von Wright's analysis of the transmission of will can be used to argue that creating the obligation is not sufficient, the knowledge provider should also enforce the obligations it created, and sanction users that do not act according to them.

There are more requirements of secure knowledgemanagement systems, which can be addressed in further research. For example, our model should be extended to cope with role-based access control using our role model [42] or exploring the delegation issues of discretionary access control using our contract model [19]. Finally, the current framework can be extended to deal not only with policies consisting of regulative rules like obligations, prohibitions, and permissions, but also with constitutive rules specifying counts-as relations and institutional facts [39]. In particular, in [38], we use countsas relations to specify local policies concerning authorizations, while global policies prescribing constitutive rules are still an open issue.

\section{REFERENCES}

[1] J. Bradshaw, S. Dutfield, P. Benoit, and J. Woolley, "KAoS: Towards an industrial strength generic agent architecture," in Software Agents. Cambridge, MA: MIT Press, 1997, pp. 375-418.

[2] A. Preece, K. Hui, W. A. Gray, P. Marti, T. J. M. Bench-Capon, D. M. Jones, and Z. Cui, "The KRAFT architecture for knowledge fusion and transformation," Knowl. Based Syst., vol. 13, no. 2/3, pp. 113-120, 2000 
[3] A. Abecker, A. Bernardi, and L. van Elst, "Agent technology for distributed organizational memories," in Proc. 5th Int. Conf. Enterprise Information Syst, 2003, pp. 3-10.

[4] B. Yu, M. Venkatraman, and M. Singh, "An adaptive social network for information access: Theoretical and experimental results," J. Appl. Artif. Intell., vol. 17, no. 1, pp. 21-38, Jan. 2003.

[5] L. van Elst, V. Dignum, and A. Abecker, "Towards agent-mediated knowledge management," in Proc. Agent-Mediated Knowledge Management, vol. 2926, Lecture Notes in Computer Science. Berlin, Germany: Springer-Verlag, 2004, pp. 1-30.

[6] R. M. van Eijk, F. S. de Boer, W. van der Hoek, and J.-J. C. Meyer, "Open multi-agent systems: Agent communication and integration," in Proc. Intelligent Agents VI, vol. 1757, Lecture Notes in Computer Science. Berlin, Germany: Springer-Verlag, 2000, pp. 218-232.

[7] M. Kolp, "Agent-based IT support for knowledge management," IAG Working Paper 29/02, 2002.

[8] S. Xu and W. Zhang, "PBKM: A secure knowledge management framework," in Proc. NSF/NSA/AFRL Workshop Secure Knowledge Management, 2004.

[9] B. Sadighi Firozabadi and M. Sergot, "Contractual access control," in Proc. Workshop Security Protocols, Cambridge, U.K., 2002, pp. 96-102.

[10] M. Bonifacio, P. Bouquet, G. Mameli, and M. Nori, "KEx: A peerto-peer solution for distributed knowledge management," in Proc. 4th Int. Conf. PAKM, vol. 2569, Lecture Notes in Computer Science. Berlin, Germany: Springer-Verlag, 2002, pp. 490-500.

[11] M. Bonifacio, R. Cuel, G. Mameli, and M. Nori, "A peer-to-peer architecture for distributed knowledge management," in Proc. 3rd Int. Symp. Multi-Agent Systems, Large Complex Systems, and E-Business (MALCEB'2002).

[12] C. Tacla and J.-P. Barthes, "A multi-agent architecture for knowledge management systems," in Proc. 2nd IEEE ISADS, 2002, pp. 1-12.

[13] D. Mundy and D. Chadwick, "Secure knowledge management," in Creating Knowledge Based Health Care Organizations. Hershey, PA: The Idea Group, 2004, pp. 321-337.

[14] L. Pearlman, V. Welch, I. Foster, C. Kesselman, and S. Tuecke, "A community authorization service for group collaboration," in Proc. IEEE Int. Workshop Policies Distributed Systems and Networks, 2002, pp. 50-59.

[15] B. Sadighi Firozabadi, M. Sergot, and O. Bandmann, "Using authority certificates to create management structures," in Proc. Workshop Security Protocols, vol. 2467, Lecture Notes in Computer Science. Berlin, Germany: Springer-Verlag, 2001, pp. 134-145.

[16] M. Blaze, J. Feigenbaum, and J. Lacy, "Decentralized trust management," in Proc. IEEE Conf. Security and Privacy, 1996, pp. 164-173.

[17] J. S. Sichman, R. Conte, C. Castelfranchi, Y. Demazeau, "A social reasoning mechanism based on dependence networks," in Proc. 11th ECAI, A. G. Cohen, Ed., 1991, pp. 188-192.

[18] A. Jones and J. Carmo, "Deontic logic and contrary-to-duties," in Handbook of Philosophical Logic, D. Gabbay and F. Guenthner, Eds. Dordrecht, The Netherlands: Kluwer, 2001, pp. 203-279.

[19] G. Boella and L. van der Torre, "A game theoretic approach to contracts in multiagent systems," IEEE Trans. Syst., Man, Cybern. C, Appl. Rev., vol. 36, no. 1, pp. 68-79, Jan. 2006.

[20] V. Kartseva, J. Gordijn, and Y.-H. Tan, "Analysing preventative and detective control mechanisms in international trade using value modelling" in Proc. 6th ACM Int. Conf. Electronic Commerce, Eds., M. Janssen, H. G. Sol, and R. W. Wagenaaar, Delft, The Netherlands, 2004, pp.5158.

[21] Y. Shoham and M. Tennenholtz, "On the emergence of social conventions: Modeling, analysis and simulations," Artif. Intell., vol. 94, no. 1/2, pp. 139-166, Jul. 1997.

[22] G. Boella and L. Lesmo, "A game theoretic approach to norms," Cogn. Sci. $Q$., vol. 2, no. 3/4, pp. 492-512, 2002.

[23] E. Goffman, Strategic Interaction. Oxford, U.K.: Blackwell, 1970.

[24] A. Newell, "The knowledge level," Artif. Intell., vol. 18, no. 1, pp. 87-127, Jan. 1982

[25] J. Broersen, M. Dastani, J. Hulstijn, and L. van der Torre, "Goal generation in the BOID architecture," Cogn. Sci. Q., vol. 2, no. 3/4, pp. 428-447, 2002.

[26] P. J. Gmytrasiewicz and E. H. Durfee, "Formalization of recursive modeling," in Proc. ICMAS, 1995, pp. 125-132.

[27] G. H. von Wright, "An essay in deontic logic and the general theory of action," Acta Philos. Fenn., vol. 21, 1968.

[28] M. S. Sloman, "Policy driven management of distributed systems," J. Netw. Syst. Manag., vol. 2, no. 4, pp. 333-360, Dec. 1994.

[29] D. Dennett, The Intentional Stance. Cambridge, MA: MIT Press, 1987.

[30] J. L. Pollock, "Defeasible reasoning," Cogn. Sci., vol. 11, no. 4, pp. 481-518, Oct.-Dec. 1987.
[31] J. Lang, L. van der Torre, and E. Weydert, "Utilitarian desires," Auton. Agents Multiagent Syst., vol. 5, no. 3, pp. 329-363, 2002.

[32] A. Anderson, "The reduction from deontic logic to alethic modal logic," Mind, vol. 67, pp. 100-103, 1958.

[33] R. Lee, "Documentary Petri nets: A modeling representation for electronic trade procedures," in Proc. Business Process Management, 2000, vol. 1806 , pp. 359-375

[34] S. Chang, Q. Chen, and M. Hsu, "Managing security policy in a large distributed web services environment," in Proc. Int. COMPSAC., Lecture Notes in Computer Science. Berlin, Germany: Springer-Verlag, 2003, pp. $610-618$

[35] G. Boella and L. van der Torre, "Normative multiagent systems and trust," in Proc. Trust Agent Societies Workshop AAMAS, vol. 3577, Lecture Notes in Artificial Intelligence. Berlin, Germany: Springer-Verlag, 2004, pp. 1-17.

[36] M. Fan, Y. Tan, and A. Whinston, "Evaluation and design of online cooperative feedback mechanisms for reputation management," IEEE Trans. Knowl. Data Eng., vol. 17, no. 2, pp. 244-254, Feb. 2005.

[37] M. Hollis, Trust Within Reason. Cambridge, U.K.: Cambridge Univ. Press, 1998.

[38] G. Boella and L. van der Torre, "Permission and authorization in policies for virtual communities of agents," in Proc. Agents and P2P Computing Workshop AAMAS, vol. 3601, Lecture Notes in Computer Science. Berlin, Germany: Springer-Verlag, 2004, pp. 86-97.

[39] J. Searle, The Construction of Social Reality. New York: Free Press, 1995.

[40] G. Boella and L. van der Torre, "Rational norm creation: Attributing mental attitudes to normative systems, part 2," in Proc. ICAIL, 2003, pp. 81-82.

[41] D. Makinson and L. van der Torre, "Permissions from an input-output perspective," J. Philos. Logic, vol. 32, no. 4, pp. 391-416, Aug. 2003.

[42] G. Boella and L. van der Torre, "Organizations as socially constructed agents in the agent oriented paradigm," in Proc. ESAW, vol. 3451, Lecture Notes in Artificial Intelligence. Berlin, Germany: Springer-Verlag, 2004, pp. 1-13.

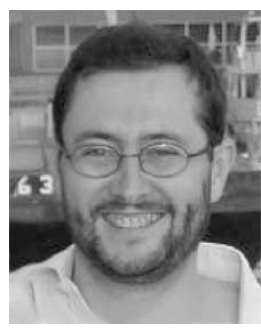

Guido Boella received the Ph.D. degree in computer science from the Università di Torino, Torino, Italy, in 2000 .

$\mathrm{He}$ is currently a Professor at the Department of Computer Science, Università di Torino. His research interests include multiagent systems, in particular, normative systems, institutions, and roles using qualitative decision theory. He organized the first workshops on normative multiagent systems (NorMAS), on coordination and organization, and the American Association for Artificial Intelligence (AAAI) Fall Symposium on roles.

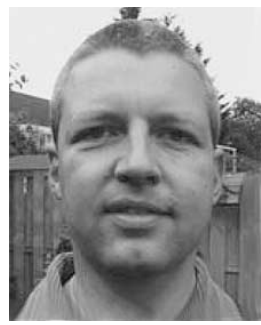

Leendert van der Torre received the Ph.D. degree in computer science from Erasmus University Rotterdam, Rotterdam, The Netherlands, in 1997.

$\mathrm{He}$ is currently a Full Professor at the University of Luxembourg, Luxembourg. He has developed the socalled input/output logics and the BOID agent architecture. His current research interests include deontic logic, qualitative game theory, and coordination and security in normative multiagent systems (NorMAS). 


\title{
A Game Theoretic Approach to Contracts in Multiagent Systems
}

\author{
Guido Boella and Leendert van der Torre
}

\begin{abstract}
Contracts are used to create new interaction possibilities among agents, and they therefore play an important role in the game theoretic analysis of agent interaction. We use normative multiagent systems to model both the contracts and the interactions. In particular, we formalize contracts as systems of regulative and constitutive norms within a larger rule-governed setting, and using recursive modelling we develop a game theory where agents make contracts. We show how agents can modify the behavior of normative systems by means of constitutive rules in the contract changing these systems, and we illustrate how agents use the game theory within contract negotiation in organizations.
\end{abstract}

Index Terms - Contracts, qualitative game theory, multiagent systems, normative systems.

\section{INTRODUCTION}

Autonomous agents negotiating deals on behalf of human traders are essential in e-commerce and e-trading systems. Many researchers focus their approaches on the game-theoretic analysis of the interaction among negotiation agents to prove macro-level properties like system stability (equilibrium) as well as efficiency (Pareto-optimality). However, the interaction structure in many multiagent systems is not completely fixed in advance to preserve the autonomy of agents. For example, as Dignum et al. [1] note, in (virtual) organizations the interaction possibilities can be changed and negotiated. For this reason, several approaches like [1]-[4] introduce the possibility for agents to stipulate contracts. A contract can be defined as a statement of intent regulating behavior among agents and organizations. Contracts have been proposed to make explicit how agents can change the interaction with and within the organization, using the legal effects of contracts involving the creation of obligations, permissions and new possibilities of interaction. From a contractual perspective, organizations can be seen as the possible sets of agreements for satisfying the diverse interests of self interested agents [2].

Hanson and Milosevic [3] distinguish various phases in the negotiation of contracts, like contract schema selection, issue identification, negotiation of values of the issues agreed upon, monitoring the performance of the contract, et cetera. As Marsh suggests in the contract negotiation handbook [5], a crucial point in the negotiation is the careful planning of the moves during the bargaining. Accordingly, agents must evaluate the effects of a contract during its negotiation both when proposing and evaluating an offer. In particular, since the

Manuscript received XXXXXX XX, 20XX; revised XXXXXX XX, 20XX Guido Boella is with Dipartimento di Informatica, Università di Torino, Italy (e-mail: guido@di.unito.it).

Leendert van der Torre is with CWI Amsterdam and Delft University of Technology, The Netherlands (e-mail: leendert@vandertorre.com).

Digital Object Identifier XXX compliance of the agents to the contract cannot be taken for granted, an agent must consider whether the other agents will fulfill their commitments or not. To make a prediction about the behavior of an agent it is necessary to consider also the reaction of the normative system [6] acting as a coordinator enforcing control by monitoring and sanctioning violations.

In this paper we develop a formal game-theoretic model for autonomous agents negotiating contracts, which we model as a normative multiagent system, that is, a "set of agents [...] whose interactions can be regarded as norm-governed; the norms prescribe how the agents ideally should and should not behave [7]." It is based on a general conceptual model formalizing contracts as so-called legal institutions, that is, as sets of norms within a larger legal setting. In this way, agents are made competent to determine the course of law within the sphere of the contracts they are allowed to create. To model the interaction among agents and the normative system in this game-theoretic setting, we apply the agent metaphor to normative systems and therefore model the interaction among agents and their normative system analogously to the interaction among real agents.

We define games using recursive modelling. Violation games model an agent deliberating whether to violate a norm or not, where the agent recursively models the normative system to predict whether its behavior counts as a violation and will be sanctioned. The set of possible behaviors includes the possibility of making contracts to change the agent's normative position based on constitutive rules that change the normative system. Negotiation games model an agent deliberating whether it will propose or accept a contract, thereby recursively modelling the other agent to find out whether it will violate the norms in the contract. In this sense, negotiation games add a recursive level of reasoning to violation games. We illustrate how agents can reason in the game theory using an example from international trade.

This paper does not address the problem of equilibrium analysis in the proposed game theory or the negotiation protocol to obtain an agreement. Instead we examine the impact of contracts on the games played by the agents and how the agents reason about the legal effects of contracts.

The layout of this paper is as follows. In Section II we discuss regulative and constitutive rules, legal institutions and how agents modify the behavior of normative systems using contracts. In Section III we discuss the foundations of our model and in Section IV we introduce our formal model applying the agent metaphor to normative systems. In Section V we present recursive modelling, the games which can be played with contracts, and an example illustrating how agents use the game theory for contracting. 


\section{Contracts as Legal institutions}

Most formalizations of normative systems identify norms with regulative norms like obligations, prohibitions and permissions. However, Searle [8] argues that there is a distinction between two types of rules.

"Some rules regulate antecedently existing forms of behaviour. For example, the rules of polite table behaviour regulate eating, but eating exists independently of these rules. Some rules, on the other hand, do not merely regulate an antecedently existing activity called playing chess; they, as it were, create the possibility of or define that activity. The activity of playing chess is constituted by action in accordance with these rules. The institutions of marriage, money, and promising are like the institutions of baseball and chess in that they are systems of such constitutive rules or conventions" ( [8], p. 131).

For Searle, regulative and constitutive norms are related via institutional facts like marriage, money and private property. They emerge from an independent ontology of "brute" physical facts through constitutive rules of the form "such and such an $\mathrm{X}$ counts as $\mathrm{Y}$ in context $\mathrm{C}$ " where $\mathrm{X}$ is any object satisfying certain conditions and $\mathrm{Y}$ is a label that qualifies $\mathrm{X}$ as being something of an entirely new sort. E.g., "X counts as a presiding official in a wedding ceremony", "this bit of paper counts as a five euro bill" and "this piece of land counts as somebody's private property". Regulative norms refer to these institutional facts. E.g., consider a society which believes that a field fenced by an agent counts as the fact that the field is the agent's property. The fence is a physical "brute" fact, while being a property is an institutional fact. Regulative norms forbidding trespassing refer to the abstract concept of property rather than to fenced fields.

Moreover, the philosopher of law Ruiter [9] shows, from the legal point of view, that legal effects of actions of the members of a legal system are complex and contracts do not concern only the regulative aspects of a legislation or the constitutive part of it. Rather, contracts are legal institutions: "systems of [regulative and constitutive] rules that provide frameworks for social action within larger rule-governed settings" [9]. This systemic view of legal institutions emerged only recently in legal studies, since legal positivism [10] mainly focused on the regulative aspects of law and its justification.

To formalize contracts as legal institutions, we have to extend Searle's model. Searle's analysis of constitutive rules has focused mainly on the attribution of a new functional status to entities like marriages, money, and property. Searle's idea is that constitutive rules "create the possibility or define that activity." In our model the role of constitutive rules is not limited to the creation of an activity and the construction of new abstract legal categories. Constitutive norms specify besides the creation of legal categories also the evolution of the system. The normative system itself specifies by means of constitutive rules how its state can be changed, who can change it, and the limits of the possible changes. In this way, complex normative systems achieve a legal regime that includes rules conferring legal powers on participants: an agent is turned into a "private legislator" (Hart [10]): "he is made competent to determine the course of law within the sphere of his contracts, trusts, wills and other structures [...] which he is enabled to build". Agents become able to design "relatively independent institutional legal orders within the comprehensive legal orders" (Ruiter [9]).

The regime of a legal institution can be defined as the set of legal consequences that flow from the existence of the institution. However, the meaning of "legal consequences" differs from what is normally understood by the term. Usually, since obligations have a conditional nature, when the condition of an obligation is satisfied, as a legal consequence the addressee of the obligation is categorically obliged to fulfill it. Legal institutions, like contracts, marriages and properties, refer to a different kind of legal consequences. E.g., the legal rule "in a marriage parents have the reciprocal obligation to take care of and support their children" is not a conditional obligation. It expresses the fact that only when a legal institution of marriage between Amy and Bob is created, the obligation is created that Amy and Bob take care and support their children. The same happens with the legal institution of contracts. When a contract comes into existence it creates obligations for the agents, i.e., new regulative norms which the normative system considers as its own. E.g., the Italian Civil Code art. 1173 (sources of obligations) specifies that obligations are created by contracts and art. 1372 (efficacy of contracts) that a contract has the strength of law (a contract is an agreement among two or more agents to regulate a juridical relationship about valuables ex art. 1321).

Therefore, contracts as legal institutions bring with them also constitutive rules creating not only new institutional facts, but also new obligations. In this way, it is possible to specify in a contract new procedures for the interaction among agents, to specify the evolution of the contract and how new obligations are created at a later stage. As Dignum et al. [1] notice, a contract specifies the events that alter the status of the contract. It is necessary to specify an interaction structure which indicates the possibilities of an agent and the consequences of its choices. The contract must specify how to proceed if a norm is violated and what the violator is expected to do. E.g., if a payment deadline is not respected, then the agent may be obliged to pay a double fee. Since we model contracts as legal institutions, this rule is not a conditional obligation, but it is an obligation created by an event specified in the contract, in the same way as the contract itself can create obligations. This is possible, because we consider a contract as a legal institution, which may be seen as a normative system inside the main normative system. As a normative system it specifies who has the power to introduce obligations.

To illustrate our model we use in this paper an example of Gordijn and Tan [11] about contracts inside a trade organization. In this example, the contracts are legal institutions within the larger context of legislation of international trade (the UN Convention on International Multimodal Transport of Goods, CIMTG). Gordijn and Tan show how the problem of trust between two agents exchanging goods for money can be solved by means of contracts offered by international trade organizations. 


\section{FOUNDATIONS OF OUR MODEL}

Inspired by the game-theoretic approach to obligations of Boella and Lesmo [12], we propose a logical multiagent framework for normative reasoning based on the philosophical foundations on strategic interaction in the work of sociologist Goffman [13]. "Strategic interaction" here means, according to Goffman, taking into consideration the actions of other agents:

"When an agent considers which course of action to follow, before he takes a decision, he depicts in his mind the consequences of his action for the other involved agents, their likely reaction, and the influence of this reaction on his own welfare" [13, p.12].

Goffman sees norms as producing a form of strategic interaction between the agent and the normative system, and gives a game-theoretic interpretation of obligations. In a normative system, "the enforcement power is taken from mother nature and invested in a social office specialized for this purpose, namely a body of officials empowered to make final judgements and to institute payments" [13, p.115]. Such a game is unusual, since "the judges and their actions will not be fully fixed in the environment, many unnatural things are possible. [...] the payment for a player's move ceases to be automatic but is decided on and made by the judges" [13, p.115]. Clearly, this approach is different from recent logical studies about norms and contracts based on modal logic like so-called deontic logic, see Section VI.

However, there are some problems to use Goffman's strategic interaction to develop a game theoretic approach to contracts. In particular, classical decision and game theory have been criticized for their assumptions of ideality. Several alternatives have been proposed that take the bounded rationality of decision makers into account. For example, Newell [14] and others develop theories in artificial intelligence using the notion of goal. Agent theory replaces probabilities and utilities by informational (knowledge, belief) and motivational attitudes (goal, desire), and the decision rule by a process of deliberation. Bratman [15] further extends such theories with intentions for sequential decisions and norms for multiagent decision making (see the discussion in Section VI). Moreover, Gmytrasiewitcz and Durfee [16] replace the equilibria analysis in classical game theory by recursive modelling. It considers the practical limitations of agents in realistic settings such as acquiring knowledge and reasoning so that an agent can build only a finite nesting of models about other agents' decisions.

"In order to solve its own decision-making situation, the agent needs an idea of what the other agents are likely to do. It can arrive at it by representing what it knows about the other agents' decision-making situations, thus modelling them in terms of their own payoff matrices. The fact that other agents could also be modelling others, including the original agent, leads to a recursive nesting of models."

Moreover, Goffman does not consider contracts, though his idea that the enforcement power is invested in a social office empowered to make final judgements and to institute payments is even more relevant when we consider besides norms also contracts. Contracts are used by agents to implement the agreements resulting from their negotiations and to change their normative situation. Social order in a multiagent system emerges from the contracts resulting from negotiations about the rights and duties of participants, rather than being given in advance. Moreover, as discussed in Section II, contracts are legal institutions, i.e., "systems of [regulative and constitutive] rules that provide frameworks for social action within larger rule-governed settings" [9], In our case, the larger setting is represented by a normative system which establishes the set of possible contracts. For this reason it is necessary to address the problem of contracts being aware of the peculiarities of legal institutions.

We emphasize here two properties of our model. First, legal institutions contain control procedures, which are policies and procedures that help to ensure that management directives are carried out [17], because, intentionally or not, an agent may fail to comply with the contract. For example, as suggested by Milosevic and Dromey [18], the specification given in a contract differs significantly from a computational specification in the expected degree of inconsistency. As Jones and Carmo observe, "importantly, the norms allow for the possibility that actual behavior may at times deviate from the ideal, i.e., that violations of obligations, or of agents' rights, may occur" [7]. We therefore represent norms as soft constraints, which are used in detective control systems where violations can be detected (you can enter a train without a ticket, but you may be checked and sanctioned), instead of hard constraints [19], which are restricted to preventative control systems that are built such that violations are impossible (you cannot enter a metro station without a ticket). Detective control is the result of the action of an agent, and consequently it is subject to errors and can be influenced by the actions of other agents.

Secondly, our model allows to analyze in detail both the decisions of agents and the creation of new regulative and constitutive norms under existing obligations. For example, Marsh [5] highlights that also the existing obligations and commitments of the agents play a role in contract negotiation, as well as the procedures they have to follow, in that they constrain both the possibility to propose and accept bids. Consequently, our model cannot only be used for the initial creation of the normative system with its contracts, but also for its evolution.

In our running example of contracts in a trade organization, there are several agents. On the one hand the seller does not want to ship the goods onto the carrier's vessel without first receiving payment from the buyer. On the other hand the buyer does not want to pay the seller before the goods have been shipped. To solve this deadlock situation banks introduced the letter of credit: an agreement that the bank of the buyer will arrange the payment for the seller as soon as the seller can prove to the bank that he has shipped the goods. The bill of lading is issued by the carrier in return for the goods that he received from the seller. According to Article 10 of the CIMTG the bill of lading as shipment document reliably indicates that the goods have been shipped in international trade procedures. This is not a regulative norm, but a new constitutive rule added by the contract. 


\section{THE CONCEPTUAL MODEL OF CONTRACTS}

The conceptual model of the normative multiagent system is visualized in Figure 1. This figure can be interpreted using classical set theory. A box $\square$ stands for a concept and is interpreted as a set. The lines and arrows - and $\rightarrow$ are interpreted as arbitrary relations over the sets they connect. A special relation is $\longrightarrow$, which is interpreted as the subset relation over the two concepts it connects: the first set is a subset of the second set. Finally, $\multimap$ says that the singleton set $o$ is an element of $S A$. Dashed boxes and lines have the same interpretation, but refer to the normative aspects of the model of multiagent systems. These four elements occur in most conceptual modelling languages, besides other elements. For example, in class diagrams in the unified modelling language (UML), $\square$ is a class interpreted as a set of objects, - and $\rightarrow$ are associations among classes, $\rightarrow$ is the "isa" relation or subsumption relation, and $\multimap$ is "part-of" or aggregation relation.

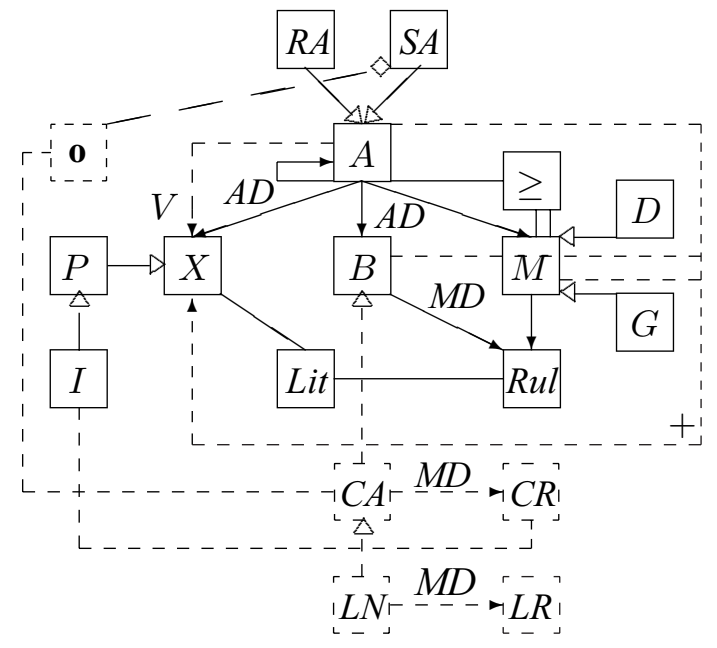

Fig. 1. Conceptual model of normative multiagent systems.

The intuitive reading of these concepts and the logical structure of the relations is detailed in the definitions below. In Section IV-A we explain the conceptual model of multiagent systems, and in Section IV-B we extend it to a normative multiagent systems by introducing violations. In Section IV-C we define how agents can change the normative system by means of constitutive rules by formalizing the constitutive rules as belief rules of the normative system. Finally in Section IV-D we define obligations in terms of desires and goals, and in Section IV-E contracts.

\section{A. Multiagent systems}

We first introduce the structural concepts and their relations. A set of propositional variables $X$ describes the different aspects of the world, and we extend it to literals built out of $X(\operatorname{Lit}(X))$ to consider also the absence of a state of affairs. Rules built out of the literals $(\operatorname{Rul}(X))$ describe the relations among the propositional variables. A rule $l_{1} \wedge \ldots \wedge l_{n} \rightarrow l$ is a pair of a set of literals built from $X$ and a literal built from $X$. The left hand side is called antecedent or body, and the right hand side is called consequent or head. Rules are used to represent the relations among propositional variables existing in the agent's mental attitudes.

Definition 1: Let $X$ be a set of variables. The set of literals built from $X$, written as $\operatorname{Lit}(X)$, is $X \cup\{\neg x \mid x \in X\}$, and the set of rules built from $X$, written as $\operatorname{Rul}(X)$, is defined by ${ }_{2}^{\operatorname{Lit}(X)} \times \operatorname{Lit}(X)$, the set of pairs of a set of literals built from $X$ and a literal built from $X$. A rule is written as $\left\{l_{1}, \ldots, l_{n}\right\} \rightarrow l$, we also write $l_{1} \wedge \ldots \wedge l_{n} \rightarrow l$ and when $n=0$ we write $\top \rightarrow l$. Moreover, for $x \in X$ we write $\sim x$ for $\neg x$ and $\sim \neg x$ for $x$.

In the multiagent system, we consider various sorts of agents. Besides real agents $R A$ (either human or artificial) we consider also socially constructed agents $S A$ like groups, normative systems and organizations. These latter agents do not exist in the usual sense of the term. Rather, they exist only as they are attributed mental attitudes by real agents. The two sets of agents are disjoint and partition the set of agents $A$.

The mental attitudes attributed to agents consist of beliefs $B$, desires $D$ and goals $G$. A mental description function $M D$ associates a rule in $\operatorname{Rul}(X)$ with each belief, desire and goal [20]. We introduce priority relations to resolve conflicts among motivations, i.e., desires and goals. A function $\geq$ associates with an agent a transitive and reflexive relation on the powerset of the motivations containing at least the subset relation. Moreover, different mental attitudes are attributed to agents by the agent description relation $A D$. It associates with each agent a set of beliefs, desires and goals. Moreover, $A D$ associates also agents with agents, because normative systems and organizations exist only as they are described as agents by other agents. Formally, a socially constructed agent $b \in S A$ exists only as some other agents attribute mental attitudes to it: $\forall b \in S A \exists a \in A: b \in A D(a)$.

Multiagent systems also contain concepts concerning informational aspects. First of all, the variables whose truth value are determined by an agent (decision variables) are distinguished from those which are not directly determined by the agent ( $P$, the parameters using Lang et al. [21]'s terminology). Besides, "institutional facts" $I$ are states of affairs existing only inside normative systems and organizations. As discussed in Section II, Searle [22] suggests that money, properties, and marriages exist only as part of social reality. Since we model social reality by means of the attribution of mental attitudes to social entities, institutional facts exist at least in the beliefs of these socially constructed agents. We associate to each agent a subset of $X$ by extending further the agent description relation $A D$. Moreover, the set of institutional facts $I$ is a subset of the parameters.

Definition 2 (MAS): A multiagent system is a tuple $\langle R A, S A, X, B, D, G, A D, M D, \geq, I\rangle$, where:

- the real agents $R A$, socially constructed agents $S A$, variables $X$, beliefs $B$, desires $D$, and goals $G$ are six finite disjoint sets. We write $A=R A \cup S A$ for all agents, and $M=D \cup G$ for motivations.

- an agent description $A D: A \rightarrow 2^{A \cup X \cup B \cup D \cup G}$ is a complete function that maps each agent to other agents that exist in its profile, to a set of variables (its decision 
variables), and to its beliefs, desires and goals. We assume that there are no cycles in the mapping of agents to agents. For each agent $a \in A$, we write $A_{a}$ for $A \cap A D(a)$, $X_{a}$ for $X \cap A D(a)$, et cetera. We write $P=X \backslash \cup_{a \in A} X_{a}$ for the parameters.

- the mental description $M D: B \cup D \cup G \rightarrow \operatorname{Rul}(X)$ is a complete function from the sets of beliefs, desires and goals to the set of rules built from $X$. For $S \subseteq B \cup D \cup G$, we write $M D(S)=\{M D(m) \mid m \in S\}$. Moreover, we write $m x \rightarrow y$ for: $m$ such that $M D(m)=x \rightarrow y$.

- a priority relation $\geq: A \rightarrow 2^{M} \times 2^{M}$ is a function from agents to a transitive and reflexive relation on the powerset of the motivations containing at least the subset relation. We write $\geq_{a}$ for $\geq(a)$.

- the institutional facts $I \subseteq \bar{P}$ are parameters.

Example 1 illustrates a highly simplified version of the running example as a multiagent system. In conceptual models used in practice as well as in the more detailed example later in this section we use meaningful names, but in Example 1 we use single letters to save space. The example describes the transaction of exchanging goods for money in terms of the motivations of the agents and the organization.

Example 1: $\langle R A, S A, X, B, D, G, A D, M D, \geq, I\rangle \quad$ with $R A=\{\mathbf{a}, \mathbf{b}\}, S A=\{\mathbf{o}\}, P=\{p, q, r, s, t\}, I=\{r, s, t\}$ and $X \backslash P, B, D, G, A D, M D$ and $\geq$ are implicitly given by the following table:

\begin{tabular}{ccccccc}
\multicolumn{1}{c}{$\mathbf{a}$} & \multicolumn{1}{c}{$\mathbf{b}$} & \multicolumn{2}{c}{$\mathbf{o}$} \\
\hline$A$ & $\mathbf{o}$ & \multicolumn{5}{c}{$\mathbf{o}$} \\
$X$ & $x_{1}$ & \multicolumn{5}{c}{$x_{2}, x_{3}$} \\
$B$ & $b_{1}$ & $x_{2} \rightarrow p$ & $b_{3}$ & $x_{3} \rightarrow p$ & $x_{4}, x_{5}$ \\
& $b_{2}$ & $x_{4} \rightarrow r$ & $b_{4}$ & $x_{4} \rightarrow r$ & $b_{5}$ & $x_{4} \rightarrow r$ \\
$D$ & $d_{1}$ & $\top \rightarrow p$ & - & & - & \\
$G$ & - & & $g_{1}$ & $\top \rightarrow x_{1}$ & $g_{2}$ & $p \rightarrow x_{1}$ \\
$\geq$ & $d_{1}>g_{1}>g_{2}$ & $g_{1}>d_{1}>g_{2}$ & $g_{2}>d_{1}=g_{1}$
\end{tabular}

This table should be read as follows. There are three agents, two real agents $\mathbf{a}$ and $\mathbf{b}$, and one socially constructed organization o. Agent a is a buyer with the desire $d_{1}$ that it receives the goods $\left(M D\left(d_{1}\right)=\top \rightarrow p\right)$, agent $\mathbf{b}$ is a seller with the goal $g_{1}$ that agent a pays for the goods $\left(M D\left(g_{1}\right)=\top \rightarrow x_{1}\right)$, and the organization has a goal $g_{2}$ that received goods are paid for. The optimal solution is that agent $\mathbf{a}$ decides $x_{1}$ and agent $\mathbf{b}$ decides $x_{2}$. Agent $\mathbf{a}$ and $\mathbf{b}$ disagree about the fact whether it is the decision $x_{2}$ or $x_{3}$ which leads to $p$. All agents agree that decision $x_{4}$ leads to institutional fact $r$. Finally, only a fragment of the priority relation is given, because it is only given for singleton motivations, whereas it is defined over sets of motivations. It says that the agents give highest priority to their own motivations, but they are social in the sense that they incorporate the other agent's motivations.

The table can be extended to deal with more detailed motivations in the obvious way. However, the example already illustrates a drawback of using only multiagent systems to describe the example, because there is no notion of obligation, violation, sanction or contract. We therefore introduce normative multiagent systems and extend the example below.

\section{B. Normative multiagent systems}

Boella and Lesmo [12] formalize the relation between multiagent systems and normative systems by attributing mental states to agents as well as to normative systems. Thus, a normative system can metaphorically be considered as a socially constructed agent. The use of the agent metaphor may be seen as an instance of Dennett's intentional stance [23] and is inspired by the interpretation of normative multiagent systems as dynamic social orders. According to Castelfranchi [24], a social order is a pattern of interactions among interfering agents "such that it allows the satisfaction of the interests of some agent", like a shared goal or a value that is good for (almost) all the agents. For example, the interests may include the avoidance of accidents or a fair society. In this approach the obligations of the agents can be formalized as desires or goals of the normative system. This representation may be paraphrased as "your wish is my command", because the desires or wishes of the normative system are the obligations or commands of the real agents. The agents attribute to the normative system also the ability to autonomously enforce the conformity of the agents to the norms by means of sanctions.

The advantage of the agent metaphor is that game theory can be used to describe the interaction among agents and the normative system in terms of games. For example, an agent considers whether its actions will lead to a reaction of the normative system such as sanctions. An agent can understand when it can evade sanctions by for example ensuring that the normative system does not observe its behavior, or by bribing the system. Moreover, a legislator can play a game with the normative system and another agent to see whether a new norm it introduces will be complied with, and which kind of sanctions it has to associate with the norm to achieve the desired behavior. In Section V we give an example of a game in contracting.

A normative multiagent system contains a normative system $\mathbf{o} \in S A$, which we formalize as a socially constructed agent. Moreover, it contains a norm (violation) description $V: A \times \operatorname{Lit}(X) \rightarrow X_{\mathbf{o}} \cup P$, a function from agents and literals to the decision variables of the normative system together with the parameters. We write $V_{a}(x)$ for the variable representing that $x \in \operatorname{Lit}(X)$ is a violation by agent $a \in A$.

Definition 3: A normative multiagent system is a tuple $\langle R A, S A, X, B, D, G, A D, M D, \geq, I, \mathbf{o}, V\rangle$ extending a multiagent system with:

- a socially constructed agent $o \in S A$ representing the normative system, such that for all real agents $a \in R A$, we have o $\in A_{a}$.

- a (partial) function $V: A \times \operatorname{Lit}(X) \rightarrow X_{\mathbf{o}} \cup P$ from agents and literals to the decision variables of the normative system and the parameters. We write $V_{a}(x)$ for $V(a, x)$.

Example 2 (Continued): The socially constructed agent o in Example 1 is now interpreted as an organization constituting the normative system. Moreover, assume that $V$ is defined as follows. $V_{\mathbf{a}}\left(\neg x_{1}\right)=s, V_{\mathbf{b}}(\neg p)=t$, and $V_{x}(y)=$ undefined for all other values of $x$ and $y$. Thus, if $s$ is the case, then $\neg x_{1}$ is recognized as a violation of agent $\mathbf{a}$, and if $t$ is the case, then $\neg p$ is recognized as a violation of agent $\mathbf{b}$. 


\section{Counts-as conditionals and self-modification}

Socially constructed agents like normative systems and organizations are able to change themselves to adapt to new situations. Self modifying normative multiagent systems contain additions to the agent description, also known as expansions, which are defined as $+: A \times(B \cup D \cup G) \rightarrow X$, i.e., as mappings from mental attitudes to propositional variables for each agent. Deletions (also known as contractions) can be defined analogously, and revisions can be defined as a combination of a deletion and an addition. In this paper we do not consider the formalization of deletion and revision, nor any other update to the normative multiagent system, to keep the formal machinery to a minimum.

Since institutional facts $I$, and among them the additions to the mental state attributed to the normative system, are parameters not directly controlled by the agent, we use belief rules of the normative system to express how they can be made true. Rules concerning beliefs about institutional facts are called constitutive rules and represent the "counts-as" relations $(C A)$ introduced by Searle [22] (see Section II). We do not define $C A$ in terms of the other concepts, but we include $C A$ in the extended normative multiagent systems, to cater for the possibility that there are also belief rules of the normative system not being counts-as conditionals, but still implying institutional facts.

Definition 4 (SNMAS): A self modifying normative multiagent system is represented by a tuple $\langle R A, S A, X, B, D, G, A D, M D, \geq, I, \mathbf{o}, V,+, C A\rangle$ extending a normative multiagent system with:

- Additions are a partial function $+: A \times(B \cup D \cup G) \rightarrow X$, such that for all $m \in B \cup M$, if $+_{\mathbf{o}}(m)$ is defined, then $+_{\mathbf{o}}(m) \in I$. We write $+_{a}(m)$ for $+(a, m)$.

- Counts-as conditionals $C A \subseteq B_{\mathrm{o}}$ or constitutive norms are beliefs of the normative system $\mathbf{0}$, such that constitutive rules $C R=M D(C A)$ are rules whose heads are institutional facts in $I$.

- Constitutive norms with legal effects $(L N)$ are the beliefs of the normative system of which the heads of the rules $L R=M D(L N)$ are additions.

The update of a $S N M A S$ by a set of literals $L \subseteq \operatorname{Lit}(X)$ is $A D_{a}^{\prime}=A D_{a} \cup\left\{m \mid+{ }_{a}(m) \in L\right\}$.

Definition 5 (Counts-as): Given a self modifying normative multiagent system SNMAS as $\langle R A, S A, X, B, D, G, A D, M D, \geq, I, \mathbf{o}, V,+, C A\rangle$, we write $S N M A S=$ counts-as $\mathbf{s}_{\mathbf{o}}(Y, p)$ if $Y \subseteq \operatorname{Lit}(X), p \in I$ and $\exists m \in C A$ such that $M D(m)=Y \rightarrow p$.

The running example illustrates counts-as conditionals.

Example 3 (Continued): If $C A=\left\{b_{5}\right\}$, then we have $S N M A S=$ counts-as $\mathbf{o}\left(x_{4}, r\right)$. Moreover, if there is a $b_{6} \in B$ not associated with any agent, with $M D\left(b_{6}\right)=\neg p \rightarrow V_{\mathbf{b}}(\neg p)$, and $+_{\mathbf{o}}\left(b_{6}\right)=r,+_{x}(y)=$ undefined for other values of $x$ and $y$, then $b_{5} \in L N$ is a constitutive norm with legal effects, and we have $S N M A S=$ counts- $a s_{\mathbf{o}}\left(x_{4},+_{\mathbf{o}}\left(b_{6}\right)\right)$. If the normative system decides $x_{4}$, then it counts as the addition of the rule $\neg p \rightarrow V_{\mathbf{b}}(\neg p)$, i.e., that if the goods are not received, then it is a violation of agent $\mathbf{b}$.

\section{Obligations}

Before we can define contracts, we must introduce obligations. We consider obligations of agent a only, which are defined in terms of goals and desires of the addressee of the norm $\mathbf{a}$ and of a normative system $\mathbf{o}$. Obligations of agent $\mathbf{b}$ can be defined analogously.

The definition of obligation contains several clauses. The first clause says that the obligation is in the content of the desires and in the goals of normative system o ("your wish is my command"). The second and third clause can be read as "the absence of $x$ is considered as a violation". The association of obligations with violations is inspired by Anderson's reduction of deontic logic to alethic modal logic [25]. The third clause says that the normative system desires that there are no violations. The fourth and fifth clause relate violations to sanctions and assume that normative system $\mathbf{0}$ is motivated to apply sanctions only as long as there is a violation; otherwise the norm would have no effect. Finally, for the same reason, we assume in the last clause that the agent does not like the sanction.

Definition 6 (Obligation): Let $\quad S N M A S=$ $\langle R A, S A, X, B, D, G, A D, M D, \geq, I, \mathbf{o}, V,+, C A\rangle \quad$ be a self modifying normative multiagent system. Agent $\mathbf{a} \in A$ is obliged to decide to do $x \in \operatorname{Lit}\left(X_{\mathbf{a}} \cup P\right)$ with sanction $s \in \operatorname{Lit}\left(X_{\mathbf{o}} \cup P\right)$ if $Y \subseteq \operatorname{Lit}\left(X_{\mathbf{a}} \cup P\right)$ in $S N M A S$, written as $S N M A S \models O_{\text {ao }}(x, s \mid Y)$, if and only if:

1) $Y \rightarrow x \in M D\left(D_{\mathbf{o}}\right) \cap M D\left(G_{\mathbf{o}}\right)$ : if normative system $\mathbf{0}$ believes $Y$, then it desires and has as a goal that $x$.

2) $Y \cup\{\sim x\} \rightarrow V_{\mathbf{a}}(\sim x) \in M D\left(D_{\mathbf{o}}\right) \cap M D\left(G_{\mathbf{o}}\right)$ : if normative system $\mathbf{o}$ believes $Y$ and $\sim x$, then it has the goal and the desire $V_{\mathbf{a}}(\sim x)$ : to recognize it as a violation by agent $\mathbf{a}$.

3) $\top \rightarrow \neg V_{\mathbf{a}}(\sim x) \in M D\left(D_{\mathbf{o}}\right)$ : normative system o desires that there are no violations.

4) $Y \cup\left\{V_{\mathbf{a}}(\sim x)\right\} \rightarrow s \in M D\left(D_{\mathbf{o}}\right) \cap M D\left(G_{\mathbf{o}}\right)$ : if normative system $\mathbf{o}$ believes $Y$ and decides $V_{\mathbf{a}}(\sim x)$, then it desires and has as a goal that it sanctions agent a with $s$.

5) $Y \rightarrow \sim s \in M D\left(D_{\mathbf{o}}\right)$ : if normative system o believes $Y$, then it desires not to sanction, $\sim s$. This desire of the normative system expresses that it only sanctions in case of violation.

6) $Y \rightarrow \sim s \in M D\left(D_{\mathbf{a}}\right)$ : if agent a believes $Y$, then it desires $\sim s$, which expresses that it does not like to be sanctioned.

The following example illustrates an obligation of agent a. To increase readability of our running example, from now on we use long names for variables. We use pay instead of $x_{1}$, and we write shipped for an institutional fact following from $x_{2}$.

Example 4 (Cont.): SNMAS $=O_{\text {ao }}($ pay, san|shipped $)$, agent $\mathbf{a}$ is obliged to pay ( pay $\in X_{\mathbf{a}}$ ) in case the requested good has been shipped (shipped $\in I$ ) or else it is sanctioned with san $\in \operatorname{Lit}\left(X_{\mathbf{o}}\right)$, if:

$$
\begin{aligned}
g_{3}, d_{2} & \text { shipped } \rightarrow \text { pay } \in G_{\mathbf{o}}, D_{\mathbf{o}} \\
g_{4}, d_{3} & \text { shipped } \wedge \neg \text { pay } \rightarrow V_{\mathbf{a}}(\neg \text { pay }) \in G_{\mathbf{o}}, D_{\mathbf{o}} \\
d_{4} & \top \rightarrow \neg V_{\mathbf{a}}(\neg \text { pay }) \in D_{\mathbf{o}} \\
g_{5}, d_{5} & \text { shipped } \wedge V_{\mathbf{a}}(\neg \text { pay }) \rightarrow \text { san } \in G_{\mathbf{o}}, D_{\mathbf{o}} \\
d_{6}, d_{7} & \text { shipped } \rightarrow \neg \text { san } \in D_{\mathbf{o}}, D_{\mathbf{a}}
\end{aligned}
$$




\section{E. Contracts}

The creation of a contract is represented by an institutional fact $c \in I$. The creation of the contract $c$ is introduced as an intermediary between the agreement and its legal effects, because it allows decoupling the conditions of the creation of the institutional facts from additions of its legal effects. For example, consider a contract $c$ created by the signatures of two agents. Using the decoupling a new way of creating the contract by means of an electronic signature can be specified, maintaining the same rules specifying its legal effects. Since a contract counts as several additions, $c$ works as an abstraction. The contract unifies all its legal effects, rather than connecting the signatures of the agents directly with the additions.

In our model contracts change the norms of a normative system or organization according to what is specified by the normative systems itself, because they are part of the beliefs attributed to the system. A contract is created only if there is some fact - either a brute fact in the world or another institutional fact - counting as $c$ for the normative system o. The effect of creating a contract is to modify the mental attitudes of the normative system. Usually, it adds some rules to the beliefs $B_{\mathbf{o}}$, the desires $D_{\mathbf{o}}$, or the goals $G_{\mathbf{o}}$ by an addition $+_{\mathbf{o}}(m)$ where $m \in B \cup M$. The additions are institutional facts: they are made true only if the normative system $\mathbf{o}$ believes that they are made true by the creation of the contract. E.g., $b \in C A \subseteq B_{\mathbf{o}}$ such that $M D(b)=c \rightarrow+_{\mathbf{o}}(m)$ is a constitutive rule read as " $c$ counts as the addition $+_{\mathbf{o}}(m)$." In summary, a contract is defined as follows.

Definition 7 (Contract): Given a self modifying system $\langle R A, S A, X, B, D, G, A D, M D, \geq, I, \mathbf{o}, V,+, C A\rangle$ called $S N M A S$, we write $S N M A S \models \operatorname{contract}(c, E \mid Y)$ where $E \subseteq\{i \in I \mid \exists m: i=+(m)\}$ and $Y \subseteq \operatorname{Lit}(X)$ if:

1) $c \in I$ is an institutional fact representing that the contract has been created.

2) $S N M A S \models$ counts- $a s_{\mathbf{o}}(Y, c): Y$ counts as the creation of the contract.

3) $S N M A S=$ counts- $a s_{\mathbf{o}}\left(c,+_{\mathbf{o}}(m)\right)$ for each $+_{\mathbf{o}}(m)$ in $E$ : the creation of the contract counts as the set of additions $E$.

Our running example about contracts in trade organizations illustrates how a contract can create the obligation to pay for shipped goods. The contract is created by a signature of agent $\mathbf{a}$, and has as legal effects three goals and desires.

Example 5: [Continued] Assume that agent a signing the contract, represented by sign $\in X_{\mathbf{a}}$, is a sufficient condition for the contract $c \in I$ to be created. Moreover, assume that SNMAS contains $d_{4}, d_{6}$ and $d_{7}$ of Example 4, representing that agent a does not like to be sanctioned and organization $\mathbf{0}$ does not like violations and sanctions. Finally, assume that the creation $c$ of the contract achieves the following effects on the mental attitudes of the organization $\mathbf{o}$. It adds the goal and desire that shipped goods are paid for $\left(g_{3}, d_{2}\right)$, the goal and desire to consider the lack of payment for shipped goods as a violation $\left(g_{4}, d_{3}\right)$, and the goal and desire to sanction violations $\left(g_{5}, d_{5}\right)$.

Under these assumptions, the creation of the obligation to pay for shipped goods as part of the contract created by the signature is represented by $S N M A S \mid=\operatorname{contract}(c, E \mid \operatorname{sign})$, when the following holds.

$$
\begin{aligned}
& E=\left\{+_{\mathbf{o}}\left(g_{3}\right),+_{\mathbf{o}}\left(d_{2}\right),+_{\mathbf{o}}\left(g_{4}\right),+_{\mathbf{o}}\left(d_{3}\right),+_{\mathbf{o}}\left(g_{5}\right),+_{\mathbf{o}}\left(d_{5}\right)\right\} \\
& S N M A S=\text { counts-as } \mathbf{o}(\text { sign }, c)\left(b_{7} \text { sign } \rightarrow c \in B_{\mathbf{o}}\right) \text { and, } \\
& S N M A S=\text { counts-as }\left(c,+_{\mathbf{o}}(m)\right) \text { for each }+_{\mathbf{o}}(m) \text { in } E: \\
& \left\{\quad b_{8} c \rightarrow+_{\mathbf{o}}\left(g_{3}\right), \quad b_{9} c \rightarrow+_{\mathbf{o}}\left(d_{2}\right),\right. \\
& b_{10} c \rightarrow+_{\mathbf{o}}\left(g_{4}\right), \quad b_{11} c \rightarrow+_{\mathbf{o}}\left(d_{3}\right), \\
& \left.\quad b_{12} c \rightarrow+_{\mathbf{o}}\left(g_{5}\right), \quad b_{13} c \rightarrow+_{\mathbf{o}}\left(d_{5}\right)\right\} \subseteq B_{\mathbf{o}}
\end{aligned}
$$

The following example illustrates that the contract can create a counts-as conditional specifying an institutional fact to be used in the interaction. The contract specifies that a document called bill-of-lading counts as the institutional fact that the goods have been shipped.

Example 6 (Continued): Assume that the contract specifies that the fact that the good has been shipped is an institutional fact shipped $\in I$, which holds if there is some document like the so-called bill of lading (bill $\in P$ ) issued by a third party [11]. With this addition of the contract we have $S N M A S \models$ contract $\left(c, E \cup\left\{{ }_{0}\left(b_{14}\right\} \mid\right.\right.$ sign $)$ : the constitutive rule $b_{15} c \rightarrow+_{\mathbf{o}}\left(b_{14}\right) \in B_{\mathbf{o}}$ creates another constitutive rule $M D\left(b_{14}\right)=$ bill $\rightarrow$ shipped, which is added to the beliefs of the organization $\mathbf{o}$ by the addition $+_{\mathbf{o}}\left(b_{14}\right)$ as a consequence of the contract $c$.

We could also add the other agents to the example, such as the trusted third party in the above example, or the shipper and the banks. Moreover, due to the uniform representation of facts and the creations of norms, these constitutive norms can be nested to formalize arbitrarily complex creations. As Searle [22] observes, this nesting of counts-as conditionals leads to the complexity of social reality in which we live. For example, Example 5 and 6 illustrates that constitutive rules created by contracts can eventually introduce new obligations and new constitutive rules. In this way a contract can specify how new obligations may arise during the interaction of the agents. Likewise, contract frames can be defined as contracts describing the creation of other contracts.

Example 7 (Continued): Assume that if an agent does not pay the fee for a shipped good, it is obliged to pay a double sum of money $\left(\right.$ pay $\left._{2}\right): O_{\mathbf{a o}}\left(\mathrm{pay}_{2}, \mathrm{san}_{2} \mid \mathrm{T}\right)$ [1]. This obligation is not a preexisting conditional obligation: it is created as a legal consequence of an event, the sanction san for not having paid the fee. The sanction san, in this case, rather than being a direct punishment for agent a, counts as the action of creating a second obligation. Note that this obligation does not exist until the normative system recognizes a violation and applies the sanction san. This part of the contract is thus represented by the constitutive rules creating further constitutive rules about the creation of goals and desires (where $\operatorname{san}_{2} \in X_{\mathbf{o}}$ is a sanction both feared by agent $\mathbf{a}$ and not desired by organization o). E.g., some of the clauses of the obligation are as follows.

$$
\begin{aligned}
& b_{16} c \rightarrow+_{\mathbf{o}}\left(b_{19} \text { san } \rightarrow+_{\mathbf{o}}\left(g_{6} \top \rightarrow \text { pay }_{2}\right)\right) \\
& b_{17} c \rightarrow+_{\mathbf{o}}\left(b_{20} \text { san } \rightarrow+_{\mathbf{o}}\left(g_{7} \neg p a y_{2} \rightarrow V_{\mathbf{a}}\left(\neg p a y_{2}\right)\right)\right) \text {, } \\
& b_{18} c \rightarrow+_{\mathbf{o}}\left(b_{21} \text { san } \rightarrow+_{\mathbf{o}}\left(g_{8} V_{\mathbf{a}}\left(\neg \text { pay }_{2}\right) \rightarrow \operatorname{san}_{2}\right)\right)
\end{aligned}
$$

Due to space limitations, in the following we restrict ourselves to the creation of the obligation in Example 5 and the creation of the counts-as conditional in Example 6. 


\section{GAMES With CONTRACTS}

In this section we define violation games in which an agent recursively models the normative system. Violation games are the simplest and most frequent kind of games and involve only two agents. Negotiation games in which an agent models another agent recursively modelling the normative system, can be defined in our conceptual model of normative multiagent systems too. More complex games like negotiation games extend violation games with additional levels of recursion.

\section{A. Recursive modeling}

The game theory is based on an agent decision model that uses belief rules to calculate the effects of decisions, and desire and goal rules to evaluate decisions. This is also where the updates of the rules are incorporated. Since we only consider rule additions and no other updates of the normative multiagent system, we assume that all relevant beliefs, desires and goals together with their priorities are already present in the system, such that we only have to adapt the agent description $A D$.

To define the effects of decisions, we first address the question how rules are applied such that the normative system is modified. Even without counts-as conditionals, there are several ways to define the application of rules. This is studied, for example, by Makinson and van der Torre using so-called input/output logics [26], where the input of a set of propositional formulas produces as output a set of propositional formulas using a set of rules expressed as pairs of propositional formulas. Here we use so-called reusable output, where the input is not necessarily part of the output, rules can be applied one after the other, and without so-called reasoning by cases. The following definition extends reusable output logic to incorporate a way to deal with additions. Instead of working with a fixed set of rules, at every step of the calculation of $o u t^{i}$ the set of rules $R^{i}$ can be updated too. The output of applying a set of rules $R$ on a set of literals $S$ with additions $E$ is written as $\operatorname{out}(S, R, E)$.

Definition 8 (Applying rules): The set of rule additions defined on $X$ and $I$, written as $\operatorname{Add}(I, X)=I \times \operatorname{Rul}(X)$, is the set of all pairs of institutional facts and rules. We define out : $2^{\operatorname{Lit}(X)} \times 2^{\operatorname{Rul}(X)} \times 2^{\operatorname{Add}(I, X)} \rightarrow 2^{\operatorname{Lit}(X)}$ as a function from a set of literals, a set of rules and a set of rule additions to a set of literals. $\operatorname{out}(S, R, E)$ is the closure of a set of literals $S$ under the rules $R$ updated by rule additions $E$.

- $R^{0}(S, R, E)=R$

- out ${ }^{0}(S, R, E)=\emptyset$

- $R^{k+1}(S, R, E)=R^{k}(S, R, E) \cup$ $\left\{r \in \operatorname{Rul}(X) \mid(i, r) \in E\right.$ and $\left.i \in S \cup o u t^{k}(S, R, E)\right\}$

- out ${ }^{k+1}(S, R, E)=$ out $^{k}(S, R, E) \cup$

$\left\{l \mid L \rightarrow l \in R^{k}(S, R, E)\right.$ and $\left.L \subseteq S \cup o u t^{k}(S, R, E)\right\}$

- $\operatorname{out}(S, R, E)=\cup_{o}^{\infty}$ out $^{k}(S, R, E)$

Decisions of agents are sets of literals built from decision variables which do not imply a contradiction. The input/output logic is used in the game theory to define the closure of a decision under a set of belief rules. When calculating the effects of decisions, the rule additions are the inverse of the addition function + mapped to rule descriptions. We describe the new belief rules of agent $\mathbf{a}$ as additions $E_{\mathbf{a}}^{B}$. We exclude the cases in which agent a makes a decision which normative system o does not hold possible, since we do not incorporate how normative system $\mathbf{0}$ revises its beliefs in case of such a surprise.

Definition 9 (Decisions): Let the set of belief additions of agent a be $E_{\mathbf{a}}^{B}=\left\{(i, M D(b)) \mid b \in B,+_{\mathbf{a}}(b)=i\right\}$, the belief extension of agent a of a set of literals $S \subseteq \operatorname{Lit}(X)$ be $S \cup \operatorname{out}\left(S, B_{\mathbf{a}}, E_{\mathbf{a}}^{B}\right)$, and analogously for normative system $\mathbf{o}$. The set of decision profiles $\Delta$ is the set of sets $\delta=\delta_{\mathbf{a}} \cup \delta_{\mathbf{o}}$ with $\delta_{\mathbf{a}} \subseteq \operatorname{Lit}\left(X_{\mathbf{a}}\right)$ and $\delta_{\mathbf{o}} \subseteq \operatorname{Lit}\left(X_{\mathbf{o}}\right)$ such that the belief extensions of agent $\mathbf{a}$ and of normative system $\mathbf{o}$ do not contain a variable and its negation.

The logic is illustrated in an extension of Example 3.

Example 8 (Continued): The effects of the beliefs of $a$ are $\operatorname{out}\left(B_{\mathbf{a}},\left\{x_{4}, \neg p\right\}, E_{\mathbf{a}}^{B}\right)=\left\{r=+_{\mathbf{o}}\left(b_{6}\right), s=V_{\mathbf{b}}(\neg p)\right\}$ : if the normative system decides $x_{4}$ and the goods are not received, then it is a violation.

We assume that agent a only considers its own motivations (and is in this sense selfish), which can be relaxed in the obvious way, and that normative system o considers its own motivations including the new rules. The unfulfilled motivations of decision profile $\delta=\delta_{\mathbf{a}} \cup \delta_{\mathbf{o}}$ are the motivations whose body is part of the closure of the decision under the belief rules but whose head is not. Given a decision $\delta_{\mathbf{a}}$, a decision $\delta_{\mathbf{o}}$ is optimal for normative system $\mathbf{o}$ if it minimizes the unfulfilled motivational attitudes in $D_{\mathbf{o}}$ and $G_{\mathbf{o}}$ according to the $\geq_{\mathbf{o}}$ relation. The decision of agent $\mathbf{a}$ is more complex. When agent $\mathbf{a}$ takes its decision $\delta_{\mathbf{a}}$ it minimizes its unfulfilled motivational attitudes. But when it considers these attitudes, it must not only consider its decision $\delta_{\mathbf{a}}$ and the consequences of this decision. It must consider also the decision $\delta_{\mathbf{o}}$ of the normative system $\mathbf{o}$ and its consequences, for example that it is sanctioned by normative system o. So agent a recursively considers which decision normative system o will take depending on its different decisions $\delta_{\mathbf{a}}$.

Definition 10 (Recursive modelling): Let $S_{\mathbf{a}}(\delta)$ and $S_{\mathbf{o}}(\delta)$ be the belief extensions of decision profile $\delta$ for agent a and $\mathbf{o}$ respectively, and unfulfilled motivations of agent $\mathbf{a}$ and normative system $\mathbf{o}$ defined as follows.

$$
\begin{aligned}
& U(\delta, \mathbf{a})=\left\{m \in M_{\mathbf{a}} \mid M D(m)=l_{1} \wedge \ldots \wedge l_{n} \rightarrow l,\right. \\
& \left.\left\{l_{1}, \ldots, l_{n}\right\} \subseteq S_{\mathbf{a}}(\delta) \text { and } l \notin S_{\mathbf{a}}(\delta)\right\} \\
& U(\delta, \mathbf{o})=\left\{m \in M_{\mathbf{o}} \cup\left\{m^{\prime} \in M \mid+_{\mathbf{o}}\left(m^{\prime}\right) \in S_{\mathbf{o}}(\delta)\right\} \mid\right. \\
& M D(m)=l_{1} \wedge \ldots \wedge l_{n} \rightarrow l \text { and } \\
& \left.\left\{l_{1}, \ldots, l_{n}\right\} \subseteq S_{\mathbf{o}}(\delta) \text { and } l \notin S_{\mathbf{o}}(\delta)\right\}
\end{aligned}
$$

- A decision profile $\delta_{\mathbf{a}} \cup \delta_{\mathbf{o}}$ is optimal for normative system $\mathbf{o}$ if and only if there is no decision of the normative system $\delta_{\mathbf{o}}^{\prime}$ such that $U\left(\delta_{\mathbf{a}} \cup \delta_{\mathbf{o}}, \mathbf{o}\right)>_{\mathbf{o}} U\left(\delta_{\mathbf{a}} \cup \delta_{\mathbf{o}}^{\prime}, \mathbf{o}\right)$.

- A decision profile $\delta_{\mathbf{a}} \cup \delta_{\mathbf{o}}$ is optimal for agent $\mathbf{a}$ and normative system $\mathbf{o}$ if and only if it is optimal for normative system $\mathbf{o}$ and there is no decision $\delta_{\mathbf{a}}^{\prime}$ such that for all decision profiles $\delta_{\mathbf{a}} \cup \delta_{\mathbf{o}}^{\prime \prime}$ and $\delta_{\mathbf{a}}^{\prime} \cup \delta_{\mathbf{o}}^{\prime}$ optimal for normative system o we have $U\left(\delta_{\mathbf{a}} \cup \delta_{\mathbf{o}}^{\prime \prime}, \mathbf{a}\right)>_{\mathbf{a}} U\left(\delta_{\mathbf{a}}^{\prime} \cup \delta_{\mathbf{o}}^{\prime}, \mathbf{a}\right)$.

Negotiation games involving all three agents $\mathbf{a}, \mathbf{b}$ and $\mathbf{o}$ can be defined analogously. For example, to find out whether agent a will fulfill its obligations, agent $\mathbf{b}$ has to consider his beliefs about agent a's beliefs about normative system o, extending the violation game with another level of recursion. 


\begin{tabular}{|c|c|c|c|}
\hline & $\delta_{\mathbf{a}}$ & out $(\delta, B, E)$ & o's perspective \\
\hline $\mathbf{o}_{1}$ & $V(\ldots)$, san & sign, bill, $c, E=\{+g(\ldots), \ldots\}$, shipped & $\top \rightarrow \neg V(\ldots)$, shipped $\rightarrow \neg$ san \\
\hline $\mathbf{O}_{2}$ & $V(\ldots), \neg \operatorname{san}$ & sign, bill, $c, E=\{+g(\ldots), \ldots\}$, shipped & $\top \rightarrow \neg V(\ldots)$ \\
\hline $\mathbf{O}_{3}$ & $\neg V(\ldots)$, san & sign, bill, $c, E=\{+g(\ldots), \ldots\}$, shipped & shipped $\wedge V(\ldots) \rightarrow$ san, shipped $\rightarrow \neg$ san \\
\hline $\mathbf{O}_{4}$ & $\neg V(\ldots), \neg \operatorname{san}$ & sign, bill, $c, E=\{+g(. .),. \ldots\}$, shipped & \\
\hline $\mathbf{a}_{1}$ & $\mathrm{~V}(\ldots)$, san & sign, bill & $\top \rightarrow \neg$ pay, shipped $\rightarrow \neg$ san \\
\hline $\mathbf{a}_{2}$ & $\mathrm{~V}(\ldots), \neg$ san & sign, bill & $\top \rightarrow \neg$ pay \\
\hline $\mathbf{a}_{3}$ & $\neg \mathrm{V}(\ldots)$, san & sign, bill & $\top \rightarrow \neg$ pay, shipped $\rightarrow \neg$ san \\
\hline $\mathbf{a}_{4}$ & $\neg V(\ldots), \neg \operatorname{san}$ & sign, bill & $\top \rightarrow \neg p a y$ \\
\hline $\mathbf{a}_{5}$ & $V(\ldots)$, san & sign, bill & shipped $\rightarrow \neg$ san \\
\hline $\mathbf{a}_{6}$ & $\mathrm{~V}(\ldots), \neg \mathrm{san}$ & sign, bill & \\
\hline $\mathbf{a}_{7}$ & $\neg \mathrm{V}(\ldots)$, san & sign, bill & shipped $\rightarrow \neg$ san \\
\hline $\mathbf{a}_{8}$ & $\vdots \quad \neg \neg(\ldots), \neg$ san & sign, bill & o's perspective \\
\hline $\mathbf{O}_{5}$ & $\lambda^{V(\ldots)}, \operatorname{san}$ & sign, bill, $c, E=\{+g(\ldots), \ldots\}$, shipped & shipped $\rightarrow$ pay, $\top \rightarrow \neg V(\ldots)$, shipped $\rightarrow \neg$ san \\
\hline $\mathbf{O}_{6}$ & $V(\ldots), \neg \operatorname{san}$ & sign, bill, $c, E=\{+g(\ldots), \ldots\}$, shipped & shipped $\rightarrow$ pay, $\top \rightarrow \neg V(\ldots)$, shipped $\wedge V(\ldots) \rightarrow$ san \\
\hline $\mathbf{O}_{7}$ & $\neg V(\ldots)$, san & sign, bill, $c, E=\{+g(\ldots), \ldots\}$, shipped & shipped $\rightarrow$ pay, shipped $\wedge \neg$ pay $\rightarrow V(\ldots)$, shipped $\rightarrow \neg$ san \\
\hline $\mathbf{O}_{8}$ & $\neg \neg(\ldots), \neg \operatorname{san}$ & sign, bill, $c, E=\{+g(\ldots), \ldots\}$, shipped & shipped $\rightarrow$ pay, shipped $\wedge \neg$ pay $\rightarrow V(\ldots)$ \\
\hline
\end{tabular}

Fig. 2. An example of the game between agent a and organization $\mathbf{0 .}$

\section{B. Example}

The violation game concerns a trade contract that creates the obligation to pay for shipped goods, enforced by a sanction, and the constitutive rule saying that the bill of lading counts as the institutional fact that the goods have been shipped, as discussed in Example 5 and 6. The extensive game tree visualized in Figure 2 is based on the rules discussed in the examples thus far, together with additional rules and priorities among rules discussed below. Both agent a and organization o believe that agent a has already signed the contract and the bill of lading bill $\in P$ has been issued.

$\left\{b_{22} \top \rightarrow\right.$ sign, $b_{23} \top \rightarrow$ bill $\} \subseteq B_{\mathbf{a}}$

$\left\{b_{24} \top \rightarrow\right.$ sign, $b_{25} \top \rightarrow$ bill $\} \subseteq B_{\mathbf{o}}$

For agent $\mathbf{a}$, not being sanctioned has a higher priority than not paying. For organization o, in case of conflict counting behavior as a violation has a higher priority than not doing so, and sanctioning violations has a higher priority than not doing so.

$$
\begin{aligned}
& \{\text { shipped } \rightarrow \neg \text { san }\}>_{\mathbf{a}}\{\top \rightarrow \neg \text { pay }\} \\
& \left\{\text { shipped } \wedge \neg \text { pay } \rightarrow V_{\mathbf{a}}(\neg \text { pay }),\right\}>_{\mathbf{o}}\left\{\top \rightarrow \neg V_{\mathbf{a}}(\neg \text { pay })\right\} \\
& \left\{\text { shipped } \wedge V_{\mathbf{a}}(\neg \text { pay }) \rightarrow \text { san }\right\}>_{\mathbf{o}}\{\text { shipped } \rightarrow \neg \text { san }\}
\end{aligned}
$$

Figure 2 should be read as follows. The branches represent decisions of the agents. Real agent $\mathbf{a} \in R A$ has two alternatives, to pay or not to pay. Organization $\mathbf{o} \in S A$ has four alternatives, to count agent a's behavior as a violation or not, and to sanction it or not. A complete path from left to right thus represents a decision profile. The leaves of the tree represent the belief extensions of the agents for the related decision profile, calculated using the input/output logic. As discussed in Examples 5 and 6, the believed consequences of the normative system contain the legal effects of the contract, represented by a set of additions. It also includes the consequences of the added constitutive norm, leading to the occurrence of shipped in some nodes. The rightmost column represents the unfulfilled desires and goals, calculated by considering for each relevant rule whether the body of the rule is implied by the believed effects of the decision, without the head being implied. For the normative system also the added rules of the contract are considered, occurring as the unfulfilled desires and goals in the right most column of the figure. The viewpoint of organization $\mathbf{0}$ is visualized in rectangular boxes on the bottom and the top, and the viewpoint of agent $\mathbf{a}$ is in between.

The violation games adopt the viewpoint of agent a deciding whether to pay its fee or not, for which it recursively models the organization $\mathbf{o} \in A_{\mathbf{a}}$. For each node we visualize the optimal decision in boldface. Consider first the viewpoint of the organization visualized in rectangular boxes. The top four lines indicate that if agent a pays, then it does not count it as a violation and does not sanction the agent (organization $\mathbf{0}$ prefers profile $o_{4}$ to profiles $o_{1}-o_{3}$ ), and the bottom four lines indicate that if agent a does not pay, then it sanctions the agent (organization o prefers profile $O_{5}$ to profiles $o_{6}-o_{8}$ ). Consequently agent a can choose among only profile $a_{4}$ and profile $a_{5}$, of which it chooses profile $a_{4}$. Note that the belief extensions and unfulfilled motivations of $a_{1}-a_{3}$ and $a_{6}-a_{8}$ do not have to be calculated to obtain this result.

The example illustrates why the definition of obligation contains six clauses. Clauses 2-6 are all necessary to derive that selfish agent a will fulfill the obligation. Clause 1 of the definition is not necessary in this example, but it is used to derive the same result for respectful agents internalizing the goals of the normative system as their own goals. There are many ways in which the example can be modified such that no longer can be derived that agent a will pay, including many subtle variants of fraud and deception. 


\section{RELATED WORK}

In this paper we use a qualitative game-theoretic approach different from the classical quantitative one based on utility functions, probability distributions, and equilibria analysis. Starting with the pioneering work of Herbert Simon and others in the 50s, many alternative conceptualizations and formalizations of decision making have been proposed in artificial intelligence and agent theory as a response to the ideality assumptions of classical decision and game theory. For example, utility functions have been replaced by goals and desires, probability functions by beliefs, and decision rules by a process of deliberation. See Dastani et al. [27] for a comparison between classical decision and game theory and alternative qualitative theories developed in artificial intelligence and agent theory.

More complex games have been defined for normative multiagent systems introduced in this paper. Due to space limitations, in this paper we only consider two stage games. In [28] we define games with multiple agents and multiple stages, but without contracts or self-modification. Based on a hierarchy, agents model the behavior of the next agent in the hierarchy. Moreover, in [29] we define norm creation games from the viewpoint of the normative system recursively modelling an agent to find out whether it will violate the new norm, which just like negotiation games extend violation games with another level of recursion.

Moreover, normative multiagent systems have been studied which are in some aspects more general than the one used in this paper. For example, in [28] we define multiple normative systems playing the role of local and global authorities. The central question concerning the interaction among normative systems is how the global authority uses global policies to control the local authorities with their local policies. The local authorities are again not forced but motivated, for which we consider a kind of nested obligations and permissions.

There are several other issues in normative multiagent systems relevant for the game-theoretic approach to contracts. In [30] we study how sanctions are negotiated among agents in the context of negotiating the distribution of obligations. Instead of defining obligations as a set of explicitly given goals and desires, we can also use an input/output logic to state that the mental attitudes logically follow from the mental states of the agents. Moreover, permissions, prohibitions and rewardbased obligations can be defined in terms of motivational attitudes in a similar way. A first version of our formal model of self-modifying normative multiagent systems can be found in [31], and a preliminary formalization of our game theoretic approach to contracts can be found in [32].

There are several other proposals to formalize contracts in the area of multiagent systems. Inspired by Sandholm and Lesser [33], Teague and Sonenberg [34] discuss the impact on reputation of levelled commitment contracts, i.e., contracts where each party can decommit by paying a predeterminate penalty. While reputation is beyond the scope of this paper, our model of contracts can specify not only decommitment penalties, but also explicit procedures for the agents to withdraw from the contract by means of constitutive rules.
Pacheco and Carmo [4] and Dignum et al. [1] define the clauses of a contract as conditional obligations, whereas we use constitutive rules creating obligations when the contract is created or when another relevant event happens. Dignum et al. propose the language $L C R$ for modelling contracts. They define contracts as tuples composed of agents, contract clauses, stages and interactional structure. They also give a definition of obligations in terms of violations, but they do not take a subjective perspective and they do not consider the decision problem of an agent subject to obligations.

Daskalopulu and Maibaum [35] model contracts as processes having states representing the legal relations among agents. They introduce obligations as consequences of the unfulfillment of other obligations. However, they do not consider the role of constitutive rules in contracts and the fact that violations are recognized only as an effect of the activity of the normative system.

Vasconcelos et al. [36], [37] and others call normative systems regulating the normative position of a multiagent system e-institutions. E-institutions establish interaction conventions and social consequences of actions, and enforce the satisfaction of commitments. These concepts could receive a foundation in terms of legal institutions as we propose in this paper.

Besides deontic logic, other formalisms have been proposed to model contracts, for example, Petri nets [38] and state transition graphs [2].

In the context of e-business Hanson and Milosevic [3] propose a framework for dealing with several aspects of contracts, from negotiation to validation, from monitoring the execution to recovering from violations. As discussed in Section I, we agree with Milosevic and Dromey [18] that a specification of an organization given by contract differs significantly from a computational specification in that the compliance cannot be taken for granted. Even if Milosevic and Dromey [18] are aware that contracts are not exhausted by obligations and permissions, they define them only in terms of deontic concepts. Our view of contracts as legal institutions emphasizes the role of constitutive norms besides regulative ones. Hanson and Milosevic [3] define contract negotiation as a process of making and adjusting offers among potential agents willing to be involved in an economic transaction until an agreement is reached or the process is terminated without an agreement. We adopt a similar perspective on contract negotiation and we focus our work on the problem of evaluating offers according to the commitments made by the agents and the procedures created by constitutive norms.

Besides works on modelling contracts, a large literature is devoted to the problem of the automated negotiation of contracts, and, in particular, to provide protocols of interaction which are self enforcing and have desirable properties, like, for example, the maximization of the sum of the outcomes of the agents [33], [39], [40]. This topic is beyond the scope of this paper focusing on reasoning about contracts rather than on searching the space of possible contracts. Many works assume that the execution of the contract is carried out without violations. This assumption is too strong, as noticed by Milosevic and Dromey [18]. 


\section{CONCLUSIONS AND FUTURE WORK}

The role of norms and contracts in the interaction of agents is a major aspect of e-commerce and e-trading systems. Agents must reason about the fulfillment of norms, the possible violations and what to do to repair such violations. However, classical game and decision theory presume a fixed set of interaction possibilities. Since contracts can be used to change the interaction possibilities, norms and contracts pose an important challenge to the game theoretic analysis of agent interactions. In this paper we explain and formalize how agents can modify the behavior of normative systems via contracts.

Our approach to modify the behavior of normative systems by means of contracts is based on constitutive rules changing the normative system, using the distinction between regulative and constitutive norms developed by Searle [8], and the concept of legal institutions as developed by Ruiter [9]. Legal effects of actions of the members of a legal system are complex and contracts do not concern only the regulative aspects of a legislation, i.e., the rules of behavior specified by obligations, or the constitutive part of it, i.e., the rules introducing institutional facts such as bidding in an auction, but contracts are systems of regulative and constitutive rules that provide frameworks for social action within larger rulegoverned settings. Therefore contracts as legal institutions bring with them constitutive norms creating not only institutional facts, but creating also new regulative and constitutive norms. In this way, it is possible to specify in a contract new procedures for the interaction among agents.

The game theoretic approach to contracts we develop in this paper builds on the notion of strategic interaction developed by Goffman. Inspired by Goffman we adopt the idea that agents can play games with normative systems just as they play games with other agents, thus modeling the normative system as a socially constructed agent, and inspired by Searle we represent the regulative norms and constitutive norms as motivational and informational attitudes of this socially constructed agent. This leads to a relatively simple model of a complex social phenomena, which can be further developed in agent theory to develop e-commerce and e-trading systems. Moreover, we introduce a qualitative game theory based on recursive modelling.

Our formalization distinguishes between the structure of normative multiagent systems and games played in it. Starting from Boella and Lesmo [12]'s observation that a normative system behaves like an agent monitoring and sanctioning violations, we develop an ontology of social reality, including not only normative systems but also groups and organizations. This ontology is based on the idea that social entities can be modelled as agents which are attributed mental attitudes. The metaphor allows us to define regulative rules as goals of the normative system and constitutive rules as its beliefs. We introduce self-modifying normative multiagent systems to define constitutive norms with legal effects, which update the beliefs and motivations of the normative system. In these normative multiagent systems, obligations are defined using six clauses, distinguishing among the motivational and regulative constituents of obligations. The creation of contracts is represented by an institutional fact that works as an abstraction between the agreement and its legal effects. Contracts seen as legal institutions are based on a tight integration of constitutive and regulative rules.

Moreover, we develop a qualitative game theory where agents are allowed to make contracts to change their normative positions, and we explain and show how agents can use the game theory. By using recursive modelling and applying the agent metaphor to normative systems we define violation games among an agent and the normative system in which the agent predicts the behavior of normative systems, and we use an example from international trade to illustrate how agents can use the game theory. Negotiation games involving for example all three agents $\mathbf{a}, \mathbf{b}$ and $\mathbf{o}$ can be defined analogously. For example, assume that agent $\mathbf{b}$ also has to sign the contract before it comes into force, and it is deliberating whether to sign it or not. One of the crucial factors in signing is whether agent $\mathbf{b}$ believes that agent $\mathbf{a}$ will fulfill its obligations, or violate them. Agent $\mathbf{b}$ therefore recursively models agent $\mathbf{a}$. However, to find out whether agent a will fulfill its obligations, agent $\mathbf{b}$ has to consider his beliefs about agent $\mathbf{a}$ 's beliefs about organization $\mathbf{o}$, as detailed in the violation game discussed in Section V-B. The negotiation game therefore extends the violation game with another level of recursion.

The qualitative game theory is not restricted to selfish agents minimizing their sanctions, based on clauses 2-6 of the definition of obligation, but it can also be used for respectful agents internalizing the goals of the normative system as their own goals, based on clause 1 . The example also suggests many ways in which the example can be modified such that no longer can be derived that agent a will pay, including many subtle variants of fraud and deception.

In this paper we use normative system and organization interchangeably, though there are several additional issues to be studied in organizations. For example, as Milosevic and Dromey [18] suggest, contracts are strictly related to roles. Contracts are used for assigning roles: they create the obligations of the holder of a role starting from the description of the role. The notion of role allows also to structure the normative system in various types of agents, like those with the task of monitoring violations and applying sanctions. Analogously new roles can be added to normative systems regulating the contract negotiation process to act as mediators or coordinators of contracts. Legal institutions, like contracts, can even create new normative systems, for example, contracts creating organizations (such as the legislation on societies). It is thus necessary that such contracts are able to specify obligations and permissions about obligations and permissions created by an organizations.

The use of normative multiagent systems is not restricted to contract negotiation. Many theories and applications of multiagent systems such as electronic commerce, virtual communities, theories of fraud and deception, of trust dynamics and reputation, secure knowledge management, et cetera, can fruitfully employ the notion of a normative system regulating an agent society. Each of these applications comes with its own characteristic properties, which are subject of further research. 


\section{REFERENCES}

[1] V. Dignum, J.-J. Meyer, and H. Weigand, "Towards an organizationaloriented model for agent societies using contracts," in Procs. of $A A$ MAS'02. ACM Press, 2002, pp. 694-695.

[2] C. Dellarocas and M. Klein, "Contractual agent societies: Negotiated shared context and social control in open multiagent systems," in Social Order in MAS, R. Conte and C. Dellarocas, Eds. Kluwer, 2001, pp. 113-134.

[3] J. Hanson and Z. Milosevic, "Conversation-oriented protocols for contract negotiations," in Procs. of EDOC'03, Brisbane (AU), 2003, pp. 40-49.

[4] O. Pacheco and J. Carmo, "A role based model of normative specification of organized collective agency and agents interaction," Autonomous Agents and Multiagent Systems, vol. 6, pp. 145-184, 2003.

[5] P. Marsh, Contract Negotiation Handbook. Aldershot: Gower, 1984.

[6] C. Alchourrón and E. Bulygin, Normative Systems. Wien: SpringerVerlag, 1971.

[7] A. Jones and J. Carmo, "Deontic logic and contrary-to-duties," in Handbook of Philosophical Logic, D. Gabbay and F. Guenthner, Eds. Kluwer, 2001, pp. 203-279.

[8] J. Searle, Speech Acts: an Essay in the Philosophy of Language. Cambridge (UK): Cambridge University Press, 1969.

[9] D. Ruiter, "A basic classification of legal institutions," Ratio Juris, vol 10(4), pp. 357-371, 1997.

[10] H. Hart, The Concept of Law. Oxford: Clarendon Press, 1961.

[11] J. Gordijn and Y.-H. Tan, "A design methodology for trust and value exchanges in business models," in Procs. of BLED Conference, 2003, pp. 423-432.

[12] G. Boella and L. Lesmo, "A game theoretic approach to norms," Cognitive Science Quarterly, vol. 2(3-4), pp. 492-512, 2002.

[13] E. Goffman, Strategic Interaction. Oxford: Basil Blackwell, 1970

[14] A. Newell, "The knowledge level," Artificial Intelligence, vol. 18, pp. $87-127,1982$.

[15] M. Bratman, Intentions, plans, and practical reason. Harvard (Massachusetts): Harvard University Press, 1987.

[16] P. J. Gmytrasiewicz and E. H. Durfee, "Formalization of recursive modeling," in Procs. of ICMAS'95, 1995, pp. 125-132.

[17] V. Kartseva, J. Gordijn, and Y.-H. Tan, "Designing control mechanisms for network organisations," in Procs. of ICEC'04. IEEE Press, 2004.

[18] Z. Milosevic and G. Dromey, "On expressing and monitoring behaviour in contracts," in Procs. of EDOC'02, Lausanne (CH), 2002, pp. 3-14.

[19] Y. Shoham and M. Tennenholtz, "On the emergence of social conventions: Modeling, analysis and simulations," Artificial Intelligence, vol. 94, no. 1-2, pp. 139-166, 1997.

[20] J. Broersen, M. Dastani, J. Hulstijn, and L. van der Torre, "Goal generation in the BOID architecture," Cognitive Science Quarterly, vol. 2(3-4), pp. 428-447, 2002.

[21] J. Lang, L. van der Torre, and E. Weydert, "Utilitarian desires," Autonomous Agents and Multiagent Systems, pp. 329-363, 2002.

[22] J. Searle, The Construction of Social Reality. New York: The Free Press, 1995.

[23] D. C. Dennett, The Intentional Stance. Cambridge, MA: The MIT Press, 1987.

[24] C. Castelfranchi, "Engineering social order," in Procs. of ESAW'00. Berlin: Springer Verlag, 2000, pp 1-18.

[25] A. Anderson, "The logic of norms," Logic et analyse, vol. 2, 1958.

[26] D. Makinson and L. van der Torre, "Input-output logics," Journal of Philosophical Logic, vol. 29, pp. 383-408, 2000.

[27] M. Dastani, J. Hulstijn, and L. van der Torre, "How to decide what to do?" European Journal of Operational Research, vol. 160(3), pp. 762$784,2005$.

[28] G. Boella and L. van der Torre, "Local policies for the control of virtual communities," in Procs. of IEEE/WIC WI'03. IEEE Press, 2003, pp. $161-167$.

[29] _ - "Rational norm creation: Attributing mental attitudes to normative systems, part 2," in Procs. of ICAIL'03. Edinburgh: ACM Press, 2003, pp. $81-82$.

[30] - "The distribution of obligations by negotiation among autonomous agents," in Procs. of ECAI'04. IOS Press, 2004, pp. 13-17.

[31] _ "Regulative and constitutive norms in normative multiagent systems," in Procs. of KR'04. AAAI Press, 2004, pp. 255-265.

[32] — "Contracts as legal institutions in organizations of autonomous agents," in Procs. of AAMAS'04. ACM Press, 2004, pp. 948-955.

[33] T. Sandholm and V. Lesser, "Issues in automated negotiation and electronic commerce: Extending the contract net framework," in Procs. of ICMAS'95, San Francisco (CA), 1995, pp. 328-335.
[34] V. Teague and L. Sonenberg, "Investigating commitment flexibility in multiagent contracts," in Game Theory and Decision Theory in AgentBased Systems, S. Parsons, P. Gymtrasiewicz, and M. Wooldridge, Eds. Kluwer, 2002, pp. 267-292.

[35] A. Daskalopulu and T. Maibaum, "Towards electronic contract performance," in Procs. of Legal Information Systems Applications, 2001, pp. 771-777.

[36] M. Esteva, J. Rodriguez-Aguilar, C. Sierra, and W. Vasconcelos, "Verifying norm consistency in electronic institutions," in Procs. of Workshop on Agent Organizations at AAAI'04, San Jose (CA), 2004.

[37] W. Vasconcelos, J. Sabater, C. Sierra, and J. Querol, "Skeleton-based agent development for electronic institutions," in Procs. of AAMAS 2002. ACM press, 2002, pp. 696-703.

[38] R. Lee, "Documentary Petri nets: A modeling representation for electronic trade procedures," in Business Process Management, LNCS 1806 Berlin: Springer Verlag, 2000, pp. 359-375.

[39] D. Reeves, M. Wellman, and B. Grosof, "Automated negotiation from declarative contract descriptions," Computational Intelligence, vol. 18(4), pp. 482-500, 2002.

[40] J. S. Rosenschein and G. Zlotkin, Rules of Encounter. Designing Conventions for Automated Negotiation among Computers. Cambridge, MA: MIT Press, 1994

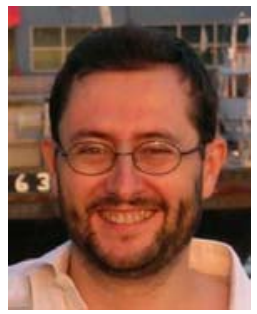

Guido Boella received the $\mathrm{PhD}$ degree at the University of Torino in 2000.

He is currently a researcher at the Department of Computer Science and the Center for Cognitive Science of the University of Torino. His research interests include multiagent systems, in particular, normative systems, institutions and roles using qualitative decision theory. He organized the first workshops on normative multiagent systems, on coordination and organization, and the AAAI Fall Symposium on roles.

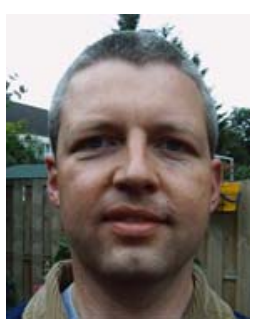

Leendert van der Torre received the $\mathrm{PhD}$ degree from Erasmus University Rotterdam in 1997. $\mathrm{He}$ is currently researcher at CWI Amsterdam and lecturer at Delft University of Technology. He has developed the so-called input/output logics and the BOID agent architecture. $\mathrm{He}$ is coordinator of the BOID project and of the CWI node of the ArchiMate project. His current research interests include deontic logic, qualitative game theory and coordination in normative multiagent systems. 


\title{
On the ontological status of plans and norms
}

\section{Guido Boella, Leonardo Lesmo and Rossana Damiano}

\author{
Dipartimento di Informatica \\ Centro di Scienze Cognitive \\ Centro per l'Ontologia Teorica e Applicata \\ Università di Torino \\ \{guido, lesmo, rossana\}@di.unito.it
}

\begin{abstract}
This article describes an ontological model of norms. The basic assumption is that a substantial part of a legal system is grounded on the concept of agency. Since a legal system aims at regulating a society, then its goal can be achieved only by affecting the behavior of the members of the society. We assume that a society is made up of agents (which can be individuals, institutions, software programs, etc.), that agents have beliefs, goals and preferences, and that they commit to intentions in order to choose a line of behavior. The role of norms, within a legal system, is to specify how and when the chosen behavior agrees with the basic principles of the legal system. In this article, we show how a model based on plans can be the basis for the ontological representation of norms, which are expressed as constraints on the possible plans an agent may choose to guide its behavior. Moreover, the paper describes how the proposed model can be linked to the upper level of a philosophically well-founded ontology (DOLCE); in this way, the model is set in a wider perspective, which opens the way to further developments.
\end{abstract}




\section{Introduction}

The role of ontologies in computer science is to provide a formal description of the world. They aim at enabling a machine to find out relevant information from documents, to recognize described situations, to draw conclusions on the basis of known facts, etc. In this context, it is not relevant that the description refers to the world 'as it is' (true ontology, in the classical view), but what is important is that it represents the world as it is conceived by sentient beings (epistemology): so far as an ontological structure is embodied in a computational agent, it represents the way that agent sees the world, but to the extent where that structure is shared among a community of agents, it represents the way the community sees the world, and if the ontology is also used in $\mathrm{man} / \mathrm{machine}$ communication, it must share with humans some basic ideas about the way the world is organized [Breuker \& Winkels, in press].

So, an ontology must be properly founded, in order to make possible this multiple sharing. But often ontologies are used in applications concerning specific domains, so that the proper foundations must be linked to domain conceptualizations. However, it is not clear how this link can be achieved. A first problem concerns the layman/expert point of view. Obviously, a physician has a very different view of what is a human body (and how it works) than a non-physician. So, it may be assumed that an ontology devoted to the medical field must be able to express the view of the physician, in order to put at her/his disposal, when needed, the vast body of knowledge concerned, for instance, with the liver, or with what an illness is ${ }^{1}$. On the other hand, a physician must be able to talk with her/his patients: this often requires a rephrasing of complex medical descriptions in (possibly imprecise, but understandable) non-technical terms. And s/he must also be able to understand what are the symptoms, even if they are not described by the patient in the most proper way. This happens in many fields, and it is especially important in the legal field, where many legal concepts are inextricably linked to the commonsense view of the world. This produces two, partially related, effects: first, in legal documents, as well as in the Statutes, there may appear terms (as window, water, tree), which are not defined in the legal systems, so that it is assumed that the definition is borrowed from a general ontology; second, some concepts, which are of common use (such as property, person, duty), are changed by a legal system by means of a body of definitions and norms appearing in the system, so that the jurist's view of a 'person' is not exactly the same as the layman's view.

The goal of this paper is twofold: first, to show how commonsense concepts (in particular, action and behavior) can be linked to legal concepts (in particular, norms and rules); second, to show how the resulting structure can be linked to a properly founded top-level ontology. It must be clear that we do not aim at covering the ontological status of all types of rules. For instance, in a legal system, there are rules concerning the definition of concepts (e.g. Non Governative Organization) and rules concerning the way a given institutional role can be established (e.g. the Secretary of an NGO), and which are its attributions. We are not concerned with definitions, since we assume they are given in advance, and already included in the ontology. But we are (at least partially) concerned with institutional roles and their attributions, since institutional roles, as authorities, are a (predefined) category of people, and their attributions define what they can and cannot do. Also, we are not concerned with the way a Court gives a verdict, which is a very complex process, but we are concerned with the presence, in the ontology, of all the concepts required to describe the facts and the evidence available to the court. In other words, this paper faces a small but relevant fraction of

\footnotetext{
1 A complex network of physiological causes and effects with respect to "I feel bad".
} 
the legal knowledge ${ }^{2}$ : it covers the norms of conduct, i.e. the norms that specify what a legal actor can and cannot do. The claim is that these rules express constraints on actions, and their relevance stands on the view that a legal system aims at organizing a society in such a way that the rights and duties of individuals and organizations are properly balanced, on the basis of superior principles. And these rights and duties are expressed in terms of norms of conduct. The article shows that the same approach can be applied to the modelling of legal powers; in this case, the constraints refer to the plans that must be executed to establish new rights and duties.

We must point out that we do not aim just at describing norm-related concepts, but also at showing how norms affect behaviour. In other words, the formalization of prescriptive rules is assumed to be available to an agent that must do things, and that must choose what to do: this agent uses the rules to decide what he will do next; in principle, he will choose a line of action that complies with the existing rules, but in some cases he may decide that a rule is not worth being respected, either because it is in conflict with other norms, or because the risk of being sanctioned is low. Although this behaviour is only partially moral, it is the way many rational agents choose to act: it must be properly modelled, since it explicitly includes the concept of sanction, and, implicitly, guides the legislator to properly choose the sanction associated with a given rule.

The paper is organized as follows: the next section presents the upper-level of the ontology (DOLCE), and our formalization of plans; in the third section, the representation of norms is described; the fourth section shows how power and authorities fit our approach; in section 5, we present a case study addressing the legal concept of 'fruit'; then, we compare our approach with other ontological models of the legal systems and, in section 7, we present some ontological models of plans; the conclusions close the paper.

\section{The upper-level of the ontology, and the plans}

In order to introduce our model of plans and norms into a well-founded ontology, we have chosen, as our starting point, the DOLCE ontology [Gangemi et al. 02]. The main advantage of DOLCE is the clear philosophical foundation of its basic categories: they have been selected as the result of a research effort that has lasted for some years, and the motivations for the final choices can be found in the referenced papers, and can be traced back to various previous proposals [Guarino 98] [Gangemi et al. 01] [Welty \& Guarino 01] [Guarino \& Welty 02]. Most of the structures addressed in the paper are based on the KIF implementation reported in [Masolo et al. 2003].

\section{DOLCE}

Without entering in any detail (the interested reader is addressed to the original papers), we report here some brief comments on the top-level of DOLCE, which seem necessary in order to explain the connections to plans and norms. Some of the concepts defined in DOLCE are depicted in fig.1.

\footnotetext{
2 "Since the norm is the most salient construct of the legal domain, we have selected it as the point of departure for our intermediate representation" [Kralingen at al. 99, p.1128]
} 


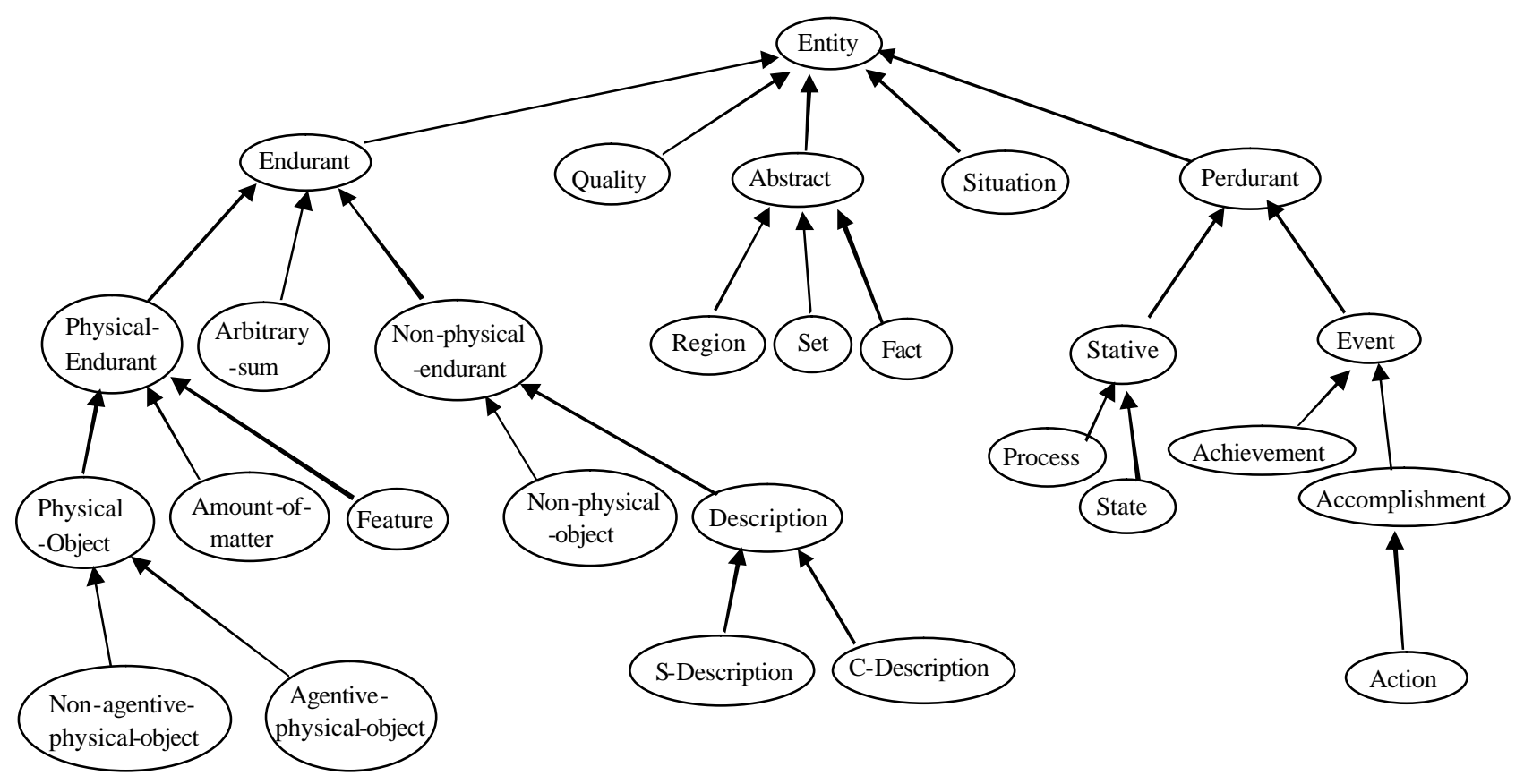

Fig.1 - A portion of the upper level of DOLCE (from [Masolo et al. 2003])

The upper-level categories are Abstract, Quality, Situation, Endurant, and Perdurant. The assumption about the existence of abstract entities and qualities is rather common (with the known philosophical intricacies). The distinction between Endurants ${ }^{3}$ and Perdurants can be specified by citing the authors:

"Endurants are wholly present (i.e. all their proper parts are present) at any time they are present. Perdurants, on the other hand, just extend in time by accumulating different temporal parts, so that, at any time they are present, they are only partially present, in the sense that some of their proper temporal parts (e.g. their previous or future phases) may be not present" [Gangemi et al. 02, \$2.1]

In our words, which could not be shared by the authors, this means that the most relevant structuring of endurants is in space (so they extend in full across time), while the most relevant structuring of perdurants is in time (so they are cut in parts by the flow of time).

Situation refers to states of affairs (SOA). According to the authors, other names used in the literature for a Situation are setting, Gestalt, or configuration. Example of Situation are a clinical condition, a murder, and a speed excess case. It is interesting to note that in the Documentation of Situation (in the KIF knowledge base [Gangemi et al. 2003, p.195]), it is stated that situations "could be equivalently modelled as a special complex perdurant defined through its relations to qualities, regions, and endurants". Situations satisfy Descriptions, in particular S-Descriptions (situation description), see fig.1. In our interpretation, a situation is the 'actual' counterpart of a representation (description), i.e. a piece of reality, if one is a realist.

\footnotetext{
${ }^{3}$ In the text, we sometime use the category names in the plural. Although this is imprecise, we believe it makes the reading more fluent.
} 


\section{Plans}

Descriptions, which are non-physical endurants, are particularly important in this article, since, in DOLCE, plans are S-Descriptions (see fig.2). So, Plans are particular descriptions of situations. This placement reflects a view where plans describe something; although we would rather take a plan as the situation which is described, in order to maintain the compatibility with DOLCE, we focus in this article on the description view. However, we must point out that we do not consider an actual plan as something occurring in the world, but rather as a 'mental' situation. It consists in the abstract object (the 'idea') that is associated with 'potential' occurrences of events. Consequently, DOLCE's Plan is taken as the mental description of this object, a description which (in DOLCE's terms) is represented-by a MentalInformationObject which, in its turn, is realized-by a given configuration of brain neurons. This is a very strong ontological commitment, which would require a lengthy discussion which, for the sake of brevity, we must omit here.

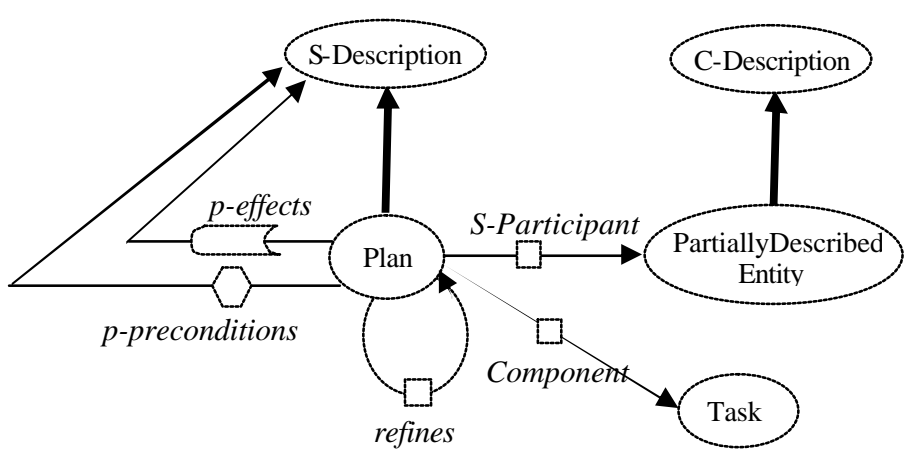

Fig.2 - The placement of Plan in the ontology

What is known about Plans in DOLCE is that they are composed of Tasks and include some Functional-Roles; moreover, there is a Planning-activity, which is sequenced-by various types of Tasks. Actually, we must be clear about what we mean by Plan. In particular, we must keep apart three different views on plans:

1. The description(s) which an agent is reasoning about while he is trying to decide what to do (one or more partially specified plan schemas).

2. The intention, i.e. a single, possibly still underspecified, sequence of steps which the agent has decided will drive her/his behaviour.

3. The execution of a plan, i.e. the actual, real-world, events that the agent carries out.

What appears in fig. 2 is the first view of plans, so that it can be imagined as a tree-like structure, where some decisions have been already taken, while others are suspended. For example, I may know about plans for travelling to far cities. And I may have the goal of being in Rome tomorrow. So I can examine the plans "go to a far city by train" and "go to a far city by plane" (two different plan schemas). If I have chosen the second schema, then I have certainly fixed the "airport of arrival' and the 'airport of departure' (i.e. two of the participants in the plan), but, possibly, the departure time is just given as 'some time in the morning of tomorrow before 8.00 a.m.', i.e. an underspecified description of the 'departure time' participant. Also note that a different plan is obtained when I have reserved the seat, since at that time the "departure time" has been fixed: this new plan is a plan 'more specific' than (refines, in DOLCE's terms) the original one (see fig.3). 


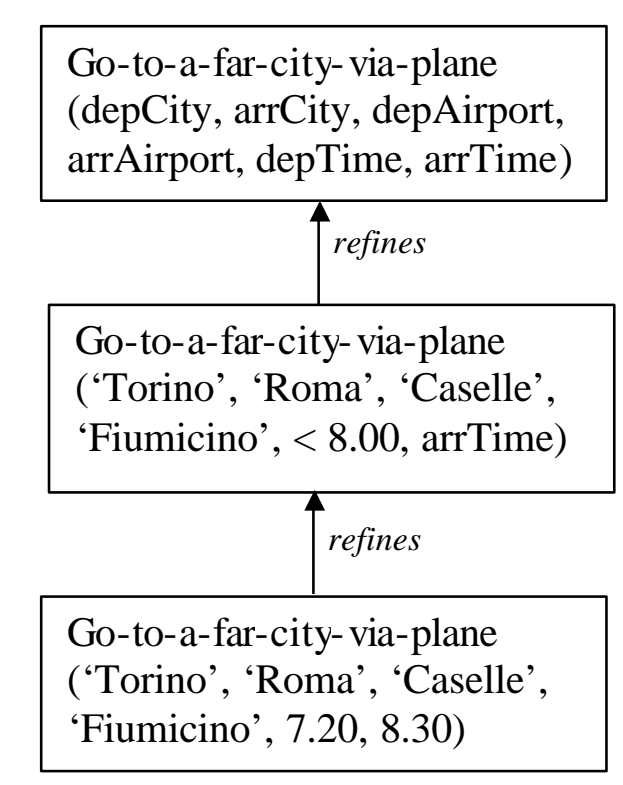

Fig.3 - Plans and more-specific plans

With respect to fig.2, we note that:

- Apart from the standard subcategory arcs (thick arrows), some other arcs have been introduced. They refer to relations in the definition, and are shown as thin arrows with a small box. The shapes that substitute the boxes in p-preconditions and p-effects are just a shorthand, to keep more compact the next figures. Each relation has a name shown near the box; the arrow enters a category, which specifies the range of the relation; this is an adaptation of the KL-One notation [Brachman \& Schmolze 85], which complies with the KIF implementation of DOLCE; in Appendix 1, we report an example of how a standard ontological language (i.e. LOOM [MacGregor 91], [MacGregor \& Brill 91]) can be used to express the concepts and relations appearing in the drawings.

- The range of p-preconditions and p-effects is S-Description. This is a partial description of a state; it can be seen as an underdetermined state, where some characteristics are fixed, while others are left unspecified. In DOLCE, precondition is a relation between $S$-Descriptions and situations. We believe that is more homogeneous to maintain a relation between $S$-Descriptions; of course, the $S$-Description which acts as p-precondition will be satisfied-by DOLCE's precondition. For example, the plan of going Rome by plane is executable if the $S$-Description "The Rome Airport is open" is satisfied by the current Situation.

- $s$-participant specifies the entities involved in the plan; its range is PartiallyDescribedEntity. This is the role taken by a $C$-Descriptions (i.e. the Descriptions which describe anything which is not a Situation) in a planning context; $C$-Descriptions describe entities having some properties, or being in some relations with other known entities (the event that happened to me in some place at a given time, the platypus I saw in the Sydney park). Participants in plans need not be completely specified (I will take $a$ taxi), but they can be described more or less precisely (I will take a yellow taxi with an old, experienced driver). The extension of a PartiallyDescribedEntity is a set of Endurants, such that the actual participant in the plan must belong to this set. The dotted oval associated with PartiallyDescribedEntity refers to its being a material role (see [Guarino \& Welty 00]): no new entity has come into being, but an existing 
one plays a new role (as when a person enrols in a University s/he takes the material role of student).

Of course, many features of the plan ontology have been left out, but the goal of this paper is mainly to address the role of norms in the planning framework, so only the concepts necessary to enable us to specify the relationship between plans and norms have been described.

\section{Planning}

As stated above, plans are the objects that are managed in the process of planning. In Appendix B, the interested reader may find a sketch of an ontological representation of how planning may be carried out. In fig.4, instead, we refrain from going into the structure of the planning action, but we simply represent the elements that affect that action. Intuitively, the idea is that Thinking is a Perdurant which has as participants the descriptions embodied in the brain. In particular, Planning is that type of Thinking that works upon the mental representation of plans. It produces new Plans by considering ${ }^{4}$ known Plans (going to Rome by bus), by specializing existing Plans (the taxy company has been chosen), or by discarding Plans (see the comments about the PLANET system in Section 7). What is shown in fig. 4 is just the final outcome of this process, i.e. the fact that a plan has become an Intention. No Intention concept is currently defined in DOLCE, but has-BDI-on is a modality-target relation holding between an Agent-role and a course (of events). Since Plans are composed of Tasks, i.e. a specific type of Course, and who plans plays an Agent-role with respect to the planning action, we call Intention the role assumed by the sequence of Tasks which the planning agent has chosen. Correspondingly, we call has-intention-on the subrelation of has-BDI-on which refers to intentions (wrt. beliefs and desires). Although this is the actual picture in DOLCE (and we comply with it in fig.4), in order to simplify the subsequent figures, we will merge the Plan and the associated Complex-Task. Consequently, in the following, we assume that Plan embodies also the Tasks it is composed of.

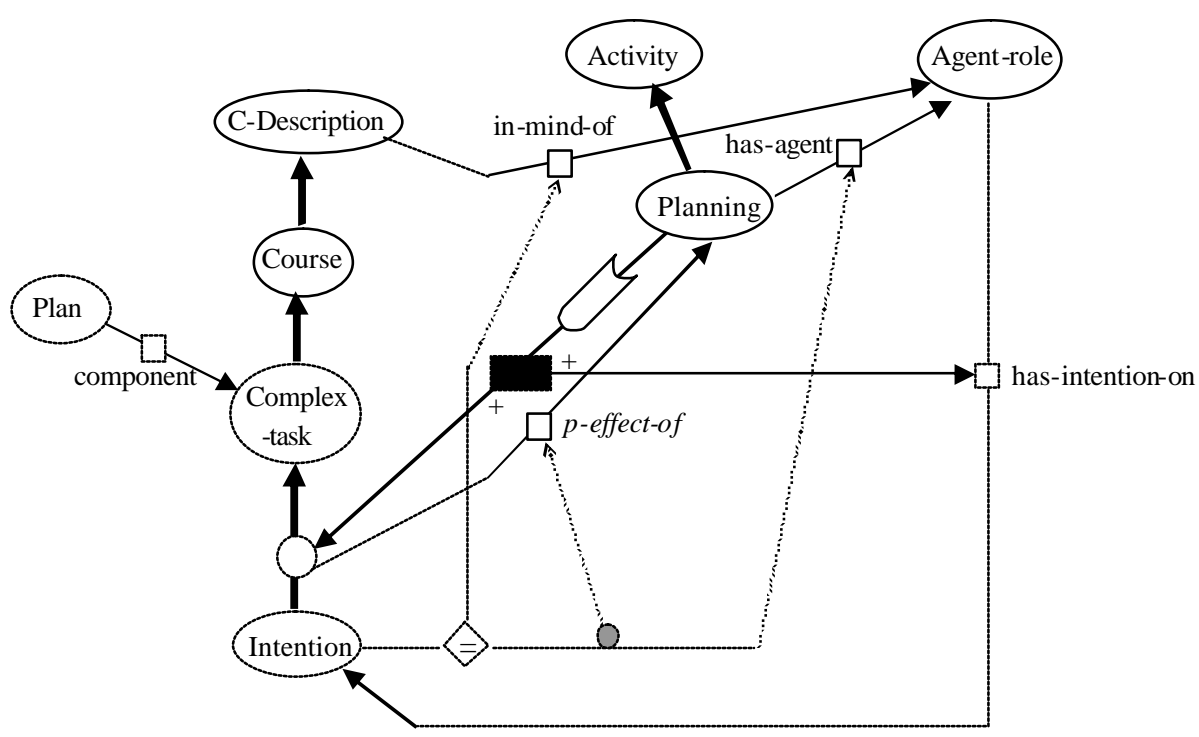

Fig.4 - The Planning activity

\footnotetext{
${ }^{4}$ By 'considering', we mean here the process of moving around (e.g. from LTM to STM) pieces of descriptions.
} 
Notes to fig. 4:

- There are two effects of Planning: a Complex-task associated with a Plan has become an Intention, and the planning agent holds that intention (has-intention-on). The black rectangle on the effects arc is just used to keep together the various effects (it is just a logical And); the " + " on the arcs exiting the black rectangle mean that these are positive effects: they did not hold before the action, and they hold after the action.

- The in-mind-of relation holds among a (mental) C-description and an Agent-role. It means that the Endurant playing that role has in its brain a physical representation of that description.

- Intention is a material role that is played by the ComplexTask. The circle on the arc has no special function, except as a graphical means to specify that that arc is one of the effects.

- The diamond on the arc exiting from Intention is a constraint on the relations: it specifies that the two elements identified by the dotted arrows exiting the '=' box must be the same individual. The first of them is the Agent-role such that the entity playing that role 'has in mind' the Intention, and the second is the Agent-role of the Planning which has produced that Intention ${ }^{5}$. In other word, in this model, agents plan for themselves (p-effect-of is just the inverse relation of $p$-effects).

What is not represented in Fig.4 is the role of Intention in driving behaviour: agents always act; so, if the current time is $t_{i}$, then agent $A$ is doing something at $t_{i}$ and will try to do something at $t_{i+1}$; what is that something is specified by $A$ 's current intention.

\section{Utilities}

Since, during planning, an agent considers many Plans, but just one of them becomes his Intention, he needs some way to decide which one of two (or more) Plans is the best. The model presented here is based on the assumption that:

- The choice is based on the agent's preferences;

- The preferences are applied to states that can be originated (either directly or indirectly) by the execution of the plan;

- From a computational point of view, applying the preferences to a state amounts to evaluating the utility of that state;

In our view, these three criteria characterize the rationality of agents. The use of utilities in the planning activity has been described elsewhere [Boella \& Lesmo 02]. Here, some comments are needed on the second point. In fact, the reason why the preferences are not necessarily applied to (i.e. the utility is not necessarily evaluated on) the state directly resulting from the execution of the plan, is one if the key features of our approach to norms.

In general, the execution of a plan has certain effects on the environment (as specified by the plan p-effects). But the new situation that obtains can also produce further foreseeable changes. This is especially true in case the world is populated by other agents, who may interact with the planning agent. So, it seems reasonable to try to imagine how the world would be after some sequence of reactions from the environment. This is similar to what happens (in a very simplified way) in twoplayers games, where a player must evaluate his moves in terms of the subsequent possible moves of the opponent; it is clear that the ability of foreseeing in advance the evolutions of the game (for two or more moves) is one of the keys for finding a successful strategy.

\footnotetext{
${ }^{5}$ More formally, by taking relations as functions, we have: $\forall \mathrm{x}$ (Intention $(\mathrm{x}) \rightarrow$ (in-mind-of( $\mathrm{x})=$ has-agent(p-effect-of( $\mathrm{x}))$ )

Of course, one can preserve relations, but the formula becomes less readable.
} 
So, the utility of a plan is evaluated on the basis of the state that obtains after the execution of the plan, and after some re-actions of the environment to the state that obtains (we assume here a twolevel lookahead: plan execution + reaction). The major point is that the environment is assumed to be able to react to the breach of norms; so, in order to model the behaviour of an agent, what is needed is a way to specify how the environment reacts to such a situation. Note that we have used the very general term 'environment'; this leaves unspecified which are the relevant component of such an environment. Currently, we assume the existence of a Normative-Agent-Role, who has to enforce the respect of law. However, this agent can be a single individual (a policeman) or a social body (the set of other agents surrounding the planning agent). Moreover, the presence of normative agents does not exclude that the respect of a norm is a value 'per se', i.e. it is embodied in preferences of the agent.

\section{Norms}

Since hw is a social construction, its bases lie on the interaction among agents. It has convincingly been argued that these interactions can be governed by some 'legal relations', and that all legal relations can be expressed in terms of four basic relations [Hohfeld 19] [Allen \& Saxon 95]. So, we start our presentation by showing how the basic legal relations fit our overall framework (see also [Boella et. al 01]). The four legal relations are: DUTY, RIGHT, NO-RIGHT, and PRIVILEGE. They are defined in terms of two interacting agents and of an action, in the following way:

1. $\mathrm{X}$ has the RIGHT that $\mathrm{Y}$ performs $\mathrm{A}$, iff in case $\mathrm{Y}$ does not perform $\mathrm{A}, \mathrm{X}$ may react with an action $\mathrm{R}$ such that $\mathrm{Y}$ is negatively affected by $\mathrm{R}$.

2. Y has the DUTY to perform A for X, iff the same condition as the one above holds (i.e. DUTY is the "opposite" of RIGHT, where the roles of the two agents are interchanged).

3. $\mathrm{X}$ has NO-RIGHT that $\mathrm{Y}$ performs $\mathrm{A}$, iff in case $\mathrm{Y}$ does not perform $\mathrm{A}, \mathrm{X}$ may not react with an action $\mathrm{R}$ such that $\mathrm{Y}$ is negatively affected by $\mathrm{R}$ (so NO-RIGHT is the "inverse" of RIGHT, where the possible reaction is not allowed, i.e. negated).

4. Y has the PRIVILEGE to perform A with respect to $\mathrm{X}$, iff in case $\mathrm{Y}$ does perform $\mathrm{A}, \mathrm{X}$ may not react with an action $\mathrm{R}$ such that $\mathrm{Y}$ is negatively affected by $\mathrm{R}$ (so, PRIVILEGE is the "converse" of the "inverse" of the "opposite" of RIGHT, where we take "converse" to refer to the execution of A by $\mathrm{Y}$, instead to the failure to execute A, as in all other relations).

Three comments. First, there are three parameters that come into play: the perspective (i.e. if we look at the relation from the point of view of X or Y; what Hohfeld calls "opposites"), the possibility to react ("inverse"), and the fact that the definition refers to Y doing or not A (what we have called "converse"). It seems that "converse" is not strictly required, and it is introduced just to conform to a linguistic view (the existence of the term "privilege"). And in fact, just four relations are defined and not all eight combinations. Second, what can be done and what cannot be done have to be interpreted in terms of legal rules. This can be extended to general moral rules, but cannot be interpreted in terms of factual ability of agents. So, nobody has the RIGHT to rob you just because if you don't give him the money, he "can" react by hurting you. Furthermore, also in case you actually have a RIGHT towards somebody, your reaction to non-compliance may not be "any" reaction, but it is further constrained. Third, in the common use of the terms that refer to the legal relations, they are not always 'directed' [Herrestad \& Krogh 95], i.e. they do not involve two agents, but just one. The correct interpretation of single-agent relations is obtained from DUTY and PRIVILEGE by quantifying over X: for DUTY, there exists an X that may react (or a set of X's, having predefined features, e.g. policemen); for PRIVILEGE no such $\mathrm{X}$ does exist (notice that the negation has wider scope than the quantifier). What one should not say is "I have the right to do that", since in this context the correct expression is "I have the privilege to do that".

We can now return to our planning-intention perspective. From this perspective, Hohfeld's approach tells us that, in case an action is a single actor one there are two possibilities: constraints 
and absence of constraints (DUTY and PRIVILEGE). But duties can also be 'directed' (and in such a case, they can be called 'rights' from the perspective of the other agent) and negated (no-rights): again, constraints and absence of constraints, although expressed in a slightly different way. It may be argued that privileges ${ }^{6}$ are useless (although it is useful to have a linguistic term for them, of course): if no constraint exists about an action, then the privilege is automatically obtained, without having to state it explicitly: privileges should not be part of legal (or moral) systems. However, they are useful for at least three reasons. First, they help in determining if an agent is liable for having done something: if the privilege is stated explicitly, then one does not need to search for all possible constraints related to the involved action. Second, rules are often organized in a taxonomy; so a rule could state that all agents of a given category (call it $\mathrm{C}$ ) and being in a given situation type (S), have the duty to do A; but it may be the case that the rule does not apply to a specific subcategory of $\mathrm{C}$ (i.e. C') or does not apply in a given subtype of S (i.e. S') or both (Lex Specialis). So, one can say that, although, in general, the members of $\mathrm{C}$ have the duty to do $\mathrm{A}$, the members of $\mathrm{C}$ ' (or the members of $C$, if they are in a situation of type $S^{\prime}$ ) have the privilege not to do A. Note that we are not claiming this is the way an actual legal system works, but rather an idealization of it. In general there could not be an exhaustive coverage of all situations, so that in real systems, privileges are even more important than in their idealized counterparts. The third, and perhaps the most important reason for the explicit statement of privileges depends on the hierarchical organization of the authorities able to enact laws: if a higher power wants to prevent that a lower power unduly constrains the behavior of the entities subject to the law, it can state that these entities have some privileges: if the Constitution states that citizens have the privilege to express freely their political opinion, then no state or regional law can state the opposite (at least in principle).

With respect to the placement of the legal relations in the ontology, DOLCE states that they are subcategories of Modal-Description (which are S-Descriptions), and have as a component a Functional-role (e.g. an Agent-role), which is in a specific Modal-relation with some Course (i.e. a course of events). The modal relations are has-right-on, has-not-right-on, has-duty-of, hasprivilege-of. All Modal-Descriptions are parts of an S-Description including two components: the first of them is a Functional-role and has as Modality-Target the second one (a Course). In other words, a Duty, for instance, is the description of a situation involving some action (a course of events is something that describes an action) which an agent (role) has the duty to perform ${ }^{7}$. This approach seems to be logic-biased, where a Modal-Description recalls a formula in deontic logic. On the contrary, we are more agent-oriented, so that we need to associate with legal relations some more information, i.e. the author of the norm and the sanction. Also, the relation between Norm and Plan has to be made more explicit. Moreover, power-related relations (see Section 4) are also viewed as Modal-Descriptions, while it seems more reasonable, according to the usual analyses, to take them as relating an agent to another legal relation, which is the $p$-effect of the associated plan.

With respect to norms, in DOLCE, Norm is a subcategory of Regulation; Norms have the function of constraining Perdurants, and their relationship with legal relations is rather indirect. In our model, we assume that the legal relations are subcategories of Norm in that they specify (describe) particular constraints on behaviour (activities).

Let us now introduce an example. As we have seen, a Plan has p-preconditions and p-effects. Let's consider a plan schema. Its p-preconditions pose some constraints on the applicability of the plan. Therefore, the p-preconditions for cooking spaghetti with garlic, oil, and chili pepper (GO\&CP), is to have spaghetti, oil, chili pepper, water (to boil the spaghetti), a pan, and some fire (a very easy recipe). As it is usual, p-preconditions are conditions that prevent the action from being executable

\footnotetext{
${ }^{6}$ Often called explicit permissions [Boella \& van der Torre 03]

${ }^{7}$ Note, however, that in [Gangemi et al. 2003], Duty, Right, etc. are subcategories of Regulation, i.e. sisters of Law and Obligation.
} 
(physical, or practical conditions). But it could happen that the King of ThatKingdom has prohibited this recipe. Or, perhaps, this prohibition applies just to dinners with more than 7 participants, or to dinners served after midnight, and so on. Also, in some situations (here and now) garlic could not be a nice ingredient (because of its side-effects on your breath); or you know that one of your guests does not like chili pepper. All of these are not p-preconditions: if you have all the things you need, you can cook them in all situations described above (maybe you are a spaghetti revolutionary, or you are not very polite). Anyway, these rules constrain your freedom to cook spaghetti GO\&CP; you can pay for that: the King may imprison you, you may lose a kiss from your love (who does not appreciate, for this night, the smell of your mouth), or your guests may decide that they will never accept another invitation from you.

The "norms" we have exemplified above are not p-preconditions, but constraints on plans. We stress that in all of these examples, the utility of that recipe is reduced by the presence of the norm, so, it is possible that in absence of the norms, your intention for tonight dinner is to cook spaghetti GO\&CP, but in presence of one or more of these norms, your intention is to prepare rice with mushrooms: you have chosen a different plan, whose utility is lower than the plan of spaghetti without norms, but is higher than the plan of spaghetti with norms (since the norm reduces the utility). The problem, now, is how to express the norms.

Let us consider the case of the King's prohibition in its basic form (independent of the number of participants and of daytime). The rule involves three or four elements: the involved plan, the authority who posed the norm, and the agent who is taken as responsible for the execution of the action; the fourth element is not strictly required, but it is very common: it is the sanction ${ }^{8}$ the agent will undergo in case he does not respect the rule. So, a norm is not a plan, but 'refers' to a plan, in the sense that it (as a description) includes (is related to) a description of the action (i.e. the plan) which is forbidden (or permitted).

In fig.5, we introduce the basic notions related to norms, and apply them to our spaghetti example. In this example, we introduce instances. They are represented as rectangles. However, see below for some comments on the distinction between classes and instances. Instances are linked to the category which they belong to via a thick dotted arrow. In the figure, it is stated that

1. Norms are a subcategory of S-Description. This should not be confused with the written law which is just a physical way to express the decription: it is a medium for communicating the norm, not the norm itself. In fact, not all norms are written: they can be transmitted via verbal traditions and customs.

2. Norms have a topic ${ }^{9}$, the Plan the norm is about.

3. Norms have a polarity. Its range is the abstract set composed of the two elements pos and neg. If the value of polarity is pos, then the norm refers to something an agent must do, otherwise (as in our example), it refers to something he must not do. In other words, if the polarity is pos, an agent $X$ has the DUTY to do the action; if it is neg, it has the DUTY not to perform the action. ${ }^{10}$

4. Every Norm has a bearer. More precisely, it has a set of bearers: usually, norms apply to categories of agents, and not to single individuals. As usual, a relation arc enters another concept: that concept is associated to the set of potential bearers of the given norm.

5. Every Plan has an actor, who is the 'main character' of the plan. Although the actor of the plan is conceptually distinct from the planning agent, we assume, in the current model, that

\footnotetext{
${ }^{8}$ For different positions on the role of sanctions in law, see [Kelsen 48] and [Hart 61].

9 The term topic is inspired to [Ross 68]'s "theme".

${ }^{10}$ The fact that only DUTY appears in the figure is not intended to mean that all other relations are reduced to DUTY + polarity. Only DUTY is relevant in this example, but the other relations are there as sisters of DUTY.
} 
the two roles are played by the same individual (see fig.4, and the comments in the text) ${ }^{11}$. In the present example, we assume that the host of the dinner is the actor of the plan of serving GO\&CP; so, he also is the agent that must decide about the recipe for the dinner (the planning agent).

6. The actor of a plan, if that plan happens to be the topic of the Norm, and if that actor is of the right category, becomes an (individual) bearer of the rule. Of course, this applies also during the planning phase, so that the planning agent, which is a potential bearer, may discard the plan not to become an actual bearer. The identification of the actor with the bearer may appear as an oversimplification, but see [Kralingen et al. 99, p.1136]: "The fourth slot is reserved for the agent who performs the act (the agent and the norm subject must be the same person)".

7. Every Norm is associated with a sanction ${ }^{12}$, which is a PlanWithPatient, i.e. belongs to that subclass of Plan which is in some has-patient relation with an individual playing a PatientRole.

8. The bearer of the Norm is the has-patient of its sanction. Of course, this does not take into account the notion of responsibility, but we assume that being an agent who freely chose a given line of behavior is the basis for further elaborations of this concept.

9. The author of a norm is an Authority (see Section 6).

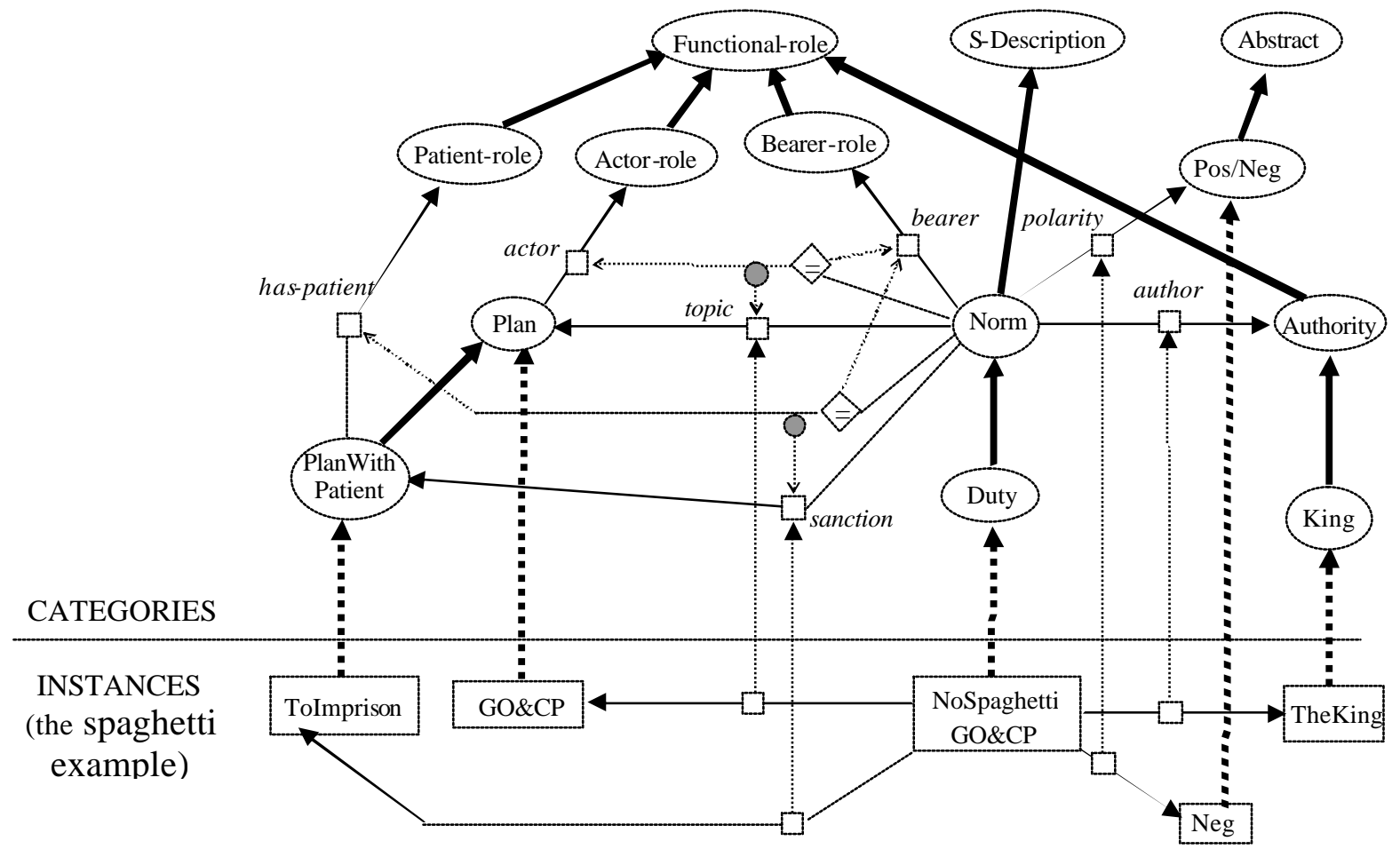

Fig.5 - Norms and plans

\footnotetext{
${ }^{11}$ Since Planning is an Action, it is the domain of the has-agent relation; however, since Planning is a mental action, its agent could more properly be called author rather than actor, since the effect is a mental representation. Consequently, we could say that the author of a plan and its actor do coincide. The 'invented' plan could actually involve more than one 'actor', but we simplify the matter by calling actor the primary participant (the host of the dinner, and not the cook, the waiters, etc).

${ }^{12} \mathrm{We}$ are dealing here with 'normative' rules. In other cases, the sanction may be replaced by a reward.
} 
With respect to the distinction between categories and instances, a few words are in order. The intuition is rather simple, and well known from decades of research on AI and KR: in standard model theory there are individuals and relations; an instance is an individual of the model, while a category corresponds to a one-place relation (expressed in logics as a one-place predicate). So, we have to define the individuals in our model. Clearly enough, the King is an individual (in particular, an Endurant), as well as the abstract entity neg. What about norms and plans? Is the single norm NoSpaghettiGO\&CP an individual? It depends on the way we use it in a reasoning framework. If we have to discuss a case, then we have to apply the norm to the description of the case; in doing that, we must bind the variables (roles) associated with the norm; in particular, we must bind the bearer. In doing that we obtain an entity which is more specific than NoSpaghettiGO\&CP, but seems to be of the same 'type'. So, it seems more appropriate that NoSpaghettiGO\&CP be a category, whose instances are the various applications of the norm to real cases. However, if we refer to the norm as a description, then NoSpaghettiGO\&CP is an individual, i.e. a single individual description, which may be in a refines relation to another individual norm description. The case of plans is even more striking: is the plan $G O \& C P$ an individual or a set, i.e. the set of all executions of the plan? The solution we propose is the same as above, but here we have three levels, instead of two: there are various descriptions of the $G O \& C P$ plan (instances of Plan); there is the execution of the plan (an instance of a Perdurant); but we also have the particular plan the planning agent has chosen (its Intention, which is a Complex-Task; see fig.4).

\section{Authorities: the Legal Relations concerning Power}

Traditionally, law scholars like [Hart 61] distinguish between primary laws, whose purpose is to direct the behavior of citizens, and secondary laws, which, among other functions, serve to the maintenance and dynamic management of the normative system. These rules form a "subsystem of rules for change": rules which have legal effects and which are instrumental to the primary system, in that they regulate the regulation (e.g., art. 2 of Italian Civil Code: "the creation of laws [...] is regulated by constitutional laws (Cost. 70)"). This subsystem, according to Hart, does not include only the rules of change which specify how new laws are introduced or old ones removed, but it also includes rules about "powers for the private citizen". These rules are at the basis of civil code and regulate testaments and contracts; for Hart they allow the exercise of limited legislative powers by the citizens. These rules do not create or remove general laws but they introduce and remove individual obligations and permissions: e.g., in the Italian Civil Code, art. 1173 (sources of obligations) specifies that obligations are created by contracts (a contract being an agreement among two or more parties to regulate a legal relationship about valuables by art. 1321).

In Hohfeld's framework, this distinction is reflected by the introduction, beyond the four Legal Relations we discussed in Section 3 (RIGHT, DUTY, NO-RIGHT, PRIVILEGE), of the relations of POWER, INABILITY, LIABILITY, IMMUNITY. They concern actions affecting the existing Legal Relations. In our terms, the topic of this particular type of rules is that subcategory of Plan whose instances have, among their effects, a modification of a Legal Relation. As we have seen in fig.5, Duty is viewed as a special subcategory of Norm. Accordingly, it seems reasonable to assume that also Powers are particular subcategories of Norm. So, we get:

1. Duty, Right, No-Right, Privilege, Power, Inability, Liability, Immunity are specific subcategories of Norm (just Duty, Privilege, and Power are shown in the figure).

2. Some Plans have as their effect ConstraintsOnBehavior, which is a specific subtype of $S$ Description. They are JurPlans and LawPlans ${ }^{13}$. The difference is that JurPlans establish new IndividualConstraintsOnBehavior, while LawPlans establish new Norms. An example

\footnotetext{
${ }^{13}$ This is consistent with [Hart 61], p.33, who characterizes power-conferring norms as recipes for creating duties.
} 
of JurPlan is 'renting a car', where, if the plan is executed correctly and by a person who can do it (according to an existing BasicPower norm), new IndividualConstraintsOn Behavior are set up, regulating the behavior of the two parties (duties and privileges of the renter and of the client). On the contrary, the introduction of a new norm stating that car rental may be made just to people older than 20 is the execution of a LawPlan

3. Not every agent may execute JurPlans or LawPlans. An agent who can do that is said to have Power. For example, the owner of a car rental agency has the Power to rent a car, and, according to the hypothetical rule mentioned above, anybody older than 20 (possibly, with a driving license) has the Power to hire a car from an agency. Both of these Powers are BasicPowers, since they enable the execution of plans that establish individual duties. On the contrary, only people (or institutions) having some LawPower can modify the minimal age for renting a car, since this implies a modification of the legal system.

The result of this conceptual analysis is depicted in fig.6. The ontological modeling of point 3 above does not appear in the figure, but it is (partially) reported in fig.7.

We can now turn to the four Legal Relations concerning Power. Their interconnections seem less clear than the ones among non-powers.

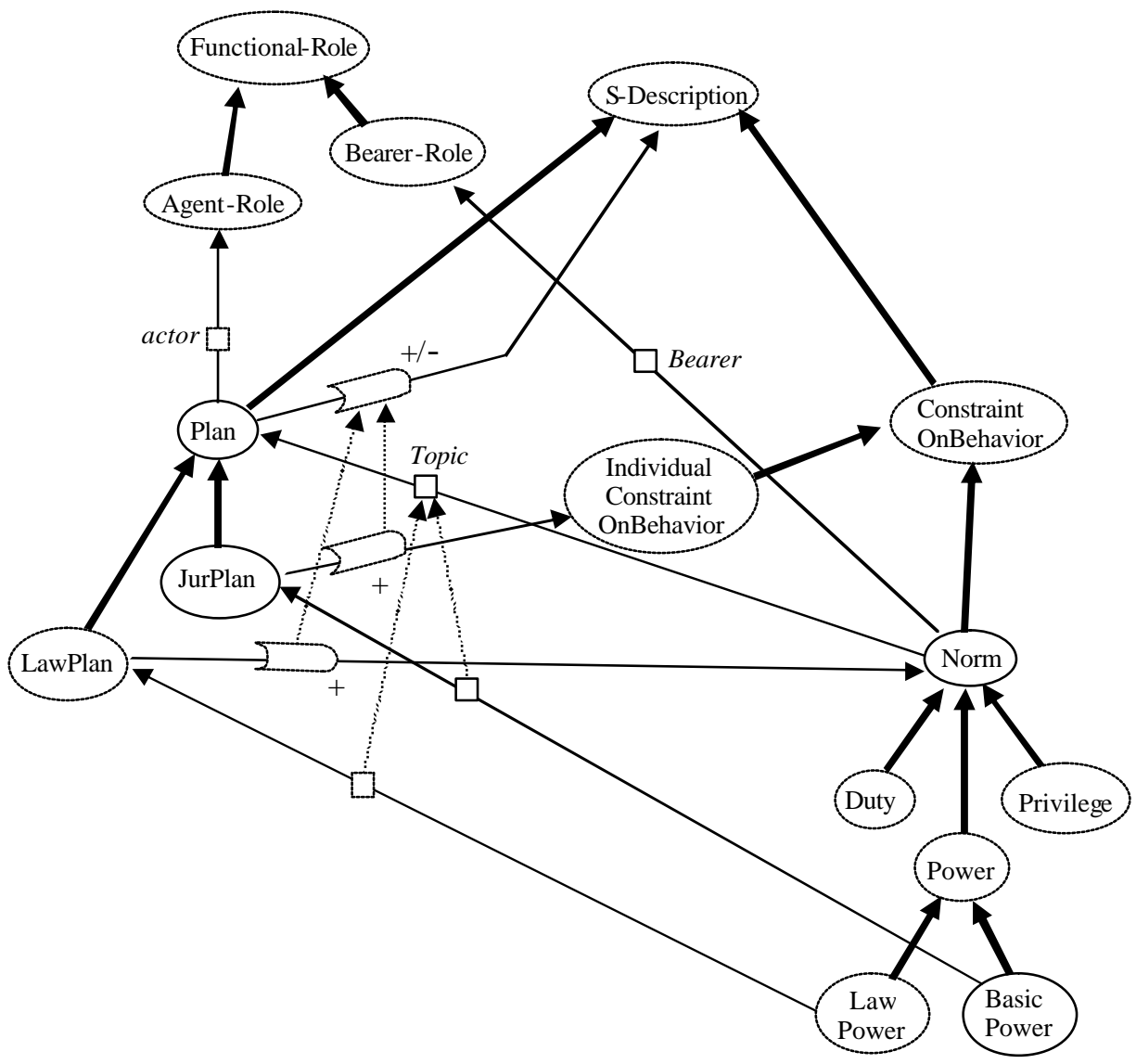

Fig. 6 - Norms concerning Power 
5. $\mathrm{X}$ has the POWER to bring about that $\mathrm{P}$ (where $\mathrm{P}$ is some Legal Relation), iff there exists an action $\mathrm{A}$ such that in case $\mathrm{X}$ does perform $\mathrm{A}, \mathrm{P}$ is established. In other words $\mathrm{A}$ has among its effects P.

6. $\mathrm{X}$ has the INABILITY to bring about that $\mathrm{P}$ (where $\mathrm{P}$ is some Legal Relation), iff there exists no action $\mathrm{A}$ such that in case $\mathrm{X}$ does perform $\mathrm{A}, \mathrm{P}$ is established.

7. $\mathrm{P}$ has the LIABILITY of being brought about by $\mathrm{X}$ iff there exists an action $\mathrm{A}$ such that in case $\mathrm{X}$ does perform $\mathrm{A}, \mathrm{P}$ is established.

8. $\mathrm{P}$ has the IMMUNITY of being brought about by $\mathrm{X}$ iff there exists no action $\mathrm{A}$ such that in case $\mathrm{X}$ does perform $\mathrm{A}, \mathrm{P}$ is established.

Although the picture is simpler than in case of non-power relations (6 is the 'inverse' of 5, 7 is the 'opposite' of 5, and 8 is the 'opposite-inverse' of 5), there is just one agent involved, and the terms LIABILITY and IMMUNITY apply to Legal Relations, while in ordinary langua ge they are more commonly used to refer to agents. When one says that agent $\mathrm{Y}$ is 'liable', it must be assumed that the speaker refers to a legal relation $\mathrm{P}$ involving $\mathrm{Y}$, so that ' $\mathrm{Y}$ is liable to $\mathrm{P}$ ' is interpreted as 'there is some agent $\mathrm{X}$ and some action Act, such that if $\mathrm{X}$ performs Act, then a Legal Relation (P) involving $\mathrm{Y}$ is established' (or, alternatively, $\mathrm{Y}$ is liable to $\mathrm{P}$ iff there exist an agent $\mathrm{X}$ such that $\mathrm{P}$ has the LIABILITY of being brought about by X; remember that P 'involves' Y).

Consequently, since all other relations are easily defined in terms of POWER, we can now focus on it. In principle, Power does not tell us very much: there is some action that an agent can perform that has some effect concerning Legal Relations. But, apart from the final qualification about the type of effect, this is true for every action: this is the very definition of an action (or a plan, if the action is complex): a plan is something an agent may execute to achieve some effect.

So, it is sufficient that such an action be defined, in order to obtain Power: if the action does not exist, then no Power exists; if the action does exist, then there is the Power; but provided that the preconditions are satisfied! And the origin of Power seems to be in the preconditions. As we have already noticed, usually, preconditions express factual constraints for the execution of an action: if there is no lamp, you cannot turn on the light. But in this case, something different is involved: there are some preconditions that are 'conventional', in the sense that even if they do not hold, the action appears to be executable. So, if a judge looks at a person while walking in the street, and tells him: "I condemn you to two years", the 'condemn' action seems to have been executed, but, of course, this is not so. But where do these further constraints come from? The answer is that there needs to be some Norm (or a set of norms) that defines the action. So, a Power depends on the existence of norms defining an action modifying a Legal Relation. Under this reading, the Hohfeldian definition applies without changes: what is important is just to note that the action A, whose execution can bring about $\mathrm{P}$, is not a 'natural' action, but it is a 'conventional' action suitably defined.

We must remark that what is in order here is not the introduction of a Norm (an action which always require an Authority), but the introduction of a Plan, as, for instance, the 'selling and buying' plan. The fact is that, according to the previous discussion, there is no way to define this plan, other than having a (set of) norm introducing it, i.e. no JurPlan or LawPlan can exist, unless it is the topic of some Norm. In other words, an agent cannot 'invent' the plan 'selling and buying', as he could 'invent' a new plan for organizing a party: the latter can be invented, because its effects are factual effects (if the party comes out to be a nice party, the plan is a good plan, since it has achieved the effects it was invented for); but the former cannot be invented, since it will never have any effect at all, unless some authority establishes which are these effects, and under which conditions they are achieved (see fig.6). 
For instance (see fig.6), a LawPlan for the definition of new types of contracts can be executed for defining UnsafeContract. This execution has as its effect the creation of a Norm (the one ruling UnsafeContract). This is a Power (the one that is possessed by those who can use UnsafeContract) i.e. a type of Norm, but it does not seem that there is a newly defined Plan involved. However, each Norm refers (topic) to a Plan; so, the same holds for Power; in our example, it is the Plan that must be executed for entering a UnsafeContract. In general, it is not possible to introduce a Norm without the associated topic, and the topic of a Power is the Plan that must be executed to exercise the Power. Note also that we are referring to the creation of norms, but of course nothing changes substantially in case of modification or cancellation of norms

The definition of UnsafeContract was an example of application of LawPower. On the contrary, the execution of the Plan associated with UnsafeContract is just an application of a JurPower. In this case, what come to existence are just one or more instances of Legal Relations (e.g. new Duties and Privileges). It is clear that the first type of Power has a much greater impact on the society, so that it must be much more constrained. So, the Authority which can do that is restricted to be a carefully defined group of people (or the King, in our spaghetti example). Of course, the degree of impact is different for different laws, so that different levels of authority are usually defined.

The task of determining "who has power" is much more complex than it might appear. Each and every institutional organization (ranging from the Constitutional Court to the lowest levels) has received its POWER on the basis of some set of norms, stating which are the attributions of these organizations and how they are composed. So, they find their proper place within an established social environment. But what about illegal organizations, for instance Mafia? How can we model the fact that a local Boss has established that all food shops of a given area must pay 1000 Euro per month for the protection? It seems that this is not so different, from an ontological point of view: even in this case, a rule has been introduced, together with its restrictions ("food" shops, "a given area"), and plan (pay 1000 Euro), in this example with a POS polarity. So, there should have been an exercise of POWER; but which is the source of this POWER? It may be argued that it is force and violence, but perhaps it is more precise to say that it comes from a parallel social organization whose grounding is on force and violence. Of course, the fact that, hopefully, there will be greater resistance to this rule than to legal rules, depends on an evaluation on the source of the POWER (commonly agreed Constitutional origin rather than violence), but this does not affect the ontological status of the rule itself. So, we have that Authority (i.e. the owner of POWER) gets its status from a social body, a source which is something different from the Authority.

With respect to BasicPower, it must be added that all agents are full of BasicPower. As legal persons, we are owners of some properties, so that we can do things with them, as selling them, whose effects are guaranteed by the legal system, or more precisely, by the BasicPower it assigns to us (unfortunately just in most cases, not always, see [Sen 99] and the concept of capabilities he introduces). Now, we can decide that Authorities are agents having a Power, in which case all of us are Authorities with respect to our home or our credit card, or that the term Authority applies just to agents having some LawPower. This latter usage seems more akin to the standard one, so we adopt it.

Before turning back to the technical issues, we must observe that some rules exist independently on any POWER. They are the rules of tradition (not to be confused with the rules coming from written religious texts). They are rules the origin of which is unclear: rules to which everybody in a society usually conforms, just because they exist, and because who does not respect them is not respected by other people. This situation does not seem to pose any special problems for the ontological framework we are proposing: the basic structure of the norm is exactly the same as before. Simply, the author of the rule is undefined. But a sanction usually exists, and it is the probable reaction of 
the other members of the society (in extreme cases, nobody wants to have any contact with you any more).

Now, we show the placement of Authority in the ontology. Note that Authority is a material role, since individuals or groups can become or cease to be authorities, according to the application of some POWER. The main ideas are depicted in Figs. 7 and 8.

In fig.7, we see that the Author of a Norm is the Actor of the LawPlan whose effect is that Norm. In fig.8, $\mathbf{t}$ is shown how one becomes an Authority, i.e. as an effect of a special type of LawPlan named AuthorityCreationPlan. Notice that the second point means that a given material role (in this case Authority) comes to be true for an individual as an effect of some action (as Intention in fig.4).

What is not represented in the figure is that Authority is organized in levels. This could enable us to state that an Authority may be created just by another 'more powerful' authority. However, it may be argued that in many cases the creator of an authority need not be an ancestor in the hierarchy: for instance the commission for the final examination of new judges need not be viewed as more powerful than the judge, if by MorePowerful we intend that the decisions of the first prevail on the decisions of the second.

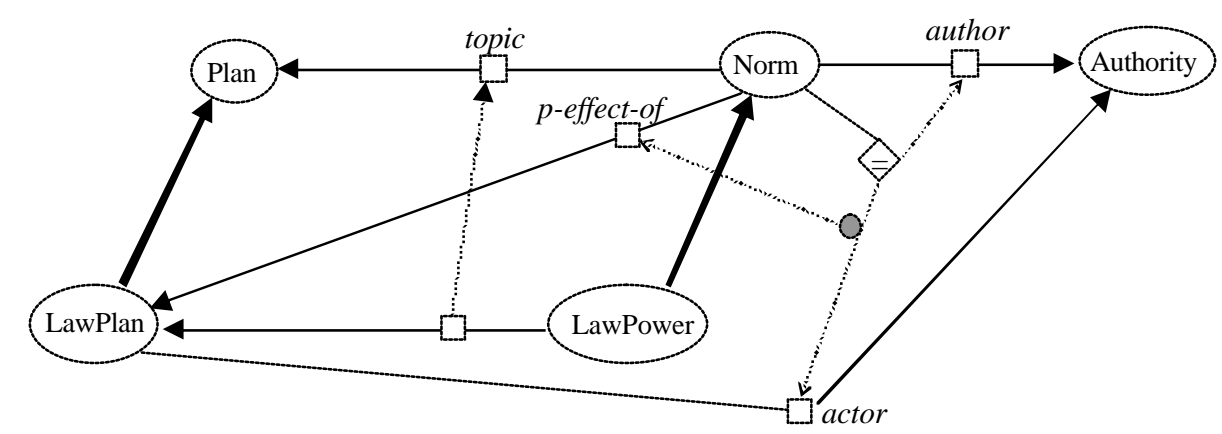

Fig. 7 - Authorities: what they do: the author of the Norm is the same as the actor of the LawPlan that brought about (effect-of) the Norm. This is obtained by exercising some LawPower, which has as topic the executed LawPlan. Note that there are two Norms involved here: the first one is the LawPower (a specific type of Norm) which has been executed; the second one is the 'new' Norm that has been created.

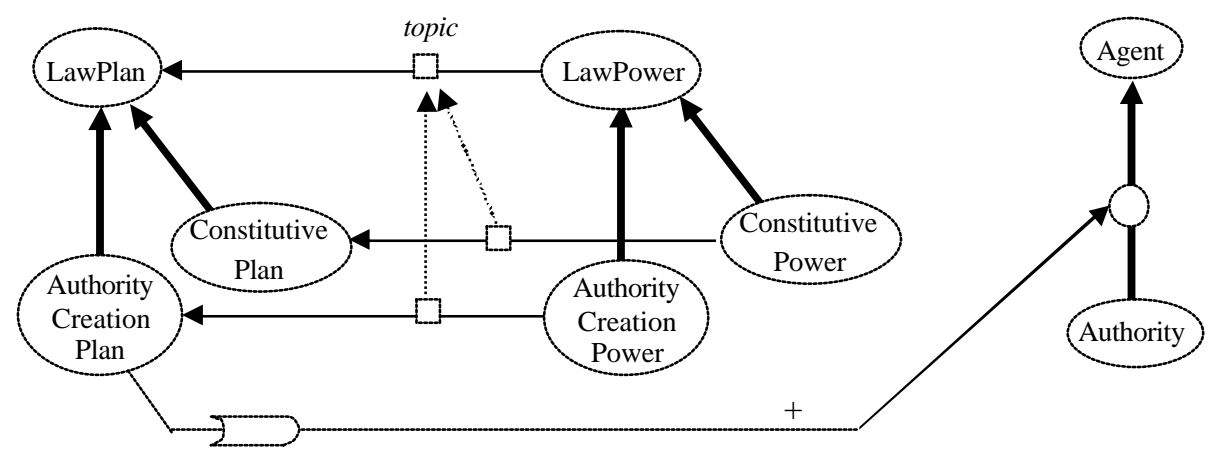

Fig. 8 - Authorities: how they are created. The fact that some Agent becomes an Authority is the effect of a particular LawPlan (called Authority-Creation-Plan). Another example of LawPower is the ConstitutivePower. 
One can wonder if, as there exist AuthorityCreationPlans that create Authorities, i.e. roles able to exercise a LawPower, there also exists something able to create entities to which some JurPower is assigned. In fact, they do exist, and could be named ConstitutivePlans. ConstitutivePlans have as their effect the creation of new Legally-Constructed-Person (of a given type). As such, this new Legally-Constructed-Person can execute some actions that have legal effects. For instance, if a new company is created as the effect of some legal procedure, the effect is that this new entity inherits all the properties of the general type Company, including their internal composition and their ability to do things (as hiring personnel, signing contracts, etc.). Of course, not everybody has the JurPower to create a new Company, but only some legal entity, to which this JurPower was assigned by another, previously established Norm. Notice that we have stated that all of this is an exercise of JurPower, and not of LawPower; accordingly, the entity who can create a company need not be an Authority. This may be seen as contrasting the common use of the term 'authority', but it seems to be consistent with the idea that Authorities create general rules, which is not the case for the creation of an individual instance of Company. A very different case is the one where some organism created the very concept of Company, with all of its attributions (Privileges, Duties, and so on). This is a true application of LawPower, a task of some pre-defined Authority. ${ }^{14}$

\section{A case study: fruits}

In this section, we report the result of a preliminary study on a legal concept, i.e. fruits (see Italian Civil Code, art. 820). A Fruit is something that is obtained by somebody as the result of his ownership of something else. For instance, you obtain the apples if you are the owner of an apple tree, or you obtain some money if you own a house and you rent it out. Notice that the presence of fruits does not affect the ownership of the thing. This section aims at presenting knowledge about fruits appearing in the Italian Civil Code. In the next section, we will report the representation of a norm concerning fruits, in order to get a first rough idea about the impact of norms on the ontology.

\section{Goods}

Goods are defined as a subcategory of Endurants ${ }^{15}$ (see fig.8). We partition the goods in two subcategories: the goods that may undergo a property relation (SubjectToProperty), and the ones which cannot (NotSubjectToProperty). The individuals of the first category can play two roles: goods that, at some time, are a property of somebody (Asset), and goods which are temporarily not owned by anybody (ResNullius). The PropertyRel relation involves a Legally-Constructed-Person (the owner) and a SubjectToProperty good (the owned thing: ownee). An Asset is the object of a SubjectToProperty good, for which there is a PropertyRel relation (i.e. the prop-of role is restricted to have at least one filler: 1,n). On the contrary, a ResNullius is not involved in any PropertyRel: prop-of is restricted to have no filler $(0,0)$.

\footnotetext{
14 Actually, an organization as a Company can also create an internal system of rules that constrain and organize the behavior of its personnel. So, in principle, it can also be seen as an Authority. A partial solution is to create a special type of LegalSubject which refers to aggregates having a rich internal organization, some of whose internal organism have the role of Authorities.

${ }^{15}$ As one referee pointed to us, the specification of what may constitute a Good from a legal point of view requires a more careful analysis. It is unclear, for instance, whether a Description may be a Good, while it is certain that some kinds of Activities (i.e. services) certainly are Goods. Since the focus of this example is on Fruits, and, at least in principle, any Good can produce Fruits, we leave open the problem of a better characterization of Goods.
} 


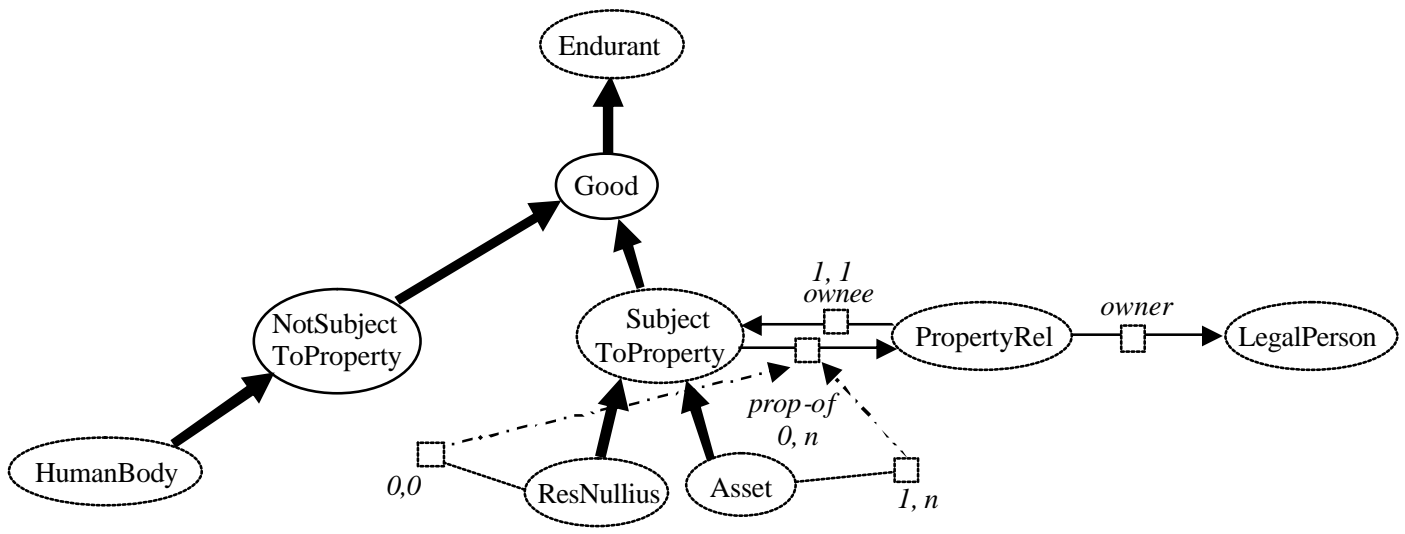

Fig. 9 - Goods

\section{Fruits}

Fruits are Goods. There are two types of Fruits: NaturalFruits and CivilFruits. NaturalFruits are goods that are "detachable" parts of some "original" good, and which originated out of the original good via some natural process (e.g. the growth of real fruits - apples, peaches, etc. - the birth of a lamb out of its mother, etc.). In this paper, we will not discuss natural fruits any more.

On the contrary, CivilFruits are Assets, which have changed their owner, after some more or less complex procedure. The general idea is that some agent Al owns (and possesses) some Good G1; then, he can decide to exchange the possession (maintaining the ownership) against some Good G2, usually for some limited period of time. A second agent $A 2$ gets the possession of $G 1$ and gives $G 2$ to the first agent. In this situation, G2 is a CivilFruit of G1. The simplest example is the one of (house) rental, where $G 1$ is the rented house, $A 1$ is the owner of the house, $A 2$ is the tenant and $G 2$ (the CivilFruit) is the amount paid for the rent. All of this is ruled by some JurPlan (as a Contract), which must be executed by the involved parties (A1 and A2). As seen before, in order to be authorized to execute a JurPlan, an agent must have some JurPower. In the example of house rental, this power has been given to the owner $A l$ by the original act of buying the house. On the contrary, the power exercised by $A 2$ is due just to his being a Legally-Constructed-Person, and to his power on the money he pays for the rental. Of course, all of this produces new legal relations, in particular, new Privileges for A2; these are the Privileges associated with the Possession relation, which has been created as an effect of the execution of the Rent contract.

In fig.10, we have depicted some of the concepts discussed above. In particular, it has been made explicit that:

1. CivilFruit, since it is an Asset, has a owner (linked to it via the PropertyRel concept)

2. CivilFruits come from some other Good, which we have called ExploitedGood (which is a role taken by the Good when it is used in a FruitProducingContract; see point 10 below)

3. The owner of the ExploitedGood is the same as the owner of the CivilFruit

4. There exists a special type of Contract involving exactly two contractors (Contract\#2).

5. A subtype of type of Contract\#2 is FruitProducingContract. The two contractors are called the giver and the receiver.

6. FruitProducingContract involves two Goods, called the primary and the secondary (in this case, the names are not so clear; we had to keep apart the good which change its possession, as the rented house - the primary - from the good that changes ownership - the secondary). 
7. A first effect of a FruitProducingContract is that a new instance of the Possess relation is created (PossessX in the figure), whose possessor is the receiver of the FruitProducingContract, and whose possessee is its primary.

8. A second effect of a FruitProducingContract is that a new instance of PropertyRel (PropertyY) is established.

9. The owner of PropertyY is the same as the giver of the FruitProducingContract which produced the creation of PropertyY, while the ownee of PropertyY is the secondary of FruitProducingContract.

10. The primary of FruitProducingContract, which is an Asset, as an effect of an execution of FruitProducingContract takes the material role of ExploitedGood, as well as the secondary takes the role of CivilFruit.

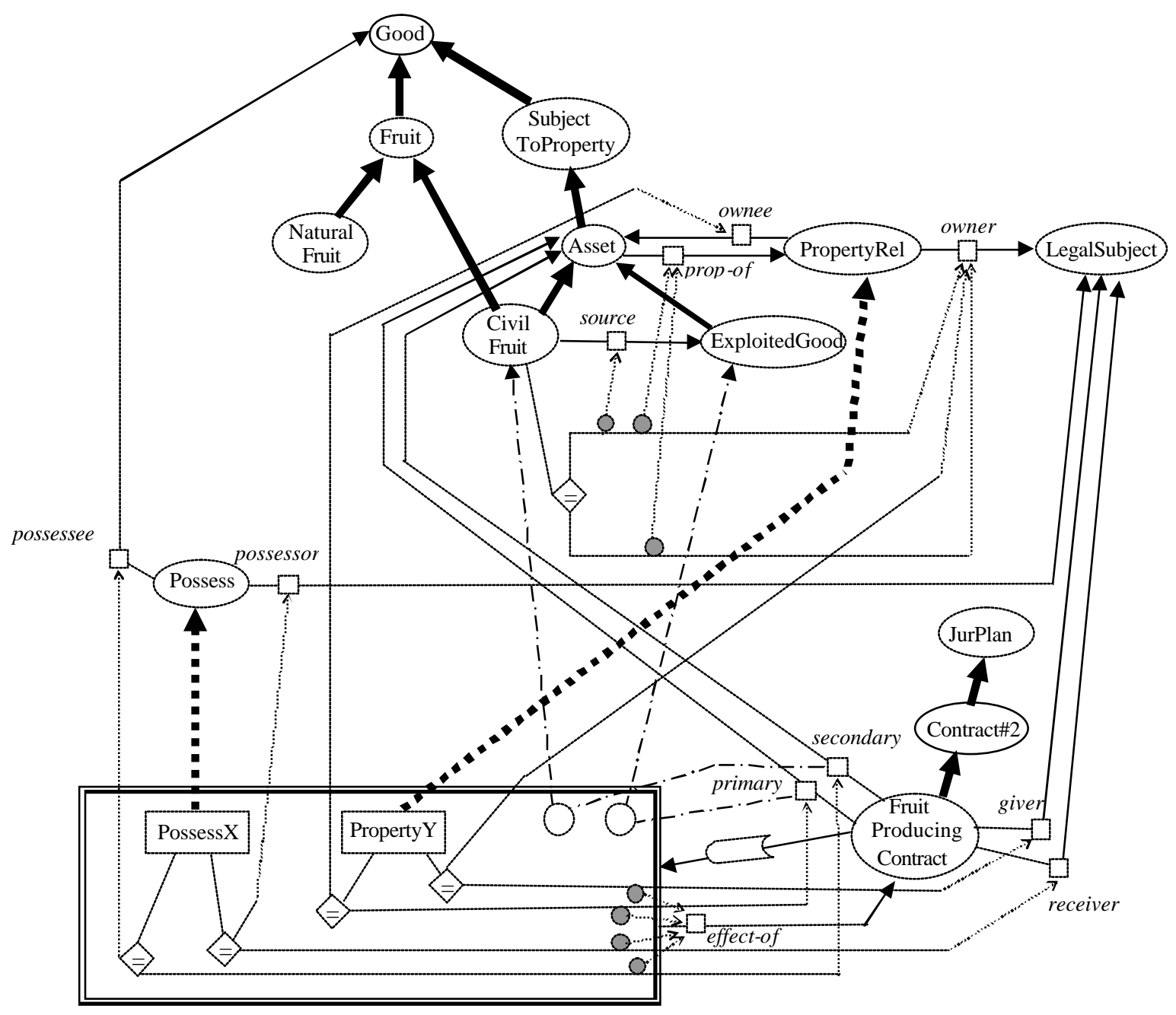

Fig. 10 - Fruits

It is clear that this is just a simple example. However, it makes explicit some interesting features; in particular, items 2 and 10 above. Item 2 implies that there is no CivilFruit without an ExploitedGood; item 10 specifies how CivilFruits come into being; note that this is not a definition, unless one states that the only way for an Asset to become a CivilFruit is as an effect of FruitProducingContract plan, which is not stated here. But apart from contracts other than 
FruitProducingContract, what can easily be made is to introduce specializations of FruitProducingContract. So, we can have ApartmentRental, CarRental, LandRental, BankDeposit, etc. all of which share (inherit) the general features of FruitProducingContract. In particular, all of them have in common the effect that some Asset becomes a CivilFruit.

\section{A norm on fruits}

As an example of norms concerning fruits, we consider part of the article 1477 of the Italian Civil Code: the seller must give the buyer the possible fruits produced by the sold asset (the ExploitedGood). More precisely, if the owner of a Good, which is currently playing the role of ExploitedGood sells that Good, then also the associated Fruits must be given to the buyer.

In fig. 11 we show the relevant part of the representation. The focus of the example is the Norm instance Give-FruitW, which is a Duty that comes into being as an effect of the execution of SellExploitedGood, which is a JurPlan. This instance is analogous to the instance of Property that refers to the fact that the buyer is now the owner of the sold item, in the sense that both of them are effects of a selling action. Note also that the Norm mentioned in the previous paragraph is not represented explicitly, since it is actually part of the definition of the Power associated with the definition of SellExploitedGood. In fact, as stated in section 4, the issuer of the Italian c.c. article 1477 had the power to (implicitly) define the SellExploitedGood action (although no name was given to it). Within this definition, the effect of creating the Duty of transferring the CivilFruits was included, and this is what is depicted in the figure.

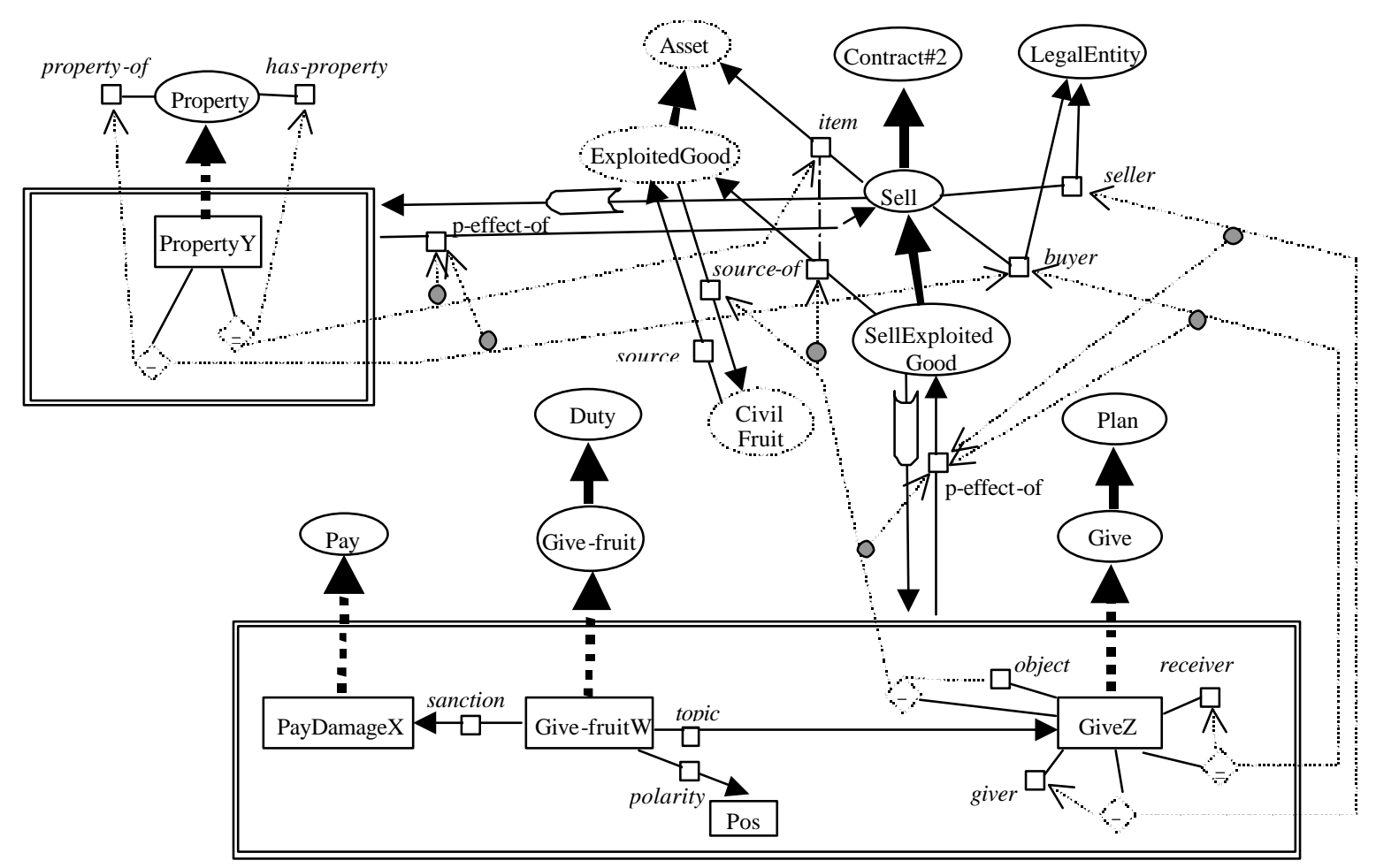

Fig. 11 - A norm about selling ExploitedGoods 
1. Selling is a specific type of Contract\#2 (and hence a JurPlan).

2. Among the effects produced by Selling there is a change of Property: a new instance of Property is established (shown in the figure) as well a previous property is cancelled (not shown in the figure).

3. In case the sold item is an ExploitedGood, a specific subcategory of Sell is defined: SellExploitedGood.

4. Beyond the (inherited) effects produced by Sell, SellExploitedGood produces a further effect: a new Duty (Give-fruitW). The bearer is the same legal subject who plays the role of seller of the Selling action, but this need not be specified explicitly, since it is inferred from the general rule that the bearer is the has-agent of the topic of the Norm (i.e. the giver).

5. On the contrary, it is explicitly stated that the giver of the Civil-Fruit is the same as the seller of the ExploitedGood.

6. The Assets which must be given by the seller to the buyer are the CivilFruits that are produced by the asset which has been sold.

7. According to the Italian civil code, when a duty created by an obligation is violated by an agent, he must repay for the damage created to the beneficiary: the sanction associated to the Give-fruit duty is thus to repay the buyer for the damage due to not giving the fruits of the sold assets.

\section{Ontological models of the legal system}

Classical formalizations of legal reasoning are based on deontic logic, as [von Wright 51] [Alchourron 93] [van der Torre \& Tan 95] [Prakken \& Sergot 97], to quote just a few examples. More recently, ontologies have been adopted as a means to give structure to the legal domain, beyond the basic deontic primitives. Ontologies have been used in a wide range of applications, from legal advice (see the application of the CLIME ontology to Maritime Law [Winkels et al. 99] [Boer at al. 01], the representation of cadastral data and norms ruling the Real Property Transactions [Stuckenschmidt et al. 01] and the reasoning about the eligibility to legal aid for the indigent [Zeleznikow \& Stranieri 01]), to the access to legal information ([Winkels et al. 00], [Tiscornia 01]). Various studies aimed at proposing ontological primitives [Mommers 01] or at comparing and evaluating different approaches to legal ontologies [Visser \& Bench-Capon 98] [Bench-Capon 01]. In this section, we overview three models, which seem more relevant for setting the context of the present work.

In various papers, Breuker, Valente and Winkels (BVW) have described a 'functional model' of legal systems. The model, which is very articulated, and covers many aspects of legal knowledge and reasoning, is based on the idea that

"the legal system as a whole (and therefore each of its components) exists to accomplish a certain function, in order to obtain certain social goals. The legal system is thus viewed as an entity with a certain internal structure, behaving in an environment, and that was designed to work in a certain way in order to be able to accomplish specified social goals. The main function of the legal system is to change or influence society in specific directions, determined by certain social goals." [Breuker et al. 97]

The goal of BVW's research activity is to provide a general framework for law, including the legal reasoning necessary to assess the cases. The knowledge required has been classified according to its function: Normative knowledge (it prescribes behavior and defines a standard of comparison for the 
social reality), Meta-legal knowledge (it organizes the relative position of norms, coping with conflicts, validity, etc.), World knowledge (it provides the background on which norms are built and deals with both entities and behaviours), Responsibility knowledge (it refers to the links between causal relations and legal responsibility of agents), Reactive knowledge (how the legal system reacts to the behaviour of agents; i.e. with sanctions or rewards), and Creative knowledge (enabling the system to define new entities and new types of entities). A general schema of legal reasoning involves taking in input a case, 'translating' its description in legal terms (i.e. according to legal categories), then 'matching' it against the existing norms in order to verify if there has been a violation, then assessing the responsibilities of agents and possibly choose the reactions of the legal system.

It is clear that BVW's perspective is much wider than ours. The present article, in fact, contributes mainly to the definition of the links between Normative knowledge and World knowledge (with a possible future impact on Responsibility knowledge). What is especially relevant in the present context is BVW's view of model-based reasoning; their approach moves away from pure he uristic reasoning towards a full representation of the principles and contents of legal knowledge. But they claim that full modelbased reasoning is not adequate for the task at hand, so that "weaker versions of model-based reasoning that do not require full envisioning" [Valente et al. 99, p.1082] are preferable $^{16}$. It cannot be denied that full-blown model based reasoning is hardly feasible in a domain as complex as legal reasoning. However, it seems that some further steps towards this end can be useful to approach the goal of linking legal concepts to world knowledge.

In particular, we follow the suggestion of matching real-world situations against legal knowledge, but we also claim that this match can produce deeper insights on what happened and why, if the mental processes that lead people to behave in a given way are properly taken into account. Also in this respect we follow BVW, who state that:

"Primary norms address individual behavior (instances of behavior) in the world by means of reference to patterns of behavior" [Valente et al. 99, p.1093].

and

“... in law the causal reasoning is contingent upon notions of responsibility, i.e. one has to take into account also the concepts 'intention' and 'knowledge/belief'” [Valente et al. 99, p.1102].

Our view is that many of the concepts strictly linked to the notion of agency (i.e. behavior, intention, knowledge/belief; see the research on BDI agents) are relevant in legal modelling, but they still lack an adequate formalization in terms of ontological primitives. We claim that BDI models, together with their involvement of desires and intentions, are one of the essential bricks required to expand and extend BVW's analysis. In fact, in assessing a case, it is not only necessary to decide if one or more norm have been broken, but it is also necessary to understand the reasons why an agent did break them. And this cannot be done, unless one has a view of the reasoning mechanism that led the agent to choose that specific line of behavior. This is also made clear in the description of Agent causation in [Lehmann et al. 03]:

"The agreement around cases of agent causation is not reached as easily as in case of physical causation. This is due to the problem of detecting the beliefs, desires and intentions of the agent that starts the physical process. Usually a non intentional action does not fully qualify the agent that performs it as an agential cause, despite him being the physical cause" [Lehmann et al. 03, pp.21-22].

The present article tries to specify which is the link between an agent's intentions, her/his behavior and the norms that affect them. In this sense, it can be seen as an extension of BVW's model,

\footnotetext{
${ }^{16}$ On this point see also [Bench-Capon \& Visser 96].
} 
although our focus is more on the reasoning of the agent than on the reasoning of the legal system that has the task of inferring (abducing) it from the agent's behavior.

Similar comments apply to the system described in [Kralingen et al. 99], where the "conceptual frame-based ontology" includes three types of frames: norm frames, act frames, and conceptdescription frames. In the description of the Norm Frames, the authors state:

"A legal subject attempts to stay within the law and attempts to avoid the breach of norms. The reason for this behaviour, whether it is social pressure or threat of sanctions, is not important to our discussion of norms" [Kralingen et al. 99, p.1133]

And, in fact, the norm frames include five slots, which describe the subject of the norm, the legal modality of the norm (duty-imposing, permission or power-conferring), the action to which the norm refers to (What must be done or forborne?), and some extra conditions of application (place and time). As we have seen, we assume that a sixth slot is required, i.e. the sanction. In our view, this is essential in the task of explaining how the existence of a norm affects the behavior of agents, who have to take into account the various aspect of the situation (including the existence of norms and possible sanctions) in order to choose a line of behaviour. Actually, the act frame includes three slots that are related to agent behaviour: cause, aim, and intentionality, but it is not clear if they have any impact on the actual model. In any case, the glosses reveal that these terms are used in a rather different way than we do (see Section 4).

In order to give an example of the importance of a deep model of actions, we may refer to the domain used in [Kralingen et al. 99], i.e. the Imperial College Library Regulation (ICLR). One of the norms refers to the maximum number of books that can be borrowed. In particular, it states that that number cannot be exceeded; it is split into two norms, the first ones specifies that a borrower cannot take a book, if s/he has already reached the limit; the second one says that the librarian should not lend a book to a person who has reached the limit. Since the system has the goal of assessing if some norm has been broken, this means that, in fact, norms can be broken. But why? If this can happen, then the librarian must have had some reasons for doing that (an error or a principled choice). In our view, the librarian must also have some reason for not always breaking the rule (sanctions); but if he did that, it could depend on the fact that the borrower was told by her thesis advisor that she had to read a book before the day after, that she has reached the limit, that she lives very far from the library, and that she promised to bring back one or more book the day after. In such a case, we assume that the librarian made the right choice, even if it goes against the norm. And this must be taken into account when the case is assessed, if any action has to be taken against him.

A third model which is relevant here is the one presented in [Hage \& Verheij 99]. It is based on three conceptual primitives, i.e. states of affairs, events and rules. States of affairs concern any type of piece of reality (as 'John is a thief', or 'A minor cannot make a valid will'), and are changed by events (as 'John taking away the car of Gerald' or 'An international treaty being ratified'). There are subcategories of events:

"A special kind of events are acts: events that consist of the intentional behaviour of an individual. A special category of acts are the so-called juristic acts" [Hage \& Verheij 99, p.1049].

In our view, one of the most important features of Hage \& Verheij proposal is the parallel existing between the pair <event,causality> and the pair <rule,constitution>. The events change the state of affair 'obtaining' (i.e holding) before the event into a new state of affair obtaining after the event. And the new state of affairs is causally related (via its 'effects') to the event. On the contrary, two state of affairs (that occur simultaneously) are related via a constitution relation (called supervenience) if there is a rule establishing the existence of this relation. For instance, the event 'signing of a contract' is causally related to the state of affair 'the contract is signed', and 'the two 
contractors are under a contractual bond' supervenes to the latter. It seems that the concept of supervenience is strictly related to what Martha Pollack [Pollack 90] called 'generation': in her case, the difference is mainly a matter of description, one of the example being 'press the switch' and 'turn the light on': they are not different actions (the agent has not done two different things), but the first only produces the second if some conditions apply (there is electric power, the wires are not cut, etc.). In the case of supervenience, we have that the conditions for the existence of the relation are not physical, but conventional: it is not different to have the contract signed and to have two individuals under a contractual bond, provided that there is a rule stating the correspondence; so, it is mainly a problem of description of a state of affairs which actually is a single one. The 'conventionality' of 'supervenience' is of paramount importance, and has been discussed in Section 4. What is also interesting is that the chain of (inverse) supervenience starting from the signing of a contract passes through 'one of the two contractors (A) is under an obligation towards the other (B)', and ends in 'A ought to perform some action': again an explicit reference to agent behaviour.

According to the previous discussion, we claim that actions, the way people use their knowledge about actions, and the way people decide which goal(s) to pursue via their actions, are all elements that have a basic role in defining a legal ontology. The next section is devoted to the introduction of a few proposals about the representation of plans in ontologies.

\section{Ontological models of plans}

Although planning is one of the main topics of AI research, not many attempts have been made to provide a principled formalization of planning concepts. Presumably, one of the main difficulties stands on the fact that knowledge about planning is mostly embodied in procedures, which are notoriously hard to represent declaratively (as an ontology demands ${ }^{17}$ ).

The input to a planning procedure (planner) includes at least the following elements: a set of goals of the planning agent, an expressions of the preferences of the agent, a description of the current situation, and background knowledge. The latter, in turns, includes both knowledge about entities and relations existing in the world, and knowledge about the actions that can be performed in the world (with conditions for their execution and, possibly non-deterministic, effects). The output of a planning procedure is an intention, i.e. a sequence (more or less detailed) of actions that the agent has decided to execute; so, an intention is a specific plan of actions which the agent has committed to. In principle, the planner has to produce all possible plans that enable the agent to achieve one or more of his goals (using the goals, the current situation and the background knowledge), and then must choose one of them (using the preferences), which becomes the agent intention. Of course, the process cannot be carried out in this way, because of the combinatorial explosion in the number of candidate plans; so actual planners become rather complex, because of the need to reduce the search space via heuristics and interleaving of various steps.

\footnotetext{
${ }^{17}$ It is worth stating here again that we are using the world 'ontology' acritically. J.Breuker pointed to us that "Planning is a problem solving task, so the perspective is an epistemological, rather than an ontological one". This comment reflects the view that any 'role' taken by a physical entity in a process concerns epistemology, i.e. the way people conceptualize the process. We share Breuker's view, but we cannot disregard that mental processes, as planning, have, as a physical substratum, the agents' brains, which are ontological objects. However, the literature in the field of philosophy of mind concerning the relationships between mind and brain is vast and growing at increasing speed. Consequently, we simply declare that we agree that a 'description' of mental processes in terms of intentionality, beliefs, etc. should more properly fall within the domain of epistemology, however, we keep the term 'ontology' as the only term that can be used without an in-depth discussion, which is outside the scope of this article.
} 
The most extensive attempt to formalize the notion of plan has been made within the Planning Initiative of DARPA, resulting in SPAR (Shared Planning and Activity Representation) [Polyak \& Tate 98, 99]. The results of this study are impressive, and their main features are the coverage of the formalization (which includes also probabilities and uncertainty) and range of sources which have contributed to the final model, among which representation languages (as PIF [Lee et al. 96]), tools (as Prodigy [Carbonell et al. 91]) and true ontologies (as Ozone [Smith \& Becker 97]); see [Polyak \& Tate 98, pp.3-4] for a complete listing. However, the strength of SPAR seems to bring with itself some weaknesses: the fact that various requirements come mainly from industrial environments (e.g. the need for standards in process specifications), and the necessity to quickly approach the core of planning has made the top-level ontology somewhat confused, including as sisters (under the topmost concept 'entity') concepts rather dishomogenous, as Environment, Activity, TimePoint, Issue, EvaluationCriterion, WorldModel, WorldModelSpecification, to quote just some of them. It seems clear that many of the concept and features appearing in the SPAR proposal are of paramount importance; but is seems equally clear that the connections to a foundational ontology need be refined.

A model which suffers from some of the drawbacks of SPAR (i.e. unclear connections to a top-level ontology) is PLANET [Gil \& Blythe 00]. However, it includes a very nice feature, i.e. an explicit representation of the partial structures which must be built and managed during the planning process. PLANET includes four main structures: planning-problem-contexts, which describe the background knowledge, the constraints that the resulting plans must satisfy, and the initial situation, plan-task-descriptions, which refers to plan templates (i.e. knowledge about actions) and plan tasks (i.e. instantiated portions of plans), goal-specifications, which describes the goals the planner must try to achieve, and plans. We want to stress here just that there is a relation between plans and planning-problem-context called candidate-plans, which carries information about the suitability of the plan for that problem; the possible values of this feature are unexplored, rejected, feasible, selected. So, it is modelled the fact that plans are built as solution hypotheses for a problem, and are then evaluated, on the basis of some criteria, in order to make a final choice. This is especially important in our model too, where norms have exactly the role of further constraints on plans, affecting the final choice of the agent.

\section{Conclusions}

In this article, we have presented an ontological model of norms, based on the behaviour of agents. We have shown that norms can be characterized as constraints on behaviour, i.e. as statements specifying what an agent can and cannot do, and what happens when a norm is broken. The basic idea is that agents choose a line of behaviour according to the utility they may gain; and usually the breach of a norm produces a decrease in the estimated utility, because of the risk of being sanctioned.

In order to include in the ontology knowledge about behaviour, we have provided a sketch of an ontological representation of plans and intentions, showing how a Norm can affect the planning process: the presence of a Norm can urge an agent to adopt a given line of behaviour (complying with the norm) instead of another (breaching the norm). This is obtained by introducing the notion of utility and by letting the agent determine his intention according to the expected maximal utility.

Although the article has mainly addressed primary norms, by also showing how they are related to the basic Hohfeldian Legal Relations, it has also shown that the proposed model covers authorities, i.e. it enables one to express explicitly the role of authorities in a legal system, and the way they are created. Finally, we have shown how the model applies to a specific case study (Goods and Fruits). 
Two final comments concern the degree of coverage of the article with respect to the various components of a legal system and the complexity of the representation. As we have stated in the introduction, the goal of this paper is not to develop a general model of a legal system (see the models described in Section 6), but only to give an ontological characterization of a specific aspect, i.e. the role of primary norms. In principle, norms are just a small portion of such a model, although they are a very important component, as we have argued in Section 2. Representing norms in an ontologically plausible way is the first step towards the implementation of a general model, which can be grounded on the behaviour of agents; the agents are the entities which populate the societies that are being regulated by legal systems and adopting them as the foundation for the representation is, in our view, the main contribution of this work. Of course, other agents do exist, most noticeably the judges and the members of the courts, that must decide about the behaviour of other agents; also judges (as all agents) are subject to norms, but they also have the role of assessing cases; this is a particular activity, that is not addressed in this paper, but that can be arguably assimilated to a kind of diagnostic reasoning: given some evidence (symptoms), classify the situation according to the categories appearing in the system. Since, in doing that, the court must respect some norms, it seems reasonable to assume that the model applies to it equally well, provided that a suitable representation is associated with the process of assessing the case.

Finally, the complexity of the representation. It is a matter of taste if a graphical representation (as the one we have adopted in this paper) is more or less readable than an equivalent representation respecting the linear syntax of a terminological language (see Appendix 1). However, independently of the graphical way the ontology is presented, there is the usual contrast between the expressivity of the language and the computational complexity of the reasoning procedures. We have argued in favor of a language including some constructs that makes the language computationally hard. Although this is not the focus of the paper, we must stress that this appears to be unavoidable, unless one is able to show that the same concepts can be represented with simpler constructs, or that the knowledge expressed by these concepts can be left out of the ontology without loss of information. We believe that neither is the case, so that we hope that suitable heuristics can help a reasoner to obtain results within acceptable time limits.

\section{References}

[Alchourron 93] C. Alchourron: Philosophical foundations of deontic logic and the logic of defeasible conditionals. In Meyer, J.-J., and Wieringa, R. (eds): Deontic Logic in Computer Science: Normative System Specification. John Wiley \& Sons, 1993, 43-84.

[Allen \& Saxon 95] L.E. Allen and C.S. Saxon: Better Language, Better Thought, Better Communication: The AHohfeld Language for Legal Analysis. Proc. of 5th Int. Conf. on Artificial Intelligence and Law, ACM Press, 1995

[Bench-Capon \& Visser 96] Trevor J.M. Bench-Capon, Pepijn R.S. Visser: Deep Models, Ontologies, and Legal Knowledge Based Systems. Proc. JURIX '96 : Foundations of legal knowledge systems. R.W. van Kralingen, H.J. van den Herik, J.E.J. Prins, M. Sergot, and J. Zeleznikow (eds.), Tilburg University Press, 1996, 3-13.

[Bench-Capon 01] Trevor J.M. Bench-Capon: Task Neutral Ontologies, Common Sense Ontologies and Legal Information Systems. Proc. $2^{\text {nd }}$ Workshop on Legal Ontologies at Jurix 2001, 15-19.

[Boella et al. 01] Guido Boella, Leonardo Lesmo, Lyda Favali: The Definition of Legal Relations in a BDI Multiagent Framework, Proc. of AI*IA 01, Bari, Springer Verlag, Berlin, 2001, 225-236.

[Boella \& Lesmo 02] Guido Boella, Leonardo Lesmo: A game theoretic approach to norms. Cognitive Science Quarterly 2, 2002, 492-512.

[Boella \& van der Torre 03] Guido Boella, Leendert van der Torre: Obligations and permissions as mental entities. Proc. of IJCAI Workshop on Cognitive Modeling of Agents and Multi-Agent Interactions, Acapulco, 2003. 
[Boer et al. 01] Alexander Boer, Rinke Hoekstra, Radboud Winkels: The CLIME Ontology. Proc. $2^{\text {nd }}$ Workshop on Legal Ontologies at Jurix 2001, 37-47

[Brachman \& Schmolze 85] Ronald J. Brachman and J. Schmolze. An overview of klone. knowledge representation system. Cognitive Science 9, 171-216, 1985

[Breuker at al. 97] Joost Breuker, André Valente and Radboud Winkels: Legal Ontologies: A Functional View. Proc. of the First International Workshop on Legal Ontologies, 1997, 23-36.

[Breuker \& Winkels, in press] Joost Breuker and Radboud Winkels: Use and Reuse of Legal Ontologies in Knowledge Engineering and Information Management. AI \& Law, this issue.

[Carbonell et al. 91] Jaime G. Carbonell, Oren Etzioni, Yolanda Gil, Robert Joseph, Craig A. Knoblock, Steven Minton, Manuela M. Veloso: PRODIGY: An Integrated Architecture for Planning and Learning. SIGART Bulletin 2, 1991, 51-55.

[Gangemi et al. 01] Aldo Gangemi, Domenico M.Pisanelli and Geri Steve. A Formal Ontology Framework to Represent Norm Dynamics. $2^{\text {nd }}$ International Workshop on Legal Ontologies, University of Amsterdam, 2001

[Gangemi et al. 02] Aldo Gangemi, Nicola Guarino, Claudio Masolo, Alessandro Oltramari, Luc Schneider: Sweetening Ontologies with DOLCE. Proc. EKAW 2002, Siguenza, Spain, 2002.

[Gangemi et al. 03] Aldo Gangemi, Maria Teresa Sagri, Daniela Tiscornia: Metadata for Content Description in Legal Information. Proc. LegOnt 2003 workshop, Edinburgh, 2003.

[Gil \& Blythe 00] Yolanda Gil and Jim Blythe: PLANET: A Shareable and reusable ontology for representing plans. Proc. AAAI 2000 Workshop on Representational Issues for Real-world Planning Systems, 2000.

[Guarino 98] Nicola Guarino: Some ontological principles for designing upper level exical resources. In Proc. First Int. Conf. on Language Resources and Evaluation (LREC 1998), Granada, 1998.

[Guarino \& Welty 00] Nicola Guarino, Christopher Welty: A Formal Ontology of Properties. Proc. EKAW 2000, 97-112.

[Guarino \& Welty 02] Nicola Guarino, Christopher Welty: Evaluating Ontological Decisions with OntoClean. Communications of the ACM 45, 61-65, 2002 .

[Hart 61] H. Hart: The Concept of Law. Clarendon Press, Oxford, 1961.

[Herrestad \& Krogh 95] H. Herrestad, C. Krogh: Obligations directed from bearers to counterparties Proc. of $5^{\text {th }}$ International Conf. on Artificial Intelligence and Law, ACM Press, 1995

[Hohfeld 1919] W.N. Hohfeld: Fundamental Legal Conceptions as Applied in Judicial Reasoning and Other Legal Essays, Yale Univ. Press, New Haven, Conn., 1919.

[Kelsen 45] Hans Kelsen: General Theory of Law and State (Wedberg trans.), New York, Russell \& Russell, 1945.

[Kralingen et al. 99] Robert W. van Kralingen, Pepjin R.S. Visser, Trevor J.R. Bench Capon, H. Jaap van den Herik: A principled approach to developing legal knowledge systems. Int. J. Human-Computer Studies 54, 1999, 1127-1154.

[Lee et al. 98] J. Lee, M. Gruniger, Y. Jin, T. Malone, A. Tate, G. Yost: Process Interchange Format and Framework. The Knowledge Engineering Review 13, Special Issue on Ontologies (eds. M. Uschold, A. Tate): http://ccs.mit.edu/pif/

[Lehmann et al. 03] Jos Lehmann, Joost Breuker, Bob Brouwer: Causation in AI \& Law. ICAIL 2003 Workshop on Legal Ontologies \& Web Based Legal Information Management. Edinburgh, June 2003, 134.

[Masolo et al. 03] Claudio Masolo, Stefano Borgo, Aldo Gangemi, Nicola Guarino, Alessandro Oltramari: WonderWeb Deliverable D18. Ontology Library. Final Report (ver. 1.0, 31-12-2003)

[MacGregor 91] Robert M. MacGregor. "Using a Description Classifier to Enhance Deductive Inference. Proc. $7^{\text {th }}$ IEEE Conf. on AI Applications, 1991, 141-147.

[MacGregor \& Brill 91] Robert M. MacGregor and David Brill: Recognition Algorithms for the Loom Classifier. Proc. 10 $0^{\text {th }}$ National Conf. on Artificial Intelligence (AAAI 92), 1992, 774-779.

[Pollack 00] Martha E. Pollack: Plans as Complex Mental Attitudes. In P.R. Cohen and J. Morgan and M.E. Pollack (eds ): Intentions in communication, MIT Press, 1990, 77-103.

[Polyak \& Tate 98] Steve Polyak, Austin Tate: Planning Initiative: Shared Planning and Activity Representation - SPAR, Version 0.1a. DARPA Internal Report, April 98.

[Polyak \& Tate 99] Steve Polyak, Austin Tate: Planning Initiative: Shared Planning and Activity Representation - SPAR. DARPA Internal Report, March 1999. 
[Prakken \& Sergot 97] H. Prakken, M. Sergot: Dyadic deontic logic and contrary-to-duty obligations. In Nute, D. ed., Defeasible Deontic Logic. Kluwer, 1997, 223-262.

[Ross 68] Alan Ross: Directives and norms. Humanities Press, New York 1968.

[Sen 99] Amartya Sen: Development as Freedom. Oxford University Press 1999

[Smith \& Becker 97] S. F. Smith, M.A. Becker: An Ontology for Constructing Scheduling Systems. Proc. AAAI-97 Spring Symposium on Ontological Engineering, 1997.

[Stuckenschmidt et al. 01] Heiner Stuckenschmidt, Erik Stubkjaer, Christoph Schlieder: Modeling Land Transactions: Legal Ontologies in Context. $2^{\text {nd }}$ Workshop on Legal Ontologies at Jurix 2001, 58-66

[Tiscornia 01] Daniela Tiscornia: Ontology-driven Access to Legal Information, DEXA 2001- Workshop LISA, Legal Information Systems and Application, Munich, September 2001.

[Valente et al. 99] Andrè Valente, Joost Breuker, Bob Brouwer: Legal modelling and automated reasoning with ON-LINE. Int. J. Human-Computer Studies 54, 1999, 1079-1125.

[Visser \& Bench-Capon 98] Pepijn R.S. Visser, Trevor J.M. Bench-Capon: A comparison of Four Ontologies for the Design of Legal Knowledge Systems. Artificial Intelligence and Law 6, 1998, $27-57$.

[van der Torre \& Tan 95] Lendeert van der Torre, Yao-Hua Tan: Cancelling and overshadowing: two types of defeasibility in defeasible deontic logic. Proc. $14^{\text {th }}$ Int. Joint Conference on Artificial Intelligence (IJCAI' 95), Morgan Kaufman, 1995, 1525-1532.

[von Wright 51] G. H. von Wright: Deontic logic. Mind 60, 1951, 1-15.

[Welty \& Guarino 01] Chirstopher Welty, Nicola Guarino: Supporting Ontological Analysis of Taxonomic Relationships. Data and Knowledge Engineering 39, 2001, 51-74

[Winkels et al. 99] Radboud Winkels, D. J. B. Bosscher, Alexander Boer, Joost Breuker: Generating exception structures for legal information serving. Proc. ICAIL 99, New York, 1999, 182-189.

[Winkels et al. 00] Radboud Winkels, D. J. B. Bosscher, Alexander Boer, Rinke Hoekstra: Extended Conceptual Retrieval. In J.A. Breuker et al. (eds): Legal Knowledge and Information Systems 2000, IOS Press, Amsterdam, 2000, 85-98.

[Zeleznikow \& Stranieri 01] John Zeleznikow and Andrew Stranieri: The use of Legal Decision Support Systems at Victoria Legal Aid. Proc. of ISDSS2001, Sixth Int. Conf. on Decision Support Systems, Brunel University, London, 2001, 186-192.

\section{Appendix A: Example of LOOM representation}

In order to give an idea of the actual meaning of the structures presented in the figures, we report here a LOOM version of the contents of fig. 10, assuming that the semantics of LOOM is clearly defined (see [http://www.isi.edu/isd/LOOM/LOOM-HOME.html]). Some comments are reported within the definitions, using the :annotations slot. In order to simplify the match between the figure and the LOOM definition, we have split the latter into three boxes, which have no representational function. The fourth box reports an example of a LOOM query; when executed, it retrieves all civil fruits known to the system. This last box has been included to show that, although LOOM does not allow for role constraints, they can be enforced in a query, as constraints on the data to be retrieved.

PropertyRel, and its roles:

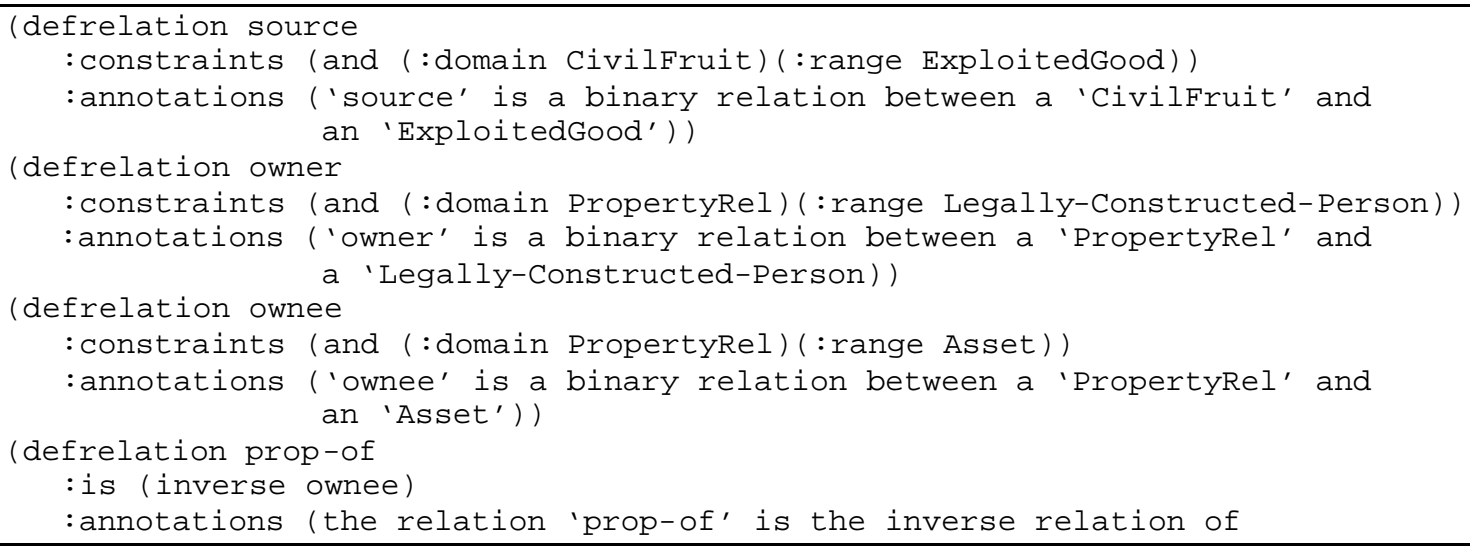




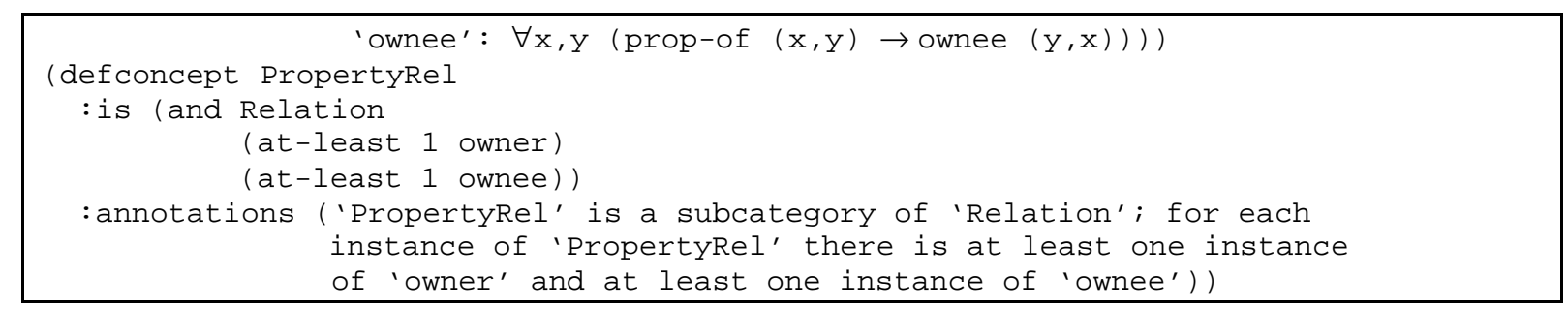

Goods and Fruits:

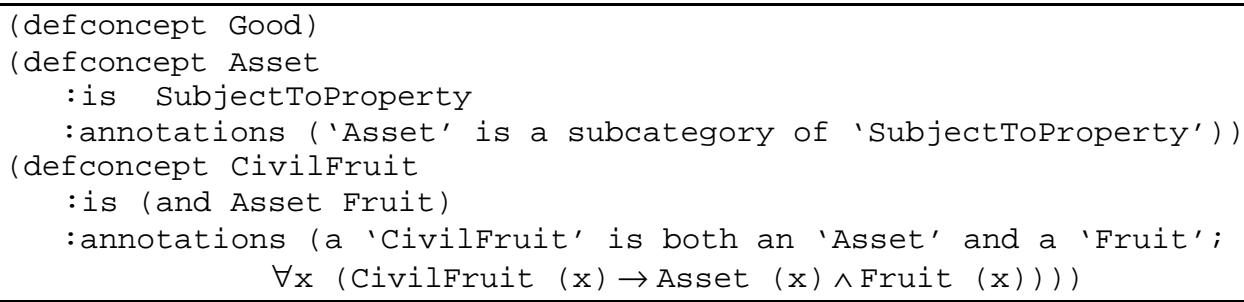

Contracts between two subjects:

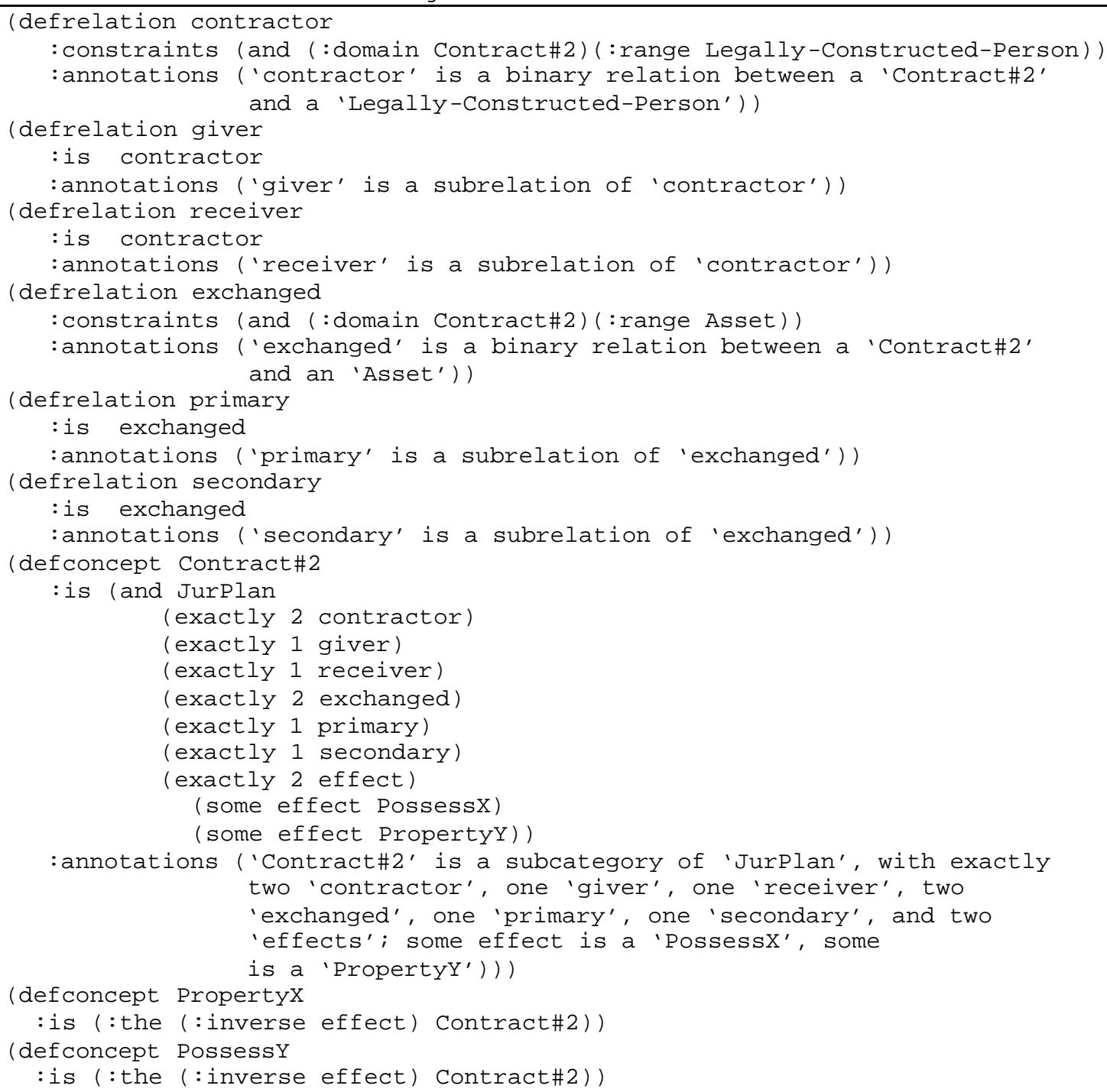

Retrieving data about fruits:

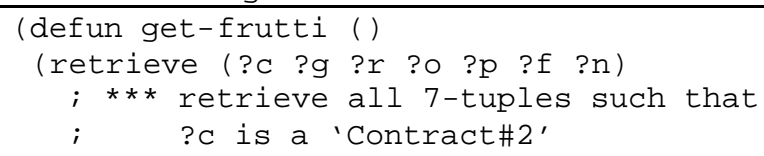




\begin{tabular}{|c|c|}
\hline $\begin{array}{l}\text {; } \\
; \\
; \\
; \\
;\end{array}$ & $\begin{array}{l}\text { ?g is its 'giver' } \\
\text { ?r is its 'receiver' } \\
\text { ?o is its 'primary' } \\
\text { ?f is its 'secondary' } \\
\text { ?n is the resulting 'PossessX' } \\
\text { (and (Contract\#2 ?c) } \\
\text { (giver ?c ?g) } \\
\quad \text { (receiver ?c ?r) } \\
\quad \text { (primary ?c ?o) } \\
\text { (PropertyRel ?p) } \\
\text { (ownee ?p ?o) } \\
\text { (owner ?p ?g) } \\
\text { (secondary ?c ?f) } \\
\text { (PossessX ?n) } \\
\text { ((:inverse effect) ?n ?c) } \\
\text { (possessor ?n ?g) } \\
\text { (possessee ?n ?f) (Civilfruit ?f) } \\
\text { (source ?f ?o))) }\end{array}$ \\
\hline
\end{tabular}

\section{Appendix B: The planning process}

In this appendix, we present a simple model of the planning process. This has to be viewed as a preliminary attempt at an ontological representation of a complex activity as planning. We describe here the features that are depicted in the figure below. Note that the content of the figure is a sort of procedure; although a procedure is inherently a 'procedural' representation of a process, its expression in a programming language also has a declarative (descriptive) flavour, since it can be inspected as a static object (for instance by a compiler). In order to provide an ontological representation, we need the equivalent of some basic programming concepts, as input parameter, variable, sequence of operations, loop, etc. A first proposal appears in DOLCE in the section related to the Task concept [Masolo et al. 03, pp.238-241]. Here, we have adopted the following graphical conventions:

1. Planning is linked to its 'body' (the large box) via a dashed arrow. This notion corresponds to the 'decomposition' of a complex action in its steps. In DOLCE, we have that a Course (which is a Description) sequences a Perdurant. Since a Task is a subcategory of Course, the large box in the figure can be taken as the complex Task that sequences (the dotted upward large arrow) the Planning Perdurant.

2. The sequence of operations is represented as lines ending in a black diamond. Some lines having this form enter the 'body' box: they are the input data, and the small box they enter is the parameter name (ex. GoalsX); some of these arrows exit the box: they are the result (the return values).

3. The sequence of steps is an alternation of ovals (e.g. FindPlans) and rectangles (e.g. PlansX); the former are sub-procedures (steps, or SubTasks), while the latter are intermediate results (local variables).

4. In particular, FindPlans retrieves from the knowledge of the planning agent all the Plans that have as p-effects one or more of the Goals in GoalsX.

5. The Cycle-Until box expresses a loop (in DOLCE, it is a subcategory of ComplexTask): all the steps inside the box are repeated until the exit condition (StartExecution?) is satisfied. The predicate is assumed to be true if a single plan has remained in the focus (FocusedP) and the initial steps of the focused plan are executable; this last condition holds if that step refers to a 'basic action', i.e. to an action that can drive the actuators of the agent.

6. EvaluateAndChoose aims at evaluating the foreseeable utility of the different alternatives (see §2.4); it gets as input the preferences of the planning agent (Prefs $X$ ). 
7. Expand takes into account all remaining focused plans (which may be more than one in case no commitment has been made), and finds ways for executing non-basic actions. After Expand, the resulting Plans are more specific than the original ones.

8. It is specified (via the usual constraints on roles) that the input goals (GoalsX) are the ones of the planning agent. A similar specification should be given for the preferences; we have omitted it because of space constraints.

9. Finally, it is specified that the final plan which is the result of the whole process (IntendP) is the one that becomes the intention of the planning agent (has-intention-on). This is the connection (effects of planning) to the concepts appearing in fig.4.

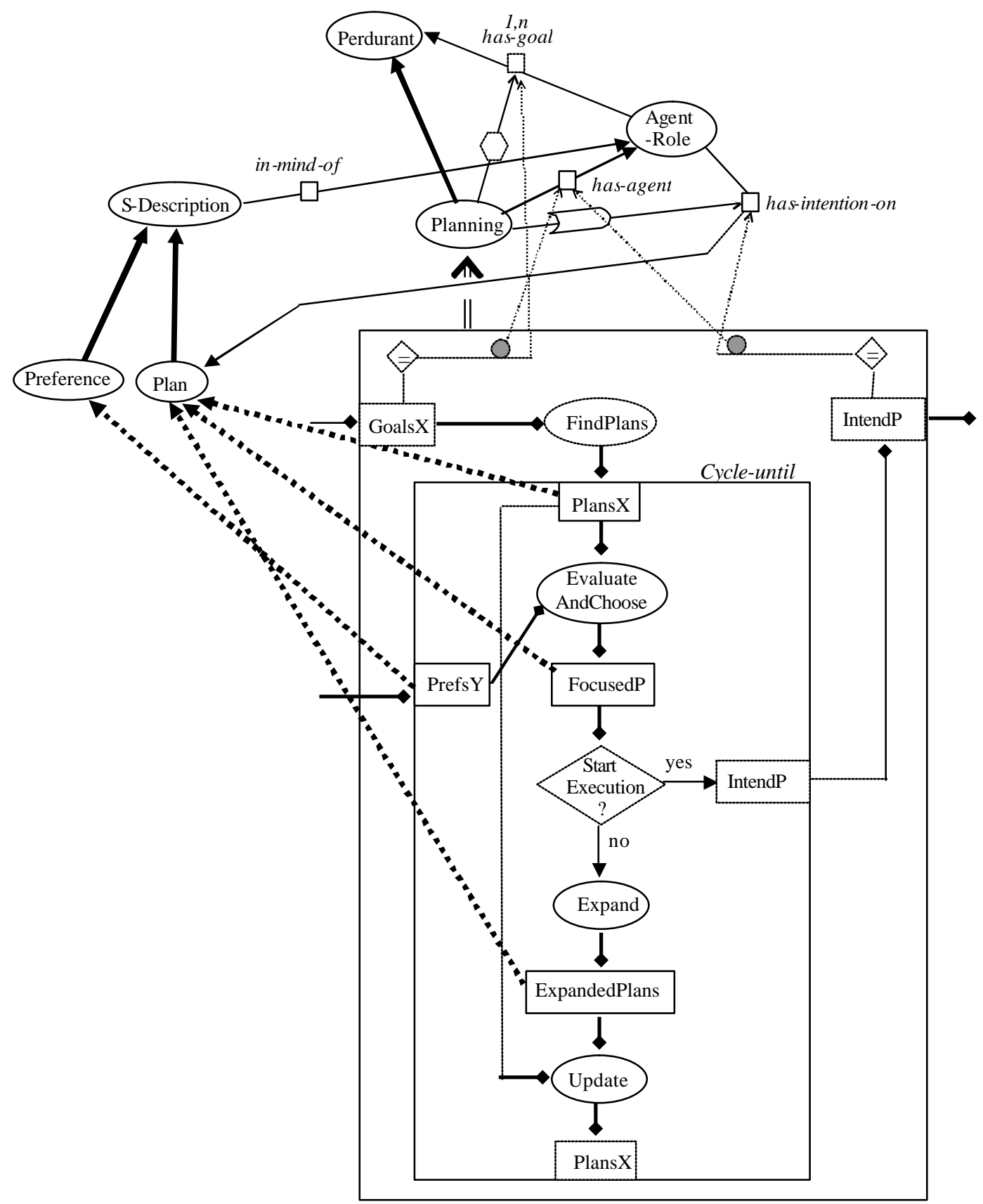

Fig. 12 - Structure of the Planning activity 


\title{
Coordination and Organization
}

\section{Definitions, Examples and Future Research Directions}

\author{
Guido Boella ${ }^{1}$ \\ Università di Torino \\ Italy \\ Leendert van der Torre ${ }^{2}$ \\ University of Luxembourg \\ Luxembourg
}

\begin{abstract}
Coordination languages and models like Linda and Reo have been developed in computer science to coordinate the interaction among components and objects, and are nowadays used to model and analyze organizations too. Moreover, organizational concepts are used to enrich the existing coordination languages and models. We describe this research area of "organization and coordination" by presenting definitions, examples, and future research directions. We highlight two issues. First, we argue for a study of value-based rather than information-based coordination languages to model the coordination of autonomous agents and organizations. Second, we argue for a study of the balance between enforced control and trust-based anticipation to deal with security aspects in the coordination of organizations.
\end{abstract}

Key words: Coordination, organization, electronic commerce, normative systems, multi-agent systems, secure systems.

\section{Introduction}

In human society, organizations embody a powerful way to coordinate complex behavior. Various models of organizations exist, from bureaucratic systems based on norms to competitive systems based on markets. Moreover, organizational concepts structure the behavior of complex entities in a hierarchy of encapsulated entities. For example, roles structure departments, which in turn structure organizations. Moreover, organizations structure interorganizational virtual organizations. Organizations specify also the interaction

1 Email: guido@di.unito.it

2 Email: leendert@vandertorre.com

This paper is electronically published in

Electronic Notes in Theoretical Computer Science

URL: www.elsevier.nl/locate/entcs 
and communication possibilities of each of these entities, abstracting from the implementation of their behavior.

In agent theory and computer science, organizational models in which organizations are autonomous entities have become popular to model coordination in open systems, and practical applications to organizational modeling are being widely developed. This is due also to the need to ensure social order within applications like Web Services, Grid Computing, and Ubiquitous Computing. In these settings, openness, heterogeneity, and scalability pose new challenges for traditional organizational models. For example, organizational and individual perspectives must be integrated and the models must dynamically adapt to organizational and environmental change.

In particular, organizational concepts are used to enrich coordination languages and models developed in computer science to coordinate the interaction among components and objects, like Linda [13] and Reo [1]. Moreover, these languages and models are nowadays used to model and analyze human organizations too. In this survey paper we raise the following questions:

(i) What are "coordination" and "organization" in the context of the first workshop on coordination and organization?

(ii) What are typical examples of research topics concerning both coordination and organization?

(iii) Which results have been obtained thus far in this research area, and what are the future research directions?

An example in electronic commerce illustrates the use of coordination techniques for modeling, analyzing and simulating human organizations, and an example in object oriented programming languages illustrates the use of roles for coordination. We raise the questions which properties a coordination language should have to model organizations, how such a coordination language can be used, which organizational concepts can be used in coordination languages and models, and how these concepts should be used.

We highlight two future research directions. First, coordination in computer science is concerned with information, whereas organizations are concerned with values. Values have properties information does not have, since, for example, it cannot be duplicated, or it can perish. Coordination languages have to be developed that deal with these properties. Second, security aspects play an important role in coordination and organization. Whereas traditional security concerns have focussed on building control mechanisms such as cryptographic methods, organizational coordination focusses on incentives to motivate agents, and trust and anticipation play a central role too.

The layout of this paper follows the research questions. In Section 2 we present some definitions, in Section 3 the two examples from electronic commerce and object oriented programming languages, and in Section 4 the future research directions including value-based and secure coordination. 


\section{Definitions}

\subsection{Coordination}

For completeness, Figure 1 presents the dictionary description of the noun 'coordination' and the verb 'to coordinate', and Figure 2 presents the various uses of 'to coordinate' in the lexical reference system Wordnet [16]. Here we focus on the use of 'coordination' in computer science.

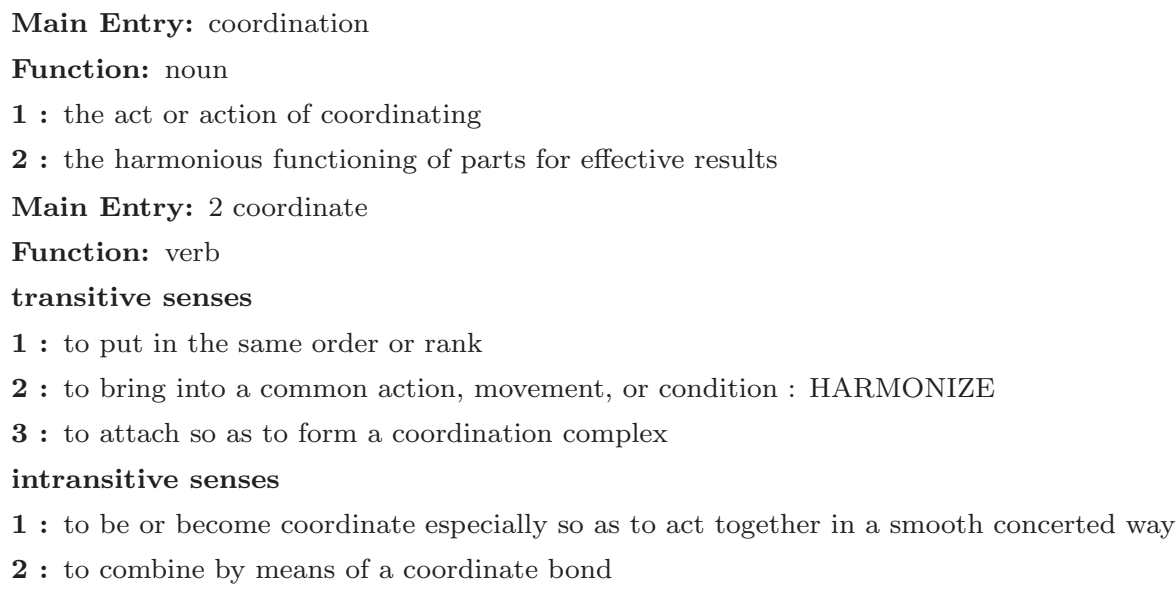

Fig. 1. Merriam Webster: 'coordination' and 'to coordinate'

An often used definition due to Malone defines coordination as the "management of dependencies among independent activities" [28]. This definition is used in particular in business and economic contexts. It emphasizes that instead of the activities themselves the interaction among these activities are central, and that the activities are autonomous.

In agent theory coordination has been defined as "the process by which an agent reasons about its local actions and the (anticipated) actions of others to try to ensure the community acts in a coherent manner [22], "the activity that involves the selection ordering and communication of the results of agent activities as that an agent works effectively in a group setting" [27], as "a process in which agents engage in order to ensure a community of individual agents acts in a coherent manner" [30], and in computational intelligence as "a way of adapting to the environment" [35].

Finally, a more technical definition in computer science due to Arbab defines coordination as "the study of the dynamic topologies of interactions among Interaction Machines, and the construction of protocols to realize such topologies that ensure well-behavedness" [2].

- the skillful and effective interaction of movements

- the regulation of diverse elements into an integrated and harmonious operation

- the grammatical relation of two constituents having the same grammatical form

Fig. 2. WordNet: S: (n) coordination 
Main Entry: 1 organization

Function: noun

$1 \mathbf{a}$ : the act or process of organizing or of being organized

b : the condition or manner of being organized

2 a : ASSOCIATION, SOCIETY < charitable organizations >

b : an administrative and functional structure (as a business or a political party); also : the personnel of such a structure

Main Entry: 2 organization

Function: adjective

1 : characterized by complete conformity to the standards and requirements of an organization <an organization man>

Fig. 3. Merriam Webster: 'organization'

\subsection{Organization}

An organization is a formal group of people with one or more shared goals. In sociology 'organization' is understood as planned, coordinated and purposeful action of human beings in order to construct or compile a common tangible or intangible product or service. This action is usually framed by formal membership and form (institutional rules). Sociology distinguishes the term organization into planned formal and unplanned informal (i.e., spontaneously formed) organizations.

Management is interested in organization mainly from an instrumental point of view. For a company organization is a means to an end in order to achieve its goals. In this sense organizations can be distinguished into two fundamentally different sets: Organizations whose goal is to generate certain services and/or to produce goods (factories, service enterprises, etc.) or to bring about certain effects in its surrounding world (e.g., authorities, police, political parties, interest groups, trade unions, etc.), and organizations whose goal is to change individuals (e.g., schools, universities, hospitals, prisons).

- a group of people who work together

- (also arrangement, system) an organized structure for arranging or classifying; "he changed the arrangement of the topics"; "the facts were familiar but it was in the organization of them that he was original"; "he tried to understand their system of classification"

- (also administration, governance, governing body, establishment, brass) the persons (or committees or departments etc.) who make up a body for the purpose of administering something; "he claims that the present administration is corrupt"; "the governance of an association is responsible to its members"; "he quickly became recognized as a member of the establishment"

- (also constitution, establishment, formation) the act of forming something; "the constitution of a PTA group last year"; "it was the establishment of his reputation"; "he still remembers the organization of the club"

- the act of organizing a business or an activity related to a business) "he was brought in to supervise the organization of a new department"

- the activity or result of distributing or disposing persons or things properly or methodically; "his organization of the work force was very efficient"

- (also system) an ordered manner; orderliness by virtue of being methodical and well organized; "his compulsive organization was not an endearing quality"; "we can't do it unless we establish some system around here"

Fig. 4. WordNet: S: (n) organization 


\section{Examples}

In this section we present two examples of research on coordination and organization. On the one hand we illustrate how research on coordination can be used within research on human organizations, by illustrating the use of coordination languages in electronic commerce. On the other hand we illustrate how research on organizations in multi-agent systems can be used within research on coordination, by discussing the use of roles for coordination in object.

\subsection{Electronic commerce}

Many social and economic constructs can be seen as mechanisms for coordination, for example marriage can be seen as a mechanism to coordinate selection, and money can be seen as a mechanism to coordinate the exchange of goods among three parties (known as Wicksell triangle). When someone wants to buy a pack of milk, he takes his cash and goes to the store. The shop-keeper and the buyer directly inspect and exchange our goods and cash, and that is the end of the story. If he wants to buy the next season's entire milk production of a dairy farm, or purchase a house, although the principle is the same as buying a pack of milk, we need to use constructs such as an escrow service, because the transaction now involves multiple steps and spans over time. All activities involved need to be coordinated such that in the end we still get the all-or-nothing effect of a transaction.

Therefore these social and economic constructs can be modeled or formalized using coordination languages developed in computer science. Using a more precise mathematical language forces one to better understand what is going on with constructs such as escrow, Letter of Credit, or other trust instruments. Moreover, formal descriptions and analysis can be used to support the design of coordination mechanisms in electronic commerce. As an example we consider here mechanisms for the coordination of value exchanges in economic and business contexts. As a running example we consider the well known documentary credit procedure of a Letter of Credit $[10,26,24]$.

Suppose we have a potential transaction between a customer and a supplier which is located in a remote and unfamiliar part of the world. Hence there are no common conventions or trade procedures. In such circumstances a lack of trust is warranted. The supplier does not want to ship the goods without first receiving payment, but the customer does not want to pay before the goods have been shipped. To solve this deadlock situation banks introduced the letter of credit procedure. A letter of credit is an agreement that the bank of the customer, called the issuing bank, will arrange a credit to guarantee payment as soon as the supplier can prove that the goods have been shipped. The supplier can prove this by presenting the shipping documents, such as a bill of lading, to his own bank, the corresponding bank. The shipping documents are issued by the carrier of the goods, as soon as the goods have in fact been shipped. The corresponding bank transfers the shipping documents to 
the issuing bank, which only delivers the shipping documents to the customer after payment. With the shipping documents the customer can then reclaim the goods from the carrier.

A possible way of modeling the procedure is depicted in Figure 5, adapted from [26]. This figure should be read as follows. It indicates the order in which documents must be exchanged, which provides information about the dependencies among actions: which actions should occur only provided some other actions have occurred. For example, the objective of step 4 is to let the supplier know that a credit has been secured, and that he can safely start shipping the goods. Similarly, the objective of the shipping documents, is to provide evidence that the goods have been transferred.

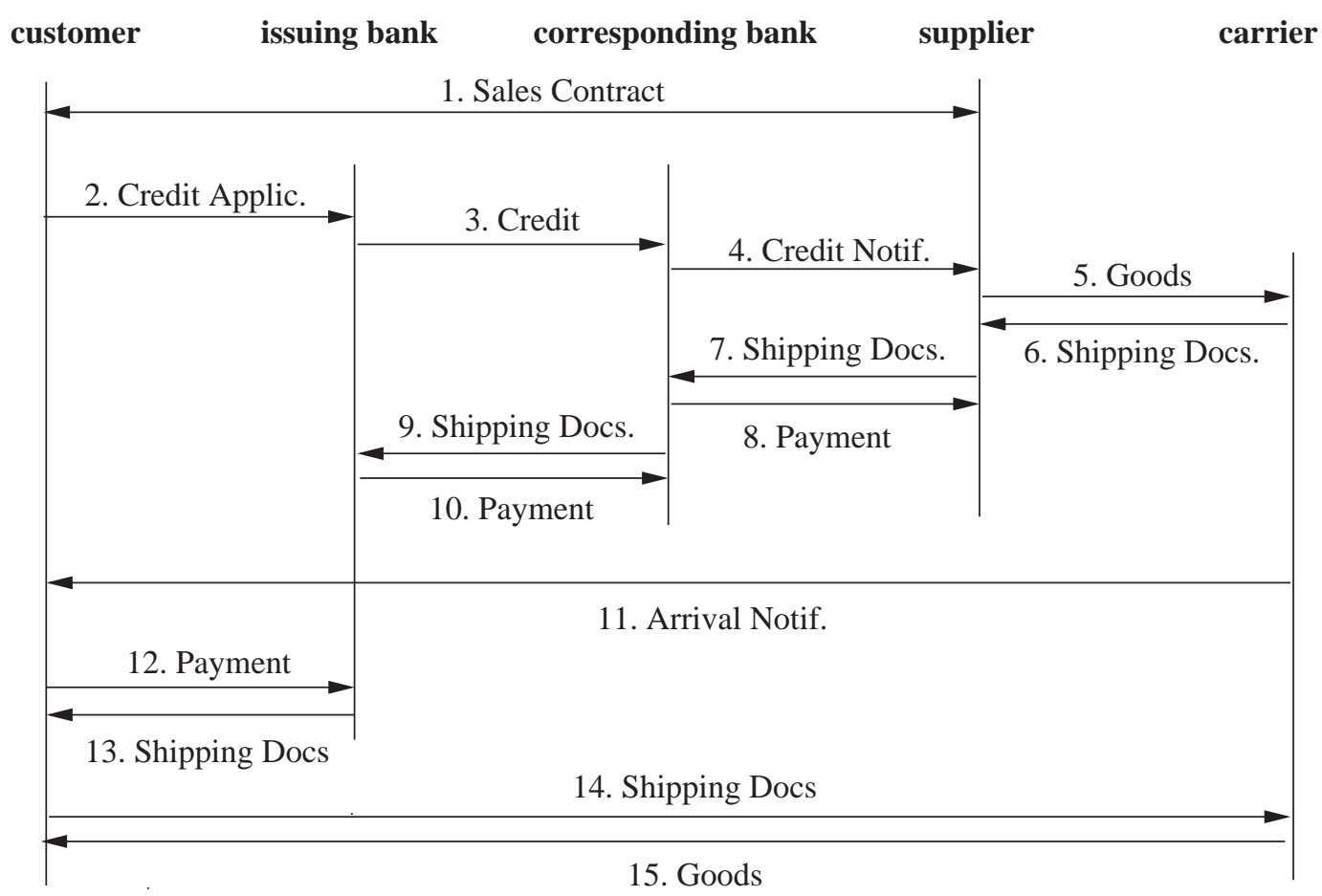

Fig. 5. Interaction Diagram of the Letter of Credit Procedure

One aspect that is not covered by this diagram is the amount of value that is transferred. For example, payment 12 by the buyer should be large enough to cover payment 10, as well as the fees of the issuing bank for supplying the service. Such issues are better addressed in the value perspective. Gordijn et al. $[18,19]$ have been advocating the value perspective for requirements engineering in electronic commerce. They developed the $\mathrm{e}^{3}$-value tool, which makes it possible to calculate the respective values of exchanges for different scenarios. The central idea is the principle of reciprocity: whatever happens, the net value of all exchanges must be equal to zero. Kartseva et al. [24] give a value-based account of the Letter of Credit procedure.

Consider a value based analysis of the trading practice of a Letter of Credit in Figure 6. The Bill of Lading is issued by the carrier, as soon as the goods 
are shipped. The supplier's bank transfers the Bill of Lading to the customer's bank, which only gives the Bill of Lading to the customer after payment. The customer's bank receives a fee for the letter of credit service ( $L o C$ fee), part of which goes to the supplier's bank for handling the payment $(P f e e)$. The Bill of Lading is an example of a multi-modal transport document that has an evidentiary effect. Compared to the interaction diagram, we also consider a shipping fee the supplier pays to the carrier, and an extension with a merchant role. The customer can claim the shipped goods from the carrier in return for the Bill of Lading, or sell the Bill of Lading to some other company, which can then claim the shipped goods.

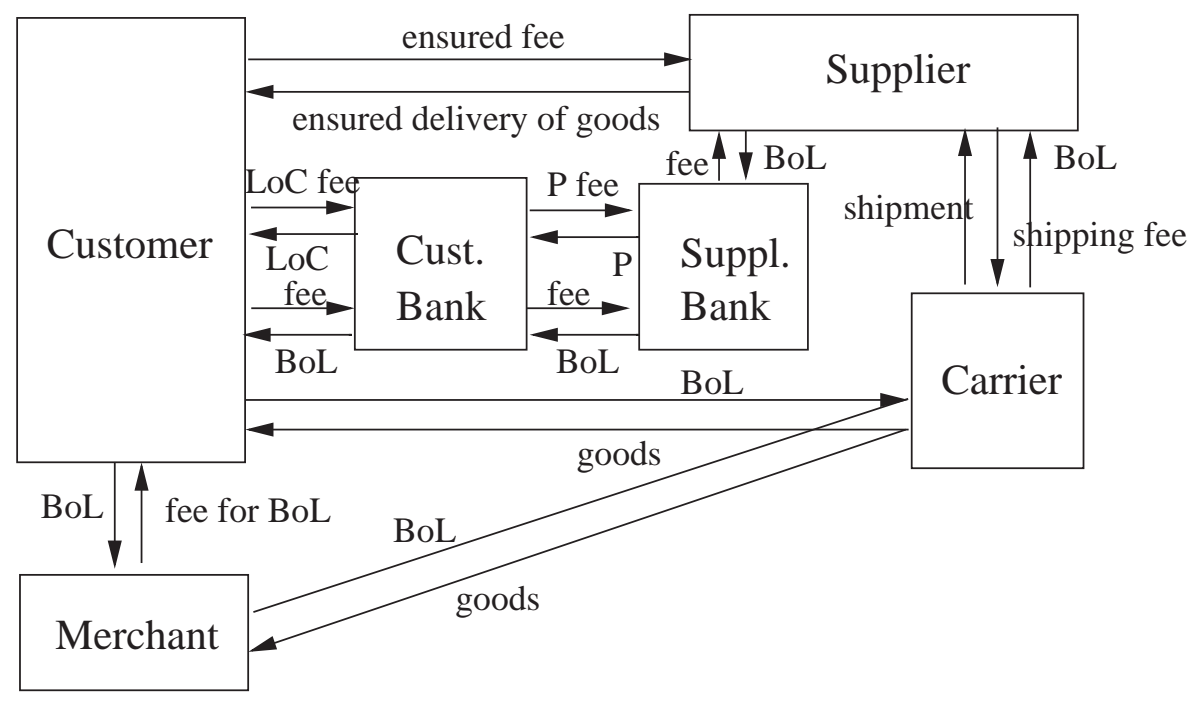

Fig. 6. Value network

Depending on what it is that you want to model with a multi-agent-system, it may or may not make sense to insist on having explicit agents representing letter of credit or escrow companies. Recognizing that their role is simply to coordinate, one can simplify the complexity of what a buyer and seller has to deal with in the real world, and simply present a view to them as if buying a house is the same as buying a pack of milk: everything needed by each party must be present and verified before the actual exchange takes place; i.e, we have an atomic transaction. We can leave it to be the responsibility of the exogenous coordination mechanism to ensure that the integrity of this complex transaction is preserved. However, if the intention is to model what happens in the real world, then we will not see an explicit role or agent for banks in this case. The atomic transaction implicitly does the same job, but eliminates their role. So, we want to have another model that includes some explicit construct representing the letter of credit company. Comparing what buyers and sellers must do in their blissfully ignorant versions versus their real-life models, clarifies the exact role of letter of credit in coordination and conduct of these transactions, as well as what exactly needs to become endogenous. 


\subsection{Roles for coordination of objects}

In order to constrain the autonomy of agents and to control their emergent behavior in multiagent systems, the notion of organization has been applied [17,37]. According to Zambonelli et al. [37] "a multiagent system can be conceived in terms of an organized society of individuals in which each agent plays specific roles and interacts with other agents".

The notion of role refers to the structure of social entities like institutions, organizations, normative systems, or groups $[6,17,37]$. Roles are usually considered as a means to distribute the responsibilities necessary for the functioning of the organization. Moreover, roles allow the uncoupling of the features of the individuals playing roles from those of their roles. Finally, roles are used to define common interaction patterns, and embed information and capabilities needed to communication and coordination [11]. E.g., the roles of auctioneer and bidder are defined in an auction, each with their possible moves.

The use of roles in multi-agent systems has not only been beneficial to the construction of multi-agent systems. It has also led to a better understanding of what is a role, which are its properties and how to formalize them.

Baldoni et al. [4,5] propose to use the notion of role as it has been developed in multi-agent systems to coordinate objects and components in the traditional object oriented paradigm rather than agents in multi-agent systems. As object orientation is a way to handle the complexity arising from the large number of interactions in a system, roles can be a further representation mechanism to achieve the same purpose in environments which are more varied and dynamic, and need to accommodate more local freedom and initiative, scenarios common in multi-agent systems [23,36]. Introducing roles in object oriented languages is a way to bring back to object orientation the fruits of applying organizational concepts to agents.

Baldoni et al. [4] propose to introduce roles in an extension of Java. The distinguishing features of roles in their model are the foundation of roles, their definitional dependence from the institution they belong to, and the powers attributed to the role by the institution. Baldoni et al. [4], thus, call powerJava their extension of Java.

To understand these issues they propose the following example. Consider the roles student and professor. Roles are always roles of some institution. A student and a professor are always roles of some university. Without the university the roles do not exist anymore: e.g., if the university goes bankrupt, the actors (e.g. a person) of the roles cannot be called professor and students anymore. The institution (the university) also specifies the properties of the student, which extend the properties of the person playing the role of student: the university specifies its enrolment number, its email address, its scores at past examinations, and also how the student can behave. For example, the student can give an exam by submitting some written examination. A student can make the professor evaluate its examination and register the mark 
because the university defines both the student role and the professor role: the university specifies how an examination is evaluated by a professor, and how it maintains the official records of the examinations. Otherwise the student could not have an effect on the professor. But in defining such actions the university empowers the person who is playing the role of student: without being a student the person has no possibility to give an examination and make the professor evaluate it.

This example highlights the following properties that roles have in the model of $[8,9]$ :

Foundation: a (instance of) role must always be associated with an instance of the institution it belongs to (see Guarino and Welty [21]), besides being associated with an instance of its player.

Definitional dependence: the definition of the role must be given inside the definition of the institution it belongs to. This is a stronger version of the definitional dependence notion proposed by Masolo et al. [29], where the definition of a role must use the concept of the institution.

Institutional empowerment: the actions defined for the role in the definition of the institution have access to the state and actions of the institution and of the other roles: they are powers.

To introduce this view of roles in Java is necessary to address the following issues:

(i) A construct defining the role with its name, who can play it and its powers in the institution.

(ii) The implementation of a role, inside an institution and according to the specification of its powers, so to make the role definitionally dependent on the institution.

(iii) How an object playing a role can exercise the powers its role gives to it in the institution to which the role belongs.

Figure 3.2 shows by mean of the above example the use of roles in powerJava. First of all, a role is specified (role - left column) by indicating who can play the role (playedby) and which are the powers acquired by playing the role (exam(), giving an exam in this case). Second, a role is implemented inside an institution as a sort of inner class which realizes the role specification (definerole). The inner class implements all the methods required by the role specification as it were an interface.

In the right column of Figure 3.2 the use of powerJava is depicted. First, the candidate player $\mathrm{p}$ of the role is created. Its type must be compatible with the requirements of the role (Person). Before the player can play the role, however, an instance of the institution hosting the role must be created first (a University $u$ ). Once the University is created, the player $\mathrm{p}$ can become a Student too. Note that the Student is created inside the University $u$ ( $u$.new Student $(p)$ ) and that the player $p$ is an argument of the constructor 


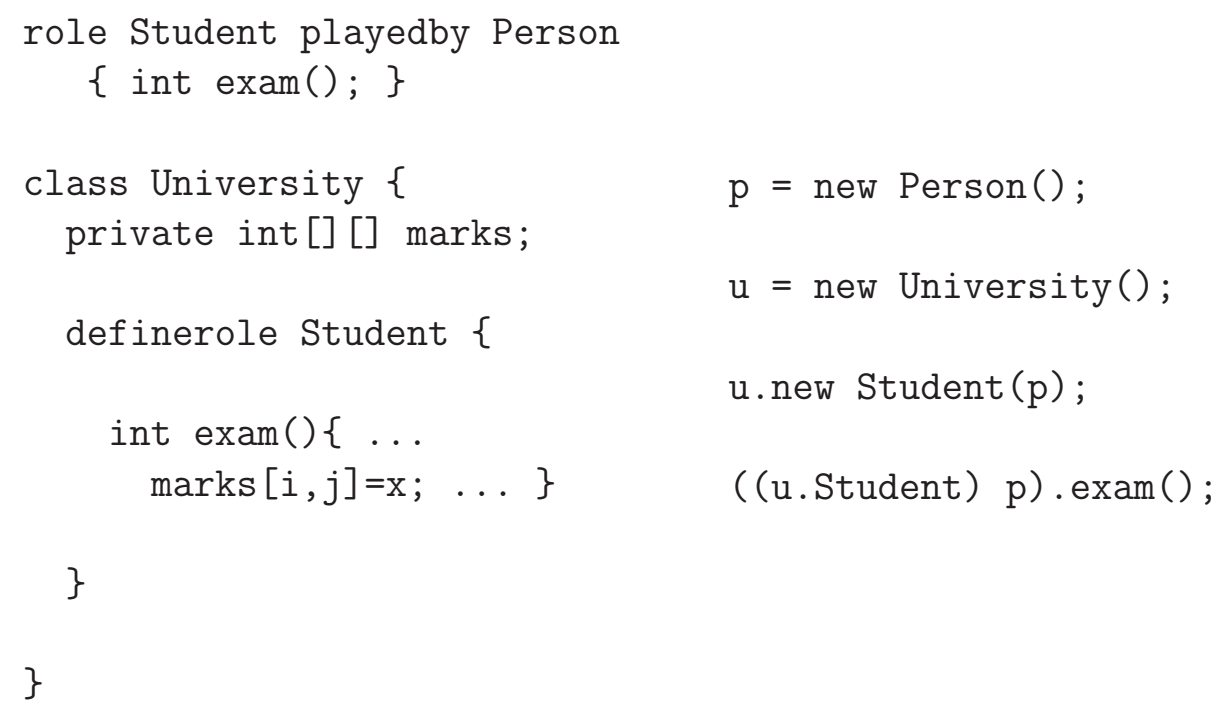

Fig. 7. A role Student inside an University.

of role Student.

The player $\mathrm{p}$ maintains its identity and if it has to act as a Student it must first be classified as a Student by means of a so-called role casting ( (u.Student) p). Note that $\mathrm{p}$ is not classified as a generic Student but as a Student of University $u$. Once $p$ is casted to its Student role, it can exercise its powers, in this example, giving an exam (exam()). Such method is called a power since, in contrast with usual methods, it can access the state of other objects, since its namespace shares the one of the institution defining the role. Thus it can access the state of the institution it belongs to and of the other roles in the institution. In the example, the method exam() can access the private state of the University and assign a value to the private variable marks: giving an exam successfully counts as the University assigning a mark to the Student in the registry of exams.

Powers are used to coordinate objects. In Baldoni et al. [5] it is shown how roles can be used for coordination purposes. First, the interaction among objects is dealt with only inside the institution which specifies how they coordinate with each other. This is possible since powers allow roles to access the state of the institution and of the other roles. In our example, the coordination between a Student and a Professor can be made inside the institution. This allows to achieve a separation of concerns between the core behavior of an object (which is given inside its class) and its interaction capabilities, which depend on the context of the interaction and, thus, are specified in the institution.

Second, the player of the role does not need to know which is the identity of the other players it must coordinate with, but it has to refer only to the roles. In our example, a Student does not need to know the identity of player of the Professor role it has to interact with, but it needs only to know which is the Professor of the course it attends. 


\section{Directions for future research}

We distinguish four directions for future research. The first direction concerns research questions concerning the gap between computer science where the coordination languages and models have been developed, and the social sciences where organizations are being studied. The second kind of questions concerns the use of coordination languages for human organizations, and the third kind concerns the use of organizational concepts for coordination. A separate class of questions concerns security issues for the coordination of organizations.

\subsection{Computer science and the social sciences}

Coordination as considered in this paper is a concept studied in computer science, whereas organization is a concept borrowed from the social sciences economics, business administration, and sociology. This gives rise to our first research question.

Question 1 What are the fundamental distinctions underlying these two concepts?

Coordination in computer science is concerned with information, whereas the letter of credit example illustrates that organizations are concerned with values. Values have properties information does not have, since, for example, it cannot be duplicated, or it can perish. Coordination languages have to be developed that deal with these properties. Porter, a business scientist who studied the notion of value chains and applied it to strategy [33], defines value as follows. "Economic Value for a company is nothing more than the gap between price and cost, and it is reliably measured only by sustained profitability. To generate revenues, reduce expenses, or simply do something useful by deploying internet technology is not sufficient evidence that value has been created." [34, p.65]

\subsection{Coordination languages for organizations}

The second research direction concerns the use of coordination languages, tools and models, as developed in the coordination community in computer science, for human organizations. Coordination techniques are used in formal models of organizations to analyze or simulate them.

Question 2 What kind of properties should a coordination language for organizations have?

Dastani et al. [15] argue that coordination for organizations should be exogenous, in the sense that coordination is defined in terms of interactions rather than agents, because organizations are defined in terms of roles rather than agents. They explain and promote the use of the exogenous coordination language Reo, developed by Arbab at the Center of Mathematics and Computer Science in Amsterdam. 
Groenewegen et al [20] argue that the coordination language should be able to describe and relate global and local behaviors, and they promote Paradigm as an organization-oriented coordination language. Global behaviors described in Paradigm provide flexibility in arranging computation as well as coordination. According to the authors, this flexibility is an organizational, organic and human like characteristic, usually absent in system specification.

Omicini et al [32] argue that the coordination language should be able to describe artifacts. Human intelligence has evolved along with the use of more and more sophisticated tools, and that therefore agent intelligence should not be considered as separated by the agent ability to perceive and affect the environment. In contrast, agent intelligence is strictly related to the artifacts that enable, mediate and govern any agent (intelligent) activity.

Question 3 How to use the coordination languages for human organizations?

An example of a coordination problem is the model integration problem. In enterprise architecture, for example, many distinct models are used to describe an organization, for example using the unified modelling language (UML) or the Zachman framework for enterprise architecture, and it is an open problem how these models can be related [25].

\subsection{Organizational concepts for coordination}

Organizational concepts are used frequently for coordination purposes in different areas of Computer Science. For example, roles are used in access control, conceptual modelling, programming languages and patterns. Contracts are used in design by contract, and services are used in web services and service level agreements. Message based communication is used in networking. However, most coordination languages refer mostly to different kinds of metaphors, like blackboards, shared dataspaces, component composition and channels.

Question 4 Which organizational concepts can be used for coordination?

Argente et al. [3] discuss how to go from human to agent organizations, with examples from electronic institutions and virtual organizations. She compared human organizational taxonomies with approaches to coordination of agents, with the aim to employ organizational theory to develop multi-agent systems based on organizational meta models.

Carabelea and Boissier [12] propose to coordinate agents in organizations using social commitments, describing how one can use social commitments to represent the expected behavior of an agent playing a role in an organization. Norms play a central role in many social phenomena such as coordination, cooperation, decision-making, etc.

The example of powerJava [4,5] illustrates the use of roles, which are adopted in agent oriented software methodologies and programming languages like GAIA, TROPOS, 3APL, etc, to define the organizational structure in ob- 
ject oriented languages. For example, roles allow to distribute responsibilities and obligations, and to require the suitable know how to their players. However, open problems are how to transform organizational theories of roles in computational theories and exploring mechanisms about how to assign agents to roles, how to design organizations in terms of roles, monitoring of roles, etc.

Colman and Hun [14] explain how to use association aspects to implement organizational contracts. They show how a coordination system can be implemented by a separate concern, and how association aspects can be used to create contracts that bind roles together in an organization. These contracts allow performance to be specified and monitored. They also define organiser roles that control, create, abrogate and reassign contracts.

Question 5 How to use these concepts in coordination languages and models?

The above mentioned papers also illustrate how organizational concepts can be used.

\subsection{Security}

Traditionally research on coordination and organization has been concerned with cooperative agents, but in open systems security concerns must be addressed. Omicini et al. [31] argue that security and coordination are in some sense complementary.

\section{Question 6 How are coordination and organization related to security?}

In electronic commerce, security is concerned with, for example, potential loss of value, sanctions and control systems. Whereas traditional security concerns have focussed on building control mechanisms such as cryptographic methods, organizational coordination focusses on incentives to motivate agents, and trust and anticipation play a central role too. In [7] we raise the question under what circumstances we need to introduce a control system by considering the following three scenarios. For example, when you buy a ticket to the theater, you buy the right to view a performance that will be delivered later on. The buyer trusts the seller concerning the validity of the ticket. It is an open problem how to balance enforced control and trust-based anticipation to deal with security aspects in the coordination of organizations.

\section{Summary}

Coordination languages and models like Linda and Reo have been developed in computer science to coordinate the interaction among components and objects, and are nowadays used to model and analyze organizations too. Moreover, organizational concepts are used to enrich the existing coordination languages and models.

Coordination is the "management of dependencies among independent activities" [28], which emphasizes the interaction among these activities, and 
their autonomy. Various more precise or technical definitions are given in agent theory and computer science. An organization is a formal group of people with one or more shared goals. In sociology 'organization' is understood as planned, coordinated and purposeful action of human beings in order to construct or compile a common tangible or intangible product or service. Management is interested in organization mainly from an instrumental point of view. For a company organization is a means to an end in order to achieve its goals.

We illustrate how research on coordination can be used within research on human organizations, by illustrating the use of coordination languages in electronic commerce. Many social and economic constructs can be seen as mechanisms for coordination, because transaction typically involve multiple steps and span over time, and constructs such as an escrow service coordinate the activities involved such that in the end we still get the all-or-nothing effect of a transaction. Using a more precise mathematical language forces one to better understand what is going on with constructs such as escrow, Letter of Credit, or other trust instruments.

Moreover, we illustrate how research on organizations in multi-agent systems can be used within research on coordination, by discussing the use of roles in object oriented programming languages. The institution roles belong to coordinates the interaction of the players of the roles independently of their core behavior. In this way for the objects to be coordinated it is only necessary that they fulfill the requirements specified by a role.

We raise the questions which properties a coordination language should have to model organizations, how such a coordination language can be used, which organizational concepts can be used in coordination languages and models, and how these concepts should be used. We highlight two issues. First, we argue for a study of value-based rather than information-based coordination languages to model the coordination of autonomous agents and organizations. Second, we argue for a study of the balance between enforced control and trust-based anticipation to deal with security aspects in the coordination of organizations.

\section{Acknowledgements}

Thanks to all speakers and participants of the first international workshop on coordination and organization (CoOrg05), co-located with the Coordination conference (COORD05) in Namur, to Dave Clark for discussing the definitions, and to Farhad Arbab, Joris Hustijn and Yao-Hua Tan for discussions on the use of the coordination language Reo to model the letter of credit procedure, as discussed in Section 3.1, and finally to Matteo Baldoni for the discussion on powerJava in Section 3.2. 


\section{References}

[1] Arbab, F., Reo: A channel-based coordination model for component composition mathematical structures in computer science 14(3) (2004), pp. 329-366.

[2] Arbab, F., Abstract behavior types: A foundation model for components and their composition, Science of Computer Programming 55 (2005), pp. 3-52.

[3] Argente, E., V. Julian and V. Botti, Multi-agent system development based on organizations, Electronic Notes in Theoretical Computer Science (2006), this volume.

[4] Baldoni, M., G. Boella and L. van der Torre, Bridging agent theory and object orientation: Importing social roles in object oriented languages, in: Postprocs. of PROMAS'05 workshop at AAMAS'05, LNAI 3862 (2006).

[5] Baldoni, M., G. Boella and L. van der Torre, Roles as a coordination construct: Introducing powerJava, Electronic Notes in Theoretical Computer Science 150(1) (2006), pp. 1-158.

[6] Bauer, B., J. Muller and J. Odell, Agent UML: A formalism for specifying multiagent software systems, Int. Journal of Software Engineering and Knowledge Engineering 11(3) (2001), pp. 207-230.

[7] Boella, G., J. Hulstijn, Y. Tan and L. van der Torre, Transaction trust in normative multiagent systems, in: Procs. of Trust in Agent Societies Workshop at $A A M A S^{\prime} 05,2005$.

[8] Boella, G. and L. van der Torre, Attributing mental attitudes to roles: The agent metaphor applied to organizational design, in: Procs. of ICEC'04 (2004).

[9] Boella, G. and L. van der Torre, Regulative and constitutive norms in normative multiagent systems, in: Procs. of 10th International Conference on the Principles of Knowledge Representation and Reasoning KR'04 (2004), pp. $255-265$.

[10] Bons, R., "Designing Trustworthy Trade Procedures for open Electronic Commerce," Ph.D. thesis, University of Rotterdam (1997).

[11] Cabri, G., L. Ferrari and L. Leonardi, Agent role-based collaboration and coordination: a survey about existing approaches, in: IEEE Systems, Man and Cybernetics Conference, 2004, pp. 5473-5478.

[12] Carabelea, C. and O. Boissier, Coordinating agents in organizations using social commitments, Electronic Notes in Theoretical Computer Science (2006), this volume.

[13] Carriero, N. and D. Gelernter, LINDA in context, Communications of the ACM 32 (1989), pp. 444-458.

[14] Colman, A. and J. Han, Using associations aspects to implement organizational contracts, Electronic Notes in Theoretical Computer Science (2006), this volume. 
[15] Dastani, M., F. Arbab and F. S. de Boer, Coordination and composition in multi-agent systems., in: Procs. of AAMAS, 2005, pp. 439-446.

[16] Fellbaum, C., "An Electronic Lexical Database," MIT Press, Cambridge (MA), 1998.

[17] Ferber, J., O. Gutknecht and F. Michel, From agents to organizations: an organizational view of multiagent systems, in: Procs. of AOSE'03, LNCS 2935 (2003), pp. 214-230.

[18] Gordijn, J. and J. M. Akkermans, Value-based requirements engineering: exploring innovative e-commerce ideas., Requir. Eng. 8 (2003), pp. 114-134.

[19] Gordijn, J. and Y.-H. Tan, A design methodology for trust and value exchanges in business models, in: Procs. of BLED Conference, 2003, pp. 423-432.

[20] Groenewegen, L., A. Stam, P. Toussaint and E. de Vink, Paradigm as organization-oriented coordination language, Electronic Notes in Theoretical Computer Science (2006), this volume.

[21] Guarino, N. and C. Welty, Evaluating ontological decisions with ontoclean, Communications of ACM 45(2) (2002), pp. 61-65.

[22] Jennings, N. R., Coordination techniques for distributed artificial intelligence, in: Foundations of Distributed Artificial Intelligence, John Wiley \& Sons, 1996 pp. $187-210$.

[23] Jennings, N. R., On agent-based software engineering, Artificial Intelligence 117(2) (2000), pp. 277-296.

[24] Kartseva, V., J. Gordijn and Y.-H. Tan, Designing control mechanisms for network organisations, in: Procs. of ICEC'04 (2004).

[25] Lankhorst et al., M., "Enterprise Architecture At Work," Springer Verlag, Berlin, 2005.

[26] Lee, R., Documentary Petri nets: A modeling representation for electronic trade procedures, in: Business Process Management, LNCS 1806 (2000), pp. 359-375.

[27] Lesser, V. R., Reflections on the nature of multi-agent coordination and its implications for an agent architecture., Autonomous Agents and Multi-Agent Systems 1 (1998), pp. 89-111.

[28] Malone, T. and K. Crowston, The interdisciplinary study of coordination, ACM Computing Surveys 26(1) (1994), pp. 87-119.

[29] Masolo, C., L. Vieu, E. Bottazzi, C. Catenacci, R. Ferrario, A. Gangemi and N. Guarino, Social roles and their descriptions, in: Procs. of KR'04 (2004), pp. 267-277.

[30] Nwana, H., L. Lee and N. Jennings, Coordination in software agent systems, The British Telecom Technical Journal 14(4) (1996), pp. 79-88. 
[31] Omicini, A., A. Ricci and M. Viroli, An algebraic approach for modelling organisation, roles and contexts in $M A S$, Applicable Algebra in Engineering, Communication and Computing 16 (2005), pp. 151-178.

[32] Omicini, A., A. Ricci and M. Viroli, Agens faber: Toward a theory of artefacts for mas, Electronic Notes in Theoretical Computer Science (2006).

[33] Porter, M., "Competitive strategy," Free Press, 1980.

[34] Porter, M., Strategy and the internet, Harvard Business Review (2001).

[35] von Martial, F., "Coordinating Plans of Autonomous Agents," Lecture Notes in Computer Science 610, Springer, 1992.

[36] Yu, E., Agent orientation as a modelling paradigm, Wirtschaftsinformatik 43(2) (2001), pp. 123-132.

[37] Zambonelli, F., N. Jennings and M. Wooldridge, Developing multiagent systems: The Gaia methodology, IEEE Transactions of Software Engineering and Methodology 12(3) (2003), pp. 317-370. 


\title{
Interaction in Normative Multi-Agent Systems
}

\author{
Guido Boella ${ }^{1}$ \\ Dipartimento di Informatica \\ Università di Torino \\ Torino, Italy \\ Joris Hulstijn $^{2}$ \\ Faculty of Economics and Business Administration \\ Vrije Universiteit \\ Amsterdam, The Netherlands \\ Leendert van der Torre ${ }^{3}$ \\ CWI Amsterdam \\ and Delft University of Technology \\ The Netherlands
}

\begin{abstract}
The central research question of this paper is how notions developed in interactive computing such as abstract behavior types, the coordination language Reo, and Boolean circuits with registers, can be used to extend logical input/output nets, or lions for short. Lions are based on input/output logic, a deontic logic which is not used as a (non-classical) inference engine deriving output from input, but as a secretarial assistant for logically assisted transformations from input to output. We consider two extensions of input/output logics and lions. First, we consider input/output logics defined on infinite sequences (or streams) of inputs and outputs. Secondly, we consider lions with AND and register gates, formalizing the behavior of channels and connectors. We discuss also the role of interactive computing in normative multi-agent systems motivating the development of lions.
\end{abstract}

Key words: Normative systems, multi-agent systems, deontic logic, input/output logic, coordination, communication.

$\overline{1}$ Email: guido@di.unito.it

2 Email: jhulstijn@feweb.vu.nl

3 Email: torre@cwi.nl

This is a preliminary version. The final version will be published in Electronic Notes in Theoretical Computer Science URL: www.elsevier.nl/locate/entcs 


\section{Introduction}

According to many in computer science, the interaction paradigm provides a new conceptualization of computational phenomena that emphasize interaction rather than algorithms: concurrent, distributed, reactive, embedded, component-oriented, agent-oriented and service-oriented systems all exploit interaction as a fundamental paradigm $[34,5]$.

In this paper we consider Makinson and van der Torre's logical input/output nets [25], or lions for short, as a model for interactive computation. Lions are structured assemblies of input/output operations and extend Makinson and van der Torre's input/output logics $[22,23]$. They are graphs, with the nodes labelled by pairs $(G$, out) where $G$ is a normative code and out is an input/output operation. The edges of the graph represent channels, which indicate which nodes have access to which other nodes and provide passage for the transmission of local outputs as local inputs. The graph is further equipped with an entry point and an exit point, for global input and output.

We consider also two extensions of lions in this paper, both inspired by the work of Arbab and colleagues on abstract behavior types [4], the coordination language Reo [3], and Boolean circuits with registers [28].

Streams. Instead of considering one input at a time, we consider input/output logics on infinite sequences (or streams) of inputs and outputs.

Connectors. We consider AND and register gates to compose channels into connectors (or circuits).

Finally we discuss the role of interaction in normative multi-agent systems, and how it is used to motivate the further development of lions. Our investigations reveal a huge number of possible further extensions of lions, which raises the question which extensions should be studied next. Input/output logic originates from deontic logic, a branch of philosophical logic that studies logical relations among obligations, permissions and prohibitions, and which has been used to model legal and moral systems, as well as problems in computer science that involve constraints that can be violated [35]. Whereas deontic logic has been very helpful in the development of input/output logics, it does not seem very helpful to guide the development of lions. A motivation of lions comes from agent architectures and normative multi-agent systems, i.e., "sets of agents (human or artificial) whose interactions can fruitfully be regarded as norm-governed; the norms prescribe how the agents ideally should and should not behave" [21].

The layout of this paper is as follows. In Section 2 we repeat the definitions of input/output logic, and in Section 3 we explain how the concept of 'logic as a secretarial assistant' is related to interactive computing. In Section 4 we discuss input/output logics of streams and register and AND gates for channels and connectors. In Section 5 we discuss interactive computing in normative multi-agent systems. 


\section{Makinson and van der Torre's input/output logic}

Makinson and van der Torre [22] argue that the new role of logic is not to study some kind of non-classical logic, but a way of using the classical one. From a very general perspective, logic is often seen as an 'inference engine', with premises as inputs and conclusions as outputs. Instead it may be seen in another role: as a 'secretarial assistant' to some other, perhaps non-logical, transformation engine. From this point of view, the task of logic is one of preparing inputs before they go into the machine, unpacking outputs as they emerge and, less obviously, coordinating the two. The process is one of 'logically assisted transformation', and is an inference only when the central transformation is so. The general perspective is one of 'logic at work' rather than 'logic in isolation'. The brief description in this section is taken from Makinson and van der Torre's introduction to input/output logic [25]. Please refer to the original papers for further explanations and motivations $[22,23]$.

\subsection{Unconstrained Input/Output Operations}

Imagine a black box into which we may feed propositions as input, and that also produces propositions as output. Of course, classical consequence may itself be seen in this way, but it is a very special case, with additional features: inputs are themselves outputs, since any proposition classically implies itself, and the operation is in a certain sense reversible, since contraposition is valid. However, there are many examples of logical transformations without those features. In [22], the outputs either express some kind of belief or expectation, or some kind of desirable situation in the conditions given by the inputs.

Technically, a normative code is seen as a set $G$ of conditional norms, i.e. a set of such ordered pairs $(a, x)$. For each such pair, the body $a$ is thought of as an input, representing some condition or situation, and the head $x$ is thought of as an output, representing what the norm tells us to be desirable, obligatory or whatever in that situation. The task of logic is seen as a modest one. It is not to create or determine a distinguished set of norms, but rather to prepare information before it goes in as input to such a set $G$, to unpack output as it emerges and, if needed, coordinate the two in certain ways. A set $G$ of conditional norms is thus seen as a transformation device, and the task of logic is to act as its 'secretarial assistant'.

In the simplest kind of unconstrained input/output operations, a set $A$ of propositions serves as explicit input, which is prepared by being expanded to its classical closure $C n(A)$. This is then passed into the 'black box' or 'transformer' $G$, which delivers the corresponding immediate output $G(C n(A))=$ $\{x: \exists a \in C n(A),(a, x) \in G\}$. Finally, this is expanded by classical closure again into the full output out $(G, A)=C n(G(C n(A)))$. We call this simpleminded output. This is already an interesting operation. As desired, it does not satisfy the principle of identity, which in this context we call throughput, i.e. in general we do not have $a \in$ out $_{1}(G,\{a\})$, which we write briefly, 
dropping the parentheses, as out $1(G, a)$.

The input/output operation out $t_{1}$ is characterized by three rules. Writing $x \in$ out $_{1}(G, a)$ as $(a, x) \in$ out $_{1}(G)$ and dropping the right hand side as $G$ is held constant, these rules are:

- Strengthening Input (SI): From $(a, x)$ to $(b, x)$ whenever $a \in C n(b)$

- Conjoining Output (AND): From $(a, x),(a, y)$ to $(a, x \wedge y)$

- Weakening Output (WO): From $(a, x)$ to $(a, y)$ whenever $y \in C n(x)$.

However, simple-minded output lacks features that may be desirable in some contexts. In the first place, the preparation of inputs is not very sophisticated. Consider two inputs $a$ and $b$. If $x \in C n(a)$ and $x \in C n(b)$ then $x \in C n(a \vee b)$, but if $x \in$ out $_{1}(G, a)=C n(G(C n(a)))$ and $x \in$ out $_{1}(G, b)=C n(G(C n(b)))$ then we cannot conclude $x \in$ out $_{1}(G, a \vee b)=C n(G(C n(a \vee b)))$.

In the second place, even when we do not want inputs to be automatically carried through as outputs, we may still want outputs to be reusable as inputs - which is quite a different matter. Operations satisfying each of these two features can be provided with explicit definitions, characterized by straightforward rules. We thus have four very natural systems of input/output, which are

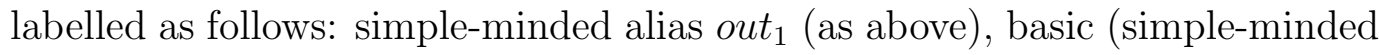
plus input disjunction: out $t_{2}$ ), reusable (simple-minded plus reusability: out $t_{3}$ ), and reusable basic (all together: out 4 ).

The three stronger systems may also be characterized by adding one or both of the following rules to those for simple-minded output:

- Disjoining input (OR): From $(a, x),(b, x)$ to $(a \vee b, x)$

- Cumulative transitivity (CT): From $(a, x),(a \wedge x, y)$ to $(a, y)$.

These four operations have four counterparts that also allow throughput. Intuitively, this amounts to requiring $A \subseteq G(A)$. In terms of the definitions, it is to require that $G$ is expanded to contain the diagonal, i.e. all pairs $(a, a)$. Derivationally, it is to allow arbitrary pairs of the form $(a, a)$ to appear as leaves of a derivation; this is called the zero-premise identity rule ID. All eight systems are distinct, with one exception: basic throughput, which we write as $o u t_{2}^{+}$, authorizes reusability, so that $o u t_{2}^{+}=o u t_{4}^{+}$. This is shown by the derivation in Figure 1, which also serves here to illustrate the proof theory of input/output logic. In the final step (OR) we are also implicitly using a replacement of classically equivalent propositions (or an application of SI).

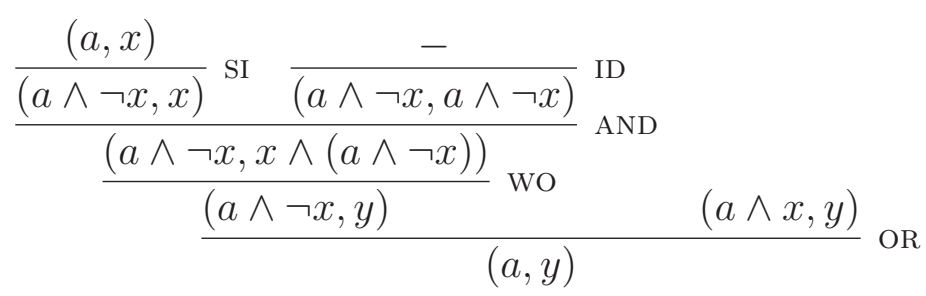

Fig. 1. Basic throughput authorizes reusability 


\subsection{Constraints}

The motivation of constraints comes from the logic of conditional norms, where we need an approach that does not presume that directives carry truth-values. Unconstrained input/output provides us with a simple and elegant construction, with straightforward behavior, but whose application to norms totally ignores the subtleties of violations and exceptions. Therefore input/output operations may be subjected to consistency constraints [23]. Our strategy is to adapt a technique that is well known in the logic of belief change - cut back the set of norms to just below the threshold of making the current situation contrary-to-duty. In effect, we carry out a contraction on the set $G$ of norms.

Specifically, we look at the maximal subsets $G^{\prime} \subseteq G$ such that $\operatorname{out}\left(G^{\prime}, A\right)$ is consistent with input $A$. In [23], the family of such $G^{\prime}$ is called the maxfamily of $(G, A)$, and the family of outputs out $\left(G^{\prime}, A\right)$ for $G^{\prime}$ in the maxfamily, is called the outfamily of $(G, A)$. To illustrate this, consider the set of norms $G=\{(\top, \neg(f \vee d)),(d, f \wedge w)\}$, where $\top$ stands for any tautology, with the contrary-to-duty input $d$. Using simple-minded output, maxfamily $(G, d)$ has just one element $\{(d, f \wedge w)\}$, and so outfamily $(G, d)$ has one element, namely $C n(f \wedge w)$.

Although the outfamily strategy is designed to deal with contrary-to-duty norms, its application turns out to be closely related to belief revision and nonmonotonic reasoning when the underlying input/output operation authorizes throughput. When all elements of $G$ are of the form $(T, x)$, then for the degenerate input/output operation out $t_{2}^{+}(G, a)=$ out $_{4}^{+}(G, a)$, the elements of $\operatorname{outfamily}(G, a)$ are just maxichoice revisions, in the sense of belief revision as defined by Alchourrón, Gärdenfors and Makinson [1]. These coincide, in turn, with the extensions of the default system of Poole [26]. More surprisingly, there are close connections between $\mathrm{out}_{3}^{+}$and the default logic of Reiter [27], see [23] for a further discussion on constraints in input/output logics and their relation to constraints in belief revision and non-monotonic reasoning.

\subsection{Permissions}

Moreover, input/output logics also provide a convenient platform for distinguishing and analyzing several different kinds of permission [24]. They give a clear formal articulation of the well-known distinction between negative and positive permission. In philosophical discussion of norms it is common to distinguish between two kinds of permission, negative and positive. Negative permission is easy to describe: something is permitted by a code iff it is not prohibited by that code, i.e. iff nihil obstat. In other words, taking prohibition in the usual way, something is negatively permitted by a code iff there is no obligation to the contrary. From the point of view of input/output logic, negative permission is straightforward to define: we simply put $(a, x) \in$ negperm $(G)$ iff $(a, \neg x) \notin$ out $(G)$, where out is any one of the four input/output operations that we have already discussed. 
Positive permission is more elusive. As a first approximation, one may say that something is positively permitted by a code if and only if the code explicitly presents it as such. Makinson and van der Torre distinguish what they call forward and backward permission as two distinct kinds of positive permission. Forward permission answers to the needs of the citizen, who needs to know whether an action that he is entertaining is permitted in the current situation. It also corresponds to the needs of authorities assessing the action once it is performed. If there is some explicit permission that covers the action in question, then it is itself implicitly permitted. On the other hand, backward permission fits the needs of the legislator, who needs to anticipate the effect of adding a prohibition to an existing corpus of norms. If prohibiting $x$ in condition a would commit us to forbid something that is implicit in what has been expressly permitted, then adding the prohibition is inadmissible under pain of incoherence, and the pair $(a, x)$ is to that extent protected from prohibition.

Intuitively, forperm tells us that $(a, x)$ is permitted whenever there is some explicitly given permission $(c, z)$ such that when we treat it as if it were an obligation, joining it with $\mathrm{G}$ and applying the output operation to the union, then we get $(a, x)$. Permissions are thus treated like weak obligations, the only difference being that while the latter may be used jointly, the former may only be applied one by one. Backperm tells us that $(a, x)$ is permitted whenever, given the obligations already present in $G$, we can't forbid $x$ under the condition $a$ without thereby committing ourselves to forbid, under a condition $c$ that could possibly be fulfilled, something $z$ that is implicit in what has been expressly permitted.

The proof theory of the various kinds of permissions contains various unexpected properties and proof-theoretic novelties. For example, whenever out satisfies a Horn rule, then the corresponding negperm operation satisfies an inverse one. Forperm and backperm are very different operations. Whereas forperm satisfies SI, backperm satisfies weakening of the input WI. Like negative permission, backperm satisfies the inverse rule of any Horn rule satisfied by out; but forperm satisfies instead a subverse rule. See [24] for the details.

Permissions in the context of constraints have not been considered yet.

\subsection{Lions}

Structured assemblies of input/output operations, called logical input/output nets, or lions for short, are graphs, with the nodes labeled by pairs $(G$, out) where $G$ is a normative code and out is an input/output operation (or recursively, by other lions). The relation of the graph indicates which nodes have access to others, providing passage for the transmission of local outputs as local inputs. The graph is further equipped with an entry point and an exit point, for global input and output. Lions have not been extended with constraints or permissions yet. 


\section{Lions for exogenous coordination}

Despite its origin in deontic logic, input/output logic is clearly related to functionality descriptions of components in interactive computing. For example, it has been shown [15] how the proof rules of input/output logic, and thus input/output operations, are related to Treur's functionality descriptions of interactive reasoning components [33]. Also constraints and lions can be interpreted in the context of interactive computing. At this moment it is not clear to us whether there is an analogue to permissions in interactive computing.

Moreover, the secretarial role of logic formalized by input/output logic can be related to discussions in interactive computing. For example, Arbab [2] argues that "coordination models and languages can also be classified as either endogenous or exogenous. [...] Exogenous models and languages provide primitives that support coordination of entities from without. In applications that use exogenous models primitives that affect the coordination of each module are outside the module itself." Typical examples of exogenous coordination are protocols enforced by the environment.

In this section we consider the proof rules of input/output logics as properties that can be enforced on the components by exogenous coordination. In particular, it has been suggested by Makinson and van der Torre that the identity rule corresponds to a forward loop, and that the cumulative transitivity rule corresponds to a feedback loop. We use lions to make this idea more precise. The problem can be phrased as follows. How far can the various ways of strengthening the input/output operation out to $_{1}$ out $_{n}(\mathrm{n}=2,3,4)$ with out without + , be simulated by integrating other familiar devices into the system as a whole? Before we introduce the definitions, we consider two examples.

\subsection{The identity rule}

Consider the lion in Figure 2. This figure should be read as follows. There are only three nodes, the start and end nodes without input/output logics associated with them, and a single node with which a component and therefore an input/output logic is associated. Moreover, there is an edge from the start node to the end node.

Informally, the behavior of the lion can be defined as follows, using some of Arbab's terminology.

- An edge between two nodes is called a channel from source to sink. The behavior of a channel is that the input of the sink contains (at least) the output of the source.

- When there are several channels ending in a node, then the input of this node is the union (or logical closure) of all the inputs. So at the right hand side of the black box in Figure 2, the output of the channel and the output of the component are combined.

- When there are several channels leaving a node, then the node's output is 


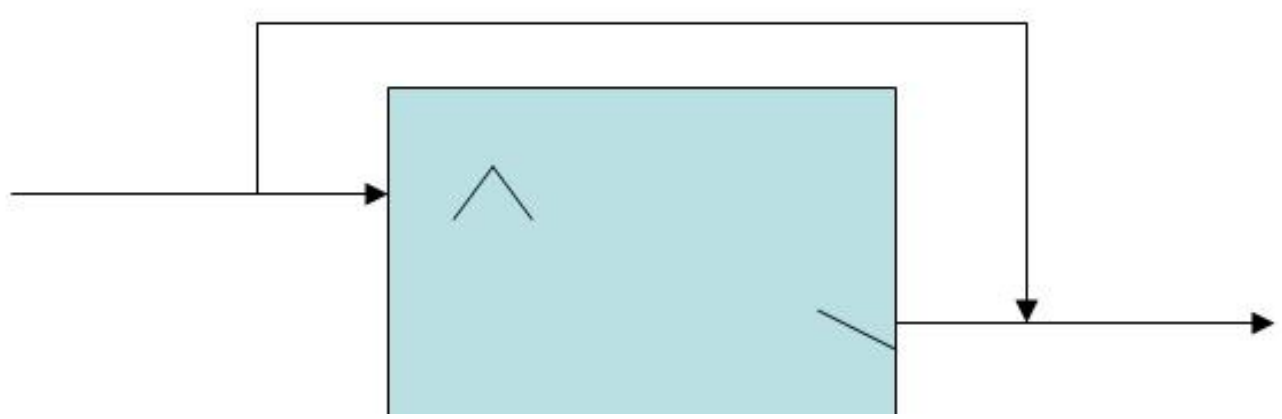

Fig. 2. Single component with feed forward (ID)

replicated to all channels.

For example, assume that the box in Figure 2 is described by a simple-minded operation out $_{1}(G, A)$, then the output of the lion is $C n\left(\right.$ out $\left._{1}(G, A) \cup A\right)$, which happens to be out $(G, A)$. So the component is exogenously (i.e., without being aware of it or able to influence it) coordinated so that the system as a whole behaves like a throughput operation, regardless of whether the component itself behaves as an input/output operation or a throughput operation.

\subsection{Cumulative transitivity}

As a second example, we consider the more complex case of a feedback loop in Figure 3, which leads to cumulative transitivity of the associated input/output logic. Again there are three nodes in the lion, but this time there is a feedback loop of the intermediate (or exit) node to the start (or intermediate) node.

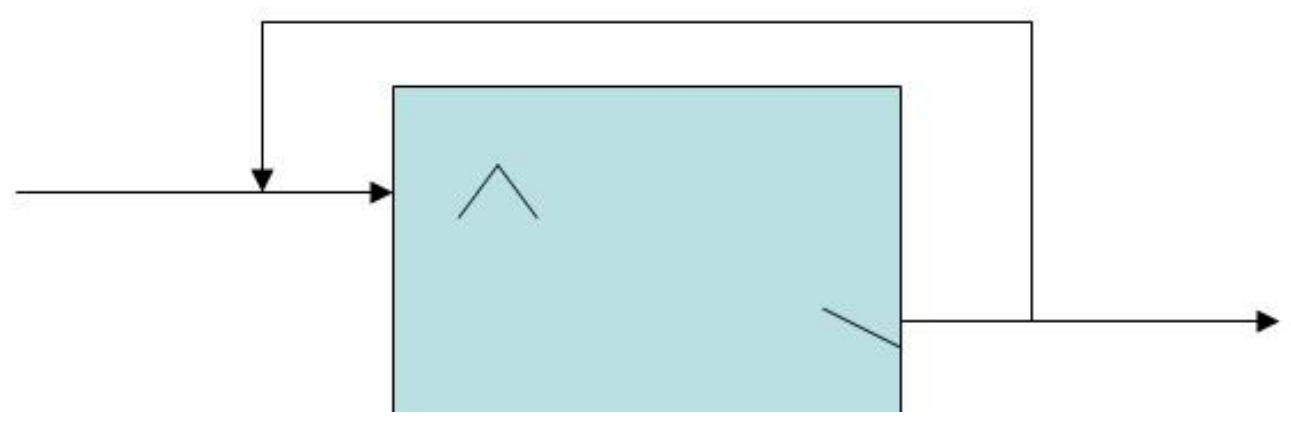

Fig. 3. Single component with feedback (CT)

The lion contains a cycle, and its behavior is therefore more complicated than the behavior of the lion in Figure 2. As usual in networks with feedback loops, we describe the behavior of the lion using a fixed point definition. The output of the lion is the least set of formulas such that the input of the black box contains at least its output. This is formalized in Definition 3.2 below.

For example, assume that the black box in Figure 3 can be described by a simple-minded operation out $1(G, A)$, and therefore the output of the lion is 
$\cap\{C n(G(B)) \mid A \subseteq B=C n(B) \supseteq G(B)\}$, which happens to be $\operatorname{out}_{3}(G, A)$. So the component is exogenously coordinated such that the system behaves like a reusable output operation, regardless of the fact whether the component itself behaves as a simple-minded or reusable operation.

To consider more complicated examples, we define lions formally based on the above intuitions. For simplicity we do not define recursive lions. Note that we do not require that all nodes of the lion are reachable from the start node, though this seems a reasonable extension.

Definition 3.1 Let $L$ be a propositional logic. A logical input/output net or lion is a tuple $\langle N, s, e, E, G, O\rangle$ where $N$ is a set of nodes, $s, e \in N$ the start and end node of the lion, $E \subseteq N \times(N \backslash\{s\})$ a set of edges, $G:(N \backslash\{s, e\}) \rightarrow 2^{L \times L}$ is a complete function from the set of nodes to sets of norms defined over $L$, and $O:(N \backslash\{s, e\}) \rightarrow\left\{\right.$ out $_{1}, \ldots$, out $\left._{4}^{+}\right\}$a complete function associating an input/output operation to nodes.

The behavior of a lion is defined as a least fixed point, which due to monotonicity (and the Knaster-Tarski theorem) exists and is unique.

Definition 3.2 Let $L$ be a propositional logic, $A$ a set of formulas of $L$, and $\langle N, s, e, E, G, O\rangle$ a lion. Moreover, let $i n(n)=\{m \mid(m, n) \in E\}$ be the set of nodes connected to $n$. A possible behavior of the lion is a function $b: N \rightarrow 2^{L}$ from the nodes to sets of propositional formulas such that:

(i) $b(s)=C n(A): A$ is the behavior of the start node, and

(ii) $b(n)=O(n)(G(n), b(i n(n)))$ for $n \in N \backslash\{s, e\}$, where we write $b(M)=$ $\cup_{m \in M} b(m)$ for $M \subseteq N$ : the behavior of an internal node is defined by the associated input/output logic, and

(iii) $b(e)=C n(b(i n(e)))$ : the behavior of the end node is the union of the outputs of the nodes connected to the end node.

Moreover, consider the case in which the black box in Figure 2 is not simpleminded output, but reusable output out $_{3}(G, A)$. The behavior of the lion is $C n\left(\right.$ out $\left._{3}(G, A) \cup A\right)$, which happens again to be equivalent to out $t_{3}^{+}(G, A)$ [23]. Likewise, we consider the case in which the black box in Figure 3 is simpleminded throughput out $t_{1}^{+}(G, A)$. The output of the lion is again out $_{3}^{+}(G, A)$.

\subsection{The disjunction rule}

Whereas the identity rule and the cumulative transitivity rule are naturally modeled as feed forward and feedback loops, this is not the case for the disjunction rule. In the semantics of input/output logics, this rule corresponds to reasoning by cases. We can consider reasoning by cases as a kind of exogenous coordination, in the sense that we can ensure that a component behaves like it is reasoning by cases, when it is not really doing so. The idea is that in the wrapper around the component, we need to generate the cases, and then we collect the outputs again. 
We cannot model this using lions as defined above. In general, there seem to be two ways in which this can modeled using a network, since it can be modeled using a set of copies of the component running in parallel, or using a single component dealing with the cases sequentially. For example, consider the network in Figure 4 in which the logical language contains three propositional atoms $p, q, r$ and the input of the component is $p$. Then there are four cases which should be considered, $q \wedge r, q \wedge \neg r, \neg q \wedge r$, and $\neg q \wedge \neg r$.

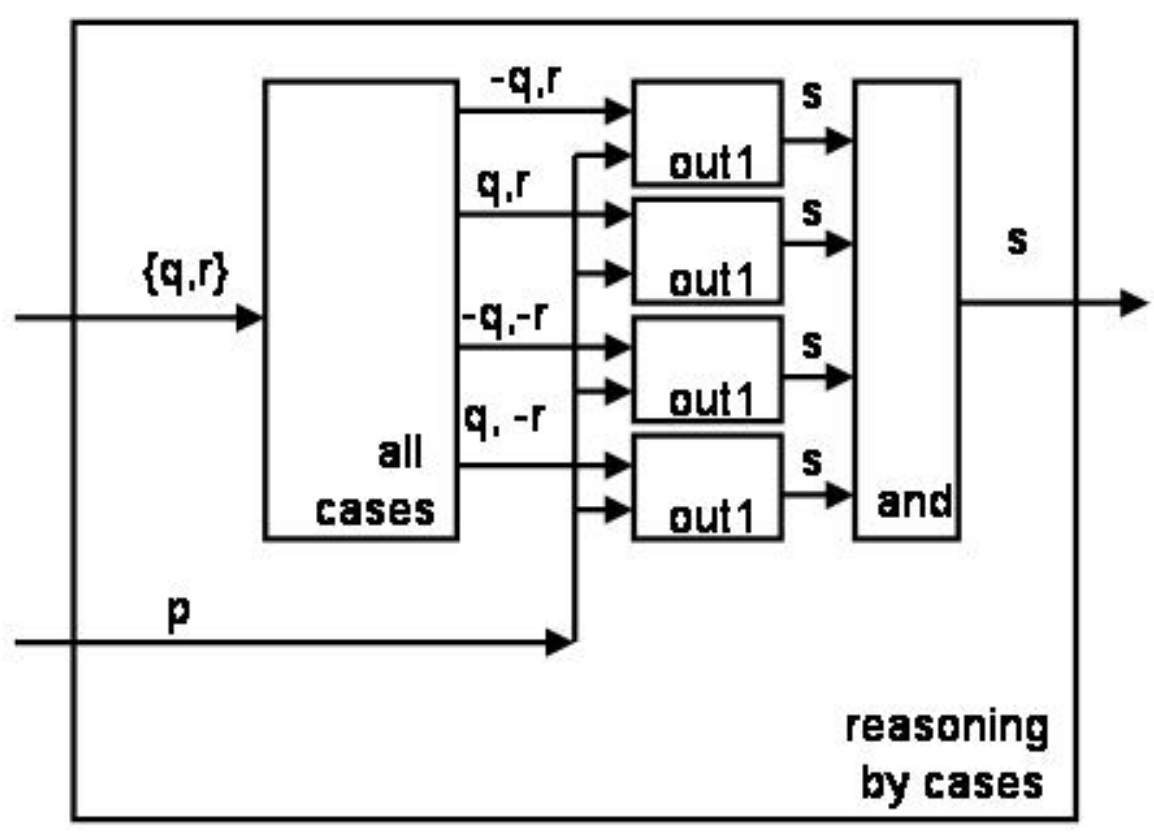

Fig. 4. Reasoning by cases as exogenous coordination

However, a drawback of this approach is that the number of cases is unknown and can be high or even infinite. The alternative of doing the cases in sequence uses a single component, by generating the cases one after the other, sending them through the component, and collecting them afterwards. This deals with the unknown number of cases, but not with an infinite number. Moreover, after each case we have to reset the component.

There are more problems related to reasoning by cases in the context of lions. For example, if the black box in Figure 2 is not simple-minded output, but basic output out $2(G, A)$, then the behavior of the lion is weaker than out $_{2}^{+}(G, A)$. For example, out $t_{2}^{+}(\{(a, x)\}, \emptyset)=C n(a \rightarrow x)$, whereas the corresponding lion only derives the propositional tautologies. Roughly, the reason is that we only have feed forward for the whole lion, whereas in basic throughput we have feed forward for each case. For example, in Figure 4 we need feed forward from the node 'all cases' to the AND gate.

Due to these problems, one may be tempted to ignore the disjunction rule. However, we would like to emphasize the importance of this reasoning pattern in many kinds of reasoning. For example, a variant of reasoning by 
cases known as the sure thing principle is a key principle in Savage's classical decision theory in the foundations of statistics [29].

\subsection{Combining components}

As an example of a lion with multiple components, consider the simple sequence of two components in the $\mathrm{BD}$ architecture in Figure 5. This lion represents the goal generation component of an agent architecture with a component outputting beliefs (B) and a component outputting desires (D), also see Section 5.1 for this interpretation.

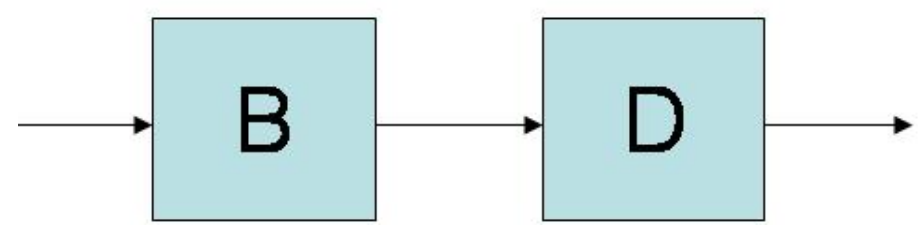

Fig. 5. BD

If $\mathrm{B}$ and $\mathrm{D}$ are input/output operations, then also the lion is an input/output operation. As David Makinson observed (personal communication), we can say quite generally the following. Take any lion $L=\langle N, s, e, E, G, O\rangle$. Define $G^{\prime}$ to be the set of all ordered pairs $(a, x)$ such that $x$ is in the output of $L$ (i.e. $x \in b(e))$ when $L$ is given input $a$ (i.e. $a \in b(s)$ ), Then $G^{\prime}=$ out $_{1}\left(G^{\prime}\right)$. Reason: so defined, $G^{\prime}$ is closed under SI, AND, WO.

Moreover, the properties of the lion depend on the properties of the components. For example, when both $\mathrm{B}$ and $\mathrm{D}$ satisfy identity, then the lion satisfies the identity rule. Likewise, when both B and D satisfy cumulative transitivity, then the lion satisfies this rule. However, when B satisfies the disjunction rule, then the lion does not have to satisfy the disjunction rule.

Moreover, consider the BD architecture with a feedback loop over both components in Figure 6. Using the semantic Definition 3.2 we can show that this architecture is different from the architecture in Figure 5 when the components do not satisfy cumulative transitivity, and they are not equivalent either when the components satisfy cumulative transitivity.

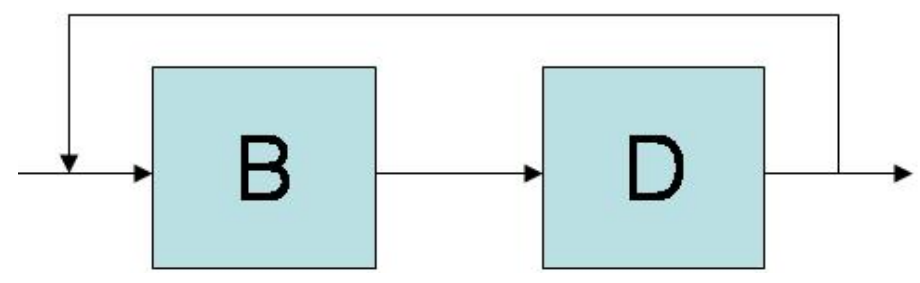

Fig. 6. BD with feedback

The proof theory of lions has not been studied yet, and neither the equivalence of lions. 


\section{Extensions to lions inspired by interactive computing}

There are many ways in which lions can be extended to cover a wider range of interactive computing. In this section we consider two of those extensions based on input/output logics for streams, and connectors.

\subsection{Input/output logic for streams}

In his section we are inspired by the notion of abstract behavior type (ABT), which has been proposed by Arbab [4] as a proper foundation model of coordination - more precisely, as a higher-level alternative to abstract data type (ADT). The ABT model supports a much looser coupling than is possible with the ADT's operational interface. The point is that ABTs do not specify any detail about the operations that may be used to implement such behavior or the data types the system may manipulate for its realization - just like input/output logic does not specify the black box. ABTs are modelled as input/output operations on infinite sequences of data, called streams, which suggests that input/output logics should not only consider transformations at one moment in time, but also transformations over time. In this section we consider this extension.

\subsubsection{Changing the base logic}

The first input/output logic for streams we consider replaces the propositional base logic by a logic of streams, and then applies input/output logic on this logic of streams. We write $\alpha$ for an infinite sequence (called a stream) of propositions $\left\langle p_{0}, p_{1}, p_{2}, \ldots\right\rangle$, and we define Boolean connectives as point-wise applications of the connectives. Thus $\neg \alpha=\neg\left\langle p_{0}, p_{1}, p_{2}, \ldots\right\rangle=\left\langle\neg p_{0}, \neg p_{1}, \neg p_{2}, \ldots\right\rangle$. Moreover, for binary connectives, we have $\left\langle p_{0}, p_{1}, p_{2}, \ldots\right\rangle \wedge\left\langle q_{0}, q_{1}, q_{2}, \ldots\right\rangle=$ $\left\langle p_{0} \wedge q_{0}, p_{1} \wedge q_{1}, p_{2} \wedge q_{2}, \ldots\right\rangle$, etc. Likewise, $\left\langle p_{0}, p_{1}, p_{2}, \ldots\right\rangle \in C n\left(\left\langle q_{0}, q_{1}, q_{2}, \ldots\right\rangle\right)$ if and only if $p_{i} \in C n\left(q_{i}\right)$ for $i=0,1,2, \ldots$.

Definition 4.1 Let $L$ be a propositional language, let $\alpha, \beta, \gamma$ be infinite sequences (called streams) of elements of $L$, and let boolean connectives on such streams be defined as point-wise application of the connectives on elements of the streams.

Moreover, let the norms in $G$ be pairs $\left\{\left(\alpha_{1}, \beta_{1}\right), \ldots,\left(\alpha_{n}, \beta_{n}\right)\right\}$, read as 'if input $\alpha_{1}$, then output $\beta_{1}$ ', etc., and consider the following proof rules strengthening of the input (SI), conjunction for the output (AND), weakening of the output (WO), disjunction of the input (OR), and cumulative transitivity (CT) and Identity (Id) defined as follows:

$$
\begin{array}{lcc}
\frac{(\alpha, \gamma)}{(\alpha \wedge \beta, \gamma)} S I & \frac{(\alpha, \beta),(\alpha, \gamma)}{(\alpha, \beta \wedge \gamma)} A N D & \frac{(\alpha, \beta \wedge \gamma)}{(\alpha, \beta)} W O \\
\frac{(\alpha, \gamma),(\beta, \gamma)}{(\alpha \vee \beta, \gamma)} O R & \frac{(\alpha, \beta),(\alpha \wedge \beta, \gamma)}{(\alpha, \gamma)} C T & \overline{(\alpha, \alpha)} I d
\end{array}
$$


The following four output operators are defined as closure operators on the set $G$ using the rules above.

$$
\begin{array}{ll}
\text { out }_{1}: \mathrm{SI}+\mathrm{AND}+\mathrm{WO} & \text { (simple-minded output) } \\
\text { out }_{2}: \mathrm{SI}+\mathrm{AND}+\mathrm{WO}+\mathrm{OR} & \text { (basic output) } \\
\text { out }_{3}: \mathrm{SI}+\mathrm{AND}+\mathrm{WO}+\mathrm{CT} & \text { (reusable output) } \\
\text { out }_{4}: \mathrm{SI}+\mathrm{AND}+\mathrm{WO}+\mathrm{OR}+\mathrm{CT} & \text { (basic reusable output) }
\end{array}
$$

Moreover, the following four throughput operators are defined as closure operators on the set $G$.

$$
\text { out }_{i}^{+}: \text {out }_{i}+\mathrm{Id} \text { (throughput) }
$$

We write $\operatorname{out}(G)$ for any of these output operations, and out $^{+}(G)$ for any of these throughput operations, we write $G \vdash_{i o l}(\alpha, \beta)$ for $(\alpha, \beta) \in$ out $(G)$, or, depending on context, $(\alpha, \beta) \in$ out $^{+}(G)$, and we write $G \vdash_{i o l} G^{\prime}$ iff $G \vdash_{i o l}$ $(\alpha, \beta)$ for all $(\alpha, \beta) \in G^{\prime}$.

Makinson and van der Torre study only the given eight input/output operations, but this set of input/output logics is not thought to be exhaustive. For example, we believe that also operations not satisfying strengthening of the input may be of interest, and the extension of simple-minded output with the transitivity rule (derive $(a, y)$ from $(a, x)$ and $(x, y))$ may have some applications. In throughput systems, assuming the simple-minded rules, the transitivity rule is equivalent to cumulative transitivity, but this is not the case in general. Moreover, the replacement of propositional logic by a logic of streams may again lead to the study of new inference rules. However, we do not consider such alternatives in this paper.

In a similar way, we can generalize the definitions of constraints, permissions and lions to point-wise operations on streams. For example, for two streams $\alpha=\left\langle p_{0}, p_{1}, p_{2}, \ldots\right\rangle$ and $\beta=\left\langle q_{0}, q_{1}, q_{2}, \ldots\right\rangle$, we say that $\alpha$ and $\beta$ are conflicting if there is an index $i$ such that $p_{i} \wedge q_{i}$ is inconsistent. Again, the use of streams may lead to new kinds of constraints.

\subsubsection{Stronger alternatives for the input/output logic of streams}

Alternatively, we can define an input/output logic on streams as if every moment in time, the box is a traditional input/output logic, i.e.,

$$
\operatorname{out}\left(G,\left\langle p_{1}, p_{2}, p_{3}, \ldots\right\rangle\right)=\left\langle\operatorname{out}\left(G, p_{1}\right), \operatorname{out}\left(G, p_{2}\right), \operatorname{out}\left(G, p_{3}\right), \ldots\right\rangle
$$

where the first out is defined on streams as in Definition 4.1, and the second out is a traditional input/output operation defined on propositions.

For a simple example of how the two treatments of streams can give different outputs, consider a component which has $\langle q, q, \bar{\top}\rangle$ amongst the outputs 
of input $\langle p, p, \bar{\top}\rangle$, and $\langle r, r, \bar{\top}\rangle$ amongst the outputs of input $\langle p, q, \bar{\top}\rangle$, where $\bar{T}$ is an infinite sequence of tautologies. In the input/output logic of streams of Definition 4.1, we do not necessarily have $\langle q \wedge r, q, \bar{T}\rangle$ as part of the output of input stream $\langle p, p, \bar{\top}\rangle$. However, this is the case in the stronger alternative defined in the previous paragraph.

The second treatment always gives at least as much output as the first, which follows from a property of the logic of streams as we have defined it. For example, consider the conjunction rule that derives $(a, p \wedge q)$ from $(a, p)$ and $(a, q)$ at every moment in time, then we can also derive the pair of streams $\left(\langle\bar{a}\rangle,\left\langle p_{0} \wedge q_{0}, p_{1} \wedge q_{1}, p_{2} \wedge q_{2}, \ldots\right\rangle\right)$ from $\left(\langle\bar{a}\rangle,\left\langle p_{0}, p_{1}, p_{2}, \ldots\right\rangle\right)$ and $\left(\langle\bar{a}\rangle,\left\langle q_{0}, q_{1}, q_{2}, \ldots\right\rangle\right)$, i.e., we can derive $(\alpha, \beta \wedge \gamma)$ from $(\alpha, \beta)$ and $(\alpha, \gamma)$.

As an intermediate solution, we can still define the input/output logic on streams as an input/output operation at every moment in time, but allow for the possibility that the set of norms is updated, i.e.,

$\operatorname{out}\left(\left\langle G_{1}, G_{2}, G_{3}, \ldots\right\rangle,\left\langle p_{1}, p_{2}, p_{3}, \ldots\right\rangle\right)=\left\langle\operatorname{out}\left(G_{1}, p_{1}\right), \operatorname{out}\left(G_{2}, p_{2}\right), \operatorname{out}\left(G_{3}, p_{3}\right), \ldots\right\rangle$

This leaves it open how the set of norms can be updated. For example, in the BD architecture in Figure 5 or 6 , it is left open how the sets of beliefs and desires are updated after outputs are generated.

\subsubsection{Other input/output logics of streams}

Thus far, we have assumed integer time, in the sense that the input and output streams may be seen as a function from the natural numbers to propositions. In other words, we have implicitly assumed that there is a clock such that every tick of the clock, a new output is generated from the input. This can be generalized to real time by making time explicit. For example, an abstract behavior type defines an abstract behavior as a relation among a set of timeddata-streams. We do not further consider this extension here.

\subsection{Connectors}

Thus far we have defined only a single type of channel in a lion connecting the output of a component to the input of another component. However, this is clearly a limitation. Consider, for example, standard Boolean circuits with AND, OR and NOT gates. How does this correspond to lions? For example, in Figure 4 an AND gate only outputs formulas which occur in each case. So if one channel has $C n(p \wedge q)$ and another channel $C n(p)$, then the output of the AND gate is $C n(p)$. Likewise, an OR gate outputs formulae that are in at least one of the input sets, and thus can be used to collect all inputs. Note that under this set-theoretic interpretation, somewhat misleadingly, the logical closure of the output of the OR gate is the consequence set of the conjunction of its inputs. Since the input of a component collects all the outputs of the nodes connected to it, this may implicitly be seen as an OR gate. Moreover, a NOT gate can be defined as a special kind of component. An AND gate 
seems to extend the expressive power of lions.

Moreover, once we use streams, we can use more complicated channels. A typical example is a register channel, which delays the throughput of data. For example, a register(1) outputs its input with a delay of one clock tick. Such a channel contains a buffer, with an initial value which is outputted as the first element of the output stream.

\subsubsection{Register gates}

For example, consider the lion with registers in Figure 7. We have a circuit which splits the input, then one direct link and on via register(1). Then it again merges with an OR port. We have the following behavior, assuming that initially there is a tautology in the register:

$$
\operatorname{out}\left(\left\langle p_{1}, p_{2}, p_{3}, \ldots\right\rangle\right)=C n\left(\left\langle p_{1}, p_{1} \wedge p_{2}, p_{2} \wedge p_{3}, \ldots\right\rangle\right)
$$

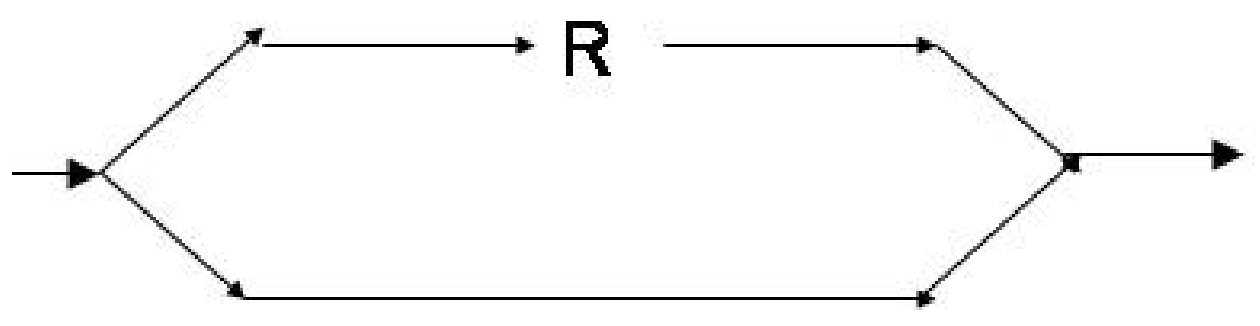

Fig. 7. Connector with register

\subsubsection{AND gates}

Consider now the extension of lions with AND gates, with the obvious behavior. Moreover, consider the lion in Figure 8, which is the result of replacing the OR gate in Figure 7 by an AND gate. We have the following behavior, if initially there is a contradiction in the buffer:

$$
\operatorname{out}\left(\left\langle p_{1}, p_{2}, p_{3}, \ldots\right\rangle\right)=C n\left(\left\langle p_{1}, p_{1} \vee p_{2}, p_{2} \vee p_{3}, \ldots\right\rangle\right)
$$

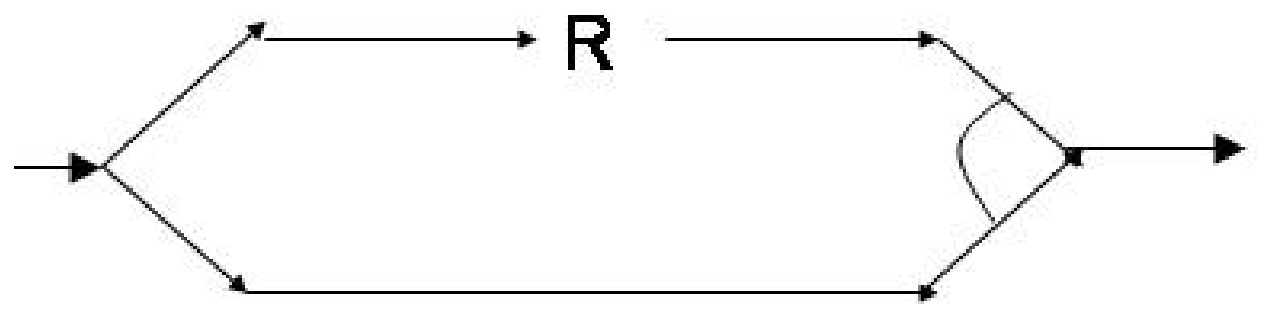

Fig. 8. Connector with register and AND gate 


\subsubsection{Further extensions}

Further inspiration for channels can be found in channel theory [7], based on Dretske's theory of information flow [19], and so-called situation theory, which originated in natural language semantics. Channel theory has studied signalling systems, in which a series of tokens at the physical level, is interpreted as carrying a certain meaning at the semantic level. Dretske defines a communication channel as "that set of existing conditions (on which the signal depends) that either (1) generate no relevant information, or (2) generate redundant information (from the point of view of the receiver) [19, p 115]". In other words: the channel defines a number of background conditions that allow communication, such as for example a shared vocabulary, but it does not add any additional information.

Moreover, several kinds of channels have been studied in the coordination language Reo. Some interesting extensions are the following.

- Channels generally have an input and an output end, but they can also have two input ends, or two output ends, which can be useful to synchronize channels.

- A new kind of gate, called a merger, will accept the input from one of the several channels leading into it, but not all of them at the same time. In the context of lions, such a gate may be called an exclusive choice gate.

- Context dependent channels, for example lossy channels which lose their inputs whenever there is no corresponding take action at the sink side of the channel.

It is an open question which of these channels form a useful extension for lions. As a guidance for further development of lions we propose their use in normative multi-agent systems, which we discuss in the following section.

\section{$5 \quad$ Normative multi-agent systems}

In this section we discuss the relevance of interactive computing in general, and lions in particular, for research on agent theory and normative multiagent systems. A pioneering approach which has inspired us is the work of Jan Treur and colleagues, on the design of interacting reasoning components [33]. Our discussion in this section is based on some of our own research. In the BOID agent architecture, interaction among the various mental attitudes like belief and desire, results in certain types of behavior. In a multi-agent system, a kind of qualitative game theory is used to describe and analyze the interaction among agents. In normative multi-agent systems, norms are used to coordinate the interaction among agents. Finally, in agent communication protocols, interaction is coordinated through social commitments. 


\subsection{BOID agent architecture}

In the BOID agent architecture [13], the behavior of an agent is characterized by the interaction among components that represent the mental attitudes belief, obligation, intention and desire. It extends the BD architecture given in Figure 5 and 6 with components for intentions (I) and obligations (O). The desires, obligations and intentions generate the goals of the agent. Moreover, the BOID architecture extends the BD architecture with the notion of priorities, such that for a social agent, obligations override desires. See [14] for a logical analysis of the interaction among mental attitudes in the BOID architecture.

A challenging issue in the formalization of the BOID architecture is the formalization of planning in this architecture, and in particular the interaction between goal generation and planning [18]. Goal generation is characterized as deductive reasoning and therefore can be formalized as a lion, but planning is characterized as means-end or abductive reasoning, which is less easily formalized as a lion. This issue has been studied using argumentation theory in [20]. Planning has been formalized as a deductive reasoning using so-called practical reasoning rules in the agent programming language 3APL, and an attempt to introduce such practical reasoning rules in the BOID architecture is given in [17].

A second challenge is the extension of the BOID architecture with streams of inputs and outputs. One central issue is whether mental attitudes persist over time, an issue discussed for example by the commitment strategies in the BDI literature.

\subsection{Multi-agent systems}

Interaction plays a central role in the design and analysis of multi-agent systems. In particular, the interaction is often based on game-theoretic approaches borrowed from economics and bounded reasoning as studied in artificial intelligence. In these approaches, an agent itself can be seen as a black box, with the agent's observations as input, and the actions that constitute its behavior as output. When an abstract behavior type characterizes a certain type of agent behavior, it is referred to as an agent type [13].

Whereas classical game theory is based on an equilibrium analysis, theories in artificial intelligence and agent theories are often based on the notion of recursive modeling. Agents model other agents when making decisions, but the number of levels in such recursive models is finite. Equilibrium analysis can be seen as reasoning about an infinite number of levels, which conflicts with the idea of bounded rationality.

\subsection{Normative multi-agent systems}

In normative multi-agent systems, norms are used to coordinate the interaction among intelligent agents. Various kinds of norms have been studied, such 
as regulative norms, permissive norms, constitutive norms, social laws, etc. Norms are modeled in terms of interaction, but the set of norms or normative system is often modeled as a component, interacting with the agents. The Boella-van der Torre model of normative multi-agent systems [9] is based on a more general notion of agent than is usually understood. This model makes it possible to attribute mental attitudes to normative systems, which means that the normative system is itself modeled as an agent - it is called a socially constructed agent. This has two important advantages:

(i) Obligations can be defined in the BDI framework. The desires or goals of the normative system are the obligations of the agent. This contributes to the open problem whether norms and obligations should be represented explicitly, for example in a deontic logic, or they can also be represented implicitly.

(ii) The interaction between an agent and the normative system can be modeled as a game between two agents. Consequently, methods and tools used in game theory such as equilibrium analysis can be applied to normative reasoning.

For example, a qualitative game theory can be developed as follows. The bearer of the obligation models the decision making of the normative system, which may itself be based on a model of the bearer of the norm. Hence the term recursive modeling. In so called violation games, the agent bases its decision on the consequences of the normative system's anticipated reaction, using a profile of the system's beliefs, desires and goals. In particular, the agent is interested in the question whether the normative system will consider its decision as a violation of the norm and thus sanction the agent. So called recursive games add one level of recursion to the game. They can be used by a legislator, to decide which norms should be created, or what the level of sanction should be. For example, in contract negotiation games, agents recursively model other agents to find out whether they would obey the new conditions and norms of the contract or not.

\subsection{Norms}

Regulative norms like obligations can be defined as follows. If agent $\mathrm{A}$ is obliged to make sure that $a$, then agent $\mathrm{N}$ may decide that the absence of $a$ counts as a violation of some norm $n$ and that agent A must be sanctioned. In particular, this means that

(i) Agent $\mathrm{A}$ believes that agent $\mathrm{N}$ desires that $\mathrm{A}$ does $a$ (objective)

(ii) Agent A believes that agent $\mathrm{N}$ desires $\neg V(n)$, that there is no violation of norm $n$, but if agent $\mathrm{N}$ believes $\neg a$ then $\mathrm{N}$ has the goal $V(n), \neg a$ counts as a violation (detection).

(iii) Agent A believes that agent $\mathrm{N}$ desires $\neg s$, not to sanction, but if agent $\mathrm{N}$ decides $V(n)$ then it has as a goal that it sanctions agent A by doing $s$. 
Agent $\mathrm{N}$ only sanctions in case of violation. Moreover, agent $\mathrm{A}$ believes that agent $\mathrm{N}$ has a way to apply the sanction (sanctioning)

(iv) Agent A desires $\neg s$ : it does not like the sanction (deterrence)

The clauses deal with various circumstances. For example, the first clause expresses the objective of the norm. The second clause ensures that normative systems will only detect actual violations. Similarly, the third clause prevents arbitrary sanctions. The last clause shows that the sanction is undesirable, and hence will deter agents from violating the norm.

We can define different agent types. For example, respectful agents simply desire to fulfill the norm as such, whereas for selfish agents the only motivation to comply with obligations is the fear for sanction or the desire for reward. Intermediate agent types are possible too. A selfish agent exploits the beliefs and goals of the normative system to try and violate an obligation without being detected or sanctioned.

In addition to regulative norms, we stress the importance of constitutive norms [31]. Just as the rules of chess constitute the game by defining legal moves, constitutive norms generate institutional facts, that allow individuals to function in society. Constitutive norms take the form of 'counts as' rules. For example, a theater ticket counts as evidence of the right to occupy a seat. Constitutive norms apply only under certain circumstances and are intimately linked to an authority or institution. Thus constitutive rules are of the form "event or fact $x$ counts as event or fact $y$ under circumstances $c$ in institution $i$ ".

Coordination in normative multi-agent systems [9] uses besides norms also more complex concepts like contracts [11] and roles [10].

\subsubsection{Contracts}

Coordination can be achieved without requiring a fixed agent architecture, but by means of roles and norms as incentives for cooperation. For this purpose, some approaches allow agents to stipulate contracts. A contract can be defined as a statement of intent that regulates behavior among organizations and individuals.

Contracts have been proposed to make explicit the way agents can change the interaction with and within the society: they create new obligations or permissions and new possibilities of interaction. From a contractual perspective, an organization can be seen as the possible set of agreements for satisfying the diverse interests of self interested individuals. In [11] we define a contract as a triple:

(i) An institutional fact $c \in I$ representing that the contract has been created.

(ii) A constitutive rule which makes the institutional fact $c$ true.

(iii) A set of constitutive rules having as antecedent the creation $c$ of the con- 
tract and as consequent creation actions modifying the mental attitudes of the organization.

\subsubsection{Roles}

Multi-agent systems are often proposed as a solution for the organizational design of open systems. A key notion in the structure of an organization is that of role. Roles specify the activities of an organization to achieve its overall goal, while abstracting from the individuals that will eventually execute them. A role is usually described in terms of normative descriptions, expectations, standardized patterns of behavior, social commitments, goals and planning rules. The normative description specifies the obligations that any agent who plays the role (called the actor) should obey. Goals are his intrinsic motivations. Roles, thus, seem to be strictly related to the notion of agent: they are described using notions like actions, goals and obligations.

In the Boella-van der Torre model, we attribute mental attitudes to roles and use this model for several problems. First, if we design an organization in terms of roles by attributing mental attitudes to them, we can design it just as we would design an organization in terms of agents. However, the organization does not depend on the agents operating in it: agents are replacable. Tasks can be delegated to roles, with a greater flexibility: it would be not sufficient to describe roles as machines. Second, if we consider the problem of assigning roles to actors and we attribute mental attitudes to roles, then the problem becomes finding a match between the beliefs representing expertise of the role and the beliefs of the actor. Third, if we consider the governance of organizations, then the attribution of mental attitudes to roles makes it easier to verify whether an agent is acting according to the overall plan of the organization: if he is acting according to the role's beliefs and achieving the role's goals, then he is fulfilling his responsibilities.

The issue discussed in the formalization is which beliefs and goals are attributed to a role. Not all the beliefs of the organization are beliefs of a role due to the role assignment problem: the less beliefs are ascribed to a role, the easier it becomes to assign agents to this role. Similarly for goals - not all the goals of an organization are the goals of a role due to the governance problem: to distribute responsibilities over the agents, and to control the running system. Consequently, the organizational design aims at defining roles with minimal sets of beliefs and goals. Note that not only the beliefs and goals of an organization may not correspond to goals of the role, but also vice versa: a goal of a role may not be a goal of the organization.

\subsection{Communication}

In the communication of computer systems, interaction is regulated by a protocol: a set of rules that define which messages are allowed by which participants at which stage of the interaction. Well known examples are the Contract Net 
Protocol, that regulates a distributed procedure for allocating tasks, or protocols for negotiation. Protocols have a normative aspect: they consist of rules specifying what an agent should and shouldn't do (prescription); based on those rules other agents can form expectations about the behavior of other participants (prediction).

In agent theory there are at least two kinds of semantics. One is based on the paradigm of mental attitudes. it makes specific assumptions about the internal architecture of the agents. The other is based on social commitments, and makes no assumptions about the agents. This approach is more in line with the paradigm of interactive computing. In this section we discuss the two approaches, and also propose a synthesis [8].

\subsubsection{Speech act theory}

Within the autonomous agents and multi-agent systems community, agent communication standards have been developed for structuring messages and for simple interaction protocols. The backbone of these standards is formed by speech act theory $[6,30]$. The semantics of a speech act is commonly given by the preconditions and intended effect on the mental state of an agent, expressed using modal operators for belief and intention. Early research in AI [16] showed that the systematic analysis of speech acts in terms of preconditions and postconditions could be modeled straightforwardly in terms of planning operators. That means that a sequence of messages makes sense, in case it can be interpreted as a plan to achieve some goal.

Various aspects of speech act theory for agents have been standardized by an organization called FIPA, and this standardization effort has been a relative success, although it has been criticized heavily. A point of criticism is that it is impossible to verify the correct usage of a speech act, since for most realistic multi-agent settings the mental state of an agent is inaccessible. Agents may well be lying. So unless a sincerity principle is postulated - which makes no sense under common assumptions regarding multi-agent systems it is impossible to verify protocols [36]. What is needed instead is a semantics that is based on public information about what agents are committed to, on the basis of what they have said.

\subsubsection{Social semantics}

For this reason, Singh [32] proposes a social semantics. It is based on the notion of commitment. Commitment binds a speaker to the community and, unlike mental attitudes, commitments have a public character. In some cases commitments generate a kind of obligation. For example, when a speaker asserts a proposition, the speaker generally becomes committed to subsequently defending the proposition, when challenged by another dialogue participant.

These two approaches - mentalistic and social - are portrayed as competing alternatives, but it is quite possible to combine them. The combination rests on the following key idea: if we attribute mental attitudes to the roles of the 
participants, rather than to the individual agents that enact the roles, the mental attitudes do get a public character, just like commitments.

Such a combination has the following advantages. On the one hand applications which take place in a restricted known cooperative environment can re-use much of the work done on FIPA compliant agent communication. On the other hand, stronger obligations can be added when required. In particular, in competitive or other non-cooperative circumstances, no sincerity principle needs to be assumed. Instead an obligation to defend a publicly attributed belief, as suggested above, must be added. However, the basic bookkeeping and the semantics of the speech acts themselves, can remain the same.

\subsubsection{Synthesis}

The method we adopt is to model dialogue as a game in which agents play roles. Speech acts are moves in the game and their preconditions and effects refer to the mental states attributed to the roles, not to the mental states of the agents themselves. This approach presupposes that mental attitudes can be attributed to roles as well as to agents. Following [10,12], we describe roles as agents with mental attitudes, albeit of a different kind, since they are not autonomous.

Agents playing roles in the game can affect the state of the game, the state of the roles they play and the state of the other roles. This is possible thanks to constitutive rules. Constitutive rules explain how an utterance by a player counts as a move in the game and how the move affects the beliefs and goals of the roles. In contrast with agents' beliefs and goals, beliefs and goals of roles have a public character in that the game defines them, and it also defines how they evolve during a dialogue according to the moves played by the agents. Moreover, since roles provide a description of expected behavior they have a prescriptive character: the player of a role becomes committed to the beliefs and goals of the role created during the dialogue. Finally, the constitutive rules can describe, if necessary, how obligations are created during the dialogue.

Since such dialogue games consist of both constitutive and regulative rules, they can be considered as a normative multi-agent system. Just as for roles, our view of normative systems is based on the agent metaphor: a normative system can technically be considered as an agent to which mental attitudes are attributed. Moreover, normative systems, like organizations, can be articulated in roles.

\subsubsection{Example}

1. $s$ : The president will win the election. $\quad$ inform $(s, r, p)$

2. $r$ : But there is fraud, so the president will not win.

3. $s$ : Fraud? But why! 
4. $r$ : Fair enough, no fraud.

So you're right.

$$
\begin{array}{r}
\operatorname{retract}(r, s, \operatorname{inform}(r, s, q)) \\
\operatorname{accept}(r, s, p)
\end{array}
$$

The example is explained as follows.

Turn 1: role $s$ informs $r$ that $p$. The rules of role $s$ now state that $p$ is a consequence of $s$ 's beliefs. The rules of role $r$ state that the belief $p$ can be attributed to $r$ unless $r$ challenges $s$ 's inform.

Turn 2: the agent playing role $r$ challenges $s$ 's information by means of an argument of the form $q,(q \supset \neg p)$.

Turn 3: $s$ is risking losing the game. Unless it does something, it will get into a contradiction, since $r$ 's argument supports $\neg p$, which is in contrast with its current beliefs $(p)$. It has some alternatives: retracting its first inform or challenging $r$ 's argument. So $s$ decides to challenge $r$ 's challenge by asking for justification (why).

Turn 4: $r$ retracts the inform about $q$, thus giving up its challenge to $p$, and subsequently accepts $p$.

This example shows that indeed utterances can be modeled as constitutive rules, with the preconditions and postconditions of the mentalistic approach, reinterpreted as the mental attitudes of the roles.

\section{Summary}

In this paper we consider logical input/output nets, or lions for short, as models of interactive computing. Lions are based on input/output logic, a deontic logic not used as a non-classical inference engine deriving output from input, but as a secretarial assistant for transformations from input to output. We present a few definitions, but no formal results.

The central research question of this paper is how notions developed in interactive computing such as abstract behavior types, the coordination language Reo, and Boolean circuits with registers, can be used to extend input/output logics and lions. In particular, we consider two extensions of input/output logics and lions.

First, we consider input/output logics defined on infinite sequences (or streams) of inputs and outputs. The first extension replaces the propositional logic underlying input/output logic by a logic of streams, and the second extension strengthens this logic by applying the input/output logic on every input. Yet another alternative applies an input/output operation at every propositional input, but assumes that the set of norms can change over time.

Secondly, we consider lions with AND and register gates, formalizing the behavior of channels and connectors. The extension illustrates how gates from Boolean circuits can be used to extend lions, and how operators for streams can be introduced to model behavior over time. We also observe that more complicated channels can be introduced, such as lossy channels. 
Our discussion has highlighted a number of future research directions for lions. A proof theory for lions can be developed, constraints can be added to lions, and permissions can be introduced to distinguish two kinds of outputs. The introduction of time in streams introduces new possibility of studying new proof rules of input/output logics, and the introduction of new kinds of channels and gates introduces a variety of new conceptual and formal issues.

Given the huge number of possible extensions, we feel that we need guidance to develops lions, just like the development of input/output logic has been guided by issues in deontic logic. We are motivated to build normative multi-agent systems on top of lions, based on the idea that interaction plays a crucial role in normative multi-agent systems. In the agent architecture the behavior of an agent is determined by the interaction among the mental attitudes, in a multi-agent system game theory is used to describe and analyze the interaction among agents, in normative multi-agent systems norms are used to coordinate interactions among agents, and in communication interaction is central in models based on social commitments.

One particular extension we believe can be guiding the further development of lions for normative multi-agent systems is the development of value webs, which are networks that describe the exchange of value. For example, a value web may model the exchange of goods for money, possibly including third parties such as shippers and banks. We suspect that when not only information but also values are transported over channels, new issues arise.

We also believe that the research on input/output logics, lions and normative multi-agent systems can be an inspiration for the wider study of interactive computing. In particular, we like to emphasize three issues:

Logic The use of logic as a secretarial assistant, which facilitates exogenous coordination.

Concepts Concepts of normative multi-agent systems to study interaction like contracts and roles.

Communication Communication based on social commitments, and its relation to the mentalistic FIPA approach.

\section{Acknowledgements}

Thanks to David Makinson for the introduction of logical input/output nets (personal communications) and for commenting on an earlier version of this paper, and to Farhad Arbab and his colleagues at CWI for many inspiring discussions on the issues raised in this paper.

\section{References}

[1] Alchourrón, C., P. Gärdenfors and D. Makinson, On the logic of theory change: partial meet contraction and revision functions, The Journal of Symbolic Logic 
50 (1985), pp. 510-530.

[2] Arbab, F., What do you mean, coordination?, Bulletin of the Dutch Association for Theoretical Computer Science, NVTI (1998), pp. 11-22, available on-line http://www.cwi.nl/NVTI/Nieuwsbrief/nieuwsbrief.html.

[3] Arbab, F., Reo: A channel-based coordination model for component composition, Mathematical Structures in Computer Science 14 (2004), pp. 329-366.

[4] Arbab, F., Abstract behavior types: A foundation model for components and their composition, Science of Computer Programming 55 (2005), pp. 3-52.

[5] Arbab, F., Coordination of interacting concurrent computations, in: Interactive Computation: The New Paradigm, Springer, 2005.

[6] Austin, J. L., "How to Do Things with Words," Harvard University Press, Cambridge, Mass, 1962.

[7] Barwise, J. and J. Seligman, "Information Flow," Cambridge University Press, 1997.

[8] Boella, G., J. Hulstijn and L. van der Torre, A synthesis between mental attitudes and social commitments in agent communication languages, in: Proceedings of IAT 2005 (2005).

[9] Boella, G. and L. van der Torre, Attributing mental attitudes to normative systems, in: Proceedings of the second international joint conference on autonomous agents and multi agent systems (AAMAS'03), 2003, pp. 942-943.

[10] Boella, G. and L. van der Torre, Attributing mental attitudes to roles: The agent metaphor applied to organizational design, in: Procs. of ICEC'04 (2004), pp. 130-137.

[11] Boella, G. and L. van der Torre, Contracts as legal institutions in organizations of autonomous agents, in: Proceedings of the Third International Joint Conference on Autonomous Agents and Multi Agent Systems (AAMAS'04), 2004, pp. 948-955.

[12] Boella, G. and L. van der Torre, Regulative and constitutive norms in normative multiagent systems, in: Procs. of 9th International Conference on the Principles of Knowledge Representation and Reasoning, 2004, pp. 255-265.

[13] Broersen, J., M. Dastani, J. Hulstijn and L. van der Torre, Goal generation in the BOID architecture, Cognitive Science Quarterly 2(3-4) (2002), pp. 428-447.

[14] Broersen, J., M. Dastani and L. van der Torre, Realistic desires, Journal of Applied Non-Classical Logics 12(2) (2002), pp. 287-308.

[15] Broersen, J., M. Dastani and L. van der Torre, Beliefs, obligations, intension and desires as components in agent architectures, International Journal of Intelligent Systems (2005).

[16] Cohen, P. and H. Levesque, Intention is choice with commitment, Artificial Intelligence 42 (2-3) (1990), pp. 213-261. 
[17] Dastani, M. and L. van der Torre, Programming BOID agents: a deliberation language for conflicts between mental attitudes and plans, in: Proceedings of the Third International Joint Conference on Autonomous Agents and Multi Agent Systems (AAMAS'04), 2004, pp. 706-713.

[18] Dastani, M. and L. van der Torre, What is a normative goal? towards goalbased normative agent architectures, in: Postproceedings RASTA02, LNAI 2934, Springer, 2004 pp. 210-227.

[19] Dretske, F., "Knowledge and the Flow of Information," MIT Press, 1981.

[20] Hulstijn, J. and L. van der Torre, Combining goal generation and planning in an argumentation framework, in: Proceedings NMR'04, 2004, pp. 212-218.

[21] Jones, A. and J. Carmo, Deontic logic and contrary-to-duties, in: D. Gabbay, editor, Handbook of Philosophical Logic, Kluwer, 2002 pp. 203-279.

[22] Makinson, D. and L. van der Torre, Input-output logics, Journal of Philosophical Logic 29 (2000), pp. 383-408.

[23] Makinson, D. and L. van der Torre, Constraints for input-output logics, Journal of Philosophical Logic 30 (2001), pp. 155-185.

[24] Makinson, D. and L. van der Torre, Permissions from an input/output perspective, Journal of Philosophical Logic 32 (4) (2003), pp. 391-416.

[25] Makinson, D. and L. van der Torre, What is input/output logic?, in: Foundations of the Formal Sciences II: Applications of Mathematical Logic in Philosophy and Linguistics, Trends in Logic 17, Kluwer, 2003.

[26] Poole, D., A logical framework for default reasoning, Artificial Intelligence 36 (1988), pp. 27-47.

[27] Reiter, R., A logic for default reasoning, Artificial Intelligence 13 (1980), pp. 81132.

[28] Rutten, J., Algebra, bitstreams and circuits, Technical Report SEN-R0502, CWI Amsterdam (2005).

[29] Savage, L., "The foundations of statistics," John Wiley \& Sons, New York, 1954.

[30] Searle, J., "Speech Acts: an Essay in the Philosophy of Language," Cambridge University Press, Cambridge (UK), 1969.

[31] Searle, J., "The Construction of Social Reality," The Free Press, New York, 1995.

[32] Singh, M. P., A social semantics for agent communication languages, in: F. Dignum and M. Greaves, editors, Issues in Agent Communication: Proceedings of the IJCAI Workshop on Agent Communication Languages, LNCS 1916, Springer, 2000 pp. $31-45$. 
[33] Treur, J., Semantic formalisation of interactive reasoning functionality, International Journal of Intelligent Systems 17 (2002), pp. 645-686.

[34] Wegner, P., Why interaction is more powerful than algorithms, Communications of the ACM 40 (1997), pp. 80 - 91.

[35] Wieringa, R. and J.-J. Meyer, Applications of deontic logic in computer science: A concise overview, in: J.-J. Meyer and R. Wieringa, editors, Deontic Logic in Computer Science: Normative System Specification, John Wiley \& Sons, Chichester, England, 1993 pp. 17-40.

[36] Wooldridge, M. J., Semantic issues in the verification of agent communication languages, Journal of Autonomous Agents and Multi-Agent Systems 3 (2000), pp. $9-31$. 


\title{
Roles as a Coordination Construct: Introducing powerJava
}

\author{
Matteo Baldoni ${ }^{1}$ Guido Boella ${ }^{2}$ \\ Dipartimento di Informatica \\ Università degli Studi di Torino (Italy) \\ Leendert van der Torre ${ }^{3}$ \\ CWI Amsterdam and Delft University of Technology (The Netherlands)
}

\begin{abstract}
In this paper we apply the role metaphor to coordination. Roles are used in sociology as a way to structure organizations and to coordinate their behavior. In our model, the features of roles are their dependence on an institution, and the powers they assign to players of roles. The institution represents an environment where the components interact with each other by using the powers attributed to them by the roles they play, even when they do not know each other. The interaction between a component playing a role and the role is performed via interfaces stating the requirements to play a role, and which powers are attributed by roles. Roles encapsulate their players' capabilities to interact with the institution and with the other roles, thus achieving separation of concerns between computation and coordination. The institution acts as a coordinator which manages the interactions among components by acting on the roles they play, thus achieving a form of exogenous coordination. As an example, we introduce the role construct in the Java programming language, providing a precompiler for it. In order to better explain the proposal, we show how to use the role construct as a coordination means by applying it to a dining philosophers problem extended with dynamic reconfiguration.
\end{abstract}

\section{Introduction}

Coordination, according to Malone and Crowston [16], is managing dependencies among independent activities. Coordination models and languages

1 Email: baldoni@di.unito.it

2 Email: guido@di.unito.it

3 Email: torre@cwi.nl

This is a preliminary version. The final version will be published in Electronic Notes in Theoretical Computer Science URL: www.elsevier.nl/locate/entcs 
all aim at providing frameworks for enhancing modularity, reuse of existing components, portability and language interoperability.

Papadopoulos and Arbab [23] distinguish two different approaches to coordination: the data-driven approach and the control-driven one. The difference rests in who drives the evolution of computation. In the former approach computation evolves driven by the data involved in the coordination, while in the latter one processes evolve according to events following state changes. Control-driven languages allow to achieve a more complete separation - even at the syntactic level - between coordination and computational concerns, which are dealt with by different processes. The state of the computation can, thus, be defined by the coordinated patterns that the processes involved in the computation adhere to. Moreover, control-driven approaches allow to treat the computational parts as black-boxes with clearly defined input/output interfaces. Finally, control-driven approaches allow a dynamic reconfiguration of the system, since the components specifying initial and evolving configurations are separated from the ones performing the actual computation.

The various coordination models and languages rely on distinct metaphors, like the shared dataspace, the blackboard model, the actor model, the chemical model, the channel model, etc. One reason for the diversity of metaphors is that coordination is an emerging area with an interdisciplinary focus going from economics to operational research, from organization theory to biology.

One basic metaphor in social theory and organization theory is the role metaphor. Roles are often defined as descriptions of expected behavior, and are used in organization theory to distribute responsibilities, obligations and rights among the agents working in an organization and, above all, to distribute institutional powers among them [22]. For example, an agent in the role of director of an organization has not only the right but also the power to sign buy orders on the behalf of the organization itself. Moreover, the agent playing the role of director has the power to commit the director to new responsibilities, as well as the power to commit to new duties the other members of the organization by ordering them. Finally, players of roles, exercising their powers, can change the structure itself of the organizations, by, e.g., merging departments, introducing new roles, hiring new employees, etc. Thus, roles, as entities endowed with powers, are used as a means to coordinate the behavior of an organization.

The research question of this paper is: "How to introduce and use the role metaphor in control-driven coordination?" To answer this question, our methodology is to start from our work on conceptual modelling [9], ontological analysis [7] and social reality of multiagent systems [8] to develop a new view on roles. Then, based on this model of roles we introduce a new role programming construct in a real programming language like Java; to prove its feasibility we translate it to pure Java by means of a precompilation phase. Finally, we show how the new language can be used for coordination purposes since it emphasises the separation of interactional aspects from the core 
behavior of a class and it allows the exogenous coordination of components.

It is beyond the scope of this paper to provide a formal semantics of the new constructs or to define the associated type theory. Moreover we do not address in this paper other issues related to roles like the problem of method delegation or of roles playing other roles, but we leave them for future work.

The structure of this paper is as follows. In Section 2 we describe our definition of roles. In Section 3 we discuss how coordination can benefit from our definition of roles. In Section 4 we introduce the new language powerJava and in Section 5 we use it to model the dining philosophers example. Conclusion ends the paper.

\section{Properties of roles}

The characteristic features of roles in our model are their foundation, their definitional dependence from the institution they belong to, and the powers attributed to the role by the institution. To understand these issues we propose an example. Consider the roles student and teacher. A student and a teacher are always a student and a teacher of some school. Without the school the roles do not exist anymore: e.g., if the school goes bankrupt, the actors (persons) of the roles cannot be called teachers and students anymore. The institution (the school) also specifies the properties of the student, which extend the properties of the person playing the role of student: the school specifies its enrolment number, its email address, its scores at past examinations, but also how the student can behave. For example, the student can try an exam by submitting some written examination. A student can make the teacher evaluate its examination and register the mark because the school defines both the student role and the teacher's role: the school specifies how an examination is evaluated by a teacher, and maintains the official records of the examinations. Otherwise the student could not have any effect on the teacher. In defining such actions the school empowers the person who is playing the role of student: without being a student the person has no possibility to give an examination and to make the teacher evaluate it.

The above example highlights the following properties that roles have in our model of normative multiagent systems $[9,7,8]$ :

Foundation: an instance of role must always be associated with an instance of the institution it belongs to (see Guarino and Welty [12]), besides being associated with an instance of its player.

Definitional dependence: The definition of the role must be given inside the definition of the institution it belongs to. This is a stronger version of the definitional dependence notion proposed by Masolo et al. [18], where the definition of a role must include the concept of the institution.

Institutional empowerment: the actions defined for the role in the definition of the institution have access to the state and actions of the institution 
and to the other roles' state and actions: they are powers.

Finally, following Steimann [24]'s analysis of roles, in our approach a role can be played by different kinds of actors. For example, the role of customer can be played by instances both of person and of organization, i.e., two classes which do not have a common superclass. The role must specify how to deal with the different properties of the possible actors. This requirement is in line with UML, which relates roles and interfaces as partial descriptions of behavior. This requirement compels to avoid modelling roles as dynamic specializations as, e.g., $[2,11]$ do. If customer were a subclass of person, it could not be at the same time a subclass of organization, since person and organization are disjoint classes. In the same way, person and organization cannot be subclass of customer, since a person can be a person without ever becoming a customer [12].

\section{Roles and coordination}

According to Arbab [3], extending the traditional Object Oriented model (OO) towards coordination of components presents some difficulties. They are related to the underlying notion of abstract data type with its idea of providing a set of operations in its interface while encapsulating data structures and the implementation of operations.

Components in $\mathrm{OO}$ are often seen as fortified collections of classes and objects with their interfaces. As a consequence the interactions among and the composition of components must use the same mechanisms as in the interaction among objects. The problem is that the method invocation semantics of the message passing metaphor in OO requires a very tight coupling between the caller and the callee objects. Amongst other things, the caller of a method of another object must know how to find this object and the syntax and semantics of the method. So, a component, to use another component, has to know it in advance, so that it can transfer control to it. The reason is that the operational interface of abstract data types induces an asymmetrical semantic dependency of consumers of operations on providers of operations: a consumer makes the decision on what operation to perform and it relies on the provider to carry out the operation. This reduces the "pluggability" of pre-existing components in a new system. Moreover, in inter-components interaction, method invocation does not allow to reach a minimum level of "control from the outside" of the participating components.

To resolve this problem, a different view of inter-component communication is advocated: untargeted passive messages exchanged with the environment that carry no control information in that they do not imply method invocation. For example, messages can be exchanged through channels. In contrast with targeted messages of method invocation, with untargeted messages the sender is not required to know who is the receiver of a message. In this way, a third party is given the possibility to set up the interaction 
between the sender and a receiver of his choice. We believe that the notion of role we propose in this paper can contribute to the solution of these problems in the inter-components interaction in OO. The role metaphor isolates the interaction between components at the level of the roles they play.

It is possible to draw a comparison between roles and the IWIM (Idealized Worker Idealized Manager) model [10,4,23]. Components playing roles are the workers carrying out the computation, while the institutions are the managers coordinating the other processes. The major difference is that institutions (managers) do not directly manipulate components (workers), but they coordinate them through the roles they play, which represent the state of the component inside the institution. Symmetrically, the components do not interact with other components, but they interact with the institution and with the other roles only through the powers offered by the roles they play. Roles give to their players the powers to interact with the other roles and the institutions. At the same time these powers, which are modelled as methods, are inside and defined by the institution, so components are not required to know their implementation, while they can be invoked on them when acting in a role.

By means of roles it is possible to connect the output of a component to the input of another component without requiring them to be aware of the connection, and to encapsulate the modalities of this connections, like concurrency synchronization. In this way we achieve the separation of concerns: components which act as the primary unit of computation in a system, and institutions which specify interaction and communication patterns between the components by means of roles.

Since powers are methods inside and defined by the institution they have the possibility to access both the institution and the other roles in it: hence, they can also reconfigure the interaction between the components playing the role. In the same way, the institution itself can modify these interconnections, thus achieving an exogenous coordination of the components composing the system. The implementation of the powers in the institution contains the information necessary to interact with the other components which play roles in the institution. At this level the interaction can be carried out by the standard intra component method invocation, since all the roles belong to the same component as the institution. Results of invocation can be then delivered to the calling component according to the preferred protocol, e.g., synchronous or asynchronous.

Finally, the interaction between components and their roles is relieved from the asymmetry of method invocation. Even if roles are object instances as any other, they represent a perspective on the object playing them. To invoke a method of a role, i.e., a power, the player of the role does not need a reference to the role instance. Rather, it needs only to specify which roles it is playing and in which institution and, then, it can invoke the method. The association between the player instance and the related role instance is managed in a 
transparent way by the framework we propose in the next section.

In this paper we illustrate the proposed powerJava language by means of the dining philosophers problem as a running example. It can be seen as a case of coordination between two types of components: resources to be concurrently shared by consumers, and consumers of resources which periodically need the output of a specific pair of resources to perform their computation. Resources provide data which are feeded as input to the consumers. Consumers do not know which resources are feeding them and how the concurrent access to the resources is synchronized. We assume that resources and consumers do not have any knowledge about the participants to the dinner, how the participants are disposed around the dinner table and how the resources have to be shared. Finally, consumers should not care about how to join and leave the dinner: they just exercise powers offered by the roles they play. All these coordination issues are dealt with by the roles and by the institution roles belong to, which constitutes at the same time the manager coordinating the workers via roles and the environment where the interaction takes place. Hence, the description of the coordination part of the system is given in a transparent and exogenous way and the components are plugged without requiring any knowledge of it.

\section{Introducing roles in Java}

In this section we discuss how our definition of roles can be introduced in Object Oriented programming languages. Since, as discussed, the role-asspecialization approach is not possible, we define roles as instances which can be associated runtime to objects which can play those roles. However, the extension of an object to roles must be transparent to the programmer.

Since powers are a distinguishing feature of roles, we call our language for coordination powerJava. To introduce roles in powerJava we have to address the following issues:

(i) The construct defining roles.

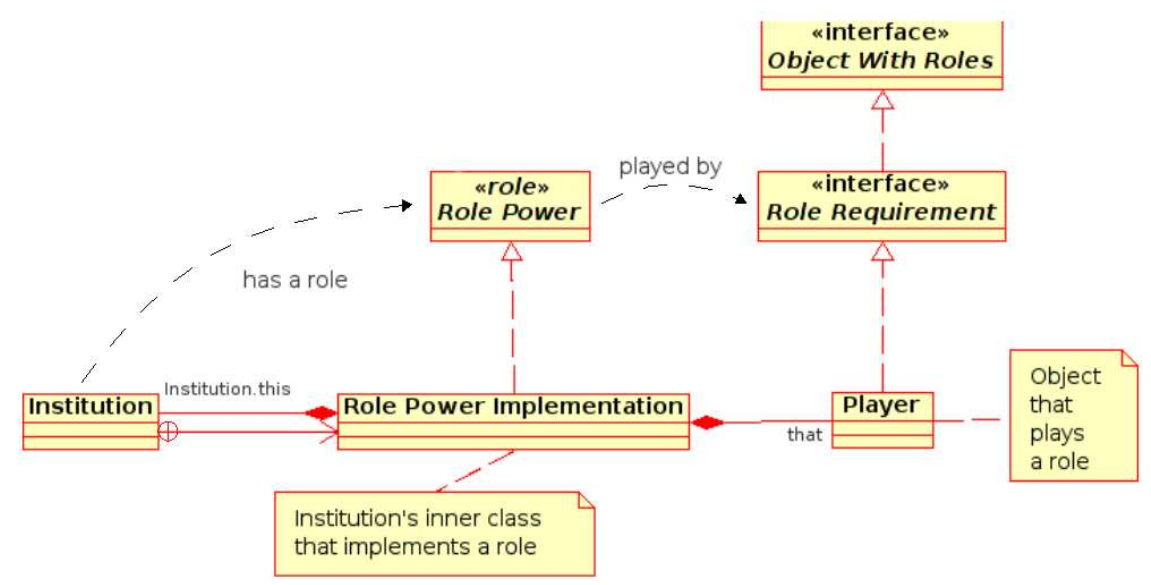

Fig. 1. Roles and Institutions. 
(ii) The implementation of a role inside an institution according to its definition.

(iii) A way to invoke the powers, which a role has in an institution, from an object playing that role.

To achieve our goals, we need very limited modifications of the Java syntax (see Figure 3). In Figure 1 we summarize the overall model using a UML diagram. Roles require to specify both who can play the role and which powers are offered by the institution in which the role is defined. The objects which can play the role might be of distinct classes, so that roles can be specified independently of the particular class playing the role. This is a form of polymorphism. For example a role customer can be played both by a person and by an organization.

Our proposal is to define the role construct as a sort of "double-sided" interface which allows the connection of a player to an institution. The interface is double in that it specifies:

The methods that a class must offer for playing the role (requirements).

In order to play a role, a class must offer some methods. These are specified in the role by an interface.

The methods offered to objects playing the role (powers). An object of a class, offering the required methods, plays the role: it is empowered with new methods as specified by this part of the role definition.

This "double face" pervades the life of a role: first, a role is defined with its requirements and powers, then its powers are implemented in a class which connects a role with a player satisfying its requirements, and, finally, the class implementing the role is instantiated passing to the constructor an instance of an object satisfying the requirements.

Requirements of a role in Java correspond to the notion of interface, specifying which methods must be defined in a class playing the role. As for interfaces, this mechanism of partial descriptions allows the polymorphism necessary for a role to be played by different classes.

The definition of a role (roledef in Figure 3) using the keyword role is similar to the definition of an interface: it is in fact the specification of the powers acquired by the role in the form of abstract methods signatures (interfacebody). The only difference is that the role definition does not allow even static variables to be declared, and it refers also to another interface, that in turn gives the requirements which an object, willing to play that role, must conform to. Such an interface is identified by the keyword playedby. In the example of Figure 4, the role definition Philosopher specifies the powers (eat, think, start, and leaveTable), whilst the playedby interface PhilosopherReq specifies its requirements (putData and processData).

This "double face" of a role definition captures the idea of Guillen-Scholten 

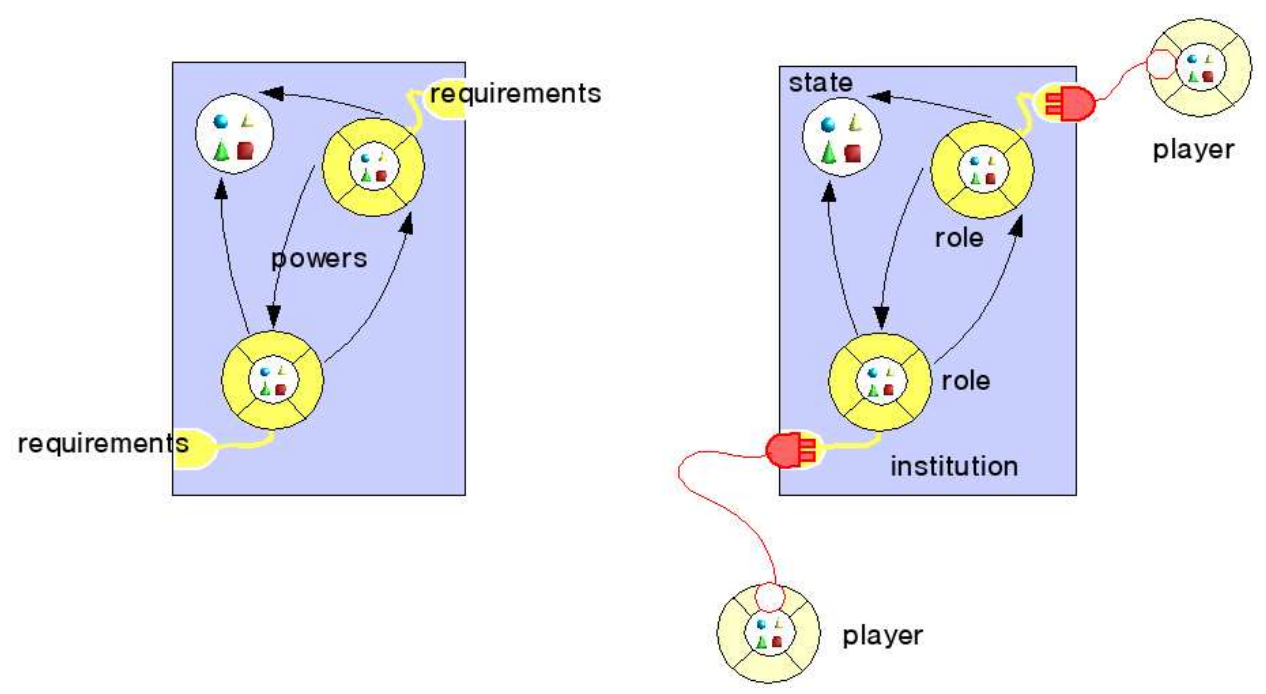

Fig. 2. The players will interact according to the acquired powers (they will follow the protocol implemented by the institution and its roles).

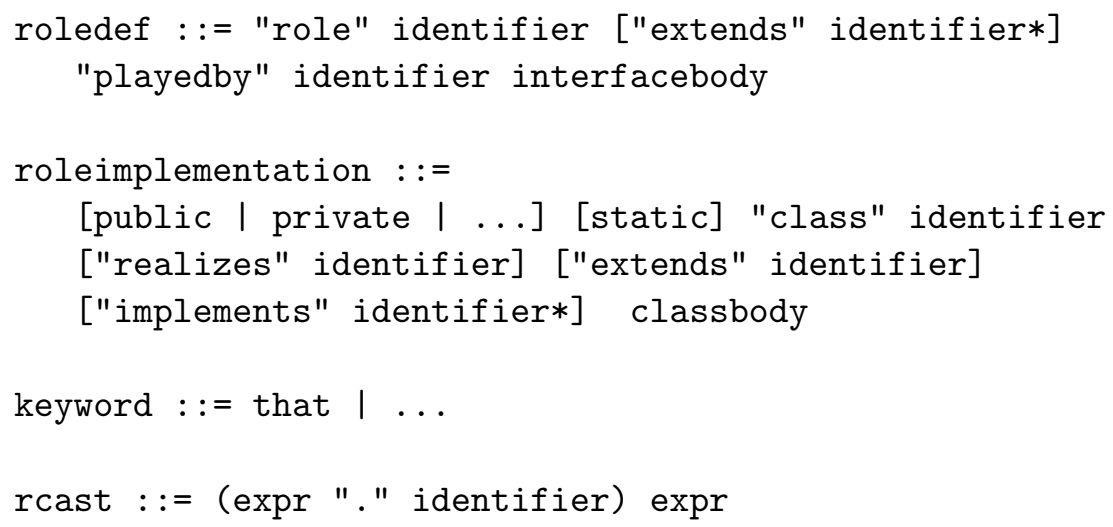

Fig. 3. The extension of Java syntax in powerJava.

et al. [13] that the concept of interface for an object is different from the corresponding notion for a component. An interface of an object involves only a one-way flow of dependencies from the object providing a service to its clients. In contrast, the interface of a component involves a two way reciprocal interaction between the component and its environment. Analogously, roles in our model allow the usability of components by specifying two interfaces: the interface required by a component to plug in the system in a role and the interface specifying which services it can provide to the system once it plays its role. The difference is that powers are implemented in the role and not in the component, since the role encapsulates how the component interacts with the rest of the system. In this way, the component can offer services integrated in the system while being developed independently without requiring any knowledge of it (see Figure 2).

The implementation of the requirements is given inside the class of the object playing the role. The implementation of the powers must be necessarily 


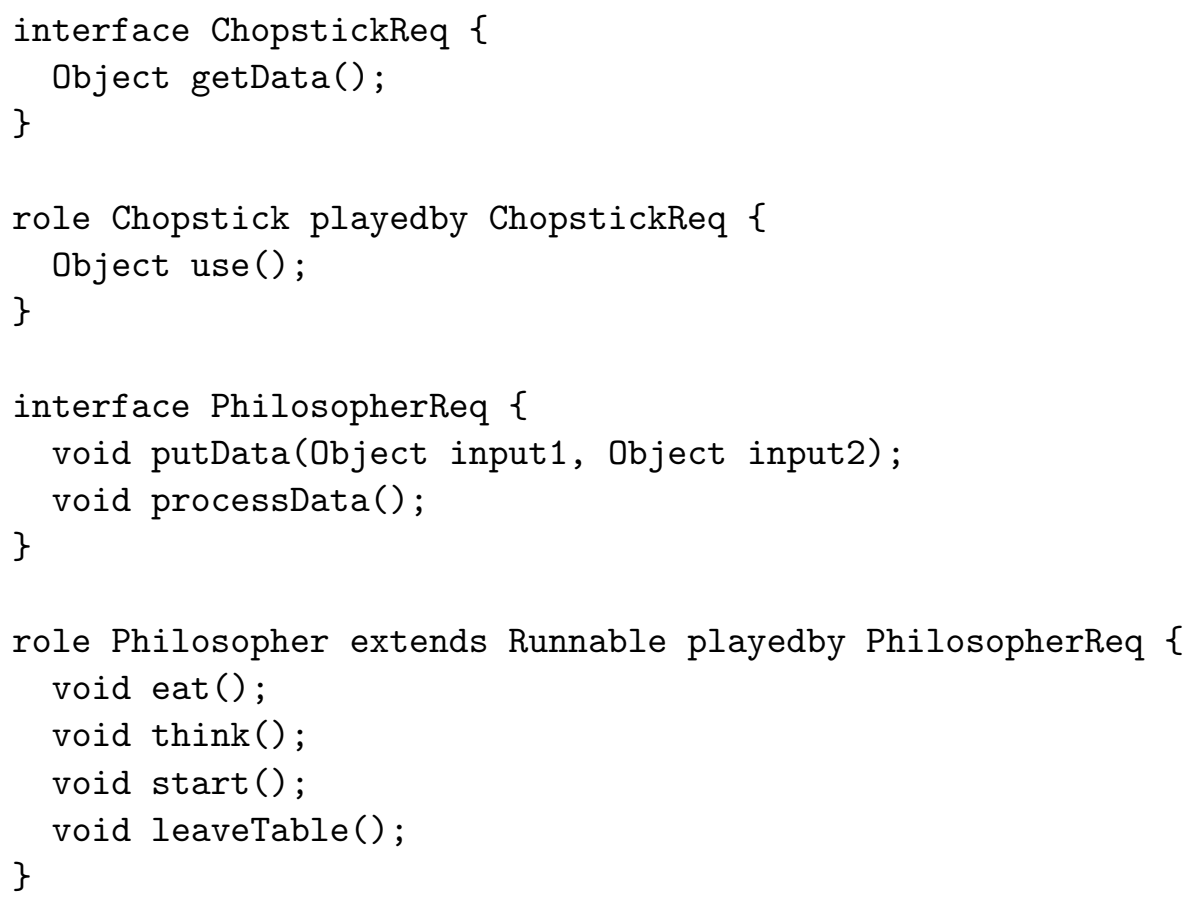

Fig. 4. Role definitions.

given in the definition of the class defining the institution of the role.

To implement roles inside an institution we extend the notion of Java inner class, by specifying with the new keyword realizes the name of the role definition that the class is implementing (see Figure 3). In powerJava, an inner class that realizes a role must implement the corresponding role definition in the very same way as a class implements an interface. For example, in Figure 5 and 6, the inner class PhilosopherImpl of the institution Table realizes the role Philosopher.

As a Java inner class, a role implementation has access to the private fields and methods of the outer class (in our case the institution) and of the other roles defined in the outer class; this possibility does not disrupt the encapsulation principle since all roles of an institution are defined by who defines the institution itself (see, e.g, the method eat in Figure 6). In other words, an object that has assumed a given role, by means of it, has access and can change the state of the corresponding institution and of the sibling roles. In this way, we realize the powers envisaged by our analysis of the notion of role. Moreover, since an inner class is a class, it can extend other classes (unless they implement roles), it can be an institution itself and thus, have, its own role implementations, etc.

The behavior of a role instance depends on the player instance of the role, so in the method implementation the player instance can be retrieved via a new reserved keyword: that, which is used only in the role implementation. See, for example, the implementation of method think in PhilosopherImpl. The value of that is initialized when the constructor of the role implementation is 


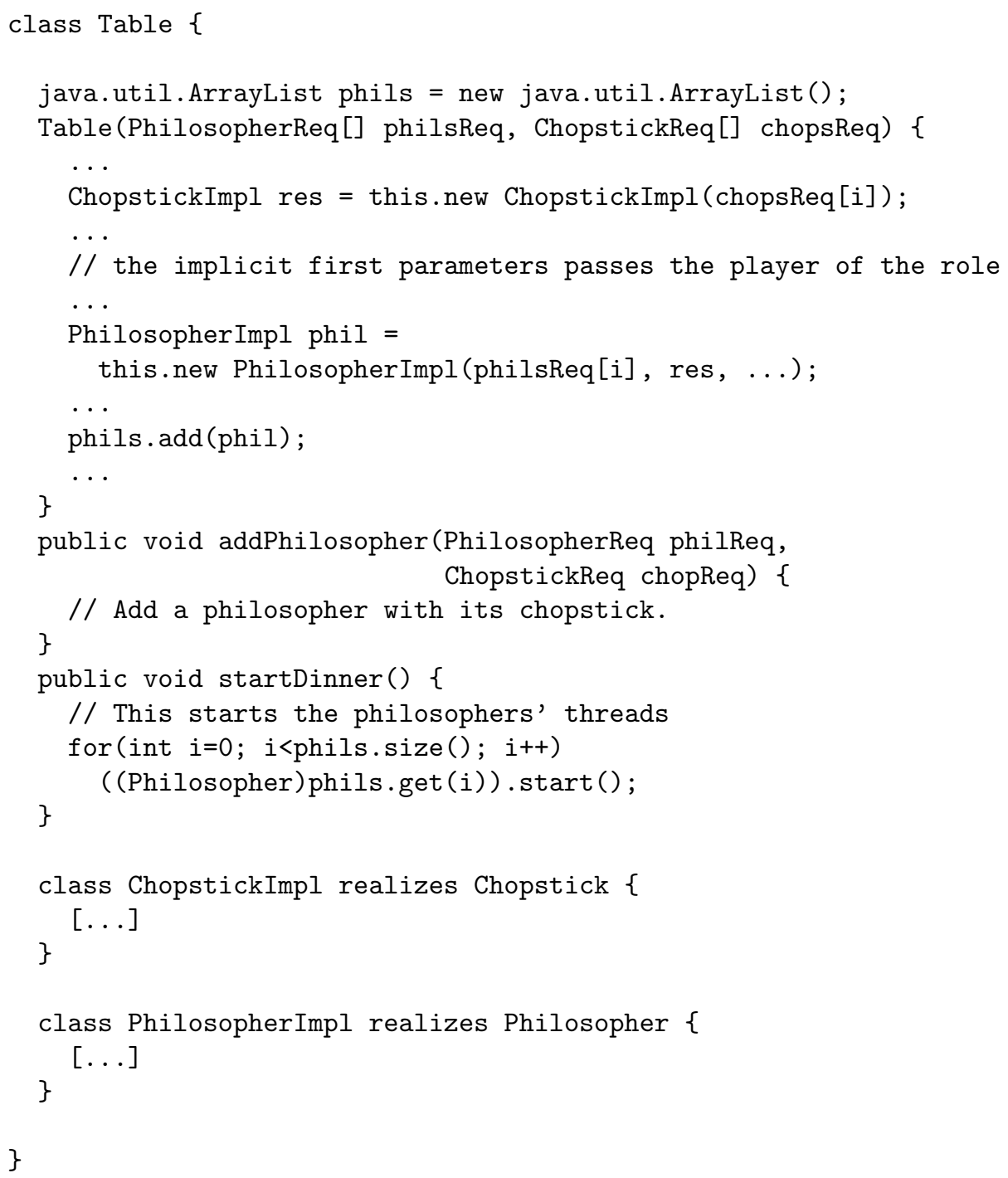

Fig. 5. The definition of an institution and its roles.

invoked. The referred object has the type defined by the role requirements.

All the constructors of all roles have an implicit first parameter which must be passed as value the player of the role. ${ }^{4}$ The reason is that to construct a role we need both the institution the role belongs to (the object the construct new is invoked on) and the player of the role (the first implicit parameter). For this reason, the parameter has as its type the requirements of the role: e.g., the constructor PhilosopherImpl has a first parameter of type PhilosopherReq. At the moment it is not possible to create an instance of a role without any player associated with but this could be object of further investigation.

A role instance is created by means of the construct new and by specifying the name of the inner class implementing the role which we want to instanti-

4 The parameter is added by the precompiler of powerJava. See the website http://www . powerjava.org for details. 


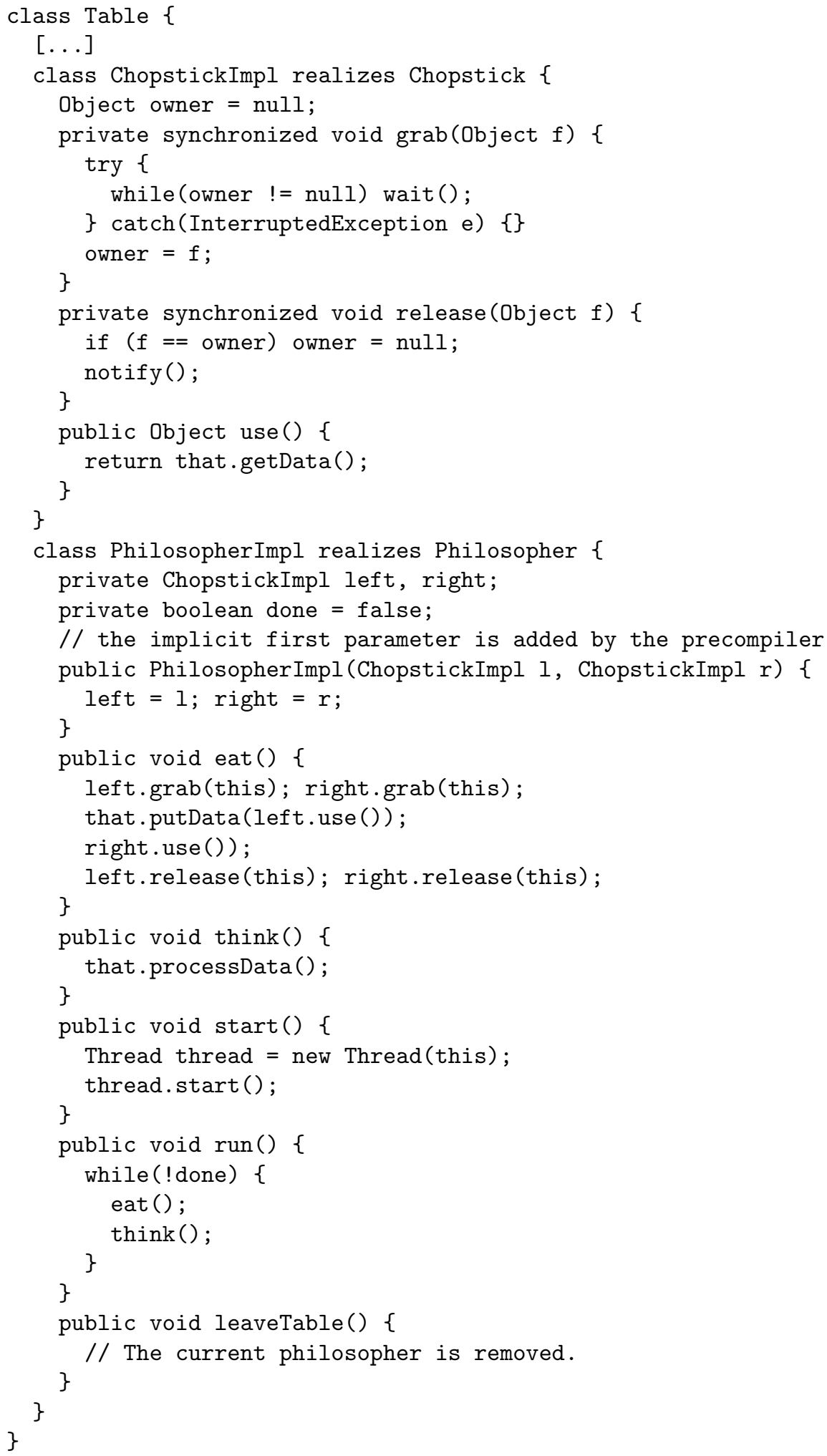

Fig. 6. The definition of an institution and its roles (continued). 


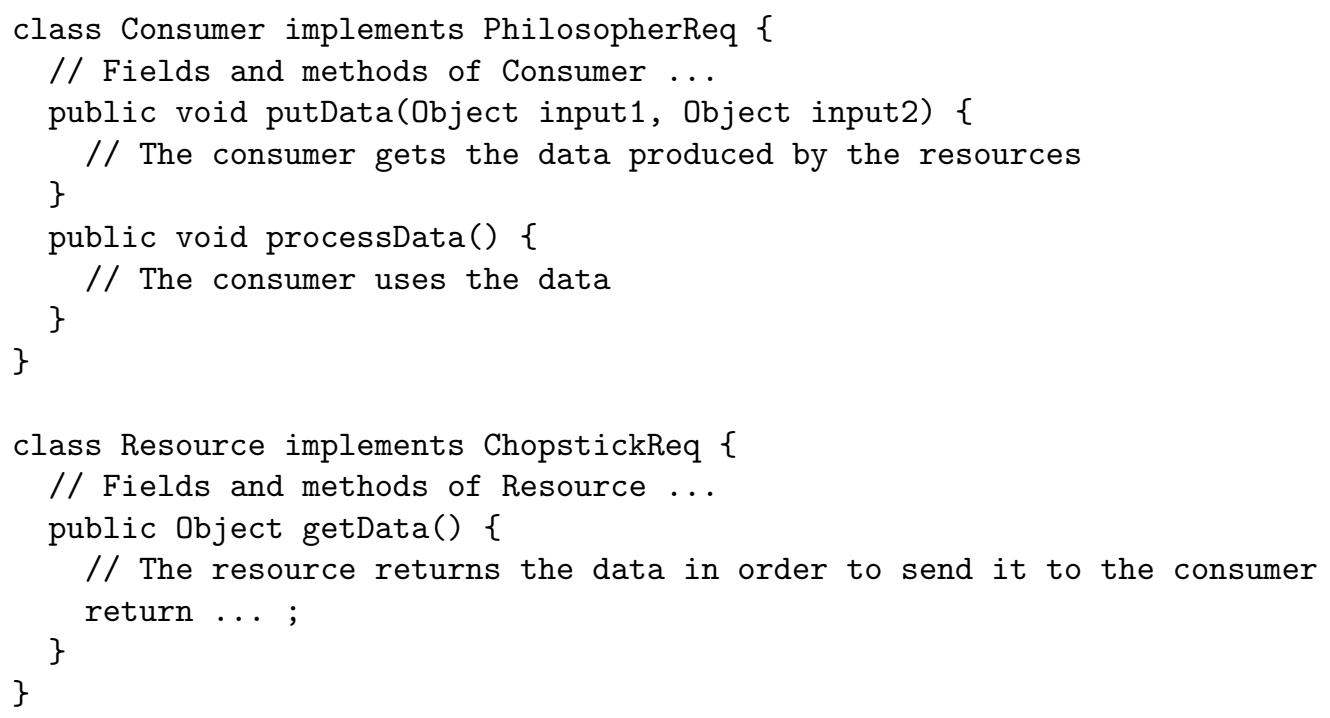

Fig. 7. Classes playing roles.

ate. This is like it is done in Java for inner class instance creation. Note that it is not possible to directly instantiate a role: first of all, roles are used like interfaces, secondly, roles can be implemented in different ways in the same institution. For example, the class Table could contain different implementations the role Philosopher. In Figure 5, a philosopher is created by this.new PhilosopherImpl (philsReq[i],...), where the parameter philsReq[i] is an object implementing the requirements PhilosopherReq.

In order for an object to play a role it is sufficient that it conforms to the role requirements. Since the role requirements are implemented as a Java interface, it is sufficient that the class of the object implements the methods of such an interface. In Figure 7, the class Consumer can play the role Philosopher, because it conforms to the interface PhilospherReq by implementing its methods.

Differently than other objects, role instances do not exist by themselves and are always associated to their players: when it is necessary to invoke a method of a philosopher it is sufficient to have a reference to its player object; it is not necessary to know which is the role instance played by the object. Methods can be invoked from the players, given that the player is seen in its role (e.g., PhilosopherImpl). To do this, we use the Java idea of casting with a difference: the object is not casted to a type. Casting is done in powerJava by casting the player of the role to the role implementation we want to refer to. It is not possible, however, to cast to a role (e.g., Philosopher since it could have been implemented by different role implementations in the same institution: hence, the casting would have been ambiguous.

However, since roles do not exist outside an instance of the institution defining them, in order to specify a role, it is necessary to specify the institution it belongs to. In the syntax of powerJava the structure of a role casting is captured by rcast in Figure 3. For instance, in Figure 8 


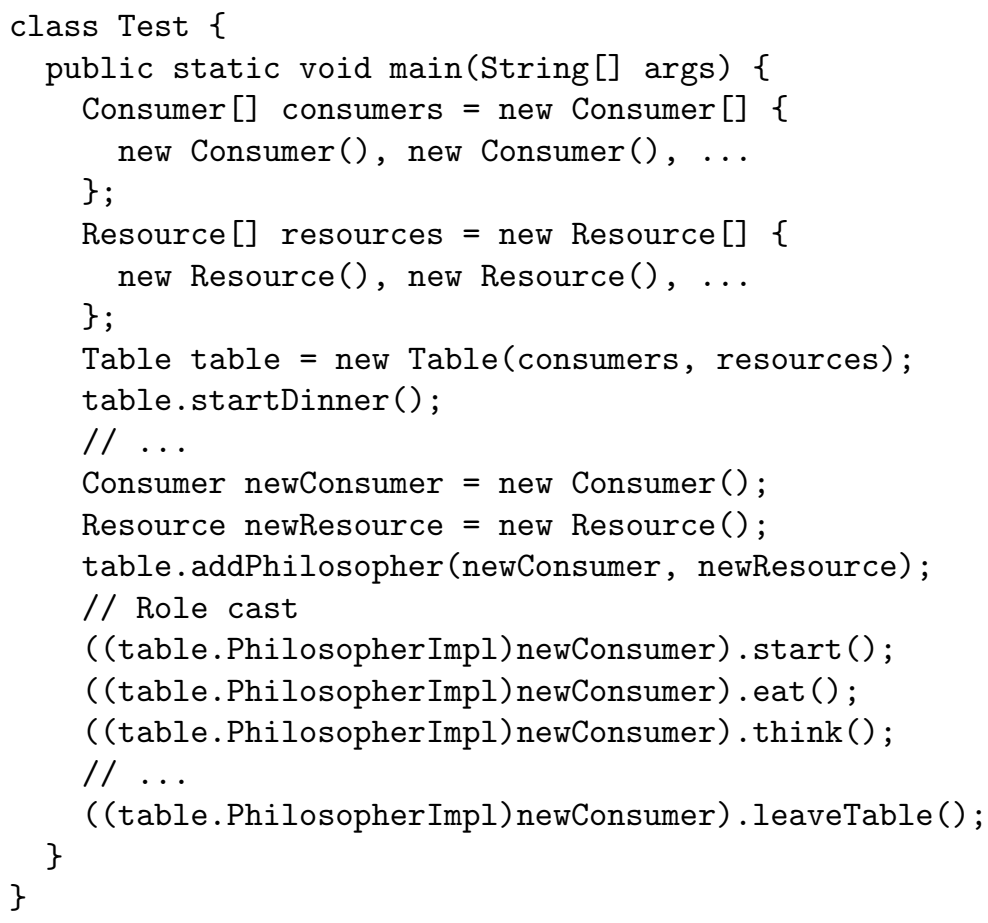

Fig. 8. How the main sets up the dinner.

\section{((table.PhilosopherImpl) newConsumer).eat()}

takes the Philosopher role played by the newConsumer in the institution table and invokes on it methods like eat which are powers of the role. In this way, the Consumer component is relieved from the burden of having an explicit reference to the role instance it is playing: the role is directly accessed via its player.

We call this role casting. Type casting in Java allows to see the same object under different perspectives while maintaining the same structure and state. In contrast, role casting views an object as having a different, even if related, state and different behaviors. This is because, it conceals a delegation mechanism: the player instance hiddenly delegates the role instance the execution of the method. The delegated object can only act as allowed by the powers of the role, but it can access the state of the institution via its powers.

Finally powerJava allows the definition of roles which can be further articulated into other roles. For example, a school can be articulated in teaching classes (another social entity) which are, in turn, articulated into student roles. In this way, it is possible to create a hierarchy of managers and workers as suggested by the IWIM model $[10,4,23]$ : at the bottom level are component workers which do no coordinate any other component, while at the upper levels there are components managing other components (either workers or other managers). 


\section{Example}

In order to illustrate how roles can be used for coordination purposes, we use, as well as Arbab did [3], the dining philosophers example, presenting it in powerJava; we do this by introducing roles in the implementation that is proposed in the Java tutorial [26]. To fully explain the potential of roles we extend the dining philosophers introducing some reconfiguration issues. In particular, we assume that new components playing the role of philosophers and new resources playing the role of chopsticks can join the table, as well as that philosophers can leave at any moment. The example is modelled by means of five kinds of objects:

(i) The dinner Table, which constitutes the environment where the components interact, and which coordinates them. The table is an institution which is organized in two types of roles: philosophers and chopsticks.

(ii) Philosophers offer to the consumers playing them four powers: the method for eating after grabbing the chopsticks (eat), the method for thinking (think), the method for starting the dinner intermixing thinking and eating (start), and the method for leaving the table (leaveTable).

(iii) Chopsticks offer the resources playing them a method for being used by other components (use) to get the data from the resource playing the role.

(iv) The Resources are components, whose interface only offers a method for getting data from them (getData).

(v) The Consumers are components, which must offer a way to pass the data to them (putData(Object input1, Object input2)) and to perform a computation on them (processData).

The Table is the coordination environment maintaining an ordered list of the philosophers sitting at it with their chopsticks and the implementation of the two roles. Its constructor takes two arrays, one containing objects that can play the philosopher role, the other containing objects that can play the chopstick role (in our example, a Consumer and a Resource which implement respectively the PhilosopherReq and ChopstickReq interfaces). Using these objects, the table creates the instances of the required roles, puts the philosophers around the table and connects them with their chopsticks (see Figure 9).

The implementations of Chopsticks and Philosophers encapsulate the fields relating them to each other (left and right) and the methods for accessing in a concurrent way to the shared resources: this information is hidden to the components playing them. However, their powers ensure that the components are made interact with each other in a way which prevents deadlocks and interferences.

In particular, the implementation ChopstickImpl of the role Chopstick implements not only the powers of that role, but also suitable private meth- 
ods for grabbing (grab) and releasing (release) chopsticks in a synchronized way. These methods block and reactivate processes accessing chopsticks which are already used (owner) by a philosopher or by the table. Note that these methods are private, but, since they are defined in an inner class, they are still visible to the other roles (indeed, they are used in the implementation of the powers of a Philosopher, see, e.g., the method eat).

The implementation PhilosopherImpl of the role Philosopher implements the powers eat, think, start, and leaveTable. The method eat allows a consumer, playing the role Philosopher, to participate to the dinner. The method first gets the chopsticks, it takes the expected data by using the chopsticks (left.use() and right.use()), and then invokes putData with the obtained information as parameters, thus passing the results to the consumer. Observe that the consumer will get an outcome without being aware of the information source (a sort of channel of information). Finally, it releases the chopsticks. Note that this method must be defined inside the role and not in its player since the player component is not required to know how to get, use, and release the chopstick; the component uses the data while the data source management is encapsulated in the role. In other words, the two classes representing the players of the roles need only implementing the methods specified by the interfaces as their requirements. These classes do not make any reference to each other, nor to the coordination structure of the environment they interact into: all these aspects are dealt with by the

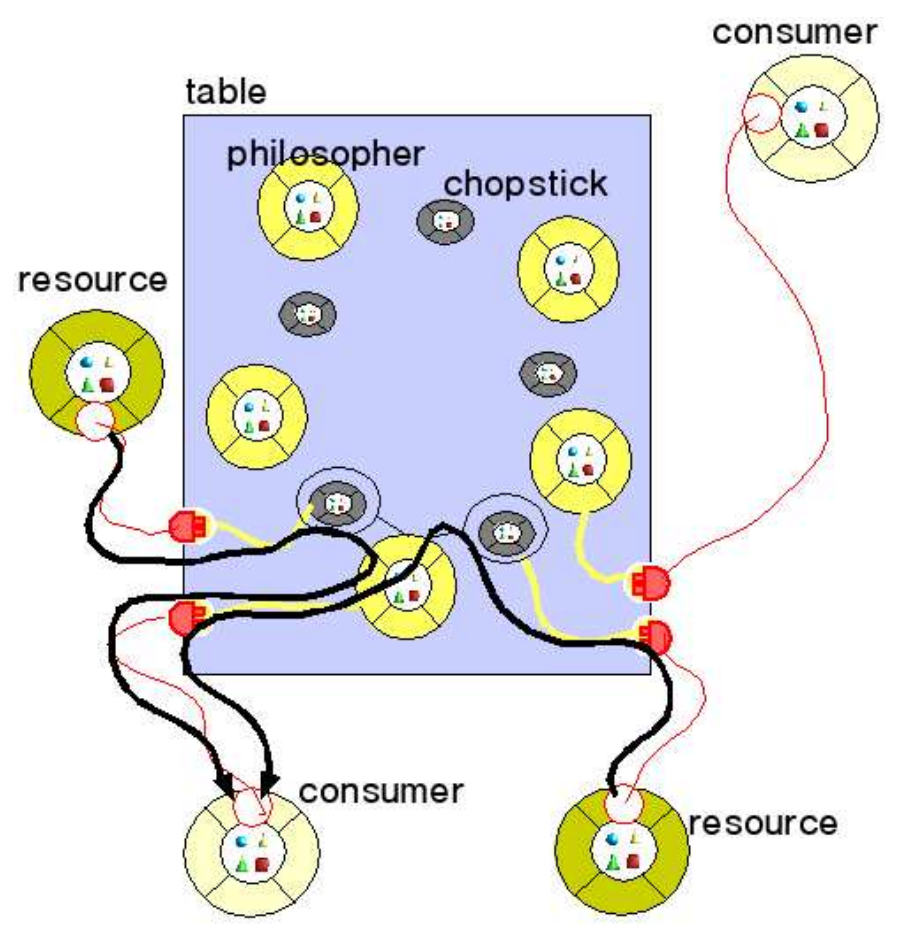

Fig. 9. The table is the coordination environment, coordination between resources and consumers is carried on through the roles. 


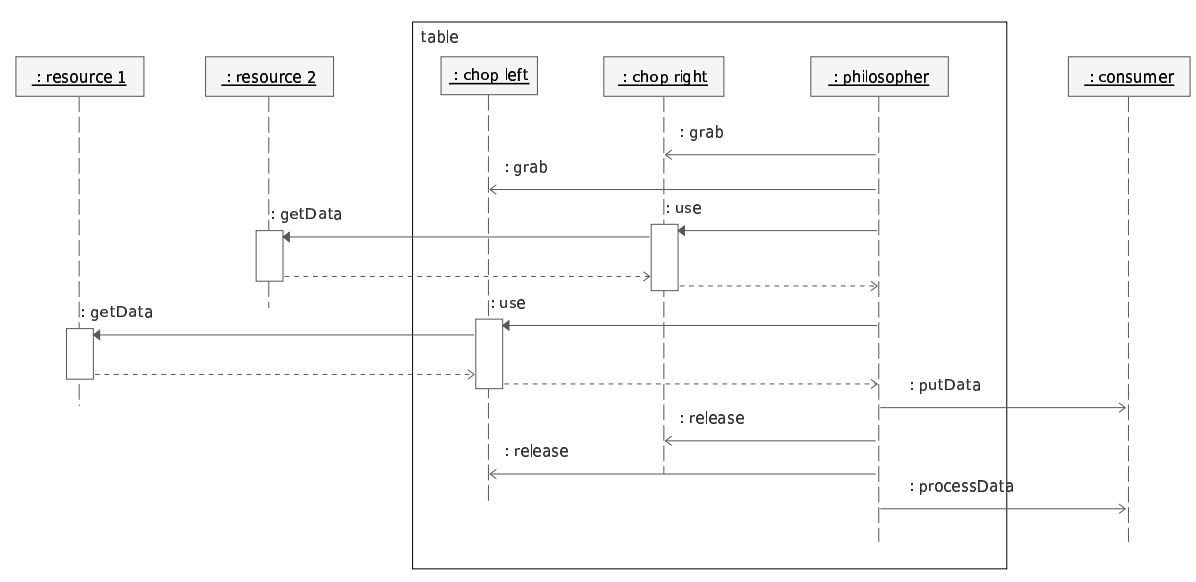

Fig. 10. Roles, institution and coordination.

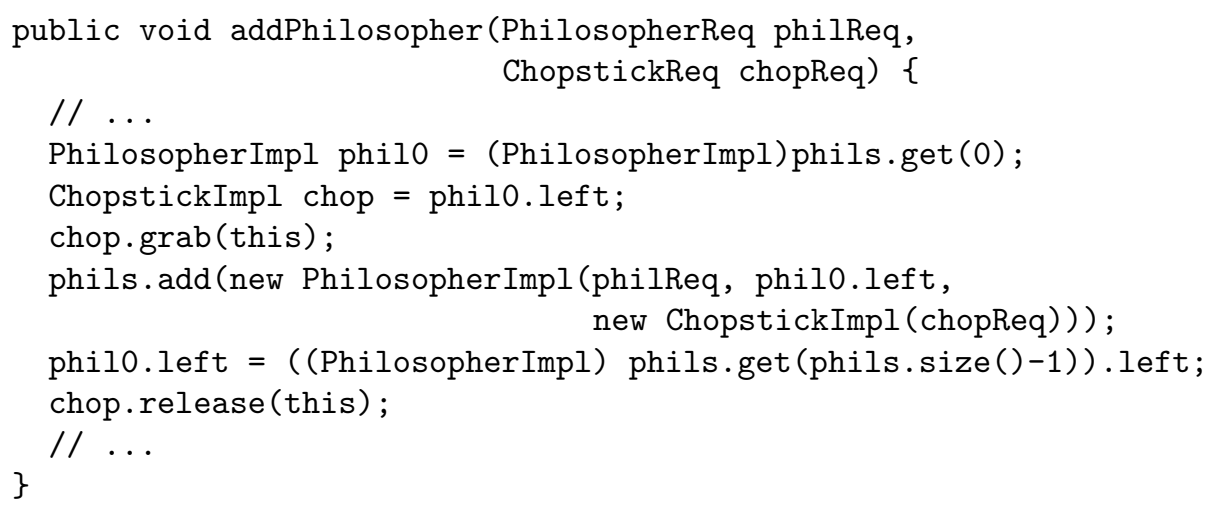

Fig. 11. Sketch of reconfiguring the dinner table by adding a philosopher.

roles they play thanks to the precompiling phase (see the underlying model in Figure 1). Figure 10 visualizes the sequence diagram of the example.

The class Resource implements the interface ChopstickReq, which contains the method for getting the data: getData. The class Consumer implements the interface PhilosopherReq, which allows it to play the role of Philosopher. It implements putData, to read data produced by the information sources and the method to process them (processData). We implemented here synchronous communication for simplicity, but nothing prevents implementing an asynchronous version, e.g., by adding a buffer to the role's state. A Consumer accesses its role of PhilosopherImpl by casting itself to that role in the table, e.g.: ((table.PhilosopherImpl)newConsumer).start() in Figure 8.

Our philosophers are able to reconfigure the system using the method addPhilosopher(PhilosopherReq philReq, ChopstickReq chopReq) of Table and the method leaveTable() of Philosopher. addPhilosopher has two objects as parameters, one able to play the Philosopher (Figure 11) role and the other one able to play the Chopstick role and adds them at the end of the table. To do so, essentially the table grab the chopstick of the first 
philosopher (not to use them, but to prevent others from using them), creates a new philosopher and a new chopstick role and adjusts the connections between the philosophers and their chopsticks. Finally it releases the reserved chopstick (which is now distributed among the remaining philosophers). In contrast with the former method, leaveTable (Figure 12) is the method directly invoked on a Consumer in its role of Philosopher (see main in Figure 8 ) in order to leave the table.

The main in Figure 8 simply constructs the required arrays of Consumers and Resources, invokes the constructor of the Table which creates the coordination environment, and starts the dinner of the philosophers. Finally, it adds a further philosopher invoking the method addPhilosopher on the table. The computation of the corresponding role played by the new consumer is started after the proper role cast, and, after a while, it leaves the table by invoking leaveTable on its role:

\section{((table.PhilosopherImpl)newConsumer). leaveTable()}

In summary, the interaction between the Consumer and Resource components is realized without requesting them to know each other to be able to invoke their methods, to know the configuration they are involved into, and the connections among the components (i.e., which the chopsticks of each philosopher are and how they are distributed around the table), and how to reconfigure the system. Roles are the connectors which relate the components to the system and among each other. The interconnections among the components can be changed by the institution or by the roles themselves, while the components remain unaware of the reconfiguration. In our model we can replace any component with another version of it without having to change any other component or the coordination scheme of the system. Finally, the coordination scheme of the system that is independent of the computation parts of components can also be updated without the necessity to change the components of the system.

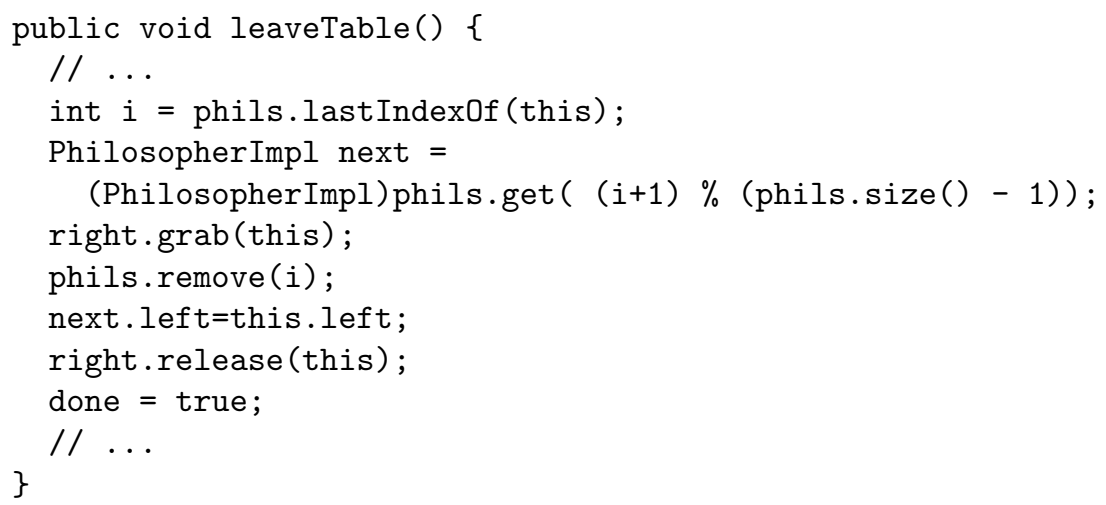

Fig. 12. Sketch of how a philosopher leaves the table. 


\section{Conclusion and related work}

In this paper we show how to introduce the role metaphor in Object Oriented languages and how to use it in control-driven coordination. Component objects interact with each other only via the roles they play in an institution which constitutes the interaction environment, thus achieving the separation of concerns, and exogenously coordinates the behavior of the system. We show how Java can be extended with roles, and how the resulting language powerJava can be used to model the dining philosophers problem enriched with reconfiguration issues. Many characteristics of this proposal of role definition have origin in the multi-agent systems research area. In [5] details about this relationship are discussed.

As Guillen-Scholten et al. [13] do for channels, we extend Java to show how it is possible to model components in a self-contained way in a widely used Object Oriented language. We implement a precompiler to pure Java for the language powerJava, using the tool javaCC, provided by Sun Microsystems [1]. The precompiler together with the complete example, more information on the language and on its translation to Java can be found at http://www . powerjava.org. Briefly, each role specification is translated into couple of interfaces, while role implementations are translated into inner classes, whose constructors are extended appropriately. Players are modified in order to manage a list of roles and role casting is translated into an instruction that allows finding the corresponding roles inside these lists (using the name of the role and the instance of the institution), then delegating this object for the execution of the power.

Roles definition has a strong relationship with the specification of a communication protocol $[17,15]$. Indeed, roles (as entities endowed with powers) are a means to coordinate the behavior within an organization. Playing a role means acquiring specific powers (given by the organization); the players interact according to the acquired powers. In other words, the players follow the protocol implemented by the institution and its roles in order to interact with each other. The institution itself is an abstraction of the protocol. Protocols as institutions can be collected together in order to constitute a library of protocols (coordination patterns). The designers must verify and prove properties of their coordination pattern just once [27], specifying which requirements should be satisfied in order to play the role involved in the protocol (to be "plugged" in the pattern, see Figure 2).

The notion of role used in powerJava has some similarities with the notion of agent coordination context developed by Omicini [21]. Agent coordination contexts are based on the control room metaphor. According to this metaphor, an agent entering a new environment is assigned its own control room, which is the only way in which it can perceive the environment, as well as the only way in which it can interact. In our terminology, an environment is an institution and a control room defines the powers of an agent working in an environment. 
Our approach shares the idea of gathering roles inside wider entities with languages like Object Teams [14] and Ceasar [19]. These languages emerge as refinements of aspect oriented languages aiming at resolving practical limitations of other languages. In contrast, our language starts from a conceptual modelling of roles and then it implements the model as language constructs. Differently than these languages we do not model aspects. The motivation is that we want to stick as much as possible to the Java language. However, aspects can be included in our conceptual model as well, under the idea that actions of an agent playing a role "count as" actions executed by the role itself. In the same way, the execution of methods of an object can give raise by advice weaving to the execution of a method of a role. On the other hand, these languages do not provide the notion of role casting we introduce in powerJava. By using role casting, it is possible to play a role at any point of the code and not only inside specific methods, as instead in [14]. Therefore, flexibility is increased.

Our notion of role, as a double-sided interface, bears some similarities with Traits [20] and Mixins. However, the latter are distinguished because roles are used to extend instances and not classes, with a few exceptions, e.g., [6]. Another difference of our approach, with respect to others that we have mentioned and, in particular, [14], stands in the use of interfaces. There is a wide agreement that variables should be declared with interfaces as their types, not classes. In fact, in this way a greater modularity is obtained. This is particularly important in the development of frameworks where classes must fit in at various points in the design and in component-based programming [25].

\section{References}

[1] Java compiler compiler [tm] (javaCC [tm]) - the java parser generator, Sun Microsystems, https://javacc.dev.java.net/.

[2] Albano, A., R. Bergamini, G. Ghelli and R. Orsini, An object data model with roles, in: Procs. of VLDB'93, 1993, pp. 39-51.

[3] Arbab, F., Abstract behavior types: A foundation model for components and their composition, in: Formal Methods for Components and Objects, LNCS 2852, Springer Verlag, Berlin, 2003 pp. 33-70.

[4] Arbab, F., The IWIM Model for Coordination of Concurrent Activities, in: Procs. of Coordination (1996), pp. 34-56.

[5] Baldoni, M., G. Boella and L. van der Torre, Bridging Agent Theory and Object Orientation: Importing Social Roles in Object Oriented Languages, in: R. H. Bordini, M. Dastani, J. DIx and A. Seghrouchni, editors, Proc. of the International Workshop on Programming Multi-Agent Systems, ProMAS 2005, Utrecht, the Netherlands, 2005, to appear. 
[6] Bettini, L., V. Bono and S. Likavec, A core calculus of mixin-based incomplete objects, in: Procs. of FOOL Workshop, 2002.

[7] Boella, G. and L. van der Torre, An agent oriented ontology of social reality, in: Procs. of FOIS'04, Torino, 2004, pp. 199-209.

[8] Boella, G. and L. van der Torre, Attributing mental attitudes to roles: The agent metaphor applied to organizational design, in: Procs. of ICEC'04 (2004), pp. 130-137.

[9] Boella, G. and L. van der Torre, Regulative and constitutive norms in normative multiagent systems, in: Procs. of KR'04 (2004), pp. 255-265.

[10] Ciancarini, P. and Hankin, C., editors, iCoordination Languages and Models, First International Conference, Coordination'96 (1996), Cesena, Italy.

[11] Gottlob, G., M. Schrefl and B. Rock, Extending object-oriented systems with roles, ACM Transactions on Information Systems 14(3) (1996), pp. 268 - 296.

[12] Guarino, N. and C. Welty, Evaluating ontological decisions with ontoclean, Communications of ACM 45(2) (2002), pp. 61-65.

[13] Guillen-Scholten, J., F. Arbab, F. de Boer and M. Bonsangue, A channel based coordination model for components, ENTCS 68(3) (2003).

[14] Herrmann, S., Object teams: Improving modularity for crosscutting collaborations, in: Procs. of Net.ObjectDays, 2002.

[15] Huget, M. P. and J. Koning, Interaction Protocol Engineering, in: H. Huget, editor, Communication in Multiagent Systems, LNAI 2650 (2003), pp. 179193.

[16] Malone, T. and K. Crowston, The interdisciplinary study of coordination, ACM Computing Surveys 26 (1994), pp. 87-119.

[17] Mamdani, A. and J. Pitt, Communication protocols in multi-agent systems: A development method and reference architecture, in: Issues in Agent Communication, LNCS 1916 (2000), pp. 160-177.

[18] Masolo, C., L. Vieu, E. Bottazzi, C. Catenacci, R. Ferrario, A. Gangemi and N. Guarino, Social roles and their descriptions, in: Procs. of KR'04, 2004.

[19] Mezini, M. and K.Ostermann, Conquering aspects with caesar, in: Procs. of the 2nd International Conference on Aspect-Oriented Software Development (AOSD) (2004), pp. 90-100.

[20] N. Scharli, O. N., S. Ducasse and A. Black, Traits: Composable units of behavior, in: S. Verlag, editor, LNCS, vol. 2743: Procs. of ECOOP'03, Berlin, 2003, pp. $248-274$.

[21] Omicini, A., Towards a notion of agent coordination context, in: D. C. Marinescu and C. Lee, editors, Process Coordination and Ubiquitous Computing, CRC Press, 2002 pp. 187-200. 
[22] Ouchi, W., A conceptual framework for the design of organizational control mechanisms, Management Science 25(9) (1979), pp. 833-848.

[23] Papadopoulos, G. and F. Arbab, Coordination models and languages, Advances in Computers 46 (1998), pp. 329-400.

[24] Steimann, F., A radical revision of UML's role concept, in: Procs. of UML2000, 2000, pp. 194-209.

[25] Steimann, F., W. Siberski and T. Kühne, Towards the Systematic Use of Interface in Java Programming, in: Proc. of 2nd Int. Conference in Java Programming, 2003, pp. 13-17.

[26] Sun Microsystems, "The Java Tutorial," Http://java.sun.com/docs/books/tutorial/.

[27] Walton, C., Model checking agent dialogues, in: Int. Workshop on Declarative Agent Languages and Technology, 2004, pp. 156-171. 


\title{
A Plan Based Agent Architecture for Interpreting Natural Language Dialogue
}

\author{
Liliana Ardissono, Guido Boella and Leonardo Lesmo
}

Dipartimento di Informatica, Università di Torino, Corso Svizzera n.185, 10149 Torino, Italy.

email: \{liliana,guido,lesmo\}@di.unito.it

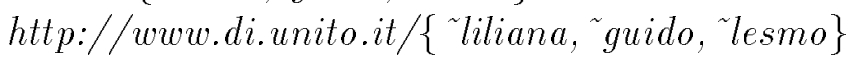

This work is going to appear in the

\author{
International Journal of Human-Computer Studies, ACADEMIC PRESS \\ (http://www.academicpress.com/www/journal/hc.htm)
}

\begin{abstract}
This paper describes a plan-based agent architecture for modeling NL cooperative dialogue; in particular, the paper focuses on the interpretation dialogue and explanation of its coherence by means of the recognition of the speakers' underlying intentions. The approach we propose makes it possible to analyze and explain in a uniform way several apparently unrelated linguistic phenomena, which have been often studied separately and treated via ad-hoc methods in the models of dialogue presented in the literature. Our model of linguistic interaction is based on the idea that dialogue can be seen as any other interaction among agents: therefore, domain-level and linguistic actions are treated in a similar way.

Our agent architecture is based on a two-level representation of the knowledge about acting: at the metalevel, the Agent Modeling plans describe the recipes for plan formation and execution (they are a declarative representation of a reactive planner); at the object level, the domain and communicative actions are defined. The Agent Modeling plans are used to identify the goals underlying the actions performed by an observed agent; the recognized plans constitute the dialogue context, where the intentions of all participants are stored in a structured way, in order to be used in the interpretation of the subsequent dialogue turns.
\end{abstract}

Keywords: Dialogue Processing, Plan and Goal Recognition, Agent Modeling, Natural Language Interpretation.

\section{Introduction}

This paper presents a computational model of dialogue that conceives dialogue as an interaction among autonomous agents. Each agent has his own beliefs, goals, and intentions and collaborates with his interlocutors: the cooperation implies that, at each step of the interaction, the agents share some goals and act to reach them; moreover, the presence 
of such shared goals is what supports the coherence of dialogue. During the dialogue, each agent must be able to recognize the explicit goals set forth by his partners, and to discover some of their implicit intentions. Then, the agent can balance what he has understood about the other agents against his own goals, in order to choose his next step in the interaction.

The computational framework we adopt is based on plans and models the activity of an agent as plan formation and execution; in order to behave properly, the agent needs knowledge about the actions that can be brought about in the domain of interest and knowledge about the linguistic actions that can be executed to interact with other agents. Moreover, he needs knowledge about the way a plan can be built, out of the available actions, and executed to reach a given goal. Although two substantially different types of knowledge are involved (knowledge about domain/linguistic actions and knowledge about planning), we represent them uniformly: in both cases, plan recipes express the relevant information.

The choice of modeling agent behavior in a plan-based, declarative way is due to the basic assumption that the knowledge structures underlying linguistic behavior are common to the two phases of language understanding and language production. So, an agent must resort to the same piece of knowledge both to understand, for instance, a polite request ("Saresti cosi gentile da aprire la finestra?", i.e. "Would you be so kind as to open the window?") ${ }^{1}$ and to produce the same request in appropriate circumstances. Note that also the plan formation activity must be properly recognized by a hearer, as is shown by the classical case of applicability condition check ("Is the library open?", Allen (1983)). Therefore, also knowledge about how to build plans is necessary for both the phases of understanding and producing behavior.

A consequence of the choice of assimilating dialogue as general cooperative interaction is that we model dialogue avoiding specific knowledge about the admissible sequences of turns. This is accomplished by treating the turns as instances of the possible continuations of an interaction: a turn is coherent with respect to the previous part of the dialogue if it can be explained as the means for achieving one of the goals that have been set forth (implicitly or explicitly) in the dialogue and which are still pending.

The solution given in most dialogue systems is to have the list of possible turn sequences (e.g. question-answer and request-refusal) by means, for example, of a dialogue grammar. However, the specifications of all the possible continuations of a dialogue are

\footnotetext{
${ }^{1}$ Our prototype has been developed for the Italian language: it is based on the GULL syntactic and semantic interpreter (Lesmo \& Torasso 1985, Lesmo \& Terenziani 1988, Di Eugenio \& Lesmo 1987, Ardissono et al. 1991), that takes as input Italian NL sentences. However, this paper focuses on the analysis of dialogue behavior, which is largely independent of the syntactic and semantic properties of a given language; therefore, from now on, all the examples will be reported in English for the reader's convenience.
} 
open-ended, too rigid, and lack a common justification. An approach that allows an explanation for an answer to a question, or a refusal to a request, on the basis of deeper unifying principles of rational behavior, as the ones embodied in our model, overcomes all these problems and, again, assimilates linguistic behavior as a general agent activity.

We will show how our goal-based model of dialogue can explain adjacency pairs, insertion sequences, presequences, grounding processes and various other phenomena, like acknowledgements, overanswering, and subdialogues due to problems in the interpretation process; this model also sheds light on the phenomenon of repairing to misunderstandings.

Our architecture includes two levels of plans: a metalevel, describing recipes for plan formation and execution, and an object level, where domain and linguistic actions are defined. All the actions contribute to changing the state of the world: the domainlevel actions are used to modify the surrounding physical situation, the linguistic actions are used to modify the beliefs of the partners, while plan-management recipes involve a modification of the agents' own intentions about what to do next. So, in modeling dialogue, turns can be interpreted as actions having the same status as the domain actions performed to achieve specific world states. We call the metalevel "Agent Modeling" (AM) level, and the recipes therein AM plans; the Agent Modeling plans have the object-level actions as their arguments.

In a sense, the Agent Modeling plans are the planner of our system: they embody the strategies that lead an agent to adopt a particular line of behavior, i.e. to choose a given sequence of (domain and/or linguistic) actions to reach his goal(s). Following the ideas developed in the research about agents after the first definitions of intention (Bratman et al. 1988), the Agent Modeling level describes behavior in terms of a "reactive planner" (like those in Georgeff \& Ingrand (1989) and Firby (1987)): in order to satisfy a goal, the agent chooses a more or less complex action, checks its preconditions, executes a suitable recipe down to the elementary actions; it also monitors whether the action succeeds and it replans any action whose execution fails. ${ }^{2}$

Since the agent is embedded into a community of agents, he can ask for cooperation, as well as offer his own collaboration to his partners: therefore, we also model the collaborative behavior described in Cohen \& Levesque (1991), as notifying the partners about how the execution of an action proceeds.

The main difference with respect to other planners is that we chose to describe the planner in a plan-based declarative language. The plan-based architecture allows us to apply the plan recognition algorithms developed so far (Carberry 1990b, Lambert 1993) in order to recognize and trace both the object-level plans carried on by the agent, and

\footnotetext{
${ }^{2}$ The term "reactive planner" is used here to denote reactive deliberative systems, also defined as "practical reasoning systems" (Jennings et al. 1998); on the contrary, we are not referring to the stimulusdriven reactive agents like the "Subsumption architecture" described in Brooks (1991).
} 
his planning behavior; this approach enables us to identify the planning phase, the monitoring activity, etc. performed by the agent through the observation of the object-level (domain and linguistic) actions he is doing. The Agent Modeling plans are the glue that keeps together the various object-level actions by specifying how the turns of the dialogue contribute to the higher goals shared by the agents.

During the interpretation process, the recognized instances of plans are recorded to contribute to the formation of the agent's internal representation of the ongoing dialogue. The agent also maintains the instances of his own Agent Modeling plans (i.e. the AM actions used to plan his behavior), as well as the object-level actions considered in these plans. In this way, the plan-based representation allows the agent to maintain in the context the trace of his own intentional activity, which can be exploited for understanding if and how his partners contribute to his own goals.

The declarative representation of the planning knowledge enables the system to model in the same way the two interacting agents. This is in contrast with the traditional view, where it is assumed that the user and the system play asymmetrical roles in dialogue. In particular, in such a view the observed agent (the system's "user") is supposed to devise and execute his own plans to reach his own goals. So, the interaction can be continued coherently, on the basis of the recognition of the user's plans. This view seems unbalanced: it can be applied correctly just in case there is a fixed role for each participant: the user asks and the system helps. Although this assumption is largely correct, it poses some limits to the generality of the model: in an interaction of this fixed type, different models have to be defined for the two roles. In our approach, where a uniform "agent" model is defined, the "function" of the turns of both speakers can be specified in the same way: they can be a continuation of a previous activity, or they can be linguistic acts, performed to satisfy an overt or implicit desire of the partner, and this is usually, but not necessarily, the system's role.

To summarize, the idea is to treat linguistic and domain-level actions uniformly, and to feed them to a reactive (collaborative) planner represented declaratively, in order to get a more finely-grained dialogue model. Moreover, if we enable an agent to recognize his partner's behavior in terms of his planning activity, we achieve a better understanding of his dialogue contributions, where his domain and linguistic actions are explained uniformly in terms of the same goal-directed behavior.

The paper is organized as follows: section 2 discusses how the work done in other research areas supports the idea that dialogue phenomena depend upon the speakers' intentions; then, in section 3 we sketch informally the main ideas underlying our work and in section 4 we present a variety of phenomena which can be explained by our solution. Sections 5 and 6 present the plan libraries used by the system and the interpretation 
process, respectively. Finally, a related work section and the conclusions close the paper.

\section{The intention-based approach to dialogue modeling}

It is widely accepted that dialogue depends on the intentions of the agents engaging in an interaction. This idea has been developed in Linguistics, Philosophy and Artificial Intelligence.

Among the first supporters of this conception of dialogue, Levinson (1981) highlights that the relevant replies to a turn depend on the background activity of the interactants. The main argument he advances is that after a question we can find not only answers, but also some other questions about the relevance of the original question, the negation of its presuppositions, or a proposal for solving the higher-level goals of the speaker who asked the question. Moreover, he notices the problem of replies concerning the perlocutionary effects of a speech act, which are, by definition, loosely related to the speaker's linguistic activity.

Also Searle (1992) agrees with this conception and advances his own explanation: he suggests that the difficulty in constraining the space of the possible replies to a speech act is due to the fact that the relevance of a contribution cannot depend only on the previous speech acts: in fact, illocutionary acts are defined in terms of goals (e.g. a request is an attempt to make the hearer perform an action) and there is no intrinsic goal in conversation.

In the AI community, dialogue has been modeled in some works by considering only the relation among speech acts and relying on the observable regularities in human dialogue: for instance, see the finite-state grammar model of Winograd \& Flores (1986). However, the role of intentions and planning in dialogue has been recognized by several researchers, such as Allen \& Perrault (1980) and Hobbs \& Evans (1980). Also Cohen (1994) challenges the idea that dialogue is a rule-based behavior: he claims that "no definitions are given for the transition types, no reasons are provided for the observed transitions between states and no explanation is given of why or how a particular subgraph constitutes a dialogue unit" (Smith \& Cohen (1996), page 29). In Smith \& Cohen (1996) the semantics of speech acts is specified in terms of the activity of forming and maintaining teams aimed at achieving a speaker's goals. In this view, a sequence of speech acts is admissible when the speakers' contributions aim at satisfying some goal prescribed by the team activity established previously. In turn, team activity is defined in terms of mutual beliefs about goals, and weak mutual goals (Cohen \& Levesque 1991): an agent involved in the collaboration should inform the partner that the goal has been achieved, is unachievable, or irrelevant, as soon as he becomes aware of this fact. Given this definition of joint intention, Smith \& Cohen (1996) model speech acts as "attempts" to form a team 
for pursuing a goal with the minimal acceptable result of making mutually believed the intention to form the team. ${ }^{3}$

Also Grosz \& Sidner (1990) support the role of intentions in dialogue modeling: in particular, in a development of their work, Lochbaum proposes a dialogue conception where the agent's intentions guide his behavior and the "partner recognition of those intentions aids in the latter's understanding of utterances" (Lochbaum (1994), page 1). In fact, an agent engages in dialogues and subdialogues for reasons deriving from the mental state requirements of action and collaboration. Lochbaum (1994) and Lochbaum (1995) provide a computational model of dialogue based on the Shared Plans operator defined in (Grosz \& Sidner 1990, Grosz \& Kraus 1996); Lochbaum's model aims at recognizing the intentional structure of dialogue and at using it in discourse processing.

Moreover, the notion of intention has been exploited to model human-computer dialogue in Bretier \& Sadek (1997) and Goerz \& Ludwig (1998): both works describe (belief, desire, intention) agent architectures for modeling dialogue as rational agent interaction.

The intention-based nature of dialogue is indirectly supported also by some of the "grammar-based" approaches to dialogue processing: for example, Stein \& Maier (1994) explain the behavior of their finite state dialogue automata in terms of agents' expectations or goal satisfaction. Moreover, they superimpose on the automaton a formalism based on the Rhetorical Structure Theory (Mann \& Thompson 1987) to explain the relations between speech acts; however, in Moore \& Paris (1993), the rhetorical relations have been explained in terms of a speaker's goals directed towards the receiver's understanding. Note also that Smith \& Cohen (1996) aim at proving that the structure of such dialogue grammars follows from the logical relationships arising from the definition of communicative actions in terms of team formation.

Other intention-based approaches to dialogue modeling have discussed and analyzed the importance of considering also the agents' metalevel activity for modeling their interaction with other agents (Litman \& Allen 1987, Ramshaw 1991, Carberry et al. 1992): this metalevel activity is important to understand behaviors like plan exploration.

\section{An architecture based on plans}

In our architecture, the actions which an agent can execute are of three types and, correspondingly, are defined as belonging to three different sets (libraries): the Agent Modeling (AM) library, the Speech Act (SA) library, and the Domain library (D).

While the AM library has a truly distinguished role (as outlined in Section 1, it is a "metalevel"), the SA library and the Domain library are kept apart just because the former is

\footnotetext{
${ }^{3}$ This is a minimal success condition because believing that the speaker wants the hearer to act is only a motivation for the hearer to do it.
} 
domain-independent, while the contents of the latter depend on the application domain.

Currently, the system does not include a generation module, so it is clearer to consider the implemented prototype as an external observer: it takes as input from the keyboard the utterances of both speakers and produces the representation of a dialogue context containing the interpretations of turns, together with the coherence relations among them. ${ }^{4}$ In the following description, we assume that the input sentences are produced either by the user $(U)$ or by a consultant $(C)$, while the system observes the exchange.

When a sentence is given as input, the system realizes that a speech act has been done. The Agent Modeling library specifies that an action is usually carried out as a step of a more general plan; therefore, a more general linguistic action, having the observed speech act as a step, is looked for. This process is iterated upwards, under the control of the AM plans; at a certain point, by analyzing the metalevel knowledge, the system realizes that a top-level speech act (e.g. a request) can be related to a domain-level action: the upward search is switched from the Speech Act library to the Domain library, but nothing else changes. Again, the AM library is inspected to determine the activities concerning plan formation and execution: so, it is possible to decide whether the recognized speech act is related to plan formation (e.g. the possible ways to carry out an action are being inspected) or plan execution (e.g. a request to register for an exam has been put forward).

When the subsequent dialogue turns are received, they are interpreted in exactly the same way. During this analysis, instances of actions in the plan libraries, including AM actions, speech acts, and domain-level actions, are recognized and maintained in a separate structure, to record a complete picture of the observed linguistic event. This structure, an extension of the Context Models described in Carberry (1990b), is the detailed representation of the speakers' activity, as recognized by the system: it specifies what they are doing and why, according to the system's comprehension.

Figure 1 sketches the representation of a turn in the pictorial form that will be used throughout the paper: in the figure, the box labeled with AM1 represents the structure containing the metalevel actions recognized while interpreting the turn; the boxes labeled as D1 and SA1 represent the structures containing the recognized Domain-level actions and speech acts. The action instances occurring in the interpretation structures are usually represented by specifying their names, the values to which their arguments are bound (such values may be actions in the case of AM actions) and possibly their preconditions (see the arcs labeled with "prec"), constraints ("constr") and effects ("eff"). The dotted arrows relating AM actions to object-level actions (i.e. domain-level actions and speech acts) represent the links between a metalevel action instance and its

\footnotetext{
${ }^{4}$ This approach has clear limitations: for example, it imposes the underlying assumption that no misunderstandings occur among the speakers. Nevertheless, this is a temporary, simplifying solution, to be maintained until the generation phase will be implemented in our system.
} 


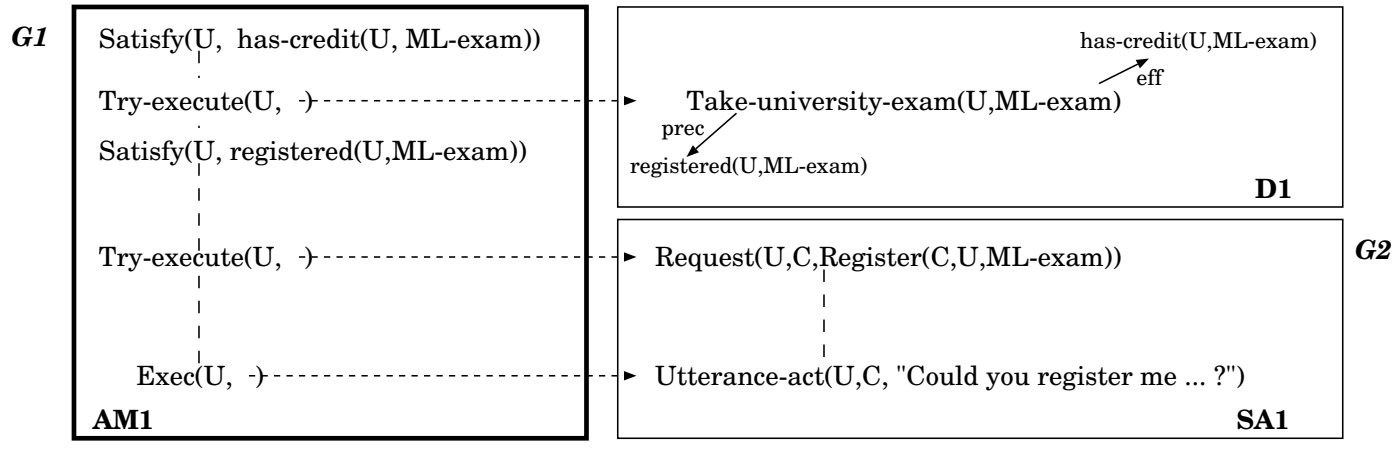

FigURE 1. Sketch of an turn representation structure.

arguments: for instance, the bottom "Exec" action in AM1 has "U" as its first argument and "Utterance-act(U, C, "Could you register me ...?")" as its second argument. Instead, the dashed lines between actions contained in the same box relate complex action instances to their substeps, omitting intermediate steps, which are not shown because only the relevant portion of the interpretation structures is provided.

During the interpretation of a dialogue, the system maintains two models containing the interpretation of the turns by the interactants: the user's (agent) model (UAM) and the consultant's one $(C A M)$. When a turn is interpreted, the system expands the speaker's model with the turn representation. Then, in order to represent explicitly the coherence of the dialogue, the system tries to connect the turn to some contextual goal; this operation succeeds only if the main goal $G 1$ (see Figure 1) which explains the reason why the turn has been produced also appears in the $U A M$ or in the $C A M$. For example, given a turn of the consultant $C$, there are two possibilities:

- If $G$ appears in the $U A M$, then he has decided to cooperate with $U$ and has produced a turn to satisfy $G$.

- Otherwise, $G$ is contained in the $C A M$, so $C$ is continuing some of his own activities, possibly because there are no other goals belonging to $U$ to be satisfied.

In the second case, the plan structures contained in the $C A M$ and representing $C$ 's activity are extended with the model of the last turn; this integration is the classical "focusing" step introduced in Carberry (1990b). The search for this goal $G$ is carried out by the system; if it succeeds, a link is established between the turn representation and the $U A M$ or the $C A M$; this link relates the two occurrences of the top-level goal $G$.

Note that $G$ does not necessarily need to be satisfied by $C: G$ is the top goal of the turn representation because $C$ 's reply has been produced while attempting to satisfy $G$; the only requirement is that $C$ 's reply is "in some way" connected (i.e. contributes) to $G$, where the possible ways are defined by the system's knowledge about plans, as encoded in the Agent Modeling library. For example, after a request by a speaker $U$, a clarification 
requested by $C$ to $U$ doesn't satisfy $U$ 's goal, but it is a coherent reply, because it can be explained as an attempt to satisfy one of the preconditions required to fulfill $U$ 's desires, perhaps a precondition on $C$ side.

This sequence of steps can be exemplified by the following exchange. Figure 2 shows very sketchily the evolution of the Agent Modeling models, in order to point out the interactions between them. We report the complete representation of this dialogue model in Section 6. In the following paragraphs, we provide the details required to understand the role of the different substructures and how are they actually built and linked together.

\section{Example 3.1:}

$\mathrm{U}-1$ : Could you register me for the ML exam?

$\mathrm{C}-1$ : What is your name?

U-2: Mario Rossi.

C-2: Ok; you are registered!

U-3: Can you tell me the exact date of the exam?

The analysis of the user's first turn (U-1) leads the system to initialize a UAM including a speech act model ( $S A 1)$. The initialization is driven by the interpretation process under the control of the Agent Modeling plans; they enable the system to determine that an indirect speech act was performed with the goal of expressing a request. This request concerns the domain action "register for an exam" (the goal that the partner registers $U$ is $G 2$ in Figures 1 and 2), that probably makes true a precondition of the action "take an exam" (contained in the structure D1, with the goal "having a credit for the exam", G1): the AM plans specify that a first step in executing an action is to check its preconditions and, if needed, to make them true. ${ }^{5}$

In Step 2, the system observes C-1 and initializes the consultant's Agent Model (CAM). The turn is interpreted as a question that aims at satisfying the precondition "know name" of the requested action "register for an exam". So, the action "Register" is replicated in the $C A M$ ( $D 2$ with effect $G 2^{\prime}$ in Figure 2), with a suitable link connecting $G 2$ and $G 2^{\prime}$ $(L 1)$. This turn initiates a subdialogue and advances the new subgoal "know name", denoted as $G 3$ in the $C A M$.

In Step 3, U-2 is observed. Here, there are two alternatives: either the user $U$ is continuing his activity, or he is trying to satisfy one of the consultant's goals. Here, $U$ is contributing to $G 3$, because providing the requested information makes it shared among the interactants. A new structure (AMS, linked to $S A 3)$ is inserted in the $U A M$ with the top goal "know name" (G3').

\footnotetext{
${ }^{5}$ In this case, the action is "Take-University-exam"; since the user knows that he is not already registered, the check does not cause the execution of any observable actions.
} 

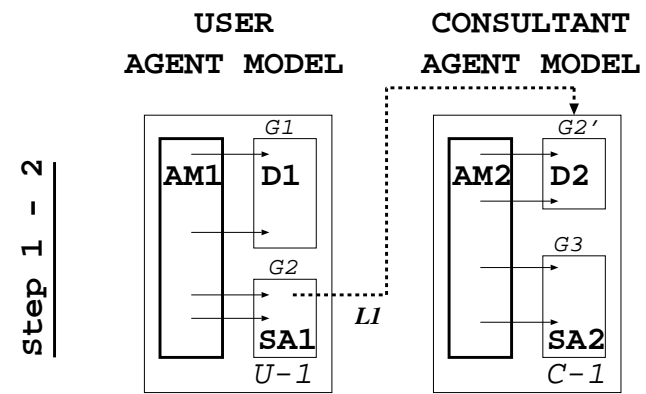

U-1: Could you register me for the $M L$ exam?

C-1: What is your name?

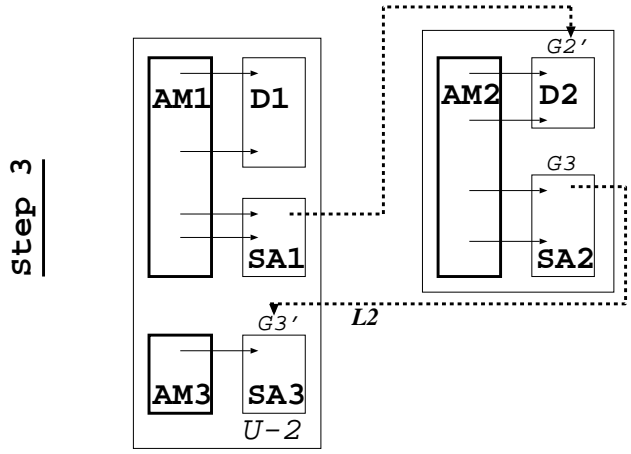

G1: has-credit (U, ML-exam)

G2: Register (C, U, ML-exam)

G3: $\operatorname{Know}(\mathrm{C}$, name $(\mathrm{U}))$

G4: $\operatorname{Know}(U$, registered (U, ML-exam))

G5: Inform (C,U, date (ML-exam))

U-2: Mario Rossi

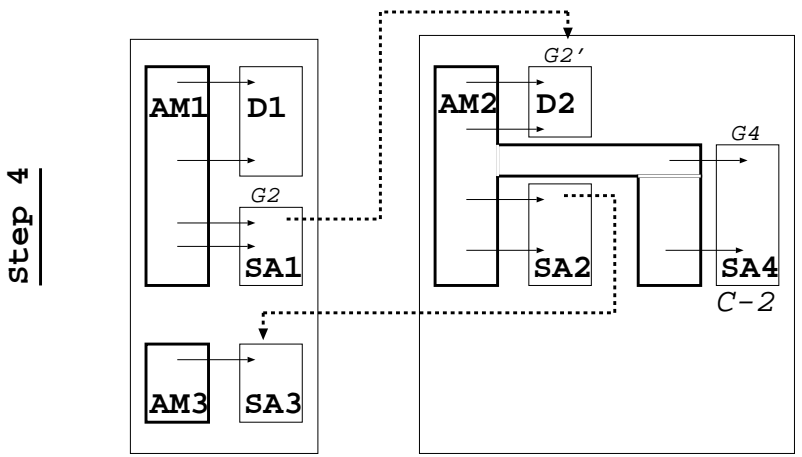

C-2: OK; you are registered!

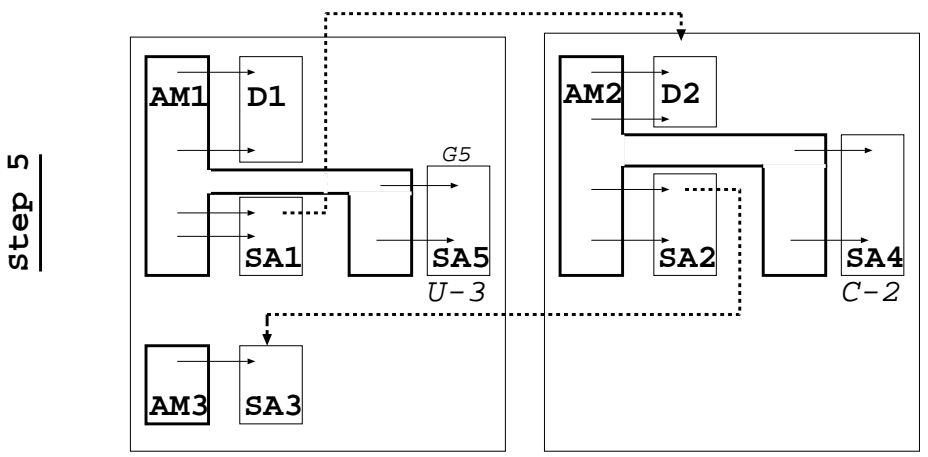

U-3: Can you tell me the exact date of the exam?

Figure 2. Evolution of $U A M$ and $C A M$ models during the interpretation of Example 3.1. 
In Step 4, C-2 signals the user $U$ that the requested action has been executed successfully. C-2 satisfies G4, a classic "notification" goal (Cohen \& Levesque 1991); the actions described in the Agent Modeling library prescribe that, after the execution of an object-level action, an agent acquires the goal to notify his partner. A new speech act representation ( $S A$ / $)$ is inserted in the $C A M$ to account for the linguistic interpretation of $\mathrm{C}-2$, and it is related to $A M 2$ as the last step of the Agent Modeling action describing how to perform the object-level actions.

In the last step, U-3 is a standard continuation step: the user has accepted the completion of the previous subtask and proceeds with his overall plan, by checking another precondition of the domain-level action in focus (achieved by pursuing the goal G5, which is linked to $A M 1$ ).

As stressed before, the architecture outlined in this section is the one that underlies the current implementation. To let the system really interact with the user coherently, we have to exploit the Agent Modeling plans for satisfying the goal that the system adopts after it has interpreted the user's turn, so that the system takes the role of the consultant $C$. Given the ability of the system to generate sentences, the instances of the executed metalevel and object-level plans can then be inserted in the CAM: in this way, the system has a model of its own activity, against which it interprets the user's turns in order to understand how they contribute to it.

It should be observed that the relationships existing among the various substructures present in the agent models (AMs) reflect the intentional/attentional structure of the dialogue as defined in Lochbaum (1994) after the work described in Grosz \& Sidner (1986). In developing these dialogue structures, we focus on an analysis of the content of utterances, and have not yet exploited cue words. It is clear that exploiting both sources of information would make our system more robust.

The approach we have adopted requires that, given a set of dialogue phenomena to model, the intentional aspects of agent behavior necessary to explain them are represented in our Agent Modeling plans. In this way, the recognition algorithm (and eventually the generation one) can be reduced to a device which works on complex and hierarchical plan operators, but needs no other knowledge about the agent's goal-directed behavior.

Currently, we have explored a number of phenomena deriving from the acquisition of a single high-level goal; on the other hand, we have not yet considered the problems deriving from the acquisition of multiple high-level goals, and the cases where agents carry on multiple plans in parallel. The next section describes a set of phenomena which are explained by the dialogue model we have adopted. 


\section{The coherence of an interaction}

In our work, we aimed at analyzing dialogue coherence on the basis of general principles, which can be exploited in order to model different phenomena uniformly. In Castelfranchi \& Parisi (1980) and Castelfranchi \& Falcone (1997), some principles were defined in order to model the acquisition of intentions among cooperating agents. We found that the cooperation notion introduced in those works was particularly useful for defining and modeling dialogue coherence in a framework of agent interaction, where domain-level actions, as well as speech acts, can be performed. Therefore, we have exploited that notion in our computational model of dialogue, which has been defined under the hypothesis that speakers are cooperating agents, and are capable of means-ends reasoning to reach the (joint) goals acquired while interacting with their interlocutors. In the following discussion, we will see that the exploitation of Castelfranchi, Parisi and Falcone's cooperation notion within our framework allows us to model uniformly the production of domain-level actions and speech acts, including communicative actions usually classified as meta-communication (e.g. grounding acts).

Our framework models a goal-based notion of coherence in an interaction, where the decision about which sequences of actions are possible is based on the existence of a relation between the goal underlying the last turn and some previous open contextual goal, belonging to the speaker or to his partner. If the goal belongs to the partner, the speaker may have identified it because it was communicated explicitly by the partner (by means of an illocutionary act), or may have inferred it although it was not stated overtly (Allen \& Perrault 1980, Allen 1983)).

Following the notion of cooperation in terms of goal delegation and goal adoption defined in Castelfranchi \& Parisi (1980) and Castelfranchi \& Falcone (1997), we consider an utterance coherent with the previous context if its receiver $A$ can interpret it as the means of the speaker $B$ to achieve a goal $g$ such that:

1. Goal adherence: $g$ is a goal that $A$ has expressed explicitly that he wants $B$ to achieve; in other words, $B$ has adopted exactly one of the goals he has been delegated by $A$ to achieve. For example, in:

A: I need a book. Where is the library?

B: It is over there.

$B$ 's response contributes to the satisfaction of $A$ 's overt goal of knowing where the library is.

2. Goal adoption: $g$ is a goal that $B$ has inferred $A$ is going to aim at; $A$ has not delegated $B$ explicitly to achieve $g$, but $B$ infers it by reasoning about $A$ 's plans. For instance, in: 
A: I need a book. Where is the library?

B: The library is closed.

$B$ provides $A$ with extra-helpful information which satisfies his future goal of checking whether the library is open. ${ }^{6}$

3. Plan continuation: $g$ contributes to a plan that $B$ is carrying on. This relation covers the typical cases where the agent continues a domain-level (or communicative) plan (Carberry et al. 1992); moreover, it covers the continuation of Agent Modeling plans, as in the following example:

B: Where is the library?

A: It's over there.

B: Is it open today?

We assume that an agent continues the execution of his own plans only when there is no other local goal of the partner to be achieved.

These three relations are defined to model cooperative dialogues. However, as shown in Castelfranchi (1992), the set of coherence relations can be extended straightforwardly to consider also the interactions among conflicting agents, who perform actions to contrast the other agents' goals.

In the following, we will describe these three relations in detail; we will show how different dialogue phenomena can be understood as instances of such relations. Our approach is based on the recognition of the intentions underlying an agent's observed actions and on the ability to appropriately relate such intentions to the interactants' pending contextual goals.

\subsection{Goal adherence}

The goal adherence relation models every contribution that derives from an agent's intention to cooperate with another agent to reach the goals communicated explicitly by $\operatorname{him}^{7}$ It covers the direct correspondences between speech acts, like the adjacency pairs studied by conversationalists (e.g. question-answer and request-accomplishment, see Sacks et al. (1974)); it also covers other more complex phenomena, among which are the occurrence of subdialogues after a question (e.g. the insertion sequences: questionquestion-answer-answer, see Schegloff (1972)) and some notifications. Consider the following example:

\footnotetext{
${ }^{6}$ Note that our goal adoption relation is more restrictive than that of Castelfranchi \& Falcone (1997): it corresponds to their literal conservative adoption.

${ }^{7}$ Note that the goal pursued by the interlocutor is inferred from the speaker's utterances in both the goal adherence and adoption cases. However, the difference is that the goal adherence relation covers the situations where the interlocutor infers that the speaker has explicitly communicated that he wants to delegate him to achieve the goal.
} 


\section{Example 4.1.1}

A: Could you give me a pass for the CS laboratories?

B: a) Are you enrolled in a program of the CS Department?

$\mathrm{B}: \mathrm{b})$ (he checks on the computer whether $A$ is in the enrollment list)

B: c) Can you show me your enrollment card?

B: d) Here you are (he gives $A$ the pass)

B: e) No, you are not enrolled in any program of the CS Department.

Here, $A$ requests the clerk $B$ to give him a pass (he does so indirectly, by means of a surface question); 4.1.1.(a - e) is a list of alternative replies of $B$ to the request. B's reactions derive from his intention of executing the requested action, but they are intrinsically different: 4.1.1.a is a question; 4.1.1.b is a domain-level action; 4.1.1.c is a request (expressed as a surface question); finally, 4.1.1.(d,e) are assertions playing opposite roles. Nevertheless, under the hypothesis that the interlocutors are cooperating, it is possible to recognize the inner rationality of each reaction, and to explain how it contributes coherently to the interaction. The explanation is provided by analyzing the turns from the point of view of agent behavior, as described in our Agent Modeling plans. All the descriptions below are based on the (cooperation) hypothesis that $B$ is trying to satisfy A's overt goal to be given a pass:

4.1.1.a: the Agent Modeling plans prescribe that, to execute an action, an agent $(B)$ must first check its preconditions; as specified in the Domain library, "Give-pass" has the precondition that the addressee is enrolled in a program of the CS Department; therefore, the question "Are you enrolled ... ?" can be interpreted as a way to check this precondition.

4.1.1.b: as above, but the "check precondition" activity is carried out by executing a domain-level action ("reading the enrollment list") instead of a linguistic action.

4.1.1.c: again, an attempt to check a precondition; the domain knowledge must specify that somebody can read from the enrollment card which program a student is enrolled in, and that before reading something you have to see it. Moreover, showing $y$ to $x$ has the effect that $x$ sees $y$. The Agent Modeling plans describe how these domain-level relations can be used for checking the mentioned precondition.

4.1.1.d: this behavior follows from the conditional goal of "notifying" the successful execution of a plan, after its completion; the notification task is the last step of the Agent Modeling plan for achieving goals. If the object-level plan succeeds and it is executed on behalf of another agent, that agent should be informed about the success.

4.1.1.e: the converse of 4.1.1.d: during the execution of the requested action, $B$ has discovered that a constraint of the action "Give-pass" is false and, since he is collaborating with $A$, he informs the partner that the action cannot be executed. 
The goal adherence relation also models acknowledgements to information acts, repetitions, and other grounding behaviors (Traum \& Hinkelman 1992): we interpret the illocutionary act of "informing" as expressing the speaker's communicative intention that the hearer update his beliefs with the new information. Therefore, the hearer's subsequent reactions can be interpreted as positive, or negative, notifications regarding the updating action. Consider the following example:

\section{Example 4.1.2}

A: Dr. Smith is in office number 214.

B: a): $\mathrm{mhm}$

$\mathrm{B}: \mathrm{b}): \mathrm{Oh}$

B: c): 214 .

Here, $B$ recognizes $A$ 's goal of making mutually believed the information conveyed in his statement; so, similar to what happens for domain actions (see turn C-2 in Example 3.1 , he notifies $A$ that he has updated own his beliefs. The goal of letting $A$ know that $B$ now believes the information makes $B$ plan a suitable action for grounding the dialogue, depending on the context where the "updating" action occurs: for example, differently from the unmarked 4.1.2.a, the reaction "oh" in 4.1.2.b is used when $B$ previously believed that Dr. Smith was not in that office (see Heritage (1984)); finally, in 4.1.2.c, B performs a grounding act by repeating the main information conveyed in $A$ 's sentence (see Clark \& Schaefer (1989)). ${ }^{8}$

\subsection{Goal aDOption}

Goal adoption models spontaneous cooperative behavior; it covers many dialogue phenomena, among which: extra-helpful behavior, the displays of behavioral and conversational cooperation among agents, the occurrence of presequences, subdialogues, notifications and acknowledgments for grounding purposes; this relation is also related to repairs of misunderstandings. We have treated these phenomena in Ardissono et al. (1997) and Ardissono et al. (1998).

First, we consider overanswering, a phenomenon widely studied in the plan recognition literature about information-seeking dialogues: see Allen (1983) and Carberry (1990b). In overanswering, the receiver of a question volunteers some extra-helpful information which was not asked explicitly by the information seeker. The rationale behind this fact is that, by reasoning about the partner's plans, the speaker infers that the partner will also aim

\footnotetext{
${ }^{8}$ Many subdialogues addressing the believability of statements can result from the failure of the "Update" action; for example, the execution of "Update" can detect the presence of a previous belief incompatible with the new information (Lambert 1993):

A: CS144 is taught by Prof. Smith

B: But wasn’t Prof. Smith on sabbatical?
} 
at other goals (not only concerning knowledge) because they are crucial to his plans. For instance, compare the two alternative responses in the following example:

\section{Example 4.2.1}

A: Could you give me a pass for the CS laboratories?

B: a): Sorry, we have finished the passes for today. Ask your tutor for a written permission to enter the laboratory.

B: b): Sorry, today the laboratories are closed.

In 4.2.1.a, the clerk $(B)$ notifies the student that it is impossible to perform the requested action (similar to 4.1.1.e); then, he suggests an alternative plan to satisfy $A$ 's goal of entering the labs. Note that $B$ performs a request deriving from his adoption of $A$ 's higher-level goal to enter the labs, and therefore, to have a permission for that. On the other hand, $B$ 's request does not derive from the goal expressed explicitly by $A$, which is that $B$ provide him with a pass.

In 4.2.1.b, $B$ 's reply does not satisfy $A$ 's request of being given a pass, nor his goal of having it, but that information is useful to $A$ : $B$ has understood $A$ 's plan to work in the labs and he adopts the goal that $A$ knows that they are closed; in fact, it is possible to work in the labs only when they are open, and $A$ will have to check this constraint before performing the action.

As pointed out in Airenti et al. (1993), the cooperation among agents can be clearly divided into behavioral and conversational: an agent might refuse his cooperation at the domain level, but he may cooperate at the shared conversational activity. In particular, goal adoption can explain the turns where an agent states his willingness to cooperate at the behavioral level, as well as refusals. For example:

\section{Example 4.2.2}

A: Could you give me a pass for the CS laboratories?

$\mathrm{B}: \mathrm{a}$ ): Here you are (he gives $A$ the pass).

B: b): Ok, just one moment.

B: c): Sorry, but I am busy now.

In 4.2.2.a, $B$ directly executes the action and notifies $A$ about his success.

In 4.2.2.b, $B$ anticipates $A$ 's intention to know whether $B$ is going to perform the requested action by letting him know that he's just about to do it. In fact, when an agent tries to make somebody else intend to perform an action, the agent's success is not guaranteed: as for any other action, he may fail (Smith \& Cohen 1996). This uncertainty causes the agent to check whether the intended effect of his attempt has been achieved. In this specific example, $B$ 's utterance provides $A$ with the feedback he needs before $A$ solicits him.

On the contrary, in 4.2.2.c, $B$ refuses to cooperate at the domain level (i.e. to perform 
the requested action), but he cooperates conversationally by telling $A$ that he does not intend to help him.

A particular role is played by the goal adherence and adoption relations when the object-level plan in execution is a linguistic action: as for domain-level actions, the constraints of linguistic actions must be checked, by means of other speech acts. This provides an explanation of the phenomenon known in conversational analysis as presequences (Schegloff 1979, Levinson 1981). Various conditions are monitored in the preparatory activity before the execution of a linguistic action:

- The speaker monitors the receiver's attention, which is a precondition of the action of uttering a sentence with a communicative goal. In the following dialogue, an agent $A$ tries to attract his partner's attention; in 4.2.3.a, $A$ checks whether the precondition of the uttering action holds, while in 4.2.3.b he makes it true by calling his partner:

\section{Example 4.2.3}

A: a) Are you listening to me?

A: b) John!

B: Yes!

A: Can you give me a pencil?

- In the case of requests, a speaker has to check that the preconditions of the requested action hold; in the following dialogue, $A$ anticipates his request for a pass by asking $B$ whether he has any:

\section{Example 4.2.4}

A: Do you have any passes for the laboratories?

B: Yes.

A: May I have one?

B: Here you are.

- In the case of actions of informing, the speaker has to check whether the hearer already knows the information he wants to communicate to him (this is a constraint of the action of informing):

\section{Example 4.2.5}

A: Do you know where I've been yesterday?

B: No.

A: I've been to Liza's party.

If the above dialogues represent typical presequence patterns, in collaborative interactions the receivers of a turn initiating a presequence often anticipate their partners, 
so producing more compact dialogues. This behavior is only possible if agents perform a plan recognition activity to infer their partners' goals and adopt them. Consider the following examples:

\section{Example 4.2.6}
A: John!
B: Yes, tell me.

\section{Example 4.2.7}

A: Do you have any passes for the laboratories?

B: a) Here you are ( $B$ gives $A$ a pass)

B: b) Do you want one?

\section{Example 4.2.8}

A: Do you know where I've been yesterday?

B: No, where?

In 4.2.6 and 4.2.8, $B$ invites the first speaker to talk; in 4.2.7.a, he anticipates $A$ 's expression of his intention to have a pass by satisfying it directly; finally, in $4.2 .7 . \mathrm{b}, B$ anticipates $A$ by offering him the pass.

The goal adoption relation also explains subdialogues, notifications and acknowledgements occurring when the receiver of an utterance interprets it. In fact, if a speaker $A$ performs an utterance act, he has the goal that the receiver $B$ interprets it. Therefore, if $B$ is cooperative, he adopts the interpretation of the utterance as a joint goal, and performs an "Interpret" action. This goal involves the identification of the syntactic and semantic interpretation of the utterance, and also of its illocutionary force; moreover, since the utterance represents a turn of a dialogue, also the coherence relation with the previous intentional context has to be determined. So, the coherence of subdialogues concerning interpretation problems and the notification of the successful execution of the interpretation action are explained by the goal adoption relation, too. These notifications can be performed as in 4.2.9.d, or by means of backchannels, ${ }^{9}$ as in 4.2.9.e (see Schegloff (1982)).

\section{Example 4.2.9}
A: The professor teaching the AI course is on sabbatical.
B: a): What does sabbatical mean?
$\mathrm{B}: \mathrm{b})$ : The professor of the advanced course?
B: c): Why are you telling me?
B: d): Ok, I've understood.

\footnotetext{
${ }^{9}$ Backchannels are also used for notifying the speaker that the receiver is paying attention to him: in such a case, they are explained by a goal adoption relation satisfying this specific precondition of uttering a sentence; compare this fact with the discussion about Example 4.2.3.
} 


\section{B: e): mhm}

The "Interpret" action has the precondition that the receiver knows the meaning of all the words occurring in the sentence and he can resolve the references unambiguously (see Heeman \& Hirst (1995)). In 4.2.9.a above, $B$ tries to satisfy the first precondition by asking $A$ what is the meaning of "sabbatical"; instead, in 4.2.9.b, $B$ tries to disambiguate the reference of the phrase "AI course". These replies initiate two subdialogues and they resemble turn $\mathrm{C}-1$ of Example 3.1. Also 4.2.9.c initiates a subdialogue aiming at understanding the reason behind $A$ 's statement.

\subsection{Plan CONTINUATion}

This relation explains the coherence of a turn representing the continuation of an agent's course of action, which may concern a metalevel or an object-level plan.

The ordinary continuation of a plan, as analyzed in most plan recognition frameworks, corresponds to the sequential execution of a domain-level recipe: for example, a student who wants to have credit for an exam first does the laboratory work and then he takes the exam. On the other hand, Example 3.1 provides two instances of plan continuation at the Agent Modeling level: the user's turns U-1 and U-3 contribute to the satisfaction of the applicability conditions of the "Take-university-exam" action; the consultant's turn C-1 aims at learning the values of the arguments of the requested action; C-2 represents a notification of the successful execution of the action.

The continuation of an agent's metalevel activity is useful for explaining the occurrence of requests for grounding a dialogue: for instance, the grounding actions of Example 4.1.2 can be solicited by the speaker if he wants to know whether his partner has understood or not; see the example from Traum \& Hinkelman (1992): ${ }^{10}$

\section{Example 4.3.1}

( $A$ and $B$ discuss about a plan to move some orange tanks from town to town)

A: so there's an engine at Avon, right?

B: right

Finally, plan continuation can be performed for linguistic actions, too: although in the paper we have considered "single-step" linguistic actions, our speech act library also includes recipes for complex linguistic actions, which are composed of more than one linguistic action. We have introduced these complex speech acts to model structured discourse, where a speaker may utter a sequence of speech acts related in an intentional structure for rhetorical purposes (Maybury 1993, Moore \& Pollack 1992). For example,

\footnotetext{
${ }^{10}$ As noted by a reviewer, in Traum and Hinkelman's example, only this interpretation of "right?" is considered; the phrase also can be interpreted as an expression of doubt about the truth of the proposition, a phenomenon we do not currently consider.
} 
in order to motivate a request, a speaker may select a recipe composed of an assertion about the underlying domain task and the real request:

\section{Example 4.3.2}

A: I have to work in the labs. May I have a pass to enter them?

A turn composed of such a sequence of speech acts must be interpreted by identifying a single discourse plan which explains both utterances. This interpretation process is the same as the one performed to recognize a sequence of actions belonging to a single domain-level plan.

\section{Representation of actions}

The domain, linguistic and agent modeling actions belong to three separate plan libraries, which store the pre-compiled recipes for executing complex actions.

The Domain plan library describes the recipes for reaching the domain goals in a restricted domain; we chose the University domain as a testing domain; see Ardissono et al. (1993) and Ardissono \& Sestero (1996).

The Speech Act library describes the speech acts (and the discourse plans) with particular attention to the direct and indirect approaches to communication and politeness techniques (Ardissono et al. 1995a, Ardissono et al. 1995b).

The Agent Modeling library describes a reactive planner used for modeling agent behavior and will be described in detail later on. ${ }^{11}$

We are also working on the definition of some object-level (domain-independent) actions representing "internal" activities of agents: among them, there are the "Update" action, that represents the updating of an agent's beliefs, and the "Interpret" action, that represents the interpretation of the turns of a dialogue.

All the libraries are organized on the basis of a Decomposition and a Generalization Hierarchy (Kautz 1991, Weida \& Litman 1992). The first hierarchy specifies how a complex action can be performed by executing simpler actions. The Generalization Hierarchy supports feature inheritance among actions: this hierarchy allows the representation, at a higher level of abstraction, of generic actions associated with different behaviors, depending on the context where they are executed. For example, the generic action that represents taking an exam can be specialized into an action for taking a theoretical or an experimental exam, which have different recipes: for instance, the latter contains the step of doing laboratory work.

Actions are represented by means of the same formalism in the three plan libraries, so that the same procedures can be used to work and reason about them. There are four

\footnotetext{
${ }^{11}$ In Ardissono et al. (1996), a preliminary version of the metalevel plans was introduced and it was referred to as "Problem-Solving" library.
} 
1) A generic action from the Domain library (Take-university-exam):

$\begin{array}{ll}\text { name: } & \text { Take-university-exam } \\ \text { roles: } & ((\text { agt, a, person)(obj, ex, exam)) } \\ \text { constraints: } & \text { enrolled(a, Turin-university) } \\ \text { preconditions: } & \text { registered(a, ex) } \\ \text { effects: } & \text { has-credit(a, ex) } \\ \text { specific-actions: } & \text { ((Take-expe-exam (a, ex) } \\ & \text { where experimental(ex)) } \\ & \text { (Take-theor-exam (a, ex) } \\ & \text { where theoretical }(\mathrm{ex})))\end{array}$

2) A simple action from the Speech Act library (Surface-imperative):

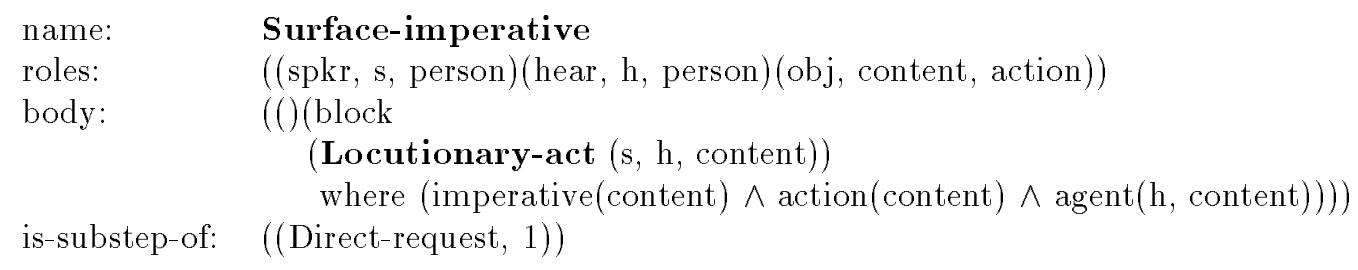

3) A complex action from the Agent Modeling library (Try-execute):

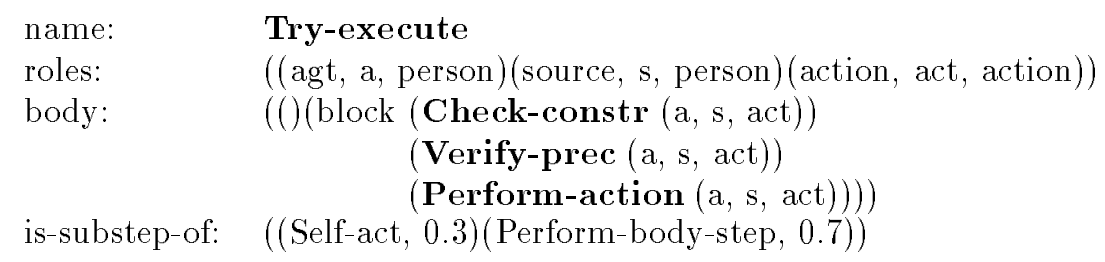

Figure 3. Representation of some of the actions of the libraries.

action types, depending on their position in the hierarchy:

- Complex actions: a complex action $A$ includes a decomposition formed by some substeps $B_{1}, B_{2}, \ldots, B_{n}$, where $n>1$. There is no action $C$ more specific than $A$.

- Simple actions: a simple action $A$ includes a decomposition formed by a single substep D. In Pollack's terms, D generates $A$ (Pollack 1990). There is no action $C$ more specific than $A$.

- Generic actions: for each generic action $A$ there are some actions $C_{1}, C_{2}, \ldots, C_{n}$ (with $n \geq 2$ ) such that $A$ is more generic than $C_{i}$ for $1 \leq i \leq n$. Generic actions have no decompositions.

- Elementary actions: they have no decompositions and no specializations.

The definitions above are rather standard. The only thing to notice is that we decided to keep apart the Decomposition and Generalization hierarchies: decomposition and specializations cannot be specified for the same action; moreover, decompositions are not 
inherited. Basically, we adopted this restriction in order to simplify the management of feature inheritance in our framework. ${ }^{12}$

In any of the three plan libraries, the representation of actions includes the following features:

- the action name;

- the roles (participants) of the action, characterized by:

- a role name: ${ }^{13}$

- an internal variable associated with the role;

- the type restriction on the role fillers;

- the type declaration of other variables occurring in the action (if any);

- the applicability conditions:

- Wh-restrictions: they constrain the relations holding among the role fillers. For instance, the domain-level restriction "has-office(teacher office)" constrains the two variables to take values such that "office" denotes the office of "teacher".

- Constraints: they are conditions that specify in which contexts the action can be executed; like the wh-restrictions, constraints cannot be made true if they do not hold; i.e. they cannot be adopted as subgoals by an agent.

- Preconditions: they are conditions of actions like constraints, but if the agent believes they are false, he can adopt them as subgoals and try to satisfy them.

- the effects of the action, i.e. the conditions that may become true after it is executed successfully. If an action execution fails, its effects do not affect the world state.

- the output variables, bound as a side effect of the execution of the action. They are representation-internal: their main purpose is to assign values to variables, in order to enable the execution of a subsequent step of the plan.

- the more generic action, i.e. the name of the immediate ancestor of the action in the Generalization Hierarchy.

- the more specific actions: i.e. the list of the actions more specific than the action described (in the Generalization Hierarchy); each more specific action is characterized by a condition distinguishing it from its alternatives.

${ }^{12}$ See (Weida \& Litman 1992, Devanbu \& Litman 1996) for an action representation language which takes into account inheritance of recipes, and other features, like temporal constraints.

${ }^{13}$ The role names have no particular impact within the representation of the action, but they are fundamental for establishing the correspondence between actions and linguistic expressions: role names must correspond exactly to the $\theta$-roles of the verb (where such a verb exists). 
- the decomposition: the decomposition (body) of an action contains a number of steps, representing occurrences of simpler actions, which have to be executed in order to perform the main action. In the decomposition, the parameters of the steps can be shared with those of the main action, or they can be strictly related to the action parameters by explicit wh-restrictions. The variables shared between an action and its steps are unified when the action instance is created; the others are bound to values which satisfy the wh-restrictions where they occur.

The body of a complex action may consist of a linear sequence of steps, or of a cyclic block; in this case, we can have either an iterative execution of the action body ("for each" loop) with a loop variable which takes its value from a given range; or, we can have a "while" loop, that terminates when the loop predicate becomes false. ${ }^{14}$ In each step of the recipe, there may occur further restrictions relating the step parameters with those of the action whose decomposition is described.

In the decomposition of an action, there can be some conditional steps, which are executed only when their execution condition holds. The execution condition of a step " $s$ " is reported in the "if" subfield of the occurrence of " $s$ " in the body of the main action.

- the "is substep of" list: this is a list of < action-name, probability> pairs which express the probability that the described action occurs as a step of a higher-level action, denoted as "action-name".

Figures 3 and 9 show the schemata of some actions in the representation formalism of our system. Figures 5, 6, 7 and 8 show some portions of the plan libraries in a pictorial and simplified form: the graphical notation is described in Figure 4. In the figures, the capitalized names are used to denote constants. Note that, for space reasons, the role labels have been omitted in all the figures representing the plan libraries and the interpretation of utterances.

\subsection{The Domain plan library}

This plan library describes the knowledge which guides an agent in reaching his domain goals, like earning a degree in Computer Science, taking the exams, and so on. It contains the precompiled recipes describing the typical well-formed plans to achieve some domain goal (currently, it includes about 100 actions); since the structure of this plan library and its content are very similar to other well-known solutions, like Carberry (1990b), we will just sketch the recipe for taking an exam. Figure 5 shows pictorially the plan for the

\footnotetext{
${ }^{14}$ Providing a formal definition of our representation of actions is future work. See DeGiacomo \& Lenzerini (1995) for a definition of a logic of actions which includes features like conditional actions and repetition of actions.
} 


\begin{tabular}{|c|l|}
\hline & $\begin{array}{l}\text { Action A has as a decomposition } \\
\text { the plan composed of the } \\
\text { actions B', ..., B'". }\end{array}$ \\
\hline
\end{tabular}

\begin{tabular}{|c|c|}
\hline${ }_{\mathrm{B}}^{\mathrm{A}}$ cond & $\begin{array}{l}\text { "While": to complete A, B must } \\
\text { be repeated until cond is false }\end{array}$ \\
\hline cond $\longleftarrow$ constr A & $\begin{array}{l}\text { "Constraint": if cond is false, A } \\
\text { cannot be executed }\end{array}$ \\
\hline cond $\longleftarrow$ prec $\mathrm{A}$ & $\begin{array}{l}\text { "Precondition": if cond is false, A } \\
\text { cannot be executed, but the agent } \\
\text { may act to make cond true }\end{array}$ \\
\hline $\mathrm{A} \stackrel{\text { eff }}{\longrightarrow}$ cond & $\begin{array}{l}\text { "Effects": cond becomes true after a } \\
\text { successful execution of A }\end{array}$ \\
\hline$\underset{[\mathrm{x}<-]}{\mathrm{A}}$ & $\begin{array}{l}\text { As a side effect of the execution of } \\
\mathrm{A} \text {, the variable } \mathrm{x} \text { is assigned a } \\
\text { value }\end{array}$ \\
\hline
\end{tabular}

FigurE 4. The symbols used in the figures of actions.

"Take-university-exam" action, which is specified into two alternative actions, according to the type of the exam to be taken; for the real representation of this action, see the first action schema in Figure 3.

1. "Take-expe-exam" describes the recipe for taking an experimental exam: first, the agent must do laboratory work ("Do-lab-work"); then, he must take an oral exam ("Take-oral-exam"). In order to do the laboratory work, he must enter the labs ("Enter") and implement a prototype ("Write-program"). The "Enter" action has the constraint that the labs are open and the precondition that the agent has the pass for accessing them; the action also has the effect that, after having performed it successfully, the agent is at the labs.

2. "Take-theor-exam" describes the recipe for taking a theoretical exam. In that case, the agent must write a report on a topic of interest for the exam ("Write-report"); then, he must take the written and the oral parts of the exam ("Take-written-exam" and "Take-oral-exam").

\subsection{The speech act library:}

In this library, about 50 linguistic actions are represented, like in the Domain plan library, as actions which an agent tries to perform in order to change the world state: in this case, the hearer's beliefs and intentions. We model communication by introducing an action hierarchy where the higher-level actions regard the speakers' activity aimed at making interlocutors acquire intentions; the lower-level actions describe the means which can be 


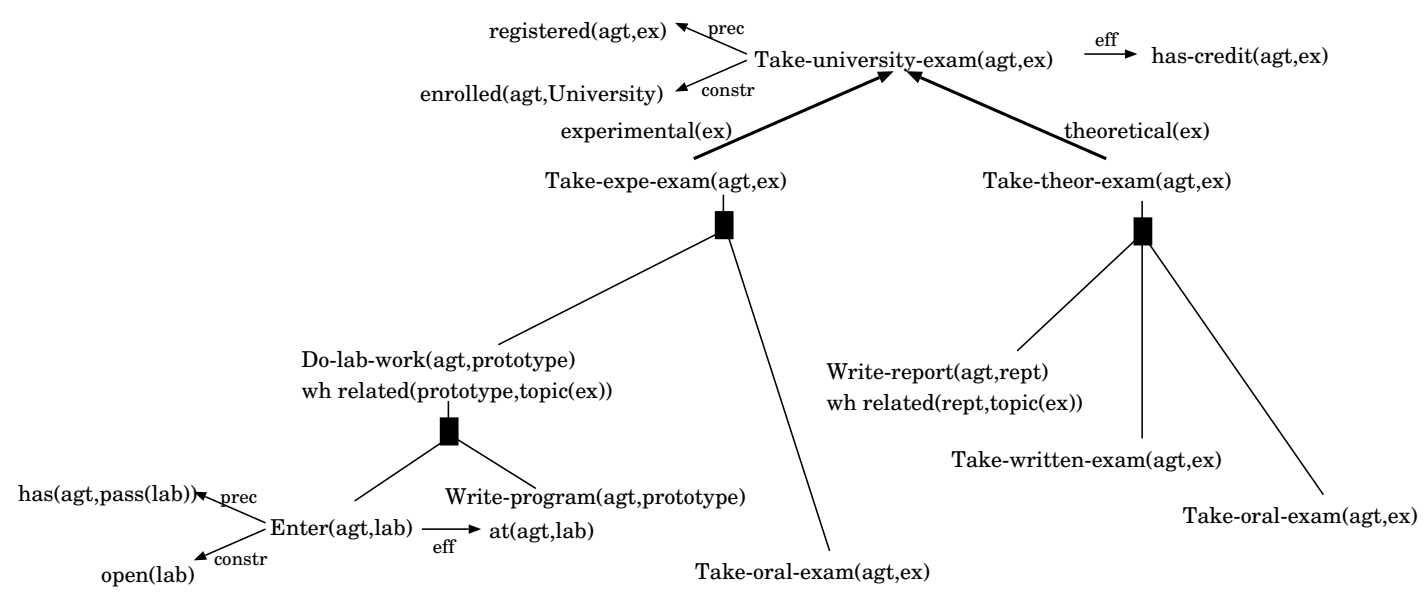

Figure 5. The "Take-university-exam" recipe.

adopted in order to perform the higher-level actions, developing them down to the surface forms which can be used in utterances.

- At the top level of the hierarchy there is the "Get-to-do" action, which describes the agent's behavior to induce the hearer to act in a certain way (Smith \& Cohen 1996). "Get-to-do" has the effect that the hearer intends to act as specified in its "content" parameter. Note that we model standard declarative (information-providing) sentences as expressions of the intention that the partner believe something; more precisely, as an intention that he perform an "Update" action on his own beliefs; therefore, "Update" can be the argument of "Get-to-do", like any other object-level action: in this case the "Get-to-do" will be decomposed into an action of informing.

"Get-to-do" has some more specific actions, representing alternative ways to induce people to act: they are associated with different motivations for acting (e.g. bare cooperativity, fear) and they are not necessarily linguistic. Figure 6 shows only one more specific action, "Cooperative-get-to-do", which relies on the hearer's willingness to adopt the speaker's intention: this restriction is specified in the condition "cooperative(hear, spkr, content)" that distinguishes "Cooperative-get-to-do" from the other alternative actions more specific than "Get-to-do".

- The actions more specific than "Get-to-do" are performed by means of one or more illocutionary acts. By definition, an illocutionary act establishes a minimal effect: making the interactants share that the speaker has the communicative intention that he intends that the hearer intends to act in a certain way. For example, "Cooperative-get-to-do" is performed by means of a request to execute an action. "Request" has the effect that the interlocutors mutually believe that the speaker has the communicative intention that the speaker intends that the hearer intends 


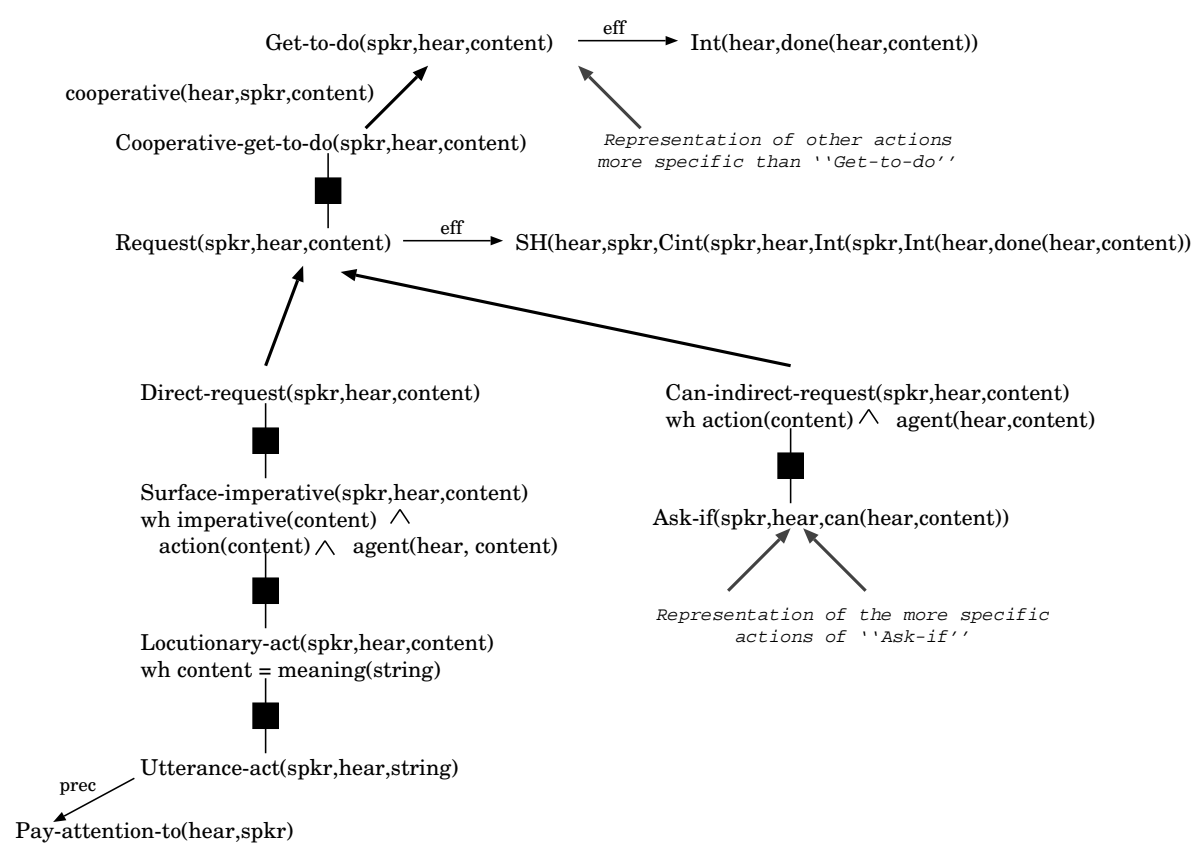

Figure 6. A fragment of the Speech Act library representing requests.

to perform the action described by "content". ${ }^{15}$

- The illocutionary acts are specialized into the means for performing them, which include indirect forms: most illocutionary acts can be expressed via different surface forms, according to the adopted politeness strategy (Ardissono et al. 1995a). Figure 6 shows a direct and an indirect way of performing requests:

- "Direct-request" describes the case where the speaker does not bother about the hearer's face (Brown \& Levinson 1987); its direct decomposition is a surface speech act in imperative mood.

- "Can-indirect-request" is more polite: it is executed by questioning the hearer about his capability of doing the requested action, as in "Could you open the door?": this is represented by the occurrence of "Ask-if" in its decomposition: note that the third argument of "Ask-if" is "can(hear, content)", while it was "content" in "Can-indirect-request".

\footnotetext{
${ }^{15}$ Consider the effect of the "Request" action in Figure 6. The communicative intention operator, "Cint", was introduced in Airenti et al. (1993) to model the Gricean notion of communication (Grice 1957): $\operatorname{Cint}_{x y} p \equiv \operatorname{Int}_{x} S H_{y x}\left(p \wedge \operatorname{Cint}_{x y} p\right)$.

In the formula, "SH" is the modal operator of mutual belief: $S H_{x y} q \equiv \operatorname{Bel}_{x}\left(q \wedge S H_{y x} q\right)$. If an agent $x$ has the communicative intention $p$ towards another agent $y$, then $x$ has the intention that it is a common belief among the two that $p$ holds, and that $x$ had that communicative intention towards $y$. So, $x$ wants it mutually believed that $p$, and that she wanted to communicate it.

The "done" operator in the formula has two parameters: an agent and an action performed by the agent; the operator maps the action on the world state after the action has been executed successfully.
} 
Actually, "Ask-if" is also the way of asking real questions; so, it also appears in the decomposition of the "Obtain-info" action, the illocutionary act used to acquire information. Moreover, "Ask-if" has different more specific actions for asking polite questions. In this way, progressively more polite speech acts can be obtained; for example, consider the request "I would like to ask you if you could lend me some money", expressed by means of an assertion that performs a polite question.

- All the surface speech acts are generated by the "Locutionary-act" action: as described in Austin (1962), this is the action of communicating a propositional content. The surface speech acts are distinguished from one another by the illocutionary force indicator devices occurring in their propositional content; these devices are typically the sentence mode (e.g. declarative, interrogative), the verb mood and the occurrence of modals. For example, as shown in Figure 6, the "Surface-imperative" act is generated by a "Locutionary-act" if the syntactic/semantic representation of the propositional content contains the feature "imperative mood" and concerns an action having the hearer as its agent: "imperative(content) $\wedge$ action(content) $\wedge$ agent(hear, content)" in the wh-restriction. ${ }^{16}$

- In turn, "Locutionary-act" is generated by an "Utterance-act", where the input sentence, which is contained in the parameter "string" (that is bound to a text) has "content" as its meaning: see the restriction "content = meaning(string)".

Note that the "meaning" predicate has a dual role in the interpretation and generation task; in the interpretation phase, as in the current implementation, this predicate is satisfied by invoking the parser and the semantic interpreter (Di Eugenio \& Lesmo 1987); so, its "string" parameter is bound to the input string, while "content" becomes bound after the analysis. Dually, in a generation process, "content" should be bound to the semantic representation of the locutionary act and the "meaning" predicate would produce a call to a surface linguistic generator, which would bind "string" to the result of the generation task.

\subsection{The agent modeling library}

The Agent Modeling actions represent the problem solving recipes used by an agent to plan and execute actions for reaching his goals. In the plan recognition perspective, such recipes are used to interpret an observed behavior of an agent, under the assumption that all agents act following the same recipes for plan formation and execution.

\footnotetext{
${ }^{16}$ In the figure only the link with the surface imperative is represented, but "Locutionary-act" occurs with different restrictions in the decomposition of other actions: "Surface-assertion", "Surface-ynquestion" and "Surface-wh-question".
} 
The AM plans are precompiled and contain about 50 actions. We have defined the Agent Modeling plans as recipes describing the behavior of the modeled agent in a complete way; i.e. they are the only knowledge source describing the system's goal-directed behavior: in this way, we can recognize the intentions underlying many dialogue phenomena which occur between agents who cooperate at a single high-level goal; at the same time, however, we avoid a further level of knowledge necessary for building the Agent Modeling recipes themselves. ${ }^{17}$

The fixed nature of our Agent Modeling plans requires that they take into account all the possible outcomes of a given behavior; therefore, the AM plans may contain conditional steps; moreover, many AM actions have several more specific actions, each one suited to a different execution context.

The Agent Modeling plans defined in the library have an homogeneous structure: any step of the plan "enables" a subsequent one, although this is not made explicit in the representation; e.g. the step of executing a plan comes after the one for finding an executable plan. For this reason, although these plans are precompiled, they are not just scripts describing typical behavior patterns.

In principle, we could have justified the relation between the various steps by defining their effects and preconditions (as in fact happens in the D and SA libraries); for instance, referring to the steps of "Satisfy" in Figure 7, "Find-plans" could have been defined as having the effect "have(a, plans)" and "Cycle-do-action" as having the precondition "have(a, plans)". However, this information cannot be used by the system, since, differently from the object-level actions, the Agent Modeling actions do not undergo a standard "planning and execution" process. In fact, since AM plans define the planner, this could have led to an infinite recursion, if applied to themselves. So the enablement information is left implicit, but it stands at the basis of the AM plans that have been devised.

The main Agent Modeling actions are "Satisfy", which describes the whole activity of an agent who reasons and acts to achieve an object-level goal, and "Try-execute", which describes the execution of a selected action.

\section{The "Satisfy" action.}

The "Satisfy" action, shown in Figure 7, represents the behavior of an agent working to achieve a goal. "Satisfy" models a fanatical behavior, in fact an agent who adopts a goal " $g$ " tries to make true the desired state of affairs until he comes to believe that he has succeeded, or that it is impossible to achieve the goal (Rao \& Georgeff 1992).

\footnotetext{
${ }^{17}$ Our system does not deal with agent behavior caused by the presence of multiple high-level intentions: e.g. we do not model agents who carry on different unrelated plans in parallel, abandon some of these plans, change their high-level commitments; these events are related to phenomena like interruptions and temporary topic changes, typically signaled by the occurrence of cue words and other linguistics markers which help to identify changes in the agents' intentions.
} 


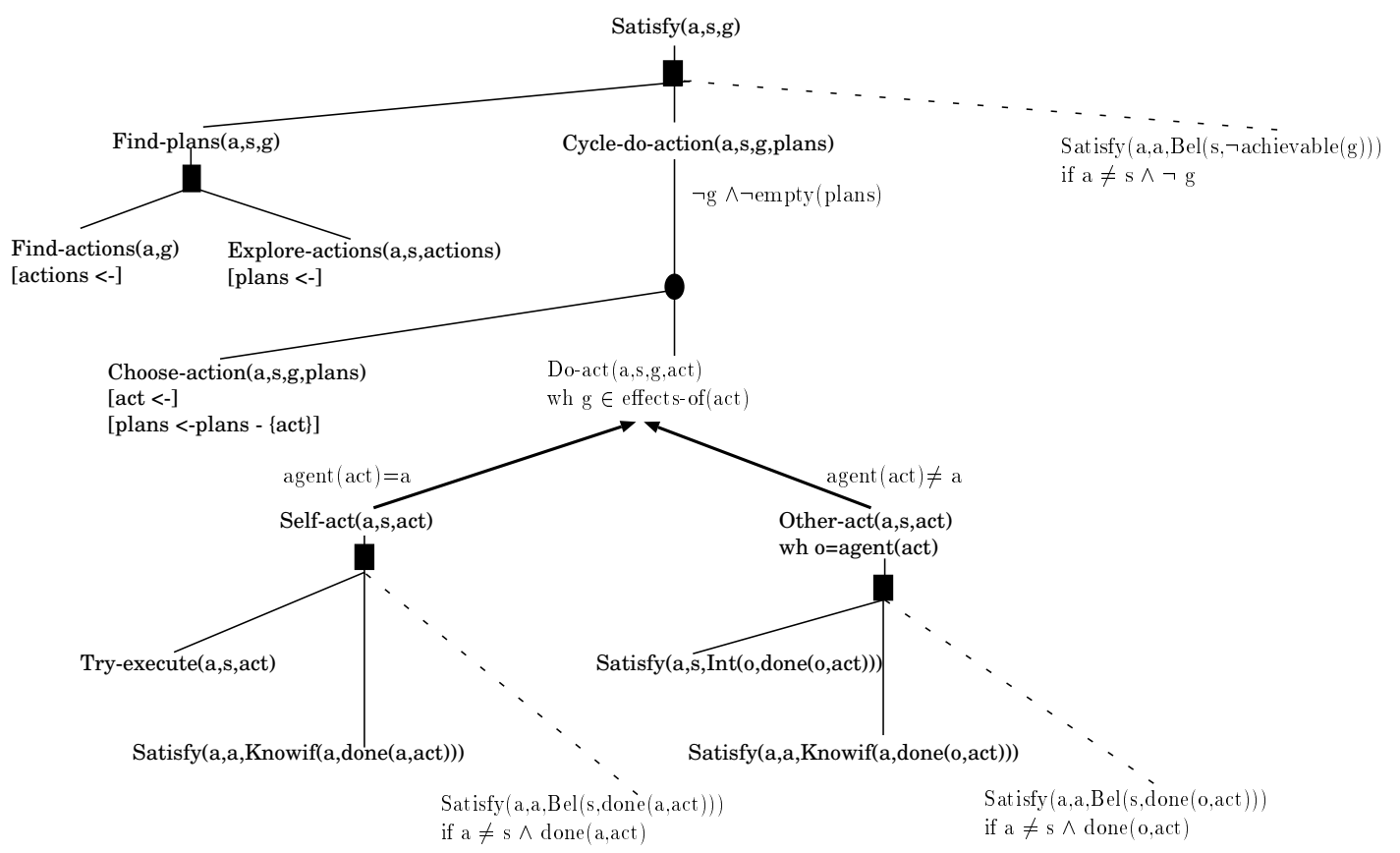

Figure 7. The "Satisfy" agent modeling action.

While the first parameter of "Satisfy", " $a$ ", represents the modeled agent, and the third one the goal " $g$ " to be achieved, the second parameter, " $s$ ", is used to express the fact that the agent " $a$ " is committed to a joint intention with " $s$ "; as discussed in Cohen \& Levesque (1991), when an agent " $a$ " pursues a goal for the sake of a "source" " $s$ ", he adopts some supplementary goals, in order to keep " $s$ " informed about the execution of the action. When he is acting for his own sake, the " $s$ " parameter is bound to the same value as " $a$ ". The "source" parameter occurs in most of the Agent Modeling actions to model this form of cooperation. "Satisfy" has three steps:

1. "Find-plans" consists of:

a) Picking out the domain or linguistic actions having the desired goal " $g$ " as an effect ("Find-actions"); ${ }^{18}$

b) Exploring these actions in order to obtain a set of (possibly partial) plans for " $g$ " ("Explore-actions"). In the exploration task, which recalls the exploration strategies described in Ramshaw (1991) and Carberry et al. (1992), the feasibility of the actions is checked and they are partially expanded, if they are complex; moreover, the preferred plans are selected.

2. "Cycle-do-action" describes the execution of the selected plans, until either the de-

${ }^{18}$ Some of the actions appearing as elementary in the AM library (e.g. like "Find-actions", "Chooseaction", etc.) could be expanded by means of complex subplans; see Sestero (1998). 
sired state of affairs " $g$ " is reached, or all the alternatives have failed. ${ }^{19}$ At each iteration, one of the candidate actions is selected in the "Choose-action" step; this step, which is currently present in a simplified form, also should contain the check that the action is compatible with the other goals of the agent (Bratman et al. 1988). After the selection of "act", the recipe continues with its execution: "Do-act" describes this execution. Note that the wh-restriction " $\mathrm{g} \in$ effects-of(act)" is of paramount importance during the plan-recognition activity. Two continuations may follow, depending on which agent has been selected by " $a$ " as the agent of the action "act" to be performed:

a) If the agent of "act" is " $a$ " himself, " $a$ " performs "Self-act", by means of "Tryexecute".

b) Otherwise, " $a$ " performs "Other-act": " $a$ " acquires the AM goal to obtain that another agent " $o$ " intends that the selected action is executed (by " $o$ " himself): "Satisfy(a, s, Int(o, done(o, act $))) "{ }^{20}$ In order to satisfy this goal, the agent " $a$ " can choose to perform a suitable linguistic action more specific than "Get-to-do", aiming at inducing his partner to act. These possibly complex communicative acts are subject to the same planning and (reactive) executing process as the domain-level actions, by means of "Try-execute".

In both cases, after the execution of the action, which may have been performed by the agent " $a$ " or by his partner, " $a$ " checks whether the action was successful or not: see the two occurrences of "Satisfy" in the body of "Self-act" and "Other-act". In fact, the successful execution of an action does not necessarily imply that its effects hold, so that its result must be monitored. As an example, when an agent takes an exam, if he gets a negative mark he does not get the credit for that exam: for this reason, before considering his plan completed, he still needs to check the effect of the "Take-university-exam" action. In particular, if " $a$ " has asked his partner to execute an action, the execution of this step can lead him to adopt linguistic behavior like soliciting feedback from the partner. This is one of the reactive aspects of the planner, that must recover from a failure in action execution by replanning its behavior. Also other parts of the Agent Modeling library contain actions for checking whether the object-level actions have been performed successfully.

The third (conditional) step occurring in "Self-act" and "Other-act" describes one of the subsidiary goals due to the presence of a joint intention between " $a$ " and " $s$ ": if " $a$ " has successfully executed the action on behalf of " $s$ " (e.g. "done(a, act)" in

\footnotetext{
19 "Cycle-do-action" is represented in detail in Figure 9, where the cycle decomposition is denoted by the "cycle-block" label, followed by the loop condition.

${ }^{20}$ For the meaning of the "done" operator, see footnote 15.
} 


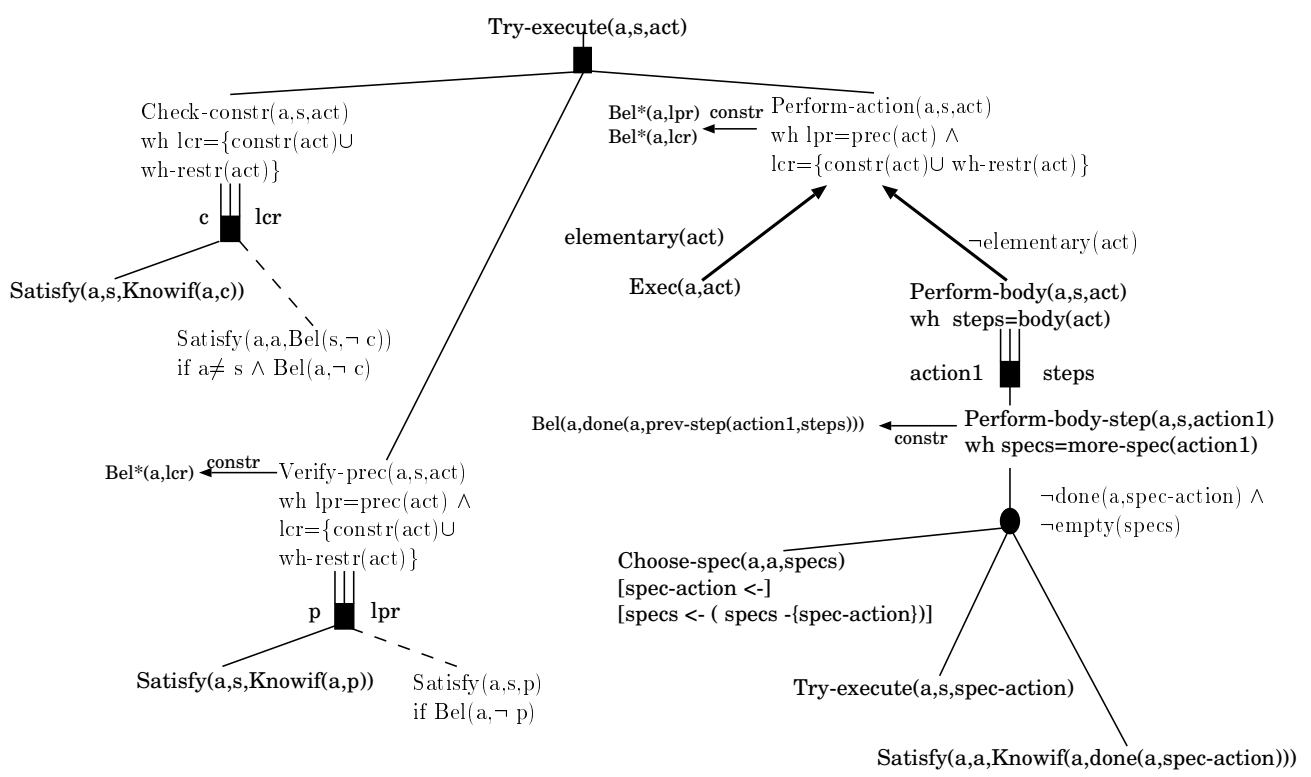

Figure 8. The "Try-execute" agent modeling action.

the figure), he acquires the goal to let the partner know about the success. This goal may lead " $a$ " to a further planning and to the execution of a suitable action in order to inform the partner accordingly.

3. The third step of "Satisfy" models another subsidiary commitment induced by a joint intention between " $a$ " and " $s$ ": if all the actions for the initial goal " $g$ " have failed to reach their effects and the agent " $a$ " shares " $g$ " with a partner " $s$ ", then he will execute a "Satisfy" action on the goal of letting " $s$ " believe that " $g$ " cannot be achieved: "Satisfy(a, a, Bel(s, ᄀachievable(g)))".

\section{The "Try-execute" action.}

The "Try-execute" action, shown in Figure 8, describes the whole process of executing an object-level action (denoted as "act" in the figure) to which may be associated a partial plan previously built in the exploration phase ("Find-plans"); this process consists of checking the prerequisites for its object action and performing the action steps, if there are any.

1. First, the agent checks whether the constraints of "act" are true ("Check-constr(a, s, act)"), which is done via a sequence of "Satisfy(a, s, Knowif(...))" actions. If any of these conditions is discovered to be false, the agent possibly notifies his partner about the failure and gives up the execution of the "Try-execute" plan; in fact, the constraint "Bel*(a, lcr)" ${ }^{21}$ of the subsequent Agent Modeling step of "Tryexecute" ("Verify-prec") is true only if the agent believes that the constraints of the

${ }^{21}$ The "Bel*" operator means that " $a$ " believes that each of the conditions in the "lcr" list is true. 


\section{Cycle-do-action:}

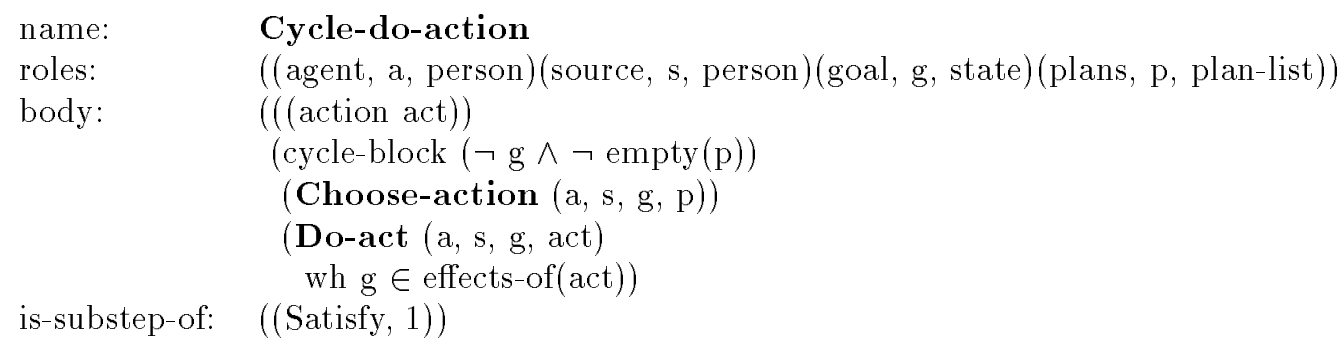

\section{Check-constr:}

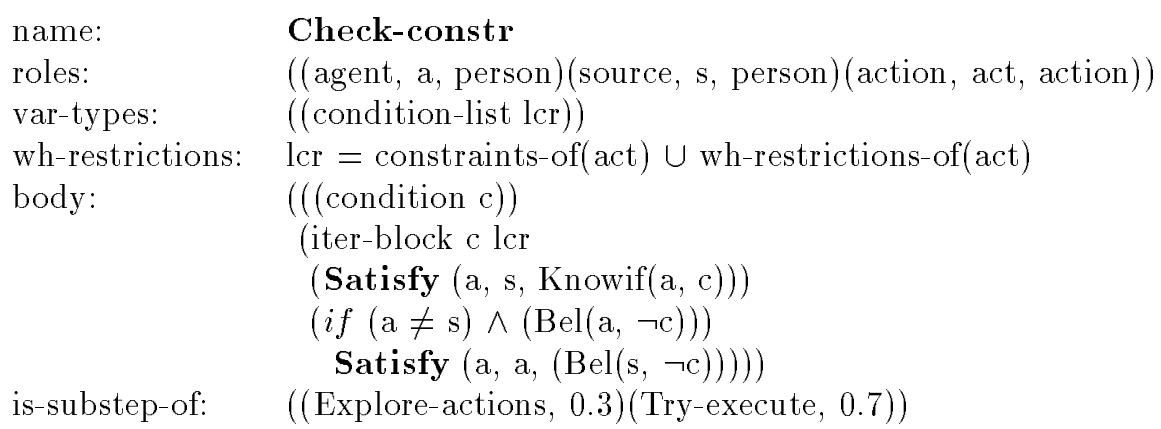

Figure 9. Detailed representation of Agent Modeling actions.

object-level action are true; as already discussed, "a" knows the truth values of the conditions in "lcr" because he has previously checked them, when he has performed "Check-constr". ${ }^{22}$

2. The preconditions of an action can be adopted as subgoals by the agent; so, the "Verify-prec" plan checks the truth value of these conditions and also tries to satisfy them, if they are false (see the second step of "Verify-prec"). Differently from "Check-constr", the goal of informing a partner that a precondition cannot be obtained comes indirectly from the fact that the goal of making the condition true is shared with him: in Figure 8, "Verify-prec" shares the "source" parameter with its conditional step "Satisfy(a, s, p)".

Checking preconditions and making them true is another reactive aspect of the Agent Modeling plans and enables the planner to repair to the changes of the environment around him by replanning the agent's subgoals.

3. "Perform-action" models the execution of actions: if its object "act" is elementary, it is executed directly ("Exec(a, act)"); otherwise, "Try-execute" is called recursively on its steps, by means of the "Perform-body" action. The execution of the body of an object-level action stops and fails if there is a failure in the execution of any of its steps. ${ }^{23}$ When a failure occurs after a portion of the body of the action

\footnotetext{
${ }^{22}$ Although in Figure 7 the "Explore-actions" plan is not shown, "Check-constr" may occur as a step of "Explore-actions", too: see Figure 9.

${ }^{23}$ In Figure 8 (see the constraint of "Perform-body-step"), the "prev-step(action1, steps)" function
} 


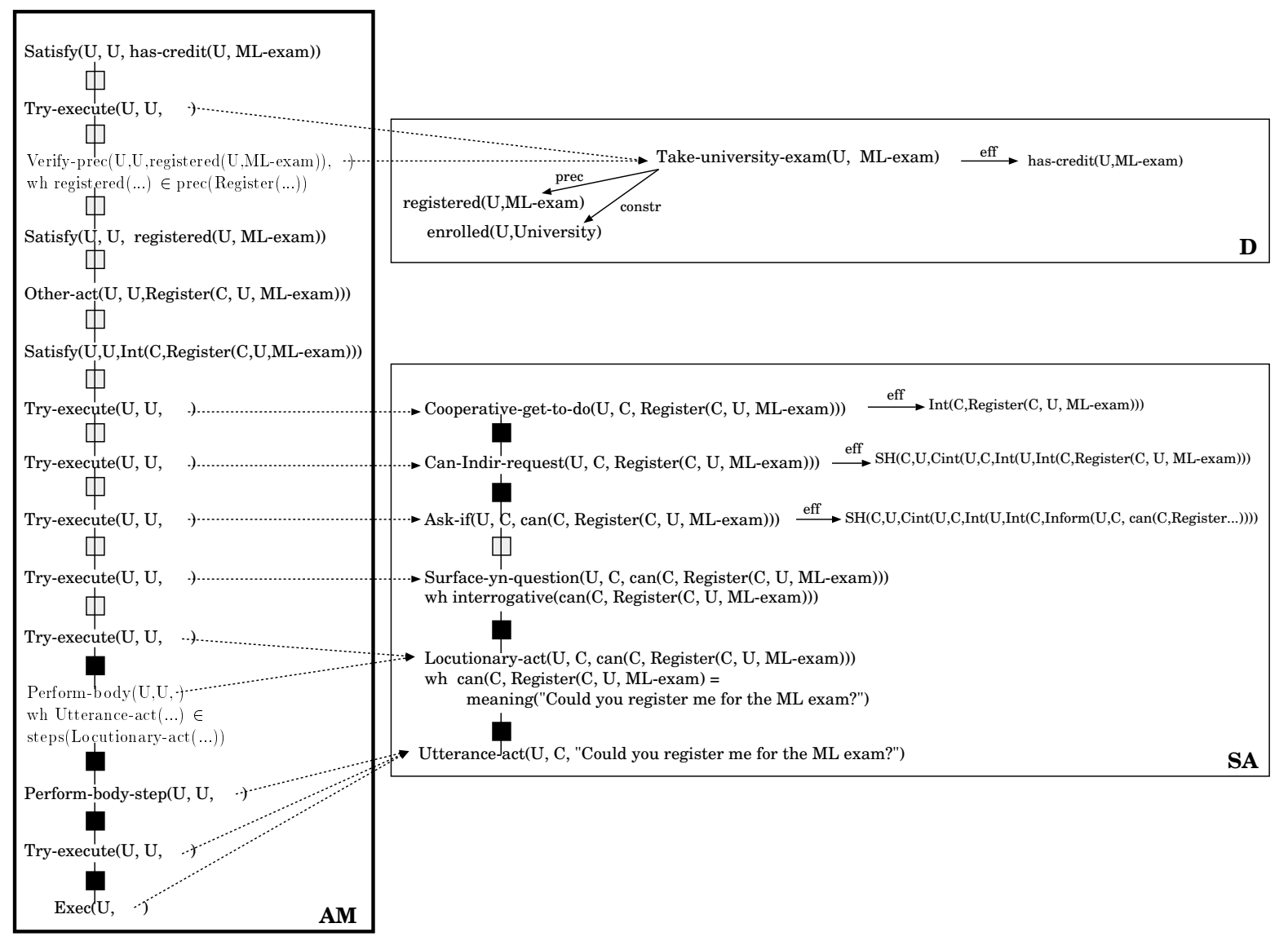

Figure 10. Local interpretation of the turn: "Could you register me for the ML exam?"

has been performed, only the effects of the steps successfully executed hold.

"Perform-body-step" manages the execution of a step: each alternative way to execute the step is tried (if there are any), until the step has been completed, or no alternatives exist. In particular, if the step is a generic action, one of its more specific actions is selected for execution ("Choose-spec"); then, the selected action is performed by means of a "Try-execute"; finally, the agent satisfies the goal of knowing whether the action was indeed successful.

\section{The interpretation process}

As already mentioned, our current prototype is limited to the interpretation task; for this reason, the system always acts as the receiver of an utterance, switching its role at every turn; moreover, the system maintains the interpretation of the turns of both speakers within a single overall context of the dialogue.

The system takes as input, in textual form, each utterance and interprets it from the perspective of the current receiver; it also tries to relate the turn to the previous turns,

selects the step before "action 1 " in the recipe for "act". 
in order to see if the utterance is a coherent continuation of the dialogue. Meanwhile, the system updates the representation of the dialogue context by adding to it the interpretation of the new turn, together with the hypothesized coherence relation which links the turn to the previous ones (see section 4).

The input of a sentence is represented in the action formalism as an instance of the Agent Modeling action "Exec", having as argument an instance of the SA (speech act) action "Utterance-act" which, in turn, has as argument the input text: for instance, "Exec $(U$, Utterance-act $(U, C$, "Could you register me for the ML exam?" $)) "$.

The interpretation process has the goal to find a connection with the representation of the previous part of the dialogue (that we call Dialogue Model - DM), making explicit the coherence relation. However, this connection is not direct, but involves some inferences on the observed "Exec" action, in order to identify the "local" goals that led the agent to produce the utterance. So, what is actually connected to the existing Dialogue Model is not the observed "Exec", but a more complex structure representing the result of these inferences. Since this structure is built by a process called Upward Expansion (see the next section), we will call it Upward Expanded Structure (UES).

For example, consider Figure 10, which shows the interpretation of the sentence "Could you register me for the ML exam?", after having established the top-level local goal that the speaker has credit for an exam. ${ }^{24}$ This goal is represented by the "Satisfy $(U, U$, hascredit( $U$, ML-exam $)$ )" action instance at the top of the Agent Modeling branch, which is linked to the observed "Exec" via a chain of decomposition links. ${ }^{25}$ The figure shows that the instances of the Agent Modeling actions have object-level action instances among their arguments. In turn, the object action instances are themselves related by decomposition links, so that the turn interpretations will be represented as two-level structures: the AM boxes contain chains of Agent Modeling action instances, while the D (domain) and SA (speech acts) boxes contain chains of instances of object-level actions. The dotted arrows from the AM box to the object-level boxes link the Agent Modeling action instances to their object-level arguments; note that more than one AM action instance may be related to the same object-level action instance. Although we usually represent the connections between an Agent Modeling action and its object-level argument as a link between two structures, in Figures 10 and 18, because of typographical reasons, we have in some cases included the referenced object-level action in the related AM action.

The Dialogue Model (DM) is a sequence of turn interpretations linked by coherence relations, where the root of each turn interpretation is related to some action occurring in

\footnotetext{
${ }^{24}$ In this figure, the grey boxes in the decomposition links are a shorthand: in fact, we have shown only the occurrences of the Agent Modeling actions most relevant in this example. The missing actions can be reconstructed using the action libraries described in Section 5.

${ }^{25}$ In the "Satisfy" action, the "source" parameter is bound to the speaker $U$ of the utterance, because we assume that he is trying to reach a goal of himself.
} 


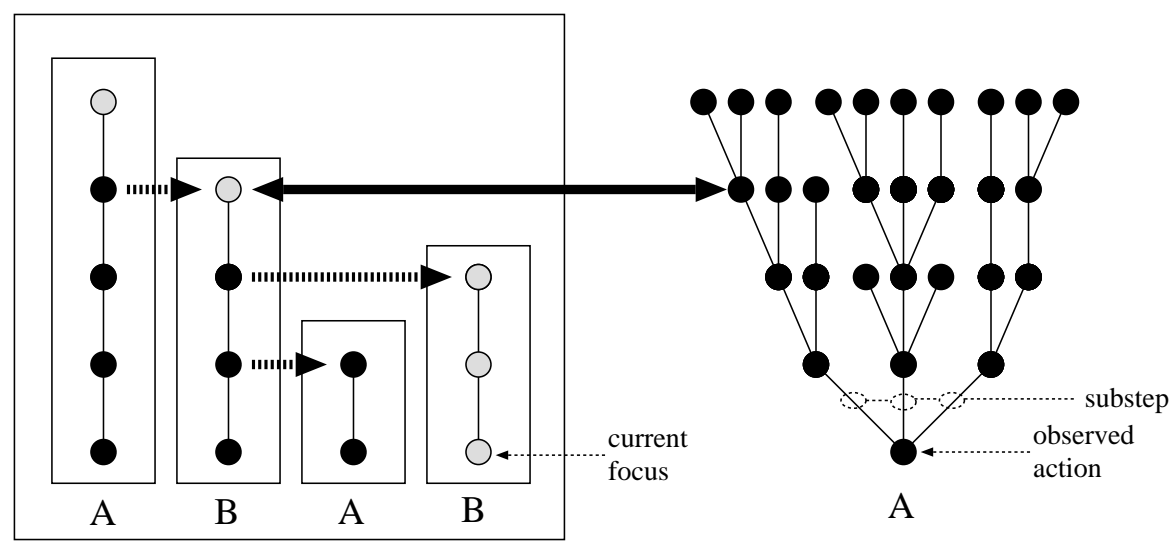

Dialogue Model (DM)

FigurE 11. The problem of searching for coherence.

the interpretation of a previous turn. ${ }^{26}$ In the next sections we describe how this complex structure is built.

\subsection{Basic Algorithm}

The algorithm for extending the dialogue model with the new input aims at guaranteeing that the coherence of the interaction is maintained, where the concept of coherence has been discussed in the previous sections. However, it is clear that the search space is huge since, in principle, any action for which the recognized one can act as a substep (even at many levels of depth) could be the right one for searching a connection among the actions in the dialogue model. The problem is described intuitively in Figure 11, where the box on the left is the Dialogue Model built until the previous turn, with various sub-boxes which represent the turn interpretations (see Figure 2). The reversed tree on the right (which could actually be a graph) is the set of the actions for which the action (presumably a Surface Speech Act) observed in the last turn can act as a substep. The thick arrow is the connection to be found.

Traditionally, the problem has been split into two parts (see Carberry (1990b)):

1. The generation of the structure appearing on the right in Figure 11. In fact, what is initially available is just the bottom node, "Observed Action", and the structure must be generated incrementally by traversing the libraries. This phase has been called Upward Expansion.

\footnotetext{
${ }^{26}$ Much work has been reported in the literature to define how a dialogue can be segmented in turns, and which are the boundaries among turns. In our framework, a turn corresponds to the sequence of sentences typed in by the user as a unique input stream. However, if more than one sentence is present in the stream, the interpretation is performed separately for each sentence. To simplify the following discussion, when we refer to interpretation of a turn we intend the sequential interpretation of each single utterance in the turn.
} 
The interpretation algorithm

1. Set Dialogue-Node to the Current Focus of the Dialogue model /*___ Loop: Focusing _____

2. Find any connection between Observed-Action and Dialogue-Node

2.1 If just one connection is found, establish it as the proper connection, and extend the Dialogue Model accordingly

2.2 If more than one connection is found, select the best one, establish the selected one as the proper connection, and extend the Dialogue Model accordingly

2.3 If none is found, set Dialogue-Node to the parent of the current Dialogue-Node

2.3.1 If Dialogue-Node is now void (i.e. we were already on the root of the Dialogue Model) then fail.

2.3.2 Otherwise, go to step 2

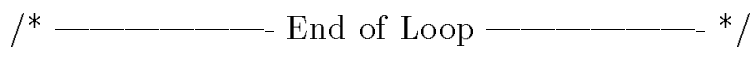

FIgURE 12. Algorithm for the interpretation of a turn.

2. The search for a connection between any newly generated node (action) of such a structure and any action in the Dialogue Model. This phase has been called Focusing.

In our approach, the picture is made somewhat more complex by the fact that there are three different libraries to take into account, and among them the Agent Modeling library has a distinguished role. Before entering the details, the algorithm can be roughly described as in Figure 12, where the terms in boldface will be discussed in more depth.

The algorithm starts from the literal interpretation of the last turn ("Observed Action") and from the literal interpretation of the previous turn ("Dialogue-Node", which is the value of the "Current Focus" of the Dialogue Model); then, it tries to find a path ("Find any connection") between "Observed Action" and "Dialogue-Node". The search is carried out by looking for all the parents (and then, if needed, the ancestors) of "Observed Action", and seeing if they match the current focus. Since the search is computationally expensive, it is partially supported by an (off-line) precompilation of the libraries, which builds an index of the paths existing between pairs of action schemas. In case no path is found, so that no connection can be set up, then "Dialogue-Node" is set to the parent of the "Current Focus" and the search is restarted. This process is iterated until a connection is found (the coherence has been established) or the root of the dialogue model is reached (the system judges the dialogue incoherent, according to its knowledge). 
Given an instantiated AM action Curr Act, let curr Act be its action schema.

1. Let genAct* be the set of action schemas more generic than curr Act (including curr Act itself).

2. Let parentActs be the set of all action schemas for which one of the elements in gen Act* is a substep.

3. For each upAct $\in$ parent Acts create an instance $U$ pAct, by binding its parameters.

Figure 13. The Upward Expansion algorithm.

\subsection{Details}

In this section, we aim at making clear the meaning of the boldface words appearing in the sketch of the interpretation algorithm shown in Figure 12. Since the major complexities, with respect to other approaches, are due to the presence of the Agent Modeling actions, we must make more precise their role in the algorithm. This can be obtained by starting the description from the way the Upward Expansion phase is carried out.

\subsubsection{Nodes reachable via (inverse) decomposition links from a given node (Upward Ex- pansion)}

Although we tried to make the basic ideas clear by keeping Figure 11 as simple as possible, we must note that the result of "observing an action" is not only an instantiation of a node in the Domain (or Speech Act) library, but it also includes an instantiation of an "Exec" action, coming from the Agent Modeling library, as shown in Figure 14.a.

Starting from this structure, we must obtain the Upward Expanded Structure shown in Figure 10. This is obtained via the Upward Expansion algorithm in Figure 13: given an action Curr Act (a member of Current-Actions in the Interpretation Algorithm in Figure 12) to be expanded, the Upward Expansion algorithm determines the actions for which Curr Act is a possible substep. Two points have to be taken into account: the first one concerns the existence of the Generalization Hierarchy (which is handled in Step 1), while the second one is more complex, and concerns the instantiation of the actions. In fact, the algorithm first finds the action schemas that, in the library, have Curr Act as a step ("parentActs"), and then binds the parameters of these schemas in the proper way.

The binding is obtained by taking into account the bindings of the arguments of the original Curr Act, the wh-restrictions associated with "curr Act" in the body of " upAct", ${ }^{27}$ and two global variables bound to the current speaker and to his interlocutor. Note that different sources contribute to the binding of the parameters of the newly instantiated actions: while the binding for the parameters shared by Curr Act and UpAct is immediately available, for the other parameters we must take into account the wh-restrictions

\footnotetext{
${ }^{27}$ We are referring to the wh-restrictions which relate the variables of the steps of an action; see, for instance, "Cycle-do-action" in Figure 9.
} 


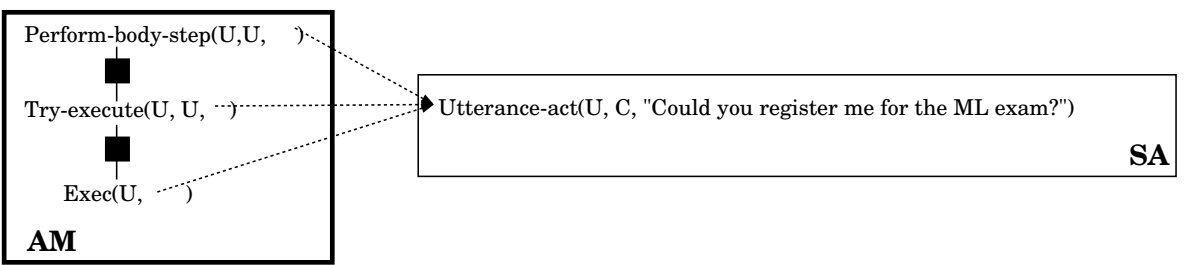

Figure 14. Two steps of the interpretation of a turn.

present in the body of $U p A c t$. In fact, these restrictions specify the relations that must exist between the parameters of UpAct and the variables occurring in its body.

Figure 14.a shows the structure given as input to the first step of Upward Expansion. As stated in the algorithm, the Agent Modeling actions undergo the Upward Expansion, since it is the AM library that specifies how the various object-level actions can be linked together. Since "Exec" occurs as a substep only in the place shown in Figure 8, i.e. to accomplish a "Try-execute" (where the abstraction to "Perform-action", more generic than "Try-execute", is accounted for in Step 1 of the Upward Expansion algorithm), "parent Acts" includes the single action schema "Try-execute". In this case, there are no problems with the parameters in the instantiation, since the three parameters of "Tryexecute" are " $a$ " (the same agent of the "Exec"), "s" (the current interlocutor), and "act" (which is bound to the same string already available in the "Exec" instantiation).

A more intricate situation arises after a further step of expansion, whose result is shown in Figure 15. In fact, here, the expansion of the Agent Modeling structures must be followed by a parallel expansion of the structure of the object-level actions. This is a consequence of the binding process on the AM action parameters, and is guided by the evaluation of the wh-restrictions present in the body of the new Agent Modeling actions to be attached.

"Perform-body-step" (which appears in Figure 14.b as the top Agent Modeling node, i.e. Curr Act in the algorithm) is a step of "Perform-body"; however, the wh-restriction in the decomposition of "Perform-body" only says that the third parameter of each instance of "Perform-body-step" must be equal to one of the steps of the object-level action which the "act" parameter of "Perform-body" is bound to ("steps = body(act)", and "action1 $\in$ steps"). In our example, the loop variable "action 1 " is bound to the "Utterance-act" of the observed utterance. This means that "Utterance-act" must occur in the body of some other action, which will play the role of third parameter in the "Perform-body" to be instantiated. In order to find a connection, we must exploit the object-level libraries. The 


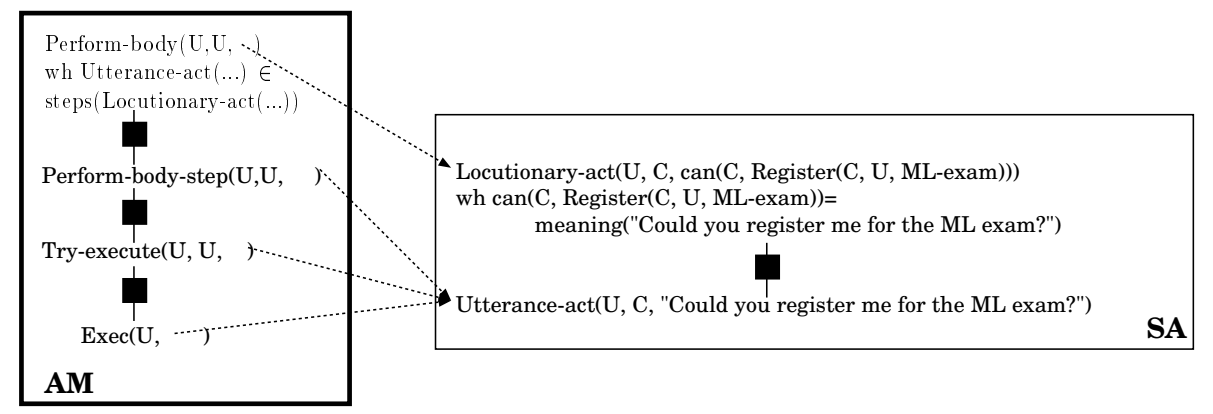

FIgURE 15. A further expansion of the structure in Figure 14.b.

Speech Act library, to which "Utterance-act" belongs, unambiguously tells us that the super-action is "Locutionary-act"; the wh-restriction in it ("content = meaning(string)"), specifies that its third parameter must be bound to the "meaning" of the input utterance, so that the parser and the semantic interpreter are activated, producing the representation in Figure $15 .^{28}$

So, the process of evaluating the action conditions in order to bind the parameters has two important side-effects: the parallel expansion of the object-level and metalevel interpretation structures and the call of the syntactic and semantic interpreter. A third noticeable result is that the evaluation process performed in Step 2 of the Upward Expansion algorithm can determine the relations between conditions and actions having them as preconditions or constraints: this task corresponds to the action identification phase described in Carberry (1990b). In fact, let's suppose that an inferred Agent Modeling action be "Satisfy(U, U, condition)"; one of the actions where "Satisfy" occurs as a substep is "Verify-prec" (see Figure 8). In particular, if we assume that "condition" is not of the form "Knowif(a,p)", only the second occurrence of "Satisfy" can match the input. ${ }^{29}$ Now, the standard Upward Expansion step tries to instantiate "Verify-prec", which is done according to the wh-restrictions of "Verify-prec": these restrictions specify that "condition" must be one of the preconditions of some to be determined object-level action ("1pr = prec(act)", and "p $\in$ lpr"); this is exactly what happens in Carberry (1990b) when the action in focus is identified from the attempt to make true one of its preconditions.

For example, consider "Satisfy(U, U, registered(U, ML-exam))" in Figure 10: "registered(U, ML-exam)" is a precondition of the domain-level action instance "Take-universityexam(U, ML-exam)". So, the Upward Expansion causes the instantiation of the Agent Modeling action "Verify-prec(U, U, Take-university-exam(U, ML-exam))", and, possibly, in the next step upward, of "Try-execute(U, U, Take-university-exam(U, ML-exam))",

\footnotetext{
${ }^{28}$ In the figures we have reduced the syntactic and semantic representation of the input sentences to logical forms for the reader's convenience without reporting details about syntactic information (such as, for example, the presence of question marks, conditional mood and so on).

${ }^{29}$ This optional occurrence concerns the possibility that if the agent believes that "condition" is not true, he tries to make it true.
} 
which is the required inference.

Finally, we observe that during the Upward Expansion, the actions need not be fully instantiated; some variables can remain unbound: they will get their value when the connection with the Dialogue Model is found.

\subsubsection{Parent of a node in the Dialogue Model}

This is a rather simple point, since it's only a matter of climbing up the right frontier of the Dialogue Model (the grey circles in Figure 11), in order to take into account all the tasks that have not been completed yet. Remember, however, that the path that is followed is always the one of the Agent Modeling actions, so that in some cases, the father of a node must be found not via a decomposition link, but via a link connecting an action of agent $A$ with an action of agent $B$ (the dotted arcs in Figure 11). Because of these interconnections, the actions found on the path can pertain to either agent, so that the agents' goals are taken into account in an interleaved way, depending on the place where the process is positioned at a given time. Consequently, the possibilities of Plan Continuation or Goal Adherence/Adoption arise automatically.

\subsubsection{Connections}

As stated in the two previous sections, the Agent Modeling actions are the backbone of both the Dialogue Model and Upward Expanded Structure (UES). So, when we say that a connection has to be found between the Dialogue Model and the (upward expanded) interpretation of the last turn, we mean that a connection must be found between "an AM node" in the dialogue model and "an AM node" in the UES.

So, let's suppose the we have to check whether there is any connection between the AM action $A_{\text {new }}$ observed or inferred from the last turn, and the AM action $A_{\text {old }}$ existing in the Dialogue Model. Clearly, we are not talking of any connection, since any connection is what needs to be found by the whole process. So we are looking for some kind of more direct connection. In the following subsections we will discuss:

- Which kind of basic connections are looked for.

- How the search process takes place.

- How the presence of the AM library affects the processing

\section{- Basic connection types}

1. The most direct type of connection is the one where there is a decomposition chain between $A_{\text {old }}$ and $A_{n e w}$; i.e $A_{n e w}$ is a substep of $A_{o l d}$ at any level of depth, as shown in Figure 16.1 (Connection type 1). 
In order for this kind of connection to apply, we assume that, although $A_{\text {old }}$ and $A_{\text {new }}$ are Agent Modeling actions, there is a corresponding decomposition chain between their arguments, i.e. the involved object-level actions.

2. In order to increase the power of the system, we also accept more complex connections. The first of them is shown in Figure 16.2.

The basic structure is analogous to the one of Figure 16.1, and in fact from the Agent Modeling perspective there is no substantial difference. However, we accept here that the argument (the object-level action) of $A_{\text {old }}$ is not connected via a decomposition chain to the argument of $A_{n e w}$; so, we have to infer the argument of $A_{\text {old }-x}$ before the connection can be found. The reason for introducing this facility is that the actions appearing in the Agent Modeling library can be complex, i.e. can have decompositions composed of more than one step. In some cases, the AM decomposition step can refer to different object-level actions which are not connected via a decomposition link, or to different conditions related to an object-level action. An example of this second case is the definition of "Other-act": the second step of this action consists of a checking of the successful execution of the domain-level action "act", by means of "Satisfy(a, a, Knowif(a, done(o, act)))" action. Of course, the notification action is not in the decomposition of the domain-level action "act". So, if we want to find a connection between $A_{\text {old }}$ (the occurrence of "Other-act") and $A_{\text {new }}$ we cannot rely only on the links between actions in the object-level libraries, but we must make a different move on the AM library in order to find $A_{\text {old }-x}$ and then let the search be based on it.

We have an example of this case in dialogue 4.3.1, where an agent $A$, after an utterance, makes a request in order to know whether his partner has understood or not ("so there's an engine at Avon, right?"). The action of requesting a notification is not in the body of the "Update" object-level action, which is the argument of "Other-act" ("Other-act(A, A, Update $(\mathrm{B}, \ldots))$ "). So, in order to connect $A_{\text {new }}$ ("right?") the system must first expand the "Other-act" action and reach $A_{\text {old }-x}$ ("Satisfy (A, A, Knowif(A, done (B, Update $(B, \ldots)))$ )") and then it can search for a connection between $A_{\text {new }}$ and $A_{\text {old }-x}$.

3. A third case concerns the direct interplay of the roles of the two agents, as shown in Figure 16.3.

Here, there is a new link, shown in the figure as a thick arrow. It is the link representing the fact that an agent is meeting a goal of his partner. In this case, we have the inverse problem as in the previous case: there is no difficulty at the object-level, since the action executed by the first agent is a substep of the action requested by the partner (a standard decomposition chain), but from the Agent 


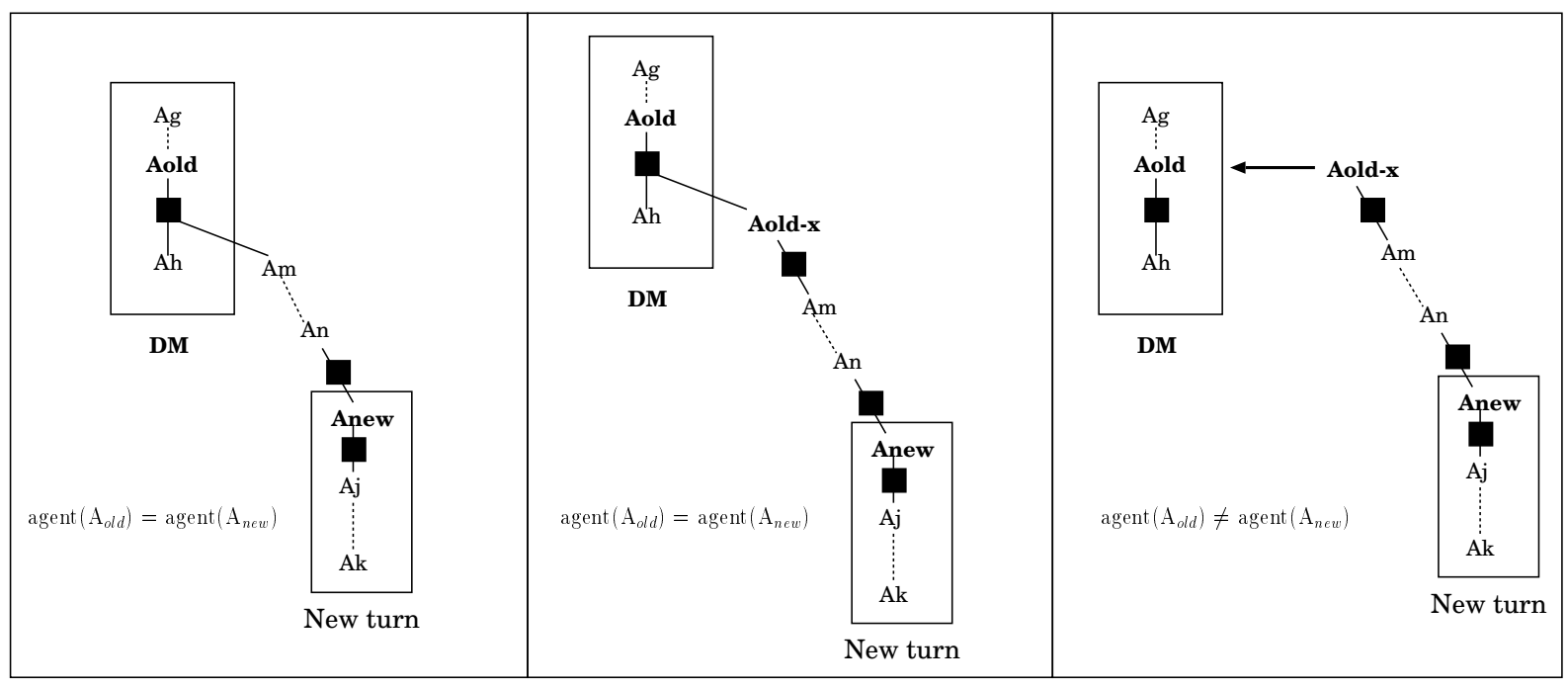

Connection type 1

Connection type 2

Connection type 3

Figure 16. Three connection types.

Modeling point of view the actions $A_{\text {old }}$ and $A_{\text {old }-x}$ must be included in two different subspaces, each concerning one of the two agents.

\section{- Search for a connection}

Although a connection has to be found between two Agent Modeling nodes, it is clear that the AM library does not provide us with much information about the possible relations between actions. So, only the object-level libraries can guide the search. For instance, if we know that, in the Upward Expanded Structure, $A_{\text {new }}$ is "Try-execute(U, U, act1)" while, in the Dialogue Model, $A_{\text {old }}$ is "Try-execute(U, U, act2)", no connection between the two actions can be imagined, unless we know which are "act1" and "act2". But even looking for an object-level path is by itself a computationally expensive process. This problem can be mitigated by a precompilation step, where all the paths are pre-computed by inspecting the (object-level) libraries. We say that the problem is just mitigated, but not solved, because paths can only be used to exclude the possibility of a connection; but if a path is found, it must still be checked in order to verify that the actual parameters (of the object-level actions) satisfy the constraints expressed in the actions along the path.

So, the basic knowledge sources for finding a connection are two sets of compiled paths, the first one concerning the Speech Act library, and the second one concerning the Domain Library. For instance, in the first set all the possible paths are stored connecting a "Request" action to a "Surface-Question", while in the second one we can find all the paths connecting "Take-university-exam" to "Enter", or to "has(agt,pass(lab))" (see Figure 5). Note that in the last case we have a path between an action and a condition; they are also stored in the compiled knowledge base. All these paths are pre-computed starting from the defined libraries. 


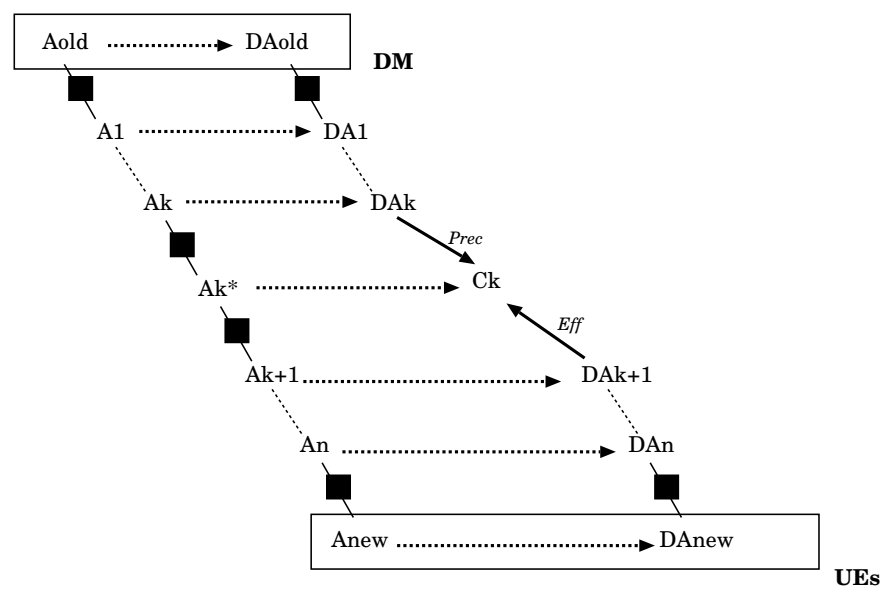

Figure 17.

Although the pre-compiled paths suggest the connections described in the previous subsection, we have extended the compilation mechanism to account for preconditioneffect relations. This is described in Figure 17. Differently from the previous figures, we have made explicit here the relations between the Agent Modeling actions and the object-level actions. It is possible to see that the AM path is a standard decomposition path, while the object-level one includes a precondition-effect link between the object-level action " $D A_{k}$ " and the object-level action " $D A_{k+1}$ " (via the condition " $C_{k}$ "). Of course, the Agent Modeling action " $A_{k *}$ " must be an instance of "Satisfy $(\ldots)$ ", in order to make explicit the fact that the AM plan has been developed considering the need to achieve the condition " $C_{k}$ ".

If the planning activity of the system were based exclusively on stored recipes, the need to account for effect-precondition connections would not arise. But we admit that in some cases the user enters a real planning behavior; ${ }^{30}$ in such a case, a sequence of actions is not justified on the basis of an existing recipe, but on the more general basis that, in order to perform an action, the agent has to make its preconditions true, and so he has to execute some action to make them true. The path shown in Figure 17 accounts for this situation, from the perspective of the recognition activity. An observed action is accepted as coherent with the previous interaction if it aims at making true a condition enabling the execution of a subsequent action that is part of the previously recognized plans.

It must be observed that the various complex connections described in this subsection and in the previous one are "orthogonal", in the sense that they may combine with each other freely. For instance, we can have a path including an effect-precondition connection (Figure 17) aimed at satisfying a goal of another agent (see Figure 16.3) not yet explicitly present in the Dialogue Model (Figure 16.2).

\footnotetext{
${ }^{30}$ See Young et al. (1994) for a planner which interleaves causal planning and action decomposition.
} 
However, since the precompilation of paths is made inside the libraries, the paths do not mix domain-level and linguistic-level actions. So, assuming that the executed action is the utterance act $U A_{\text {new }}$ (but nothing changes if it is non-linguistic), and that the current focus is a previous utterance act $U A_{\text {focus }}$, then a linguistic path is searched, which connects $U A_{\text {new }}$ and $U A_{\text {focus }}$. If the path is not found, then $U A_{\text {new }}$ is "upward expanded", and the search is repeated, using the compiled paths, starting from its parent(s).

If the search succeeds within the linguistic library, we obtain something which may be seen as a purely linguistic behavior. Examples of this situation are direct answers to questions. For instance, the connection between "what is your name?" and "Mario Rossi" in Example 3.1 (see Figure 18) is found at this level. In fact, it is an instance of a connection of type 3 (see Figure 16.3) where $A_{\text {old }}$ is "Obtain-info(C,U,name(U,name))", $A_{\text {new }}$ is "Utterance-act(U,C, "Mario Rossi")" and $A_{\text {old }-x}$ is the inferred goal of "Obtaininfo" (i.e. "Inform(U,C,name(U,name))", not shown in Figure 18).

But if no such connection can be found, then the Upward expansion reaches the top of the Speech Act library and enters the domain library. In the current implementation, we have assumed that no path can be found between an action in the domain library and a (focused) action in the SA library. So, the focus is moved up to the nearest domain node in the DM, and the search continues on the basis of the same algorithm, but applied now to domain actions.

\section{- The role of the AM library}

Much of what has been described above can be accomplished without adding the complexities of the AM library. Consider, for instance Figure 14.b. According to the described algorithm, there is no way to find a connection to

"Try-execute(U,U,Utterance-act(U,C,'Could you register ...')"

unless you can find exactly the same connection to

"Exec(U,Utterance-act(U,C,'Could you register ...')"

or to

"Perform-body-step(U,U,Utterance-act(U,C,'Could you register ...')"

So why are three different nodes useful in the AM part of the discourse model? The relevant cases depend on the fact that the AM library models the reactive behavior of agents who know that actions may fail. In particular, the recipe of the "Satisfy" action (see Figure 7) includes a "Cycle-do-action" that is repeated until a plan for achieving the goal $g$ has succeeded (see the $\neg g$ conjunct in the loop condition). The achievement of $g$ is tested in the second step of both "Self-act" and "Other-act". What is implicit in the figure is that when "Knowif(a,done(agent,act))" (where agent can be $a$ or $o$ ) is satisfied, the (possible) falsity of "done(agent,act)" is assumed to imply that $g$ is false, so that the upper loop enters a new cycle, in order to apply a different recipe for achieving $g$. 
At the AM level, all these actions appear explicitly, so that "Satisfy(a,a, Knowif (a,done(agent,act)))" is a node different from "Try-execute(a,s,act)", even if they refer to the same domain action act. This enables the system to keep apart the meta-level action of verifying the success of an action from its execution. The recognition of the actual situation is accomplished by the algorithm described above on the basis of the recipe associated with the Knowif goal. In the same way, the AM level enables the system to observe and recognize the activity of forming plans ("Find-plans"), of notifying a partner about the success of an action (the optional "Satisfy(a,a,Bel(s,done(a,act)))"), and so $\mathrm{on}^{31}$. An example of the structure resulting in these cases appears at the bottom of Figure 18. In this example, the final "OK" has been interpreted as an anticipation by $C$ of the (presumed) next $U$ 's subgoal of knowing if $C$ got the name, i.e. if the associated "Inform" linguistic action succeeded.

\subsubsection{Best paths}

In some cases, a new turn can be connected to the previous context in multiple ways; this fact means that there are different possible reasons why the new input is coherent with the discourse. In these cases, there are two alternatives: either one keeps all the hypotheses, by duplicating the Dialogue Model as many times as there are possible connections, or one makes a choice, preserving only the "most reasonable" connection and discarding all the other ones. The first alternative is computationally unfeasible, because an unmanageable number of different contexts should be maintained. The second alternative is more practicable, but it requires some means to choose the best hypothesis.

Various methods have been proposed in the literature to cope with this problem. For example, the model described in Carberry (1990a) and the one in (Bauer 1995) are based on the Dempster-Shafer theory of evidence, whose main advantage with respect to classical probability theory is its ability to keep apart uncertainty and ignorance. Although both approaches are interesting in providing the basis for modeling the process of choice, it can be observed that Carberry (1990a) allows just for local decisions, without describing a method for combining evidence coming from different sources. Instead, Bauer shows how such a combination can be carried out, but his method is not linked to any architecture for dialogue modeling. Furthermore, both approaches are concerned only partially with the basic difficulty of Dempster-Shafer's theory, i.e. the difficulty of assessing the Basic Probability Assignment (see however Bauer (1996)).

We have not faced the problem in depth, so we decided to approach this particular aspect of the overall architecture in the simplest way. This choice is also due to the fact

\footnotetext{
${ }^{31}$ The current content of the AM library is suitable for recognition (the task currently addressed), but some extra machinery would be required for using it in generation. In particular, it specifies that each executed action must be followed by a check about its success (i.e. that its expected effects actually hold). This is clearly unreasonable for many actions, such as the linguistic ones
} 
that our architecture makes the problem a bit harder: in fact, we have to take into account two sources of ambiguity: the first one is the well known ambiguity inherent to Domain Knowledge (I can want to go to the library either to get a book or to get a certificate that I am an authorized visitor of the library), or to Speech Act Knowledge (I can ask you if you can raise your hand either as a request of doing so or as an inspection about your current abilities - if I am a physician visiting you). But ambiguity arises also from the Agent Modeling Library; in particular, any action concerning a precondition can pertain to the planning phase or the execution phase (I can ask you where is the library for two reasons: either because I have already decided that I want to borrow a book and I need to know about its location - I'm just continuing the execution of a plan - or because I am considering different possibilities - the first one is to borrow the book from the library, but I'm ready to find an alternative plan if the library is too far away).

Our main choice has been to interrupt the process of finding a connection as soon as a solution has been found. This means that the most local coherent interpretation of the last turn is privileged (i.e. the one nearest to the current focus). However, even with these hard-wired preferences, some ambiguity remains. In fact, different paths could link the same action of the Upward Expanded Structure to the same action of the Dialogue Model. These paths are related to the ambiguities discussed in the previous paragraph. In these cases, we resort to a simple probabilistic evaluation. First, we must specify which is the "most local coherent interpretation of the last turn" (MLCI). We define the set of MLCI's, as the set of instances of "Satisfy" reachable from the "Observed Action" without traversing, during the Upward Expansion process, any other "Satisfy".

Notice that the locality is not evaluated as the number of steps required to reach a "Satisfy", but the measure unit is the number of "Satisfy" encountered. However, the various "Satisfy" in the MLCI have to be weighted against each other. This would involve a combination of the evidence of the different steps of Upward Expansion. In order to avoid that, we assumed that each Upward Expansion step can take advantage of a local "probability" of the possible alternatives, so that a depth first search can be adopted.

Let's suppose that $A_{n e w}$ is Upward Expanded, so that $A_{n e w_{1}}, A_{n e w_{2}}, \ldots, A_{n e w_{n}}$ are reached, with probabilities $p_{1}, p_{2}, \ldots, p_{n}$. If $p_{k}$ is the maximum of all $p_{i}(1 \leq \mathrm{i} \leq \mathrm{n})$, then $A_{n e w_{k}}$ is used for the next Upward Expansion step. When the reached action is an instance of "Satisfy", then a connection is looked for. If it is found, then the process is completed, otherwise, a backtrack occurs to the last choice point (most recent branching point), and another expansion is tried, choosing the hypothesis with highest probability, among the remaining ones. Of course, according to the basic algorithm, the search for a connection path from any "Satisfy" identified as described above starts from the current focus, so that the most local contextual connection is the first one found.

However, there still remains a possible ambiguity: multiple paths can lead from a 
given "Satisfy" in the Upward Expanded Structure to the same action in the Dialogue Model. This means that the attempt of an agent to make true a given condition (i.e. the argument of "Satisfy(...)") can be coherently interpreted in different ways. For instance, the attempt to satisfy a "Knowif(a, condition)" can be justified as a precondition check during plan construction or as the check done after each action in a reactive planner (assuming that the previous context has not already determined if the agent is in the phase of planning or execution). In these cases we simply multiply the probabilities of the single elements of a path, in order to obtain its overall score. This very rough approach is justified at least in two respects:

1. The product operator decreases the overall score for long paths; if, in general, this is a drawback, since it does not provide a good balance among the alternatives, in our case this is acceptable, because it seems reasonable to favor the shorter paths.

2. The product is a correct operation to get the probability of a joint event $A B$, just in case $A$ and $B$ are independent. Otherwise, you have to use conditional probabilities. However, the probabilities stored in our plan libraries are conditional probabilities. In fact, if the action $A$ is declared in the library to be a possible step of $A_{1}, A_{2}$, $\ldots, A_{n}$, with probabilities $p_{1}, p_{2}, \ldots, p_{n}$, what is said is that $\mathrm{p}\left(A_{i} / \mathrm{A}\right)=p_{i}$, and not that the a-priori probability of $A_{i}$ is $p_{i}$. Of course, this does not generalize to paths longer than two steps, so that we must accept approximate values in these cases. Apart from the last observation, there also is the problem of determining the correct values for the probabilities, a problem which is common to most approaches, and which we have not faced: we assigned the values according to our intuition just for testing the model.

\subsubsection{Extending the Dialogue Model}

When a connection is found, and it has been chosen as the best one, then it has already been checked that all constraints on the parameters of the action instances are respected. This check is carried out by building an instantiation of the whole path. This means that the real path is now available as a side-effect of the process of consistency verification. During this process, the variables that were left unbound in the Upward Expansion get their values on the basis of the contextual link. Nothing more is required, except for moving the instantiated path inside the Dialogue Model. This step involves:

- the update of an index that records which actions are part of the Dialogue Model.

- the update of the "Current Focus" of the Dialogue Model, which is set to the observed action ("Exec") of the new turn of the dialogue. 


\subsection{The first turn: Upward Expansion without Focusing}

When the first input is analyzed, the Dialogue Model is empty, so that the interpretation process cannot be guided by it. However, the mechanism of Upward Expansion already provides the guidelines for facing the problem. In fact, the "Observed Action" can be Upward Expanded exactly as explained in Section 6.2.4, i.e. via a shaded depth-first search driven by the conditional probabilities that an action serves as a step for a possible super-action; the only problem is when to stop the search. We believe that this is a rather hard problem in a general Knowledge Base of actions, but within a restricted domain there exists some top-level goal (e.g. graduating) where the upward movement is stopped necessarily. So, we let the Upward Expansion to reach the top of the Domain Hierarchy, and the obtained Upward Expanded Structure becomes the initial Dialogue Model.

\subsection{The system implementation}

The three plan libraries and the interpretation algorithm described in the previous sections has been fully implemented; our system is written in Lucid Common LISP and runs under the Unix operating system.

While, in order to identify the linguistic phenomena described in this paper, we have analyzed some corpora of task-oriented dialogues, ${ }^{32}$ we have not tested our system on such corpora: the main reason is that, to understand such dialogues, we should have introduced in the system a huge amount of knowledge concerning other domains. In order to avoid this effort, we have preferred to select out of them a set of significant examples, containing the linguistic phenomena of interest to us, and adapt them to our University domain; in this way, we could feed the examples to our system. We have paid special attention to include in the dialogue set all the alternative user reactions to dialogue turns which we have discussed in Section 4. In this way, we could check that the system is able to recognize the intentions underlying dialogue, highlighting the fact that, although different continuations of a dialogue are possible, many of them can be reduced to the same set of high-level intentions.

Our system can correctly interpret almost all the examples reported in Section 4; the only exceptions are:

- the instances of "offers", which we have reported as interesting linguistic examples, but we do not handle in our dialogue model (e.g. consider Example 4.2.7.b). This limitation is due to the absence in the Speech Act library of an action suitable for modeling this behavior. In particular, it is not clear whether offers can be modeled

\footnotetext{
${ }^{32}$ For instance, we have analyzed some extracts from the "Trains" corpus (Gross et al. 1993); an airlines corpus (Transcripts 1992) and some Italian corpora (de Mauro et al. 1993, Gavioli \& Mansfield 1990).
} 
as specializations of "Get-to-do" (as it is currently the case for all the speech acts), it requires the introduction of a more general top-level action in the Speech Act library.

- the examples where agents suggest alternative ways of action to their interlocutors (e.g. consider the second part of Example 4.2.1.a). In particular, in our framework, an agent can identify a plan to suggest to his partner by entering the "Find-actions" and "Choose-action" steps of the "Satisfy" Agent Modeling action. However, suggestions may occur in various situations: our model covers the cases where the agent is required to suggest a way to achieve a goal; on the other hand, it currently does not cover the autonomous acquisition of the goal to monitor the actions performed by another agent and possibly suggest a better way to achieve his goals. The treatment of such behaviors requires that supplementary goals of the interactants are modeled.

Although in this paper we have focused on speech acts, it must be noted that our system can receive as input both NL (Italian) sentences, and observations relative to the execution of domain-level actions: these action instances can be introduced from the keyboard in the internal representation used in the system (e.g. "Register(C, U, MLexam)"). So, the system also processes examples like 4.1.1.b: "the consultant checks on the computer whether the user is in the enrollment list" (page 13). ${ }^{33}$

Figure 18 shows the interpretation context which our system builds for the dialogue:

\section{Example 3.1:}

$\mathrm{U}$ : "Could you register me for the ML exam?"

C: "What's your name?"

$\mathrm{U}$ : "Mario Rossi"

C: "OK, you are registered."

Since most of the interpretation details have already been discussed in the previous sections, we will not repeat them here. It is only important to note that the notification ("OK") by agent $C$ is interpreted as "Inform $(\mathrm{C}, \mathrm{U}$, done(C, act))", where act is a free variable which has been bound after the focusing step, i.e. after the system finds that the most local pending goal was "Satisfy(U, C, Knowif(U, done(C, Update(C, name(U, "Mario Rossi")))))".

\footnotetext{
${ }^{33}$ When the system receives an action instance as input, it processes it in the same way as it happens to an "Utterance-act".
} 


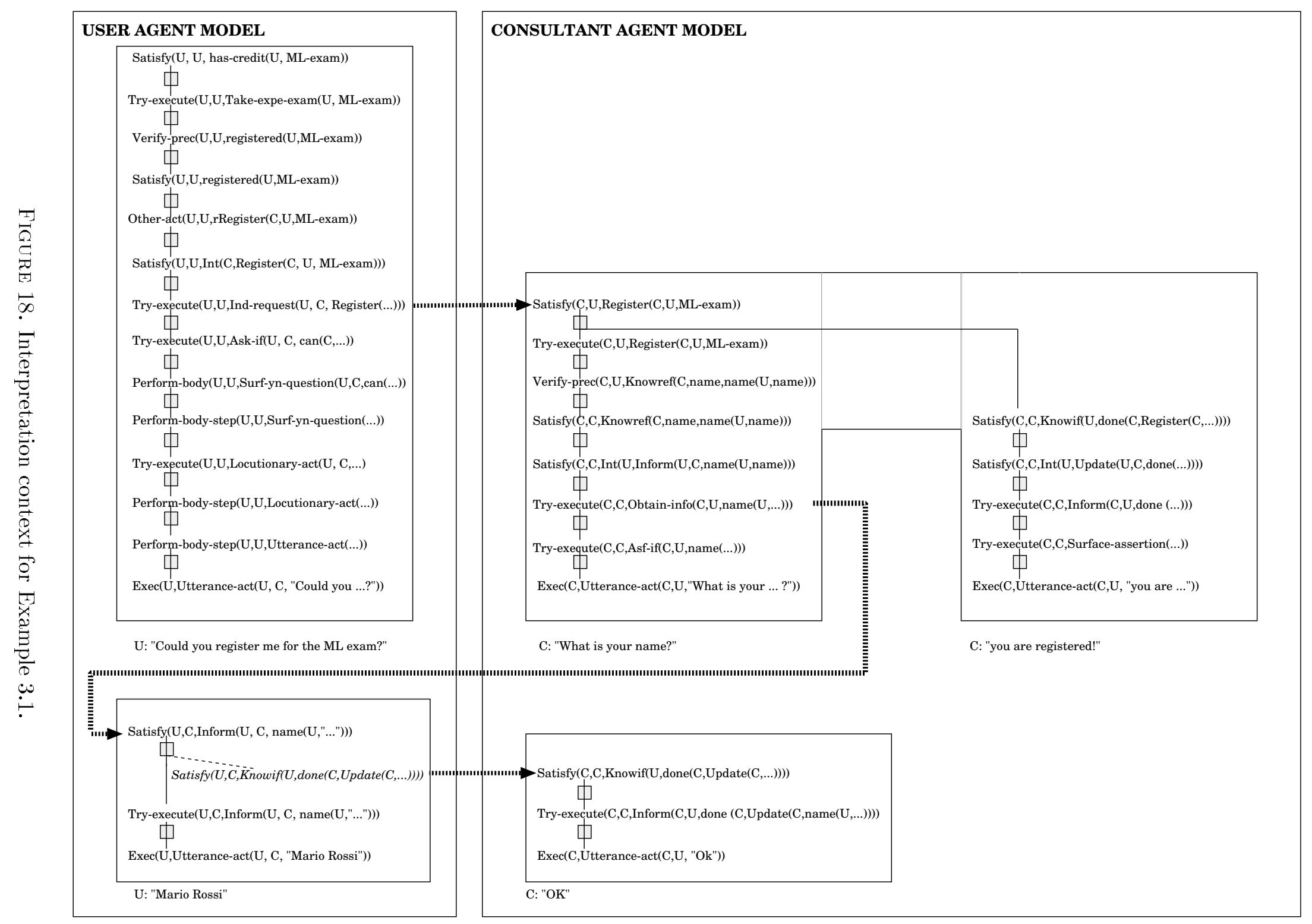




\section{Related work}

In several models of dialogue, the coherence of an agent's behavior has been related to a continuation of domain-level activity by introducing heuristic rules associated with the most plausible shifts in the focus of attention of agents, during a typical (task-oriented) interaction (Carberry 1990b, Litman \& Allen 1987). In particular, Carberry's model explains the coherence of an information-seeking dialogue with the reference, within all the turns, to the steps of a single, high-level domain plan of the information seeker, admitting smooth changes of the focus of attention. In our model, we refine this notion of coherence by identifying in which way the new actions in an interaction relate with the context: as in Carberry's approach, we identify the relation between the new goals and the ones already appearing in the context by looking for dependencies among goals. However, beyond the cases where an agent simply continues to execute one of his own plans, we treat homogeneously the turns where an agent adheres to the partner's overt goals or adopts one of the partner's identifiable (but not stated) goals.

The work of Litman \& Allen (1987) introduces a metalevel of plan operators, called "Discourse plans" in order to model types of subdialogs that cannot be accounted for by considering only domain plans. In particular, the discourse plans specify how an utterance can relate to a discourse topic, where the discourse topic is represented by the objectlevel plans. The same plan recognition techniques are applied both to metalevel and to object-level plans and the discourse context is represented as a stack of plans and they follow the stack paradigm of discourse coherence.

Litman and Allen's goals are different from ours. They aim at modelling how people communicate with each other about plans. So, their discourse-level actions specify only how people talk about plans, and how the speech acts can be used to affect the performance of the plan. Consequently, they are not interested in specifying how the plan referred to in INTRODUCE-PLAN was built, or why an agent decided to insert a new step in a plan (CORRECT-PLAN). In particular, the plans do not say why, given that an agent intends to execute a plan, he decides to execute an INTRODUCE-PLAN: they aim at establishing the coherence of a given sequence of linguistic actions, but not at generating that sequence.

On the contrary, in our approach, speech acts are considered as a support for carrying out domain plans, not as means to talk about them. And our meta-level plans express coherence in terms of planning, not in terms of plan recognition: the behavior of an agent is coherent when it conforms to rational ways to plan and execute actions. So, a request is coherent just in case an agent could have planned it for achieving his goals; and what an agent can plan is specified in the AM library.

On the other hand, Litman \& Allen (1987) anticipate our work in that some linguistic 
behaviors related to dialog coherence are modeled by the metalevel activity; in particular, the "Continue-plan" operator corresponds in our model to goal adherence and plan continuation, and "Track-plan" models acknowledgments after the execution of a domain action.

Another proposal strictly related to the one presented in this paper appears in Lambert (1993) and Carberry et al. (1993): however, our representation of speech acts is different in that we treat in a uniform way domain-level and linguistic actions. In their model, "the domain level represents the system's beliefs about the user's plan for achieving some goal in the application domain. [...] The discourse level captures the system's beliefs about each agent's communicative actions and how they contribute to one another" (Carberry et al. (1993), page 2). This leads them to build multiagent discourse plans, characterized by recipes where different speakers perform linguistic actions in an interleaved way. Instead, in our model only linguistic single-agent plans are considered. Since these plans justify the coherence of the dialogue, we do not need to specify separately the admissible sequences of turns.

As discussed in Section 2, Lochbaum (1994) and Cohen \& Levesque (1991) include dialogue phenomena in the wider perspective of interaction; however, there seems to be some dishomogeneity in their treatment of linguistic actions. In particular, in Lochbaum's work, the role of speech acts is not always described as a collaborative activity: for example, the occurrence of notifications is modeled by means of specific steps of the main loops for modeling agent behavior and for recognizing the other agents' behavior.

Although Smith \& Cohen (1996) explain notifications in a more general way, via the notion of Joint Intention, their treatment of speech acts still keeps apart linguistic actions from domain-level ones: in fact, they define speech acts in terms of attempts. They explain that a specific goal of a communicative act is to alter the receiver's mental state, in order to make him act in a desired way; but, of course, there is no certainty that the Speech Act will succeed, so the speech act is an attempt.

In our framework, we model the different goals pursued in the execution of a speech act with the "Get-to-do" and "Illocutionary-act" actions of our speech act library. In particular, while "Get-to-do" models the action which aims at making the partner intend to act, the illocutionary act establishes Smith and Cohen's "minimal success condition" (that the interactants share the belief that the speaker wants the hearer to act accordingly); finally, an illocutionary act has associated the means for performing it by means of a locutionary act. Given this structure of linguistic action, our solution enables us to reduce the planning and execution of linguistic actions to the same process of planning and executing any other actions; at the same time, the "attempt" nature of speech acts is modeled in that all these actions are performed by means of metalevel actions modeling 
a reactive planner. Note that the failure to persuade, by means of a given speech act, the hearer to perform an action, or to believe a proposition, corresponds to an unsuccessful execution of the speech act: in this case, some other action must be planned to recover from the failure. Our approach is able to manage the reported phenomena uniformly with respect to domain actions; moreover, it is consistent with the classical work on politeness (Brown \& Levinson 1987), which opens the discussion on politeness by assuming that speakers are capable of doing means-end reasoning.

In contrast to the multi-level approach to dialogue processing, Grosz, Sidner and Lochbaum only use domain-level plans to model collaborative dialogue (Grosz \& Sidner 1986, Lochbaum et al. 1990, Lochbaum 1991, Lochbaum 1995). We agree with Lochbaum that it is better not to introduce multiple plan types, but:

- Although we have three plan libraries, only the object-level and the metalevel plan libraries have functionally distinct roles.

- Our Agent Modeling plans aim at modeling intentional behavior in terms of a reactive planner model. So, they play a role similar to the Shared Plan operator in Lochbaum's framework. Although they may look more complex than the Shared Plan operator, they describe declaratively the whole knowledge for modeling behavior and they are the only responsibles for updating the agent's intentional state. On the contrary, in Lochbaum (1994), the Shared Plan operator represents a static notion of "having a plan"; so, the "augmentation" and "interpretation" processes are in charge of modifying an agent's intentional state (and of recognizing those changes, respectively). These processes work procedurally and model the occurrence of all the types of acknowledgements and disagreement statements.

Also Heeman \& Hirst (1995) consider dialogue as a goal-directed collaborative activity among agents. They propose a plan-based model for the negotiation of the meaning of referring expressions; in their model, the speakers can perform the same actions, so that the same plan operators account for how utterances are generated and understood. Heeman and Hirst propose a two-tier framework where the plans for creating referring expressions are arguments of meta-actions which describe the process of establishing the meaning of such expressions and resolving interpretation problems by means of negotiation subdialogues.

Although we don't focus on referring expressions, in our framework we manage the difficulties in the interpretation of utterances (e.g. ambiguities and unknown meanings) as any other problem arising in the process of the execution of object-level actions under the control of the Agent Modeling plans; in particular, these plans establish the goal of reacting to possible failures in action execution, from which negotiation can be derived.

The feasibility of the approaches to dialogue based on intention recognition is chal- 
lenged in McRoy \& Hirst (1995): they claim that these models lead to an unconstrained search for higher-level intentions to explain a turn; therefore, they advance the idea of relying on linguistic expectations and linguistic intentions to limit the search space. The linguistic expectations are based on the idea that any speech act raises an expectation on how the partner will react to it; so, they capture the notion of adjacency pairs; the linguistic intentions are more general, but they are still strictly tied to conventional behavior. McRoy and Hirst exploit this solution in a model for detecting and repairing to misunderstandings in dialogue. In their system, the presence of a misunderstanding is hypothesized, when a deviance from the expected behavior occurs or when the beliefs of a speaker look contradictory. They can manage the defeasibility of these expectations because their system is based on a framework supporting default and abductive reasoning. Although McRoy and Hirst's approach is one of the most akin to ours, it can be noted that we do not require that linguistic expectations are represented as separate knowledge: they derive from the fact that linguistic actions are considered as means for making partners act. Therefore, expectations appear in the dialogue context as low-level goals inferred during the recognition of speech acts. When a new turn can be interpreted as aiming at the satisfaction of such low-level goals, there is no need to proceed in searching for higher-level goals that might explain it.

The finite-state automata approaches to dialogue have been usefully exploited in applicative domains. However, as Stein \& Maier (1994) shows, these dialogue protocols alone are not sufficient to model dialogue: in fact, they introduce other devices, like dialogue scripts, to model the various phases of an information extraction dialogue, like the system's request to refine a user's query to constrain the search. In some way, their scripts merge object-level and problem-solving behavior and this fact makes them suited to model dialogues arising from fixed activities, but too specific to model general natural language interactions. Moreover scripts are not particularly suited to cope with mixed initiative dialogue, because they enforce the asymmetry between the roles of the system and of the user in human-computer dialogue.

In our framework, we model the double nature of collaboration pointed out in Airenti et al. (1993) and Traum \& Allen (1994): it is not necessary that the partner cooperates at the high-level goal concerning the planning - executing activity on domain actions; in fact, he can be engaged in conversation simply by collaborating to a goal related with the planning - execution of linguistic actions, i.e. to the conversation itself. For example, the cooperation can be limited to informing the partner that the speaker does not intend to cooperate with him at the domain level. In this way, the two cooperation forms are explained by the same model for understanding dialogue.

It must be noted that the structure of the Agent Modeling plans allows us to de- 
scribe in an explicit way the rationale behind the plan recognition heuristics introduced in Allen (1983) to identify the goals underlying an agent's behavior. Consider, for instance, the following rule: ${ }^{34}$

Precondition-Action rule:

$\operatorname{SBAW}(\mathrm{p}) \supset \operatorname{SBAW}($ act $)$, if " $p "$ is a precondition of "act"

In our model, we represent this inference by the fact that the "Try-execute" action has the "Verify-prec" step, where the preconditions of the action are checked and the agent may act to obtain them, if needed. So, if the system observes that the agent verifies a condition, it can hypothesize that the agent is trying to perform an action which has that condition as a precondition.

Allen's work also includes rules which have the opposite role with respect to the rules for goal recognition: e.g. the Action-Precondition rule (supporting inferences from the intention to perform an action to the intention that the action preconditions are satisfied) and the Action-Body rule. Similar inferences can be obtained by a generation process able to exploit our plan libraries to produce agent behavior.

\section{Conclusions}

We have described a model of linguistic interaction where dialogue is treated as a form of collaborative interaction among agents that behave directed by their beliefs, goals, and intentions.

We consider a dialogue coherent if any new turn contributes to some goal occurring in the previous part of the dialogue. In particular, the relation of a turn with the previous context can be of adherence (an agent tries to satisfy a goal explicitly set forth by one of his partners), adoption (an agent tries to satisfy a goal of a partner that the agent has inferred) or continuation (an agent proceeds in the attempt to reach his own goals).

In our framework, the representation of dialogues consists of a set of plan instances, which describe the activity of the interacting agents, connected via links that make explicit the relationships between the various turns.

In order to form plans, to recognize the plans being carried on by a partner, and to establish the proper coherence links, a set of known recipes is available. These recipes are grouped in three libraries: the Domain library, the Speech Act library, and the Agent Modeling library. In the paper, we argue that the introduction of the third library enables our model to account in a perspicuous way for a number of apparently unrelated linguistic phenomena that, in other approaches, require ad hoc treatment.

\footnotetext{
${ }^{34}$ In the formula, the SBAW notation means that the system believes that the agent A wants to obtain a condition, or to perform an action.
} 
However, some assumptions limit the power of the model. The most important of them concerns the hypothesis that all agents behave according to plans known to all of them: an agent is able to understand (i.e. to realize the coherence of) a turn of a dialogue just if it results from the execution of one or more recipes known to him. Some flexibility is given by the fact that the Agent Modeling plans represent a reactive agent that can achieve action preconditions by replanning subgoals. However, some works, like Lesh \& Etzioni (1995), have faced this problem in a more general way.

Moreover, while we currently represent interactions among agents who perform singleagent actions, we do not model more complex cases of cooperation, like multiagent plans. We hope this second restriction does not sound too limiting, since our work is focused on linguistic actions, which are typically single-agent (moreover, the problem of shared plans has already found satisfactory solutions as, for example, in Grosz \& Kraus (1996)).

The system described in this paper is focused on the interpretation of other speakers' turns; therefore, we have not addressed some aspects concerning the generation of turns, like for example those considered (Moore 1995, Chu-Carroll \& Carberry 1998). Most noticeably, in this work we do not distinguish goals from intentions; in fact, in the interpretation perspective, the system's role is just to recognize the intentions that lead the speakers to produce a turn; since the turns produced by the partner are the result of attempts to reach goals to which he has already committed, this problem is somewhat hidden.

In some more recent work, we have extended the model described in this paper to obtain a system that can recognize other agents' plans, as well as take the initiative to act on the basis of its own goals. In particular, in Ardissono et al. (1999b), we have considered the reasons underlying the selection of direct or indirect speech acts, on the basis of their characteristics, described in the Speech Act library. Morever, in Ardissono et al. (1999a), we have examined the motivations leading an agent to cooperate at the behavioral and conversational level, and we have provided an alternative solution to the obligation-based approaches to dialog (e.g. see Traum \& Allen (1994)). The concept underlying both works is that the speaker's behavior is directed by the desire to respect social goals, like avoiding that the interlocutor is offended (see Brown \& Levinson (1987)). Finally, the model of cooperation among agents has been refined in Boella et al. (1999), where a decision theoretic planning framework is exploited to allow an agent decide when it is worthwhile that he adopts some of the partner's goals and communicates with him to coordinate the shared activity.

\section{Acknowledgments}

We are grateful to the anonymous IJHCS reviewers who have helped us to improve substantially the first drafts of this paper, and to Sandra Carberry and Mathias Bawer, who have read 
the paper carefully to provide further useful comments to it. We also thank Rossana Damiano for having contributed to the implementation of a portion of our prototype and for having provided useful comments on the paper.

This work was partially supported by MURST $60 \%$ and by the Italian National Research Council (CNR), projects "Pianificazione e riconoscimento di piani nella comunicazione" (Planning and plan recognition in communication) and "Conoscenza, Intenzioni e Comunicazione" (Knowledge, Intentions and Communication).

\section{References}

Airenti, G., Bara, B. \& Colombetti, M. (1993). Conversational and behavior games in the pragmatics of discourse. Cognitive Science, 17:197-256.

Allen, J.F. \& Perrault, C.R. (1980). Analyzing intention in utterances. Artificial Intelligence, $15: 143-178$.

Allen, J.F. (1983). Recognizing intentions from natural language utterances. In Brady, M. \& Berwick, R.C., Eds. Computational models of discourse, pages 107-166. MIT Press.

Ardissono, L., Lesmo, L., Pogliano, P. \& Terenziani, P. (1991). Representation of determiners in natural language. In Proc. 12th IJCAI, pages 997-1002, Sydney.

Ardissono, L., Lombardo, A. \& Sestero, D. (1993). A flexible approach to cooperative response generation in information-seeking dialogues. In Proc. 31st Annual Meeting $A C L$, pages 274-276, Columbus.

Ardissono, L., Boella, G. \& Lesmo, L. (1995a). Indirect speech acts and politeness: A computational approach. In Proc. 17th Cognitive Science Conference, pages 316-321, Pittsburgh.

Ardissono, L., Boella, G. \& Lesmo, L. (1995b). Recognizing preliminary sentences in dialogue interpretation. In Lecture Notes in Artificial Intelligence n. 992: Topics in Artificial Intelligence, pages 139-144. Springer Verlag, Berlin.

Ardissono, L., Boella, G. \& Lesmo, L. (1996). Recognition of problem-solving plans in dialogue interpretation. In Proc. 5th Int. Conf. on User Modeling, pages 195-197, Kailua-Kona, Hawaii.

Ardissono, L., Boella, G. \& Damiano, R. (1997). A computational model of misunderstandings in agent communication. In Lecture Notes in Artificial Intelligence n. 1321: Advances in Artificial Intelligence, pages 48-59. Springer Verlag, Berlin.

Ardissono, L., Boella, G. \& Damiano, R. (1998). A plan-based model of misunderstandings in cooperative dialogue. International Journal of Human-Computer Studies, $48: 649-679$.

Ardissono, L., Boella, G., Damiano, R. \& Lesmo, L. (1999a). Conversational cooperation: the leading role of intentions. In Proc. of the AMSTELOGUE'99 Workshop on Dialogue, Amsterdam.

Ardissono, L., Boella, G. \& Lesmo, L. (1999b). Politeness and speech acts. In Proc. Workshop on Attitude, Personality and Emotions in User-Adapted Interaction, pages 41-55, Banff, Canada.

Ardissono, L. \& Sestero, D. (1996). Using dynamic user models in the recognition of the plans of the user. User Modeling and User-Adapted Interaction, 5(2):157-190.

Austin, J.L. (1962). How to Do Things with Words. Harvard University Press, Cambridge, Mass. 
BAuER, M. (1995). A dempster-shafer approach to modeling agent preferences for plan recognition. User Modeling and User-Adapted Interaction.

Bauer, M. (1996). Acquisition of user preferences for plan recognition. In Proc. 5th Int. Conf. on User Modeling, pages 105-111, Kailua-Kona, Hawaii.

Boella, G., Damiano, R. \& Lesmo, L. (1999). Cooperating to the group's utility. In Proc. of The 6th Int. Workshop on Agent Theories, Architectures, and Languages (ATAL-99), Orlando, Florida.

Bratman, M.E., Israel, D.J. \& Pollack, M.E. (1988). Plans and resource-bounded practical reasoning. Computational Intelligence, 4:349-355.

Bretier, P. \& SADEK, D. (1997). A rational agent as the kernel of a cooperative spoken dialogue system: implementing a logical theory of interaction. In Muller, J.P., Wooldridge, M.J. \& Jennings, N.R., Eds. Lecture Notes in Artificial Intelligence $n$. 1193 Intelligent Agents III, pages 189-203. Springer.

Brooks, R.A. (1991). Intelligence without reason. In Proc. 12th IJCAI, pages 589-595, Sydney.

Brown, P. \& Levinson, S.C. (1987). Politeness: some universals on language usage. Cambridge University Press, Cambridge.

Carberry,S., Kazi, Z. \& Lambert, L. (1992). Modeling discourse, problem-solving, and domain goals incrementally in task-oriented dialogue. In Proc 3rd Int. Workshop on User Modeling, pages 192-201, Wadern.

Carberry, S., Chu, J. \& Lambert, L. (1993). User-system conflict in task-oriented consultation. In Proc. IJCAI-93 Workshop: Computational models of conflict management in cooperative problem solving, Chambery.

Carberry, S. (1990a). Incorporating default inferences into plan recognition. In Proc. 8th Conf. AAAI, pages 471-478, Boston.

Carberry, S. (1990b). Plan Recognition in Natural Language Dialogue. MIT Press.

Castelfranchi, C. \& Falcone, R. (1997). From task delegation to role delegation. In Lenzerini, M., Ed. LNAI 1321. AI*IA 97: Advances in Artificial Intelligence, pages 278-289. Springer Verlag, Berlin.

Castelfranchi, C. \& Parisi, D. (1980). Linguaggio, conoscenze e scopi. Il Mulino, Bologna.

Castelfranchi, C. (1992). No more cooperation, please! In search of the social structure of verbal interaction. In Slack, J., Ortony, A. \& Stock, O., Eds. Communication from an Artificial Intelligence perspective. Theoretical and Applied Issues. Springer Verlag, Berlin.

Chu-Carroll, J. \& Carberry, S. (1998). Collaborative response generation in planning dialogues. Computational Linguistics, 24(3):355-400.

Clark, H.H. \& Schaefer, E.F. (1989). Contributing to discourse. Cognitive Science, $13: 259-294$.

Cohen, P.R. \& Levesque, H.J. (1991). Confirmation and joint action. In Proc. 12th IJCAI, pages 951-957, Sydney.

Cohen, P.R. (1994). Models of dialogue. In Ishiguro, T., Ed. Cognitive processing for voice and vision, pages 245-274. Society of Industrial and Applied Mathematics.

de Mauro, T., Mancini, F., Vedovelli, M. \& Voghera, M. (1993). Lessico di Frequenza dell'taliano Parlato. ETASLIBRI.

DeGiacomo, G. \& Lenzerini, M. (1995). Pdl-based framework for reasoning about actions. In Lecture Notes in Artificial Intelligence n. 992: Topics in Artif icial Intelligence, pages 103-114. Springer Verlag, Berlin.

Devanbu, P.T. \& Litman, D.J. (1996). Taxonomic plan reasoning. Artificial Intelligence, $84: 1-35$. 
Di Eugenio, B. \& Lesmo, L. (1987). Representation and interpretation of determiners in natural language. In Proc. 10th IJCAI, pages 648-653, Milano.

Firby, R.J. (1987). An investigation into reactive planning in complex domains. In Proc. 6th Conf. AAAI, Seattle (WA).

Gavioli \& Mansfield (1990). The PIXI corpora: bookshop encounters in English and Italian. CLUEB, Bologna, Italy.

Georgeff, M. \& Ingrand, F.F. (1989). Decision-making in an embedded reasoning system. In Proc. 11th IJCAI, pages 972-978, Detroit, Michigan.

Goerz, G. \& Ludwig, B. (1998). Modelling users, intentions and structure in spoken dialog. In Proc. Second Workshop on Human-Computer Conversation, Bellagio, Italy.

Grice, H.P. (1957). Meaning. Philosophical Review, 56:377-388.

Gross, D., Alten, J.F. \& Traum, D.R. (1993). The TRAINS91 dialogues. Technical Report TN92-1, University of Rochester.

Grosz, B.J. \& Kraus, S. (1996). Collaborative plans for complex group action. Artificial Intelligence, 86(2):269-357.

Grosz, B.J. \& Sidner, C.L. (1986). Attention, intentions, and the structure of discourse. Computational Linguistics, 12:175-204.

Grosz, B.J. \& Sidner, C.L. (1990). Plans for discourse. In Cohen, P.R., Morgan, J. \& Pollack, M.E., Eds. Intentions in communication, pages 417-443. MIT Press.

Heeman, P.A. \& Hinst, G. (1995). Collaborating on referring expressions. Computational Linguistics, 21(3):353-382.

Heritage, J.C. (1984). A change-of-state token and aspects of its sequential placement. In Atkinson, J.M. \& Heritage, J.C., Eds. Structures of Social Action. Cambridge University Press.

Hobbs, J.R. \& Evans, D.A. (1980). Conversation as planned behavior. Cognitive Science, $4: 349-377$.

Jennings, N.R., Sycara, K.P. \& Wooldridge, M. (1998). A roadmap of agent research and development. In Autonomous Agents and Multi-agent Systems, pages 275-306. Kluwer Academic Publishers, Boston.

Kautz, H. (1991). A formal theory of plan recognition and its implementation. In Brachman, R.J., Eds. Reasoning About Plans, pages 69-125. Morgan Kaufmann Publishers.

Lambert, L. (1993). Recognizing Complex Discourse Acts: A Tripartite Plan-Based Model of Dialogue. Ph.D. thesis, University of Delaware.

Lesh, N. \& Etzioni, O. (1995). A sound and fast goal recognizer. In Proc. 14th IJCAI, pages $1704-1710$, Montreal.

Lesmo, L. \& Terenziani, P. (1988). Interpretation of noun-phrases in intensional contexts. In Proc. Coling Budapest, pages 378-383, Budapest.

Lesmo, L. \& Torasso, P. (1985). Analysis of conjunctions in a rule based parser. In Proc. 23rd Annual Meeting ACL, pages 180-187, Chicago.

Levinson, S.C. (1981). The essential inadequacies of speech act models of dialogue. In Parret, M., Sbisá, M. \& Verschueren, J., Eds. Possibilities and Limitations of Pragmatics, pages 473-492. Benjamins, Amsterdam.

Litman, D. \& Allen, J.F. (1987). A plan recognition model for subdialogues in conversation. Cognitive Science, 11:163-200.

Lochbaum, K.E., Grosz, B.J. \& Sidner, C.L. (1990). Models of plans to support communication: An initial report. In Proc. 8th Conf. AAAI, pages 485-490, Boston.

Lochbaum, K.E. (1991). An algorithm for plan recognition in collaborative discourse. In Proc. 29th Annual Meeting of ACL, pages 33-38, Berkeley,CA.

Lochbaum, K.E. (1994). Using Collaborative Plans to Model the Intentional Structure of Discourse. Ph.D. thesis, Harvard University. 
Loснванм, K.E. (1995). The use of knowledge preconditions in language processing. In Proc. 14th IJCAI, pages 1260-1265, Montreal.

Mann, W.C. \& Thompson, S.A. (1987). Rhetorical structure theory: a theory of text organization. In Polanyi, L., Ed. The Structure of Discourse. Ablex Publishing Corporation.

Maybury, M.T. (1993). Communicative acts for generating natural language arguments. In Proc. 11th Conf. AAAI, pages 357-364, Washington DC.

McRoy, S.W. \& Hirst, G. (1995). The repair of speech act misunderstandings by abductive inference. Computational Linguistics, 21(4):433-478.

Moore, J.D. \& PARIs, C.L. (1993). Planning text for advisory dialogues: Capturing intentional and rhetorical information. Computational Linguistics, 19(4):651-694.

Moore, J.D. \& Pollack, M.E. (1992). A problem for RST: The need for multi-level discourse analysis. Computational Linguistics, 18(4):537-544.

Moore, J.D. (1995). Participating in explanatory dialogues. The MIT Press, Cambridge, MA.

Pollack, M.E. (1990). Plans as complex mental attitudes. In Cohen, P.R., Morgan, J. \& Pollack, M.E., Eds.Intentions in communication, pages 77-103. MIT Press.

Ramshaw, L.A. (1991). A three-level model for plan exploration. In Proc. 29th Annual Meeting of $A C L$, pages 39-46, Berkeley,CA.

Rao, A. \& Georgeff, M.P. (1992). An abstract architecture for rational agents. In Nebel, B., Rich, C., Swartout, Eds. Principles of Knowledge Representation and Reasoning: Proc. 3rd Int. Conf. (KR:92), pages 439-449, Cambridge, MA.

Sacks, H., Schegloff, E.A. \& Jefferson, G. (1974). A simplest systematics for the organization of turn-taking for conversation. Language, 50:696-735.

Schegloff, E.A. (1972). Sequencing in conversational opening. In Gumperz, J.J. \& Hymes, D., Eds. Directions in Sociolinguistics. Rinehart and Winston, New York.

Schegloff, E.A. (1979). Identification and recognition in telephone conversation openings. In Psatas, G., Ed. Everyday language: studies in ethnomethodology. Irvington, New York.

Schegloff, E.A. (1982). Georgetown university roundtable on languages and linguistics. In Tannen, D., Ed. Discourse as an interactional achievement: Some uses of 'uh huh' and other things that come between sentences. Georgetown University Press, Washington, D.C.

Searle, J.R. (1992). (On) Searle on Conversation. Benjamins, Amsterdam.

Sestero, D. (1998). Producing Responses in Goal-Endowed Dialogue Systems. Ph.D. thesis, University of Torino.

Smith, I.A. \& Cohen, P.R. (1996). Toward a semantics for an agent communications language based on speech-acts. In Proc. 14th Conf. AAAI, pages 24-31, Portland.

Stein, A. \& Maier, E. (1994). Structuring collaborative information-seeking dialogues. Knowledge-Based Systems, 8(2-3):82-93.

SRI TRANSCRIPTS (1992). Transcripts derived from audiotape conversations made at SRI international. prepared by Jacqueline Kowtko under the direction of Patti Price. Technical report, SRI, Menlo Park, CA.

Traum, D.R. \& Allen, J.F. (1994). Discourse obligations in dialogue processing. In Proc. 32nd Annual Meeting of ACL, pages 1-8, Las Cruces, New Mexico.

Traum, D.R. \& Hinkelman, E.A. (1992). Conversation acts in task-oriented spoken dialogue. Computational Intelligence, 8(3):575-599.

Weida, R. \& Litman, D.J. (1992). Terminological reasoning with constraint networks and an application to plan recognition. In Principles of Knowledge Representation and Reasoning: Proc. 3rd Int. Conf. (KR:92), pages 282-293, Cambridge, MA. 
Winograd, T. \& Flores, F. (1986). Understanding Computers and Cognition. Reading, MA: Addison-Wesley.

Young, R.M., Moore, J.D. \& Pollack, M.E. (1994). Towards a principled representation of discourse plans. In Proceedings of the Sixteenth Conference of the Cognitive Science Society, Atlanta, GA. 


\title{
A plan-based model of misunderstandings in cooperative dialogue
}

\author{
Liliana ardissono, Guido Boella and Rossana Damiano \\ Dipartimento di Infomatica, Universitri di Torino, Cono Svizzern n.185, 10149 Torino, \\ Italy. emad: $\{$ libana, guido $\}$ @di. unito it
}

This work is going to appear in the International Journal of Human-Computer Studies, Special Issue on Detecting, Reparing, and Preventing Human-Machine Miscommunication.

We describe a plan-based agent architecture that models misunderstandings in cooperative $\mathrm{NL}$ agent communication; it exploits a notion of coherence in dialogue based on the idea that the explicit and implicit goals which can be identified by interpreting a conversational turn can be related with the previous explicit / implicit goals of the interactants. Misunderstandings are hypothesized when the coherence of the interaction is lost (i.e. an unrelated utterance comes). The processes of analysis (and treatment) of a misunderstanding are modeled as rational behaviors caused by the acquisition of a supplementary goal, when an incoherent turn comes: the agent detecting the incoherence commits to restore the intersubjectivity in the dialogue; so, he restructures his own contextual interpretation, or he induces the partner to restructure his (according to who seems to have made the mistake). This commitment leads him to produce a repair turn, which initiates a subdialogue aimed at restoring the common interpretation ground. Since we model speech acts uniformly with respect to the other actions (the domain level actions), our model is general and covers misunderstandings occurring at the linguistic level as well as at the underlying domain activities of the interactants.

\section{The phenomenon of misunderstandings}

\subsection{INTRODUCTION}

In order for a dialogue to procesed successfully, it is important that the speakers maintain a consistent view of the interaction. Nevertheless, vagueness and ambiguities can intervene in a spewker's utterance and interfere with the process of interpretation of that utterance. Many researchers have recognized the role of various ambiguity types in generating misunderstandings, from different perspectives (Zaefferer 1977, Dascal 1989, Blum-Kulka \& Weizman 1988, Vendler 1994).

In the linguistic and philosophical research, misunderstandings have besen analyzed under different points of view: for example, Zaefferer (1977) and Dascal support the idea that the study of misunderstandings is related with comprehension in an indirect, external way, "much in the same way as pathological behavior is often said to be able to illuminate the nature of 'normal' behavior" (Dascal 1989). More recently, misunderstanding 
has bexn recognized by Blum-Kulke \& Weizman (1988) and Weigand (1997) as a normal phenomenon in communication, to introduce into an harmonical model of dialogue

In the research on conversational analysis, Schegoff (1987), 5chegloff (1992) has analyzed misunderstandings with respect to the sequences of turns in a dialogue, in order to identify which specific mechanisms are used by the interactants to defend the common set of beliefs necessary for the interaction to go on successfully: he calls "Bintersubjectivity" this common ground in dialogue and points out that, during an interaction, the speakers monitor their partners' rewactions and interpret them as displays of understanding / misunderstanding of the previous turns. "Having registeres the observation that, through their talk, speakers can display aspects of their understanding of prior talk, it remains to be noted that, in doing so, they can reveal understandings that the speakers of that prior talk find problematic - in other words - what they take to be misunderstanding" (Schegloff 1992). Schegloff considers various cases whtre there are problems in maintaining the intersubjectivity among the interactants; he studies different types of repairs, which can be started by the misunderstood speaker, or by the agent who has misunderstood a previous turn, when they realize that something is wrong with the interpretation. He explains that repairs are an important instrument for reconstructing the mutuality in the interactants' beliefs.

In this paper, we describe a plan-based agent model that takes into account the problem of misunderstandings in (cooperative) agent interaction. This model supports NL communication and interprets dialogue as a rational, cooperative form of interaction among agents. The coherence of a dialogue is assessed by identifying the relation among each turn and the previous interaction context: we model a goal-based notion of coherence, which builds on the idere that the explicit and implicit goals identifyable by interpreting a conversational turn can be related with the previous explicit / implicit goals of the interactants (Allen 1983, Litman \& Allen 1987, Carberry 1990). Under the assumption that the intaractants cooperate and that every turn is performed to carry on some of their goals jointly, a misunderstanding is hypothesized when the coherence of the dialogue is lost, i.e. an utterance comes which is not related with the contextual goals. In fact, the presence of an utterance that does not contribute to the goals pursued in the receiver's interpretation of the dialogue is taken as a sign that his interpretation context is different from the speaker's one (who certainly considered his own turn coherent).

The processes of analysis and resolution of a misunderstanding are rational behaviors caused by the acquisition of a supplemettary goal when an incoherent turn comes: when an agent $A$ interprets a turn and finds it incoherent with respect to the previous context, he can adopt the intention of looking for an alternative interpretation of the interaction. This choice is the consequence of another goal which he decides to pursue $A$ wants that his view of dialogue converges with his interlocutor's one. If he considers it unlikely that the speaker $B$ has performed a topic shift, or that a breakdown in cooperation has occurred, he looks for an action to modify the wrong interpretation which caused the misunderstanding. Such an action must lead one of the interactants, possibly himself, to change the interpretation context: so, $A$ restructures his own interpretation of the previous dialogue, or he induces $B$ to restructure his dialogue context, depending on who $A$ believes to have made the mistake. This commitment leads $A$ to produce a repair turn, which initiates a subdialogue aimed at restoring the common interpretation ground.

Many researchers (e.g. consider Pollack (1990), Perrault (1990), Hobbs et al (1993), 
Nagao (1993) and Hirst et al. (1994)) have used abductive frameworks for carrying on the interpretation of a dialogue and possibly restructuring the dialogue context, when a failure occurs in the integration of new utterances. In our plan-based model, the whole interpretation process is represented as the execution of interpretation (and reinterpretation) actions, as well as restructuring actions which modify the agent's dialogue interpretation; moresover, the maintenance of a correct interpretation context is a mutual goal of the interactants (Cohen \& Levesque 1991). In this sense, the actions undertaken by agents to recover from a misunderstanding are not a separate activity with respect to their normal rational behavior: they are adopted as commitments by them, when necessary. $\uparrow$ We believe that this is a major difference with respect to the other models of dialogue processing, where the treatment of misunderstandings (and general communication problems) is embedded in the normal interpretation process, or is managed by external recovery strategies (like the metarules used in Eller (1993) to restructure the dialogue context). Moreover, some of these models are mainly focused on misunderstandings in the interpretation of the illocutionary force of utterances and are constrained by a rigid notion of coherence. Instead, we claim that the misunderstanding phenomenon goes beyond dialogue and regards wider kinds of interactions; in our model, we analyze misunderstandings due to ambiguities both at the level of communicative and domain activity.

Incoherent turns are not always due to misinterpretations: also topic shifts and intentional breakdowns in cooperation should be considered. Currently, we don't model topic shifts due to the initiation of new dialogues; however, as pointed out in other works, $\ddagger$ focus and topic shifts are usually marked by the presence of "cue" words. So, they can be distinguished from misunderstandings. In our model, this means that when an unexpected change in a dialogue occurs, the presence of a cue word should trigger the hypothesis that a topic shift has occurred, before hypothesizing that a misunderstanding has occurred. So, no misunderstanding analysis would start. On the contrary, since we model cooperative dialogues, we exclude the hypothesis that a breakdown in cooperation can occur.

The paper is organized as follows: after a brief description of the phenomenon of misunderstandings (section 1.2), we will present our notion of coherence in dialogue and our approach to the repair of a misunderstanding (section 2). Then, we will describe our computational model of dialogue (section 3): the agent model (section 3.1), the interpretation process of utterances (section 3.2) and the recognition and recovery from a misalignment in dialogue (section 3.3 and 3.4). We will then provide a detailed example to show how our model works (section 4). Section 5 describes the evidence about the occurrence of misunderstandings gathered by studying a number of dialogues. Finally, section 6 compares our model to other related works and section 7 concludes the paper.

\subsection{BACKGROUND}

Schegloff (1992) describes the behavior of agents whel a misunderstanding occurs and identifies different types of repairs. He also implicitly supports the ider that misunderstandings can be distinguished according to which type of information is actually misinterpreted; for example, an incorrect identification of the referent of a description represents

f The interpretaticn and restructuring actions undergo the same planning and execntion process w the other actions, like domain-level acticns and speech acts.

f For example, consider Ohen (1984), Cohen (1987), Lútman (1986), Lítman \& Allen (1987), Grosz \& Sidner (1986) and Grosz \& Sidner (1990). 
a different level phenomenon with respect to an incorrect identification of the intended spesch act, or of the plan underlying the interaction.

In Schegloff's description, third position repair corresponds to the cases where the misunderstood agent realizes that the partner has a wrong interpretation and urges him to restructure it. For example, consider turn T4 in the following interaction (5chegloff (1992), page 1317):

Example 1:

T1: Dan: ".. See Al tends, it seems, to pull in one or two individuls on his side [..."

T2: Al: "Wr"

T3: Roger: "Well so do I."

T4: Dan: "Yesh. I'm not criticizing, I menn we'll just... "

T5: Roger: "Oh gou wanna talk ahout him"

T6: Dan: "Took, let's just talk"

In the example, Dan realizes that he has besen misunderstood by Roger and provides him with the integded interpretation of his own previous sentence by performing a third position repair in turn T4: in this way, be urges Roger to change his interpretation of the dialogue. $\uparrow$

In fourth position repair, $\ddagger$ a speaker $A$ produces a turn that is misunderstood by $B$, who replies with another turn, sequentially appropriate to his own (wrong) understanding of the first turn. $A$ doesn't realize that the two interpretations are not aligned any more and responds with a further turn, coherent in $A$ 's view of dialogue. At this point, $B$ understands his own mistake, restructures his own interpretation accordingy and informs the partner about the realignment. For example (Schegloff (1992), page 1321):

Example 2:

T1: Marty: "Loes, do you have a calendar,"

T2: Loes: "Yeah" ((reaches for her desk calendar))

T3: Marty: "Do you have one that hangs on the wall?"

T4: Loes: "Oh, you want one."

T5: Marty: "Yeah"

The recognition that a misunderstanding has occurred is triggered by the lack of coherence betwesn the interpretation of the misunderstood turn and that of the subsequent turns. Having misinterpreted, in fact, the hearer gives his contribution to dialogue in a way that is coherent in his wrong interpretation, but not from the point of view of his partner's intended meaning.

† Thrn T5 lowks like a fourth position repair by Roger, but we will discoss this aspect in section 2.2 .

$\ddagger$ Third and forrth position repairs are not nexessarily the third / fourth turns of the interaction, starting from the misunderstood turns: several turns (even kng subdialogies) can come, before an agent realizes that an interpretation problem has oxnnred. Anyway, in third powition repairs, the misunderstood speaker performs the repair turn after a "sequentially inappropriate" turn from his partner comnes (sor, the repair comess in third position, in an extended way); instead, in fonrth powition repair the misunderstander detexts his own mistake after the partner speaks again; $\mathbf{s c}$, the repair cxmes in an extended forrth powition. In Schegkff (1992), the expression "gequentially appropriate" has been used to indicate the coherence relation between a participant's understanding of a turm and his reply. 


\section{Coherence and misunderstandings in dialogue}

\subsection{COHERENCE AB A RATIONAL PHENOMENON}

We analyze dialogue from the intention recognition point of view (Cohen et al. 1981, Allen 1983, Allen \& Perrault 1980, Cohen \& Levesque 1991): when an agent acts, a relation of his action with the interaction context is looked for, to ses whether the action represents an attempt to satisfy any intention expressed explicitly by the partner, or an implicit goal which can only be inferred by reasoning on the partner's plans, or if it is a further step in a plan that the agent has already started. A different approach to the analysis of coherence was adopted by the conversational analysts, who introduced adjacency pairs (5acks et al. 1974) to model the expected continuations of an interaction: adjacency pairs are sequences of spexch acts (e.g. question-answer pairs) such that, after the first element occurs, the second one is expected. However, as pointed out in Levinson (1981), agent behavior can not directly be explainesd by means of such type of strict interactional rules. On the contrary, the intentional approach to dialogue interpretation makes it possible to adopt a flexible notion of coherence: a new contribution is considered coherent as long as a relation can be identified among the intentions underlying an action and the previous pending intentions of the interactants. Following the ideas of Castelfranchi \& Parisi (1980), we consider an uttarance coherent with the previous context if and only if its receiver can interpret it as a means of the speaker to achieve an unsatisfied goal $g$ which realizes one of the following coherence relations:

1. Gonl adherence: $g$ is one of the goals addressed explicitly by the partner in a spexch act (not necessarily in the last turn of the interaction).

Goal adherence covers the phenomena treated by adjacency pairs, but it is a more general approach than that. We represent spesch acts as actions (Austin 1962) having the effect that the interactants share the belief that the speaker intends the partner to execute a certain (domain-level or linguistic) action. An illocutionary act and the action occurring in its effect clearly correspond to the first and second components of an adjacency pair (e.g. a question is performed to induce the hearer to answer it). In the simplest case, dialogues are composed of sequences of adjacency pairs because the participants recognize each other's intentions and react to them as expected. However, other behaviors are modeled in our intentional approach: e.g. insertion sequences (Schegloff 1972), like "question-question-answer-answer" sequences (where further questions are asked before the answer to the initial question is provided) can be modeled as investigations on the actions addressed in the initial question. Asking questions about the preconditions of an action is explained as a coherent continuation, where the speaker tries to execute the action and, to do that, moves to the satisfaction of the subgoal of checking whether the action is executable or not. E.g. consider the following excerpt, taken from Merritt (1976):

T1: A: "Sell me a bottle of whisky, please."

T2: B: "Are you 18?"

Also notifications of (un)successful performance of actions (e.g. acknowledgements) follow from the satisfaction of a joint goal (see Cohen \& Levesque (1991)):

T1: A: Could you register me for the Artificinl Intelligence exam?

T2: B: $O K$, you are registered. 
2. Gonl adoption: $g$ is one of the goals that the speaker $B$ has inferred the hevarer $A$ is aiming at; this goal has not bexn expressed explicitly by $A$, but $B$ has inferred it by reasoning on $A$ 's plans. For example, in:

T1: A: "I need to borrow a book. Where is the lomry?"

T2: B: "The libmry is over there, but it is closed for the whole week"

$B$ replies to $A$ 's utterances by providing him with the information that his plan can not be executed, because one of its constraints is false. $B$ 's second utterance follows from the fact the be has adopted $A$ 's goal to know whether he may enter the library (Allen 1983). A didn't express such a goal, but it can be identifed from the fact that $A$ is checking another condition (a knowledge precondition (Morgenstern 1987)) of the plan of using the library.

3. Plan continuation: $g$ is a subgoal deriving from the course of actions already undertaken by the speaker; so, the speaker is carrying on the execution of his plan. For example, in:

T1: B: "Where is the Computer Science library?"

T2: A: "It is in the underground floor."

T3: B: "Do gou know of it is open today?"

T3 is not related with $A$ 's previous turn T2, but it can be related with T1, as another step in B's preparation for going to the library. Also turns aimed at chesking whether actions have succesded represent continuations of an agent's acting; for example, consider: "Can you give me your book? ... Did gou hear me?".

In summary, the coherence of the turns of a dialogue is assessed by identifying a structured set of goals which relate the linguistic and domain actions of the interactants. During the interpretation of a turn, a relation among the goals pursued by the turn and the contextual pending goals is searched for in a "bottom-up" way (as we will explain in Section 3.2): the turn is matched on the "nearest" pending goals and, possibly, on the other, higherlevel ones. In this way, the existence of a goal adherence relation is identified before a goal adoption relation, which in turn precedes a possible plan continuation relation. This corresponds to hypothesizing that, before carrying on one's own plan, an agent tries to satisfy his partner's open goals.

Note that this notion of coherence is not necessarily associated with cooperation among the interactants. In fact, it is possible to respect the first two conditions above, while thwarting the satisfaction of a goal $g$; as pointed out in Castelfranchi (1992), when this happens, an exchange between two debating agents can be perceived as fluent.

In our model, the "display of understanding" described by Schegloff is mirrored on the ability of producing goal-coherent turns. For example, acknowledgements, as well as the production of a coherent turn, display understanding (e.g. consider a proper answer to a question). When an agent tries to interpret a new turn from his interlocutor, he might not be able to relate it with the previous context. In that case, he should reconsider such context to ses whether there is an alternative interpretation of some turns which restores the coherence of the whole context. 


\subsection{REPAIRING TO A MIBUNDERGTANDING}

When an agent $A$ receives an incoherent turn, he commits to restoring the intersubjectivity in the dialogue; in principle, $A$ has two possibilities: the first one is to change his own view of the interaction; the second one is to persuade his partner $B$ to change his own. The appropriate strategy depends upon the alternative (coherent) interpretation(s) of the interaction that $A$ is able to find out; the existence of such an alternative interpretation is an applicability condition of the action of restoring the intersubjectivity:

1) If the last (apparently incoherent) turn becomes coherent by changing the interpretation of some previous turn $T_{i}$ uttered by himself, then $A$ can inform $B$ he should correct his interpretation of $T_{i}$ in order to adjust his view of the interaction.

2) If the last turn becomes coherent by changing the interpretation of some previous turn $T_{j}$ uttered by $B$, then $A$ will restructure his own interpretation of the dialogue.

When the hearer $A$ chooses an altarnative interpretation of a turn, he has to reconstruct the interpretation of the whole sequence of turns, in order to identify the partner's dialogue context. In fact, the speaker $B$ who has uttered the problematic turn, should have an interpretation that is coherent from his point of view, although different from $A$ 's. Empirical data (see section 5) show that agents succeed in identifying the alternative interpretations of the misunderstood turns, both in cases they have been produced or recognized by them. In fact, repairs are often composed of a part where the recognized wrong intarpretation is made explicit (e.g. in turn T4 of Example 1, "I'm not critric izing ... "). Referring to the classification of repairs in Schegloff (1992), we sese that:

1) In third position repair, the speaker of the misunderstood turn stops the diverging dialogue and formulates a repair (a request for a reconstruction of the interpretation), to induce his partner to replace the interpretation of the earlier turns. Schegloff identifies a well defined recurrent schema for formulating a repair, with a few types of realizations and a clear rationality.

2) Instead, fourth position repair looks like a notification that a change of state happened: typically, it is structured as in Example 2 (section 1.2), with a marker like "Oh" (Heritage 1984), and a sort of grounding sentence (Clark \& Schaefer 1989), where the agent informs the partner that he has finally understood what the other meant initially.

In Example 1 (5ection 1.2), it can be seen that turn T5 ("Oh you wanna trlk about him"), which follows the request to reconstruct the interpretation of the dialogue, is similar to an acknowledgement of action execution, just as it happens in a fourth turn repair. This similarity suggests that in fourth turn repair, the agent who restructures his own interpretation performs the same action triggered on another agent by a third turn repair. As a fact, both phenomena are characterized by the same underlying goal: the reconstruction of the intersubjectivity. However, the reason for adopting such a goal is different: when a third position repair is performed, the hearer is solicited by the partner's request, which typically also suggests the intended interpretation of the turn ("Yeah. I'm not criticizing, I mean we'll just ..."); instead, in fourth position repair, the hearer adopts the goal of recovering from the context misalignment and commits to changing his own interpretation autonomously. In both cases, the speaker notifies the partner that he has restructured his own dialogue context. If, instead, there is no way of realigning the subjective views of the interaction, it is not a problem: unfortunately, intersubjectivity sometimes breaks down; typically, in this case, the failure is notified to the partner. 


\section{A computational model of misunderstandings}

\subsection{REPRESENTATION OF THE AGENT KNOWLEDGE ABOUT ACTING}

Our analysis of misunderstandings is performed in a plan based architecture for generating and recognizing agent behavior, based on a two-level representation of the knowledge about acting. At the metalevel, the Agent Modeling (AM) library (Ardissono et al 1996) describes the recipes for planning and executing actions; at the object level, there are the Domain and the Spesch Act plan libraries, and some internal actions like those describing the interpretation task; the Domain library (Ardissono et al 1993) describes the recipes for obtaining the domain goals in a restricted domain; the Spesch Act Library (Ardissono et al. 1995a, Ardissono et al. 1995b) keeps the linguistic knowledge. The threse plan libraries are based on a Generalization and a Decomposition Hierarchies (Kautz 1991) and share the same representation formalism, so that the same procedures can be used on them. Moreover, plans support a declarative representation style, that can be used both for interpreting and generating the behavior of the agent. The idea of using the same structures to model interpretation as well as generation is basic to our notion of coherence, that presupposes that agents have the same Agent Modeling plans (they have the same rational behavior, although their beliefs and goals may differ substantially). The agent's knowledge also includes some prior information about the world state; such knowlesge is strictly domain dependent and regards beliefs about other agents, about conditions which hold in the world, etc. Although the presence of this type of knowledge poses a limit in the applicability of the model to general domains, it is a basic component of any system which aims at performing a deep interpretation of the interactions among agents.

\subsubsection{The object level actrons}

At the object level there are the Domain plan library and the Spesch Act Library.

The Domain plan lbbrary contains pre-compiled recipes which describe typical well formed plans to obtain domain goals. We chose the University environment as an experimental domain; so, actions regard borrowing books from the library, taking exams, and so on. The structure of this library recalls other well known solutions, so we will not describe it here

The Spech Act Ibrary contains the definition of spesch acts: they are represented, similarly to domain actions, as acts that an agent performs to try to change the world state (in this case, the hearer's beliefs). The reason for representing spesch acts at the object level is that, in this way, their planning and execution can be reduced to that of any other actions, by means of the Agent Modeling plans, and the treatment of dialogue is unified for any type of interaction. In particular, we model communication by introducing multiple (object level) actions, each of which has a different role:

- at the highest level, spesch acts are performed to induce the hearer to act in a certain way (5mith \& Cohen 1996); action "Get-to-do" represents this level and has the effect that the hearer intends to act in a certain way; $\uparrow$

- "Gett-to-do" is realized by means of one of a set of alternative illocutionary acts (e.g.

f We model the action of altering one's own beliefs as an "Update" action, which is treated w any other objext level actín. 
a request, an order, or other), which have a weaker effect: that it is shared among the interactants that the speaker has the communicative intention that be intends that the hearer intends to act in a certain way. "Get-to-do" can also be realized by mevans of a complex communicative act, composed of multiple spesch acts, which are related by rhetorical relations (Moore 1995, Barboni \& Sestero 1997);

- the illocutionary act can be performed, in turn, by mexns of different surface spesch acts (e.g. a request may be performed as an imperative sentence, a question, or other). We use direct and indirect spesch acts to describe some of the politeness techniques used in dialogue, following the taxonomy of polit thess strategies in Brown \& Levinson (1987); - the surface acts are all performed by mevans of a "Locutionary-act", which has as an argument the syntactic and semantic representation of the spesch act; in turn, this is realized by an "Utterance act" which contains a suitable text string.

As an example, we report the "Ask-if" illocutionary act (asking information about the truth value of a condition): $\uparrow$

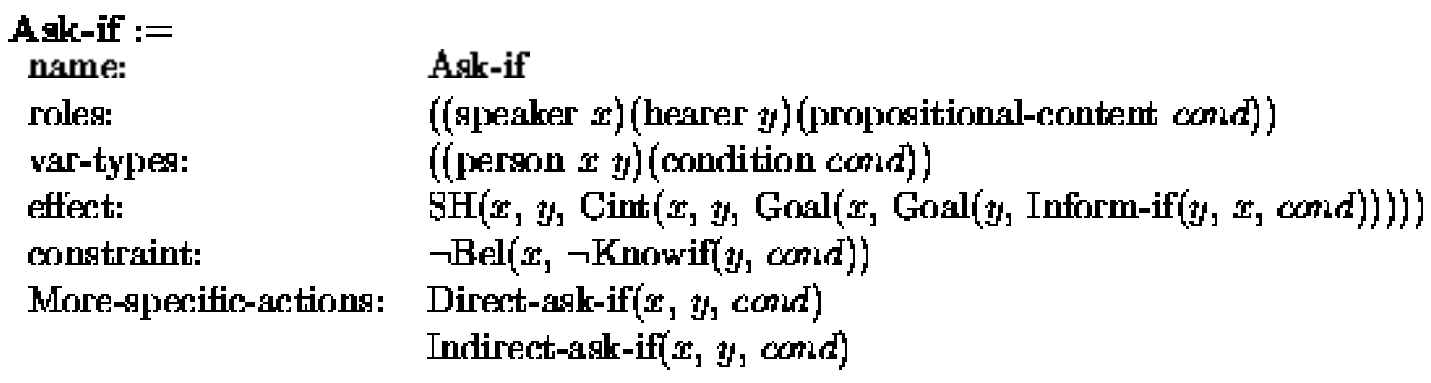

\subsubsection{The metrlevel actrons}

The Agent Modeling (AM) actions take domain actions and spesch acts as objects: the idea is that an agent who is performing a problem solving activity to obtain his goals can plan both domain and linguistic actions, according to what is better in the sperific situation (Ardissono et al. 1996).

Figure 1 shows a portion of the metalevel library: as it can be seen, most of the AM actions bave a "source" parameter (denoted as s) that represents the agent for whom the action is performed (i.e. its beneficiary). When an agent acts on his own, this parameter is bound to the agent himself; in gentral, sonrce is used to model team cooperation. The actions are represented in the figure with the following graphical notation:

- specific actions are related to more general ones by means of thick arrows, labeled with the restriction on their executability which distinguishes a specific action from its alternatives (e.g. "agent(action) $=a$ ");

- the links in the Decomposition hierarchy are represented by boxed multiple arrows (the oval below "Cycledo-action" represents a cyclic decomposition that is executed while the associated condition is true). Dashed lines denote conditional steps which are executed only when their "if" condition is true; the "wh" restrictions spesify when an action can be a step of an higher-level one;

$f$ In the action, $\mathrm{SH}$ is the mutnal belief operator; Cint is the "communicative intenticin" operator introduced in Airenti et al. (1993): Cint $\{x, y, p)$ means that $x$ has the intention that it is cxmmon belief among $x$ and $y$ that $p$ holds, and that $x$ had that communicative intention towards $y$. So, $x$ wants that it is mitually believed $p$, and that $x$ wanted to communicate it. 


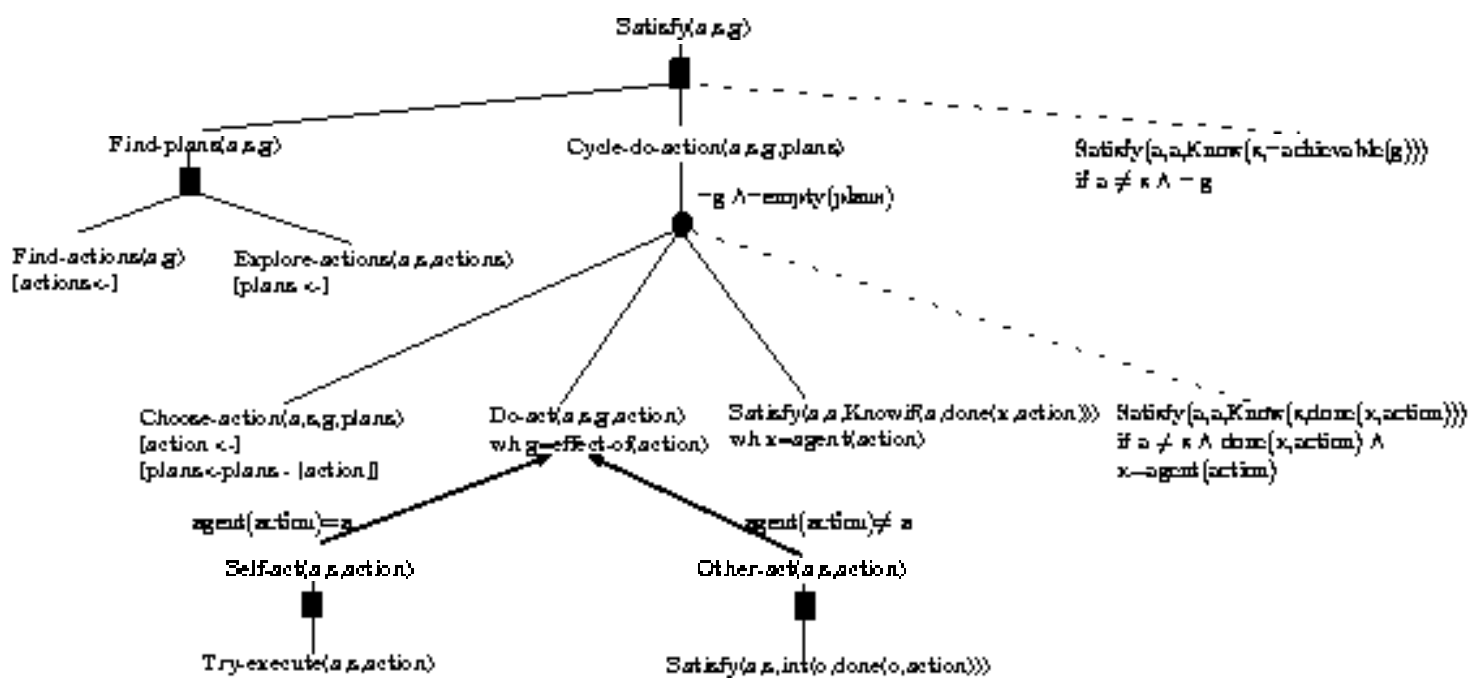

Figure 1. A portion of the AM plan library (the "Satisfy" plan).

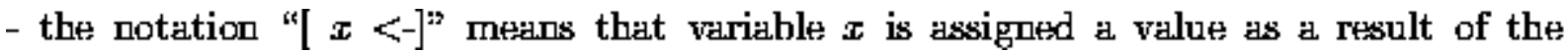
execution of the action to which the notation is associated.

The main AM action ("Satisfy $(a, \delta, g)$ ") describes the problem solving behavior of an agent $a$ who wants to satisfy a goal $g$ which he has committed to. Briefly, the agent looks for a plan which leads to $g$ (this involves finding some actions - "Find-actions" and exploring them hierarchically - "Exploreactions", if they are complex). Then, he chooses betwexn two alternative behaviors: executing it by himself ("Self-act"), or inducing another agent to perform it ("Other-act"). After that, he checks whether the action has been executed successfully by its agent (who can be himself, or his partner): step "Satisfy $(a, a$, Knowif( $a$, done $(x, a c t i o n)))$ " describes this behavior (the "done" operator maps an action on the world state after a successful execution of the action). If the action was performed successfully and the agent is cooperating with another agent, he notifies the partner about the success (sex the conditional step in the recipe). Instead, given that the execution can fail, the agent could loop in this activity ("Cycledo-action"), by choosing another alternative for $g$ and trying it. Finally, if none of the alternatives could be completed successfully and the agent is cooperating with another agent, he informs his partner that the main goal $g$ is impossible.

The other major Agent Modeling action is "Try-execute( $a, s, a c t i o n)$ " (not shown in the figure), which describes the process of executing an object level action: when an agent is committed to performing an action, he first checks whether its constraints and preconditions are satisfied (this is described by an occurrence of the "Satisfy" action in the body of "Try-execute"); if the preconditions of the action are not satisfied, the agent can adopt them as subgoals. Then, if the action can be executed, he moves to its performance, or to the execution of its steps, if it is complex. At teld, he has to check if the effects of the action hold, in order to know if he has succesded; on the contrary, if the action can not be executed (because its constraints are false), the agent can inform the partner of the problem (ses the notion of Joint Intention in agent cooperation introduced 
in Cohen \& Levesque (1991)).

Our agent model is a (Belief, Desire, Intention) BDI agent (Rao \& Georgeff 1991). The notion of commitment adopted in our model follows the notion of intention and Joint Intention described in Cohen \& Levesque (1990a), Cohen \& Levesque (1990), Cohen \& Levesque (1991), as derived from the original description of intention given in Bratman (1990). The agent's behavior is ruled by a core interpreter which loops on three phases: the interpretation of the input, the desision of which high-level goal to commit to (on the basis of which is the internal state of the agent), and the reaction, where the agent starts acting to reach the chosen goal (by means of the execution of a "Satisfy" action on the goal). In our current implementation, the agent works only in the interpretation mode, so the system plays the role of the hearer for each turn of a conversation, by switching his role among the two interactants and letting the human user play the role of the "speakri" and type sentences on the keyboard.

\subsection{INTERPRETATION OF UTTERANCES}

During an intaraction, the agent performs an incremental interpretation of the dialogue, by maintaining as a context the model of his and his partner's activities. Such dialogue context (ctx) is represented by the sequence of local interpretations of the (interleaved) turns of both speakers; in the context, the interpretation of each turn which has besen considered coherent is explicitly related with some previous turn by reporting the coherence relation (adherence, adoption and plan continuation) identified with a contextual goal. Instead, incoherent turns are unrelated with respect to the previous context.

The local interpretation of a turn is a complex structure which represents its Agent Modeling, linguistic, internal and domain level actions (Figure 3 shows one of these structures; there, the thres action types are contained in boxes labeled suitably, with the "AM", "SAM", "IM" and "DM" labels). In this interpretation structure, the AM plans relate the linguistic and domain activity, since they not only specify the steps necessary for (linguistic and domain) actions execution, but they also explain how a given spexch act contributes to the problem solving activity of an agent who is working to satisfy a domain goal.

The process of local interpretation of a turn takes in input an interpretation structure which only contains the observation that the agent has performesd an action (e.g. "Exec $(A, \mathrm{Utterance}$ act $(A, B$, "Do you have a calendar?") $)$ "); at this stage, the input utterance bas not yet besen interpreted syntactically and semantically. The interpretation process expands this structure by traveling along the plan libraries in order to find which high level goals explain the observation. Since the interpretation structures are composed of two levels, the AM actions and the object-level ones are expanded in parallel. During the expansion of a SAM (Spesch Act Model), this process builds the syntactic (Lesmo \& Torasso 1985) and semantic (Di Eugenio \& Lesmo 1987) representation of the input sentence (the "Utterance-act" action generates a "Locutionary-act" if a semantic interpretation can be found for its object text string), and identifies the performed illocutionary act.

In such a complex interpretation structure, the possible ambiguities that lead to alternative interpretations (the main cause of the phenomenon of misunderstanding) may concern several aspects: the syntactic and semantic interpretation (including references) 
of the sentence can cause the activation of more than one "Locutionary-act" hypothesis. The ambiguity in the illocutionary force leads to identify different actions representing spesch-acts. A domain action can be interpreted as a means for more than one goal (e.g. going to the library can be a step of both borrowing book and studying in the library). Even the AM plans allow to construct different hypotheses on what the agent is doing (e.g. the applicability conditions of a domain action are checked in two places: when an agent is exploring the action and when be is executing it). Finally, the same local interpretation of a turn can be related to the previous context in different ways. $\uparrow$

The interpretation and coherence-seeking processes are represented by the "Buildinterpretation" action, which is at the object level, and takes as arguments the interpreting agent $x$, the agent $y$ to which $x$ attributes the interpretation, the dialogue context for turns $t_{1}, \ldots, t_{n-1}$, and the last turn $t_{n}$. The effect of "Build-Interpretation" is to build agent $x$ 's view of $y$ 's interpretation of the dialogue, for the context $c t x_{1, n}: c t x_{1, n}$ is obtained from $c x_{1, n-1}$ by incrementing it with the interpretation of turn $t_{n}$ and, possibly, specifying the relation which links $t_{n}$ with some previous pending goal of $c t x_{1, n-1}: \downarrow$

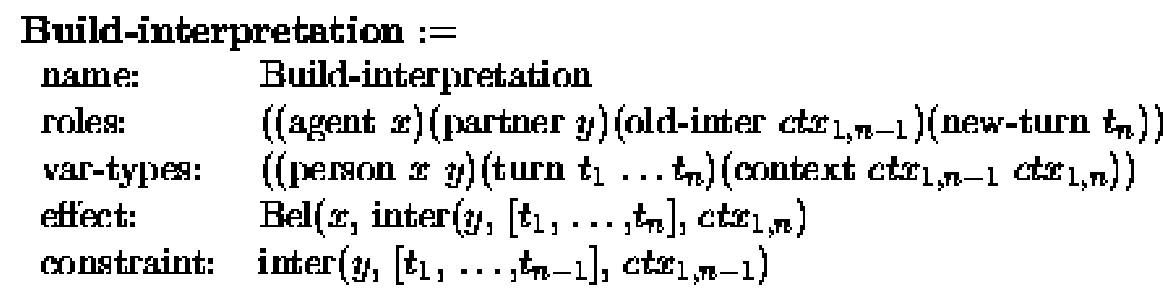

The Agent Modeling plans in a local interpretation provide the basic information to decide whether the new utterance is coherent with the previous context or not. In fact, the top AM action of a turn interpretation represents a speaker's goal $G$ which led him to produce the turn: if the turn is coherent, $G$ must appear also in the context. As described in section 2, a new utterance from a speaker $A$ can be related to the dialogue context in different ways: it can aim at the satisfaction of some goal of the partner $B$ (goal adherence / adoption), or at the continuation of the speaker's plan (plan continuation). In the first case, the goal which explains $A$ 's new turn appears in some previous turn interpretation of his partner $B$, having besen expressed explicitly by him (by means of a spesch act), or inferred by $A$ from $B$ 's overt actions. Instead, in the plan continuation case, the top Agent Modeling action of $A$ 's turn interpretation can be linked to $A$ 's previous metalevel activity (the continuation of an AM plan likely corresponds to the continuation of an object level plan, either a domain or a communicative one, or to the satisfaction of some subsidiary goal due to the cooperation with the partner).

It must be noted that the process performing the local interpretation of a turn operates in a goal directed fashion, in cooperation with the coherence-sexking process: in principle, when a new action of uttering a sentence is recognized, one could try to interpret it locally, and take the context into account only after having recognized the speaker's goal (by looking for it in the context itself). On the contrary, we use some heuristics

$f$ In this paper, we dor't deal with the problem of how one specific interpretation is chosen for a turn. In Ardisscmo \& Sestero (1996), we have discossed how maintaining a model of the speaker can help in discarding some alternatives, and how it can be useful to identify the information common to the alternative interpretaticus, as a minimal conveyed content.

$\ddagger$ The meaning of predicate "inter $\left\{y,\left[t_{1}, \ldots, t_{n}\right], d x_{1, n}\right\}^{\text {s }}$ is that agent $y$ interprets turns $t_{1}, \ldots, t_{n}$ as a context $c t x_{1, n}$. 
which start from the (speaker's and hearer's) pending contextual goals to guide the interpretation of the new contribution. This is done by looking for the possible paths which relate an object level action with the plans occurring in the interpretation of the previous context. The search for those paths makes it possible to avoid trying every expansion of the AM local interpretations towards any higher-level goals. Anyway, if the top goal of a local interpretation can not be related with some goal pending from a previous turn interpretation, there is still the chance to expand these interpretations further, in order to find some higher-level goals that explain them. So, our hegristics also search for some hightr-level goal, for which there are both a path leading to the new turn interpretation, and a path from some previous turn interpretation to the high-level goal.

Although the last turn of a dialogue may be incoherent with respect to the previous context, it is possible that the interpretation function produces a local interpretation, which remains unrelated. A reval failure in the interpretation of a turn happens only when also the local interpretation fails, maybe because the sentence is syntactically / semantically ill formed, or it is impossible to identify the performed spesch act, or the underlying domain goal.

\subsection{RECOGNITION OF A MIBUNDERGTANDING}

In the linguistic research, the term reparir has besen used in a wide sense; in fact, phenomena due to problerns in the understanding process (eg. consider questions like "Are you asking me or do you want to know?", "What do gou mean?" or problems in the identification of a referent as described in Hesman \& Hirst (1995)) have bext classified as repairs (e.g. se Schegloff (1992)). In our model these questions are related with subgoals which derive from the execution of a "Build-interpretation" action: they are not associated with the resolution of misunderstandings because the speaker has not yet committed to one interpretation of his partner's turn, but he is trying to build one.

If, during the interpretation of an input utterance, the process searching for the coheregce of the last turn fails but a local interpretation of the turn is still possible, then the interpretation action produces a context in which this local interpretation is unrelated with respect to the previous context. Since our model does not currently manage topic shifts and breakdowns in partners' collaboration, the agent $(A)$ can only hypothesize a misunderstanding. Hence, in the second phase of the main agent loop (the one where the agent descides which goal to adopt next) $A$ assumes that this interpretation hypothesis is not admissible and does not reflect what his partner has in mind. Under assumption that the partner $(B)$ is actually collaborating, this implies that the intersubjectivity in the interaction has besen lost. In this case, $A$ adopts the goal of realigning the subjective views of the dialogue; in our model, this is done by the execution of a "Satisfy" action on the goal that the $A$ 's (private) interpretation of the dialogue is the same as that of $B$, up to the misinterpreted turn $\mathbf{t}_{j}$ :

Satisfy $\left(A, B\right.$, inter $\left(A,\left[t_{1}, \ldots, t_{j}\right], c t x^{\prime}\right) \wedge$ inter $\left(B,\left[t_{1}, \ldots, t_{j}\right], c t x^{\prime \prime}\right) \wedge$ equal $\left.\left(c t x^{\prime}, c t x^{\prime \prime}\right)\right) \dagger$

Given this goal, there is a planning phase, in which the agent looks for the actions having the goal among their effects. The two alternative ways to achieve the goal are restruc-

f The "Satisfy" action does not manage complex gouls in a general way. It can however satisfy separately the subgowls of a conjunctive gow. In this particnlar formula, this is encolgh bexalse, during the diagnosis of the misunderstanding, (ne of the context variables is bond to one of the speakers' real interpretation subcontext; sor, it is not nexxssary to satisfy both the subgouls. 
turing his own context, or inducing the partnes to change his context. These alternatives correspond to two different instantiations of the same "Restructure" action.

The "Restructure" action must be executed by the agent who is misunderstanding his partner and consists of modifying his own interpretation of the turns of the dialogue to obtain the correct interpretation (i.e the one intended by the partner). 


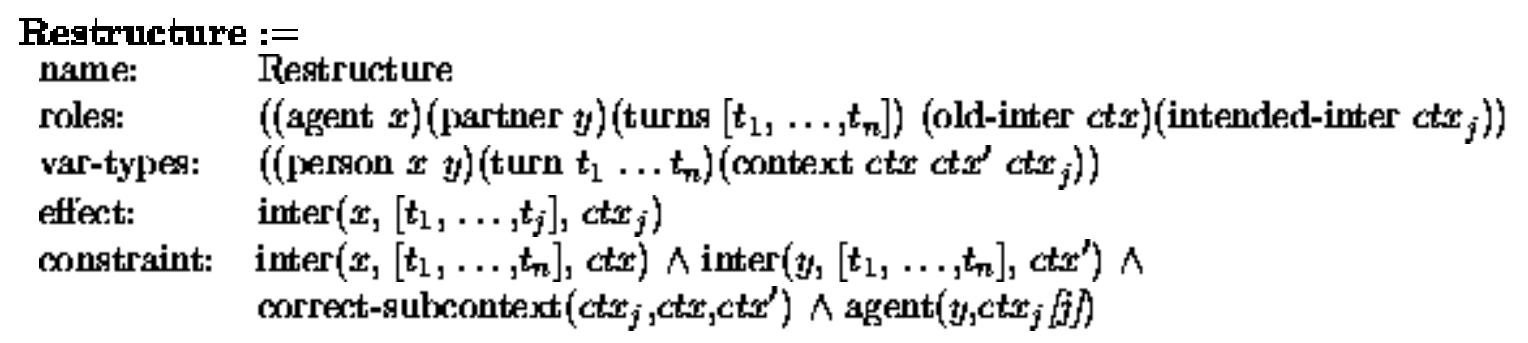

The execution of the "Restructure" action is ruled by the same Agent Modeling actions that describe the performance of the domain-level and linguistic actions. As we said in section 3.1, before committing to an action (and before executing it) an agent checks whether its constraints are true; this checking process corresponds to the diagnosis of the misunderstanding, since the constraints of "Restructure" are the following ones:

- the agent $x$ (who has to perform the action in order to correct his wrong interpretation) has an interpretation $c t x$ of the whole dialogue, up to the last turn $t_{n}$;

- an alternative interpretation $c t x^{\prime}$ of the dialogue can be attributed to $x$ 's partner $y$;

- $c t x_{j}$ (determined by comparing $c t x$ and $c t x^{\prime}$ ) is the coherent intarpretation of the misunderstood speaker, up to the misinterpreted turn $t_{j}(j<n)$;

- the agent $y$ corresponds to the speaker of the first turn that has besn interpreted differently in $c t x$ and $c t x^{\prime}$ (i.e. the last turn of $c t x_{j}$, denoted as $c t x_{j}[j)$;

To check whether the constraints of an action hold, the agent must perform a further "Satisfy" action on the goal of knowing if they are true. The truth value of a partially instantiated condition depends on whether a value for its unbound parameters is found that satisfies the condition; in this specific case, the value is the interpretation of the dialogue (i.e. the goal is "Knowref $\left(A, \operatorname{ct} x^{\prime}\right.$, inter $\left.\left(B,[\ldots], c t x^{\prime}\right)\right)$ ").

The "Knowref $(. .$.$) " goal is obtained by executing an action corresponding to the$ "Reinterpret" algorithm described in Figure 2. This action, that the agent undertakes to identify the alternative interpretations of the turns of the dialogue, is very similar to the one previously performed in the standard interpretation task. The main difference is that while in the basic interpretation process the agent considers the interpretation of his own turns as fixed, in this phase, he must consider the alternative interpretations of such turns as well: by doing this, he tries to understand why the partner believes that the interaction is coherent.

When a hearer $A$ chooses an alternative interpretation of a turn $T_{i}$, he has not only to check its coherence with the last problematic turn, but he also has to reconstruct the interpretation of the whole sequence of turns. In fact, if the speaker $B$ has uttered the last turn, he must have a wrong, but coherent view of the whole interaction, otherwise he would have started a repair.

The reinterpretation process traces back the dialogue, turn after turn and looks for a relation betwen the problematic turn and some previous (probably misinterpreted) turn. In principle, it should be possible to look for a gobal alternative interpretation of the dialogue, starting from the last contribution, like in a standard backtracking procedure. But time would be wasted in producing many candidate alternatives which fail to relate with the last turn. Instead, we exploit the newest information for pruning the inadequate hypotheses: first, we look for an alternative interpretation of a single turn $T_{i}$, which explains the last contribution; then, we propagate the change to see if the rest of dialogue becomes coherent: if the interpretations of the other turns are not adequate any more, an alternative for them is looked for. 


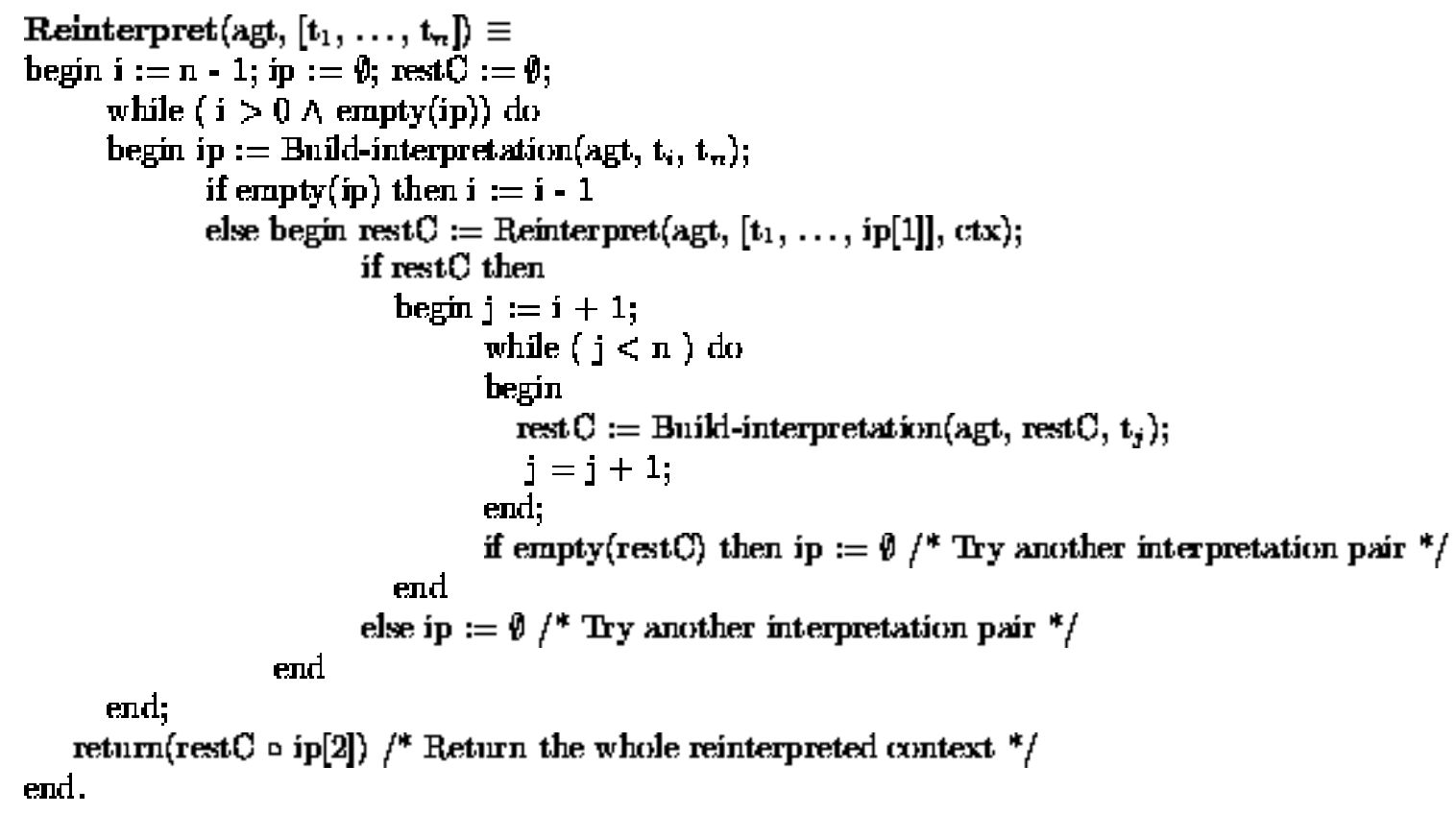

FIGURE 2. The algorithm for finding alternative interpretations of a dialogue.

"Reinterpret" has two arguments: the agent (agt) and a sesquence of turns $\left\{\left[t_{1}, \ldots\right.\right.$, $\left.t_{n}\right]$ ). When it is called on a list of uninterpreted turns $\left[t_{1}, \ldots, t_{n}\right]$, it goes backward from the problematic last turn $t_{n}$ towards the beginning of the dialogue, and stops when it finds the most recent turn $t_{i}$ for which "Build-interpretation" has found a new interpretation, coherent with that of $t_{n \cdot} \uparrow$ Then, "Reinterprets" propagates the new interpretation ip (interpretation parr) to the whole context, using the interpretation of $t_{i}$ in ip (the first component of this context, degoted by ip[1]) as a "pivot", backward by calling itself recursively, and forward by means of a loop of "Build-interpretation" on the remaining turns. When the loop stops, the new interpretation context is returned; this context is composes of the (sub)context for turns $t_{1}, \ldots t_{n-1}$ (restC in the figure) with that of turn $t_{n}$ (denoted as $i p[2]$ ).

The algorithm stops changing the previous interpretation when an alternative interpretation of a turn $t_{i}$ is found that is coherent with the interpretation of the turns $t_{1} \ldots$, $t_{i-1}$. The speaker of the last changed turn $\left(t_{i}\right)$ is the agent who has been misunderstood.

\subsection{RECOVERY FROM A MПSUNDERSTANDING}

Aftar the agent has identified the trouble source turn of the dialogue (i.e. the first misunderstood turn), be can react in two ways, according to who is the speaker of the turn: 1) If he has been misunderstood, he can try to persuade his partner to restructure his

$f$ Although "Build-interpretation" takes in input a context and a turn, there is no cxntradiction here, bexamse a single twin is by itself an elementary context. 
interpretation by performing the "Restructure" action. $\uparrow$ As describes in section 3.1.1, the (object level) action "Get-to-do", is used to induce other agents to perform actions for one's sake; so, in this case, a request to restructure the context is performed (see T4 in Example 1, section 1.2).

2) If, instead, he has misunderstood his partner, he has to execute the "Restructure" action himself. As a result of this execution, his model of the previous dialogue is changed and he can go on with the interaction, having restablished the interpretation context. In this new context, both agents share the same interpretation. Note that, before continuing the dialogue, the agent has still something to do: he notifies the partner that he has succesded in realigning the interpretation.

In both cases, the recovery goal is shared among the speakers; when everything ends up well, the agent informs the partner about the success of their aims, otherwise, if no repair is feasible, the agent warns his partner that the intersubjectivity is unrecoverable.

Our model directly supports the (positive and negative) notifications to the partner. $\neq$ In fact, the hearer is committed to the goal that the two dialogue intarpretations meet, and this goal is naturally shared with the partner, in that sperakers involved in a cooperative interaction want that their intersubjectivity is maintained.

Moreover, whth, after the occurrence of a repair, the interactants have finally restored the intersubjectivity, they can find out that some ut terances expressed betwen the trouble source turn and the repair turn may be no longer interesting. So, the pegding intentions that were created by them should be considered irrelevant, too, and no reply to them would be expected.

Some more words must be spent about the recognition of the turns expressing a request for a repair: differently from the recognition of a misunderstanding, which is triggered by a failure in the interpretation of the last turn, this type of recognition takes place in the standard interpretation process. The interpreting agent $B$ accepts this topic shift since the dialogue could not go on anymore and the partner is still collaborating with him in some way.

\section{Example}

We will show how our model works on Example 2 of section 1.2: in the interaction, Loes, who is degoted as $B$, is the receptionist and the kefper of supplies. Marty is degoted as $A$.

f In some cases, agents rexugnize that their interlocutors have misunderstood them but, for politeness reasons, they let the interaction go c⿴, without making any repais. In general, any planned action (in this case "Restructure") can be discarded if it is in conflict with other gowls of the agents. In fact, their behavior is influenced by several factors, like the relationship anong the interactants, and how much the misunderstanding can influence the subsequent talk. Here, we don't deal with these side behavions.

$\ddagger$ In principle, a notification is nexessary only when the agent has produced some turn which cxnld mislead his partner. It is a limitation of owr model the fact that it always prescribes an explicit notification. The notification goaks are managed by conditional steps of the "Satisfy" action (see the rightmost steps in Figure 1): "Satisfy $(A, A, \operatorname{Kncmu}(B$, done $\{$ Restructure $(. .)\})$.$\} " and$

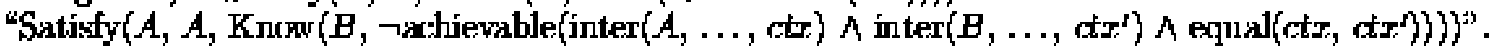




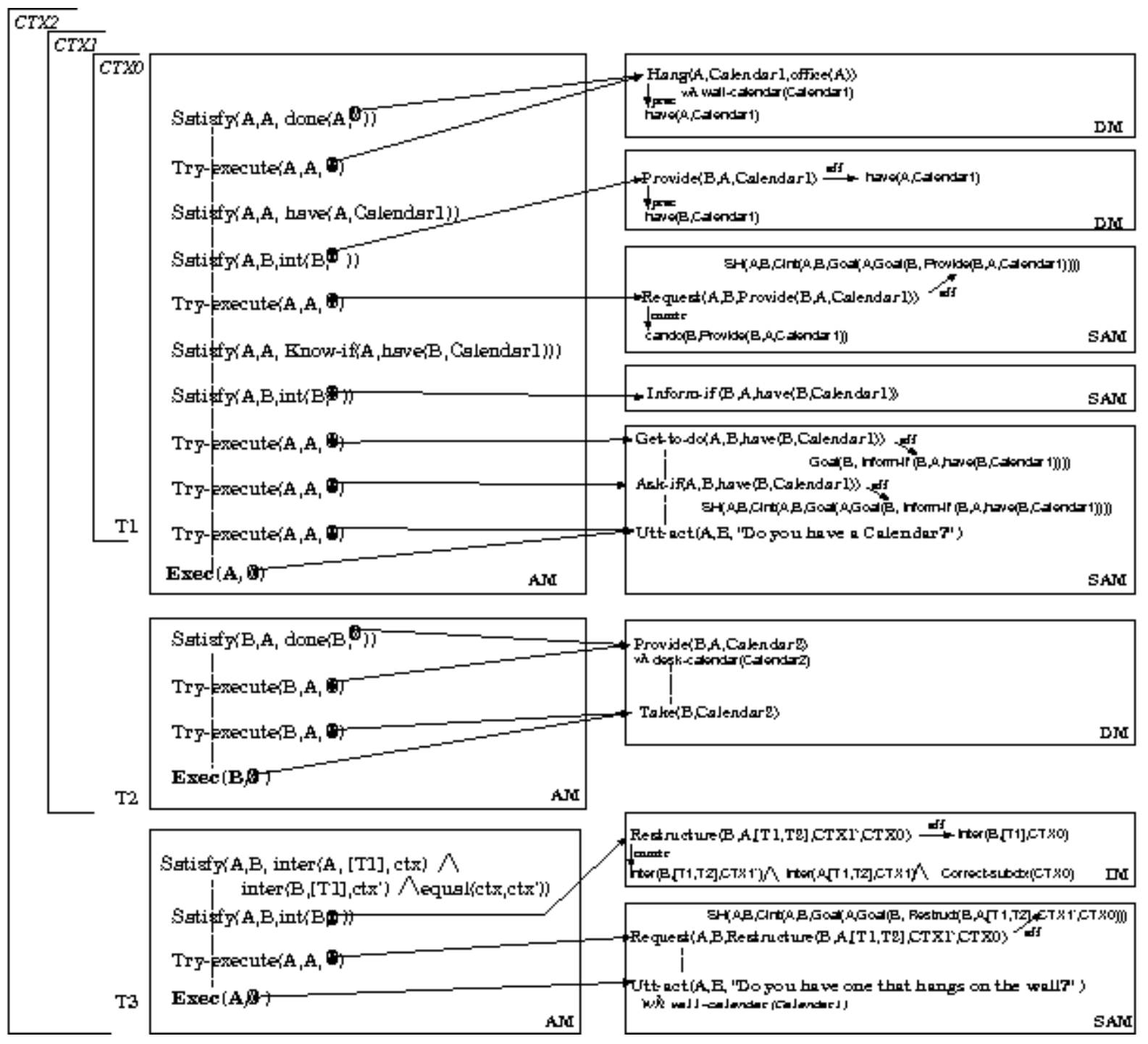

FTGURE 3. Interpretation of Example 2 from Martin's (A's) point of view.

T1: A: "Loes, do you have a calendar,"

T2: B: "Yeah" ((reaches for her desk calteldar))

T3: A: "Do gou have one that hangs on the wall?"

T4: B: "Oh, you want one."

T5: A: "Yeah"

In this interaction, there are two repairs directed towards two distinct instances of misunderstandings; the first one is a third position repair in T3 and the second one is a (different) self-repair in T4, due to the fact that $B$ recognizes a further misunderstanding from the request for repair in $\mathrm{T} 3$.

Figures 3, 4 and 5 will be used in the following to describe the different interpretations of this interaction by the two spevalkers. In the figures, we only show the most important actions of the rightmost path in the recognized Agent Modeling plans. As a fact, the structures would contain other previous AM actions, so we only report the interpretation 
branch which contains the pending goals. We will now analyze the dialogue from the different points of view of the two speakers.

Figure 3 shows $A$ 's point of view: in CTX0, $A$ has the goal of hanging a calendar in his office ("Satisfy(A, A, done(A, Hang(A, Calendar1), office(A)))" ). In order to do that, he must satisfy the precondition of action "Hang", that is "have( $A$, Calendar1)" (swe the DM containing "Hang(A, Calendar1, office(A))" in the figure); so he starts another "Satisfy" action on the goal of having a calendar. The execution of this AM action leads $A$ to plan an adequate object level action to induce $B$ to provide him with the calegdar (the "Find-plan" AM action is not shown in Figure 3). A request is a good action for that, but it is not very polite; so, $A$ refers indirectly to his intentions by checking whether $B$ can execute the action (i.e. whether she has any calendars; see the action "Satisfy(A, B, Knowif(A, have $(B$, Calendar1) $\left.))^{2}\right)$. In other words, $A$ hides his intention to execute the request by performing a question ("Ask-if(A, B, have(B, Calendar1))"), from which $B$ can infer $A$ 's intention (this way of acting is a "pre-request", an indirect politeness strategy described in Levinson (1983)).

We now analyze $B$ 's (Loes') view of the dialogue, shown in Figure 4 . While $B$ does not have any problem in interpreting $A$ 's question as a resquest for a calendar, she misinterprets her partner's high level intentions underlying the underspecified sentence (see CTX0"): she thinks that $A$ wants to borrow the calendar to have a look at it (the underlying domain action is "Read"). As a consequence of her misinterpretation, she chooses a calendar of the wrong type, a desk one: Figure 4 shows her action by associating the ("wh") restriction "desk-calendar(Calendar2)" to action "Provide(B, A, Calendar2)" in context CTX1".

Since $B$ is collahorative, she adopts $A$ 's still unexpressed intention that she gives him a calendar: this fact has been denoted in the figure by means of the dotted arc that relates the effect of the request ("SH(A, B, Cint (A, B, Goal(A, Goal(B, Provide(B, A, Calegdar2) )) ))" with action "Satisfy(B, A, done(B, Provide(B, A, Calendar2)))

Going back to Figure 3, we can examine how $A$ interprets the obstrvation that $B$ is taking a calendar ('Exec(B, Take(B, Calendar2))'). The interpretation heuristics mentioned in section 3.2 are used to perform the interpretation task: instead of identifying all the possible reasons for taking a calendar, the interpretation process tries to find a relation betwen $A$ 's goals and the new contribution. Starting from the low-level goals, taking something has nothing to do with $A$ 's explicitly expressed goal of being informed about something; however, the heuristics relate $A$ 's implicit request that $B$ provides him with a calendar with the fact that $B$ has just taken one: to provide an agent with something, you have to take the thing and pass it to the receiver.

Unfortunately, $B$ 's action can not be related with $A$ 's dialogue context CTXO because $B$ is handling the wrong type of calendar; so, the coherence-sexking procedure fails and, in CTX1, the new turn remains unrelated from subcontext CTX().

At this point, $A$ tries to restore the intersubjectivity by planning a "Satisfy" action on the goal that $A$ and $B$ have the same interpretation context on a subpart of the dialogue. $\dagger$ This results in the instantiation of an action "Satisfy(A, B, inter(A, [T1], ctx) $\wedge$ inter(B, [T1], ctx') $\wedge$ equal (ctx, ctx'))". $A$ plans a "Restructure" action and evaluates its constraints (in doing so, be applies the

f The subpart is initially unspexified, but, in this case, the only possible diakgne context that $A$ and $B$ can share is the one cxmposed of the interpretation of T1: in fact, T2 his been produced from an incorrext understanding of T1. 

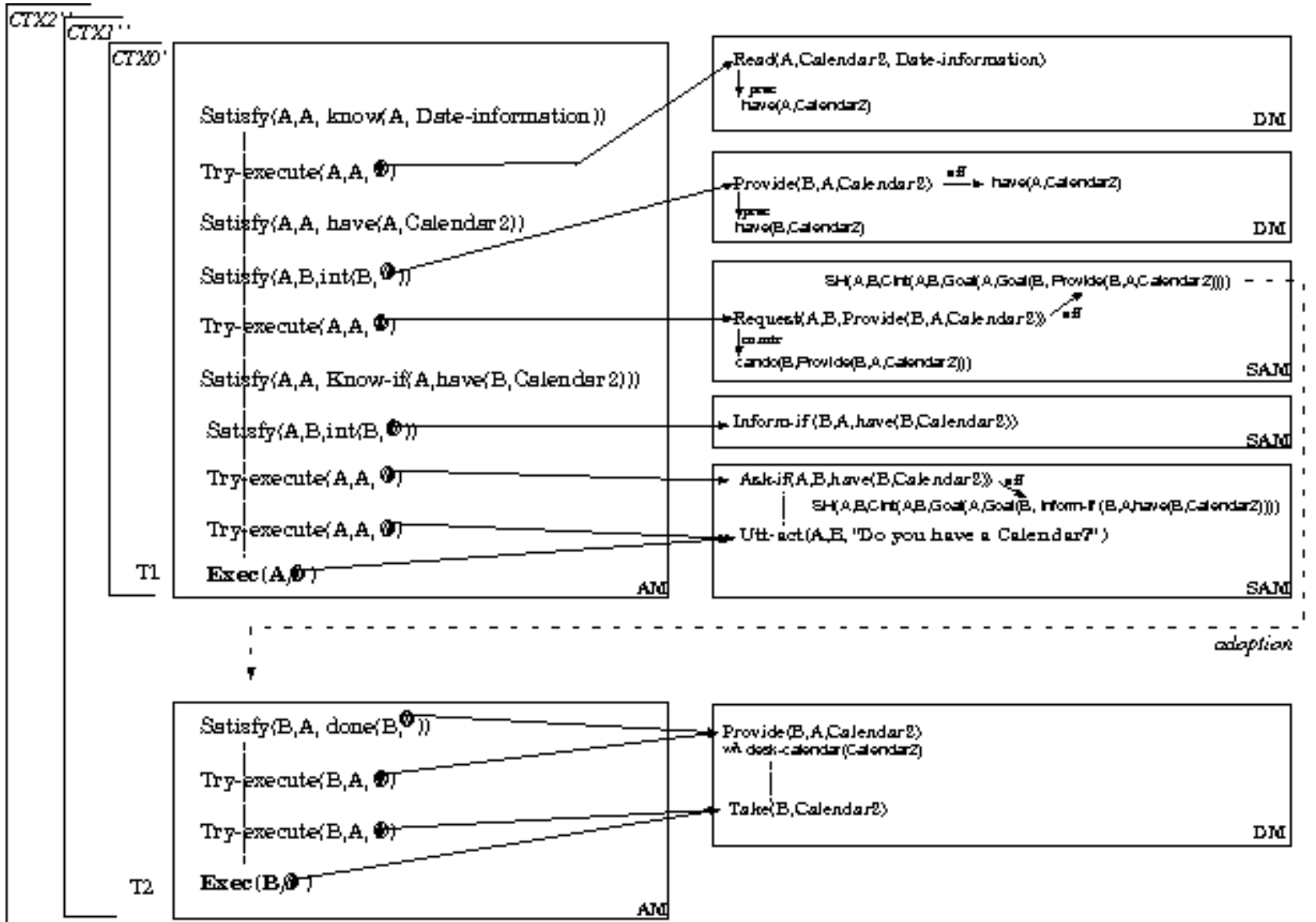

Satisfor $(\mathrm{A}, \mathrm{B}$, inter $\mathrm{A},[\mathrm{T} 1], \mathrm{ctz})$ A

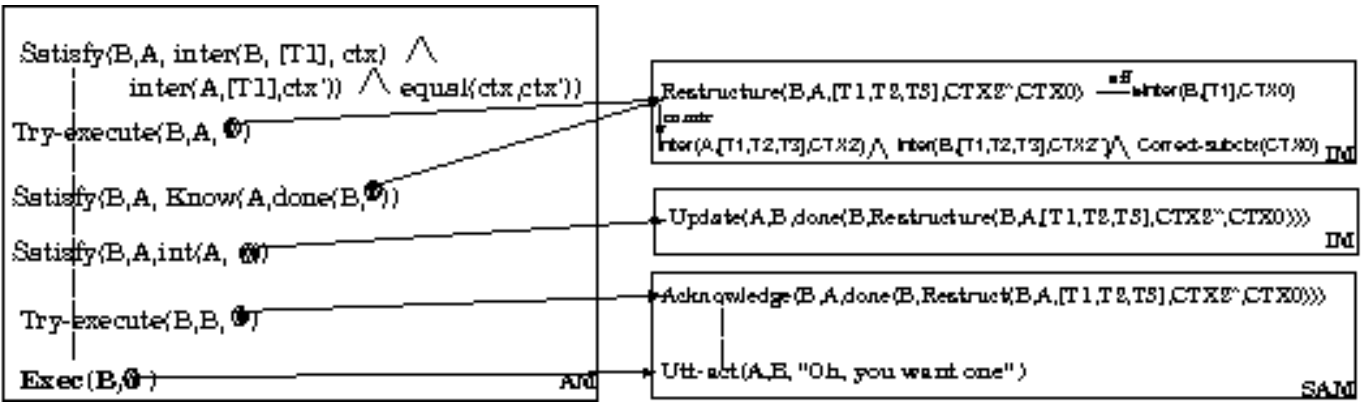

FTGURE 4. Interpretation of Example 2 from Loeg' ( $B$ 's) point of view. 


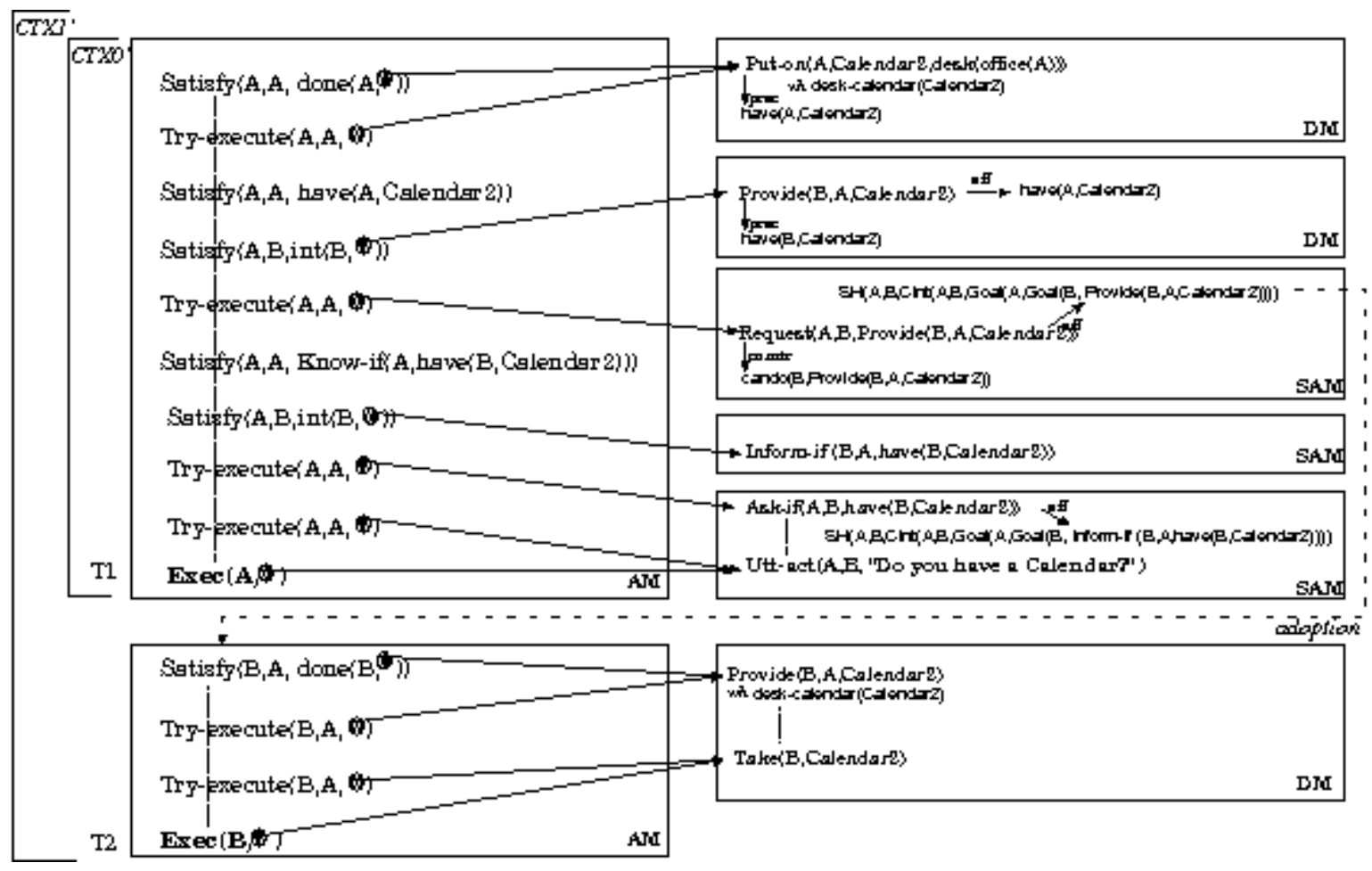

FIGURE 5. The reconstruction of turns $T 1$ and $T 2$ from Martin's point of view.

algorithm described in section 3.3, which, in this case, only makes him reinterpret locally T1 and relate it with T2).

$A$ finds an alternative interpretation of his own turn T1 which explains T2 by means of an adoption relation (ste CTX1' in Figure 5). So, he assigns the responsibility of the misalignment to $B$. In the interpretation of CTX1', which is still different from what $B$ really has in mind, $A$ attributes to $B$ the belief that he wants to put a calendar on his desk. However, $A$ has chosen the wrong hypothesis; since he is unaware of his error, he consequently formulates a request for repair ("Restructure( $B, A,[T 1, T 2], C T X 1$, CTX(1)" ) : he exploits the strategy of repeating the first turn with the addition of some more specific information about the type of calendar he wants. The new information serves to enable the partner to disambiguate between the two alternative interpretations (CTX0) and CTX0)'

Going back to B's view of the interaction (see CTX2" in Figure 4), she successfully recognizes T3 as an unrelated turn, which is a repair following an interactional breakdown. However, in this case, it is a justified topic shift: in fact, this turn satisfies the joint goal of maintaining the intersubjectivity in the dialogue.

After $B$ interprets T3, she finds out that the beliefs underlying her interpretation context CTX2" are inconsistent with $A$ 's request for restructuring. While $B$ reconstructs T3 with the newly conveyed information about the desired calendar type, she understands what $A$ thinks that she has in mind. In fact, she can recognize the "Restructure" action in $A$ 's plan only by hypothesizing that $A$ believes that the constraints of such an action are 
true: in particular, she has to reconstruct $A$ 's alternative context interpretation CTX1' (Figure 5); she can do so thanks to the fact that $A$ has specified that the calendar should be hung on the wall, in order to contrast with the "Put-on" interpretation ( $A$ believes that constraint "inter(B, $[\mathrm{T} 1, \mathrm{~T} 2], \mathrm{CTX1})$ ") is true). At the same time, $B$ succesds in identifying $A$ 's original intention, displayed in T1: banging the calendar on the wall ( $A$ believes that the other constraint "inter(A, [T1,T2], CTX1)" is true).

On the basis of all this information, $B$ adopts the goal of resolving the complex misunderstanding: she restructures har interpretation context by changing it from CTXO" to CTX0, as a consequence of the effect of "Restructure(B, A, [T1,T2,T3], CTX2", CTX0)" and then she notifies $A$ that she has understood what he meant initially (turn T4). The notification is performed by means of a "Satisfy" action on the goal of letting $A$ know that a "Restructure" action has besen successfully executed.

It must be noted that $B$ could not accept $A$ 's repair of turn T3; in fact, this would have mislead $A$ by inducing him to believe that $B$ 's dialogue context really corresponded to CTX1'. Instead, from her acknowledgment, $A$ understands that $B$ 's "Restructure" action is not the one requested by him in T3: T4 ("Oh, you want one") means that $B$ has realized only at this point that $A$ wanted to kefp the calteldar for himself, instead of borrowing it. Now, the dialogue can go on correctly and $B$ can provide $A$ with the calendar to be hung on the wall.

For Schegloff (1992), Marty's controversial turn T3 is a follow-up question that spesifies his intentions, rather than a repair for correcting Loes' interpretation. However, Schegoff does not exclude the other interpretation, since he admits the existence of repairs which do not have the recurrent linguistic features characterizing their canonical form: turn T3 could be a polite form of repair where, instead of correcting Loes explicitly, Marty responds to her with another question that addresses the interpretation problem. Anyway, Schegloff also points out the fact that, if Marty's turn T3 wire a repair, it would be addressed to a different interpretation problem (calendar type) from the one which is addressed by Loes' fourth turn (keeping or borrowing). In our model, the double misunderstanding interpretation, that Schegloff partially admits, is not problematic: in fact, thanks to the planning context in which it is embedded, the role of T3 in triggering T4 is recognized.

\section{Evidence from a corpus of conversational data}

In order to fully understand the phenomenon to be modeled, we based our work on the analysis of a corpus of data collected from natural spoken conversations. The corpus contains 63 instances of misunderstandings; all the excerpts are in Italian, except two instances taken from an English transcription. $\dagger$

The analysis of the data well evidenced the complexity of the phenomenon and highlighted some important features of natural occurring misunderstandings, such as the flex-

$f$ The transcriptions nsed for the research contist of two published cxrpora of spoken Italian, and two unpublished minor cxrpora, plus a few examples transcripted for the spexific purpowe. de Maluro et al. (1993) contains varions types of interactions, while Gavioli \& Manstield (1990) is a $(x)$ lextion of book shop encxinters (with a portion in English). The unpublished cxipors were cxllexted in 1994 by $\mathrm{C}$. Ferrus and G. Fornara for their theses: the first one ontains only instanos of mismderstundings of proper nouns, the sexond one contains transcriptions of job interviews. 
ibility of repair mechanisms in human conversation, the nesd to take different levels of misunderstanding into account and the nature of resources exploited by human conversants in accomplishing the repair task. All these fextures sarved as guidelines during the development of the model, hopefully contributing to make its treatment closer to reality.

First of all, the empirical data show the impact of misunderstanding on dialogue and confirm, apart from the theoretical expectations, the nesd for a treatment of this phenomenon in a computational model of dialogue. In fact, the episodes of misunderstanding found in 59 hours and 12 minutes of conversation amount to 52 , with a quite high rate of occurrence of one every 68.3 minutes approximately. This datum justifies the intuition that, even if they can be considered a sporadic event, misunderstandings do still play a role in conversation and consequently nesd to be treated by an appropriate mechanism (ste also Perlis \& Purang (1996)).

By observing the misunderstood turns, for example, it is possible to attempt a distinction concerning what is misunderstood. With reference to the process of interpretation of an utterance, five levels can be identified as possible object of misunderstanding, namely the phonetic level, the syntactic level, the lexical level, the semantic level and the pragmatic level. $\uparrow$ If we consider the origin level of misunderstandings, we have the following distribution: the semantic level gathers the highest amount of instances, with $54 \%$ of the total, followsd by the pragmatic level $(19 \%)$, the phonetic level $(14 \%)$ and the lexical level (13\%), while no instances mere found belonging to the syntactic level. More precisely, looking at the internal composition of the semantic level, divides into propositional content and reference (including wrong reference to the allocutor, as evidenced in Schegloff's data) the rate importance of the whole category can be attributed to the misunderstandings of the referent intended by the speaker. As far as the pragmatic level is concerned, it includes several types of misunderstandings that hadn't besen highlighted before. At this level, misunderstandings concern not only the comprehension of the illocutionary force, but also the speaker's plan, the role of participants, the grasping of the indirect meaning, the choice of the right interpretation context and the discourse topic. Interestingly, only a few instances ( 3 instances) were found in which the hearer's reaction was misunderstood, generating a wrong repair, i.e. a repair that it was not neccessary.

Regarding the causes of misunderstanding the analysis of the real examples underlines the substantial role played by ambiguity in allowing for wrong interpretations through all the levels (see Zaefferer (1977), Vendler (1994)). In detail, the responsibility for misunderstanding can be attributed to the presence of a relevant ambiguity at one of the identified levels in approximately two thirds of the instances, while only one third takes place in complete absence of ambiguity (e.g. in the presence of mishearings and other phenomena like misconceptions).

Finally, it is interesting to have a look at the position of repair with respect to the misunderstood turn. The evidence from the corpus suggests that, while most repairs are executed within two or three turns of distance from the misunderstood turn, a significant set of examples shows that repairs can be delayed at up to several turns of distance from the point where the misunderstanding has occurred. There are examples of repair at the 12th, 15th, 19th and even later, at 31st turn, confirming the data collested in Hansen et al. (1996) about the possibility for the speaker to delay the repair. Moresover, most of the analyzed repairs were performed by the misunderstood speaker on the under-

f The presence of different levels of misunderstanding has been pointed out by many researchers, like Zaefferer (1977), Dascal (1989), Schegloff (1992), Vendler (1994) and Bagzanella \& Damiano (1997). 
standing shown by the other participant (i.e. third turn repair), while only three instances can be classified as repairs initiated by one participant on his own interpretation of a previous turn. So, although this datum confirms the preference for third position repair, it underlines the need for a flexible diagnosis of misunderstanding, which doesn't rely only on a pre-ordered sequence of turns, but which is able to go backward in the dialogue in search for a misunderstood element that may reside in any previous positions and at any possible level of meaning.

\section{Related work}

First of all, some comments must be made about our representation of the context of a dialogue: our Context Models (indicated as CTXi in the figures) are rather different from the dialogue model described in Lambert \& Carberry (1991), Carberry et al. (1992) and Lambert (1993). As discussed in Ardissono et al. (1996), although both frameworks contain domain, communicative and metalevel plans, we make a different use of our (AM) metalevel and communicative plans. In Lambert's model, the problem solving plans relate communicative actions to the domain level plans pursued by the sperakers; instead, in our approach, both domain level and linguistic actions are the objects of the metalevel actions and can be performed interchangeably. Morever, our structures can be extended for answer production, adding them some supplementary information about how to execute actions (Ardissono \& Coht? 1996).

As far as the management of dialogue is concerned, other frameworks have exploited the view of dialogue as a collaborative activity among agents. For example, Chu-Carroll \& Carberry (1995a), Chu-Carroll \& Carberry (1995b) describe a framework where a system and its user collaborate to build a (correct) plan for obtaining the user's goals: in an interaction, the system and the user negotiate their beliefs, until they agres on a solution. In that work, however, no negotiation of the meaning of sentences is performed.

Dialogue has been modeled as a collaborative activity also in Hexman \& Hirst (1995), where a planning framework bas been used to interpret and recognize referential expressions. In this model, the conversational turns are seen as actions that contribute to the interaction by establishing some communicative goals, which become shared betwen the interactants. Although our work is based on the same idea, it differs in the way how it models negotiation among agents. In particular, Hesman and Hirst introduce specific actions to plan the form of a referential expression, and a number of actions which describe the reactions which an agent can adopt when he receives a referential expression: e.g. the agent can accept it, refuse it, correct it, and so on, depending on the fact that he has been able to resolve the expression, or he has encountered any troubles in the interpretation process. In any case, he starts collaborating with his partner at the resolution of the problem, by means of a negotiation subdialogue.

In our work, the interpretation of referential expressions is not separated with respect to the other linguistic phenomena: we describe a generic framework of interaction, where the various interpretation levels (syntactic, semantic, etc.) are modeled in a unique way, by means of the execution of object level interpretation actions, under the control of the Agent Modeling actions which manage the reaction to a possible failure. The idea is that an acceptance, a refusal, or other, may happen at different levels, not only at the referential one: for example, an ambiguous word may cause the hearer to build more than 
one semantic interpretation of a sentence, so that he would bave to start a clarification subdialogue with the speraker to establish the intended meaning of the utterance; in fact, this is quite similar to the case of an underspecifed referential expression. For this reason, we adopt a unique (metalevel) model for handling action execution and failure: this model rules the execution of domain actions, as well as spexch acts and interpretation actions. During the interpretation of an utterance, it supports the possible reactions to a failure (in terms of notification to the partner) at the utterance level, as well as at the locutionary and illocutionary ones.

Our representation of the utterance interpretation process as multiple object level actions does not only offer a framework for treating interpretation failures, but also for managing the phemomenon of grounding in dialogue (Traum \& Hinkelman 1992): we treat the acknowledgments that the receiver of an utterance has interpreted it successfully and accepted it (e.g. "Okay.") exactly in the same way as the acknowledgements of successful execution of a domain action, as prescribed by our Agent Modeling plans; similarly, the occurrence of requests for an acknowledgement by the speaker of an utterance (e.g. "Right?", "Ok?") are collapsed into the process by which an agent monitors that his partner has executed a requested action successfully: in the case of linguistic actions, this corresponds to checking whether the partner has beern able to perform the interpretation of the utterance and the updating of his own beliefs with the communicative effects of the utterance itself.

Some computational models of dialogue use a notion of coherence based on an analysis of the expected behavior of agents in conversation. In those approaches (for instance, consider McRoy \& Hirst (1995), Traum \& Hinkelman (1992), Traum \& Allen (1994), Danieli (1996)), the spesch acts occurring in the last conversational turn, together with the existing dialogue context, are used to predict which spesch acts the interlocutor should perform if the interaction goes well; a deviance from the expected behavior is taken as a sign that some interaction problem is occurring and the presence of a misunderstanding is hypothesized.

Our work uses a deeper notion of context, where different types of intentions (related with the interaction, as well as with the agent's domain activity and the goals deriving from his participating to a conversation) are represented explicitly and maintained as a context; they contribute to the identification of more general relations that can exist with the new input. In particular, we model linguistic expectations and underlying integtions uniformly as goals, although they fall at different levels. So, we provide a unified model of the dialogue context, where the pending goals which happeg to correspond to the linguistic expectations fall at a lower level with respect to those pertaining the underlying activity of the agents. An immediate consegquence of this is that, when a turn satisfies some lowlevel pending goals, there is no nexd to inspect the higher level ones (so, the effort spent in the interpretation task is limited). At the same time, however, when the low-level goals are not matches, the dialogue context is rich enough to be analyzed, searching for the possible relations betwen the turn and the previous part of the interaction.

Our model also differs from the above mentioned systems because of the two-level organization of our plan libraries, where the agent modeling plans rule all other actions, including interpretation and repair actions. In particular, in McRoy \& Hirst (1995), metalevel plans model the expected continuations of an interaction: following the ideas developed in Litman \& Allen (1987), the strongest expectation is that the receiver of a turn accepts 
the turn and reacts by contributing to it, but he might also start another (sub) dialogue, by introducing a new communicative goal. McRoy and Hirst extegd Litman's approach and introduce metaplans to diagnose misunderstandings and formulate repairs, when the expected behavior is violated. However, their metaplans only analyze the surface expectations introduced by performing of a spesch act; the absence of a deeper intentional analysis limits their approach to the treatment of misunderstandings on spesch acts, while our model also treats misunderstandings on domain level actions (sex the analysis of the example in section 4).

\section{Conclusions}

In this paper, we have described an agent model that provides a computational treatment of misunderstandings in dialogue. We have focused on third and fourth position repairs, and we have described how they can be detected when a turn incoherent with the dialogue context occurs. On the contrary, we have left apart first and second turn repairs, because they are rather different phenomela: third and forth position repairs are caused by the recognition that one of the interactants has committed to a wrong interpretation of the previous part of the dialogue (they are concerned with interpretation mistakes which occurred before the repair turn); instexd, first turn repairs are performed by a speaker in order to avoid that the bearer has interpretation problems; finally, second turn repairs are typically performed by the receiver of a turn, when he experiences some problems in the interpretation of his partner's turn, so he has not yet committed to a specific interpretation (e.g. they are usually related with misspellings, and other similar phenomena).

Our dialogue model consists of a plan-based representation of the knowledge about the way to reach one's own goals. This knowledge takes into account the normal planning behavior of an agent, as well as his capability to recover from problems in the interaction with other agents. Misunderstandings are treated as one of the specific problems that can occur when interacting with other agents and are dealt with by adopting the goal of maintaining the safety of the intersubjectivity in dialogue In summary, the main ideas underlying our work are:

1) Misunderstandings in dialogue are explained in the same way as problems occurring in non-linguistic interaction. In fact, in our agent model, linguistic actions are means to obtain one's goals exactly as the other (domain level) actions. The maintenance of such a general interaction context allows us to model misunderstandings without posing any limitations to the object of the misunderstanding and to the distance betwesen the misunderstood turns and the repair turns performed by the agents.

2) Different levels of interpretation have been identified as the possible objects of a misunderstanding, from the utterance level (which regards the syntactic and semantic interpretation), to the pragmatic level, which covers misunderstandings on the illocutionary force of spesch acts, and on the domain level activity underlying the linguistic behavior of agents. $\dagger$

3) The recognition of misunderstandings follows from an agent's attempt to resume the intersubjectivity, after he has not been able to interpret the last turn coherently. How-

$f$ Although our model is compatible with the presence of different levek of misunderstanding, we don't consider mishearings: they are important for speech rexxgnition, as discossed in Smith \& Hipp (1994) and Danieli (1996), but they are not a problem for our system, bexwse it takes its inpnt in textul form. 
ever, the identification of the mistake and the reinterpretation of the dialogue are fully embedded into his goal-directed behavior, in that the agents participating to an interaction share the common goal of maintaining their intersubjectivity and act to restore it, when threatened. Restoring intersubjectivity is a goal shared among the interactants because the intersubjectivity is the basis for any social action (5chegloff 1992).

4) There is a clear distinction among repairs to misunderstandings (i.e. requests of repair) and notifications that one of the interactants has just adjusted his dialogue context to recover from a misunderstanding.

5) Repairs can be the object of a misunderstanding, too; moreover, an agent can believe that he bas bexpl misunderstood by another one, so generating a repair, but he might be wrong (so, the other one could make another repair). Our model takes into account these phenomena, as it can be seen in the example described in section 4.

Our agent model is implemented in Common Lisp and runs on workstations. The interpretation of utterances is fully implemented, starting from the NL (Italian) form, to the construction of the dialogue context of the interaction; the generation of agent behavior is under development.

Some aspects of our model have to be fully developed and represent an avenue for future work:

1) A desper study of the structure of dialogue, as mentioned in section 1.1, in order to analyze subdialogues and topic shifts. The cases where a topic shift is an acceptable interpretation hypothesis, even in a cooperative environment, can be determined by the satisfaction of the previous goals or by the urgency of the newly introduced goals (e.g. consider the problem of managing interactions in highly dynamic environments, as studied in the reactive planning research).

2) The development of the linguistic strategies to perform a repair (in terms of sequences of spesch acts (Schegloff 1992)). An interesting related problem is to find the minimal information necessary to disambiguate the meaning of the trouble source turn when a misunderstanding occurs. The idea of finding some discriminating information for disambiguation purposes recalls the strategies used in plan recognition to deal with ambiguous hypotheses on the observed agent's plans (e.g. see the initiation of clarification dialogues to disambiguate the partner contribution (van Beek \& Cohen 1991, Cohen et al 1994)). 3) The ability of the speakers to talk about what is bappening in dialogue, by means of anaphoras, verbs and nouns referring to spesch acts (such as "to criticize", "to order", etc.); Goy \& Lesmo (1997) provide a first analysis of the lexical semantics of communication verbs.

4) The development of a method to analyze the "weaknesses" of one's own utterances (in terms of ambiguities and underspecification). Empirical data show that humans identify the causes of the misunderstanding very easily, and that they repair them in an effective way by "filling the gaps" of their previous turns. We believe that the type of failure arising when an agent tries to relate the incoherent turn to the previous context could be useful for an efficient search of the alternative interpretation for the misunderstood turn. 5) The ability to prevent misunderstandings by performing early repairs, like first-turn repairs. While we have not worked at this problem, there are some dialogue models which try to predict how the hearer will interpret an utterance, in his mental state. For example, Ndiaye \& Jameson (1996) use the concept of antricipation feedhack loops 
(introduced in Wahlstar \& Kobsa $(1989)$ ) to choose the "most promising" utterance that can be expressed to the user, in order to convey an information. Although this does not model the occurrence of utterances immediately followed by repairs (it is a way to plan what to say), the idea underlying anticipation fessback loops could be exploited to recognize first turn repairs in the interpretation of a turn.

6) The ability of a speaker to identify a misconception of the partner. Usually misconceptions make it impossible to interpret a turn locally (e.g. consider the problems studied in McCoy (1986) and McCoy (1988)); however, if they aren't promptly recognized, they can lead to misunderstandings. When an agent looks for an alternative interpretation of the previous dialogue, he should take into account also the possibility that a misconception of the partner led him to a different understanding of what has been said.†

7) It also must be noticed that our analysis of coherence does not take into account the role of cognitive load in dialogue interpretation. As evident from the analyzed corpora, speakers often misinterpret complex utterances, or they forget what has been previously said (Jordan \& Thomason 1996). However, this problem is currently outside our interests.

\section{Acknowledgements}

We are very grateful to Leonardo Lesmo, Carla Bayzanella and Morena Danieli for the fruitful discussions and good advice that they provided us with. We also thank them and the anonymous reviewers for having carefully helped us to improve our paper with their comments on the first version. Finally, we thank Sandra Carberry for her comments to the agent modeling architecture which represents the conceptual framework of this work. This work was partially supported by MURST 60\% and by the Italian National Research Council (CNR), project "Pianificaxione e riononscimento di piani nella comunicaxione". In particular, the analysis of the corpora which guided us in the construction of our model of misunderstanding was carried out within this project by Carla Baxsanella and her students, and we thank them a lot for their collaboration.

\section{References}

G. AIrenti, B. BARA, \& M. Colombetti (1993). Conversational and behavior games in the pragmatics of discourse. Cognitive Science, 17:197-256.

J.F. Allen \& C.R. PkrRault (1980). Analyzing intmition in utterances. A trificinl Intelligence, 15:143-178.

J.F. AlLEN (1983). Recognizing integtions from natural language utterances. In M. Brady \& R.C. Berwick, editors, Computational models of discourse, pages 107-166. MIT Press.

L. ARDIssono \& R. CoHEN (1996). Extending the role of user fesdback in plan rescognition and response generation for advice-giving systems: an initial report. In Lecture Notes in Artificial Intelligence n. 1081: Advances in Artificial Intelligence, pages 109-120. Springer Verlag, Berlin.

f For an analysis of missonceptions, see Goodman (1985), Pollack (1987), Pollack (1990), Calistri-Yeh (1991), Carberry (1986), Carberry (1987), Eller \& Carberry (1992), Eller (1993), Donaldson \& Cohen (1996). Sote that plan misconceptions have been implicitly reduced to the presence of different interpretation cxntexts in all the approwches based on the presence of buggy plan ibsurieg. This silggests that a bisic model of misunderstandings can be extended to the treatment of miscxicxpticns, by considering ako the buggy plans w alternatives. 
L. ARDissono \& D. SEgtero (1996). Using dynamic user models in the recognition of the plans of the user. User Modeling and User-Adapted Internction, 5(2):157-190.

L. Ardissono, A. LOMBardo, \& D. SEstero (1993). A flexible approach to cooperative response generation in information-seeking dialogues. In Proc. 91st Annunl Meetring $A C L$, pages 274-276, Columbus.

L. ARDissono, G. BOELLA, \& L. LesMo (1995a). A computational approach to spesch acts recognition. In Proc. 17th Cognitive Science Conference, pages 316-321, Pittshurgh.

L. ARDISSONO, G. BOELLA, \& D. SESTKRo (1995b). Recognition of preliminary sentences in dialogue interpretation. In Lecture Notes in Artificial Intelligence n. 992: Topics in Artificial Intelligence, pages 139-144. Springer Verlag, Berlin.

L. Ardissono, G. BokLla, \& L. LesMo (1996). Recognition of problem-solving plans in dialogue interpretation. In Proc. 5th Int. Conf. on User Modeling, pages 195-197, Kailua-Kona, Hawaii.

J.L. Austin (1962). How to Do Things with Words. Harvard University Press, Cambridge, Mass.

P. BARBONI \& D. BEstero (1997). Flexible response choice using problem-solving plans and rethorical relations. In Proc. 5th Congresso della Associazione Italiana per l'Tntelligenza Artificiale, Roma, Italy. to appear .

C. BAzZANELLA \& R. DaMIANo (1997). Il fraintendimento lnguistico nelle interazioni quotidiane: proposte di classificazione. Lingun e Stile, 32(3).

8. BLUM-KULKA \& E. WEIZMAN (1988). The inevitability of misunderstanding: discourse ambiguities. Text, $8(3): 219-241$.

M.E. BratMan (1990). What is intention? In P.R. Cohen, J. Morgan, \& M.E. Pollack, editors, Intentrons in communication, pages 15-32. MП Press.

P. BRown \& 3. C. Levinson (1987). Politeness: some universals on language usage. Cambridge University Press, Cambridge.

R.J. CALISTRI-YeH (1991). Utilizing user models to handle ambiguity and misconceptions in robust plan recognition. User Modeling and User-Adapted Internction, 1:289-322.

9. CARBERRY, Z. KAZI, \& L. LAYMBERT (1992). Modeling discourse, problem-solving, and domain goals incrementally in task-oriented dialogue. In Proc 9nI Int. Workshop on User Modeling, pages 192-201, Wadern.

8. CARBkRRY (1986). User models: the problem of disparity. In Proc. 11th COLING, pages 29-34, Bonn.

8. CARBerRy (1987). Pragmatic modeling: toward a robust natural language interface. Computational Intelligence, 3:117-136.

9. CaRBerRY (1990). Flan Recognition in Natumal Language Dialogue. MIT Press.

C. Castklfranchi \& D. Parisi (1980). Länguaggio, conoscenze e scopi. Il Mulino, Bologna.

C. Castelaranch (1992). No more cooperation, please! In search of the social structure of verbal interaction. In J. Slack. A. Ortony \& O. Stock, editors, Communication from an Artificial Intelligence perspective. Theoretical and Applied Issues. Springer Verlag, Berlin.

J. Chu-CarRoll \& \$. Carberry (1995a). Communication for conflict resolution in multiagent collaborative planning. In Proc. First Intemational Conference on Mulbiagent Systems, pages 49-56.

J. CHU-CARROLL \& S. CARBERRY (1995b). Response geteration in collaborative negotiation. In Proc. 99ri Annual Meetiong of the ACL, pages 136-143.

H.H. ClakK \& E.F. SChakper. (1989). Contributing to discourse. Cognitive Science, 13:259-294. 
P.R. COHEN \& H.J. Levesque (1990a). Intention is choice with commitment. Artificial Intelligence, 42:213-261.

P.R. COHEN \& H.J. Levesque (1990b). Rational interaction as the basis for communication. In P.R. Cohen, J. Morgan, \& M.E. Pollack, editors, Intentrons gin communication, pages 221-255. МПТ Press.

P.R. CoHeN \& H.J. Levesque (1991). Confirmation and joint action. In Froc. 19th IJCAI, pages 951-957, 5ydney.

P.R. Cohen, C.R. Pkrkault, \& J.F. Allen (1981). Beyond question answering. In W. Lehnert \& M. Ringle, editors, Strategies for Naturnl Language Processing, pages 245-274. Lawrence Erlbaum, Hillsdale NJ.

R. COHEN, K. SCHMDT, \& P. VAN BEEK (1994). A framework for soliciting clarification from users during plan recognition. In Proc. 4th Int. Conf. on User Modeling, pages 11-17, Hyannis, MA.

R. COHEN (1984). A computational theory of the function of clue words in argument understanding. In Proc. 10th Intemational Confenence on Computational Linguistics, pages 251-258, 5tanford, CA.

R. CoHEN (1987). Analyzing the structure of argumentative discourse. Computational Linguistics, 13:11-24.

M. DANIELI (1996). On the use of expectations for detecting and repairing human-machine misomunicaiton. In AAAI 1996 Workshop: Detectring, Repasing and Preventring Human-Machine Miscommunication, pages 87-93, Portland.

M. DAscal (1989). The relevance of misunderstanding. In M. Dascal, editor, Dialogue: an intendisciplinary approach, pages 441-459. Benjamin, Amsterdam - Philadelphia.

T. DE MaURo, F. MaNcin, M. VeDoveld, \& M. VoghrRA (1993). Lessico di Frequenza dell'Italiano Parato. ETASLIBRI.

B. Di EUGENIO \& L. LesMo (1987). Representation and interpretation of determiners in natural language. In Proc. 10th IJCAI, pages 648-653, Milano.

T. DONALDSON \& R. COHEN (1996). Addressing user misconceptions within a goaloriented, turn-taking framework in dialogue. In $A A A I 1996$ Workshop: Detecting, Repairing and Preventing Human-Machine Miscommunication, pages 98-101, Portland.

R.M. Eller. \& B. CARBerRY (1992). A meta-rule approach to flexible plan recognitionin dialogue. User Modeling and User-Adapted Interaction, 2:27-54.

R.M. ElLer. (1993). A Plan Recognition Architecture for Ml-Fomed Dialogue. Ph.D. thesis, University of Delaware.

GaviOLI \& MANSPIELD (1990). The PIXI corpom: bookshop encounters in English and Italian. CLUEB, Bologna, Italy.

B. GOODMAN (1985). Repairing reference identification failures by relaxation. In Proc. 29th Annurl Meetring ACL, pages 204-217, Chicago.

A. GoY \& L. LesMo (1997). Integrating lexical semantics and pragmatics: The case of italian communication verbs. In Proc. \&nd International Workshop on Computational Semantics, Tillburg, The Netherlands.

B.J. GROSZ \& C.L. BIDNER. (1986). Attention, intentions, and the structure of discourse. Computational Linguistics, 12:175-204.

B.J. Grosz \& C.L. SIDNkR. (1990). Plans for discourse. In P.R. Cohen, J. Morgan, \& M.E. Pollack, editors, Intentions in communication, pages 417-443. MПT Press.

B. Hansen, D. Novick, \& B. ButTon (1996). Prevention and repair of breackdowns in a simple task domain. In AAAI 1996 Workshop: Detecting, Repairing and Preventing Human-Machine Miscommunication, pages 5-12, Portland. 
P.A. HERMAN \& G. Hinst (1995). Collahorating on referring expressions. Computational Linguistics, 21(3):353-382.

J.C. HkRITAGE (1984). A change of-state token and aspects of its sequential placement. In J.M.Atkinson \& J.C.Heritage, editors, Structures of Socknl Action. Cambridge University Press.

G. HIR.sT, 马. MoRoy, P. HEEMAN, P. EDMONDs, \& D. HORTON (1994). Repairing conversational misunderstandings and non-understandings. Speech Communication, 15:213229.

J.R. HoBBs, M. \$TICKRL, D.E. APPELT, \& P. MARTIN (1993). Interpretation as abduction. Artificial Intelligence, 63:69-142.

P.W. JORDAN \& R.H. THOMASON (1996). Refining the categories of miscommunication. In AAAI 1996 Workshop: Detecting, Repairing and Preventing Human-Machine Miscommunication, pages 13-20, Portland.

H. KaUtz (1991). A formal theory of plan recognition and its implementation. In R.J. Brachman, editor, Rensoning About Plans, pages 69-125. Morgan Kaufinann Publishers.

L. LAMBeri \& B. CARBerRy (1991). A tripartite plan-based model of dialogue. In Proc. 29th Anrunl Megting of ACL, pages 47-54, Berkeley,CA.

L. LAMBerr (1993). Recognizing Complex Discourse Acts: A Tripartite Plan-Based Model of Dialogue. Ph.D. thesis, University of Delaware

L. LEsMo \& P. TORAsso (1985). Analysis of conjunctions in a rule based parser. In Proc. 29rd Annual Megting ACL, pages 180-187, Chicago.

B.C. Levinson (1981). The essential inadequacies of spesch act models of dialogue. In M. Parret, M. Sbisa, \& J. Verschueren, editors, Possibilities and Limitations of Pragmatics, pages 473-492. Benjamins, Amsterdam.

B.C. Levinson (1983). Pmgmatics. Cambridge University Press, Cambridge.

D. LITMAN \& J. Allen (1987). A plan recognition model for subdialogues in conversation. Cognitive Science, 11:163-200.

D. LitMan (1986). Linguistic coherence: a plan-based alternative. In Proc. 24th Annual Metring of the ACL, pages 215-223, New York, NY.

K.F. MOC OY (1986). The ROMPER system: responding to object-related misconceptions using perspective. In Proc. 94th Annual Metring of the ACL, pages 97-105, New York.

K.F. MOCoY (1988). Reasoning on a highlighted user model to respond to misconceptions. Computational Linguistics, 14(3):52-63.

3.W. MoROY \& G. HIRsT (1995). The repair of spesch act misunderstandings by abductive inferegce. Computational Linguzistics, 21(4):433-478.

M. MerRITT (1976). On question following question (in service encounters). Language in Society, V:315-357.

J.D. MOORE (1995). The role of plans in discourse geteration. In D. Everett \& 5.G. Thomason, editors, Discourse: Linguistic, Computational and Philosophical Perspectives.

L. Mor.genstern (1987). Knowledge preconditions for actions and plans. In Proc. 10th IJCAI, Milano.

K. NAGAO (1993). Abduction and dynamic preference in plan-based dialogue understanding. In Proc. 19th IJCAI, pages 1186-1192, Chambery.

A. NDLAYE \& A. JaYRESON (1996). Predictive role taking in dialog: gobal anticipation fesdback based on trasmutahility. In Proc. 5th Int. Conf. on User Modeling, pages 137-144, Kailua-Kona, Hawaii. 
D. Perlis \& K. Purang (1996). Conversational adequacy: Mistakes are the essence. In AAAI 1996 Workshop: Detecting, Repairing and Preventing Human-Machine Miscommunication, pages 47-56, Portland.

C.R. Perrault (1990). An application of default logic to speesch act theory. In P.R. Cohen, J. Morgan, \& M.E. Pollack, editors, Intentions in communication, pages 160185. MIT Press.

M.E. POLLACK (1987). Some requirements for a model of the plan-inference process in conversation. In Ronan Reilly, editor, Communication Faidure in Dialogue, pages 245256. North-Holland, Amsterdam.

M.E. POLACK (1990). Plans as complex mental attitudes. In P.R. Cohen, J. Morgan, \& M.E. Pollack, editors, Intentions in communication, pages 77-103. MIT Press.

A. RAO \& M.P. GEOR.GeFF (1991). Modeling rational agents within a bdi-architecture. In Proc. 2th Int. Conf. Principles of Knowledge Representation and Reasoning (KR:91), pages 473-484, Cambridge, MA.

H. BACKs, E.A. BCHEGLOFF, \& G. JEFPRR.sON (1974). A simplest systematics for the organization of turn-taking for conversation. Langunge, 50:696-735.

E.A. SCHEGLOFF (1972). Sequencing in conversational opening. In J.J. Gumperz \& D. Hymes, editors, Directions in Sociolinguistics. Rinehart and Winston, New York.

E.A. SCHEGLOFF (1987). Some sources of misunderstanding in talk-in-interaction. Lingutstics, 25(1):201-218.

E.A. BCHEGLOFF (1992). Repair after the next turn: The last structurally provided defense of intersubjectivity in conversation. American Joumal of Sociology, $7(5): 1295-1345$.

I.A. ВМптн \& P.R. CoHeN (1996). Toward a semantics for an agent communications language based on spesch-acts. In Proc. 14th Conf. AAAI, pages 24-31, Portland.

R. Вмттн \& D. HIPP (1994). Spoken Natural Language Dialogue Systems: a Practical Approach. Oxford University Press, New York.

D.R. Traum \& J.F. Allen (1994). Discourse obligations in dialogue processing. In Proc. 9ind Annual Meeting of ACL, pages 1-8, Las Cruces, New Mexico.

D.R. Traum \& E.A. Hinkrlman (1992). Conversation acts in task-oriented spoken dialogue. Computrtional Intelligence, $8(3): 575-599$.

P. VAN BEEK \& R. COHEN (1991). Resolving plan ambiguity for cooperative response generation. In Proc. 18th IJCAI, pages 938-944, 5ydney.

Z. VeNDLER. (1994). Understanding misunderstanding. In D. Jamieson, editor, Language, Mind and Att, pages 9-21. Kluwer, Dodrecht-Boston-London.

W. WaHLITkR. \& A. KOBSA (1989). User models in dialog systems. In A. Kobse \& W. Wahlster, editors, User Models on Dialog Systems, pages 4-34. Springer Verlag, Berlin.

E. WEIGAND (1997). Misunderstanding: the standard case. Joumal of Pragmatics. to appear .

D. ZARFFERER. (1977). Understanding misunderstanding: a proposal for an explanation of reading choices. Joumal of Pmgmatics, 329-346. 